CHANNEL CHANGES AT CROSS SECTIONS OF THE POWDER RIVER

BETWEEN MOORHEAD AND BROADUS, MONTANA, 1975-88

By John A. Moody and Robert H. Meade

U.S. GEOLOGICAL SURVEY

Open-File Report 89-407

Denver, Colorado

1990

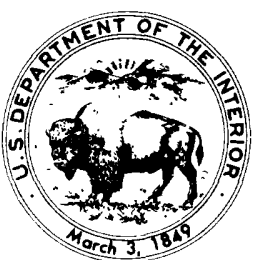


DEPARTMENT OF THE INTERIOR

MANUEL LUJAN, JR., Secretary

U.S. GEOLOGICAL SURVEY

Dallas L. Peck, Director

For additional information write to:

District Chief

U.S. Geological Survey

Box 25046, Mail Stop 415

Federal Center

Denver, CO 80225-0046
Copies of this report can be purchased from:

U.S. Geological Survey

Books and Open-File Reports Section Box 25425

Federal Center

Denver, CO 80225-0425 
Abstract-1-0- 1

Introduction--

Purpose and scope-1

Acknowledgments-- 3

Cross sections-1- 3

Survey method-1-- 8

Channel changes-- 11

Minimum riverbed elevation-1

Cross-section area of erosion and deposition-- 17

Summary-----

References cited--10 22

Cross-section data--

Description of cross section PR113- 25

Description of cross section PR116- 36

Description of cross section PR120- 45

Description of cross section PR122- 55

Description of cross section PR122A- 64

Description of cross section PR125

Description of cross section PR130- 84

Description of cross section PR136- 94

Description of cross section PR141- 104

Description of cross section PR141A- 112

Description of cross section PR147- 121

Description of cross section PR151- 131

Description of cross section PR156A- 142

Description of cross section PR156- 151

Description of cross section PR163- 161

Description of cross section PR167- 172

Description of cross section PR175- 182

Description of cross section PR180- 193

Description of cross section PR183- 203

Description of cross section PR191- 213

Description of cross section PR194- 224

Description of cross section PR200A-_. 233

Description of cross section PR206-_. 243

FIGURES

Figure 1. Map showing location of Power River drainage basin and the study reach-_.-. 2

2. Map showing location of 20 channel cross sections and tributaries of Powder River in the study reach--.--.--- 4

3-7. Graphs showing water-level elevation between April 1 and October 1 at Moorhead and at Broadus, for:

$\begin{array}{llr}\text { 3. } 1976-78 & \\ \text { 4. } 1979-81-1982-84 & 13 \\ \text { 6. } 1985-86 & \end{array}$ 
Figure 8. Map showing location of cross section PR113 in the Bradshaw Creek quadrangle-_... 26

9. Profiles of cross section PR113 from 1975 to 1980-_-n 27

10. Profiles of cross section PR113 from 1980 to 1986-.....- 28

11. Profiles of cross section PR113 from 1986 to 1988-.-.-.- 29

12. Map showing location of cross section PR116 in the Moorhead quadrangle-...- 37

13. Profiles of cross section PR116 from 1975 to $1980-\ldots$

14. Profiles of cross section PR116 from 1980 to 1986--.-- 39

15. Profiles of cross section PR116 from 1986 to 1988-.-.-.- 40

16. Map showing location of cross section PR 120 in the Moorhead quadrangle-.. 46

17. Profiles of cross section PR120 from 1975 to $1980-\ldots$

18. Profiles of cross section PR120 from 1980 to $1986-\ldots$

19. Profiles of cross section PR120 from 1986 to 1988-- 49

20. Map showing location of cross section PR122 in the Moorhead quadrangle-1 56

21. Profiles of cross section PR122 from 1975 to 1979-_-_-_-- 57

22. Profiles of cross section PR122 from 1979 to 1984-..-.-.- 58

23. Profiles of cross section PR122 from 1984 to $1987-\ldots$

24. Map showing location of cross section PR122A in the Moorhead quadrangle-- 65

25. Profiles of cross section PR122A from 1979 to 1985-1-1-- 66

26. Profiles of cross section PR122A from 1985 to 1988--.-.-- 67

27. Map showing location of cross section PR125 in the Moorhead quadrangle-1. 74

28. Profiles of cross section PR125 from 1975 to $1980-\cdots$

29. Profiles of cross section PR125 from 1980 to 1986-..- 76

30. Profiles of cross section PR125 from 1986 to 1988--- 77

31. Map showing location of cross section PR130 in the

32. Profiles of cross section PR130 from 1975 to 1980-1- 86

33. Profiles of cross section PR130 from 1980 to 1986--.-.-- 87

34. Profiles of cross section PR130 from 1986 to 1988- 88

35. Map showing location of cross section PR136 in the

Bloom Creek quadrangle-10 95

36. Profiles of cross section PR136 from 1975 to $1980-196$

37. Profiles of cross section PR136 from 1980 to 1986-_... 97

38. Profiles of cross section PR136 from 1986 to 1988-1...- 98

39. Map showing location of cross section PR141 in the

Bloom Creek quadrangle-105

40. Profiles of cross section PR141 from 1975 to 1980-106

41. Profiles of cross section PR141 from 1980 to 1984- 107

42. Map showing location of cross section PR141A in the Huckins School quadrangle-- 113

43. Profiles of cross section PR141A from 1979 to 1985- 114

44. Profiles of cross section PR141A from 1985 to 1988-.....- 115

45. Map showing location of cross section PR147 in the Huckins School quadrangle-- 122

46. Profiles of cross section PR147 from 1975 to 1980-123

47. Profiles of cross section PR147 from 1980 to 1986-...-..- 124

48. Profiles of cross section PR147 from 1986 to 1988-125 
Figure 49. Map showing location of cross section PR151 in the

Huckins School quadrangle-_... 132

50. Profiles of cross section PR151 from 1975 to $1980 \ldots$

51. Profiles of cross section PR151 from 1980 to 1986-.-.-.- 134

52. Profiles of cross section PR151 from 1986 to 1988-_..- 135

53. Map showing location of cross section PR156A in the

Huckins School quadrangle-_._. 143

54. Profiles of cross section PR156A from 1978 to 1984_....... 144

55. Profiles of cross section PR156A from 1984 to 1988--..- 145

56. Map showing location of cross section PR156 in the

Huckins School quadrangle-... 152

57. Profiles of cross section PR156 from 1977 to 1982

58. Profiles of cross section PR156 from 1982 to $1986 \ldots \ldots \ldots$

59. Profiles of cross section PR156 from 1986 to 1988-a. 155

60. Map showing location of cross section PR163 in the

Yarger Butte quadrangle_... 162

61. Profiles of cross section PR163 from 1977 to 1982

62. Profiles of cross section PR163 from 1982 to 1986_....... 164

63. Profiles of cross section PR163 from 1986 to 1988_....... 165

64. Map showing location of cross section PR 167 in the

Lonesome Peak quadrangle-_. 173

65. Profiles of cross section PR167 from 1977 to $1982 \ldots \ldots$

66. Profiles of cross section PR167 from 1982 to 1986_... 175

67. Profiles of cross section PR167 from 1986 to 1988-1...... 176

68. Map showing location of cross section PR175 in the

Lonesome Peak quadrangle-... 183

69. Profiles of cross section PR175 from 1977 to 1982- 184

70. Profiles of cross section PR175 from 1982 to 1986 _....... 185

71. Profiles of cross section PR175 from 1986 to 1988- 186

72. Map showing location of cross section PR180 in the

Lonesome Peak quadrangle-_. 194

73. Profiles of cross section PR180 from 1977 to 1982

74. Profiles of cross section PR180 from 1982 to 1986-_-_-_- 196

75. Profiles of cross section PR180 from 1986 to 1988- 197

76. Map showing location of cross section PR183 in the

Lonesome Peak quadrangle-_. 204

77. Profiles of cross section PR183 from 1977 to 1982-

78. Profiles of cross section PR183 from 1982 to 1986-_-_- 206

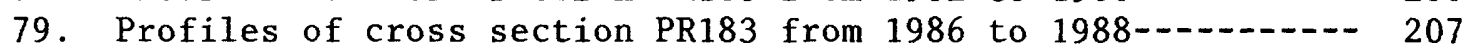

80. Map showing location of cross section PR191 in the

Eldon Mountain quadrangle-_. 214

81. Profiles of cross section PR191 from 1977 to $1982 \ldots$

82. Profiles of cross section PR191 from 1982 to 1986-_- 216

83. Profiles of cross section PR191 from 1986 to 1988-_._- 217

84. Map showing location of cross section PR194 in the

Eldon Mountain quadrangle-_... 225

85. Profiles of cross section PR194 from 1977 to 1982--.-- 226

86. Profiles of cross section PR194 from 1982 to 1986_....... 227

87. Profiles of cross section PR194 from 1986 to 1988--.-.-.- 228

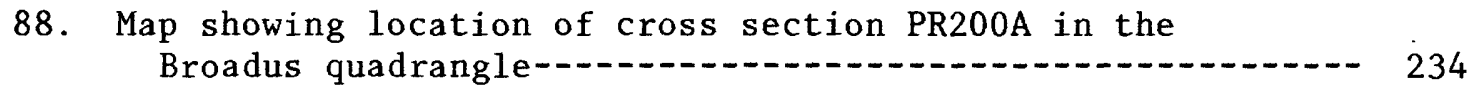

89. Profiles of cross section PR200A from 1978 to 1984- 235 
Pigure $90.9 \mathrm{ge}$

Figure 90. Profiles of cross section PR200A from 1984 to 1988-1- 236

91. Map showing location of cross section PR206 in the

Broadus quadrangle-...- 244

92. Profiles of cross section PR206 from 1977 to $1982-\cdots$

93. Profiles of cross section PR206 from 1982 to 1986-_- 246

94. Profiles of cross section PR206 from 1986 to 1988-_.-.-.- 247

Table 1. Types of reaches represented by cross sections of Powder

Page

River between Moorhead and Broadus, 1975-88--- 5

2. Vertical and geographical locations of cross sections of

Powder River between Moorhead and Broadus--.- 6

3. Vertical and geographical locations of cross sections of

Powder River between Moorhead and Broadus using the

universal transverse mercator (UTM) grid-_... 7

4. Errors in measurements of elevation and cross-section area---- 10

5. Minimum riverbed elevation at the end of the water year

for 1975-88 12

6. Changes in channel cross-section area between surveys--.-.-.- 18

7-29. Listings of horizontal stations and elevations for cross section:

7. PR113 31

8. PR116- 41

9. PR120- 51

10. PR122 61

11. PR122A- 69

12. PR125 79

13. PR130 89

14. PR136-1 99

15. PR141- 109

16. PR141A- 117

17. PR147-14 127

18. PR151-19 137

19. PR156A-1 147

20. PR156-157

21. PR163 167

22. PR167-10 177

23. PR175 187

24. PR180-199 199

25. PR183-1 209

26. PR191- 219

27. PR194-129

28. PR200A- 237

29. PR206 249 


\section{CONVERSION FACTORS}

Metric units (International System) in this report may be converted to inch-pound units by using the following conversions factors:

Multiply metric units

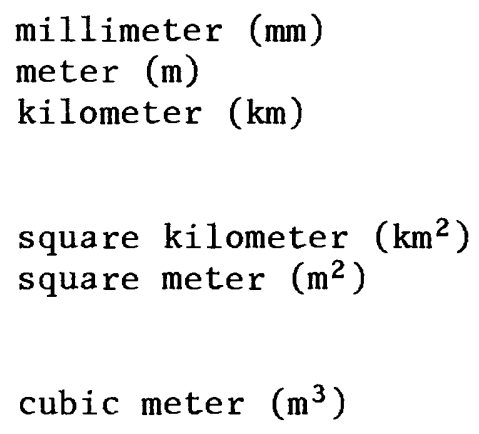

$B y$

To obtain inch-pound units

\section{Length}

0.03937

3.281

0.6214

Area

\author{
0.3861 \\ 10.76
}

Volume

35.31

\section{Flow}

35.31

35.31

Mass Flow

1.102

1.102

\section{Mass Yield}

2.854 inch

foot

mile

square mile

square foot

cubic foot

cubic foot per second cubic foot per year

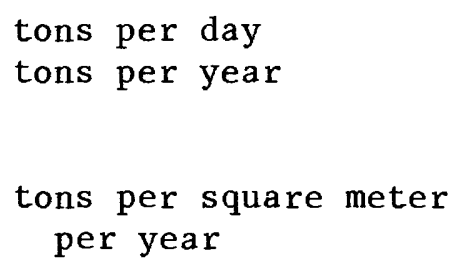

Sea level: In this report "sea level" refers to the National Geodetic Vertical Datum of 1929 (NGVD of 1929)--a geodetic datum derived from a general adjustment of the first-order level nets of both the United States and Canada, formerly called Sea Level Datum of 1929. 


\title{
CHANNEL CHANGES AT CROSS SECTIONS OF THE POWDER RIVER BETWEEN MOORHEAD AND BROADUS, MONTANA, 1975-88
}

By John A. Moody and Robert H. Meade

\begin{abstract}
Bed elevations were measured at 23 cross sections along a 95-kilometer reach of Powder River between Moorhead and Broadus, Montana. The measurements were repeated each year (except 1976, 1981, and 1983) after the peak runoff and include measurements made before and after the flood of May 1978. As a result of the flood, the average of the changes in the minimum riverbed elevation at 17 cross sections indicated a decrease in elevation of 0.15 meter, but this change was followed by a 0.17 -meter increase in the minimum riverbed elevation during the years after the flood (1978-88). There was essentially no net change in the minimum riverbed elevation for the study reach during 1977-88. The areas of erosion and deposition calculated for successive years at each cross section as a measure of channel change indicated that, during the 1978 flood, the average net channel change (deposition minus erosion) for the study reach was the erosion of 16 cubic meters of sediment per meter of channel length. After the 1978 flood, the channel changes were characterized by multiple areas of erosion, which became areas of deposition during the following year and areas of deposition which became areas of erosion. The average net channel change for the entire study reach during the years since the 1978 flood was the average deposition of about 1 cubic meter of sediment per meter of channel length. Thus, during 1977-88 there was a net erosion of about 15 cubic meters of sediment per meter of channel length within the study reach.
\end{abstract}

\section{INTRODUCTION}

Powder River flows east and northeast from the Bighorn Mountains in north-central Wyoming through northeastern Wyoming and southeastern Montana (fig. 1). At its confluence with the Yellowstone River, Powder River drains an area of $34,706 \mathrm{~km}^{2}$ and has an average discharge of about $500 \mathrm{million} \mathrm{m}^{3} / \mathrm{yr}$. Near the Wyoming-Montana State line (at the gaging station at Moorhead, Mont.), Powder River discharges an average of 2 to 3 million metric tons of suspended sediment per year, which equals a sediment yield of about 400 metric tons $/ \mathrm{km}^{2} / \mathrm{yr}$. The Powder River drainage basin includes areas of intensive production of oil and coal (Teapot Dome oil fields and part of the Gillette coal fields); but, at the present time, the primary use of river water in the basin is for irrigation. No dams or other large engineering structures obstruct the flow of Powder River or its principal tributaries. 


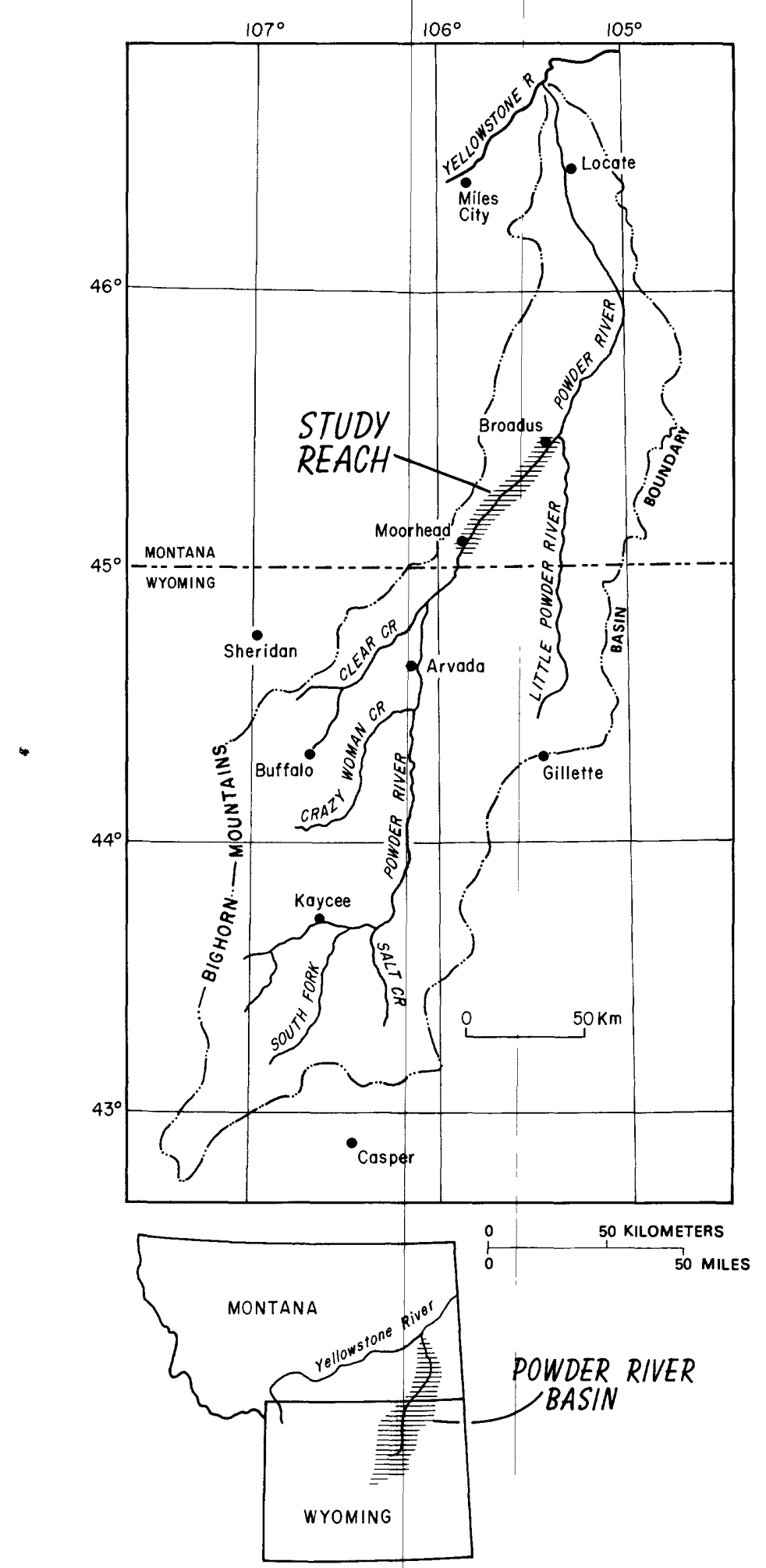

Figure 1.--Location of Powder River drainage basin and the study reach. 
In 1975, a program of measurements was begun to define long-term sediment budgets and channel changes in a reach of Powder River. This river was selected for several reasons. First, Powder River carries a large load of suspended sediment (Hembree and others, 1952), which is an indication of active sedimentary processes in the river and in its drainage basin. Second, Powder River is one of the few remaining rivers of its size in the region that is not controlled by a dam (in contrast with the Bighorn, Tongue, and Belle Fourche Rivers). Third, at the time the study began, energy-related developments were expected to have substantial effects on Powder River--including a new dam on the main stem. If a dam were built on the main stem, this study would provide before-and-after information that could define the effects of the dam. If a dam were not built (and no dam has been built as of 1988), this study would then provide a long-term record of channel changes under natural conditions.

\section{Purpose and Scope}

The purpose of this report is to present annual or biennial measurements of channel cross section and channel changes occurring under natural conditions in a river that carries a large sediment load. The reach selected for detailed study was the $95-\mathrm{km}$ reach of Powder River between Moorhead and Broadus, Mont. (fig. 1). Daily measurements of suspended sediment were made at the gaging stations at Moorhead and at Broadus, from which the annual loads of suspended sediment entering and leaving the study reach were computed (U.S. Geological Survey, 1976-89). The selected cross sections of the river were resurveyed annually or biennially, and channel changes were computed from the distances and elevations listed in this report.

\section{Acknowledgments}

We thank our friends and colleagues who helped us establish and relevel the cross sections: Ned Andrews in 1975; Eric Meade in 1977; Holly Martinson in 1979; Mike Fitzgerald and Carl Nordin in 1980; Mike Karlinger and Shirley Stewart (now Hamamoto) in 1982. James Fisher and Melvin White made an extra effort to provide needed data from the Montana District office. We thank the ranchers and landowners along Powder River for their hospitality and their continuing courtesy in allowing us repeated access to the river: Howard Best; Jim Bowers; John Daily; Slim Frandsen; Cliff Franklin; George and Hugh Fulton; Hubert, Mary, Bill, and Glenn Gay; Jean Hough; Bunk and Phil Huckins; Ray Moore; Randall Perry; Doug, Lucille, and Craig Randall; Frank and Frances Rice; Charles Russell; John, Diane, and Shirley Stuver; Roy Swope; Charles Whiton; and J.L. Wilson.

\section{CROSS SECTIONS}

Ten cross sections (identified as PR113 to PR151) were established during 1975 to monitor channel changes and sediment storage (fig. 2). The section number, for example 113 or 151 (tables 1, 2, and 3), is the river distance in kilometers downstream from the mouth of Crazy Woman Creek near Arvada, Wyo., as shown in the U.S. Geological Survey river-survey maps of 1946 (Martinson 


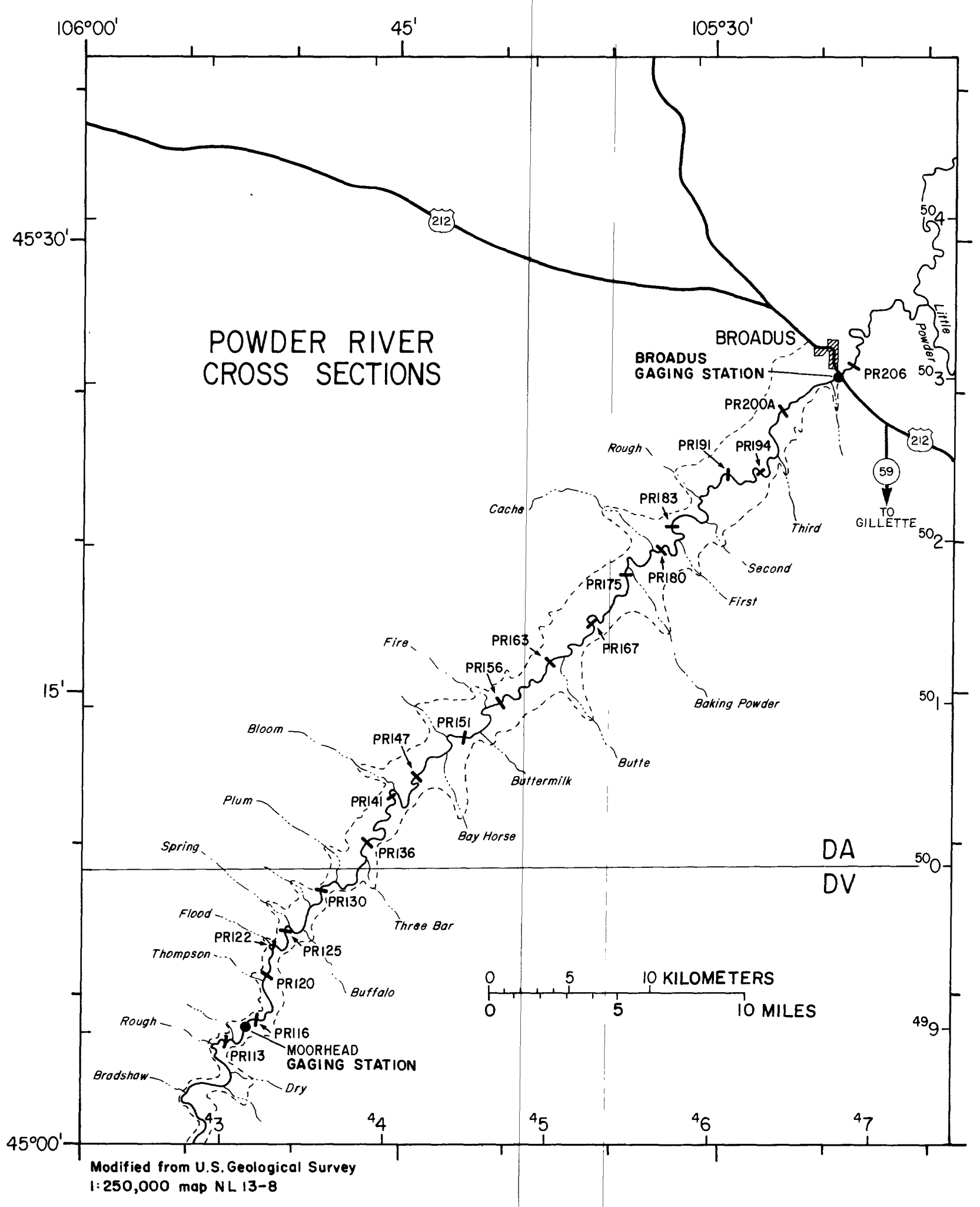

Figure 2.--Location of 20 channel cross sections and the tributaries of Powder River in the study reach. The dashed line indicates the approximate boundary of the valley. Longitude and latitude tick marks are on the outside of the border, and tick marks for the universal transverse mercator (UTM) grid are on the inside of the border. DA and DV are the identification letters for the 100,000-meter-square grid (table 3). PR113 is the cross section identification. 


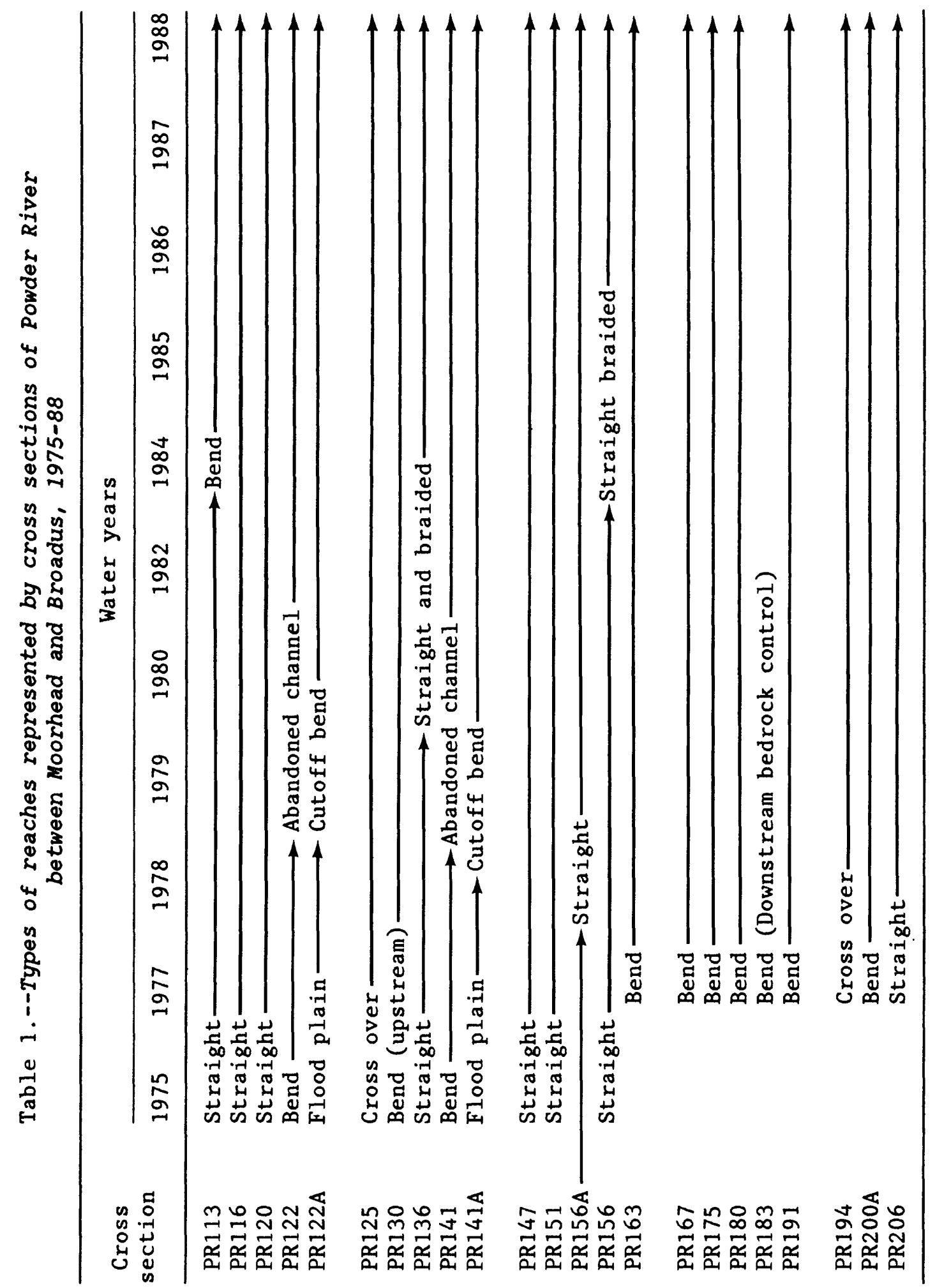




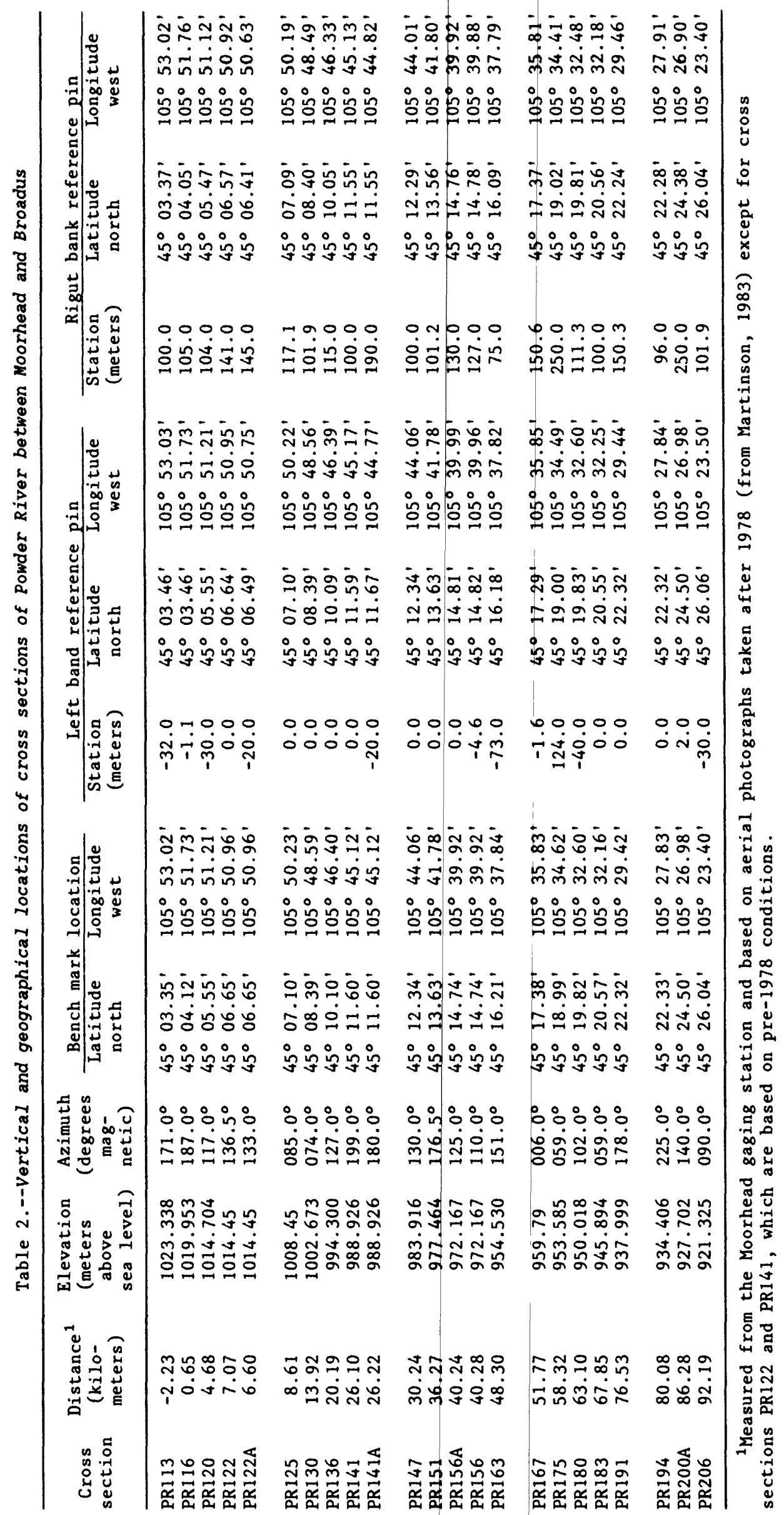




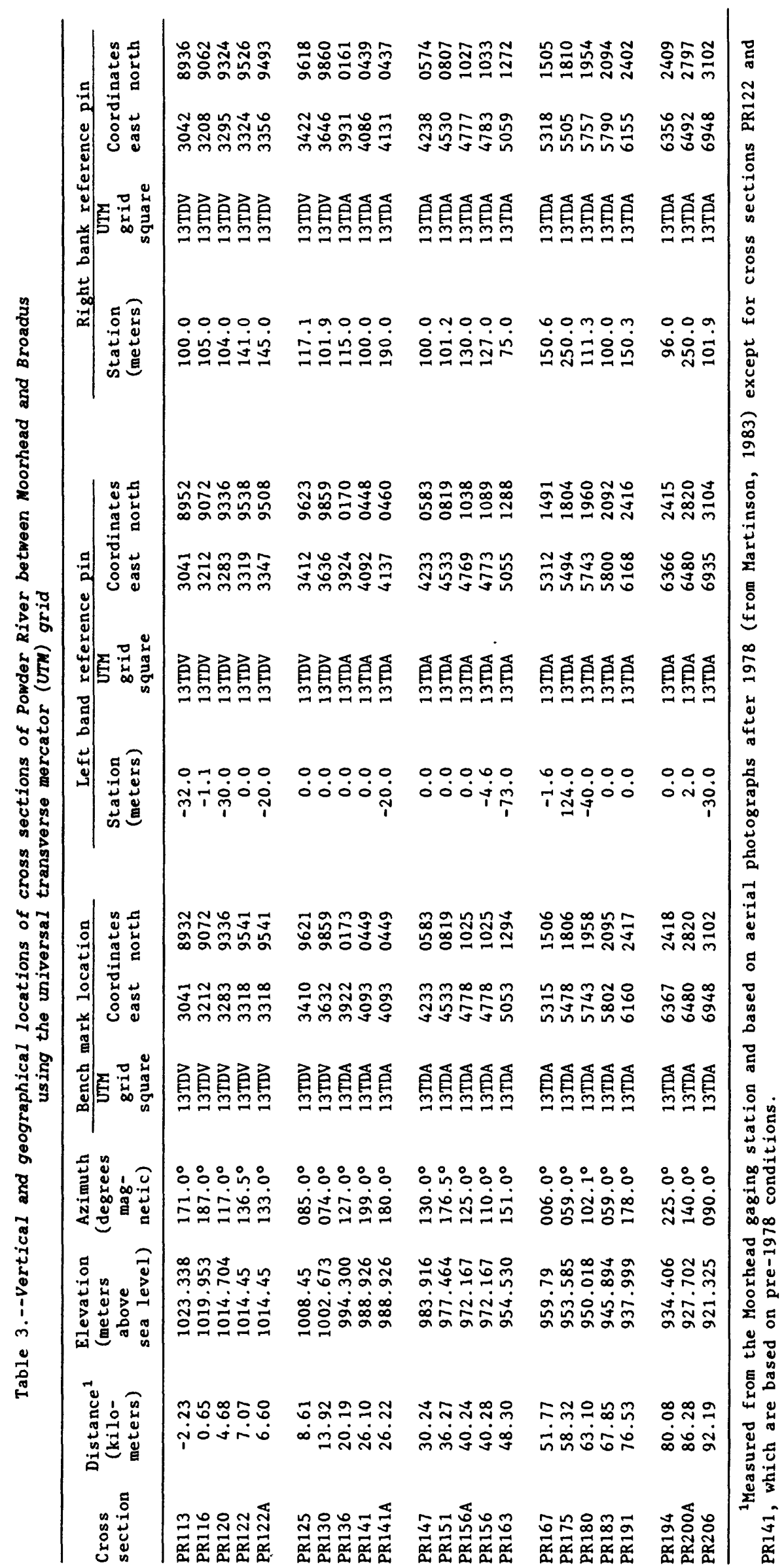


and Meade, 1983). The first cross section was located about $3 \mathrm{~km}$ upstream from the Moorhead gaging station, and the remaining sections were located 2 to $6 \mathrm{~km}$ apart for $39 \mathrm{~km}$ (river distance) downstream from the Moorhead gaging station (tables 1, 2, and 3). Ten additional cross sections (PR156 to PR206) were established during 1977 from $43 \mathrm{~km}$ to about $95 \mathrm{~km}$ (river distance) downstream from the Moorhead gaging station. These sections were spaced farther apart ( 3 to $8 \mathrm{~km}$ ), and the last section was about $1 \mathrm{~km}$ downstream from the U.S. Highway 212 bridge, where the Broadus gaging station is located.

After an intense rainfall, a major flood occurred between May 17 and 23, 1978 (fig. 3), producing peak discharges of water and suspended sediment at Moorhead of $800 \mathrm{~m}^{3} / \mathrm{s}$ and 2 million metric tons per day. Two cross sections (PR122 and PR141) located on bends were cut off during the flood, and new sections (PR122A and PR141A) were established in 1979 on the new cutoffs. These cutoffs shortened the total river distance of the study reach by about $2.7 \mathrm{~km}$. All reference marks for section PR200 were completely destroyed in the flood, and a new section (PR200A) was established in approximately the same location in fall 1978. One section (PR156) was believed destroyed during the flood, and a new section (PR156A) was established about $40 \mathrm{~m}$ upstream from the location of the original section. Later, however, the metal reference pins were found using a metal detector, and both sections have been resurveyed regularly since 1978 .

There now are 23 cross sections along Powder River spanning a total river distance of $92.4 \mathrm{~km}$. Eight cross sections represent straight reaches, eleven sections represent bends, two sections are at thalweg crossovers, and two sections represent abandoned channels. Some cross sections have evolved with time, and these changes also are listed in table 1. Twenty-one cross sections are located on an active part of the river, and two cross sections are located along meander cutoffs.

In the "Cross-section data" section at the back of this report, the township location, U.S. Geological Survey quadrangle map, access, landowners, and from whom to obtain permission for access are listed for each cross section. The horizontal location and average elevation (above sea level) for the horizontal reference pins also are listed. The location of the section is shown on part of a U.S. Geological Survey $7 \frac{1}{2}$-minute topographical map $(1: 24,000)$, and the bench mark location is shown on a large-scale map $(1: 20$, $1: 500$, or $1: 1000$ ), which includes surrounding local landmarks. All azimuths are reported in degrees magnetic. Profiles of each cross section have been plotted in the "Cross-section data" section in pairs of successive surveys to indicate the areas of erosion and deposition. Following the section profiles is a listing of the stations and elevations for each profile.

\section{SURVEY METHOD}

At each cross section a steel reference pin (about $1.2 \mathrm{~m}$ long and $12 \mathrm{~mm}$ in diameter) was driven almost flush with the ground to mark the horizontal zero point (station 0.0) on the left bank, and a second pin was driven on the right bank to mark a convenient horizontal reference distance such as 100 , 120 , or $150 \mathrm{~m}$ (see tables 2 and 3 for the geographical location of each reference pin). Two additional steel tieback pins were driven into the ground on the line of section but behind the reference pins; about 100 to $150 \mathrm{~mm}$ remained above ground to hold the cable clamps used to stretch a steel cable 


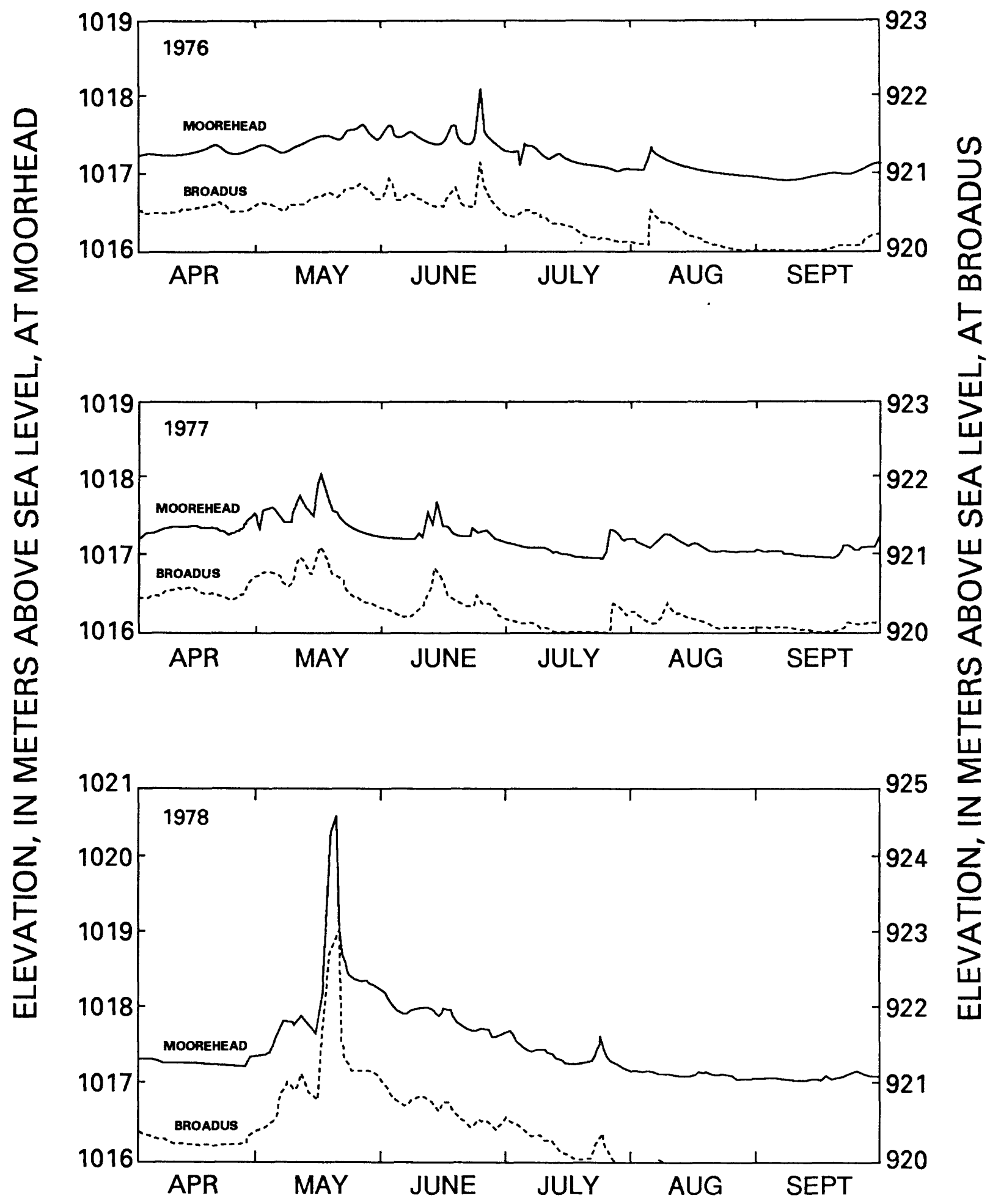

Figure 3.--Water-level elevation between April 1 and October 1 at Moorhead and at Broadus 1976-78. 
(150 $\mathrm{m}$ long and $3 \mathrm{~mm}$ in diameter) across the river. The cable was marked every meter by a brass bead, and horizontal distances were measured to $0.1 \mathrm{~m}$ by estimating between beads.

A local vertical bench mark was installed at each cross section, and the elevation above sea level (tables 2 and 3 ) was determined by leveling from U.S. Coast and Geodetic Survey bench marks shown on the U.S. Geological Survey topographical maps. The local bench mark consisted of a brass circular plate set in reinforced concrete flush with the ground. The cross-section identification number (for example, PR113) is stamped into the plate, and an arrow in the center of the brass plate points to station 0.0. The elevations of the horizontal reference and tieback pins also were determined as secondary vertical references. Vertical elevations were determined by using a tripod and level (Wild NA2 ${ }^{1}$ ) that had an automatic leveling compensator and a metric rod with markings at every $0.01 \mathrm{~m}$. Cross-section elevations were not always measured at the same horizontal locations or stations each time, nor at regularly spaced distances, but rather at high, low, and inflection points with maximum spacings of 3.0 to $5.0 \mathrm{~m}$.

The error in the elevation measurements (table 4) was estimated by repeating the measurements at three cross sections (PR141A, PR163, and PR206) a second time. The second measurement was made on a different day and in the opposite direction from the first measurement to decrease the possibility of the rodman recognizing the location where the rod was placed the first time.

Table 4.--Errors in measurements of elevation and cross-section area. Duplicate leveling surveys were made several days apart at each cross section

\begin{tabular}{|c|c|c|c|}
\hline \multirow{2}{*}{$\begin{array}{l}\text { Cross } \\
\text { section }\end{array}$} & \multirow{2}{*}{$\begin{array}{l}\text { Length } \\
\text { (meters) }\end{array}$} & \multicolumn{2}{|c|}{ Elevation error--absolute difference } \\
\hline & & $\begin{array}{c}\text { Average } \\
\text { (millimeters) }\end{array}$ & $\begin{array}{c}\text { Extreme } \\
\text { (millimeters) }\end{array}$ \\
\hline PR141A & 150 & 14 & 60 \\
\hline PR163 & 148 & 15 & 100 \\
\hline PR206 & 130 & 19 & 120 \\
\hline \multicolumn{4}{|c|}{ Cross-section area error per 100 horizontal meters } \\
\hline $\begin{array}{l}\text { Cross } \\
\text { section }\end{array}$ & $\begin{array}{c}\text { Erosion } \\
\text { (square meters) }\end{array}$ & $\begin{array}{c}\text { Deposition } \\
\text { (square meters) }\end{array}$ & $\begin{array}{c}\text { Net } \\
\text { (square meters) }\end{array}$ \\
\hline PR141A & 0.62 & 0.81 & 0.20 \\
\hline PR163 & 0.59 & 1.11 & 0.51 \\
\hline PR206 & 1.18 & 0.99 & 0.18 \\
\hline
\end{tabular}

${ }^{1}$ The use of brand, trade, or firm names in this report is for identification purposes only and does not constitute endormement by the U.S. Geological Survey. 
One source of error in elevation results from errors in estimating the horizontal distance; this error is largest where slopes are greatest, such as at bank edges. A second source of error is caused by not repeating the measurements at the same horizontal station each time the cross section is resurveyed and by assuming a linear interpolation between stations. The duplicate surveys for the three sections were plotted on graph paper, and $1 \mathrm{~mm}$ equaled $20 \mathrm{~mm}$ of real elevation. The absolute differences in elevation were read from the graph every $2.5 \mathrm{~m}$ to the nearest $10 \mathrm{~mm}$, and an average value was computed (table 4). The average absolute difference, or error, in elevation for all three sections weighted by the length of the section was $16 \mathrm{~mm}$. The extreme values of the absolute difference ranged from 60 to $120 \mathrm{~mm}$ and usually occurred where the ground slope was large--riverbanks and sandbars.

\section{CHANNEL CHANGES}

Two measurements of channel change have been used for this report: Changes in minimum riverbed elevation, and (2) changes in cross-section area of erosion and deposition. Changes in channel dimensions, island area, meander wavelength, and amplitude have been described for Powder River by Martinson (1983).

\section{Minimum Riverbed Elevation}

The minimum riverbed elevations for each cross section are listed in table 5. The largest decrease was $70 \mathrm{~cm}$ at section PR180 after the 1978 flood. The largest increase (excluding abandoned channels) was $33 \mathrm{~cm}$ at section PR183 in 1979, the year after the flood. The changes between any two surveys or for the entire survey period seem to depend more on the type of cross section (table 1) than on the nature of the river stage (figs. 3, 4, 5, 6 , and 7) between surveys. The exception was the 1978 flood year which resulted in erosion of the riverbed at 14 of 19 cross sections.

Most cross sections (PR113, PR116, PR125, PR151, PR156A, PR156, PR163, PR167, PR180, PR183, PR191, PR194, and PR200A) did not have a significant (at the 95-percent confidence level) relation between time and minimum riverbed elevation. One cross section (PR120) had a slight but significant ( $\mathrm{p}$-value = 0.0 percent) decrease in the minimum riverbed elevation of approximately $0.06 \mathrm{~m}$ during 13 years, and at PR206 the minimum riverbed elevation decreased $1.30 \mathrm{~m}$ from 1977-84 and then increased $0.56 \mathrm{~m}$ from 1984-88. Six cross sections (PR122, PR122A, PR130, PR136, PR147, and PR175) have had a significant increase in the minimum riverbed elevation since the May 1978 flood.

The average of the changes in the minimum riverbed elevation for 17 cross sections (PR113, PR116, PR120, PR125, PR130, PR136, PR147, PR151, PR156, PR163, PR167, PR175, PR180, PR183, PR191, PR194, and PR206) was computed as a measure of the channel change for the entire study reach. There was an average decrease of $0.2 \mathrm{~m}$ as a result of the May $1978 \mathrm{flood}$, but this was followed by a $0.2 \mathrm{~m}$ average increase in the minimum riverbed elevation during the 10 years after the flood. Therefore, the change in minimum riverbed elevation for the study reach over the period of this study was essentially zero. 


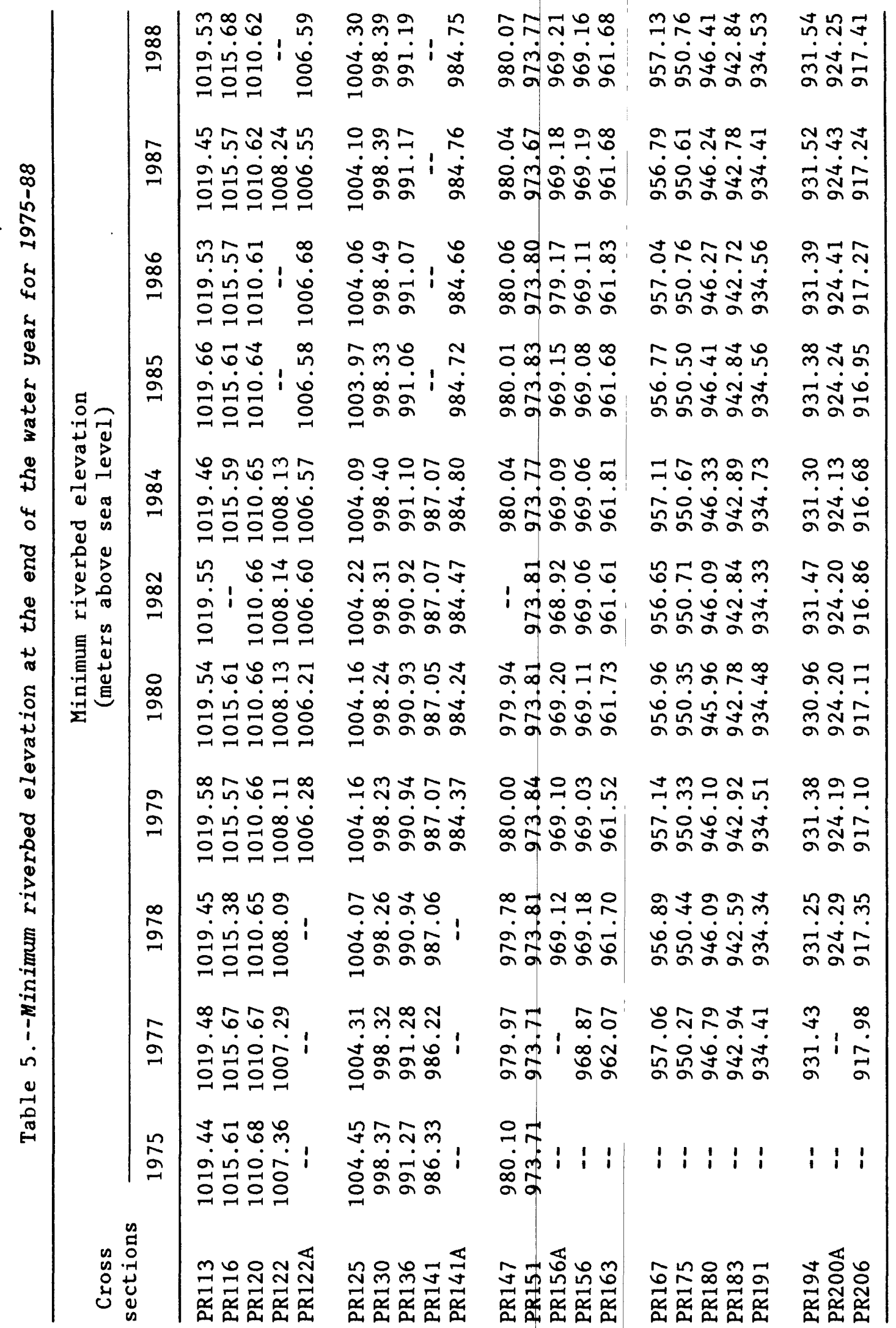




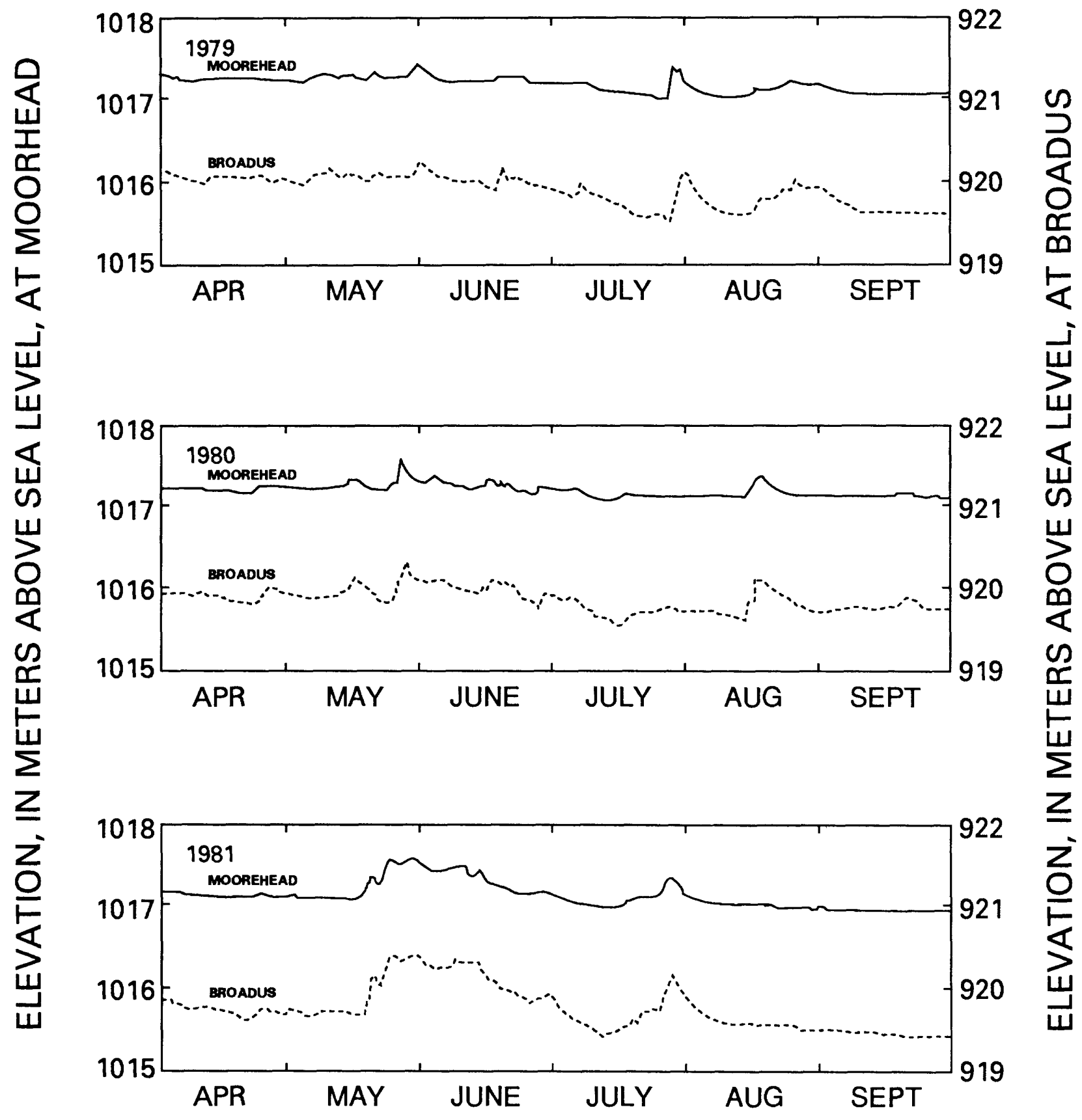

Figure 4.--Water-level elevation between April 1 and October 1 at Moorhead and at Broadus for 1979-81. 


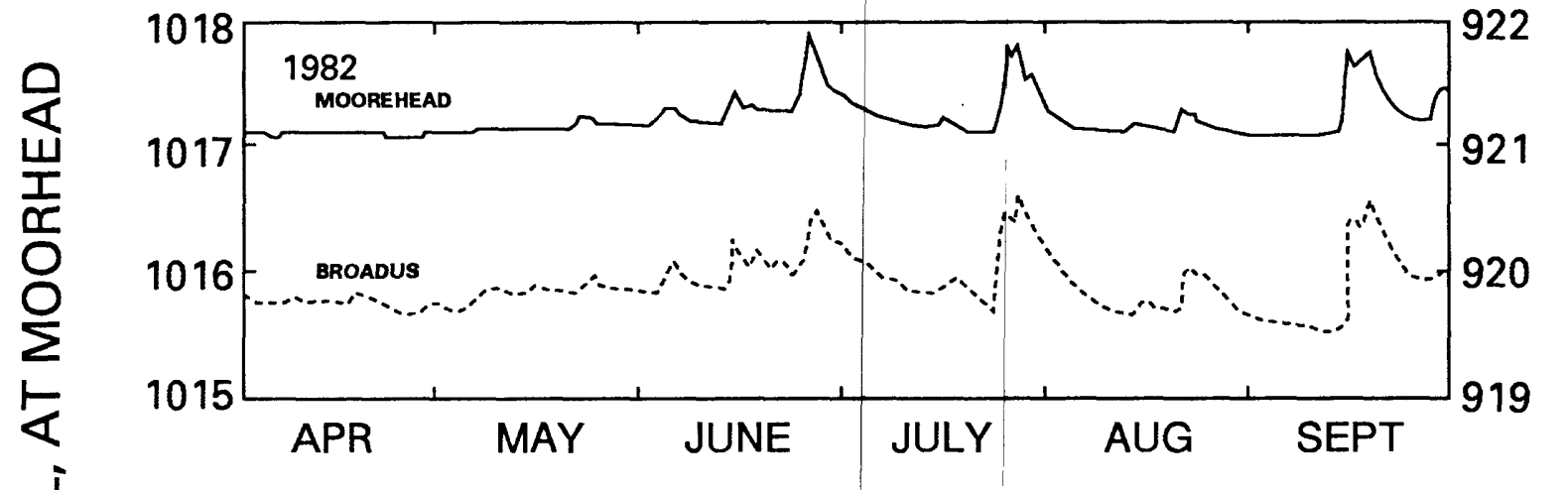

0
2
0
8
0
0
0
$\frac{1}{4}$

ए

4

$山$

$\sum_{0}^{4}$

w

م

荘

핀
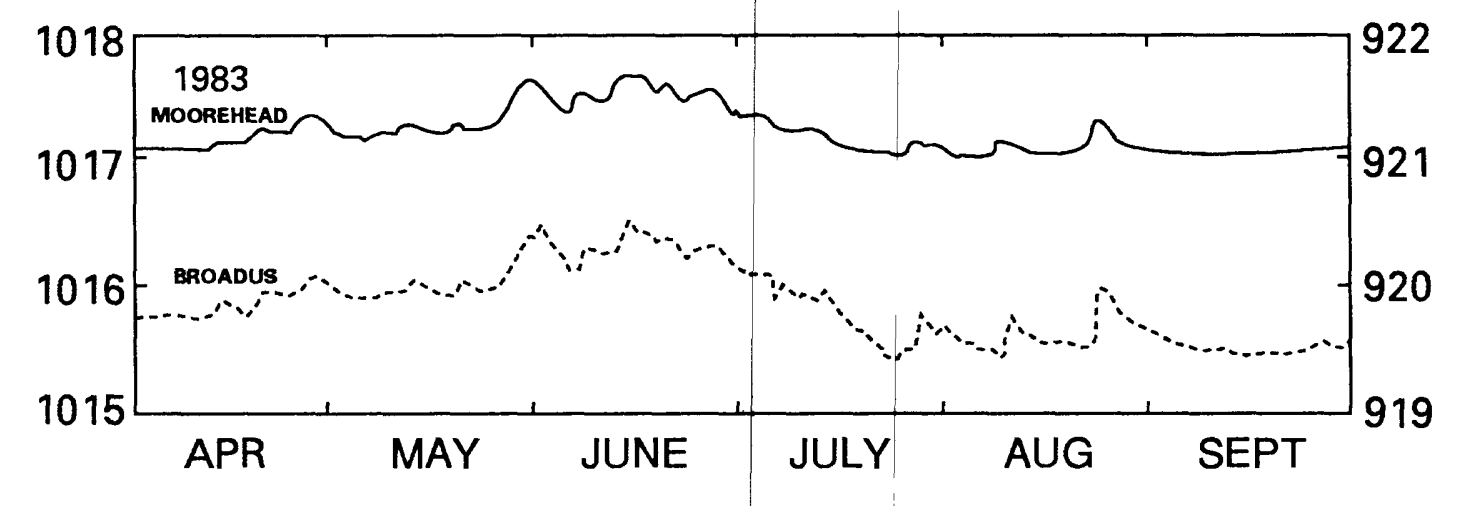

$\underline{Z}$

走

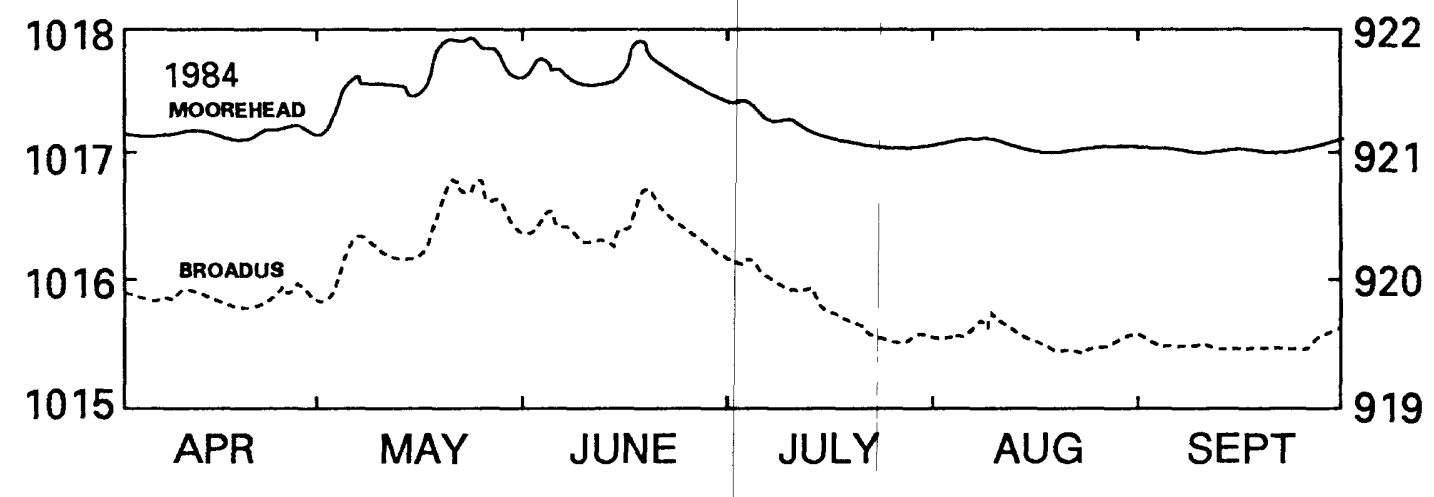

922

921

920

تं

W

$\varangle$

山

일

N

ש

$\stackrel{W}{\Sigma}$

Figure 5.--Water-level elevation between April 1 and October 1 at Moorhead and at Broadus for 1982-84. 


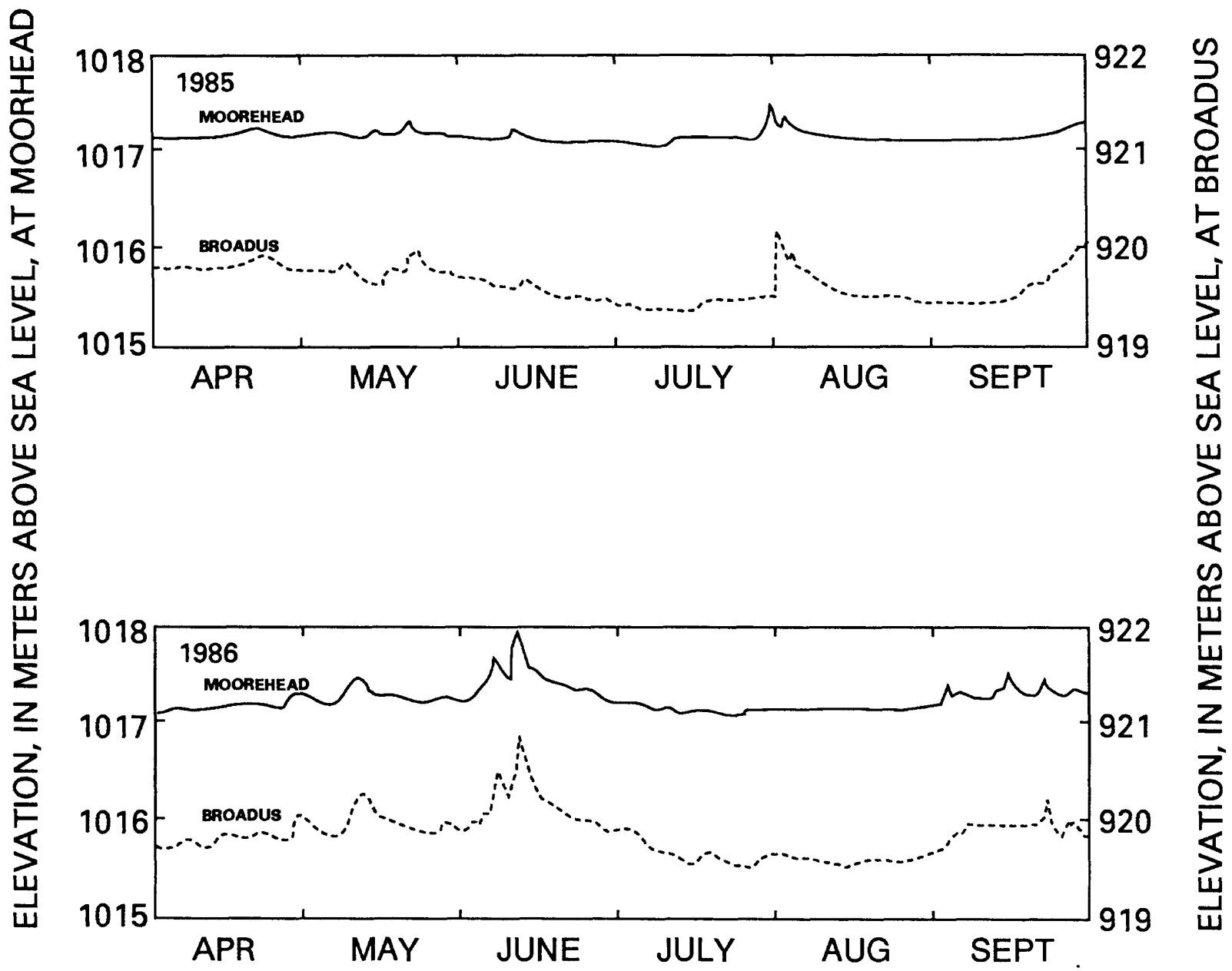

Figure 6.--Water-level elevation between April 1 and October 1 at Moorhead and at Broadus for 1985-86. 


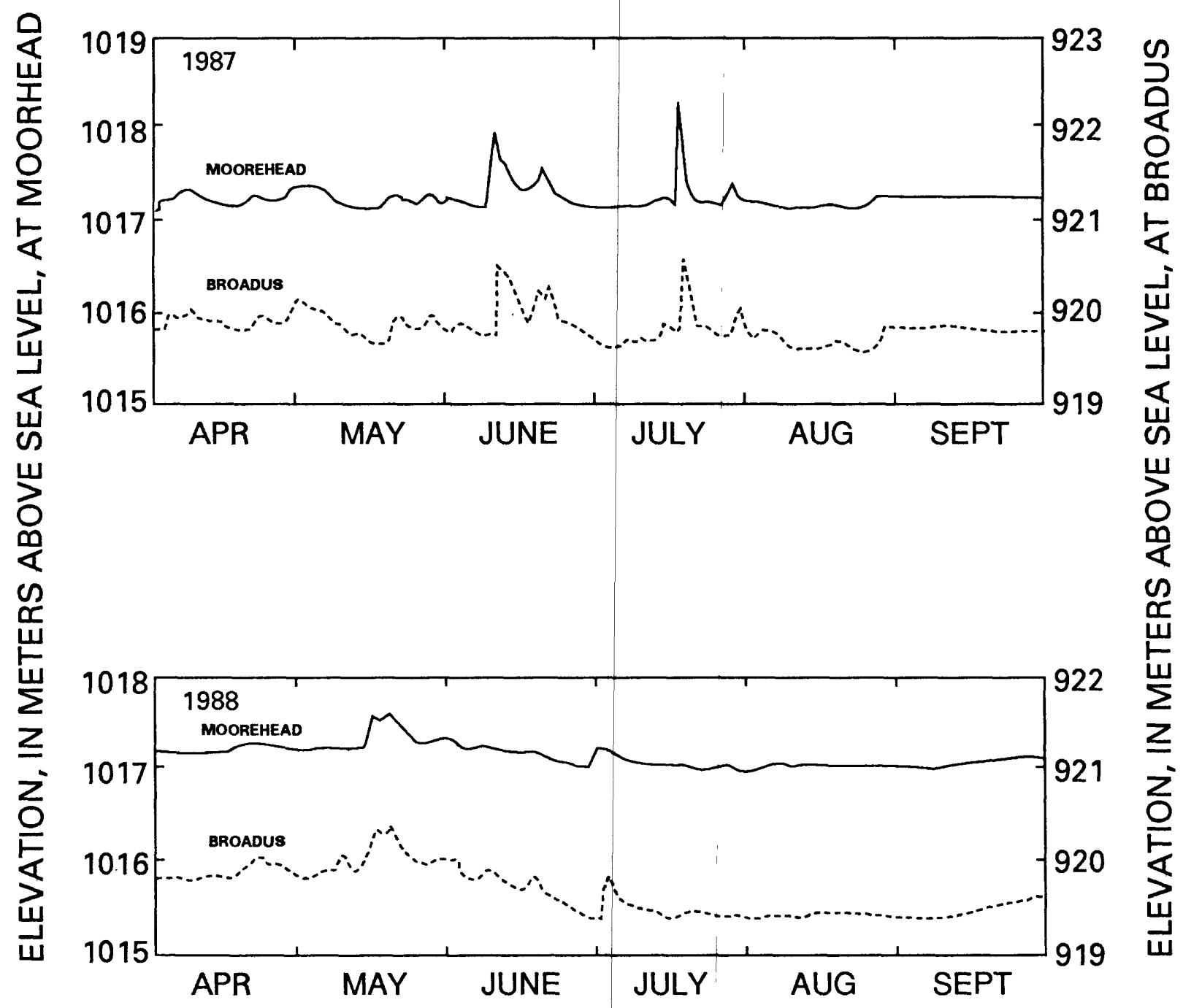

Figure 7.--Water-level elevation between April 1 and October 1 at Moorhead and at Broadus for 1987-88. 


\section{Cross-Section Area of Erosion and Deposition}

The cross-section area of erosion and deposition was computed for successive surveys by linearly interpolating the elevations between horizontal locations at every $0.1 \mathrm{~m}$ for both surveys and then summing separately the positive (deposition) and negative (erosion) differences in elevation. This method approximated the area as a sum of narrow $(0.1 \mathrm{~m}$ wide) rectangular strips. The error in this approximation was estimated by calculating the exact areas as sums of triangles and by subtracting the areas computed using the rectangular approximation for one cross section (PR147, 1984 to 1985). The difference was $0.01 \mathrm{~m}^{2}$ for area of deposition and $0.11 \mathrm{~m}^{2}$ for area of erosion. This was a 1.4-percent difference for the total area of channel change. The error due to the field leveling was determined by computing the area of erosion and deposition for the duplicate surveys of cross sections PR141A, PR163, and PR206 (table 4). The average error (for the six values of erosion and deposition) was $0.88 \mathrm{~m}^{2}$ per 100 horizontal meters of section.

The changes in cross-section area of erosion and deposition for most sections were greatest during the 1978 water year (table 6). At PR163, about $141 \mathrm{~m}^{2}$ of sediment was eroded from the left bank (about $65 \mathrm{~m}$ of bank erosion), and about $87 \mathrm{~m}^{2}$ of sediment was deposited on the right bank during the 1978 flood. About $143 \mathrm{~m}^{2}$ of sediment was deposited at cross section PR175 as the channel shifted approximately $170 \mathrm{~m}$ to the right, but the area of erosion could not be measured because the 1977 survey stopped about $30 \mathrm{~m}$ to the left of the future channel. Sections PR122 and PR141 had large quantities of deposition $\left(41 \mathrm{~m}^{2}\right.$ and $\left.72 \mathrm{~m}^{2}\right)$ as a result of cutoffs at each section during the flood; the new sections PR122A and PR141A are active sites of erosion and deposition as the river creates a new channel.

Since 1978, the changes within the channel (excluding bank erosion) at many cross sections (PR130, PR136, PR147, PR156A, PR156, PR167, PR183, and PR194) were characterized by multiple areas of erosion that became areas of deposition during the following years, and vice versa. Therefore, the total channel change (deposition plus erosion, listed in table 6) often is much larger than the net channel change (deposition minus erosion, listed in table 6). After the 1978 flood, section PR130 had about 20 times as much total channel change as net channel change, and sections PR147 and PR194 had 11 and 45 times as much total channel change as net channel change. The total channel change for the eight sections during the years after the 1978 flood was about 14 times the net channel change.

At several cross sections (PR180, PR200A, and PR206) a steady and substantial erosion has been occurring at a rate ranging from 2 to $13 \mathrm{~m}^{2}$ of riverbank as the river adjusts to changes downstream that occurred during the 1978 flood. Some sections (PR116, PR120, PR147, PR151, and PR183) have been very stable since the 1978 flood and have areas of erosion and deposition not substantially different from the estimated error in cross-section area. 


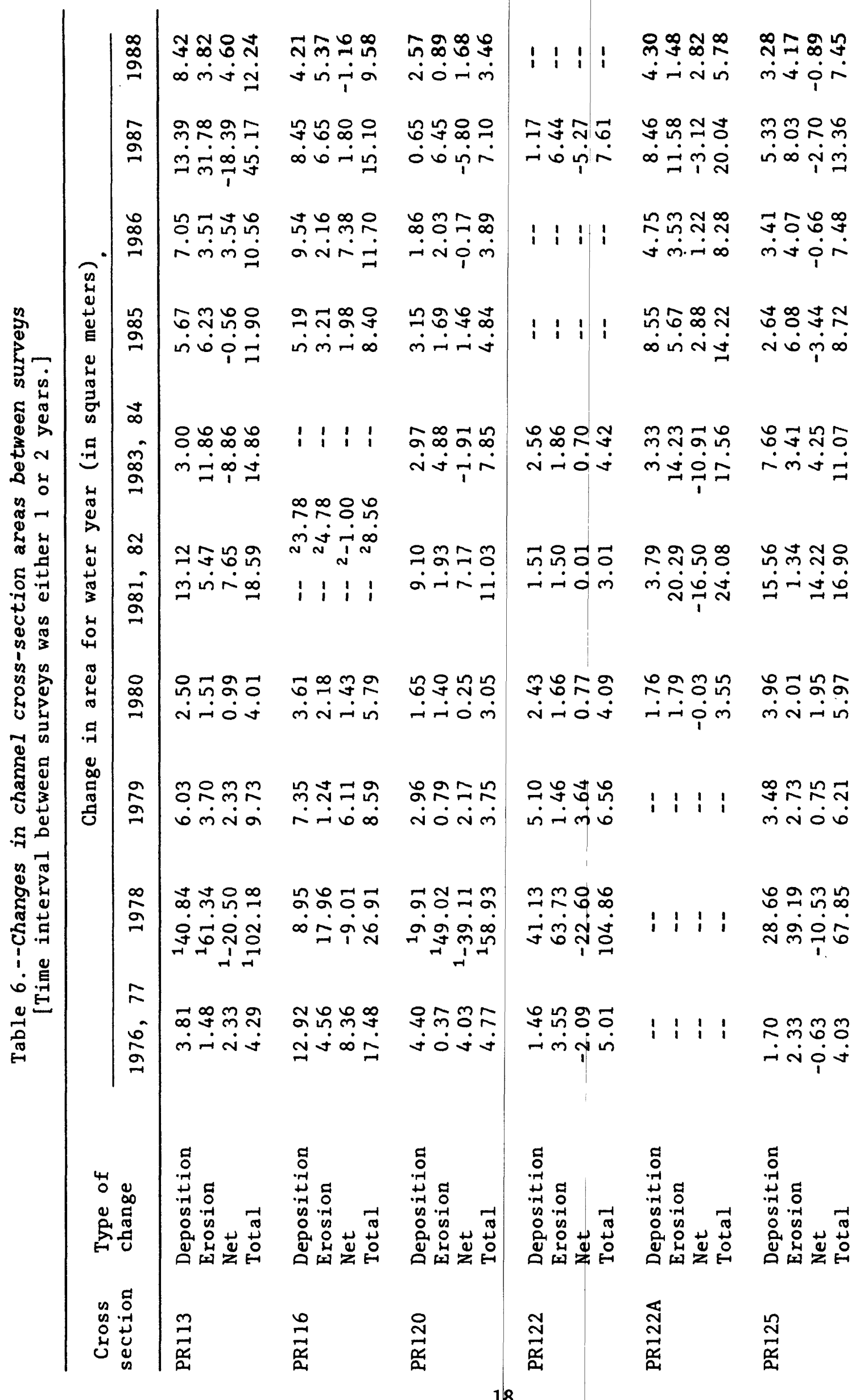




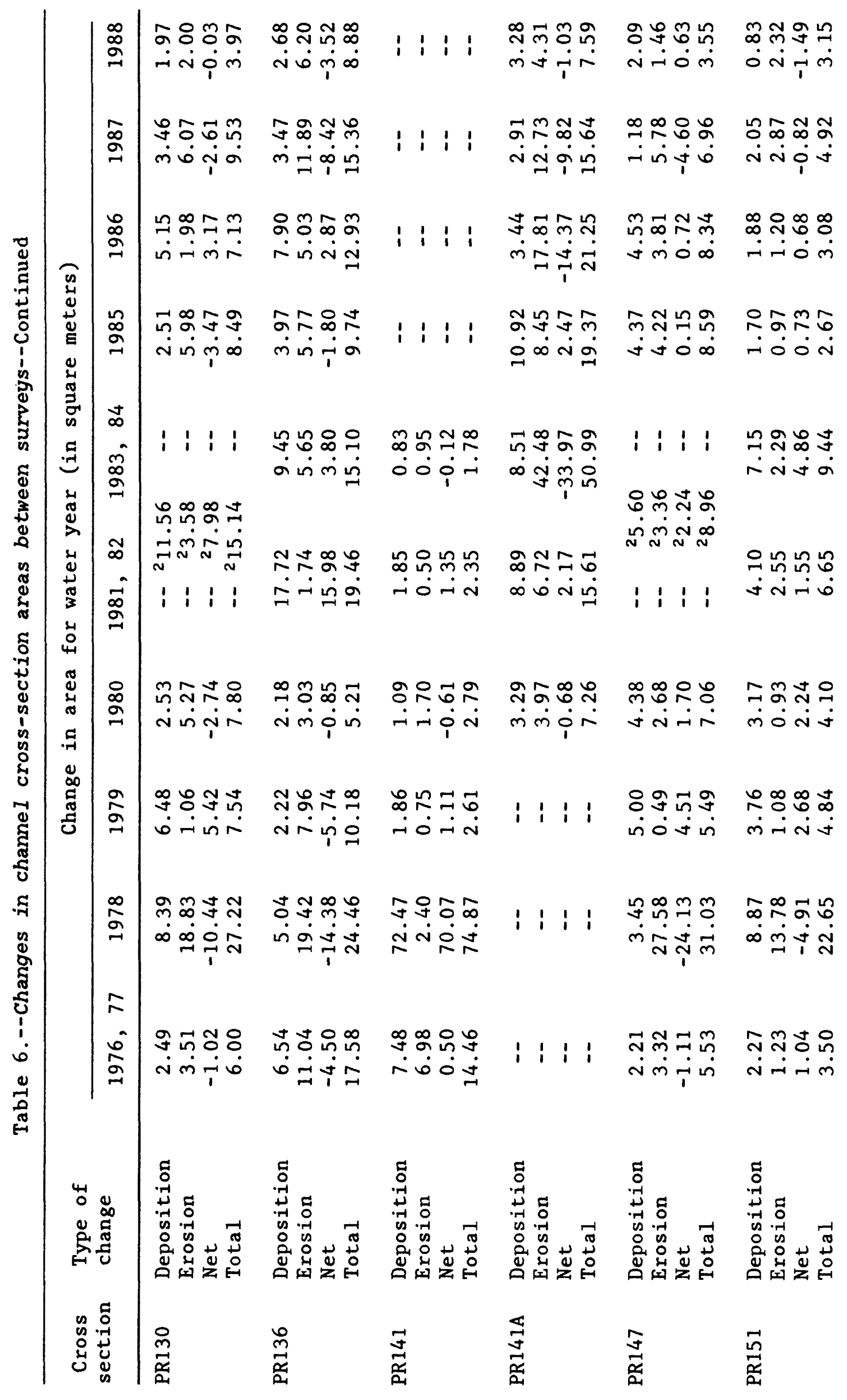




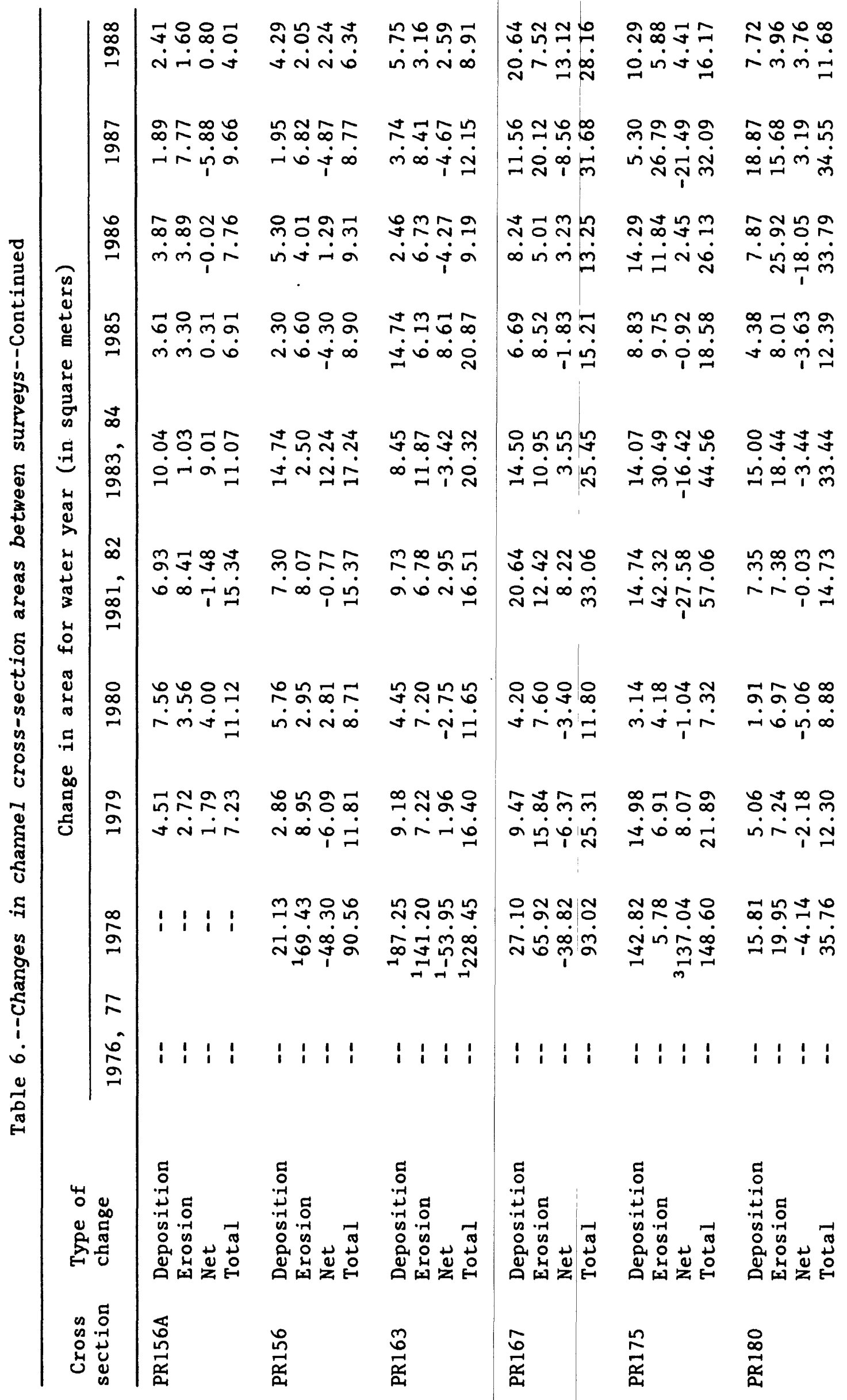




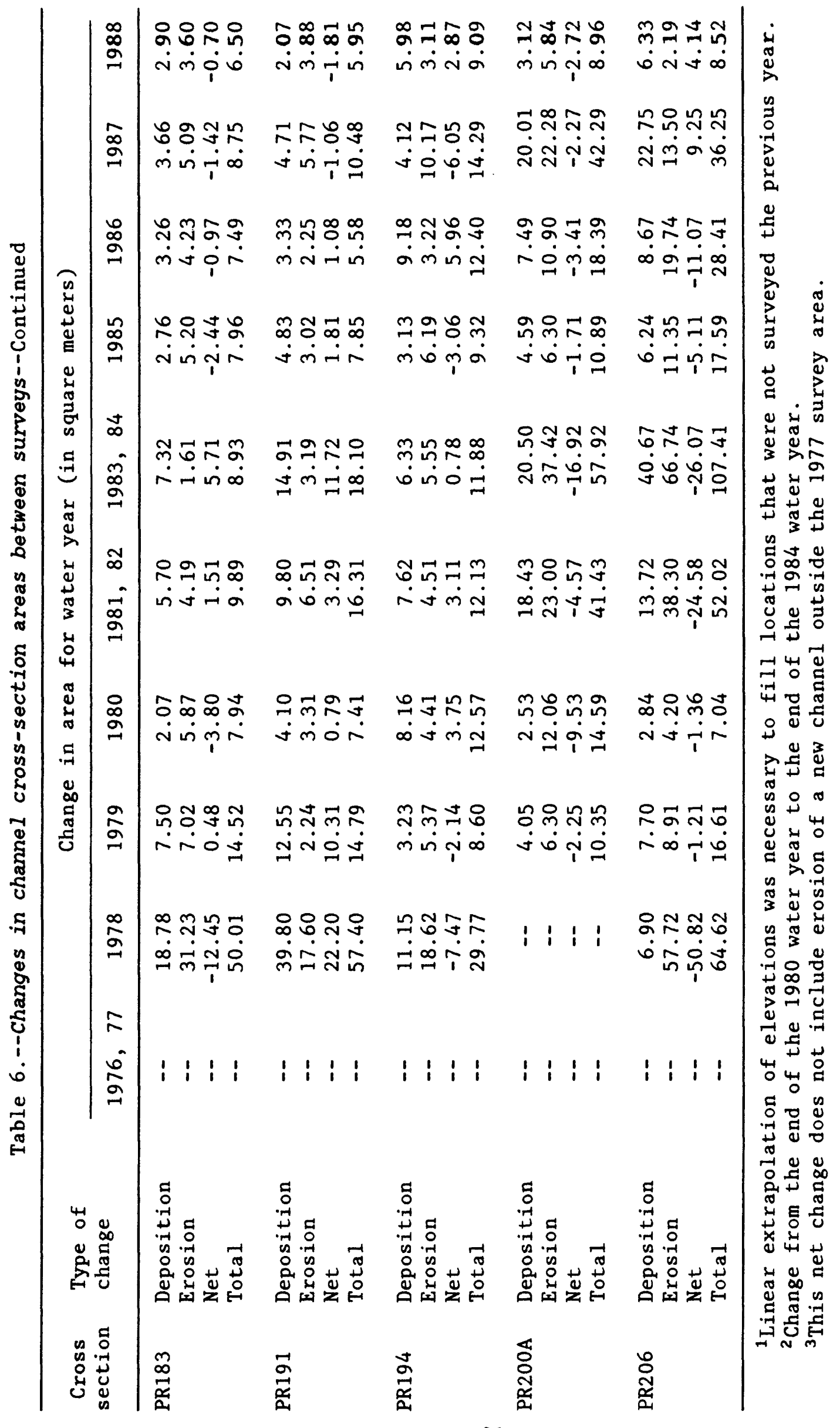


The average total channel change (deposition plus erosion, listed in table 6) during the 1978 flood was about three times the average net channel change (deposition minus erosion, listed in table 6). The average net channel change during the flood was about $16 \mathrm{~m}^{3}$ of erosion per meter of channel for 16 sections listed in table 6 for 1978 (PR122, PR141, and PR175 were not included), characterizing $95 \mathrm{~km}$ of the river. During the 10 years after the 1978 flood (1978-88), 15 of 21 sections had an average net deposition of about $15 \mathrm{~m}^{3}$ of sediment per meter of channel, and six sections had an average net erosion of about $35 \mathrm{~m}^{3}$ of sediment per meter of channel. The overall average net channel change (deposition minus erosion, PR122 and PR141 were not included) for the entire study reach since the 1978 flood has been essentially zero $\left(-0.8 \mathrm{~m}^{3}\right.$ of deposition per meter of channel length).

\section{SUMMARY}

During the period of this study (1975-88), the average minimum riverbed elevation for the study reach decreased about $0.2 \mathrm{~m}$ as a result of the May 1978 flood which eroded about $16 \mathrm{~m}^{3}$ of sediment per meter of channel length. The average minimum riverbed elevation increased about $0.2 \mathrm{~m}$ since the May 1978 flood (1978-88), and there was a negligible net deposition of about $1 \mathrm{~m}^{3}$ of sediment per meter of channel length. The overall result was that there was no significant change in the riverbed elevation but there was a net erosion of about $15 \mathrm{~m}^{3}$ of sediment per meter of channel length (equivalent to a decrease in depth of about $0.1 \mathrm{~m}$ for a 150-meter-wide channel) within the study reach from Moorhead to Broadus.

\section{REFERENCES CITED}

Hembree, C.H., Colby, B.R., Swenson, H.A., and Davis, J.R., 1952, Sedimentation and chemical quality of water in the Powder River drainage basin, Wyoming and Montana: U.S. Geological Survey Circular 170, 92 p.

Martinson, H.A., 1983, Channel changes of Powder River between Moorhead and Broadus, Montana, 1939 to 1978: U.S. Geological Survey Water-Resources Investigations Report 83-4128, $62 \mathrm{p}$.

Martinson, H.A, and Meade, R.H., 1983, Channel changes of Powder River, 1938-78, Powder River County, Montana: U.S. Geological Survey Hydrologic Investigations Atlas HA-661, scale 1:24,000, 3 sheets.

U.S. Geological Survey, 1976-89, Water resources data for Montana, water years 1975-88--volume 1: U.S. Geological Survey Water-Data Reports, MT-75-1 to MT-88-1 (published annually). 
CROSS-SECTION DATA

23

(followed by p.25) 
Description of Cross Section PR113

Location: Township 9 South/Range 48 East--section 18

U.S. Geological Survey quadrangle $(1: 24,000)$ : Bradshaw Creek

Landowners--left bank: Lloyd Sams

right bank: U.S. Government

Access: right bank

Permission from: none necessary

Reference pins

\begin{tabular}{lll}
\hline $\begin{array}{l}\text { Station } \\
\text { (meters) }\end{array}$ & $\begin{array}{c}\text { Elevation } \\
\text { (meters } \\
\text { above } \\
\text { sea level) }\end{array}$ & \multicolumn{1}{c}{ Comments } \\
\hline-32.0 & $1,022.824$ & $\begin{array}{l}0.17 \text { meter above } 1987 \text { ground } \\
\text { level. Usually was farthest from } \\
\text { the leveling instrument. }\end{array}$ \\
100.0 & $\begin{array}{l}0.48 \text { meter below } 1987 \text { ground level. } \\
0.10 \text { meter above } 1987 \text { ground level. }\end{array}$ \\
\hline
\end{tabular}




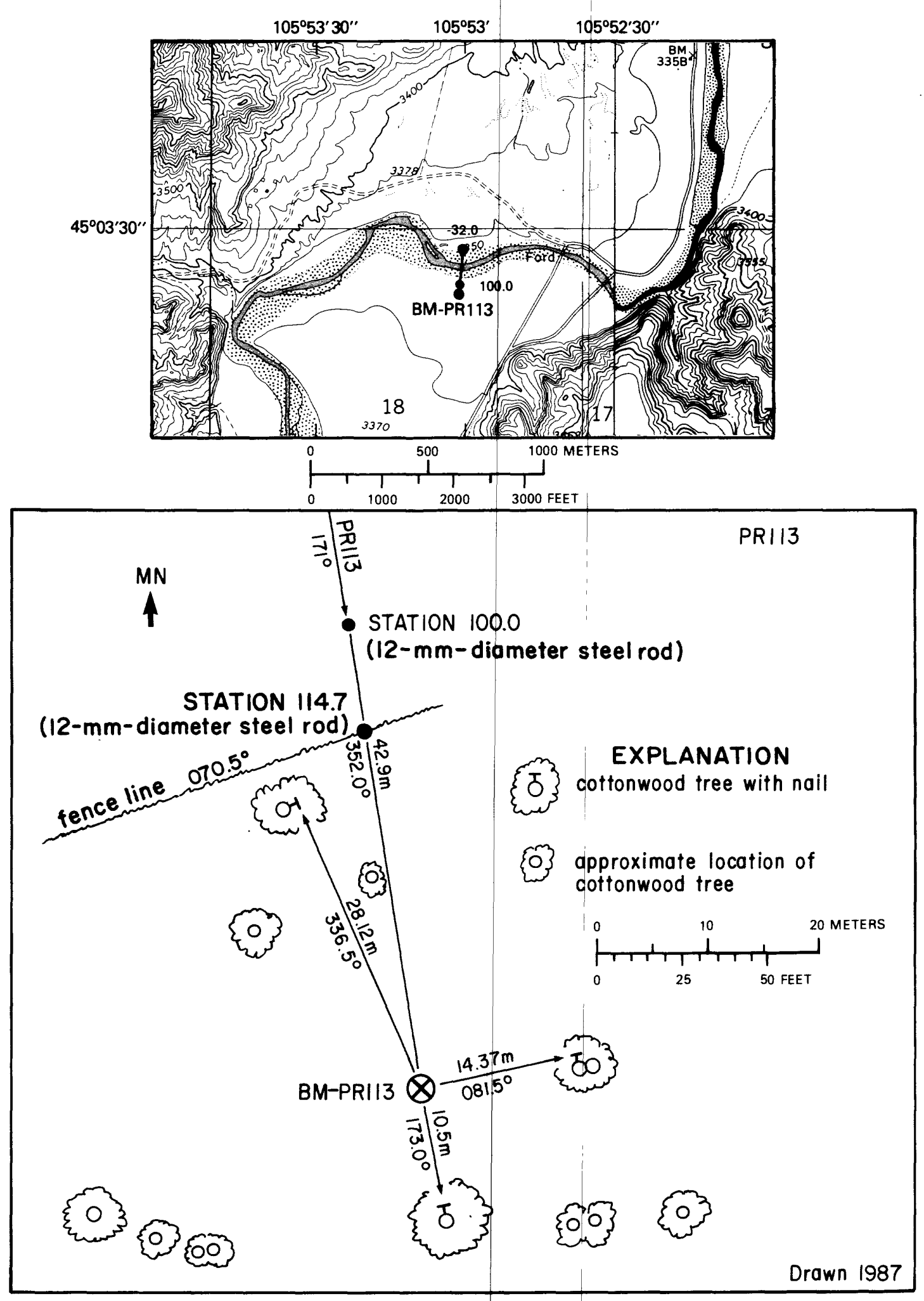

Figure 8.--Upper: Location of cross section PR113, bench mark BM-PR113, and left and right bank reference pins (tables 2 and 3) in the Bradshaw Creek quadrangle. Lower: Location of bench mark on the right bank. $M N$ is magnetic north. 

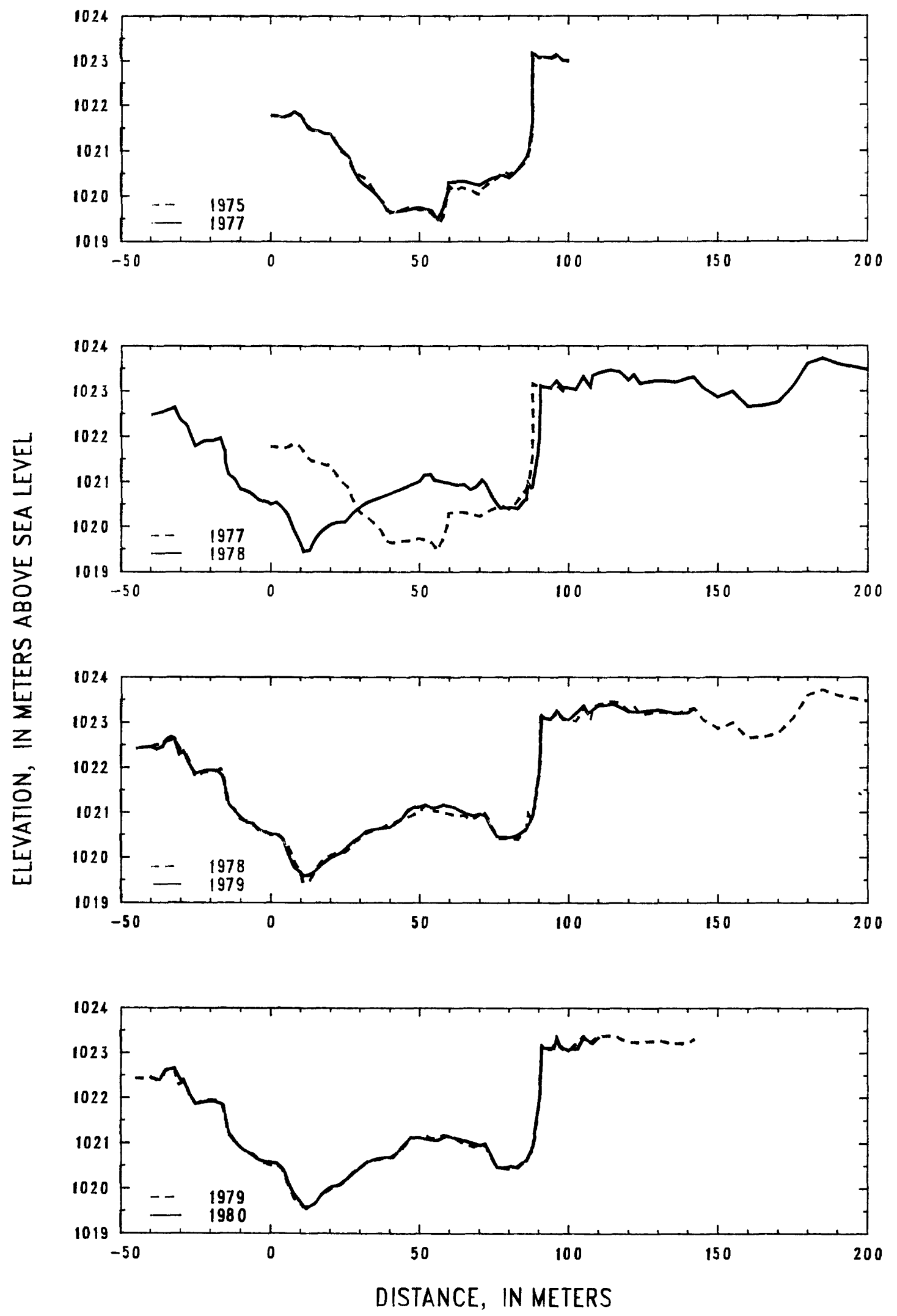

Figure 9. --Profiles of cross section PR113 from 1975 to 1980. 


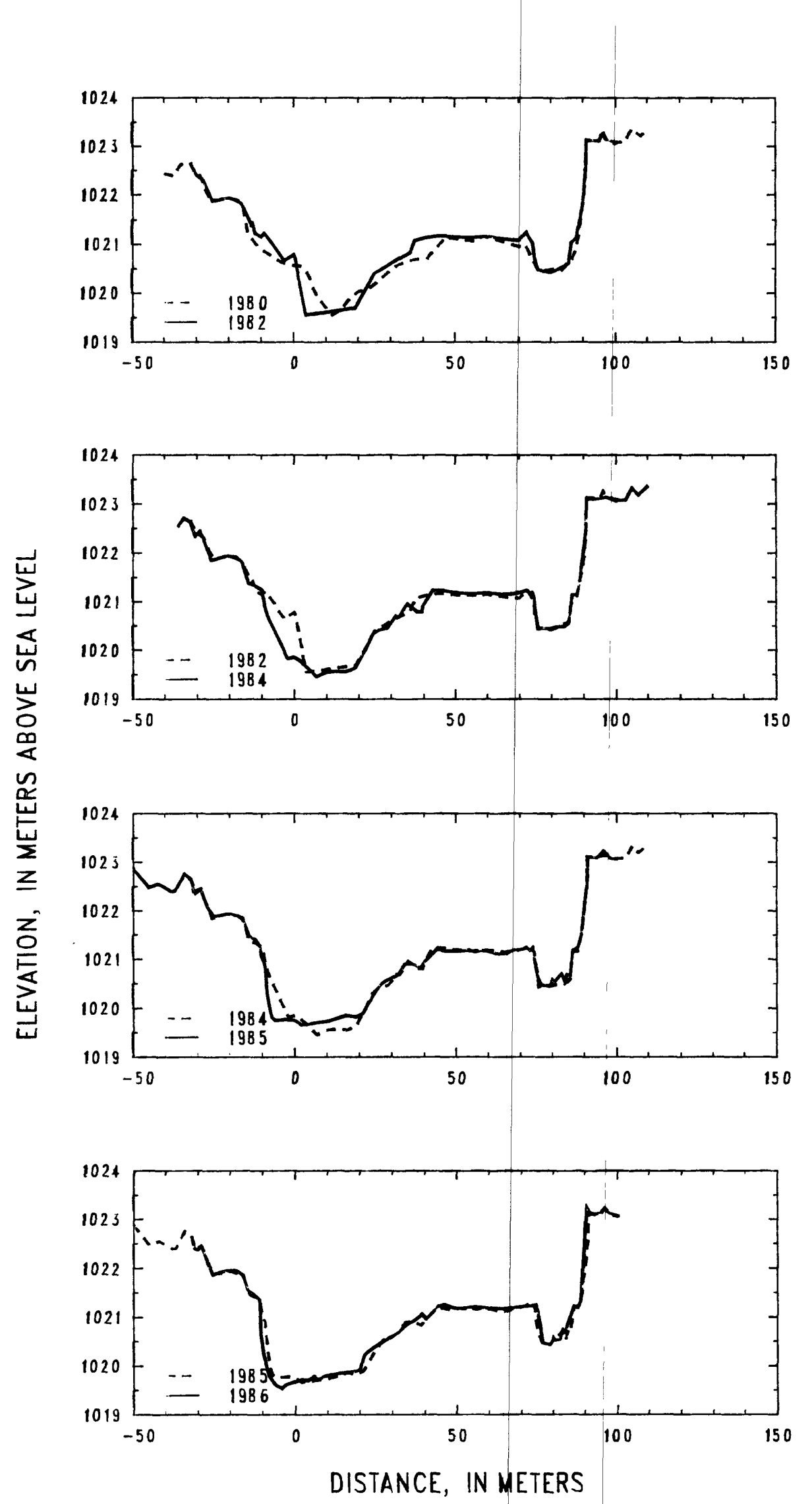

Figure 10.--Protiles of cross section PR113 from 1980 to 1986. 


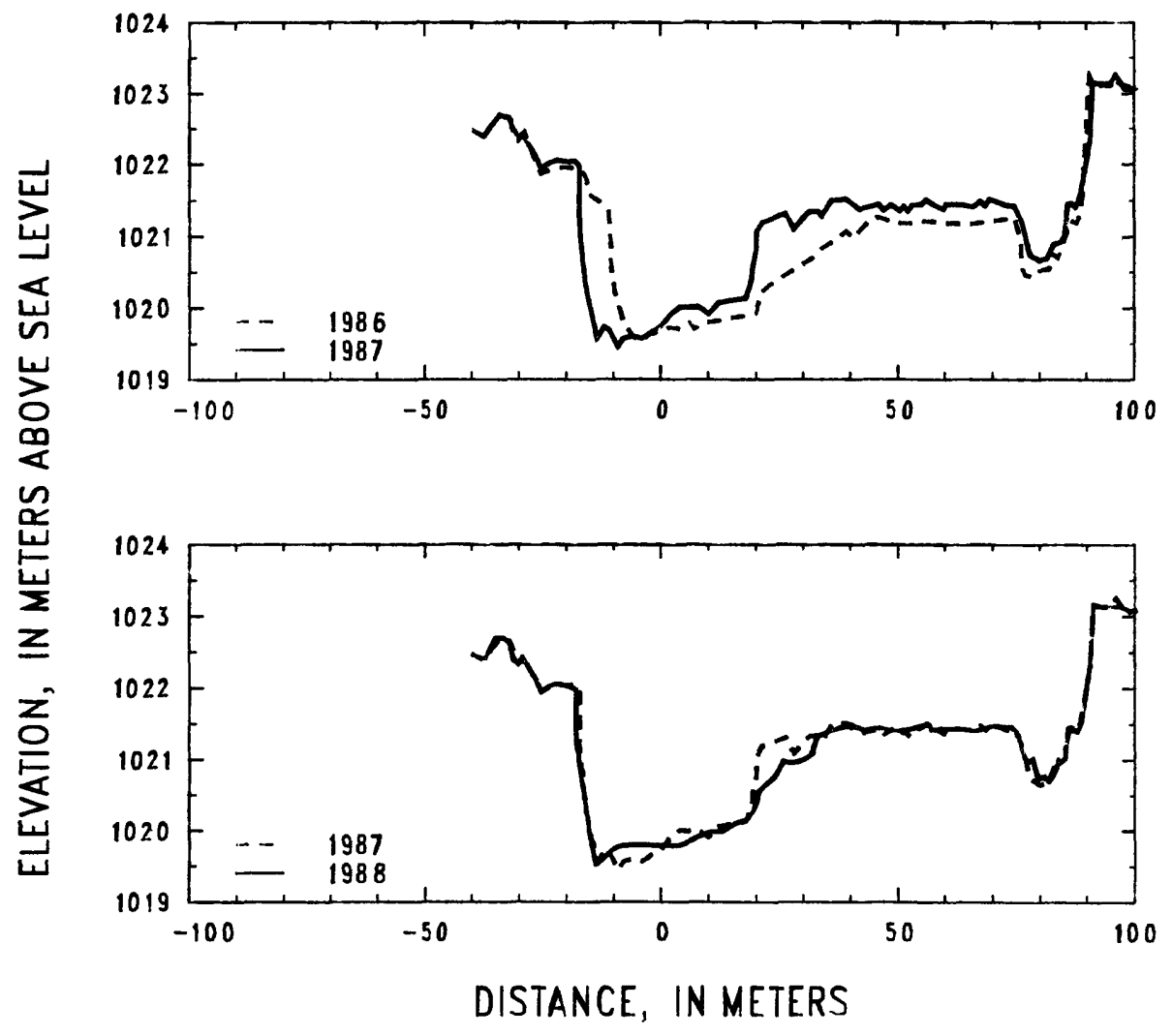

Figure 11.--Profiles of cross section PR 113 from 1986 to 1988

(followed by p. 31) 
Table 7.--Listing of horizontal stations and elevations for cross section PR113

[Sta., station, distance in meters from a reference point on

the left bank; Elev., elevation, in meters above sea level].

\begin{tabular}{|c|c|c|c|c|c|c|c|c|c|}
\hline \multicolumn{2}{|c|}{1975} & \multicolumn{2}{|c|}{1975} & \multicolumn{2}{|c|}{1977} & \multicolumn{2}{|c|}{1977} & \multicolumn{2}{|c|}{1978} \\
\hline Sta. & Elev. & Sta. & Elev. & Sta. & Elev. & Sta. & Elev. & Sta. & Elev. \\
\hline 0.0 & 1021.77 & 69.0 & 1020.05 & 0.0 & 1021.78 & 84.0 & 1020.66 & -40.0 & 1022.48 \\
\hline 2.0 & 1021.76 & 70.0 & 1020.03 & 2.0 & 1021.77 & 86.0 & 1020.85 & -35.0 & 1022.57 \\
\hline 3.0 & 1021.78 & 70.5 & 1020.13 & 4.0 & 1021.74 & 86.8 & 1020.99 & -32.0 & 1022.65 \\
\hline 4.0 & 1021.72 & 72.0 & 1020.21 & 6.0 & 1021.77 & 87.9 & 1021.61 & -30.0 & 1022.37 \\
\hline 6.0 & 1021.78 & 74.0 & 1020.34 & 8.0 & 1021.85 & 88.0 & 1023.17 & -28.0 & 1022.27 \\
\hline 8.0 & 1021.81 & 76.0 & 1020.39 & 10.0 & 1021.78 & 90.0 & 1023.08 & -25.0 & 1021.79 \\
\hline 10.0 & 1021.77 & 78.0 & 1020.55 & 12.0 & 1021.57 & 92.0 & 1023.08 & -22.0 & 1021.91 \\
\hline 11.0 & 1021.71 & 80.0 & 1020.48 & 14.0 & 1021.45 & 94.0 & 1023.06 & -19.0 & 1021.91 \\
\hline 12.0 & 1021.56 & 82.0 & 1020.54 & 16.0 & 1021.44 & 96.0 & 1023.12 & -16.6 & 1021.97 \\
\hline 13.0 & 1021.47 & 84.0 & 1020.64 & 18.0 & 1021.37 & 98.0 & 1023.00 & -15.2 & 1021.70 \\
\hline 14.0 & 1021.45 & 86.0 & 1020.78 & 20.0 & 1021.37 & 100.0 & 1023.00 & -15.1 & 1021.43 \\
\hline 16.0 & 1021.45 & 87.0 & 1021.01 & 22.0 & 1021.17 & & & -14.0 & 1021.17 \\
\hline 18.0 & 1021.38 & 88.0 & 1021.40 & 24.0 & 1021.00 & & & -12.0 & 1021.06 \\
\hline 20.0 & 1021.38 & 88.1 & 1023.17 & 26.0 & 1020.88 & & & -10.0 & 1020.84 \\
\hline 21.0 & 1021.33 & 89.0 & 1023.14 & 28.0 & 1020.54 & & & -8.0 & 1020.80 \\
\hline 22.0 & 1021.17 & 90.0 & 1023.07 & 30.0 & 1020.33 & & & -6.0 & 1020.73 \\
\hline 24.0 & 1020.97 & 92.0 & 1023.08 & 32.0 & 1020.22 & & & -4.0 & 1020.60 \\
\hline 26.0 & 1020.88 & 94.0 & 1023.05 & 34.0 & 1020.12 & & & -2.0 & 1020.56 \\
\hline 27.0 & 1020.67 & 96.0 & -1023.10 & 35.5 & 1020.03 & & & -1.0 & 1020.55 \\
\hline 28.0 & 1020.56 & 98.0 & 1023.00 & 37.0 & 1019.92 & & & 0.0 & 1020.50 \\
\hline 30.0 & 1020.45 & 100.0 & 1022.98 & 39.0 & 1019.68 & & & 2.0 & 1020.55 \\
\hline 32.0 & 1020.37 & & & 40.0 & 1019.65 & & & 4.0 & 1020.43 \\
\hline 34.0 & 1020.20 & & & 42.0 & 1019.64 & & & 6.0 & 1020.25 \\
\hline 35.0 & 1020.10 & & & 44.0 & 1019.68 & & & 8.0 & 1019.91 \\
\hline 36.0 & 1019.98 & & & 46.0 & 1019.69 & & & 10.0 & 1019.66 \\
\hline 38.0 & 1019.84 & & & 48.0 & 1019.73 & & & 11.0 & 1019.45 \\
\hline 40.0 & 1019.63 & & & 50.0 & 1019.74 & & & 13.0 & 1019.48 \\
\hline 42.0 & 1019.65 & & & 52.0 & 1019.70 & & & 15.0 & 1019.73 \\
\hline 44.0 & 1019.70 & & & 54.0 & 1019.67 & & & 17.0 & 1019.90 \\
\hline 46.0 & 1019.73 & & & 56.0 & 1019.48 & & & 20.0 & 1020.05 \\
\hline 48.0 & 1019.72 & & & 58.0 & 1019.73 & & & 22.0 & 1020.10 \\
\hline 50.0 & 1019.68 & & & 59.2 & 1020.02 & & & 25.0 & 1020.12 \\
\hline 52.0 & 1019.71 & & & 59.5 & 1020.07 & & & 28.0 & 1020.34 \\
\hline 54.0 & 1019.61 & & & 59.7 & 1020.30 & & & 30.0 & 1020.44 \\
\hline 56.0 & 1019.48 & & & 62.0 & 1020.31 & & & 32.4 & 1020.55 \\
\hline 57.0 & 1019.44 & & & 64.0 & 1020.32 & & & 35.0 & 1020.61 \\
\hline 58.0 & 1019.55 & & & 66.0 & 1020.30 & & & 40.0 & 1020.74 \\
\hline 59.0 & 1019.88 & & & 68.0 & 1020.26 & & & 45.0 & 1020.88 \\
\hline 59.8 & 1020.10 & & & 70.0 & 1020.23 & & & 50.0 & 1021.01 \\
\hline 60.2 & 1020.20 & & & 72.0 & 1020.31 & & & 51.5 & 1021.15 \\
\hline 62.0 & 1020.10 & & & 74.0 & 1020.38 & & & 53.7 & 1021.17 \\
\hline 64.0 & 1020.18 & & & 76.0 & 1020.43 & & & 55.0 & 1021.04 \\
\hline 66.0 & 1020.14 & & & 78.0 & 1020.46 & & & 57.0 & 1021.01 \\
\hline 67.3 & 1020.13 & & & 80.0 & 1020.39 & & & 59.0 & 1020.99 \\
\hline 68.0 & 1020.09 & & & 82.0 & 1020.50 & & & 61.0 & 1020.95 \\
\hline
\end{tabular}


Table 7.--Listing; of horizontal stations and elevations for cross section PR113 --Continued.

\begin{tabular}{|c|c|c|c|c|c|c|c|c|c|}
\hline \multicolumn{2}{|c|}{1978} & \multicolumn{2}{|c|}{1978} & \multicolumn{2}{|c|}{1979} & \multicolumn{2}{|c|}{1979} & \multicolumn{2}{|c|}{1980} \\
\hline Sta. & $\overline{\text { Elev. }}$ & Sta. & Elev. & Sta. & Elev. & Sta. & Elev. & Sta. & Elev. \\
\hline 63.0 & 1020.92 & 175.0 & 1023.09 & -45.0 & 1022.42 & 44.0 & 1020.86 & -40.0 & 1022.44 \\
\hline 65.0 & 1020.94 & 180.0 & 1023.61 & -40.0 & 1022.46 & 46.0 & 1021.04 & -37.0 & 1022.40 \\
\hline 67.0 & 1020.83 & 185.0 & 1023.73 & -38.0 & 1022.40 & 48.0 & 1021.12 & -35.0 & 1022.62 \\
\hline 69.0 & 1020.89 & 190.0 & 1023.60 & -36.0 & 1022.46 & 50.0 & 1021.12 & -32.0 & 1022.66 \\
\hline 71.0 & 1021.05 & 200.0 & 1023.47 & -35.0 & 1022.60 & 52.0 & 1021.17 & -30.0 & 1022.40 \\
\hline 72.0 & 1020.98 & & & -33.0 & 1022.68 & 54.0 & 1021.09 & -29.0 & 1022.41 \\
\hline 75.0 & 1020.58 & & & -32.0 & 1022.64 & 56.0 & 1021.11 & -27.0 & 1022.07 \\
\hline 77.0 & 1020.42 & & & -30.5 & 1022.29 & 58.0 & 1021.17 & -25.0 & 1021.86 \\
\hline 79.0 & 1020.44 & & & -29.0 & 1022.36 & 60.0 & 1021.13 & -22.0 & 1021.92 \\
\hline 81.0 & 1020.43 & & & -27.0 & 1022.06 & 62.0 & 1021.10 & -19.0 & 1021.93 \\
\hline 83.0 & 1020.40 & & & -25.0 & 1021.86 & 64.0 & 1021.02 & -17.0 & 1021.88 \\
\hline 85.0 & 1020.58 & & & -22.0 & 1021.93 & 66.0 & 1020.96 & -15.8 & 1021.83 \\
\hline 86.0 & 1020.61 & & & -20.0 & 1021.95 & 68.0 & 1020.92 & -14.0 & 1021.23 \\
\hline 86.5 & 1020.98 & & & -18.0 & 1021.93 & 70.0 & 1020.96 & -12.0 & 1021.03 \\
\hline 87.5 & 1020.86 & & & -16.0 & 1021.81 & 72.0 & 1020.94 & -10.0 & 1020.89 \\
\hline 88.0 & 1020.89 & & & -15.0 & 1021.56 & 74.0 & 1020.67 & -7.0 & 1020.77 \\
\hline 90.0 & 1021.74 & & & -14.0 & 1021.18 & 76.0 & 1020.46 & -4.0 & 1020.63 \\
\hline 90.4 & 1021.99 & & & -12.0 & 1021.03 & 78.0 & 1020.45 & -2.0 & 1020.59 \\
\hline 90.7 & 1023.13 & & & -10.0 & 1020.89 & 80.0 & 1020.44 & 0.0 & 1020.56 \\
\hline 92.0 & 1023.09 & & & -8.0 & 1020.77 & 82.0 & 1020.45 & 2.0 & 1020.55 \\
\hline 94.0 & 1023.08 & & & -6.0 & 1020.75 & 84.0 & 1020.53 & 4.0 & 1020.44 \\
\hline 96.0 & 1023.23 & & & -4.0 & 1020.62 & 86.0 & 1020.63 & 5.0 & 1020.33 \\
\hline 98.0 & 1023.07 & & & -2.0 & 1020.56 & 88.0 & 1020.91 & 6.0 & 1020.11 \\
\hline 100.0 & 1023.07 & & & 0.0 & 1020.51 & 90.0 & 1021.80 & 8.0 & 1019.87 \\
\hline 102.5 & 1023.03 & & & 2.0 & 1020.51 & 90.3 & 1021.93 & 10.0 & 1019.70 \\
\hline 105.0 & 1023.33 & & & 4.0 & 1020.42 & 91.0 & 1023.16 & 12.0 & 1019.54 \\
\hline 107.4 & 1023.09 & & & 6.0 & 1020.08 & 92.0 & 1023.09 & 13.0 & 1019.60 \\
\hline 108.1 & 1023.35 & & & 8.0 & 1019.78 & 94.0 & 1023.07 & 15.0 & 1019.68 \\
\hline 110.0 & 1023.41 & & & 10.0 & 1019.67 & 96.0 & 1023.26 & 17.0 & 1019.86 \\
\hline 114.0 & 1023.47 & & & 11.0 & 1019.58 & 98.0 & 1023.08 & 20.0 & 1020.03 \\
\hline 117.0 & 1023.44 & & & 13.0 & 1019.60 & 100.0 & 1023.05 & 23.0 & 1020.08 \\
\hline 120.0 & 1023.25 & & & 15.0 & 1019.69 & 105.0 & 1023.37 & 26.0 & 1020.23 \\
\hline 122.0 & 1023.37 & & & 17.0 & 1019.83 & 106.4 & 1023.19 & 29.0 & 1020.41 \\
\hline 124.0 & 1023.17 & & & 20.0 & 1019.99 & 110.0 & 1023.38 & 32.0 & 1020.56 \\
\hline 128.0 & 1023.23 & & & 22.0 & 1020.05 & 115.0 & 1023.40 & 35.0 & 1020.65 \\
\hline 132.0 & 1023.23 & & & 24.0 & 1020.12 & 120.0 & 1023.23 & 38.0 & 1020.69 \\
\hline 136.0 & 1023.20 & & & 26.0 & 1020.25 & 125.0 & 1023.24 & 41.0 & 1020.69 \\
\hline 140.0 & 1023.29 & & & 28.0 & 1020.37 & 130.0 & 1023.28 & 44.0 & 1020.91 \\
\hline 142.0 & 1023.32 & & & 30.0 & 1020.45 & 135.0 & 1023.20 & 47.0 & 1021.13 \\
\hline 145.0 & 1023.07 & & & 32.0 & 1020.57 & 140.0 & 1023.22 & 50.0 & 1021.12 \\
\hline 150.0 & 1022.87 & & & 34.0 & 1020.61 & 142.0 & 1023.31 & 53.0 & 1021.08 \\
\hline 155.0 & 1023.00 & & & 36.0 & 1020.63 & & & 56.0 & 1021.06 \\
\hline 160.0 & 1022.66 & & & 38.0 & 1020.66 & & & 59.0 & 1021.16 \\
\hline 165.0 & 1022.68 & & & 40.0 & 1020.67 & & & 62.0 & 1021.11 \\
\hline 170.0 & 1022.76 & & & 42.0 & 1020.76 & & & 65.0 & 1021.05 \\
\hline
\end{tabular}


Table 7.--Listins of horizontal stations and elevations for cross section PR113 --Continued.

\begin{tabular}{|c|c|c|c|c|c|c|c|c|c|}
\hline \multicolumn{2}{|c|}{1980} & \multicolumn{2}{|c|}{1982} & \multicolumn{2}{|c|}{1982} & \multicolumn{2}{|c|}{1984} & \multicolumn{2}{|c|}{1984} \\
\hline Sta. & Elev. & Sta. & Elev. & Sta. & Elev. & Sta. & Elev. & Sta. & Elev. \\
\hline 68.0 & 1020.99 & -32.0 & 1022.65 & 97.0 & 1023.14 & -36.0 & 1022.53 & 74.4 & 1021.14 \\
\hline 70.0 & 1020.95 & -30.0 & 1022.40 & 100.0 & 1023.08 & -34.0 & 1022.71 & 75.8 & 1020.43 \\
\hline 72.0 & 1020.99 & -28.0 & 1022.31 & 100.4 & 1023.08 & -32.0 & 1022.63 & 77.0 & 1020.46 \\
\hline 75.0 & 1020.58 & -25.0 & 1021.87 & & & -30.5 & 1022.34 & 79.5 & 1020.44 \\
\hline 76.0 & 1020.47 & -20.0 & 1021.94 & & & -29.0 & 1022.44 & 81.0 & 1020.47 \\
\hline 78.0 & 1020.46 & -16.0 & 1021.83 & & & -25.5 & 1021.84 & 84.0 & 1020.49 \\
\hline 81.0 & 1020.49 & -13.0 & 1021.38 & & & -21.0 & 1021.94 & 85.4 & 1020.57 \\
\hline 83.0 & 1020.45 & -12.6 & 1021.42 & & & -18.0 & 1021.91 & 85.7 & 1020.68 \\
\hline 85.0 & 1020.60 & -11.7 & 1021.20 & & & -16.0 & 1021.81 & 86.4 & 1021.14 \\
\hline 86.0 & 1020.62 & -10.0 & 1021.14 & & & -14.0 & 1021.37 & 88.0 & 1021.16 \\
\hline 88.0 & 1020.92 & -9.0 & 1021.22 & & & -12.0 & 1021.33 & 89.0 & 1021.44 \\
\hline 90.4 & 1022.03 & -7.0 & 1021.05 & & & -10.0 & 1021.24 & 90.8 & 1022.49 \\
\hline 91.0 & 1023.13 & -3.0 & 1020.65 & & & -9.0 & 1020.90 & 90.9 & 1023.11 \\
\hline 93.0 & 1023.10 & 0.0 & 1020.78 & & & -8.0 & 1020.68 & 93.0 & 1023.09 \\
\hline 95.0 & 1023.11 & 0.5 & 1020.70 & & & -6.0 & 1020.40 & 97.0 & 1023.14 \\
\hline 96.0 & 1023.35 & 2.0 & 1020.16 & & & -4.0 & 1020.11 & 100.0 & 1023.06 \\
\hline 97.0 & 1023.15 & 3.7 & 1019.55 & & & -2.0 & 1019.82 & 100.4 & 1023.08 \\
\hline 100.0 & 1023.05 & 19.0 & 1019.69 & & & 0.0 & 1019.85 & 103.0 & 1023.08 \\
\hline 100.4 & 1023.08 & 20.0 & 1019.80 & & & 2.0 & 1019.77 & 105.0 & 1023.33 \\
\hline 103.0 & 1023.10 & 25.0 & 1020.40 & & & 5.0 & 1019.58 & 107.0 & 1023.18 \\
\hline 105.0 & 1023.35 & 31.8 & 1020.67 & & & 7.0 & 1019.45 & 110.0 & 1023.37 \\
\hline 108.0 & 1023.22 & 36.0 & 1020.82 & & & 10.0 & 1019.55 & & \\
\hline 110.0 & 1023.36 & 37.5 & 1021.07 & & & 13.0 & 1019.57 & & \\
\hline & & 40.0 & 1021.12 & & & 16.0 & 1019.55 & & \\
\hline & & 45.0 & 1021.17 & & & 19.0 & 1019.65 & & \\
\hline & & 50.0 & 1021.14 & & & 22.0 & 1020.01 & & \\
\hline & & 55.0 & 1021.13 & & & 24.0 & 1020.30 & & \\
\hline & & 60.0 & 1021.15 & & & 26.0 & 1020.41 & & \\
\hline & & 65.0 & 1021.10 & & & 29.0 & 1020.46 & & \\
\hline & & 70.0 & 1021.07 & & & 31.5 & 1020.70 & & \\
\hline & & 72.5 & 1021.24 & & & 32.5 & 1020.68 & & \\
\hline & & 73.0 & 1021.15 & & & 35.0 & 1020.96 & & \\
\hline & & 74.4 & 1021.01 & & & 38.0 & 1020.79 & & \\
\hline & & 75.0 & 1020.68 & & & 39.6 & 1020.79 & & \\
\hline & & 76.0 & 1020.46 & & & 40.0 & 1020.93 & & \\
\hline & & 80.0 & 1020.42 & & & 42.8 & 1021.24 & & \\
\hline & & 85.5 & 1020.59 & & & 46.0 & 1021.23 & & \\
\hline & & 86.3 & 1021.03 & & & 50.0 & 1021.18 & & \\
\hline & & 88.0 & 1021.12 & & & 55.0 & 1021.16 & & \\
\hline & & 90.0 & 1021.82 & & & 60.0 & 1021.19 & & \\
\hline & & 90.4 & 1022.12 & & & 65.0 & 1021.14 & & \\
\hline & & 90.7 & 1022.56 & & & 69.0 & 1021.17 & & \\
\hline & & 91.0 & 1023.13 & & & 72.4 & 1021.23 & & \\
\hline & & 95.0 & 1023.11 & & & 73.4 & 1021.15 & & \\
\hline & & 96.0 & 1023.26 & & & 74.0 & 1021.19 & & \\
\hline
\end{tabular}


Table 7.--Listing of horizontal stations and elevations for cross section PR113 --Continued.

\begin{tabular}{|c|c|c|c|c|c|c|c|c|c|}
\hline \multicolumn{2}{|c|}{1985} & \multicolumn{2}{|c|}{1985} & \multicolumn{2}{|c|}{1986} & \multicolumn{2}{|c|}{1986} & \multicolumn{2}{|c|}{1987} \\
\hline Sta. & Elev. & Sta. & Elev. & Sta. & Elev. & Sta. & Elev. & Sta. & Elev. \\
\hline-50.0 & 1022.87 & 50.0 & 1021.17 & -32.0 & 1022.62 & 61.0 & 1021.17 & -40.0 & 1022.48 \\
\hline-45.0 & 1022.47 & 52.0 & 1021.16 & -31.0 & 1022.39 & 64.0 & 1021.16 & -37.4 & 1022.40 \\
\hline-42.0 & 1022.55 & 54.0 & 1021.19 & -30.0 & 1022.37 & 67.0 & 1021.18 & -34.0 & 1022.70 \\
\hline-40.0 & 1022.47 & 56.0 & 1021.14 & -29.0 & 1022.46 & 70.0 & 1021.21 & -32.0 & 1022.66 \\
\hline-38.0 & 1022.39 & 58.0 & 1021.18 & -27.0 & 1022.12 & 73.0 & 1021.23 & -30.2 & 1022.34 \\
\hline-37.0 & 1022.40 & 61.0 & 1021.15 & -25.4 & 1021.86 & 74.6 & 1021.26 & -29.3 & 1022.43 \\
\hline-34.0 & 1022.75 & 63.0 & 1021.10 & -24.0 & 1021.91 & 76.0 & 1020.87 & -27.0 & 1022.17 \\
\hline-32.0 & 1022.64 & 66.0 & 1021.12 & -21.0 & 1021.96 & 76.5 & 1020.53 & -25.4 & 1021.93 \\
\hline-31.0 & 1022.39 & 68.0 & 1021.19 & -18.0 & 1021.95 & 77.0 & 1020.45 & -24.0 & 1022.01 \\
\hline-29.0 & 1022.45 & 70.0 & 1021.19 & -16.0 & 1021.85 & 79.0 & 1020.42 & -22.0 & 1022.06 \\
\hline-27.0 & 1022.08 & 72.0 & 1021.25 & -14.6 & 1021.55 & 80.0 & 1020.52 & -20.0 & 1022.03 \\
\hline-25.0 & 1021.88 & 73.0 & 1021.16 & -13.0 & 1021.47 & 82.0 & 1020.54 & -18.0 & 1022.05 \\
\hline-20.0 & 1021.94 & 74.0 & 1021.23 & -11.0 & 1021.34 & 83.0 & 1020.74 & -17.2 & 1021.97 \\
\hline-16.0 & 1021.85 & 75.3 & 1020.67 & -10.8 & 1021.19 & 83.9 & 1020.68 & -17.2 & 1021.13 \\
\hline-14.0 & 1021.45 & 76.5 & 1020.47 & -10.5 & 1020.61 & 86.6 & 1021.22 & -16.0 & 1020.39 \\
\hline-12.0 & 1021.40 & 78.0 & 1020.44 & -9.7 & 1020.23 & 87.8 & 1021.18 & -13.5 & 1019.56 \\
\hline-10.7 & 1021.28 & 79.5 & 1020.47 & -8.0 & 1019.79 & 88.9 & 1021.37 & -12.0 & 1019.74 \\
\hline-10.3 & 1021.06 & 80.0 & 1020.57 & -6.0 & 1019.60 & 90.6 & 1023.24 & -11.0 & 1019.70 \\
\hline-9.3 & 1020.91 & 80.5 & 1020.50 & -4.0 & 1019.53 & 91.3 & 1023.13 & -9.0 & 1019.45 \\
\hline-8.2 & 1020.20 & 81.5 & 1020.60 & -3.0 & 1019.61 & 92.0 & 1023.11 & -8.0 & 1019.57 \\
\hline-7.0 & 1019.81 & 82.8 & 1020.70 & 0.0 & 1019.68 & 93.0 & 1023.11 & -6.0 & 1019.61 \\
\hline-6.0 & 1019.75 & 84.0 & 1020.52 & 2.0 & 1019.73 & 95.0 & 1023.11 & -4.0 & 1019.57 \\
\hline-4.0 & 1019.76 & 84.6 & 1020.69 & 4.0 & 1019.70 & 96.0 & 1023.22 & -2.0 & 1019.66 \\
\hline-2.0 & 1019.77 & 85.6 & 1020.66 & 6.0 & 1019.79 & 97.0 & 1023.14 & 0.0 & 1019.74 \\
\hline 0.0 & 1019.75 & 86.5 & 1021.18 & 7.0 & 1019.70 & 100.0 & 1023.06 & 2.0 & 1019.90 \\
\hline 2.0 & 1019.66 & 88.0 & 1021.23 & 9.0 & 1019.79 & & & 4.0 & 1020.01 \\
\hline 4.0 & 1019.67 & 89.0 & 1021.41 & 12.0 & 1019.82 & & & 6.0 & 1020.00 \\
\hline 7.0 & 1019.71 & 90.0 & 1021.99 & 15.0 & 1019.86 & & & 8.0 & 1020.01 \\
\hline 10.0 & 1019.73 & 90.9 & 1022.56 & 18.0 & 1019.88 & & & 10.0 & 1019.91 \\
\hline 14.0 & 1019.81 & 91.1 & 1023.10 & 20.0 & 1019.92 & & & 12.0 & 1020.06 \\
\hline 16.0 & 1019.86 & 92.0 & 1023.10 & 21.3 & 1020.22 & & & 14.0 & 1020.09 \\
\hline 19.0 & 1019.82 & 94.0 & 1023.08 & 23.0 & 1020.32 & & & 16.0 & 1020.11 \\
\hline 21.0 & 1019.89 & 96.0 & 1023.23 & 26.0 & 1020.45 & & & 18.0 & 1020.13 \\
\hline 23.5 & 1020.18 & 98.0 & 1023.07 & 29.0 & 1020.57 & & & 19.2 & 1020.38 \\
\hline 25.0 & 1020.33 & 100.0 & 1023.05 & 32.0 & 1020.69 & & & 19.3 & 1020.54 \\
\hline 27.0 & 1020.51 & 100.4 & 1023.07 & 34.0 & 1020.83 & & & 20.1 & 1020.78 \\
\hline 29.0 & 1020.58 & & & 37.0 & 1020.94 & & & 20.1 & 1021.04 \\
\hline 32.0 & 1020.68 & & & 39.0 & 1021.06 & & & 21.3 & 1021.19 \\
\hline 34.0 & 1020.89 & & & 40.5 & 1020.96 & ! & & 26.3 & 1021.31 \\
\hline 36.0 & 1020.91 & & & 44.0 & 1021.22 & & & 28.0 & 1021.09 \\
\hline 39.0 & 1020.82 & & & 46.0 & 1021.26 & $\vdots$ & & 30.0 & 1021.25 \\
\hline 40.0 & 1020.93 & & & 49.0 & 1021.18 & & & 31.5 & 1021.34 \\
\hline 42.0 & 1021.07 & & & 52.0 & 1021.17 & & & 33.3 & 1021.33 \\
\hline 44.0 & 1021.20 & & & 55.0 & 1021.21 & & & 34.0 & 1021.27 \\
\hline 47.0 & 1021.15 & & & 58.0 & 1021.19 & & & 36.0 & 1021.49 \\
\hline
\end{tabular}


Table 7.--Listing of horizontal stations and elevations for cross section PR113 --Continued.

\begin{tabular}{|c|c|c|c|c|c|}
\hline \multicolumn{2}{|c|}{1987} & \multicolumn{2}{|c|}{1988} & \multicolumn{2}{|c|}{1988} \\
\hline Sta. & Elev. & Sta. & Elev. & Sta. & Elev. \\
\hline 39.0 & 1021.52 & -40.0 & 1022.47 & 37.0 & 1021.44 \\
\hline 42.0 & 1021.36 & -37.0 & 1022.41 & 40.0 & 1021.49 \\
\hline 44.0 & 1021.40 & -35.0 & 1022.69 & 43.0 & 1021.41 \\
\hline 46.0 & 1021.44 & -34.0 & 1022.71 & 46.0 & 1021.44 \\
\hline 47.0 & 1021.35 & -33.0 & 1022.69 & 49.0 & 1021.39 \\
\hline 48.5 & 1021.43 & -32.0 & 1022.63 & 52.0 & 1021.42 \\
\hline 50.5 & 1021.34 & -31.0 & 1022.39 & 55.0 & 1021.48 \\
\hline 51.3 & 1021.41 & -30.3 & 1022.35 & 56.7 & 1021.48 \\
\hline 52.2 & 1021.34 & -29.0 & 1022.40 & 57.0 & 1021.42 \\
\hline 53.0 & 1021.42 & -27.0 & 1022.17 & 60.0 & 1021.43 \\
\hline 55.0 & 1021.44 & -25.4 & 1021.94 & 63.0 & 1021.43 \\
\hline 56.0 & 1021.50 & -24.0 & 1022.02 & 66.0 & 1021.41 \\
\hline 58.0 & 1021.41 & -22.0 & 1022.06 & 68.0 & 1021.43 \\
\hline 59.8 & 1021.36 & -20.0 & 1022.04 & 70.0 & 1021.48 \\
\hline 60.2 & 1021.44 & -18.0 & 1021.99 & 72.0 & 1021.46 \\
\hline 62.0 & 1021.44 & -17.9 & 1021.34 & 74.0 & 1021.45 \\
\hline 64.0 & 1021.43 & -17.3 & 1020.95 & 75.3 & 1021.36 \\
\hline 66.0 & 1021.41 & -15.4 & 1020.16 & 76.6 & 1021.06 \\
\hline 67.4 & 1021.49 & -14.5 & 1019.78 & 77.5 & 1020.96 \\
\hline 68.5 & 1021.41 & -13.7 & 1019.53 & 78.5 & 1021.02 \\
\hline 69.4 & 1021.51 & -13.0 & 1019.56 & 79.7 & 1020.74 \\
\hline 71.0 & 1021.48 & -11.0 & 1019.70 & 81.0 & 1020.77 \\
\hline 73.0 & 1021.42 & -9.0 & 1019.78 & 82.0 & 1020.69 \\
\hline 74.6 & 1021.42 & -6.0 & 1019.81 & 83.0 & 1020.78 \\
\hline 75.9 & 1021.26 & -3.0 & 1019.80 & 83.9 & 1020.99 \\
\hline 78.0 & 1020.74 & 0.0 & 1019.79 & 84.3 & 1020.95 \\
\hline 80.0 & 1020.65 & 2.0 & 1019.78 & 85.4 & 1021.02 \\
\hline 81.6 & 1020.68 & 4.0 & 1019.79 & 86.3 & 1021.44 \\
\hline 83.0 & 1020.88 & 7.0 & 1019.90 & 88.0 & 1021.40 \\
\hline 84.5 & 1020.92 & 10.0 & 1019.98 & 88.8 & 1021.52 \\
\hline 85.2 & 1021.00 & 13.0 & 1019.98 & 90.6 & 1022.30 \\
\hline 86.1 & 1021.45 & 16.0 & 1020.12 & 91.0 & 1022.51 \\
\hline 86.8 & 1021.46 & 18.0 & 1020.15 & 91.4 & 1023.16 \\
\hline 87.7 & 1021.40 & 19.0 & 1020.23 & 93.0 & 1023.13 \\
\hline 88.5 & 1021.52 & 20.0 & 1020.38 & 95.0 & 1023.13 \\
\hline 90.7 & 1022.33 & 20.6 & 1020.56 & 97.0 & 1023.15 \\
\hline 90.9 & 1022.57 & 22.0 & 1020.65 & 99.0 & 1023.07 \\
\hline 91.3 & 1023.16 & 24.0 & 1020.76 & 100.4 & 1023.08 \\
\hline 93.0 & 1023.13 & 25.7 & 1020.98 & & \\
\hline 95.0 & 1023.14 & 27.0 & 1020.95 & & \\
\hline 96.0 & 1023.27 & 29.0 & 1020.97 & & \\
\hline 98.0 & 1023.06 & 31.0 & 1021.04 & & \\
\hline 100.0 & 1023.06 & 32.0 & 1021.09 & & \\
\hline 100.4 & 1023.08 & 33.0 & 1021.33 & & \\
\hline & & 35.0 & 1021.39 & & \\
\hline
\end{tabular}


Location: Township 9 South/Range 48 East--section 8

U.S. Geological Survey quadrangle $(1: 24,000)$ : Moorhead

Landowners--1eft bank: county road right bank: Amory Hubbard

Access: left bank

Permission from: none necessary

Reference pins

\begin{tabular}{|c|c|c|}
\hline $\begin{array}{l}\text { Station } \\
\text { (meters) }\end{array}$ & $\begin{array}{l}\text { Elevation } \\
\text { (meters } \\
\text { above } \\
\text { sea level) }\end{array}$ & Comments \\
\hline-1.7 & $1,020.367$ & $\begin{array}{l}\text { Pins on the left bank were closest } \\
\text { to the leveling instrument. } \\
\text { Installed } 0.22 \text { meter above ground } \\
\text { level in } 1988 \text { after cable car was } \\
\text { removed. }\end{array}$ \\
\hline $\begin{array}{r}-1.3 \\
-1.1 \\
0.0\end{array}$ & $\begin{array}{l}1,019.995 \\
1,019.993 \\
1,019.690\end{array}$ & $\begin{array}{l}\text { Bolt in downstream concrete footing. } \\
\text { Bolt in upstream concrete footing. } \\
\text { Installed } 0.04 \text { meter above ground } \\
\text { level in } 1988 \text {. }\end{array}$ \\
\hline $\begin{array}{l}110.0 \\
112.6\end{array}$ & $\begin{array}{l}1,019.406 \\
1,019.828\end{array}$ & $\begin{array}{l}\text { Center of concrete deadman. } \\
\text { Ground level under fence } 1 \text { ine. }\end{array}$ \\
\hline
\end{tabular}




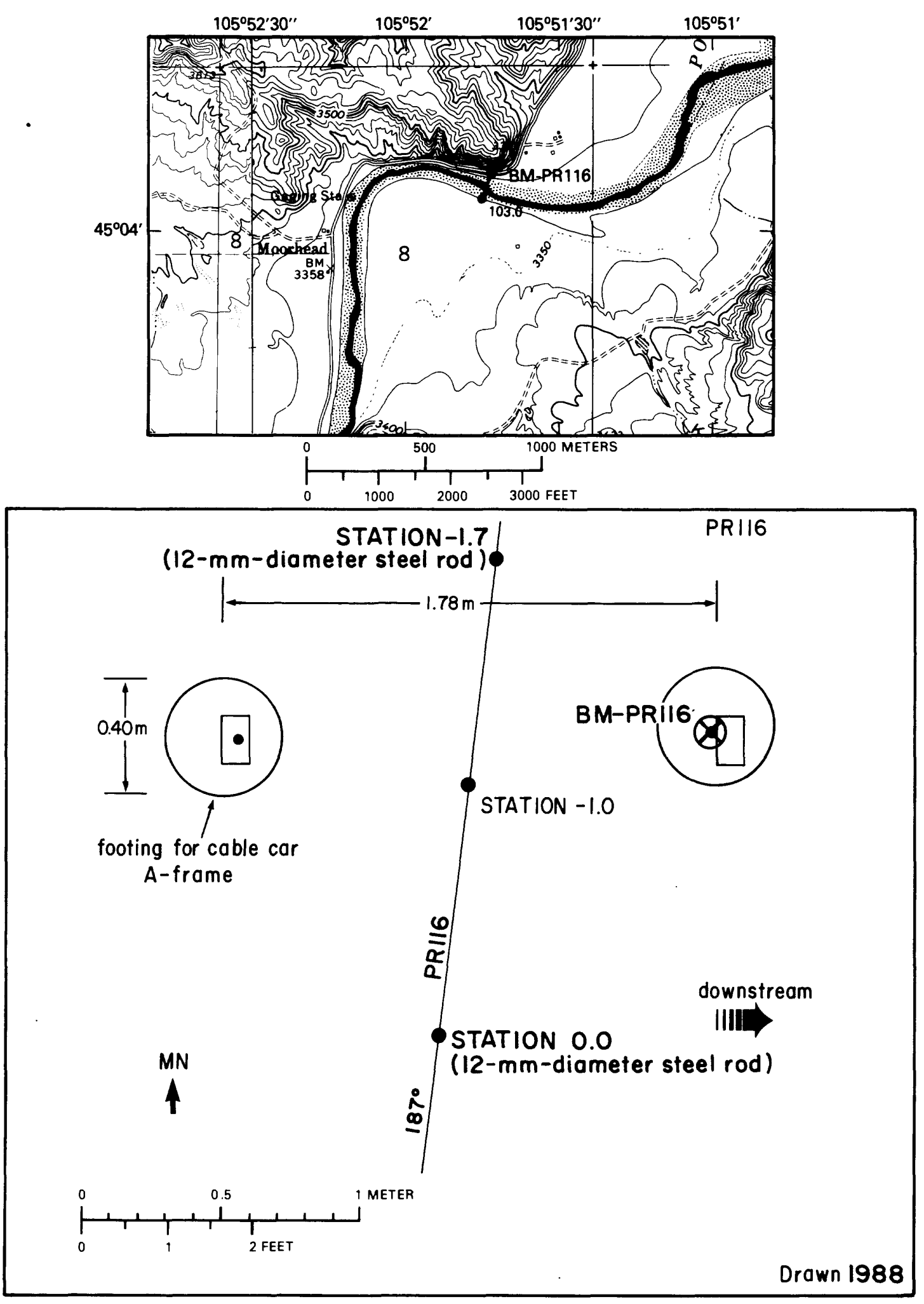

Figure 12.--Upper: Location of cross section PR116, bench mark BM-PR116, and right bank reference pins (tables 2 and 3 ) in the Moorhead quadrangle.

Lower: Location of bench mark on the left bank. MN is magnetic north. 


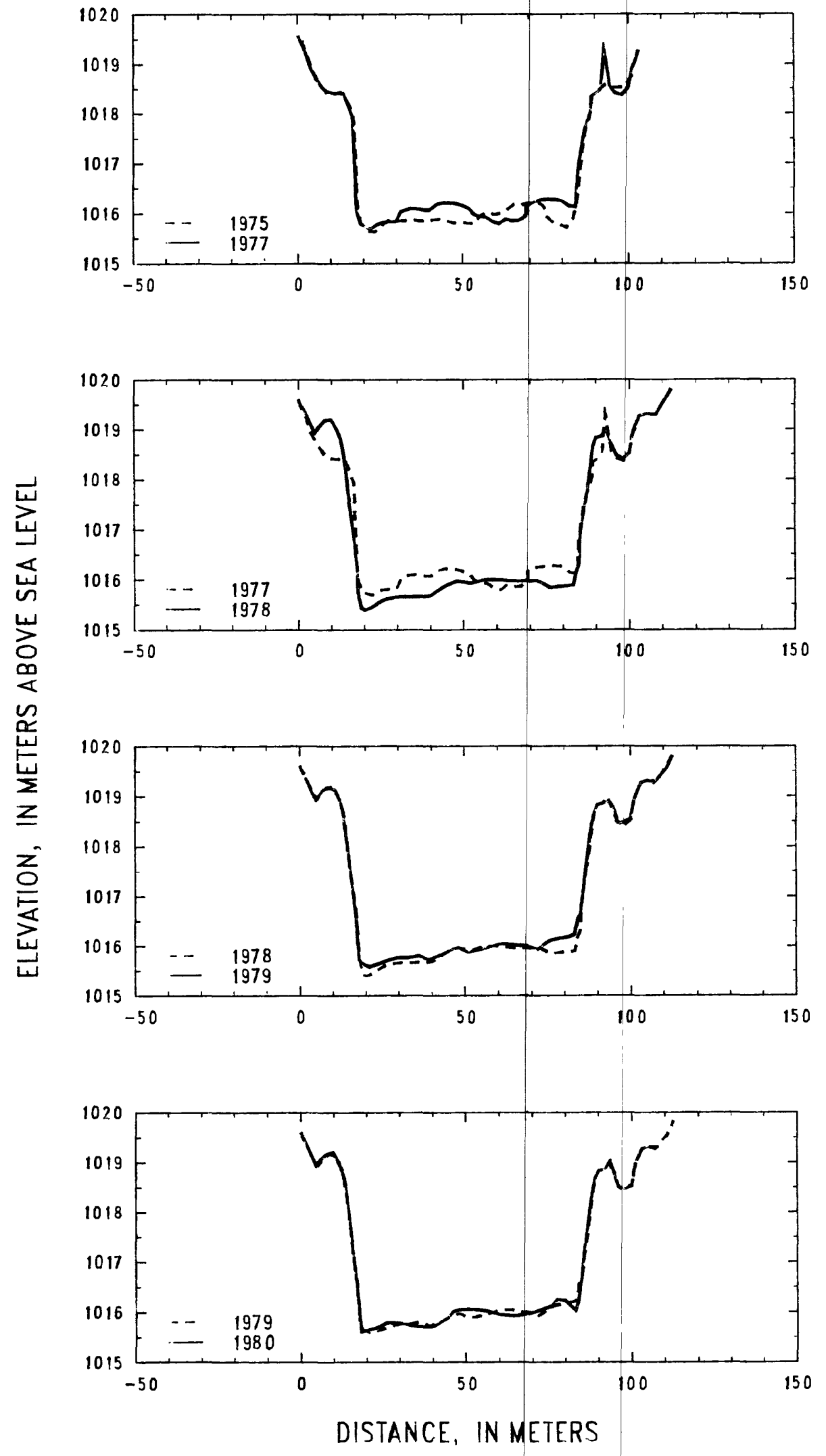

Figure 13.--Profiles of cross section PR116 from 1975 to 1980 


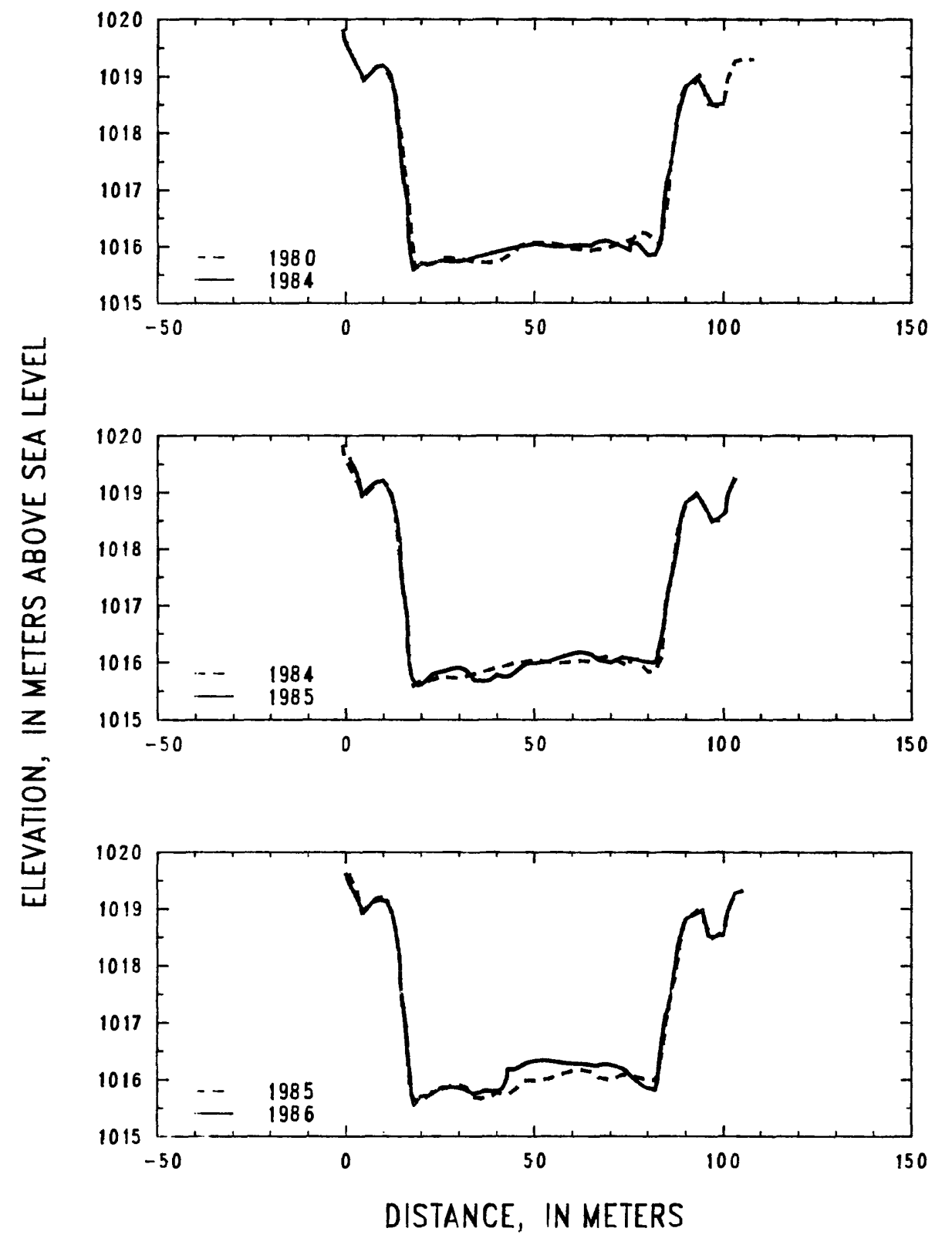

Figure 14.--Profiles of cross section PR116 from 1980 to 1986. 


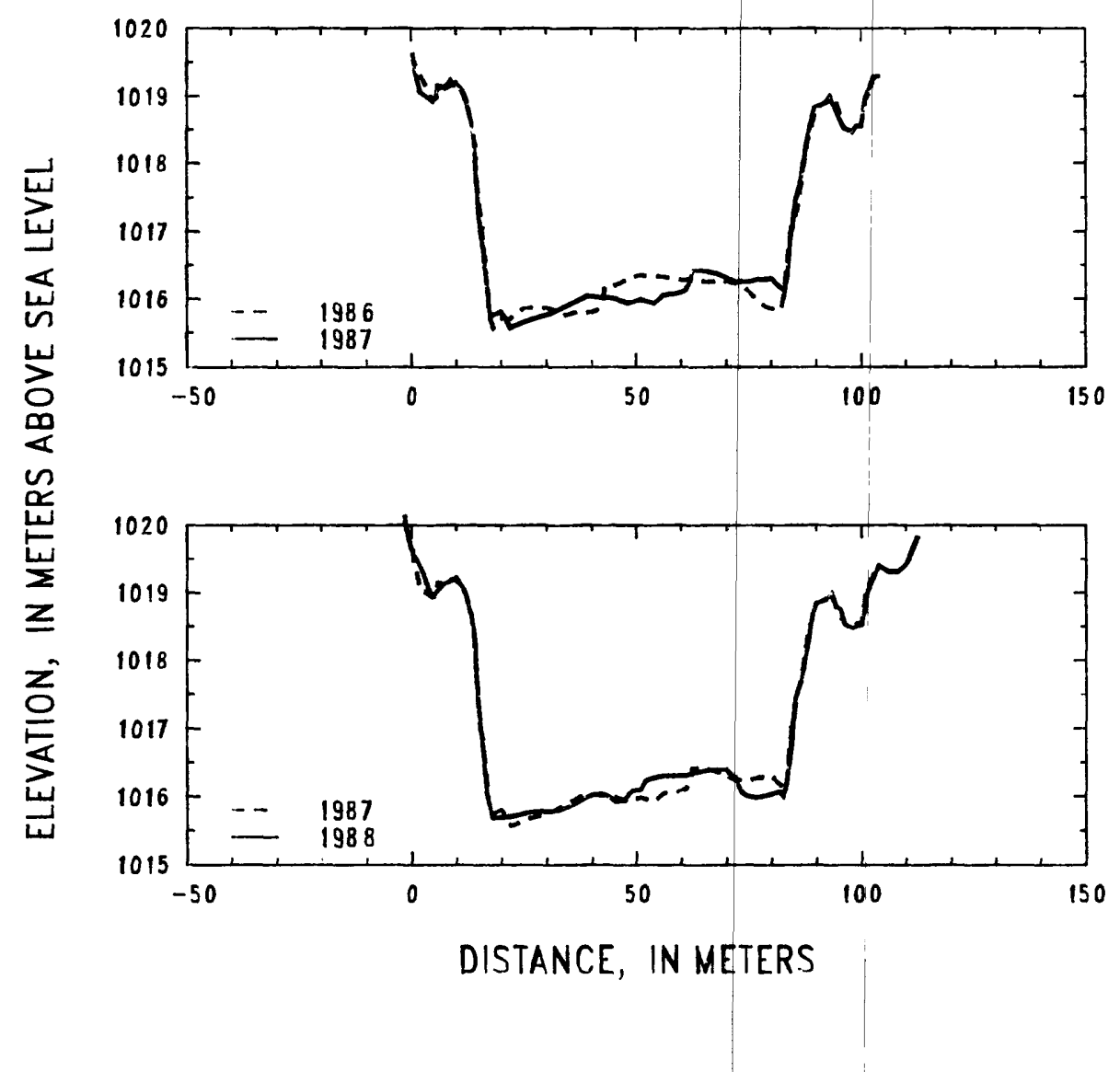

Figure 15.--Profiles of cross section PR116 from 1986 to 1988. 
Table 8.--Listing of horizontal stations and elevations for cross section PR116 [Sta., station, distance in meters from a reference point on the left bank; Elev., elevation, in meters above sea level].

\begin{tabular}{|c|c|c|c|c|c|c|c|c|c|}
\hline \multicolumn{2}{|c|}{1975} & \multicolumn{2}{|c|}{1975} & \multicolumn{2}{|c|}{1977} & \multicolumn{2}{|c|}{1977} & \multicolumn{2}{|c|}{1978} \\
\hline Sta. & Elev. & Sta. & Elev. & Sta. & Elev. & Sta. & Elev. & Sta. & Elev. \\
\hline 1.3 & 1019.46 & 77.3 & 1015.81 & 0.0 & 1019.56 & 84.1 & 1016.12 & 0.0 & 1019.59 \\
\hline 3.3 & 1019.12 & 79.3 & 1015.76 & 2.0 & 1019.31 & 84.8 & 1016.87 & 2.0 & 1019.36 \\
\hline 5.3 & 1018.73 & 81.3 & 1015.70 & 4.0 & 1018.91 & 86.8 & 1017.67 & 4.0 & 1019.05 \\
\hline 7.3 & 1018.57 & 83.3 & 1015.89 & 6.0 & 1018.68 & 88.6 & 1017.99 & 5.0 & 1018.92 \\
\hline 9.3 & 1018.40 & 83.6 & 1015.98 & 8.0 & 1018.50 & 89.0 & 1018.34 & 6.0 & 1019.02 \\
\hline 11.3 & 1018.39 & 84.3 & 1016.17 & 10.0 & 1018.41 & 91.7 & 1018.48 & 8.0 & 1019.17 \\
\hline 13.3 & 1018.43 & 86.3 & 1017.36 & 12.0 & 1018.39 & 92.8 & 1019.29 & 10.0 & 1019.20 \\
\hline 15.3 & 1018.23 & 87.3 & 1017.75 & 14.0 & 1018.41 & 94.5 & 1018.56 & 12.0 & 1018.96 \\
\hline 16.8 & 1018.00 & 88.3 & 1017.90 & 16.8 & 1017.93 & 96.0 & 1018.42 & 13.0 & 1018.77 \\
\hline 17.3 & 1017.71 & 89.3 & 1018.37 & 17.9 & 1016.03 & 98.0 & 1018.37 & 14.0 & 1018.37 \\
\hline 18.3 & 1016.01 & 91.3 & 1018.48 & 19.0 & 1015.78 & 100.0 & 1018.52 & 15.0 & 1017.71 \\
\hline 19.3 & 1015.80 & 93.3 & 1018.59 & 20.0 & 1015.73 & 101.0 & 1018.90 & 16.0 & 1017.23 \\
\hline 21.3 & 1015.65 & 95.3 & 1018.52 & 22.0 & 1015.68 & 102.0 & 1019.05 & 17.0 & 1016.76 \\
\hline 23.3 & 1015.61 & 99.3 & 1018.54 & 24.0 & 1015.77 & 103.0 & 1019.27 & 17.8 & 1016.16 \\
\hline 25.3 & 1015.71 & 101.3 & 1018.82 & 26.0 & 1015.81 & & & 18.0 & 1015.73 \\
\hline 27.3 & 1015.84 & & & 28.0 & 1015.83 & & & 19.0 & 1015.43 \\
\hline 29.3 & 1015.84 & & & 30.0 & 1015.84 & & & 20.0 & 1015.38 \\
\hline 31.3 & 1015.85 & & & 31.4 & 1016.02 & & & 21.0 & 1015.41 \\
\hline 33.3 & 1015.85 & & & 34.0 & 1016.09 & & & 22.0 & 1015.44 \\
\hline 35.3 & 1015.86 & & & 36.0 & 1016.09 & & & 24.0 & 1015.54 \\
\hline 37.3 & 1015.83 & & & 38.0 & 1016.06 & & & 26.0 & 1015.61 \\
\hline 39.3 & 1015.84 & & & 40.0 & 1016.06 & & & 30.0 & 1015.66 \\
\hline 41.3 & 1015.86 & & & 42.0 & 1016.17 & & & 34.0 & 1015.66 \\
\hline 43.3 & 1015.87 & & & 44.0 & 1016.21 & & & 38.0 & 1015.67 \\
\hline 45.3 & 1015.83 & & & 46.0 & 1016.21 & & & 40.0 & 1015.68 \\
\hline 47.3 & 1015.79 & & & 48.0 & 1016.19 & & & 44.0 & 1015.87 \\
\hline 49.3 & 1015.82 & & & 50.0 & 1016.15 & & & 48.0 & 1015.97 \\
\hline 51.3 & 1015.78 & & & 52.0 & 1016.09 & & & 52.0 & 1015.92 \\
\hline 53.3 & 1015.77 & & & 53.1 & 1015.99 & & & 56.0 & 1016.00 \\
\hline 55.3 & 1015.95 & & & 55.0 & 1015.92 & & & 60.0 & 1016.00 \\
\hline 57.3 & 1016.00 & & & 57.0 & 1015.92 & & & 64.0 & 1015.96 \\
\hline 58.3 & 1016.01 & & & 59.0 & 1015.83 & & & 68.0 & 1015.96 \\
\hline 59.3 & 1015.96 & & & 61.0 & 1015.78 & & & 72.0 & 1015.98 \\
\hline 60.3 & 1015.97 & & & 63.0 & 1015.88 & . & & 76.0 & 1015.84 \\
\hline 61.3 & 1015.99 & & & 65.0 & 1015.84 & & & 80.0 & 1015.87 \\
\hline 63.3 & 1016.06 & & & 67.0 & 1015.85 & & & 83.0 & 1015.89 \\
\hline 65.3 & 1016.16 & & & 69.0 & 1015.94 & & & 83.9 & 1016.17 \\
\hline 67.3 & 1016.15 & & & 69.2 & 1016.01 & & & 84.8 & 1016.31 \\
\hline 69.3 & 1016.19 & & & 69.3 & 1016.16 & & & 85.0 & 1016.88 \\
\hline 71.3 & 1016.19 & & & 72.0 & 1016.24 & & & 86.0 & 1017.23 \\
\hline 73.3 & 1016.19 & & & 74.0 & 1016.27 & & & 87.4 & 1017.86 \\
\hline 74.3 & 1016.15 & & & 76.0 & 1016.28 & & & 89.0 & 1018.66 \\
\hline 75.0 & 1016.07 & & & 78.0 & 1016.27 & & & 90.0 & 1018.83 \\
\hline 75.1 & 1015.99 & & & 80.0 & 1016.24 & & & 91.7 & 1018.86 \\
\hline 75.3 & 1015.93 & & & 82.0 & 1016.14 & & & 93.0 & 1018.99 \\
\hline
\end{tabular}


Table 8.--Listing of horizontal stations and elevations for cross section PR116 --Continued.

\begin{tabular}{|c|c|c|c|c|c|c|c|c|c|}
\hline \multicolumn{2}{|c|}{1978} & \multicolumn{2}{|c|}{1979} & \multicolumn{2}{|c|}{1979} & \multicolumn{2}{|c|}{1980} & \multicolumn{2}{|c|}{1980} \\
\hline Sta. & Elev. & Sta. & Elev. & Sta. & Elev. & Sta. & $\overline{\text { Elev. }}$ & Sta. & Elev. \\
\hline 94.5 & 1018.73 & 0.0 & 1019.58 & 95.0 & 1018.74 & 0.0 & 1019.60 & 100.0 & 1018.52 \\
\hline 96.0 & 1018.48 & 2.0 & 1019.35 & 96.0 & 1018.49 & 2.0 & 1019.36 & 101.0 & 1018.95 \\
\hline 98.0 & 1018.40 & 4.0 & 1019.03 & 97.0 & 1018.45 & 4.8 & 1018.93 & 103.0 & 1019.26 \\
\hline 100.0 & 1018.53 & 5.4 & 1018.94 & 100.0 & 1018.54 & 6.0 & 1019.05 & 105.0 & 1019.31 \\
\hline 101.0 & 1018.93 & 7.0 & 1019.11 & 101.0 & 1018.93 & 8.0 & 1019.17 & 108.0 & 1019.30 \\
\hline 103.0 & 1019.27 & 9.0 & 1019.17 & 103.0 & 1019.26 & 10.0 & 1019.20 & & \\
\hline 105.0 & 1019.31 & 11.0 & 1019.12 & 105.0 & 1019.31 & 12.0 & 1018.95 & & \\
\hline 108.0 & 1019.29 & 12.0 & 1018.96 & 107.0 & 1019.25 & 13.4 & 1018.67 & & \\
\hline 112.5 & 1019.80 & 13.0 & 1018.76 & 111.0 & 1019.57 & 14.2 & 1018.23 & & \\
\hline & & 14.0 & 1018.30 & 112.5 & 1019.81 & 16.0 & 1017.16 & & \\
\hline & & 15.0 & 1017.71 & & & 17.0 & 1016.61 & & \\
\hline & & 16.0 & 1017.14 & & & 18.3 & 1015.61 & & \\
\hline & & 17.0 & 1016.63 & & & 20.0 & 1015.65 & & \\
\hline & & 18.0 & 1015.71 & & & 23.0 & 1015.69 & & \\
\hline & & 19.0 & 1015.64 & & & 26.0 & 1015.80 & & \\
\hline & & 21.0 & 1015.57 & & & 30.0 & 1015.78 & & \\
\hline & & 24.0 & 1015.64 & & & 35.0 & 1015.71 & & \\
\hline & & 27.0 & 1015.72 & & & 40.0 & 1015.71 & & \\
\hline & & 30.0 & 1015.77 & & & 45.0 & 1015.92 & & \\
\hline & & 33.0 & 1015.76 & & & 46.0 & 1016.01 & & \\
\hline & & 36.0 & 1015.80 & & & 48.0 & 1016.05 & & \\
\hline & & 39.0 & 1015.72 & & & 52.0 & 1016.06 & & \\
\hline & & 42.0 & 1015.80 & & & 56.0 & 1016.03 & & \\
\hline & & 45.0 & 1015.90 & & & 60.0 & 1015.95 & & \\
\hline & & 48.0 & 1015.96 & & & 65.0 & 1015.92 & & \\
\hline & & 51.0 & 1015.87 & & & 70.0 & 1015.99 & & \\
\hline & & 54.0 & 1015.91 & & & 73.0 & 1016.06 & & \\
\hline & & 57.0 & 1015.97 & & & 76.0 & 1016.13 & & \\
\hline & & 60.0 & 1016.03 & & & 77.5 & 1016.25 & & \\
\hline & & 63.0 & 1016.04 & & & 79.8 & 1016.23 & & \\
\hline & & 66.0 & 1016.01 & & & 81.0 & 1016.15 & & \\
\hline & & 69.0 & 1015.99 & & & 82.0 & 1016.10 & & \\
\hline & & 72.0 & 1015.92 & & & 83.0 & 1016.03 & & \\
\hline & & 75.0 & 1016.09 & & & 84.0 & 1016.25 & & \\
\hline & & 78.0 & 1016.15 & & & 84.4 & 1016.55 & & \\
\hline & & 81.0 & 1016.18 & & & 84.8 & 1016.62 & & \\
\hline & & 83.0 & 1016.23 & & & 86.0 & 1017.38 & & \\
\hline & & 84.0 & 1016.56 & & & 88.0 & 1018.37 & & \\
\hline & & 84.8 & 1016.62 & & & 89.0 & 1018.69 & & \\
\hline & & 87.0 & 1017.77 & & & 90.0 & 1018.83 & & \\
\hline & & 88.0 & 1018.30 & & & 92.0 & 1018.86 & & \\
\hline & & 89.0 & 1018.67 & & & 93.5 & 1019.01 & & \\
\hline & & 90.0 & 1018.82 & & & 95.0 & 1018.72 & & \\
\hline & & 92.0 & 1018.87 & & & 96.0 & 1018.50 & & \\
\hline & & 94.0 & 1018.88 & & & 98.0 & 1018.46 & & \\
\hline
\end{tabular}


Table 8.--Listing of horizontal stations and elevations for cross section PR116 --Continued.

\begin{tabular}{|c|c|c|c|c|c|c|c|c|c|}
\hline \multicolumn{2}{|c|}{1984} & \multicolumn{2}{|c|}{1985} & \multicolumn{2}{|c|}{1985} & \multicolumn{2}{|c|}{1986} & \multicolumn{2}{|c|}{1986} \\
\hline Sta. & Elev. & Sta. & Elev. & Sta. & Elev. & Sta. & Elev. & Sta. & Elev. \\
\hline-1.0 & 1019.83 & 1.0 & 1019.61 & 85.0 & 1017.12 & 0.0 & 1019.62 & 94.4 & 1018.97 \\
\hline 0.0 & 1019.62 & 3.0 & 1019.37 & 87.3 & 1017.84 & 3.0 & 1019.19 & 96.0 & 1018.52 \\
\hline 3.0 & 1019.20 & 4.5 & 1018.93 & 89.0 & 1018.61 & 5.0 & 1018.93 & 97.0 & 1018.49 \\
\hline 5.0 & 1018.94 & 6.0 & 1019.05 & 90.0 & 1018.82 & 7.0 & 1019.10 & 100.0 & 1018.53 \\
\hline 8.0 & 1019.17 & 8.0 & 1019.18 & 93.0 & 1018.97 & 9.0 & 1019.17 & 101.0 & 1018.94 \\
\hline 10.0 & 1019.21 & 10.0 & 1019.21 & 95.0 & 1018.75 & 11.0 & 1019.14 & 102.0 & 1019.07 \\
\hline 11.8 & 1019.06 & 12.0 & 1018.99 & 97.0 & 1018.48 & 12.4 & 1018.92 & 103.0 & 1019.28 \\
\hline 13.3 & 1018.67 & 13.6 & 1018.58 & 99.0 & 1018.55 & 13.6 & 1018.56 & 105.0 & 1019.31 \\
\hline 15.3 & 1017.24 & 14.4 & 1018.15 & 100.5 & 1018.65 & 14.4 & 1018.16 & & \\
\hline 16.5 & 1016.71 & 14.7 & 1017.67 & 101.0 & 1018.95 & 14.7 & 1017.65 & & \\
\hline 16.7 & 1016.20 & 16.4 & 1016.70 & 103.0 & 1019.25 & 15.7 & 1017.09 & & \\
\hline 17.0 & 1015.90 & 16.6 & 1016.20 & & & 17.0 & 1016.08 & & \\
\hline 18.0 & 1015.59 & 17.1 & 1015.92 & & & 17.6 & 1015.71 & & \\
\hline 20.0 & 1015.70 & 17.6 & 1015.73 & & & 18.3 & 1015.57 & & \\
\hline 22.0 & 1015.68 & 19.0 & 1015.61 & & & 20.0 & 1015.71 & & \\
\hline 25.0 & 1015.75 & 20.0 & 1015.61 & & & 22.0 & 1015.70 & & \\
\hline 3.). 0 & 1015.73 & 21.5 & 1015.70 & & & 25.0 & 1015.86 & & \\
\hline 35.0 & 1015.82 & 23.0 & 1015.80 & & & 28.0 & 1015.87 & & \\
\hline 40.0 & 1015.91 & 25.0 & 1015.84 & & & 31.0 & 1015.85 & & \\
\hline 45.0 & 1015.99 & 27.0 & 1015.87 & & & 34.0 & 1015.75 & & \\
\hline 50.0 & 1016.04 & 28.0 & 1015.90 & & & 37.0 & 1015.81 & & \\
\hline 55.0 & 1015.99 & 30.0 & 1015.91 & & & 40.0 & 1015.79 & & \\
\hline 58.5 & 1016.00 & 32.0 & 1015.86 & & & 41.5 & 1015.84 & & \\
\hline 62.0 & 1016.03 & 34.0 & 1015.69 & & & 42.6 & 1015.99 & & \\
\hline 65.0 & 1016.01 & 36.0 & 1015.67 & & & 42.9 & 1016.18 & & \\
\hline 66.3 & 1016.07 & 38.0 & 1015.68 & & & 45.0 & 1016.19 & & \\
\hline 69.0 & 1016.10 & 40.0 & 1015.78 & & & 48.0 & 1016.30 & & \\
\hline 70.5 & 1016.06 & 42.0 & 1015.74 & & & 51.0 & 1016.34 & & \\
\hline 75.0 & 1015.93 & 43.5 & 1015.76 & & & 54.0 & 1016.33 & & \\
\hline 75.3 & 1016.05 & 46.6 & 1015.93 & & & 57.0 & 1016.30 & & \\
\hline 77.0 & 1016.06 & 48.0 & 1015.99 & & & 60.0 & 1016.27 & & \\
\hline 80.0 & 1015.84 & 51.0 & 1015.99 & & & 63.0 & 1016.27 & & \\
\hline 82.0 & 1015.85 & 54.0 & 1016.02 & & & 66.0 & 1016.24 & & \\
\hline 83.7 & 1016.11 & 57.0 & 1016.10 & & & 69.0 & 1016.27 & & \\
\hline 84.7 & 1017.03 & 60.0 & 1016.16 & & & 72.0 & 1016.22 & & \\
\hline 86.0 & 1017.39 & 62.0 & 1016.18 & & & 74.0 & 1016.16 & & \\
\hline 88.0 & 1018.31 & 65.0 & 1016.14 & & & 76.0 & 1016.03 & & \\
\hline 90.0 & 1018.83 & 67.0 & 1016.03 & & & 79.0 & 1015.87 & & \\
\hline 93.0 & 1018.99 & 70.0 & 1015.99 & & & 32.0 & 1015.82 & & \\
\hline 97.0 & 1018.49 & 73.0 & 1016.09 & & & 82.5 & 1015.93 & & \\
\hline \multirow[t]{5}{*}{100.0} & 1018.52 & 76.0 & 1016.06 & & & 84.7 & 1017.12 & & \\
\hline & & 80.0 & 1016.00 & & & 85.4 & 1017.23 & & \\
\hline & & 82.0 & 1016.00 & & & 86.0 & 1017.41 & & \\
\hline & & 82.5 & 1016.07 & & & 88.0 & 1018.31 & & \\
\hline & & 84.0 & 1016.53 & & & 90.0 & 1018.82 & & \\
\hline
\end{tabular}


Table 8.--Listing of horizontal stations and elevations for cross section PR116 --Continued.

\begin{tabular}{|c|c|c|c|c|c|c|c|}
\hline \multicolumn{2}{|c|}{1987} & \multicolumn{2}{|c|}{1987} & \multicolumn{2}{|c|}{1988} & \multicolumn{2}{|c|}{1988} \\
\hline Sta. & Elev. & Sta. & Elev. & Sta. & Elev. & Sta. & Elev. \\
\hline 0.0 & 1019.63 & 79.0 & 1016.28 & -1.7 & 1020.15 & 79.0 & 1016.01 \\
\hline 2.0 & 1019.06 & 80.0 & 1016.30 & 0.0 & 1019.65 & 81.0 & 1016.07 \\
\hline 4.6 & 1018.93 & 81.0 & 1016.22 & 2.0 & 1019.40 & 82.0 & 1016.07 \\
\hline 5.7 & 1018.97 & 83.0 & 1016.10 & 4.5 & 1018.95 & 82.6 & 1016.00 \\
\hline 6.0 & 1019.15 & 83.5 & 1016.31 & 5.5 & 1018.99 & 83.3 & 1016.17 \\
\hline 6.5 & 1019.15 & 84.4 & 1016.93 & 7.0 & 1019.12 & 84.6 & 1016.84 \\
\hline 6.6 & 1019.11 & 85.5 & 1017.49 & 9.0 & 1019.19 & 85.2 & 1017.39 \\
\hline 8.1 & 1019.17 & 86.5 & 1017.67 & 10.0 & 1019.23 & 86.0 & 1017.58 \\
\hline 8.7 & 1019.24 & 88.0 & 1018.36 & 12.0 & 1019.02 & 87.0 & 1017.80 \\
\hline 9.0 & 1019.18 & 89.7 & 1018.83 & 13.0 & 1018.75 & 89.0 & 1018.63 \\
\hline 10.0 & 1019.20 & 92.0 & 1018.90 & 13.7 & 1018.54 & 90.0 & 1018.85 \\
\hline 12.0 & 1019.02 & 93.2 & 1018.99 & 14.3 & 1018.19 & 92.0 & 1018.90 \\
\hline 13.5 & 1018.60 & 94.0 & 1018.84 & 14.7 & 1017.60 & 93.4 & 1019.00 \\
\hline 14.2 & 1018.16 & 96.0 & 1018.54 & 15.0 & 1017.25 & 94.5 & 1018.78 \\
\hline 14.8 & 1017.51 & 98.0 & 1018.45 & 16.0 & 1016.64 & 95.3 & 1018.75 \\
\hline 15.0 & 1017.19 & 99.0 & 1018.55 & 16.4 & 1016.23 & 96.3 & 1018.52 \\
\hline 16.0 & 1016.75 & 100.0 & 1018.56 & 17.0 & 1015.96 & 98.0 & 1018.47 \\
\hline 16.5 & 1016.30 & 101.0 & 1018.96 & 18.0 & 1015.68 & 100.0 & 1018.52 \\
\hline 17.6 & 1015.73 & 103.0 & 1019.29 & 20.0 & 1015.69 & 100.8 & 1018.76 \\
\hline 20.0 & 1015.80 & & & 23.0 & 1015.72 & 101.0 & 1018.93 \\
\hline 22.0 & 1015.56 & & & 26.0 & 1015.77 & 103.0 & 1019.26 \\
\hline 25.0 & 1015.66 & & & 29.0 & 1015.78 & 104.0 & 1019.40 \\
\hline 28.0 & 1015.71 & & & 32.0 & 1015.79 & 106.0 & 1019.31 \\
\hline 30.0 & 1015.75 & & & 35.0 & 1015.86 & 108.0 & 1019.30 \\
\hline 33.0 & 1015.84 & & & 38.0 & 1015.97 & 110.0 & 1019.42 \\
\hline 36.0 & 1015.95 & & & 40.0 & 1016.02 & 111.0 & 1019.57 \\
\hline 39.0 & 1016.04 & & & 42.0 & 1016.04 & 112.6 & 1019.83 \\
\hline 42.0 & 1016.01 & & & 43.0 & 1016.03 & & \\
\hline 45.0 & 1016.00 & & & 45.0 & 1015.93 & & \\
\hline 48.0 & 1015.93 & & & 47.0 & 1015.93 & & \\
\hline 51.0 & 1015.99 & & & 49.0 & 1016.08 & & \\
\hline 54.0 & 1015.92 & & & 51.0 & 1016.11 & & \\
\hline 56.0 & 1016.05 & & & 52.0 & 1016.23 & & \\
\hline 59.0 & 1016.07 & & & 54.0 & 1016.29 & & \\
\hline 61.0 & 1016.12 & & & 57.0 & 1016.31 & & \\
\hline 62.1 & 1016.26 & & & 60.0 & 1016.30 & & \\
\hline 62.4 & 1016.41 & & & 63.0 & 1016.35 & & \\
\hline 64.0 & 1016.42 & & & 66.0 & 1016.39 & & \\
\hline 66.0 & 1016.40 & & & 68.0 & 1016.40 & & \\
\hline 68.0 & 1016.36 & & & 70.0 & 1016.39 & & \\
\hline 70.0 & 1016.31 & & & 71.0 & 1016.30 & & \\
\hline 72.0 & 1016.23 & & & 72.0 & 1016.26 & & \\
\hline 74.0 & 1016.25 & & & 73.0 & 1016.09 & & \\
\hline 75.0 & 1016.24 & & & 74.0 & 1016.02 & & \\
\hline 77.0 & 1016.28 & & & 76.0 & 1015.98 & & \\
\hline
\end{tabular}




\section{Description of Cross Section PR120}

Location: Township 8 South/Range 48 East--section 33

U.S. Geological Survey quadrangle $(1: 24,000)$ : Moorhead

Landowners--left bank: Harold Crocker

right bank: Amory Hubbard

Access: left bank

Permission from: Hugh Fulton (T8S/R48E--32) or

George Fulton (T9S/R48E--8)

Reference pins

\begin{tabular}{lll}
\hline $\begin{array}{l}\text { Station } \\
\text { (meters) }\end{array}$ & $\begin{array}{c}\text { Elevation } \\
\text { (meters } \\
\text { above } \\
\text { sea level) }\end{array}$ & Comments \\
& & Both of these pins were across the \\
river from the leveling instrument. & \\
100.9 & $1,013.927$ & 0.16 meter above 1987 ground level. \\
104.0 & $1,014.043$ & 0.20 meter above 1987 ground level. \\
\hline
\end{tabular}




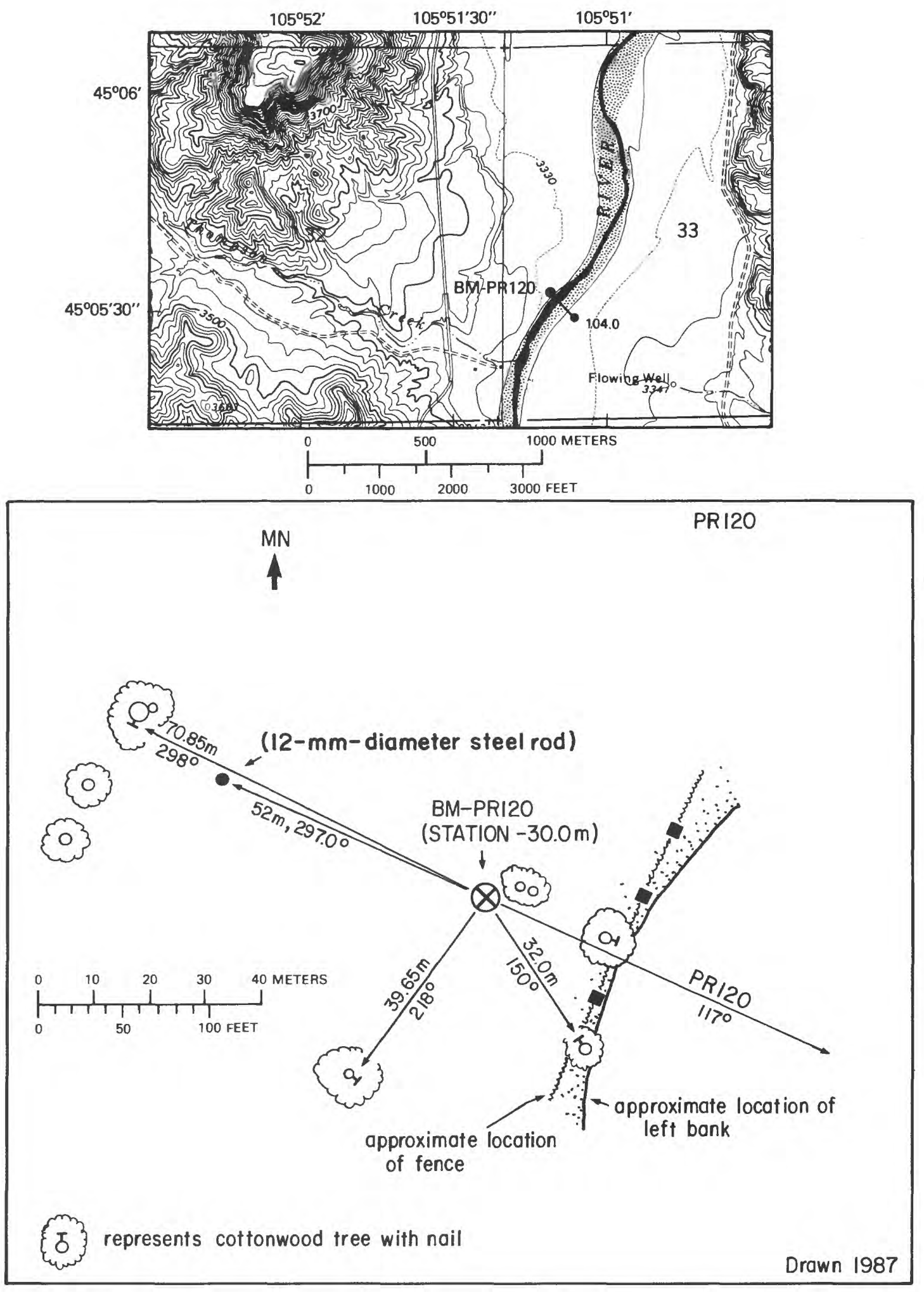

Figure 16.--Upper: Location of cross section PR120, bench mark BM-PR120, and right bank reference pins (tables 2 and 3 ) in Moorhead quadrangle. Lower: Location of bench mark on the left bank. MN is magnetic north. 

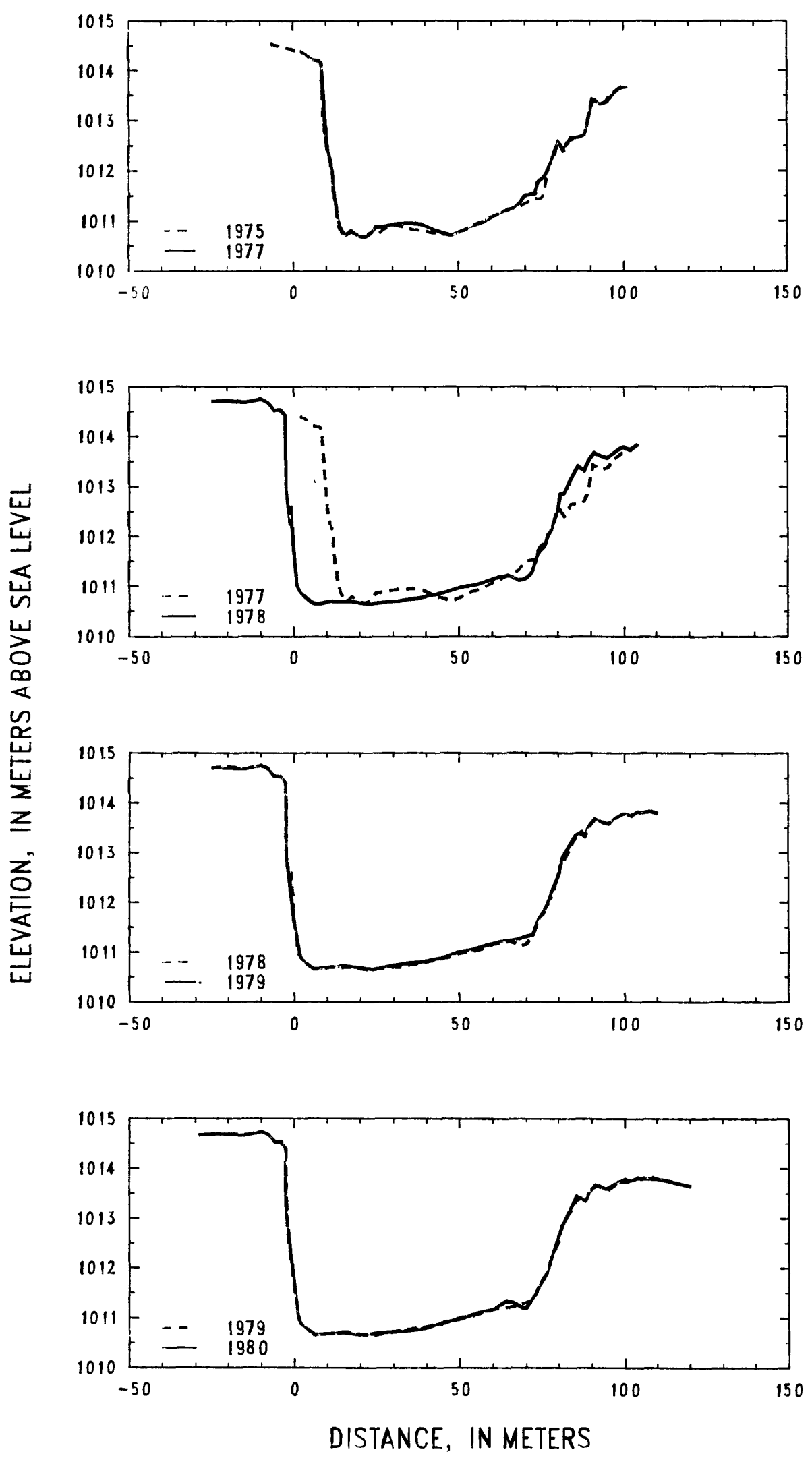

Figure 17. --Profiles of cross section PR120 from 1975 to 1980. 

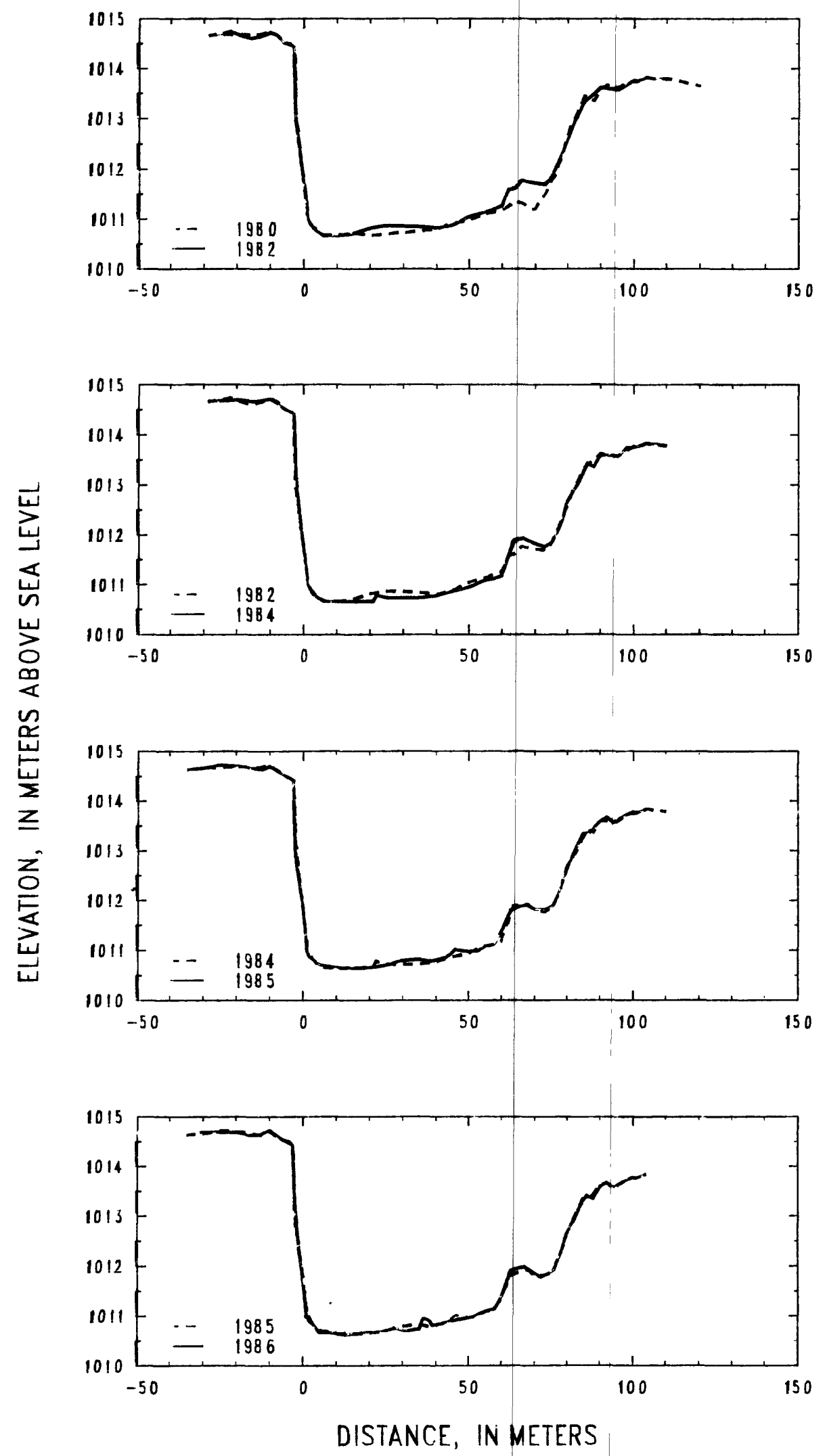

Figure 18 --Profiles of cross section PR120 from 1980 to 1986. 


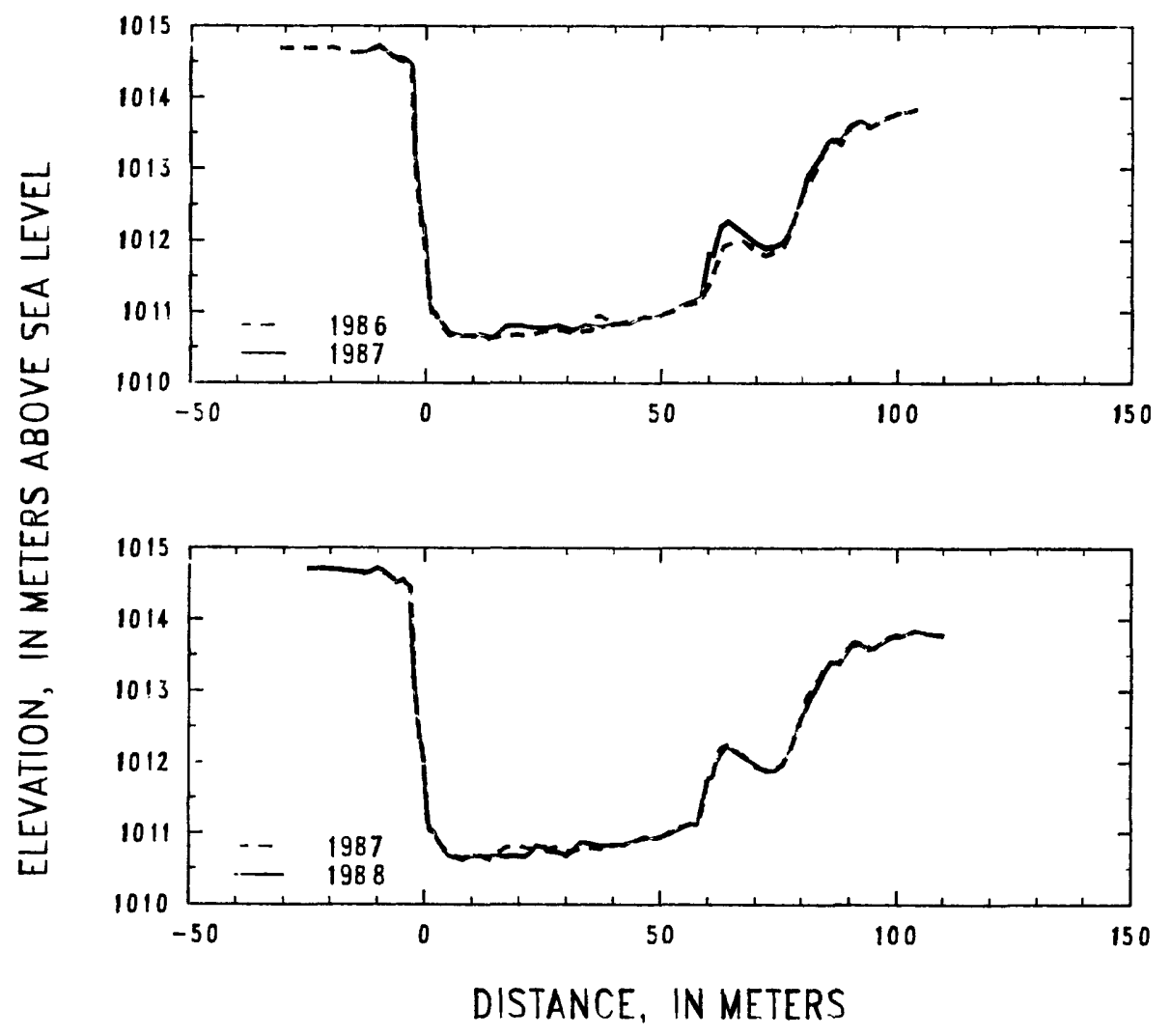

Figure 19.--Profiles of cross section PR120 from 1986 to 1988. 
Table 9.--Listing of horizontal stations and elevations for cross section PR120 [Sta., station, distance in meters from a reference point on

the left bank; Elev., elevation, in meters above sea levell.

\begin{tabular}{|c|c|c|c|c|c|c|c|c|c|}
\hline \multicolumn{2}{|c|}{1975} & \multicolumn{2}{|c|}{1975} & \multicolumn{2}{|c|}{1977} & \multicolumn{2}{|c|}{1977} & \multicolumn{2}{|c|}{1978} \\
\hline Sta. & Elev. & Sta. & Elev. & Sta. & Elev. & Sta. & Elev. & Sta. & Elev. \\
\hline-6.8 & 1014.53 & 68.0 & 1011.27 & 2.0 & 1014.40 & 74.1 & 1011.77 & -25.0 & 1014.71 \\
\hline 0.0 & 1014.42 & 70.0 & 1011.34 & 4.0 & 1014.33 & 76.0 & 1011.88 & -20.0 & 1014.73 \\
\hline 2.0 & 1014.39 & 72.0 & 1011.42 & 6.0 & 1014.22 & 78.0 & 1012.14 & -15.0 & 1014.68 \\
\hline 4.0 & 1014.33 & 74.0 & 1011.44 & 8.0 & 1014.19 & 80.2 & 1012.57 & -10.0 & 1014.76 \\
\hline 6.0 & 1014.22 & 75.0 & 1011.45 & 8.7 & 1014.14 & 82.0 & 1012.41 & -8.0 & 1014.69 \\
\hline 8.0 & 1014.21 & 76.0 & 1011.56 & 10.3 & 1012.48 & 84.0 & 1012.65 & -6.0 & 1014.53 \\
\hline 8.7 & 1014.15 & 76.7 & 1011.86 & 11.8 & 1012.07 & 86.0 & 1012.65 & -4.0 & 1014.54 \\
\hline 9.0 & 1013.38 & 77.3 & 1012.04 & 12.6 & 1011.46 & 88.0 & 1012.71 & -2.5 & 1014.42 \\
\hline 9.7 & 1012.77 & 78.0 & 1012.14 & 13.1 & 1011.29 & 89.0 & 1012.88 & -2.2 & 1012.89 \\
\hline 10.4 & 1012.42 & 79.0 & 1012.26 & 13.5 & 1011.08 & 90.6 & 1013.43 & -1.1 & 1012.34 \\
\hline 11.0 & 1012.27 & 79.9 & 1012.52 & 14.2 & 1010.91 & 93.0 & 1013.33 & -0.7 & 1012.48 \\
\hline 12.0 & 1011.94 & 80.2 & 1012.57 & 15.0 & 1010.77 & 95.0 & 1013.38 & 0.0 & 1011.70 \\
\hline 12.5 & 1011.48 & 82.0 & 1012.36 & 16.0 & 1010.71 & 97.0 & 1013.55 & 1.1 & 1011.03 \\
\hline 13.0 & 1011.27 & 83.0 & 1012.56 & 17.6 & 1010.81 & 99.0 & 1013.66 & 2.0 & 1010.89 \\
\hline 13.3 & 1011.18 & 84.0 & 1012.60 & 20.0 & 1010.69 & 100.9 & 1013.68 & 4.0 & 1010.76 \\
\hline 13.7 & 1010.96 & 86.0 & 1012.64 & 21.0 & 1010.68 & & & 6.0 & 1010.66 \\
\hline 14.0 & 1010.86 & 88.0 & 1012.71 & 22.0 & 1010.68 & & & 8.0 & 1010.65 \\
\hline 15.0 & 1010.72 & 89.0 & 1012.91 & 23.0 & 1010.71 & & & 9.0 & 1010.67 \\
\hline 16.0 & 1010.72 & 90.0 & 1013.24 & 24.0 & 1010.78 & & & 11.0 & 1010.71 \\
\hline 18.0 & 1010.77 & 91.0 & 1013.41 & 25.0 & 1010.88 & & & 14.0 & 1010.69 \\
\hline 20.0 & 1010.70 & 92.0 & 1013.40 & 27.5 & 1010.89 & & & 17.0 & 1010.71 \\
\hline 22.0 & 1010.68 & 94.0 & 1013.33 & 30.0 & 1010.93 & & & 20.0 & 1010.67 \\
\hline 23.0 & 1010.75 & 96.0 & 1013.49 & 32.0 & 1010.95 & & & 23.0 & 1010.64 \\
\hline 24.0 & 1010.77 & 98.0 & 1013.62 & 34.0 & 1010.95 & & & 26.0 & 1010.68 \\
\hline 26.0 & 1010.75 & 100.0 & 1013.70 & 36.0 & 1010.96 & & & 29.0 & 1010.70 \\
\hline 28.0 & 1010.86 & & & 38.0 & 1010.94 & & & 32.0 & 1010.71 \\
\hline 30.0 & 1010.93 & & & 38.8 & 1010.93 & & & 35.0 & 1010.75 \\
\hline 32.0 & 1010.90 & & & 40.0 & 1010.90 & & & 38.0 & 1010.77 \\
\hline 34.0 & 1010.88 & & & 42.0 & 1010.84 & & & 41.0 & 1010.83 \\
\hline 36.0 & 1010.83 & & & 44.0 & 1010.78 & & & 44.0 & 1010.86 \\
\hline 38.0 & 1010.81 & & & 46.0 & 1010.74 & & & 47.0 & 1010.91 \\
\hline 40.0 & 1010.79 & & & 48.0 & 1010.71 & & & 50.0 & 1010.98 \\
\hline 42.0 & 1010.76 & & & 50.0 & 1010.77 & & & 55.4 & 1011.04 \\
\hline 44.0 & 1010.75 & & & 52.0 & 1010.83 & & & 59.0 & 1011.13 \\
\hline 46.0 & 1010.73 & & & 54.0 & 1010.90 & & & 62.0 & 1011.20 \\
\hline 48.0 & 1010.70 & & & 56.0 & 1010.94 & & & 65.0 & 1011.22 \\
\hline 50.0 & 1010.76 & & & 58.0 & 1011.00 & & & 68.0 & 1011.13 \\
\hline 52.0 & 1010.82 & & & 60.0 & 1011.08 & & & 70.0 & 1011.16 \\
\hline 54.0 & 1010.90 & & & 62.0 & 1011.14 & & & 72.0 & 1011.29 \\
\hline 56.3 & 1010.96 & & & 64.0 & 1011.21 & & & 73.5 & 1011.58 \\
\hline 58.0 & 1011.00 & & & 66.0 & 1011.25 & & & 74.0 & 1011.68 \\
\hline 60.0 & 1011.08 & & & 68.0 & 1011.33 & & & 75.0 & 1011.75 \\
\hline 62.0 & 1011.13 & & & 70.0 & 1011.50 & & & 76.0 & 1011.84 \\
\hline 64.0 & 1011.19 & & & 72.0 & 1011.54 & & & 77.0 & 1012.05 \\
\hline 66.0 & 1011.25 & & & 73.1 & 1011.54 & & & 78.0 & 1012.12 \\
\hline
\end{tabular}


Table 9.--Listing of horizontal stations and elevations for cross section PR120 --Continued.

\begin{tabular}{|c|c|c|c|c|c|c|c|c|c|}
\hline \multicolumn{2}{|c|}{1978} & \multicolumn{2}{|c|}{1979} & \multicolumn{2}{|c|}{1979} & \multicolumn{2}{|c|}{1980} & \multicolumn{2}{|c|}{1980} \\
\hline Sta. & Elev. & Sta. & Elev. & Sta. & Elev. & Sta. & Elev. & Sta. & Elev. \\
\hline 79.0 & 1012.33 & -25.0 & 1014.70 & 87.0 & 1013.42 & -29.0 & 1014.67 & 104.0 & 1013.82 \\
\hline 80.0 & 1012.51 & -20.0 & 1014.70 & 88.0 & 1013.32 & -25.0 & 1014.69 & 106.0 & 1013.79 \\
\hline 80.7 & 1012.85 & -15.0 & 1014.68 & 89.0 & 1013.51 & -20.0 & 1014.69 & 110.0 & 1013.79 \\
\hline 82.0 & 1012.87 & -10.0 & 1014.75 & 91.0 & 1013.68 & -15.0 & 1014.66 & 115.0 & 1013.72 \\
\hline 84.0 & 1013.17 & -8.0 & 1014.67 & 93.0 & 1013.61 & -10.0 & 1014.74 & 120.0 & 1013.64 \\
\hline 86.0 & 1013.41 & -6.0 & 1014.53 & 95.0 & 1013.57 & -8.0 & 1014.67 & & \\
\hline 88.0 & 1013.32 & -4.0 & 1014.53 & 97.0 & 1013.69 & -6.0 & 1014.52 & & \\
\hline 89.0 & 1013.50 & -2.5 & 1014.40 & 99.0 & 1013.76 & -4.0 & 1014.50 & & \\
\hline 91.0 & 1013.68 & -2.4 & 1013.04 & 100.0 & 1013.79 & -2.6 & 1014.40 & & \\
\hline 93.0 & 1013.61 & -2.0 & 1012.71 & 100.9 & 1013.76 & -2.5 & 1013.16 & & \\
\hline 95.0 & 1013.57 & -1.0 & 1012.19 & 102.0 & 1013.74 & 0.0 & 1011.66 & & \\
\hline 97.0 & 1013.69 & 0.0 & 1011.63 & 104.0 & 1013.83 & 1.3 & 1011.02 & & \\
\hline 99.0 & 1013.77 & 1.0 & 1011.21 & 105.0 & 1013.80 & 2.0 & 1010.88 & & \\
\hline 100.0 & 1013.79 & 2.0 & 1010.89 & 108.0 & 1013.84 & 4.0 & 1010.78 & & \\
\hline 100.9 & 1013.75 & 4.0 & 1010.76 & 110.0 & 1013.79 & 6.0 & 1010.67 & & \\
\hline 102.0 & 1013.73 & 6.0 & 1010.66 & & & 10.0 & 1010.68 & & \\
\hline 104.0 & 1013.84 & 9.0 & 1010.68 & & & 15.0 & 1010.71 & & \\
\hline & & 12.0 & 1010.70 & & & 20.0 & 1010.66 & & \\
\hline & & 15.0 & 1010.73 & & & 25.0 & 1010.70 & & \\
\hline & & 18.0 & 1010.70 & & & 30.0 & 1010.73 & & \\
\hline & & 21.0 & 1010.67 & & & 35.0 & 1010.75 & & \\
\hline & & 24.0 & 1010.65 & & & 40.0 & 1010.80 & & \\
\hline & & 27.0 & 1010.70 & & & 45.0 & 1010.91 & & \\
\hline & & 30.0 & 1010.73 & & & 50.0 & 1010.97 & & \\
\hline & & 33.0 & 1010.77 & & & 55.0 & 1011.10 & & \\
\hline & & 36.0 & 1010.79 & & & 60.0 & 1011.17 & & \\
\hline & & 39.0 & 1010.81 & & & 64.0 & 1011.35 & & \\
\hline & & 42.0 & 1010.86 & & & 66.0 & 1011.31 & & \\
\hline & & 45.0 & 1010.88 & & & 69.0 & 1011.19 & & \\
\hline & & 48.0 & 1010.97 & & & 70.0 & 1011.20 & & \\
\hline & & 51.0 & 1011.02 & & & 72.0 & 1011.41 & & \\
\hline & & 54.0 & 1011.05 & & & 73.0 & 1011.49 & & \\
\hline & & 57.0 & 1011.12 & & & 75.0 & 1011.77 & & \\
\hline & & 60.0 & 1011.17 & & & 76.0 & 1011.86 & & \\
\hline & & 63.0 & 1011.22 & & & 78.0 & 1012.22 & & \\
\hline & & 66.0 & 1011.23 & & & 81.0 & 1012.84 & & \\
\hline & & 69.0 & 1011.30 & & & 83.0 & 1013.11 & & \\
\hline & & 72.0 & 1011.35 & & & 85.3 & 1013.45 & & \\
\hline & & 74.0 & 1011.66 & & & 88.0 & 1013.34 & & \\
\hline & & 76.0 & 1011.87 & & & 90.0 & 1013.60 & & \\
\hline & & 78.0 & 1012.22 & & & 92.0 & 1013.67 & & \\
\hline & & 80.0 & 1012.54 & & & 94.0 & 1013.58 & & \\
\hline & & 81.0 & 1012.87 & & & 96.0 & 1013.64 & & \\
\hline & & 83.0 & 1013.09 & & & 98.0 & 1013.73 & & \\
\hline & & 85.0 & 1013.34 & & & 100.9 & 1013.74 & & \\
\hline
\end{tabular}


Table 9.--Listing of horizontal stations and elevations for cross section PR120 --Continued.

\begin{tabular}{|c|c|c|c|c|c|c|c|c|c|}
\hline \multicolumn{2}{|c|}{1982} & \multicolumn{2}{|c|}{1984} & \multicolumn{2}{|c|}{1985} & \multicolumn{2}{|c|}{1985} & \multicolumn{2}{|c|}{1986} \\
\hline Sta. & Elev. & Sta. & Elev. & Sta. & Elev. & Sta. & Elev. & Sta. & Elev. \\
\hline-29.0 & 1014.67 & -29.0 & 1014.68 & -35.0 & 1014.64 & 87.0 & 1013.36 & -31.0 & 1014.69 \\
\hline-25.0 & 1014.70 & -25.0 & 1014.68 & -29.0 & 1014.67 & 90.0 & 1013.59 & -25.0 & 1014.69 \\
\hline-22.0 & 1014.75 & -20.0 & 1014.71 & -25.0 & 1014.73 & 92.0 & 1013.67 & -20.0 & 1014.69 \\
\hline-19.0 & 1014.66 & -15.0 & 1014.66 & -20.0 & 1014.70 & 94.0 & 1013.56 & -16.0 & 1014.62 \\
\hline-16.0 & 1014.61 & -10.0 & 1014.72 & -15.0 & 1014.66 & 97.0 & 1013.69 & -13.0 & 1014.64 \\
\hline-13.0 & 1014.65 & -5.0 & 1014.49 & -12.0 & 1014.63 & 100.0 & 1013.78 & -10.0 & 1014.73 \\
\hline-10.0 & 1014.73 & -2.8 & 1014.41 & -10.0 & 1014.71 & 101.0 & 1013.76 & -6.0 & 1014.52 \\
\hline-8.0 & 1014.67 & -2.2 & 1013.13 & -7.0 & 1014.56 & 104.0 & 1013.83 & -4.0 & 1014.49 \\
\hline-6.0 & 1014.52 & -0.8 & 1012.26 & -5.0 & 1014.50 & & & -3.0 & 1014.42 \\
\hline-2.9 & 1014.44 & 1.5 & 1010.99 & -2.9 & 1014.42 & & & -2.4 & 1013.16 \\
\hline-2.5 & 1013.07 & 4.0 & 1010.74 & -2.4 & 1012.98 & & & -0.9 & 1012.14 \\
\hline 0.0 & 1011.74 & 7.0 & 1010.65 & 0.0 & 1011.84 & & & -0.3 & 1011.96 \\
\hline 0.5 & 1011.54 & 10.0 & 1010.66 & 1.3 & 1011.00 & & & 1.2 & 1010.98 \\
\hline 1.5 & 1010.98 & 16.0 & 1010.66 & 2.0 & 1010.88 & & & 2.0 & 1010.99 \\
\hline 3.0 & 1010.81 & 21.0 & 1010.66 & 5.0 & 1010.72 & & & 3.0 & 1010.82 \\
\hline 6.0 & 1010.66 & 22.0 & 1010.79 & 10.0 & 1010.65 & & & 5.0 & 1010.67 \\
\hline 10.0 & 1010.66 & 25.0 & 1010.73 & 15.0 & 1010.64 & & & 7.0 & 1010.67 \\
\hline 15.0 & 1010.71 & 30.0 & 1010.73 & 20.0 & 1010.66 & & & 10.0 & 1010.65 \\
\hline 20.0 & 1010.82 & 35.0 & 1010.73 & 25.0 & 1010.71 & & & 13.0 & 1010.61 \\
\hline 25.0 & 1010.87 & 40.0 & 1010.78 & 28.0 & 1010.77 & & & 16.0 & 1010.66 \\
\hline 30.0 & 1010.86 & 45.0 & 1010.87 & 30.0 & 1010.80 & & & 19.0 & 1010.67 \\
\hline 34.0 & 1010.85 & 50.0 & 1010.95 & 35.0 & 1010.83 & & & 22.0 & 1010.67 \\
\hline 40.0 & 1010.81 & 55.0 & 1011.09 & 38.0 & 1010.78 & & & 25.0 & 1010.72 \\
\hline 45.0 & 1010.88 & 57.0 & 1011.11 & 40.0 & 1010.81 & & & 28.0 & 1010.74 \\
\hline 50.0 & 1011.05 & 60.0 & 1011.18 & 43.5 & 1010.87 & & & 31.0 & 1010.70 \\
\hline 55.0 & 1011.14 & 63.7 & 1011.89 & 45.0 & 1010.95 & & & 34.0 & 1010.73 \\
\hline 60.0 & 1011.27 & 66.5 & 1011.93 & 46.0 & 1011.02 & & & 35.2 & 1010.74 \\
\hline 62.0 & 1011.59 & 70.0 & 1011.82 & 50.0 & 1010.97 & & & 36.2 & 1010.93 \\
\hline 64.0 & 1011.62 & 73.0 & 1011.76 & 53.0 & 1010.99 & & & 36.7 & 1010.95 \\
\hline 66.0 & 1011.77 & 75.5 & 1011.87 & 55.0 & 1011.08 & & & 38.4 & 1010.89 \\
\hline 70.0 & 1011.71 & 78.0 & 1012.22 & 58.0 & 1011.13 & & & 39.0 & 1010.81 \\
\hline 73.0 & 1011.69 & 80.0 & 1012.66 & 60.0 & 1011.37 & & & 40.0 & 1010.82 \\
\hline 75.0 & 1011.83 & 83.0 & 1013.00 & 63.0 & 1011.81 & & & 43.0 & 1010.86 \\
\hline 77.0 & 1012.12 & 86.0 & 1013.43 & 65.0 & 1011.87 & & & 46.0 & 1010.92 \\
\hline 79.0 & 1012.41 & 88.0 & 1013.36 & 68.0 & 1011.93 & & & 49.0 & 1010.95 \\
\hline 82.0 & 1012.92 & 90.0 & 1013.60 & 70.0 & 1011.82 & & & 52.0 & 1011.00 \\
\hline 85.0 & 1013.33 & 93.0 & 1013.59 & 73.0 & 1011.81 & & & 55.0 & 1011.09 \\
\hline 90.0 & 1013.62 & 95.0 & 1013.57 & 75.0 & 1011.86 & & & 58.0 & 1011.14 \\
\hline 95.0 & 1013.57 & 98.0 & 1013.74 & 76.0 & 1011.92 & & & 60.0 & 1011.37 \\
\hline 100.0 & 1013.77 & 101.0 & 1013.76 & 77.0 & 1012.11 & & & 63.0 & 1011.91 \\
\hline 100.9 & 1013.75 & 104.0 & 1013.84 & 78.0 & 1012.24 & & & 64.0 & 1011.93 \\
\hline 104.0 & 1013.82 & 110.0 & 1013.78 & 79.0 & 1012.41 & & & 67.0 & 1011.99 \\
\hline \multirow[t]{3}{*}{110.0} & 1013.77 & & & 80.0 & 1012.63 & & & 70.0 & 1011.85 \\
\hline & & & & 83.0 & 1013.09 & & & 72.0 & 1011.78 \\
\hline & & & & 85.0 & 1013.34 & & & 74.0 & 1011.84 \\
\hline
\end{tabular}


Table 9.--Listing of horizontal stations and elevations for cross section PR120 --Continued.

\begin{tabular}{|c|c|c|c|c|c|c|c|c|c|}
\hline \multicolumn{2}{|c|}{1986} & \multicolumn{2}{|c|}{1987} & \multicolumn{2}{|c|}{1987} & \multicolumn{2}{|c|}{1988} & \multicolumn{2}{|c|}{1988} \\
\hline Sta. & Elev. & Sta. & Elev. & Sta. & Elev. & Sta. & Elev. & Sta. & Elev. \\
\hline 76.0 & 1011.92 & -13.0 & 1014.63 & 81.0 & 1012.90 & -25.0 & 1014.71 & 60.2 & 1011.77 \\
\hline 78.0 & 1012.24 & -10.0 & 1014.72 & 84.0 & 1013.20 & -20.0 & 1014.71 & 61.0 & 1011.79 \\
\hline 80.0 & 1012.65 & -7.0 & 1014.57 & 85.0 & 1013.35 & -15.0 & 1014.66 & 62.3 & 1012.08 \\
\hline 82.0 & 1012.89 & -4.6 & 1014.54 & 86.0 & 1013.41 & -12.0 & 1014.65 & 64.0 & 1012.24 \\
\hline 84.0 & 1013.20 & -3.1 & 1014.46 & 88.0 & 1013.39 & -10.0 & 1014.73 & 66.0 & 1012.14 \\
\hline 86.0 & 1013.41 & -2.9 & 1014.41 & 90.0 & 1013.60 & -8.0 & 1014.64 & 69.0 & 1012.01 \\
\hline 88.0 & 1013.34 & -2.4 & 1013.75 & 92.0 & 1013.67 & -6.0 & 1014.51 & 72.0 & 1011.87 \\
\hline 90.0 & 1013.59 & -2.4 & 1013.19 & 95.0 & 1013.59 & -4.5 & 1014.56 & 74.0 & 1011.88 \\
\hline 92.0 & 1013.68 & -0.8 & 1012.25 & 98.0 & 1013.74 & -4.0 & 1014.50 & 76.0 & 1011.95 \\
\hline 94.0 & 1013.57 & -0.3 & 1012.19 & 101.0 & 1013.77 & -3.1 & 1014.46 & 78.0 & 1012.22 \\
\hline 97.0 & 1013.69 & 1.0 & 1011.10 & 104.0 & 1013.84 & -2.5 & 1013.75 & 79.8 & 1012.61 \\
\hline 100.0 & 1013.78 & 1.5 & 1010.98 & & & -2.4 & 1013.22 & 80.4 & 1012.64 \\
\hline 101.0 & 1013.76 & 3.0 & 1010.89 & & & -1.0 & 1012.32 & 81.5 & 1012.88 \\
\hline \multirow[t]{32}{*}{104.0} & 1013.84 & 5.0 & 1010.70 & & & -0.4 & 1012.19 & 83.0 & 1013.04 \\
\hline & & 8.0 & 1010.64 & & & 1.0 & 1011.05 & 84.0 & 1013.18 \\
\hline & & 11.0 & 1010.69 & & & 2.0 & 1011.02 & 86.0 & 1013.41 \\
\hline & & 14.0 & 1010.62 & & & 3.0 & 1010.86 & 88.0 & 1013.38 \\
\hline & & 17.0 & 1010.80 & & & 5.0 & 1010.68 & 90.0 & 1013.60 \\
\hline & & 20.0 & 1010.81 & & & 8.0 & 1010.62 & 91.0 & 1013.68 \\
\hline & & 22.0 & 1010.78 & & & 10.0 & 1010.67 & 92.0 & 1013.67 \\
\hline & & 25.0 & 1010.75 & & & 13.0 & 1010.66 & 94.0 & 1013.58 \\
\hline & & 28.0 & 1010.80 & & & 16.0 & 1010.65 & 96.0 & 1013.62 \\
\hline & & 31.0 & 1010.73 & & & 19.0 & 1010.67 & 98.0 & 1013.73 \\
\hline & & 34.0 & 1010.81 & & & 21.0 & 1010.66 & 100.0 & 1013.78 \\
\hline & & 37.0 & 1010.77 & & & 22.0 & 1010.69 & 101.0 & 1013.76 \\
\hline & & 40.0 & 1010.83 & & & 23.6 & 1010.82 & 104.0 & 1013.84 \\
\hline & & 43.0 & 1010.83 & & & 25.5 & 1010.79 & 107.0 & 1013.79 \\
\hline & & 46.0 & 1010.93 & & & 26.0 & 1010.73 & 110.0 & 1013.77 \\
\hline & & 49.0 & 1010.92 & & & 28.0 & 1010.73 & & \\
\hline & & 52.0 & 1011.00 & & & 29.5 & 1010.70 & & \\
\hline & & 54.5 & 1011.09 & & & 30.0 & 1010.66 & & \\
\hline & & 56.0 & 1011.13 & & & 32.0 & 1010.79 & & \\
\hline & & 58.5 & 1011.20 & & & 33.3 & 1010.87 & & \\
\hline & & 60.1 & 1011.81 & & & 35.0 & 1010.86 & & \\
\hline & & 61.0 & 1011.79 & & & 37.0 & 1010.81 & & \\
\hline & & 62.5 & 1012.19 & & & 40.0 & 1010.83 & & \\
\hline & & 64.0 & 1012.25 & & & 42.0 & 1010.85 & & \\
\hline & & 66.0 & 1012.16 & & & 44.0 & 1010.87 & & \\
\hline & & 68.0 & 1012.06 & & & 46.0 & 1010.92 & & \\
\hline & & 70.0 & 1011.94 & & & 49.0 & 1010.92 & & \\
\hline & & 72.0 & 1011.88 & & & 52.0 & 1010.99 & & \\
\hline & & 74.0 & 1011.90 & & & 55.0 & 1011.10 & & \\
\hline & & 76.0 & 1011.97 & & & 57.0 & 1011.13 & & \\
\hline & & 78.0 & 1012.25 & & & 57.7 & 1011.12 & & \\
\hline & & 80.0 & 1012.63 & & & 58.3 & 1011.23 & & \\
\hline
\end{tabular}




\section{Description of Cross Section PR122}

Location: Township 8 South/Range 48 East--section 28

U.S. Geological Survey quadrangle $(1: 24,000)$ : Moorhead

Landowners--left bank: Virginia Williams

right bank: U.S. Government

Access: left bank

Permission from: Gay Ranch (T7S/R49E--20)

Reference pins

\begin{tabular}{rll}
\hline $\begin{array}{l}\text { Station } \\
\text { (meters) }\end{array}$ & $\begin{array}{c}\text { Elevation } \\
\text { (meters } \\
\text { above } \\
\text { sea leve1) }\end{array}$ & \multicolumn{1}{c}{ Comments } \\
\hline-1.2 & $1,014.76$ & 0.18 meter above 1987 ground level. \\
0.0 & $1,014.56$ & $\begin{array}{l}0.07 \text { meter above 1987 ground leve1. } \\
\text { Bent, about } 0.08 \text { meter below } 1978 \\
\text { ground level. }\end{array}$ \\
136.8 & $1,009.29$ & $\begin{array}{l}\text { Bent over at 1978 ground level. } \\
0.26 \text { meter above 1987 ground level. }\end{array}$ \\
\hline
\end{tabular}




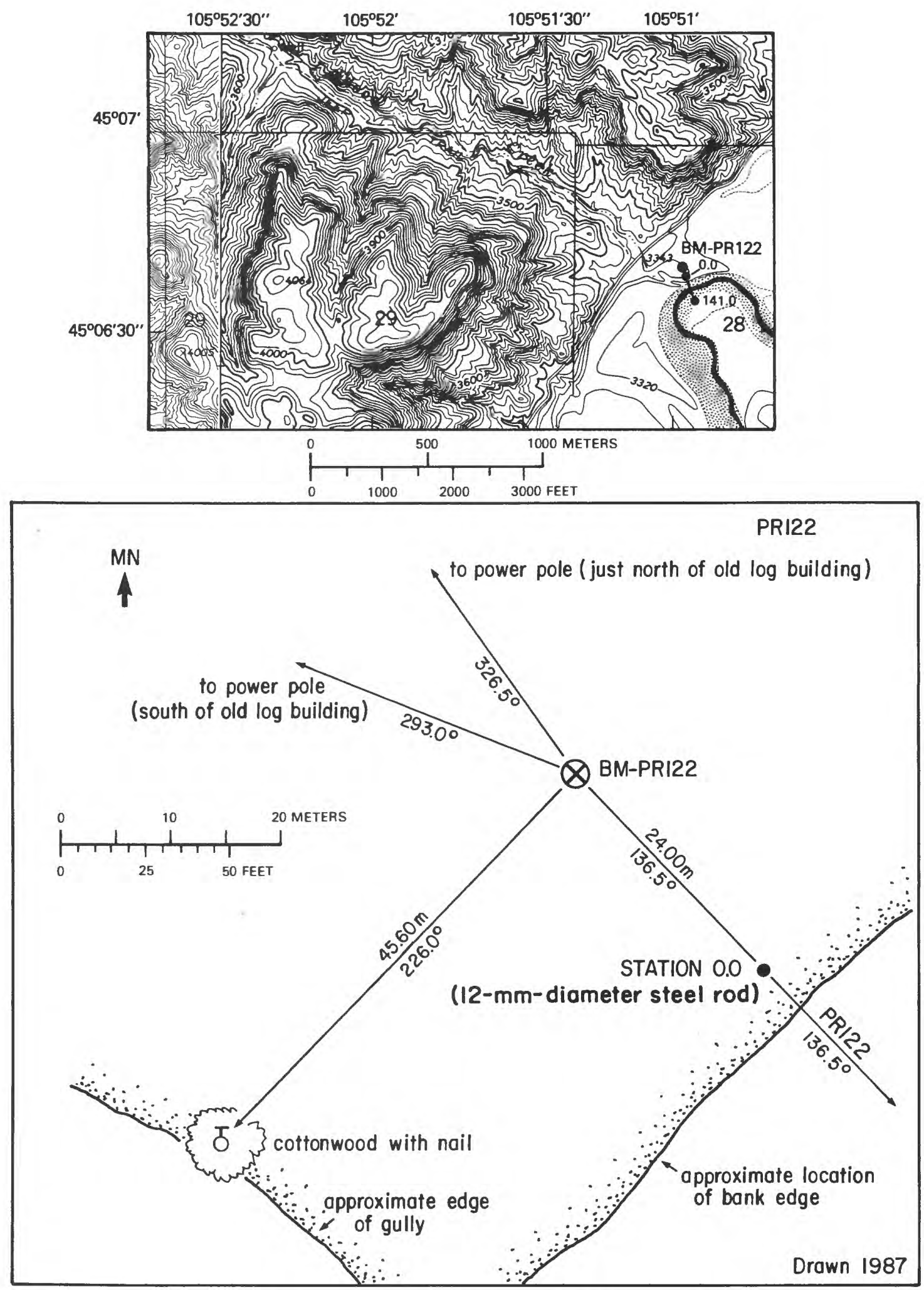

Figure 20.--Upper: Location of cross section PR122, bench mark BM-PR122, and left and right bank reference pins (tables 2 and 3 ) in the Moorhead quadrangle. Lower: Location of bench mark on the left bank. MN is magnetic north. 

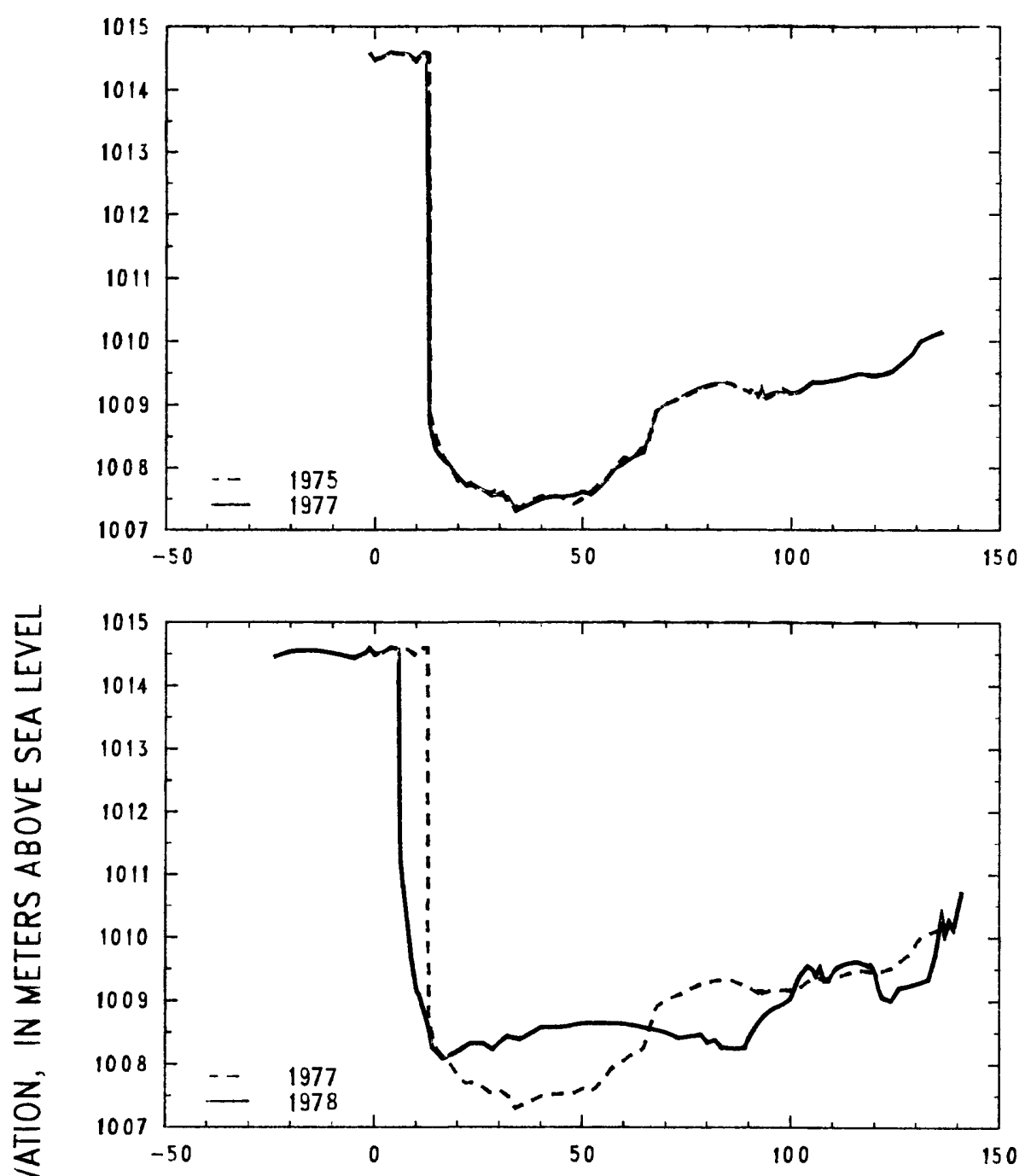

w

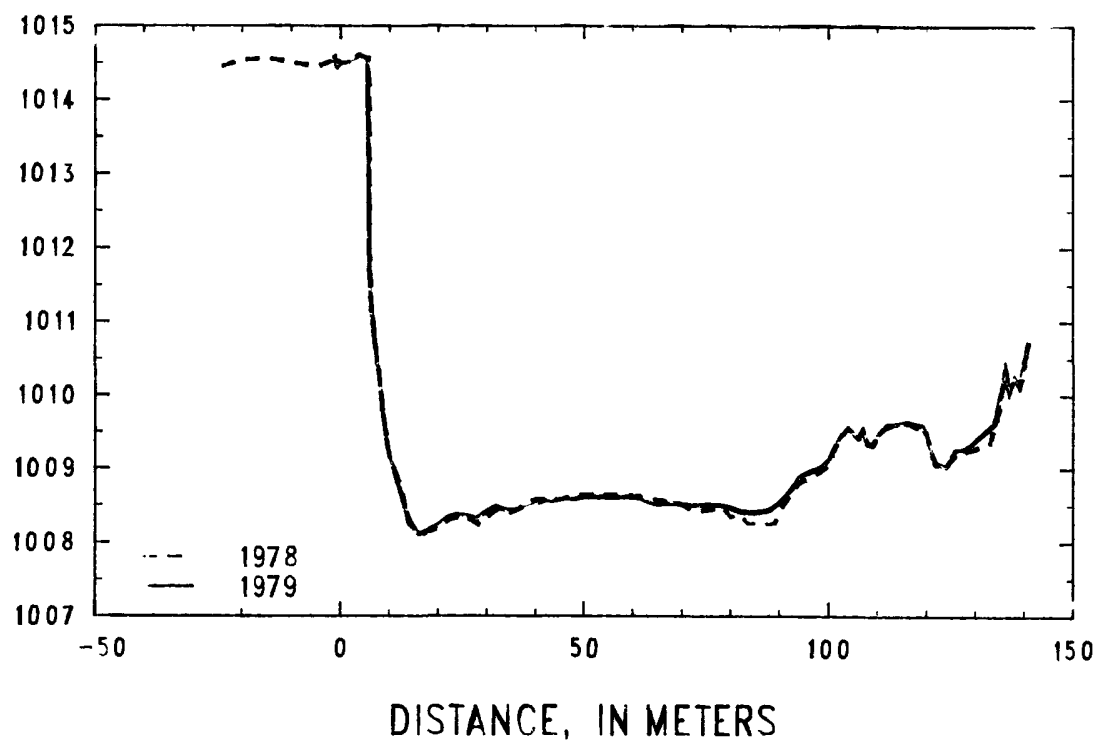

Figure 21---Protiles of cross section PR122 from 1975 to 1979. 


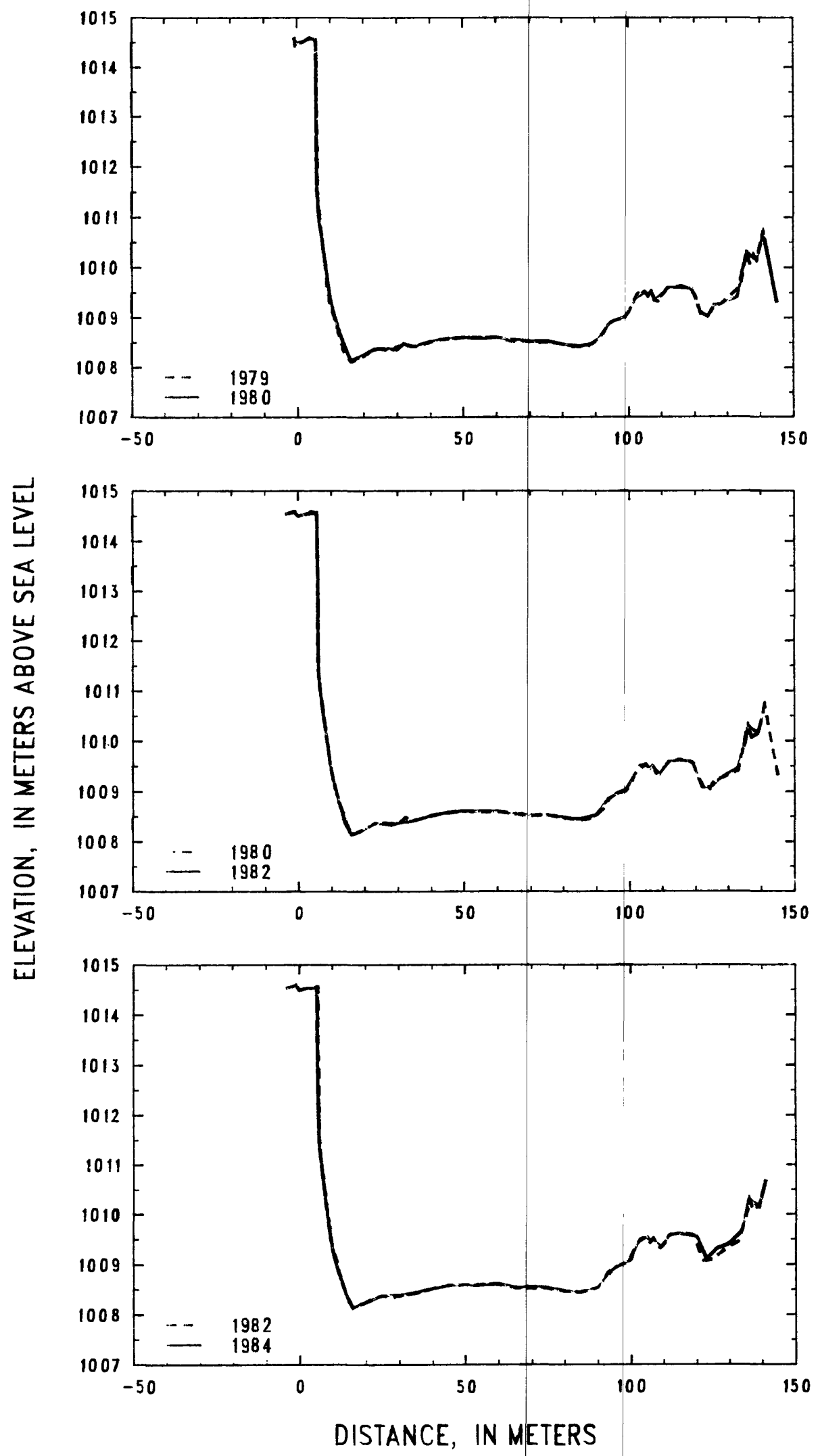

Figure 22. --Profiles of cross section PR122 from 1979 to 1984. 


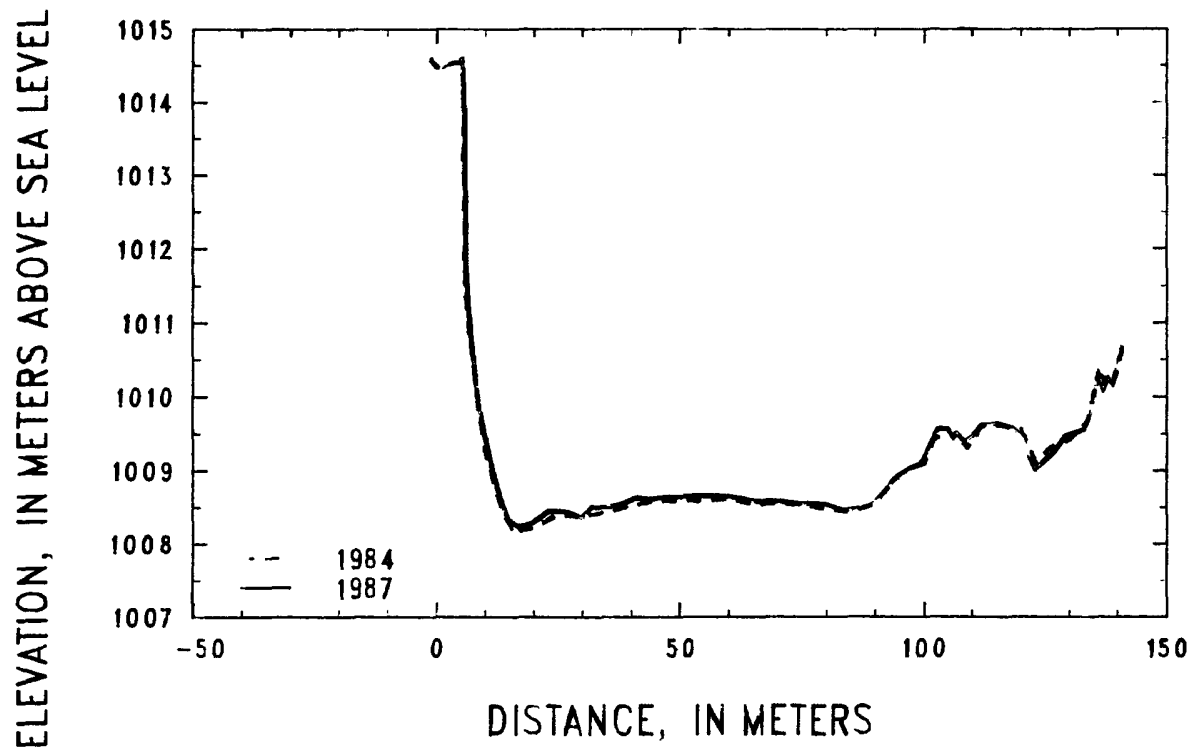

Figure 23.--Profiles of cross section PR122 from 1984101987. 
Table 10.--Listing of horizontal stations and elevations for cross section PR122

[Sta., station, distance in meters from a reference point on

the left bank; Elev., elevation, in meters above sea leve1].

\begin{tabular}{|c|c|c|c|c|c|c|c|c|c|}
\hline \multicolumn{2}{|c|}{1975} & \multicolumn{2}{|c|}{1975} & \multicolumn{2}{|c|}{1977} & \multicolumn{2}{|c|}{1977} & \multicolumn{2}{|c|}{1978} \\
\hline Sta. & Elev. & Sta. & Elev. & Sta. & Elev. & Sta. & Elev. & Sta. & $\overline{\text { Elev. }}$ \\
\hline 0.0 & 1014.47 & 78.0 & 1009.24 & -1.2 & 1014.58 & 93.3 & 1009.12 & -24.0 & 1014.45 \\
\hline 2.0 & 1014.51 & 80.0 & 1009.28 & 0.0 & 1014.48 & 96.0 & 1009.18 & -20.0 & 1014.54 \\
\hline 4.0 & 1014.60 & 82.0 & 1009.32 & 2.0 & 1014.52 & 100.0 & 1009.16 & -15.0 & 1014.56 \\
\hline 6.0 & 1014.57 & 84.0 & 1009.35 & 4.0 & 1014.60 & 101.8 & 1009.19 & -10.0 & 1014.51 \\
\hline 8.0 & 1014.56 & 86.0 & 1009.31 & 6.0 & 1014.58 & 105.0 & 1009.34 & -5.0 & 1014.43 \\
\hline 10.0 & 1014.44 & 88.0 & 1009.19 & 8.0 & 1014.57 & 108.0 & 1009.35 & -2.0 & 1014.52 \\
\hline 12.0 & 1014.61 & 89.0 & 1009.23 & 10.0 & 1014.47 & 112.0 & 1009.40 & -1.2 & 1014.59 \\
\hline 13.1 & 1014.58 & 90.0 & 1009.18 & 12.0 & 1014.60 & 116.0 & 1009.48 & 0.0 & 1014.49 \\
\hline 13.3 & 1008.87 & 91.0 & 1009.28 & 12.8 & 1014.59 & 120.0 & 1009.44 & 2.0 & 1014.51 \\
\hline 14.1 & 1008.62 & 92.0 & 1009.13 & 13.1 & 1008.73 & 124.0 & 1009.51 & 4.0 & 1014.60 \\
\hline 16.0 & 1008.23 & 93.0 & 1009.24 & 14.6 & 1008.28 & 128.0 & 1009.73 & 5.9 & 1014.56 \\
\hline 18.0 & 1008.02 & 94.0 & 1009.08 & 16.0 & 1008.16 & 129.0 & 1009.79 & 6.2 & 1011.75 \\
\hline 20.0 & 1007.78 & 96.0 & 1.009 .17 & 18.0 & 1008.02 & 131.0 & 1009.99 & 6.5 & 1011.12 \\
\hline 21.0 & 1007.73 & 98.0 & 1009.25 & 20.0 & 1007.83 & 134.0 & 1010.09 & 8.0 & 1010.24 \\
\hline 22.0 & 1007.71 & 100.0 & 1009.17 & 22.0 & 1007.69 & 136.0 & 1010.13 & 9.0 & 1009.62 \\
\hline 23.0 & 1007.75 & 102.1 & 1009.19 & 24.0 & 1007.71 & 136.2 & 1010.14 & 10.2 & 1009.15 \\
\hline 24.0 & 1007.68 & & & 26.0 & 1007.63 & & & 10.8 & 1009.10 \\
\hline 26.0 & 1007.62 & & & 28.0 & 1007.54 & & & 13.0 & 1008.62 \\
\hline 28.0 & 1007.59 & & & 30.0 & 1007.57 & & & 14.0 & 1008.26 \\
\hline 30.0 & 1007.69 & & & 32.0 & 1007.52 & & & 16.5 & 1008.09 \\
\hline 32.0 & 1007.45 & & & 34.0 & 1007.29 & & & 20.0 & 1008.20 \\
\hline 34.0 & 1007.36 & & & 36.0 & 1007.37 & & & 23.0 & 1008.33 \\
\hline 35.5 & 1007.36 & & & 38.0 & 1007.43 & & & 26.0 & 1008.33 \\
\hline 36.0 & 1007.40 & & & 40.0 & 1007.49 & & & 28.5 & 1008.23 \\
\hline 38.0 & 1007.49 & & & 42.0 & 1007.52 & & & 30.0 & 1008.33 \\
\hline 40.0 & 1007.54 & & & 44.0 & 1007.53 & & & 32.0 & 1008.44 \\
\hline 42.0 & 1007.54 & & & 46.0 & 1007.53 & & & 35.0 & 1008.39 \\
\hline 44.0 & 1007.54 & & & 48.0 & 1007.55 & & & 40.0 & 1008.58 \\
\hline 46.0 & 1007.48 & & & 50.0 & 1007.61 & & & 45.0 & 1008.58 \\
\hline 48.0 & 1007.41 & & & 52.0 & 1007.56 & & & 50.0 & 1008.65 \\
\hline 50.0 & 1007.50 & & & 53.2 & 1007.62 & & & 55.0 & 1008.65 \\
\hline 52.0 & 1007.61 & & & 55.0 & 1007.74 & & & 60.0 & 1008.64 \\
\hline 54.0 & 1007.72 & & & 57.0 & 1007.92 & & & 65.0 & 1008.57 \\
\hline 56.0 & 1007.82 & & & 60.0 & 1008.06 & & & 70.0 & 1008.50 \\
\hline 58.0 & 1008.02 & & & 62.0 & 1008.16 & & & 73.0 & 1008.41 \\
\hline 60.0 & 1008.15 & & & 64.6 & 1008.24 & & & 78.5 & 1008.47 \\
\hline 62.0 & 1008.12 & & & 67.8 & 1008.90 & & & 80.0 & 1008.34 \\
\hline 64.0 & 1008.29 & & & 70.0 & 1009.01 & & & 82.0 & 1008.38 \\
\hline 65.7 & 1008.33 & & & 74.0 & 1009.12 & & & 83.5 & 1008.26 \\
\hline 67.6 & 1008.88 & & & 78.0 & 1009.25 & & & 87.0 & 1008.24 \\
\hline 68.0 & 1008.90 & & & 82.0 & 1009.32 & & & 89.0 & 1008.26 \\
\hline 70.0 & 1009.00 & & & 86.0 & 1009.32 & & & 90.0 & 1008.41 \\
\hline 72.0 & 1009.06 & & & 90.0 & 1009.21 & & & 92.0 & 1008.64 \\
\hline 74.0 & 1009.11 & & & 92.2 & 1009.13 & & & 94.0 & 1008.80 \\
\hline 76.0 & 1009.17 & & & 92.9 & 1009.25 & & & 96.0 & 1008.88 \\
\hline
\end{tabular}


Table 10.--Listing of horizontal stations and elevations for cross section PR122 --Continued.

\begin{tabular}{|c|c|c|c|c|c|c|c|c|c|}
\hline \multicolumn{2}{|c|}{1978} & \multicolumn{2}{|c|}{1979} & \multicolumn{2}{|c|}{1979} & \multicolumn{2}{|c|}{1980} & \multicolumn{2}{|c|}{1980} \\
\hline Sta. & Elev. & Sta. & Elev. & Sta. & Elev. & Sta. & Elev. & Sta. & Elev. \\
\hline 98.0 & 1008.94 & -1.2 & 1014.59 & 80.0 & 1008.46 & -1.2 & 1014.59 & 128.0 & 1009.26 \\
\hline 100.0 & 1009.04 & -0.5 & 1014.44 & 82.0 & 1008.41 & 0.0 & 1014.48 & 133.0 & 1009.41 \\
\hline 101.8 & 1009.37 & 0.0 & 1014.49 & 84.0 & 1008.40 & 2.0 & 1014.51 & 136.0 & 1010.30 \\
\hline 104.0 & 1009.55 & 2.0 & 1014.51 & 86.0 & 1008.41 & 4.0 & 1014.59 & 139.0 & 1010.13 \\
\hline 105.0 & 1009.51 & 4.0 & 1014.60 & 88.0 & 1008.43 & 5.8 & 1014.56 & 141.0 & 1010.69 \\
\hline 106.0 & 1009.39 & 5.8 & 1014.55 & 90.0 & 1008.53 & 6.0 & 1011.54 & 145.0 & 1009.30 \\
\hline 107.0 & 1009.53 & 6.2 & 1011.46 & 92.0 & 1008.67 & 6.5 & 1011.05 & & \\
\hline 108.0 & 1009.32 & 8.0 & 1010.30 & 94.0 & 1008.88 & 10.0 & 1009.41 & & \\
\hline 109.0 & 1009.31 & 9.0 & 1009.64 & 96.0 & 1008.95 & 11.0 & 1009.09 & & \\
\hline 110.0 & 1009.45 & 10.0 & 1009.23 & 98.0 & 1008.99 & 13.0 & 1008.63 & & \\
\hline 111.0 & 1009.53 & 12.0 & 1008.80 & 100.0 & 1009.10 & 16.0 & 1008.13 & & \\
\hline 113.0 & 1009.59 & 14.0 & 1008.32 & 102.0 & 1009.40 & 20.0 & 1008.24 & & \\
\hline 116.0 & 1009.62 & 16.0 & 1008.11 & 104.0 & 1009.56 & 23.0 & 1008.36 & & \\
\hline 118.6 & 1009.55 & 16.6 & 1008.11 & 106.0 & 1009.42 & 26.0 & 1008.38 & & \\
\hline 119.0 & 1009.58 & 18.0 & 1008.17 & 107.0 & 1009.52 & 30.0 & 1008.35 & & \\
\hline 120.0 & 1009.47 & 20.0 & 1008.21 & 108.0 & 1009.34 & 32.0 & 1008.48 & & \\
\hline 121.0 & 1009.20 & 22.0 & 1008.33 & 109.0 & 1009.32 & 35.0 & 1008.42 & & \\
\hline 122.0 & 1009.05 & 24.0 & 1008.37 & 110.0 & 1009.45 & 40.0 & 1008.52 & & \\
\hline 124.0 & 1009.00 & 26.0 & 1008.36 & 112.0 & 1009.59 & 45.0 & 1008.57 & & \\
\hline 126.0 & 1009.21 & 28.0 & 1008.31 & 114.0 & 1009.60 & 50.0 & 1008.60 & & \\
\hline 128.0 & 1009.23 & 30.0 & 1008.42 & 116.0 & 1009.63 & 55.0 & 1008.58 & & \\
\hline 133.0 & 1009.34 & 32.0 & 1008.47 & 119.0 & 1009.58 & 60.0 & 1008.60 & & \\
\hline 134.6 & 1009.69 & 34.0 & 1008.42 & 120.0 & 1009.46 & 65.0 & 1008.54 & & \\
\hline 136.2 & 1010.36 & 36.0 & 1008.42 & 121.0 & 1009.24 & 70.0 & 1008.51 & & \\
\hline 137.0 & 1010.04 & 38.0 & 1008.47 & 122.0 & 1009.07 & 75.0 & 1008.53 & & \\
\hline 138.0 & 1010.26 & 40.0 & 1008.51 & 124.0 & 1009.01 & 80.0 & 1008.46 & & \\
\hline 139.0 & 1010.12 & 42.0 & 1008.57 & 126.0 & 1009.25 & 85.0 & 1008.42 & & \\
\hline 141.0 & 1010.72 & 44.0 & 1008.56 & 128.0 & 1009.26 & 89.0 & 1008.47 & & \\
\hline & & 46.0 & 1008.59 & 134.0 & 1009.61 & 92.0 & 1008.64 & & \\
\hline & & 48.0 & 1008.58 & 135.0 & 1009.91 & 94.0 & 1008.87 & & \\
\hline & & 50.0 & 1008.61 & 136.0 & 1010.30 & 96.0 & 1008.95 & & \\
\hline & & 52.0 & 1008.61 & 137.0 & 1010.06 & 99.0 & 1009.01 & & \\
\hline & & 54.0 & 1008.61 & 138.0 & 1010.25 & 101.0 & 1009.22 & & \\
\hline & & 56.0 & 1308.60 & 139.0 & 1010.14 & 102.0 & 1009.37 & & \\
\hline & & 58.0 & 1008.61 & 141.0 & 1010.72 & 105.0 & 1009.52 & & \\
\hline & & 60.0 & 1008.61 & & & 106.0 & 1009.45 & & \\
\hline & & 62.0 & 1008.58 & & & 107.0 & 1009.52 & & \\
\hline & & 64.0 & 1008.52 & & & 108.0 & 1009.34 & & \\
\hline & & 66.0 & 1008.51 & & & 110.0 & 1009.43 & & \\
\hline & & 68.0 & 1008.53 & & & 112.0 & 1009.59 & & \\
\hline & & 70.0 & 1008.52 & & & 115.0 & 1009.61 & & \\
\hline & & 72.0 & 1008.48 & & & 119.0 & 1009.58 & & \\
\hline & & 74.0 & 1008.49 & & & 122.0 & 1009.09 & & \\
\hline & & 76.0 & 1008.50 & & & 124.0 & 1009.01 & & \\
\hline & & 78.0 & 1008.49 & & & 126.0 & 1009.25 & & \\
\hline
\end{tabular}


Table 10.--Listing of horizontal stations and elevations for cross section PR122 --Continued.

\begin{tabular}{|c|c|c|c|c|c|c|c|c|c|}
\hline \multicolumn{2}{|c|}{1982} & \multicolumn{2}{|c|}{1982} & \multicolumn{2}{|c|}{1984} & \multicolumn{2}{|c|}{1987} & \multicolumn{2}{|c|}{1987} \\
\hline Sta. & Elev. & Sta. & Elev. & Sta. & Elev. & Sta. & Elev. & Sta. & Elev. \\
\hline-4.0 & 1014.53 & 135.0 & 1009.92 & -1.3 & 1014.60 & -1.3 & 1014.58 & 106.0 & 1009.44 \\
\hline-1.2 & 1014.59 & 136.0 & 1010.29 & 0.0 & 1014.49 & 0.0 & 1014.49 & 107.0 & 1009.51 \\
\hline 0.0 & 1014.49 & 137.0 & 1010.07 & 5.4 & 1014.56 & 1.0 & 1014.46 & 108.0 & 1009.37 \\
\hline 3.0 & 1014.55 & 139.0 & 1010.15 & 6.0 & 1011.43 & 3.0 & 1014.56 & 110.0 & 1009.46 \\
\hline 5.7 & 1014.57 & 141.0 & 1010.68 & 8.0 & 1010.18 & 5.4 & 1014.60 & 112.0 & 1009.60 \\
\hline 6.2 & 1011.26 & & & 10.0 & 1009.32 & 5.9 & 1013.89 & 115.0 & 1009.63 \\
\hline 8.0 & 1010.31 & & & 12.0 & 1008.81 & 6.2 & 1011.48 & 118.0 & 1009.59 \\
\hline 10.0 & 1009.41 & & & 14.0 & 1008.40 & 8.3 & 1010.08 & 120.0 & 1009.46 \\
\hline 12.0 & 1008.84 & & & 16.0 & 1008.13 & 11.0 & 1009.15 & 123.0 & 1009.01 \\
\hline 14.0 & 1008.38 & & & 18.0 & 1008.20 & 13.0 & 1008.66 & 125.0 & 1009.14 \\
\hline 16.0 & 1008.14 & & & 20.0 & 1008.24 & 15.0 & 1008.31 & 127.0 & 1009.26 \\
\hline 18.0 & 1008.18 & & & 25.0 & 1008.39 & 17.0 & 1008.24 & 129.0 & 1009.46 \\
\hline 22.0 & 1008.33 & & & 30.0 & 1008.38 & 20.0 & 1008.30 & 133.0 & 1009.54 \\
\hline 25.0 & 1008.38 & & & 35.0 & 1008.44 & 23.0 & 1008.44 & 134.5 & 1009.73 \\
\hline 28.0 & 1008.33 & & & 40.0 & 1008.52 & 26.0 & 1008.45 & 136.0 & 1010.30 \\
\hline 30.0 & 1008.38 & & & 45.0 & 1008.59 & 28.0 & 1008.41 & 137.0 & 1010.09 \\
\hline 35.0 & 1008.43 & & & 50.0 & 1008.59 & 30.0 & 1008.35 & 138.0 & 1010.24 \\
\hline 40.0 & 1008.52 & & & 55.0 & 1008.59 & 32.0 & 1008.52 & 139.0 & 1010.16 \\
\hline 45.0 & 1008.59 & & & 60.0 & 1008.62 & 35.0 & 1008.51 & 141.0 & 1010.67 \\
\hline 50.0 & 1008.60 & & & 65.0 & 1008.53 & 38.0 & 1008.54 & & \\
\hline 55.0 & 1008.60 & & & 70.0 & 1008.55 & 41.0 & 1008.64 & & \\
\hline 60.0 & 1008.60 & & & 75.0 & 1008.53 & 44.0 & 1008.60 & & \\
\hline 65.0 & 1008.53 & & & 80.0 & 1008.47 & 47.0 & 1008.64 & & \\
\hline 70.0 & 1008.52 & & & 85.0 & 1008.43 & 50.0 & 1008.63 & & \\
\hline 75.0 & 1008.52 & & & 90.0 & 1008.54 & 53.0 & 1008.66 & & \\
\hline 80.0 & 1008.47 & & & 95.0 & 1008.94 & 56.0 & 1008.66 & & \\
\hline 85.0 & 1008.44 & & & 100.0 & 1009.10 & 59.0 & 1008.65 & & \\
\hline 90.0 & 1008.53 & & & 102.0 & 1009.41 & 62.0 & 1008.62 & & \\
\hline 93.0 & 1008.83 & & & 105.0 & 1009.55 & 65.0 & 1008.57 & & \\
\hline 96.0 & 1008.96 & & & 106.0 & 1009.44 & 68.0 & 1008.57 & & \\
\hline 99.0 & 1009.04 & & & 107.0 & 1009.52 & 71.0 & 1008.57 & & \\
\hline 101.0 & 1009.27 & & & 109.0 & 1009.32 & 74.0 & 1008.54 & & \\
\hline 103.0 & 1009.50 & & & 112.0 & 1009.59 & 77.0 & 1008.54 & & \\
\hline 105.0 & 1009.54 & & & 115.0 & 1009.61 & 80.0 & 1008.53 & & \\
\hline 106.0 & 1009.42 & & & 120.0 & 1009.56 & 83.0 & 1008.45 & & \\
\hline 107.0 & 1009.51 & & & 123.0 & 1009.10 & 86.0 & 1008.47 & & \\
\hline 109.0 & 1009.32 & & & 126.0 & 1009.31 & 89.0 & 1008.51 & & \\
\hline 112.0 & 1009.58 & & & 130.0 & 1009.41 & 91.0 & 1008.63 & & \\
\hline 115.0 & 1009.63 & & & 134.0 & 1009.67 & 94.0 & 1008.89 & & \\
\hline 118.0 & 1009.59 & & & 136.0 & 1010.30 & 96.0 & 1008.99 & & \\
\hline 119.0 & 1009.56 & & & 139.0 & 1010.14 & 99.0 & 1009.07 & & \\
\hline 122.0 & 1009.07 & & & 141.0 & 1010.68 & 100.0 & 1009.17 & & \\
\hline 125.0 & 1009.10 & & & & & 102.0 & 1009.45 & & \\
\hline 128.0 & 1009.27 & & & & & 103.0 & 1009.56 & & \\
\hline 133.0 & 1009.46 & & & & & 105.0 & 1009.56 & & \\
\hline
\end{tabular}


Location: Township 8 South/Range 48 East--section 28

U.S. Geological Survey quadrangle $(1: 24,000)$ : Moorhead

Landowners--left bank: U.S. Government right bank: U.S. Government

Access: left bank

Permission from: Gay Ranch (T7S/R49E--20)

Reference pins

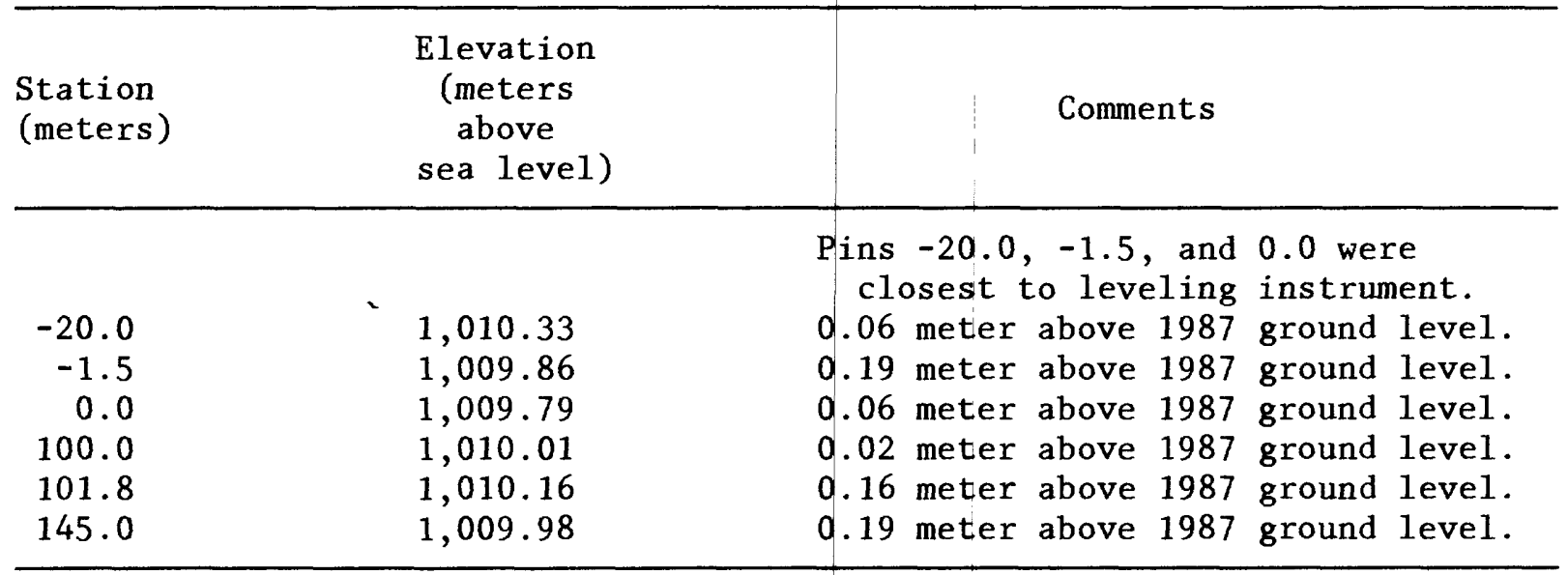




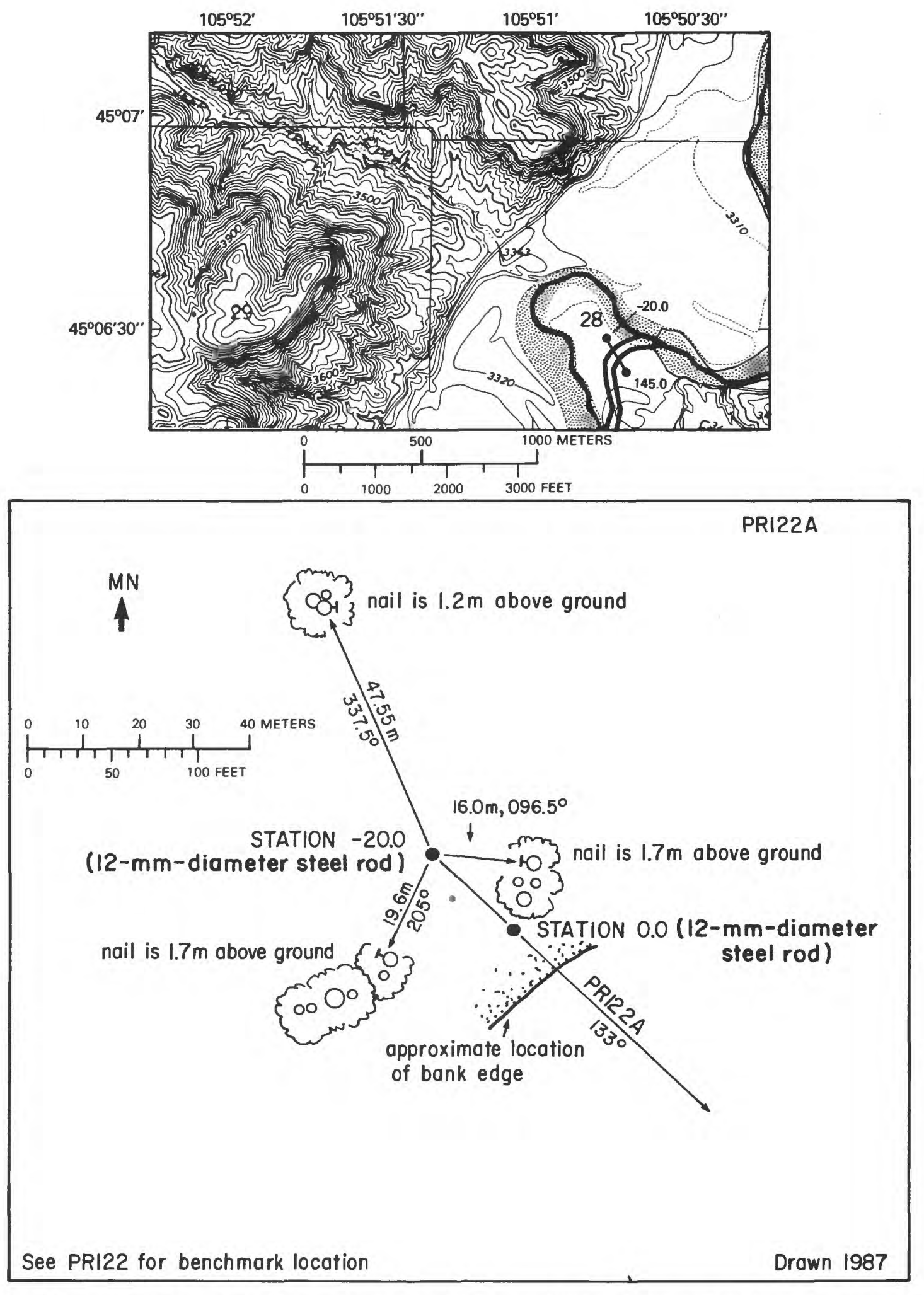

Figure 24.--Upper: Location of cross section PR122A, and left and right bank reference pins (tables 2 and 3 ) in the Moorhead quadrangle. New channel is shown in its approximate location upstream and downstream from the section. Lower: Location of secondary bench marks (12-mm-diameter steel rods) at stations 0.0 and -20.0 on the left bank. Primary bench mark is BM-PR122, as shown in figure 20. MN is magnetic north. 


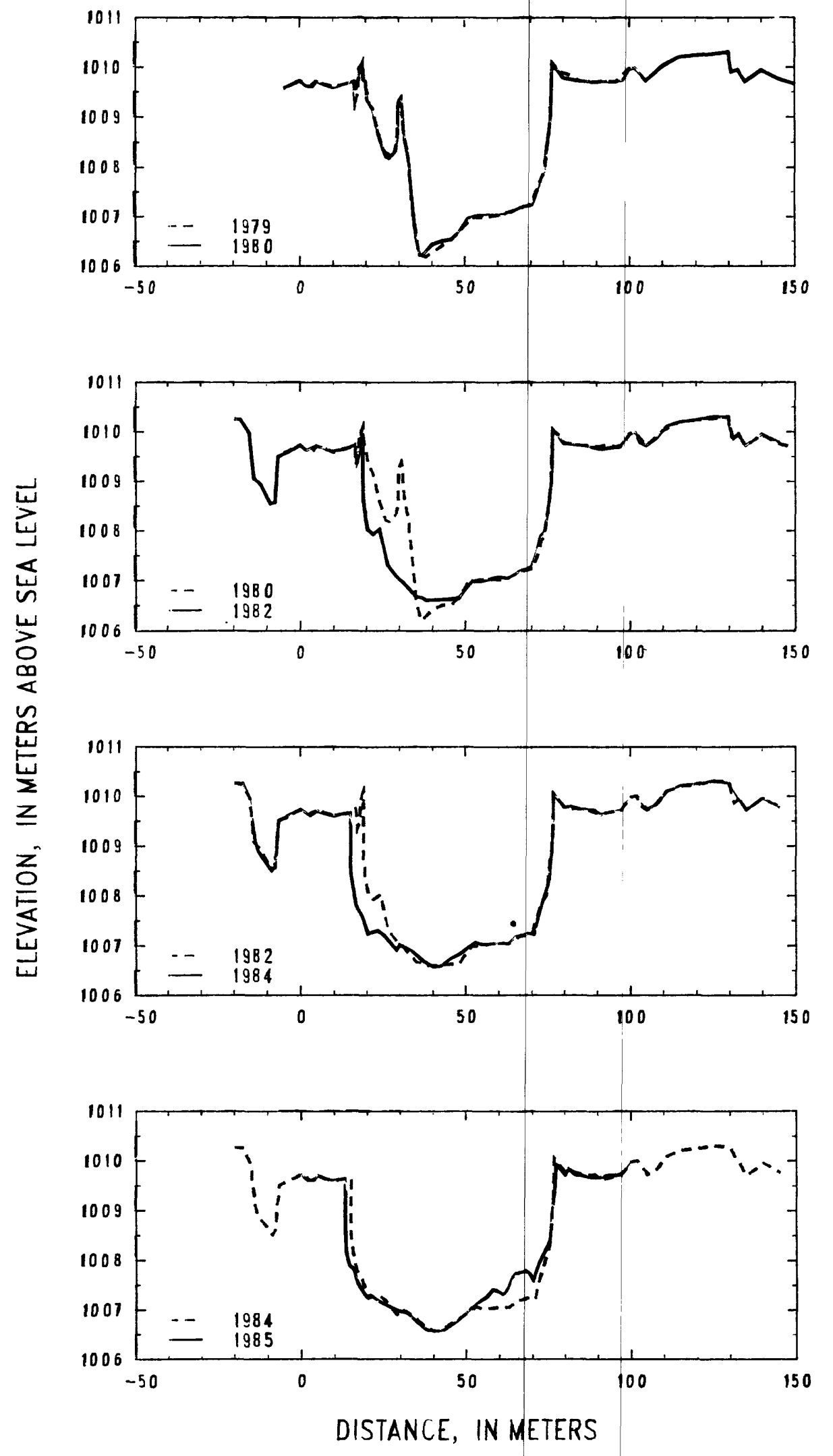

Figure 25.--Profiles of cross section PR122A from 1979 to 1985. 

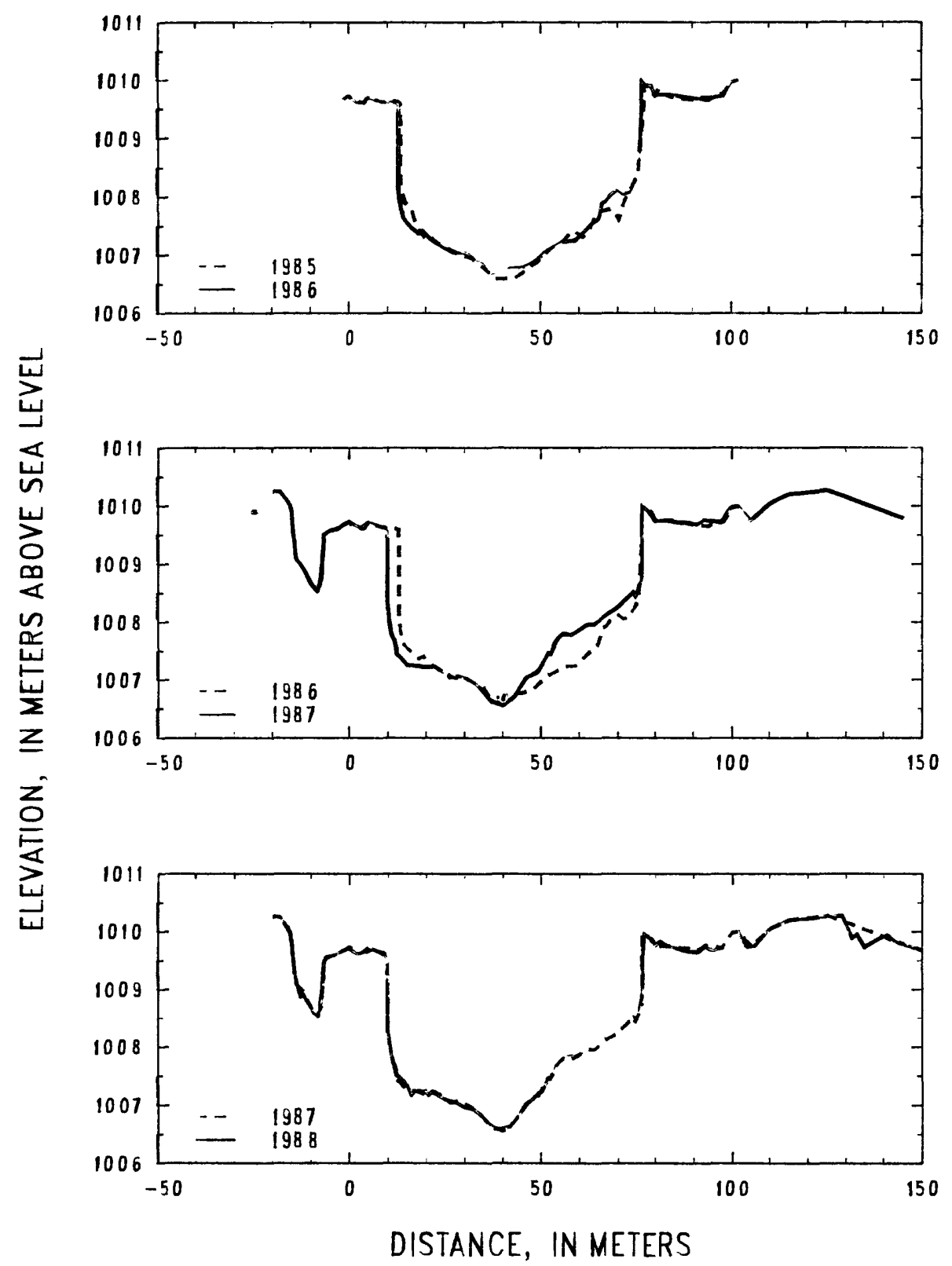

Figure 26.--Profiles of cross section PR122A from 1985 to 1988 
Table 11.--Listing of horizontal stations and elevations for cross section PR122A [Sta., station, distance in meters from a reference point on

the left bank; Elev., elevation, in meters above sea levell.

\begin{tabular}{|c|c|c|c|c|c|c|c|c|c|}
\hline \multicolumn{2}{|c|}{1979} & \multicolumn{2}{|c|}{1980} & \multicolumn{2}{|c|}{1980} & \multicolumn{2}{|c|}{1982} & \multicolumn{2}{|c|}{1982} \\
\hline Sta. & Elev. & Sta. & Elev. & Sta. & Elev. & Sta. & Elev. & Sta. & Elev. \\
\hline-1.5 & 1009.69 & -5.0 & 1009.58 & 76.6 & 1010.04 & -20.0 & 1010.27 & 76.6 & 1010.06 \\
\hline 0.0 & 1009.74 & -1.5 & 1009.68 & 78.0 & 1009.96 & -18.0 & 1010.26 & 80.0 & 1009.76 \\
\hline 3.0 & 1009.61 & 0.0 & 1009.73 & 80.0 & 1009.78 & -15.2 & 1009.98 & 83.0 & 1009.74 \\
\hline 5.0 & 1009.73 & 2.0 & 1009.62 & 82.0 & 1009.75 & -14.0 & 1009.06 & 87.0 & 1009.71 \\
\hline 10.0 & 1009.60 & 4.0 & 1009.61 & 85.0 & 1009.72 & -12.0 & 1008.95 & 91.0 & 1009.64 \\
\hline 16.7 & 1009.72 & 5.0 & 1009.72 & 89.0 & 1009.69 & -9.0 & 1008.55 & 94.0 & 1009.68 \\
\hline 16.9 & 1009.33 & 7.0 & 1009.66 & 93.0 & 1009.72 & -7.6 & 1008.58 & 97.0 & 1009.70 \\
\hline 17.7 & 1009.59 & 10.0 & 1009.59 & 96.0 & 1009.70 & -6.6 & 1009.51 & 100.0 & 1009.97 \\
\hline 18.0 & 1009.98 & 13.0 & 1009.66 & 98.0 & 1009.74 & -4.0 & 1009.58 & 101.7 & 1010.00 \\
\hline 19.2 & 1010.11 & 16.6 & 1009.72 & 100.0 & 1009.96 & 0.0 & 1009.74 & 103.0 & 1009.79 \\
\hline 19.4 & 1009.69 & 16.8 & 1009.33 & 101.7 & 1009.99 & 2.0 & 1009.63 & 105.0 & 1009.72 \\
\hline 19.9 & 1009.67 & 18.0 & 1009.91 & 105.0 & 1009.72 & 5.0 & 1009.72 & 108.0 & 1009.86 \\
\hline 21.0 & 1009.27 & 19.1 & 1010.12 & 110.0 & 1010.02 & 10.0 & 1009.61 & 111.0 & 1010.11 \\
\hline 22.0 & 1009.17 & 19.3 & 1009.74 & 115.0 & 1010.20 & 14.0 & 1009.66 & 115.0 & 1010.20 \\
\hline 25.0 & 1008.36 & 20.0 & 1009.72 & 120.0 & 1010.24 & 16.0 & 1009.73 & 120.0 & 1010.24 \\
\hline 28.0 & 1008.18 & 20.4 & 1009.36 & 125.0 & 1010.27 & 16.6 & 1009.75 & 125.0 & 1010.31 \\
\hline 29.5 & 1008.50 & 22.0 & 1009.16 & 130.0 & 1010.31 & 17.1 & 1009.35 & 129.7 & 1010.29 \\
\hline 30.0 & 1009.32 & 24.0 & 1008.57 & 131.0 & 1009.90 & 17.6 & 1009.47 & 131.4 & 1009.86 \\
\hline 30.8 & 1009.40 & 26.0 & 1008.20 & 133.0 & 1009.95 & 18.4 & 1009.99 & 133.0 & 1009.96 \\
\hline 31.5 & 1008.69 & 27.0 & 1008.17 & 135.0 & 1009.71 & 19.0 & 1010.10 & 135.0 & 1009.72 \\
\hline 33.0 & 1008.14 & 29.0 & 1008.32 & 140.0 & 1009.94 & 19.0 & 1008.64 & 140.0 & 1009.96 \\
\hline 34.2 & 1007.18 & 29.7 & 1008.61 & 145.0 & 1009.76 & 20.4 & 1008.03 & 145.0 & 1009.78 \\
\hline 36.0 & 1006.25 & 29.9 & 1009.26 & 150.0 & 1009.66 & 22.0 & 1007.93 & & \\
\hline 38.0 & 1006.17 & 30.7 & 1009.39 & & & 24.0 & 1008.04 & & \\
\hline 40.0 & 1006.27 & 31.0 & 1009.26 & & & 25.5 & 1007.60 & & \\
\hline 44.0 & 1006.45 & 31.5 & 1008.66 & & & 26.5 & 1007.31 & & \\
\hline 48.0 & 1006.66 & 33.0 & 1008.14 & & & 29.0 & 1007.09 & & \\
\hline 52.0 & 1006.97 & 35.0 & 1006.67 & & & 31.0 & 1006.98 & & \\
\hline 56.0 & 1006.98 & 36.3 & 1006.21 & & & 33.0 & 1006.83 & & \\
\hline 60.0 & 1007.02 & 37.1 & 1006.22 & & & 35.0 & 1006.68 & & \\
\hline 64.0 & 1007.10 & 40.0 & 1006.43 & & & 37.0 & 1006.65 & & \\
\hline 67.0 & 1007.17 & 43.0 & 1006.51 & & & 38.0 & 1006.60 & & \\
\hline 70.0 & 1007.24 & 46.0 & 1006.53 & & & 48.0 & 1006.64 & & \\
\hline 73.0 & 1007.72 & 49.0 & 1006.75 & & & 50.0 & 1006.82 & & \\
\hline 74.3 & 1007.89 & 51.0 & 1006.96 & & & 52.0 & 1006.99 & & \\
\hline 76.1 & 1008.97 & 54.0 & 1007.02 & & & 55.0 & 1006.99 & & \\
\hline 76.6 & 1010.09 & 60.0 & 1007.02 & & & 57.0 & 1007.03 & & \\
\hline 79.0 & 1009.89 & 63.0 & 1007.05 & & & 60.0 & 1007.07 & & \\
\hline 81.4 & 1009.85 & 66.0 & 1007.16 & & & 63.0 & 1007.04 & & \\
\hline 85.0 & 1009.72 & 68.0 & 1007.21 & & & 66.0 & 1007.18 & & \\
\hline 89.0 & 1009.70 & 70.5 & 1007.23 & & & 70.0 & 1007.26 & & \\
\hline 93.0 & 1009.72 & 72.2 & 1007.55 & & & 72.0 & 1007.64 & & \\
\hline 96.0 & 1009.71 & 73.3 & 1007.81 & & & 73.0 & 1007.89 & & \\
\hline 100.0 & 1009.97 & 74.0 & 1007.87 & & & 74.4 & 1007.99 & & \\
\hline 101.7 & 1010.00 & 76.1 & 1008.98 & & & 76.4 & 1009.11 & & \\
\hline
\end{tabular}


Table 11.--Listing of horizontal stations and elevations for cross section PR122A--Continued.

\begin{tabular}{|c|c|c|c|c|c|c|c|c|c|}
\hline \multicolumn{2}{|c|}{1984} & \multicolumn{2}{|c|}{1984} & \multicolumn{2}{|c|}{1985} & \multicolumn{2}{|c|}{1985} & \multicolumn{2}{|c|}{1986} \\
\hline Sta. & Elev. & Sta. & Elev. & Sta. & Elev. & Sta. & Elev. & Sta. & Elev. \\
\hline-20.0 & 1010.27 & 80.0 & 1009.76 & -1.5 & 1009.68 & 76.2 & 1008.94 & -1.5 & 1009.68 \\
\hline-17.4 & 1010.27 & 82.0 & 1009.79 & 0.0 & 1009.73 & 76.8 & 1009.37 & 0.0 & 1009.72 \\
\hline-15.3 & 1009.96 & 85.0 & 1009.72 & 2.0 & 1009.62 & 77.3 & 1009.92 & 2.0 & 1009.62 \\
\hline-13.8 & 1009.13 & 89.0 & 1009.71 & 4.0 & 1009.62 & 78.0 & 1009.89 & 4.0 & 1009.61 \\
\hline-13.0 & 1008.92 & 91,0 & 1009.62 & 5.0 & 1009.72 & 80.0 & 1009.72 & 5.0 & 1009.71 \\
\hline-10.4 & 1008.68 & 94.0 & 1009.68 & 7.0 & 1009.65 & 81.0 & 1009.85 & 7.0 & 1009.66 \\
\hline-8.6 & 1008.51 & 97.0 & 1009.71 & 10.0 & 1009.60 & 83.0 & 1009.73 & 10.0 & 1009.60 \\
\hline-7.6 & 1008.63 & 100.0 & 1009.98 & 12.0 & 1009.64 & 86.0 & 1009.69 & 12.0 & 1009.61 \\
\hline-6.6 & 1009.51 & 101.8 & 1010.01 & 13.0 & 1009.63 & 88.0 & 1009.67 & 12.8 & 1009.59 \\
\hline-5.0 & 1009.58 & 105.0 & 1009.73 & 13.4 & 1009.58 & 90.0 & 1009.66 & 12.8 & 1008.09 \\
\hline-1.5 & 1009.68 & 108.0 & 1009.84 & 13.4 & 1008.69 & 93.0 & 1009.71 & 13.9 & 1007.65 \\
\hline 0.0 & 1009.73 & 111.0 & 1010.10 & 13.6 & 1008.16 & 96.0 & 1009.71 & 15.0 & 1007.54 \\
\hline 3.0 & 1009.61 & 115.0 & 1010.21 & 14.6 & 1007.90 & 98.0 & 1009.78 & 17.0 & 1007.41 \\
\hline 5.0 & 1009.71 & 120.0 & 1010.25 & 16.0 & 1007.82 & 100.0 & 1009.97 & 18.0 & 1007.36 \\
\hline 7.0 & 1009.65 & 125.0 & 1010.31 & 17.1 & 1007.55 & 101.8 & 1010.00 & 19.0 & 1007.41 \\
\hline 10.0 & 1009.60 & 130.0 & 1010.26 & 19.0 & 1007.33 & & & 21.0 & 1007.27 \\
\hline 13.0 & 1009.65 & 135.0 & 1009.71 & 20.0 & 1007.26 & & & 24.0 & 1007.14 \\
\hline 15.0 & 1009.67 & 140.0 & 1009.96 & 21.0 & 1007.31 & & & 27.0 & 1007.03 \\
\hline 15.1 & 1008.50 & 145.0 & 1009.77 & 23.4 & 1007.17 & & & 30.0 & 1007.03 \\
\hline 16.8 & 1007.79 & & & 26.0 & 1007.09 & & & 31.0 & 1006.99 \\
\hline 18.4 & 1007.62 & & & 29.0 & 1006.99 & & & 33.0 & 1006.93 \\
\hline 20.3 & 1007.24 & & & 32.0 & 1006.95 & & & 35.0 & 1006.87 \\
\hline 23.2 & 1007.31 & & & 34.0 & 1006.83 & & & 37.0 & 1006.68 \\
\hline 25.0 & 1007.22 & & & 36.0 & 1006.72 & & & 40.0 & 1006.70 \\
\hline 29.0 & 1006.91 & & & 38.0 & 1006.60 & & & 42.0 & 1006.76 \\
\hline 30.0 & 1007.02 & & & 40.0 & 1006.58 & & & 45.0 & 1006.77 \\
\hline 32.0 & 1006.95 & & & 43.0 & 1006.59 & & & 48.0 & 1006.85 \\
\hline 34.0 & 1006.87 & & & 46.0 & 1006.72 & & & 51.0 & 1007.04 \\
\hline 37.0 & 1006.69 & & & 49.0 & 1006.88 & & & 53.3 & 1007.13 \\
\hline 40.0 & 1006.57 & & & 52.0 & 1007.06 & & & 55.0 & 1007.20 \\
\hline 42.0 & 1006.58 & & & 54.0 & 1007.17 & & & 57.0 & 1007.24 \\
\hline 45.0 & 1006.72 & & & 56.0 & 1007.25 & & & 59.0 & 1007.25 \\
\hline 48.0 & 1006.83 & & & 58.0 & 1007.41 & & & 61.0 & 1007.37 \\
\hline 50.0 & 1006.92 & & & 59.0 & 1007.40 & & & 63.0 & 1007.52 \\
\hline 53.0 & 1007.07 & & & 61.0 & 1007.31 & & & 65.0 & 1007.61 \\
\hline 55.0 & 1007.02 & & & 62.0 & 1007.36 & & & 66.1 & 1007.89 \\
\hline 60.0 & 1007.05 & & & 63.0 & 1007.48 & & & 67.0 & 1007.92 \\
\hline 63.0 & 1007.05 & & & 64.0 & 1007.65 & & & 68.0 & 1008.02 \\
\hline 65.0 & 1007.18 & & & 65.0 & 1007.73 & & & 70.0 & 1008.13 \\
\hline 69.0 & 1007.25 & & & 68.0 & 1007.79 & & & 71.5 & 1008.05 \\
\hline 70.8 & 1007.22 & & & 69.0 & 1007.75 & & & 73.0 & 1008.08 \\
\hline 71.8 & 1007.55 & & & 70.3 & 1007.60 & & & 75.5 & 1008.39 \\
\hline 75.4 & 1008.30 & & & 72.0 & 1007.95 & & & 76.3 & 1009.27 \\
\hline 76.4 & 1009.16 & & & 74.0 & 1008.19 & & & 76.5 & 1009.98 \\
\hline 76.7 & 1010.05 & & & 75.5 & 1008.41 & & & 77.3 & 1009.90 \\
\hline
\end{tabular}


Table 11.--Listing of horizontal stations and elevations for cross section PR122A--Continued.

\begin{tabular}{|c|c|c|c|c|c|c|c|c|c|}
\hline \multicolumn{2}{|c|}{1986} & \multicolumn{2}{|c|}{1987} & \multicolumn{2}{|c|}{1987} & \multicolumn{2}{|c|}{1988} & \multicolumn{2}{|c|}{1988} \\
\hline Sta. & Elev. & Sta. & Elev. & Sta. & Elev. & Sta. & Elev. & Sta. & Elev. \\
\hline 79.0 & 1009.90 & -20.0 & 1010.25 & 60.0 & 1007.87 & -20.0 & 1010.26 & 46.0 & 1007.01 \\
\hline 80.0 & 1009.74 & -18.0 & 1010.25 & 62.0 & 1007.95 & -18.0 & 1010.25 & 48.0 & 1007.12 \\
\hline 81.0 & 1009.73 & -16.0 & 1010.07 & 64.0 & 1007.96 & -16.0 & 1010.04 & 49.5 & 1007.22 \\
\hline 82.0 & 1009.75 & -15.1 & 1009.96 & 67.0 & 1008.13 & -15.2 & 1009.93 & 50.3 & 1007.23 \\
\hline 84.0 & 1009.75 & -13.8 & 1009.08 & 70.0 & 1008.25 & -14.0 & 1009.13 & 52.0 & 1007.46 \\
\hline 87.0 & 1009.71 & -12.0 & 1008.94 & 72.0 & 1008.39 & -12.7 & 1008.87 & 53.0 & 1007.49 \\
\hline 90.0 & 1009.68 & -10.0 & 1008.65 & 74.0 & 1008.52 & -12.0 & 1008.91 & 54.0 & 1007.67 \\
\hline 92.0 & 1009.67 & -8.3 & 1008.54 & 74.7 & 1008.44 & -9.6 & 1008.59 & 56.0 & 1007.81 \\
\hline 94.0 & 1009.65 & -7.4 & 1008.71 & 76.3 & 1008.71 & -8.3 & 1008.53 & 58.0 & 1007.85 \\
\hline 96.0 & 1009.72 & -6.6 & 1009.51 & 76.5 & 1009.99 & -7.4 & 1008.77 & 59.0 & 1007.83 \\
\hline 98.0 & 1009.73 & -5.0 & 1009.58 & 77.0 & 1009.98 & -6.6 & 1009.49 & 61.0 & 1007.93 \\
\hline 100.0 & 1009.97 & -3.0 & 1009.60 & 79.0 & 1009.83 & -6.0 & 1009.55 & 63.0 & 1007.96 \\
\hline \multirow[t]{33}{*}{101.7} & 1010.00 & -1.5 & 1009.67 & 80.0 & 1009.73 & -4.0 & 1009.59 & 66.0 & 1008.07 \\
\hline & & 0.0 & 1009.72 & 83.0 & 1009.74 & -1.5 & 1009.67 & 69.0 & 1008.21 \\
\hline & & 3.0 & 1009.62 & 86.0 & 1009.71 & 0.0 & 1009.72 & 71.0 & 1008.31 \\
\hline & & 5.0 & 1009.71 & 89.0 & 1009.71 & 1.0 & 1009.65 & 73.0 & 1008.50 \\
\hline & & 7.0 & 1009.66 & 91.0 & 1009.67 & 2.0 & 1009.61 & 74.0 & 1008.53 \\
\hline & & 9.0 & 1009.62 & 93.0 & 1009.76 & 4.0 & 1009.62 & 75.0 & 1008.47 \\
\hline & & 10.0 & 1009.54 & 96.0 & 1009.72 & 6.0 & 1009.70 & 75.6 & 1008.57 \\
\hline & & 10.0 & 1008.29 & 97.4 & 1009.71 & 8.0 & 1009.64 & 76.5 & 1008.93 \\
\hline & & 11.0 & 1007.79 & 99.0 & 1009.89 & 9.9 & 1009.52 & 76.6 & 1009.66 \\
\hline & & 12.0 & 1007.65 & 100.0 & 1009.98 & 10.0 & 1008.33 & 76.8 & 1009.72 \\
\hline & & 12.2 & 1007.45 & 101.7 & 1010.00 & 10.8 & 1007.87 & 77.0 & 1009.96 \\
\hline & & 13.4 & 1007.36 & 105.0 & 1009.74 & 12.2 & 1007.51 & 79.0 & 1009.87 \\
\hline & & 15.0 & 1007.25 & 110.0 & 1010.04 & 14.0 & 1007.42 & 80.0 & 1009.75 \\
\hline & & 18.0 & 1007.23 & 115.0 & 1010.20 & 15.0 & 1007.33 & 81.3 & 1009.83 \\
\hline & & 20.0 & 1007.21 & 120.0 & 1010.24 & 16.0 & 1007.17 & 83.0 & 1009.74 \\
\hline & & 22.0 & 1007.23 & 125.0 & 1010.28 & 17.0 & 1007.25 & 86.0 & 1009.70 \\
\hline & & 24.0 & 1007.15 & 130.0 & 1010.16 & 18.0 & 1007.22 & 88.0 & 1009.65 \\
\hline & & 26.0 & 1007.03 & 145.0 & 1009.79 & 19.0 & 1007.18 & 91.0 & 1009.64 \\
\hline & & 28.0 & 1007.07 & & & 19.6 & 1007.24 & 93.0 & 1009.74 \\
\hline & & 31.0 & 1007.00 & & & 20.5 & 1007.17 & 95.0 & 1009.66 \\
\hline & & 34.0 & 1006.86 & & & 21.3 & 1007.23 & 97.0 & 1009.70 \\
\hline & & 37.0 & 1006.62 & & & 22.0 & 1007.21 & 98.0 & 1009.75 \\
\hline & & 40.0 & 1006.55 & & & 23.0 & 1007.15 & 100.0 & 1009.96 \\
\hline & & 43.0 & 1006.71 & & & 25.0 & 1007.08 & 101.7 & 1010.00 \\
\hline & & 46.0 & 1007.03 & & & 28.0 & 1007.03 & 103.0 & 1009.78 \\
\hline & & 49.0 & 1007.13 & & & 30.0 & 1006.96 & 104.0 & 1009.73 \\
\hline & & 51.2 & 1007.37 & & & 32.0 & 1006.94 & 106.0 & 1009.75 \\
\hline & & 51.9 & 1007.46 & & & 34.0 & 1006.85 & 110.0 & 1010.05 \\
\hline & & 52.5 & 1007.44 & & & 36.0 & 1006.71 & 115.0 & 1010.20 \\
\hline & & 53.8 & 1007.65 & & & 38.0 & 1006.59 & 120.0 & 1010.23 \\
\hline & & 55.0 & 1007.75 & & & 40.0 & 1006.60 & 125.0 & 1010.27 \\
\hline & & 56.0 & 1007.80 & & & 42.0 & 1006.63 & 129.0 & 1010.27 \\
\hline & & 58.0 & 1007.78 & 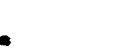 & & 44.0 & 1006.80 & 131.7 & 1009.88 \\
\hline
\end{tabular}


Table 11.--Listing of horizontal stations and elevations for cross section PR122A--Continued. 1988 Sta. Elev.

$133.0 \quad 1009.95$

$135.0 \quad 1009.72$

$138.0 \quad 1009.86$

$141.0 \quad 1009.94$

$143.0 \quad 1009.82$

$145.0 \quad 1009.78$

$150.0 \quad 1009.67$ 


\section{Description of Cross Section PR 125}

Location: Township 8 South/Range 48 East--section 21

U.S. Geological survey quadrangle $(1: 24,000)$ : Moorhead

Landowners--left bank: Virginia Williams

right bank: Amory Hubbard

Access: $\quad$ left bank

Permission from: Gay Ranch (T7S/R49E--20)

Reference pins

\begin{tabular}{|c|c|c|}
\hline $\begin{array}{l}\text { Station } \\
\text { (meters) }\end{array}$ & $\begin{array}{l}\text { Elevation } \\
\text { (meters } \\
\text { above } \\
\text { sea level) }\end{array}$ & Comments \\
\hline & & $\begin{array}{l}\text { Pins } 0.0 \text { and } 5.0 \text { were closest to } \\
\text { leveling instrument. }\end{array}$ \\
\hline 0.0 & $1,008.17$ & 0.16 meter above 1987 ground level. \\
\hline 5.0 & $1,008.01$ & 0.12 meter above 1987 ground level. \\
\hline 100.8 & $1,007.41$ & 0.11 meter above 1987 ground level. \\
\hline 105.4 & $1,007.57$ & Possibly covered by sand in 1978 . \\
\hline 117.1 & $1,007.84$ & 0.23 meter above 1987 ground level. \\
\hline
\end{tabular}




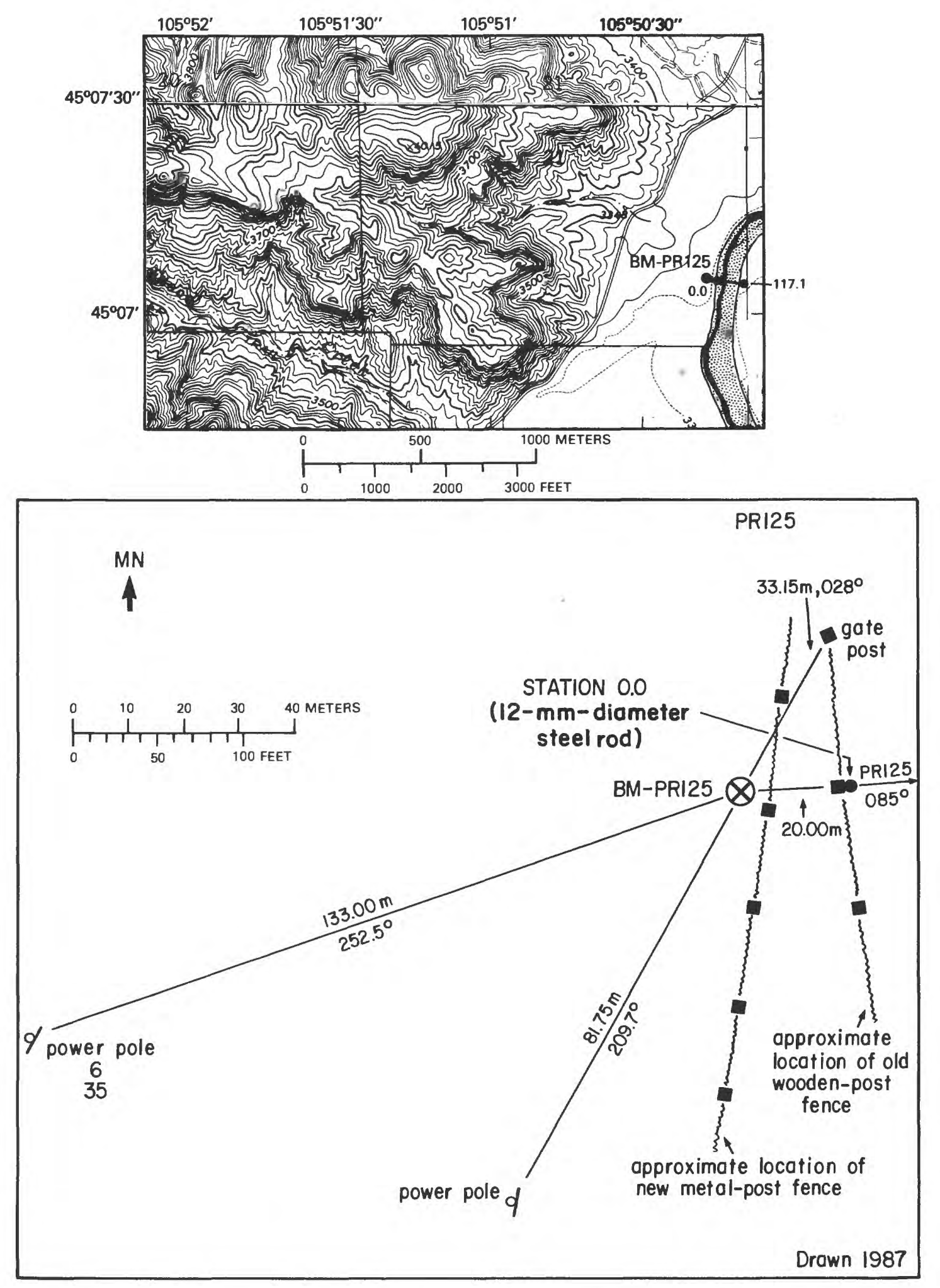

Figure 27.--Upper: Location of cross section PR125, bench mark BM-PR125, and left and right bank reference pins (tables 2 and 3 ) in the Moorhead quadrangle. Lower: Location of bench mark on the left bank. MN is magnetic north. 

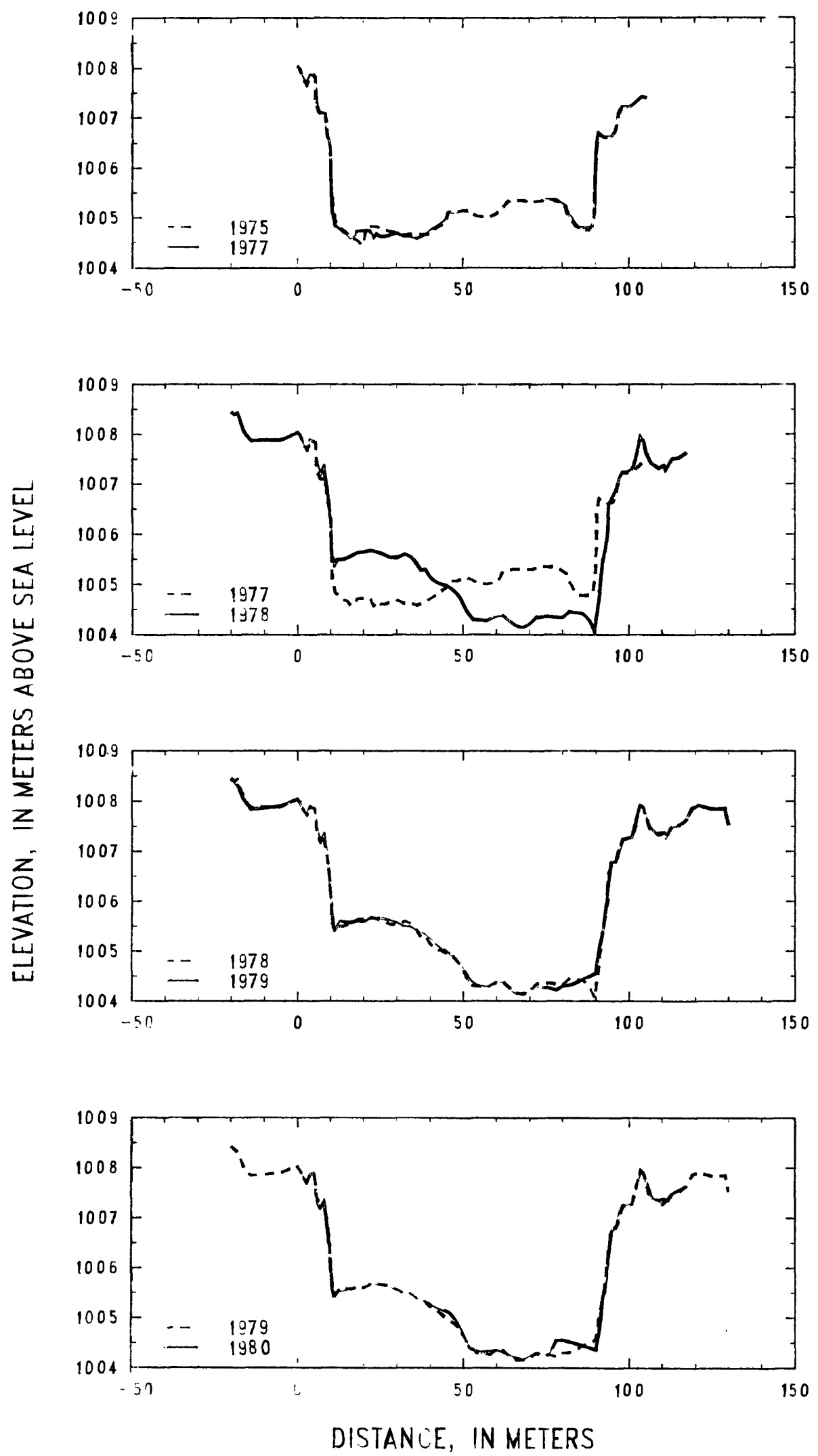

Figure 28 --Profiles of cross section PR125 from 1975 to 1980. 

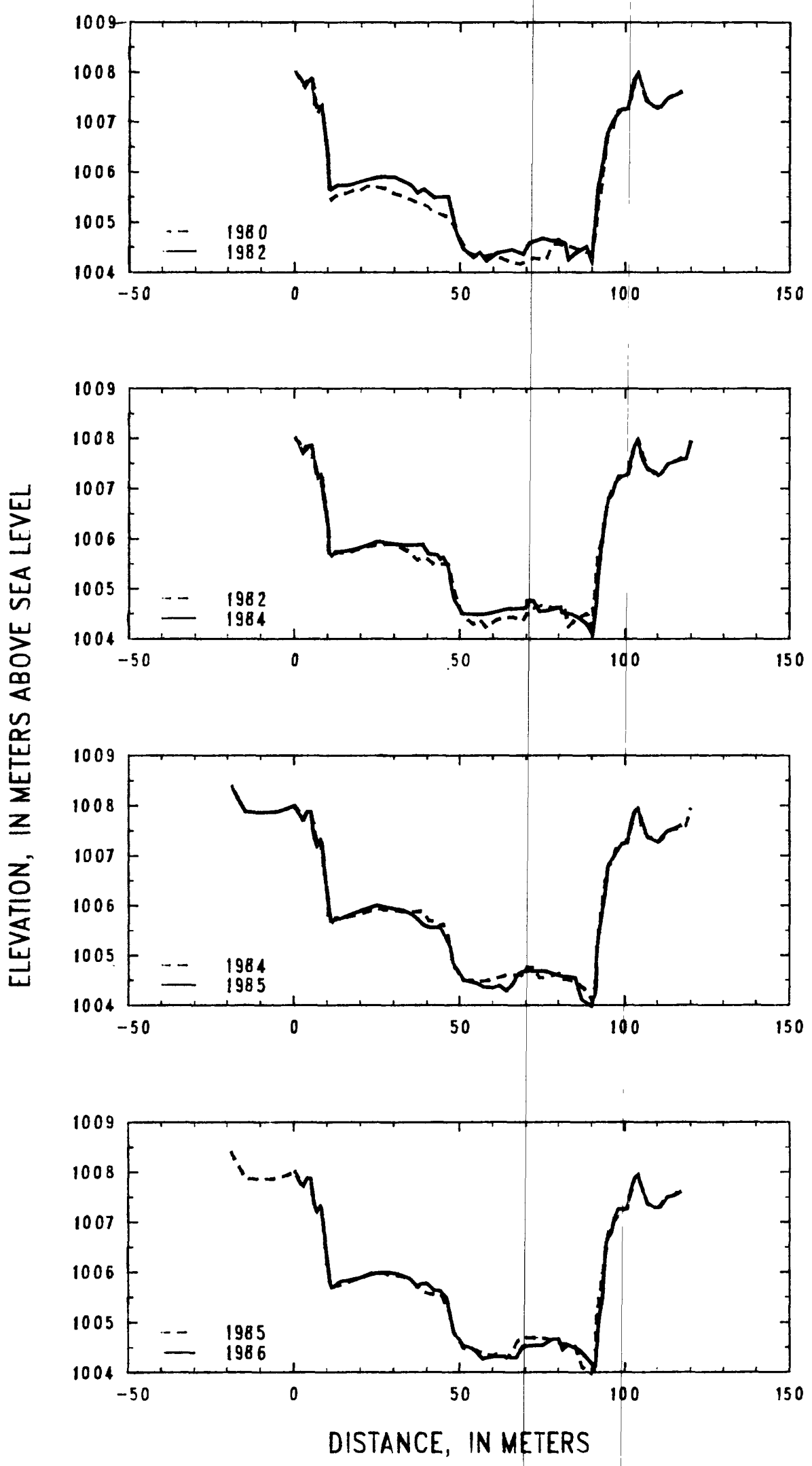

Figure 29.--Profiles of cross section PR125 from 1980 to 1986. 


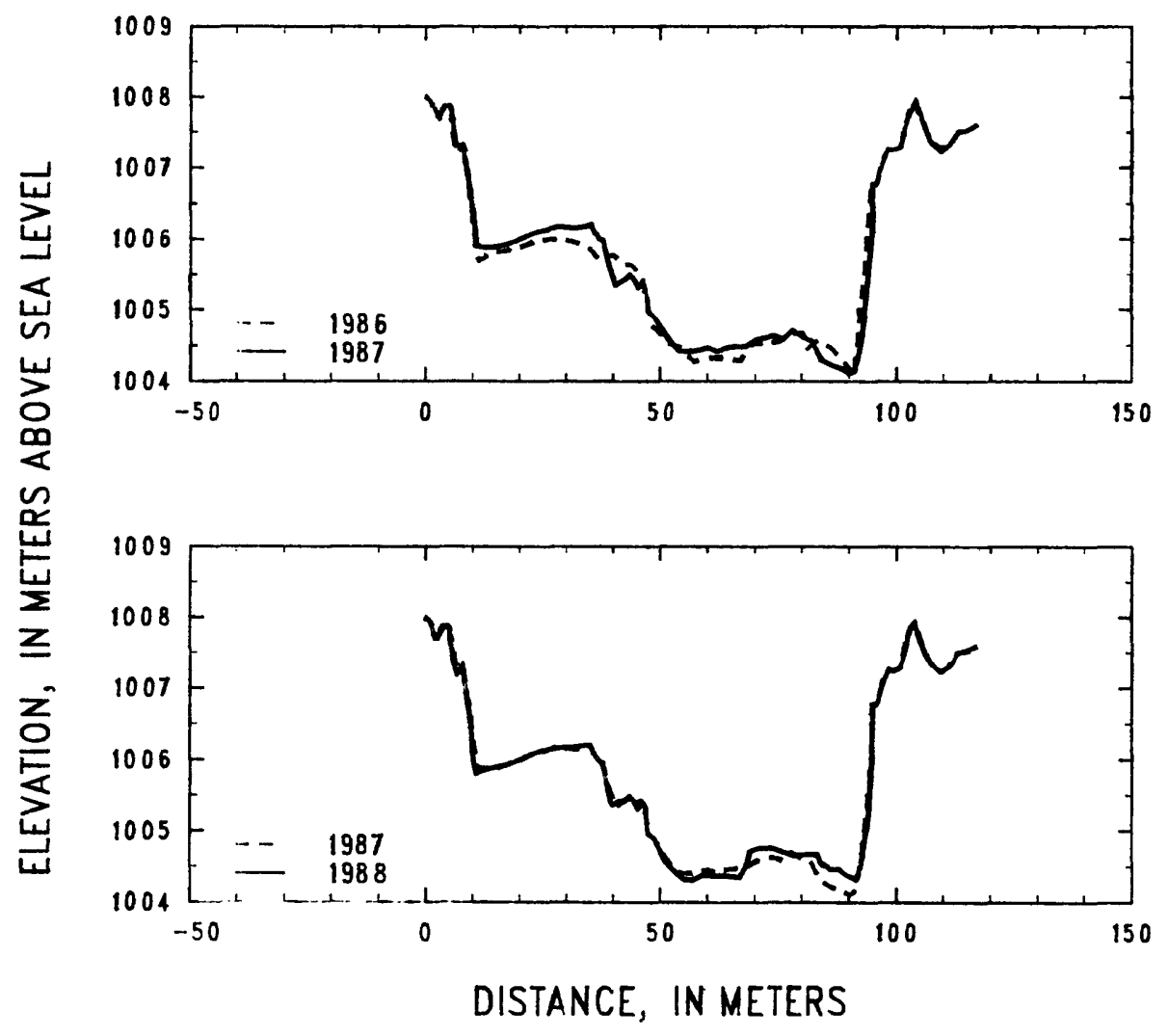

Figure 30.--Profiles of cross section PR 125 from 1986 to 1988. 
Table 12.--Listing of horizontal stations and elevations for cross section PR125 [Sta., station, distance in meters from a reference point on the left bank; Elev., elevation, in meters above sea levell.

\begin{tabular}{|c|c|c|c|c|c|c|c|c|c|}
\hline \multicolumn{2}{|c|}{1975} & \multicolumn{2}{|c|}{1975} & \multicolumn{2}{|c|}{1977} & \multicolumn{2}{|c|}{1977} & \multicolumn{2}{|c|}{1978} \\
\hline Sta. & Elev. & Sta. & Elev. & Sta. & Elev. & Sta. & Elev. & Sta. & Elev. \\
\hline 0.0 & 1008.04 & 50.0 & 1005.14 & 0.0 & 1008.04 & 70.0 & 1005.30 & -20.0 & 1008.45 \\
\hline 1.0 & 1007.96 & 52.0 & 1005.12 & 1.0 & 1007.99 & 74.0 & 1005.36 & -19.0 & 1008.39 \\
\hline 2.0 & 1007.77 & 54.0 & 1005.02 & 2.0 & 1007.78 & 78.0 & 1005.36 & -18.0 & 1008.43 \\
\hline 3.0 & 1007.66 & 56.0 & 1005.00 & 3.0 & 1007.69 & 81.2 & 1005.22 & -16.0 & 1008.02 \\
\hline 4.0 & 1007.84 & 58.0 & 1005.00 & 4.0 & 1007.86 & 82.0 & 1005.07 & -14.0 & 1007.86 \\
\hline 5.0 & 1007.86 & 60.0 & 1005.06 & 5.0 & 1007.87 & 83.5 & 1004.88 & -10.0 & 1007.88 \\
\hline 5.6 & 1007.79 & 62.0 & 1005.18 & 5.6 & 1007.82 & 85.0 & 1004.79 & -5.0 & 1007.88 \\
\hline 5.7 & 1007.41 & 63.6 & 1005.32 & 6.0 & 1007.27 & 87.0 & 1004.78 & 0.0 & 1008.03 \\
\hline 6.0 & 1007.26 & 64.0 & 1005.33 & 7.0 & 1007.10 & 89.0 & 1004.81 & 1.0 & 1007.98 \\
\hline 6.6 & 1007.12 & 66.0 & 1005.33 & 8.6 & 1007.09 & 89.9 & 1005.16 & 2.0 & 1007.81 \\
\hline 7.0 & 1007.09 & 68.0 & 1005.34 & 9.3 & 1006.64 & 90.3 & 1006.44 & 3.0 & 1007.70 \\
\hline 8.0 & 1007.16 & 70.0 & 1005.29 & 10.1 & 1006.44 & 91.0 & 1006.71 & 4.0 & 1007.88 \\
\hline 8.6 & 1007.14 & 72.0 & 1005.31 & 10.7 & 1005.13 & 93.0 & 1006.60 & 5.5 & 1007.83 \\
\hline 9.0 & 1006.82 & 74.0 & 1005.33 & 11.6 & 1004.83 & 95.0 & 1006.64 & 6.0 & 1007.38 \\
\hline 9.3 & 1006.62 & 76.0 & 1005.37 & 13.0 & 1004.76 & 96.0 & 1006.76 & 7.0 & 1007.18 \\
\hline 10.0 & 1006.53 & 78.0 & 1005.32 & 13.6 & 1004.72 & 97.0 & 1007.11 & 8.0 & 1007.33 \\
\hline 10.1 & 1006.44 & 80.0 & 1005.29 & 14.0 & 1004.69 & 98.0 & 1007.22 & 9.0 & 1006.87 \\
\hline 10.5 & 1005.58 & 81.0 & 1005.21 & 15.0 & 1004.67 & 100.0 & 1007.22 & 10.0 & 1006.41 \\
\hline 11.0 & 1005.29 & 82.0 & 1005.01 & 16.0 & 1004.59 & 100.7 & 1007.23 & 10.5 & 1005.60 \\
\hline 11.4 & 1004.97 & 84.0 & 1004.82 & 17.5 & 1004.61 & 104.0 & 1007.43 & 11.3 & 1005.38 \\
\hline 11.9 & 1004.85 & 86.0 & 1004.75 & 17.9 & 1004.71 & 105.0 & 1007.42 & 12.0 & 1005.49 \\
\hline 12.0 & 1004.81 & 88.0 & 1004.74 & 20.0 & 1004.73 & 105.4 & 1007.40 & 14.0 & 1005.50 \\
\hline 14.0 & 1004.73 & 89.0 & 1004.84 & 21.8 & 1004.73 & & & 16.0 & 1005.56 \\
\hline 15.0 & 1004.67 & 89.7 & 1005.06 & 23.0 & 1004.58 & & & 18.0 & 1005.63 \\
\hline 16.0 & 1004.59 & 90.0 & 1005.93 & 24.0 & 1004.67 & & & 20.0 & 1005.64 \\
\hline 18.0 & 1004.54 & 90.5 & 1006.42 & 25.0 & 1004.62 & & & 22.0 & 1005.68 \\
\hline 19.5 & 1004.44 & 91.0 & 1006.67 & 26.0 & 1004.61 & & & 24.0 & 1005.64 \\
\hline 20.0 & 1004.47 & 92.0 & 1006.62 & 28.0 & 1004.65 & & & 26.0 & 1005.59 \\
\hline 20.9 & 1004.80 & 93.0 & 1006.61 & 30.0 & 1004.69 & & & 28.0 & 1005.53 \\
\hline 22.0 & 1004.82 & 94.0 & 1006.60 & 32.0 & 1004.62 & & & 30.0 & 1005.52 \\
\hline 24.0 & 1004.81 & 96.0 & 1006.72 & 34.0 & 1004.63 & & & 32.5 & 1005.60 \\
\hline 26.0 & 1004.75 & 97.0 & 1007.05 & 36.0 & 1004.58 & & & 34.4 & 1005.54 \\
\hline 28.0 & 1004.71 & 98.0 & 1007.19 & 38.0 & 1004.63 & & & 36.0 & 1005.41 \\
\hline 30.0 & 1004.67 & 100.0 & 1007.21 & 40.3 & 1004.73 & & & 37.3 & 1005.27 \\
\hline 32.0 & 1004.64 & & & 42.0 & 1004.81 & & & 38.7 & 1005.29 \\
\hline 34.0 & 1004.65 & & & 44.9 & 1004.94 & & & 40.0 & 1005.14 \\
\hline 36.0 & 1004.66 & & & 45.5 & 1005.08 & & & 42.0 & 1005.03 \\
\hline 38.0 & 1004.65 & & & 48.0 & 1005.07 & & & 44.0 & 1004.98 \\
\hline 40.0 & 1004.66 & & & 51.0 & 1005.15 & & & 46.0 & 1004.93 \\
\hline 42.0 & 1004.78 & & & 54.0 & 1005.04 & & & 48.0 & 1004.81 \\
\hline 43.9 & 1004.87 & & & 57.0 & 1005.00 & & & 49.0 & 1004.73 \\
\hline 45.0 & 1004.94 & & & 60.0 & 1005.04 & & & 51.0 & 1004.46 \\
\hline 45.6 & 1005.10 & & & 61.3 & 1005.14 & & & 53.0 & 1004.29 \\
\hline 46.0 & 1005.10 & & & 61.9 & 1005.25 & & & 55.0 & 1004.28 \\
\hline 48.0 & 1005.10 & & & 65.0 & 1005.31 & & & 57.0 & 1004.26 \\
\hline
\end{tabular}


Table 12.--Listing of horizontal stations and elevations for cross section PR125 --Continued.

\begin{tabular}{|c|c|c|c|c|c|c|c|c|c|}
\hline \multicolumn{2}{|c|}{1978} & \multicolumn{2}{|c|}{1979} & \multicolumn{2}{|c|}{1979} & \multicolumn{2}{|c|}{1980} & \multicolumn{2}{|c|}{1980} \\
\hline Sta. & Elev. & Sta. & Elev. & Sta. & Elev. & Sta. & Elev. & Sta. & Elev. \\
\hline 60.0 & 1004.37 & -20.0 & 1008.43 & 62.0 & 1004.36 & 0.0 & 1008.02 & 96.2 & 1006.85 \\
\hline 62.0 & 1004.38 & -18.0 & 1008.32 & 64.0 & 1004.23 & 2.0 & 1007.81 & 97.0 & 1007.10 \\
\hline 64.0 & 1004.25 & -16.0 & 1008.01 & 66.0 & 1004.16 & 3.0 & 1007.70 & 98.0 & 1007.24 \\
\hline 66.0 & 1004.15 & -14.0 & 1007.84 & 68.0 & 1004.16 & 4.0 & 1007.87 & 100.0 & 1007.26 \\
\hline 68.0 & 1004.13 & -10.0 & 1007.87 & 70.0 & 1004.24 & 5.0 & 1007.89 & 100.9 & 1007.27 \\
\hline 70.0 & 1004.20 & -5.0 & 1007.90 & 72.0 & 1004.30 & 5.4 & 1007.84 & 103.5 & 1007.96 \\
\hline 72.0 & 1004.34 & 0.0 & 1008.04 & 74.0 & 1004.29 & 6.2 & 1007.30 & 104.4 & 1007.90 \\
\hline 74.0 & 1004.35 & 2.0 & 1007.82 & 76.0 & 1004.26 & 7.0 & 1007.19 & 107.0 & 1007.38 \\
\hline 76.0 & 1004.36 & 3.0 & 1007.72 & 78.0 & 1004.22 & 8.2 & 1007.33 & 110.0 & 1007.27 \\
\hline 78.0 & 1004.33 & 4.0 & 1007.89 & 80.0 & 1004.31 & 9.7 & 1006.50 & 113.0 & 1007.49 \\
\hline 80.0 & 1004.33 & 5.4 & 1007.85 & 82.0 & 1004.32 & 10.0 & 1006.47 & 117.1 & 1007.63 \\
\hline 82.0 & 1004.45 & 5.7 & 1007.50 & 84.0 & 1004.37 & 10.6 & 1005.62 & & \\
\hline 84.0 & 1004.43 & 7.0 & 1007.18 & 86.0 & 1004.45 & 11.1 & 1005.44 & & \\
\hline 86.0 & 1004.41 & 8.0 & 1007.32 & 88.0 & 1004.50 & 12.0 & 1005.51 & & \\
\hline 88.0 & 1004.28 & 9.0 & 1006.95 & 90.0 & 1004.57 & 14.0 & 1005.55 & & \\
\hline 89.6 & 1004.06 & 10.0 & 1006.39 & 91.4 & 1005.19 & 16.0 & 1005.58 & & \\
\hline 91.0 & 1004.73 & 10.1 & 1006.24 & 91.8 & 1005.27 & 18.0 & 1005.63 & & \\
\hline 92.0 & 1005.47 & 10.3 & 1005.91 & 93.0 & 1005.90 & 20.0 & 1005.63 & & \\
\hline 92.7 & 1005.65 & 11.0 & 1005.48 & 94.6 & 1006.78 & 22.0 & 1005.70 & & \\
\hline 93.0 & 1005.80 & 11.5 & 1005.43 & 96.0 & 1006.80 & 25.0 & 1005.68 & & \\
\hline 93.5 & 1005.99 & 13.0 & 1005.60 & 98.0 & 1007.25 & 28.0 & 1005.64 & & \\
\hline 93.8 & 1006.58 & 14.0 & 1005.59 & 100.0 & 1007.28 & 30.0 & 1005.56 & & \\
\hline 95.0 & 1006.74 & 16.0 & 1005.57 & 100.8 & 1007.28 & 33.0 & 1005.50 & & \\
\hline 96.0 & 1006.85 & 18.0 & 1005.62 & 103.4 & 1007.93 & 36.0 & 1005.41 & & \\
\hline 97.0 & 1007.10 & 20.0 & 1005.60 & 104.4 & 1007.88 & 40.0 & 1005.31 & & \\
\hline 98.0 & 1007.23 & 22.0 & 1005.68 & 106.0 & 1007.49 & 41.0 & 1005.24 & & \\
\hline 100.0 & 1007.25 & 24.0 & 1005.68 & 108.0 & 1007.37 & 45.0 & 1005.12 & & \\
\hline 100.7 & 1007.27 & 26.0 & 1005.66 & 110.6 & 1007.38 & 46.0 & 1005.09 & & \\
\hline 101.6 & 1007.39 & 28.0 & 1005.61 & 111.0 & 1007.28 & 48.0 & 1004.92 & & \\
\hline 103.5 & 1007.95 & 30.0 & 1005.56 & 113.0 & 1007.50 & 50.0 & 1004.66 & & \\
\hline 104.0 & 1007.88 & 32.0 & 1005.48 & 115,0 & 1007.53 & 52.0 & 1004.41 & & \\
\hline 104.4 & 1007.88 & 34.0 & 1005.48 & 117.1 & 1007.63 & 55.0 & 1004.30 & & \\
\hline 105.0 & 1007.66 & 36.0 & 1005.39 & 119.0 & 1007.87 & 60.0 & 1004.34 & & \\
\hline 105.4 & 1007.59 & 38.0 & 1005.35 & 121.0 & 1007.92 & 65.0 & 1004.20 & & \\
\hline 106.5 & 1007.42 & 40.0 & 1005.22 & 125.0 & 1007.84 & 68.0 & 1004.16 & & \\
\hline 109.0 & 1007.31 & 42.0 & 1005.15 & 129.0 & 1007.86 & 72.0 & 1004.28 & & \\
\hline 110.6 & 1007.37 & 44.0 & 1005.04 & 130.0 & 1007.54 & 76.0 & 1004.24 & & \\
\hline 111.0 & 1007.27 & 46.0 & 1004.95 & & & 78.0 & 1004.55 & & \\
\hline 113.0 & 1007.50 & 48.0 & 1004.84 & & & 80.0 & 1004.56 & & \\
\hline 115.0 & 1007.52 & 50.0 & 1004.64 & & & 85.0 & 1004.47 & & \\
\hline 117.1 & 1007.62 & 52.0 & 1004.40 & & & 90.0 & 1004.36 & & \\
\hline & & 54.0 & 1004.30 & & & 91.0 & 1004.72 & & \\
\hline & & 56.0 & 1004.28 & & & 92.0 & 1005.44 & & \\
\hline & & 58.0 & 1004.27 & & & 92.5 & 1005.50 & & \\
\hline & & 60.0 & 1004.37 & & & 94.7 & 1006.75 & & \\
\hline
\end{tabular}


Table 12.--Listing of horizontal stations and elevations for cross section PR125 --Continued.

\begin{tabular}{|c|c|c|c|c|c|c|c|c|c|}
\hline \multicolumn{2}{|c|}{1982} & \multicolumn{2}{|c|}{1982} & \multicolumn{2}{|c|}{1984} & \multicolumn{2}{|c|}{1984} & \multicolumn{2}{|c|}{1985} \\
\hline Sta. & Elev. & Sta. & Elev. & Sta. & Elev. & Sta. & Elev. & Sta. & Elev. \\
\hline 0.0 & 1007.99 & 100.9 & 1007.27 & 0.0 & 1008.02 & 94.5 & 1006.65 & -19.0 & 1008.40 \\
\hline 1.0 & 1007.96 & 103.0 & 1007.87 & 1.0 & 1007.95 & 95.0 & 1006.79 & -14.5 & 1007.87 \\
\hline 3.0 & 1007.72 & 104.0 & 1007.99 & 2.6 & 1007.71 & 96.0 & 1006.86 & -10.0 & 1007.85 \\
\hline 5.0 & 1007.88 & 106.5 & 1007.44 & 3.5 & 1007.84 & 97.0 & 1007.08 & -5.0 & 1007.87 \\
\hline 5.4 & 1007.87 & 109.0 & 1007.31 & 5.3 & 1007.86 & 98.0 & 1007.25 & 0.0 & 1007.99 \\
\hline 5.6 & 1007.62 & 111.0 & 1007.30 & 7.0 & 1007.20 & 99.0 & 1007.24 & 2.0 & 1007.78 \\
\hline 6.6 & 1007.30 & 113.0 & 1007.49 & 8.0 & 1007.27 & 100.8 & 1007.29 & 3.0 & 1007.71 \\
\hline 8.3 & 1007.30 & 115.0 & 1007.54 & 10.3 & 1006.21 & 103.0 & 1007.86 & 4.0 & 1007.87 \\
\hline 10.0 & 1006.43 & 117.1 & 1007.61 & 10.4 & 1005.74 & 104.0 & 1007.95 & 5.0 & 1007.88 \\
\hline 10.6 & 1005.73 & & & 11.4 & 1005.65 & 105.2 & 1007.61 & 5.3 & 1007.86 \\
\hline 11.0 & 1005.64 & & & 12.5 & 1005.75 & 107.0 & 1007.38 & 6.0 & 1007.39 \\
\hline 13.0 & 1005.73 & & & 15.0 & 1005.75 & 109.0 & 1007.34 & 7.0 & 1007.19 \\
\hline 16.0 & 1005.72 & & & 20.0 & 1005.83 & 110.0 & 1007.26 & 8.0 & 1007.32 \\
\hline 20.0 & 1005.81 & & & 25.0 & 1005.94 & 111.0 & 1007.30 & 8.4 & 1007.27 \\
\hline 25.0 & 1005.89 & & & 29.0 & 1005.90 & 113.0 & 1007.49 & 11.0 & 1005.68 \\
\hline 30.0 & 1005.90 & & & 32.0 & 1005.88 & 117.1 & 1007.58 & 12.0 & 1005.70 \\
\hline 35.0 & 1005.73 & & & 35.0 & 1005.87 & 118.5 & 1007.60 & 15.0 & 1005.76 \\
\hline 37.0 & 1005.57 & & & 38.6 & 1005.89 & 120.0 & 1007.94 & 20.0 & 1005.89 \\
\hline 39.0 & 1005.65 & & & 40.4 & 1005.70 & & & 25.0 & 1006.01 \\
\hline 42.0 & 1005.48 & & & 43.0 & 1005.68 & & & 30.0 & 1005.93 \\
\hline 46.3 & 1005.50 & & & 43.9 & 1005.59 & & & 33.0 & 1005.90 \\
\hline 48.0 & 1005.01 & & & 44.9 & 1005.63 & & & 36.0 & 1005.79 \\
\hline 49.0 & 1004.77 & & & 46.3 & 1005.45 & & & 39.0 & 1005.60 \\
\hline 51.0 & 1004.46 & & & 48.0 & 1004.80 & & & 41.0 & 1005.55 \\
\hline 54.0 & 1004.29 & & & 51.0 & 1004.50 & & & 44.0 & 1005.56 \\
\hline 56.0 & 1004.39 & & & 53.0 & 1004.49 & & & 47.0 & 1005.19 \\
\hline 58.0 & 1004.22 & & & 56.0 & 1004.47 & & & 48.0 & 1004.83 \\
\hline 61.0 & 1004.38 & & & 60.0 & 1004.52 & & & 50.3 & 1004.60 \\
\hline 64.0 & 1004.42 & & & 64.0 & 1004.60 & & & 51.0 & 1004.49 \\
\hline 66.0 & 1004.44 & & & 67.0 & 1004.61 & & & 55.0 & 1004.44 \\
\hline 69.0 & 1004.35 & & & 70.0 & 1004.62 & & & 57.0 & 1004.36 \\
\hline 71.0 & 1004.59 & & & 70.3 & 1004.77 & & & 60.0 & 1004.34 \\
\hline 75.0 & 1004.68 & & & 72.0 & 1004.76 & & & 62.0 & 1004.39 \\
\hline 78.0 & 1004.62 & & & 74.0 & 1004.55 & & & 64.0 & 1004.28 \\
\hline 80.0 & 1004.64 & & & 76.0 & 1004.56 & & & 66.0 & 1004.41 \\
\hline 82.0 & 1004.57 & & & 80.5 & 1004.64 & & & 67.2 & 1004.59 \\
\hline 83.0 & 1004.24 & & & 81.0 & 1004.52 & & & 68.0 & 1004.65 \\
\hline 85.0 & 1004.38 & & & 82.0 & 1004.51 & & & 70.0 & 1004.69 \\
\hline 88.5 & 1004.51 & & & 84.0 & 1004.49 & & & 73.0 & 1004.69 \\
\hline 90.0 & 1004.22 & & & 86.0 & 1004.42 & & & 76.0 & 1004.68 \\
\hline 92.0 & 1005.73 & & & 88.5 & 1004.29 & & & 80.0 & 1004.58 \\
\hline 92.7 & 1005.89 & & & 90.2 & 1004.09 & & & 85.0 & 1004.54 \\
\hline 94.7 & 1006.78 & & & 91.3 & 1004.61 & & & 87.0 & 1004.11 \\
\hline 97.0 & 1007.09 & & & 91.6 & 1005.12 & & & 90.0 & 1003.97 \\
\hline 99.0 & 1007.26 & & & 93.0 & 1005.94 & & & 91.3 & 1004.26 \\
\hline
\end{tabular}


Table 12.--Listings of horizontal stations and elevations for cross section PR125 --Continued.

\begin{tabular}{|c|c|c|c|c|c|c|c|c|c|}
\hline \multicolumn{2}{|c|}{1985} & \multicolumn{2}{|c|}{1986} & \multicolumn{2}{|c|}{1986} & \multicolumn{2}{|c|}{1987} & \multicolumn{2}{|c|}{1987} \\
\hline Sta. & Elev. & Sta. & Elev. & Sta. & Elev. & Sta. & Elev. & Sta. & Elev. \\
\hline 91.5 & 1005.04 & 0.0 & 1008.02 & 89.0 & 1004.26 & 0.0 & 1008.00 & 70.0 & 1004.57 \\
\hline 93.0 & 1005.83 & 1.0 & 1007.95 & 91.0 & 1004.06 & 1.0 & 1007.97 & 72.0 & 1004.61 \\
\hline 94.0 & 1006.24 & 2.0 & 1007.77 & 91.8 & 1004.60 & 3.0 & 1007.71 & 74.0 & 1004.63 \\
\hline 95.0 & 1006.78 & 3.0 & 1007.71 & 92.2 & 1005.03 & 4.0 & 1007.87 & 76.0 & 1004.58 \\
\hline 97.0 & 1007.03 & 4.0 & 1007.88 & 92.9 & 1005.31 & 5.0 & 1007.88 & 78.0 & 1004.70 \\
\hline 99.0 & 1007.23 & 5.0 & 1007.88 & 93.4 & 1005.63 & 5.4 & 1007.86 & 80.0 & 1004.61 \\
\hline 100.0 & 1007.25 & 5.3 & 1007.85 & 93.8 & 1006.10 & 6.6 & 1007.31 & 82.0 & 1004.56 \\
\hline 100.8 & 1007.26 & 6.0 & 1007.40 & 94.6 & 1006.66 & 8.0 & 1007.32 & 84.0 & 1004.30 \\
\hline 103.0 & 1007.88 & 7.0 & 1007.21 & 95.8 & 1006.77 & 10.0 & 1006.53 & 86.0 & 1004.22 \\
\hline 104.0 & 1007.95 & 8.0 & 1007.32 & 96.8 & 1007.07 & 11.0 & 1005.89 & 88.0 & 1004.18 \\
\hline 107.0 & 1007.36 & 8.4 & 1007.25 & 98.0 & 1007.26 & 13.0 & 1005.87 & 90.0 & 1004.10 \\
\hline 110.0 & 1007.27 & 10.9 & 1005.77 & 100.0 & 1007.26 & 15.0 & 1005.88 & 91.0 & 1004.13 \\
\hline 113.0 & 1007.49 & 11.5 & 1005.68 & 100.8 & 1007.29 & 17.0 & 1005.92 & 92.0 & 1004.38 \\
\hline 117.1 & 1007.60 & 14.0 & 1005.80 & 102.0 & 1007.65 & 20.0 & 1005.99 & 93.2 & 1004.89 \\
\hline & & 17.0 & 1005.84 & 103.0 & 1007.87 & 23.0 & 1006.09 & 94.9 & 1006.15 \\
\hline & & 20.0 & 1005.88 & 104.0 & 1007.95 & 26.0 & 1006.13 & 94.9 & 1006.78 \\
\hline & & 23.0 & 1005.95 & 105.0 & 1007.74 & 28.0 & 1006.17 & 95.8 & 1006.78 \\
\hline & & 26.0 & 1005.99 & 107.0 & 1007.38 & 30.0 & 1006.16 & 97.0 & 1007.07 \\
\hline & & 29.0 & 1005.99 & 109.0 & 1007.29 & 32.0 & 1006.15 & 98.0 & 1007.26 \\
\hline & & 32.0 & 1005.94 & 111.0 & 1007.30 & 34.0 & 1006.17 & 100.0 & 1007.27 \\
\hline & & 35.0 & 1005.86 & 113.0 & 1007.50 & 35.3 & 1006.21 & 100.8 & 1007.30 \\
\hline & & 37.0 & 1005.70 & 115.0 & 1007.53 & 36.0 & 1006.08 & 102.0 & 1007.67 \\
\hline & & 38.0 & 1005.76 & 117.1 & 1007.61 & 36.6 & 1006.06 & 104.0 & 1007.95 \\
\hline & & 40.0 & 1005.77 & & & 37.0 & 1006.00 & 107.0 & 1007.38 \\
\hline & & 42.0 & 1005.64 & & & 37.8 & 1005.97 & 109.4 & 1007.23 \\
\hline & & 44.0 & 1005.62 & & & 39.0 & 1005.64 & 111.0 & 1007.31 \\
\hline & & 46.0 & 1005.47 & & & 40.6 & 1005.34 & 113.0 & 1007.50 \\
\hline & & 47.0 & 1005.19 & & & 43.0 & 1005.45 & 115.0 & 1007.53 \\
\hline & & 48.4 & 1004.76 & & & 43.5 & 1005.50 & 117.1 & 1007.61 \\
\hline & & 49.8 & 1004.68 & & & 44.4 & 1005.43 & & \\
\hline & & 51.0 & 1004.54 & & & 45.3 & 1005.31 & & \\
\hline & & 54.0 & 1004.47 & & & 46.3 & 1005.39 & & \\
\hline & & 57.0 & 1004.27 & & & 47.0 & 1005.23 & & \\
\hline & & 60.0 & 1004.32 & & & 47.6 & 1004.95 & & \\
\hline & & 63.0 & 1004.32 & & & 48.6 & 1004.90 & & \\
\hline & & 65.0 & 1004.30 & & & 50.0 & 1004.75 & & \\
\hline & & 67.0 & 1004.29 & & & 52.0 & 1004.54 & & \\
\hline & & 69.0 & 1004.51 & & & 54.0 & 1004.41 & & \\
\hline & & 72.0 & 1004.54 & & & 56.0 & 1004.40 & & \\
\hline & & 75.0 & 1004.54 & & & 58.0 & 1004.42 & & \\
\hline & & 78.0 & 1004.66 & & & 60.0 & 1004.46 & & \\
\hline & & 80.0 & 1004.67 & & & 62.0 & 1004.40 & & \\
\hline & & 81.2 & 1004.45 & & & 64.0 & 1004.46 & & \\
\hline & & 83.0 & 1004.56 & & & 66.0 & 1004.48 & & \\
\hline & & 86.0 & 1004.46 & & & 68.0 & 1004.48 & & \\
\hline
\end{tabular}


Table 12.--Listing of horizontal stations and elevations for cross section PR125 --Continued.

\begin{tabular}{|c|c|c|c|}
\hline \multicolumn{2}{|c|}{1988} & \multicolumn{2}{|c|}{1988} \\
\hline Sta. & Elev. & Sta. & Elev. \\
\hline 0.0 & 1007.99 & 64.0 & 1004.37 \\
\hline 1.0 & 1007.97 & 67.0 & 1004.34 \\
\hline 2.4 & 1007.70 & 68.6 & 1004.57 \\
\hline 3.0 & 1007.71 & 69.0 & 1004.70 \\
\hline 3.5 & 1007.84 & 70.0 & 1004.75 \\
\hline 4.0 & 1007.88 & 72.0 & 1004.77 \\
\hline 5.0 & 1007.88 & 74.0 & 1004.76 \\
\hline 5.3 & 1007.85 & 76.0 & 1004.70 \\
\hline 6.1 & 1007.36 & 78.5 & 1004.65 \\
\hline 7.0 & 1007.20 & 80.0 & 1004.67 \\
\hline 8.0 & 1007.32 & 82.0 & 1004.67 \\
\hline 9.3 & 1006.72 & 83.4 & 1004.66 \\
\hline 9.8 & 1006.51 & 84.0 & 1004.56 \\
\hline 10.5 & 1005.94 & 84.5 & 1004.52 \\
\hline 11.0 & 1005.81 & 85.0 & 1004.54 \\
\hline 12.0 & 1005.84 & 86.0 & 1004.46 \\
\hline 14.0 & 1005.88 & 88.0 & 1004.46 \\
\hline 16.0 & 1005.90 & 89.0 & 1004.39 \\
\hline 18.0 & 1005.95 & 91.5 & 1004.31 \\
\hline 20.0 & 1006.00 & 92.7 & 1004.56 \\
\hline 23.0 & 1006.09 & 93.3 & 1004.88 \\
\hline 26.0 & 1006.14 & 94.0 & 1005.12 \\
\hline 29.0 & 1006.16 & 95.0 & 1006.11 \\
\hline 32.0 & 1006.18 & 95.1 & 1006.77 \\
\hline 34.0 & 1006.20 & 96.0 & 1006.80 \\
\hline 35.2 & 1006.20 & 97.0 & 1007.05 \\
\hline 36.0 & 1006.08 & 98.4 & 1007.28 \\
\hline 37.0 & 1005.99 & 99.5 & 1007.24 \\
\hline 37.8 & 1005.96 & 100.8 & 1007.29 \\
\hline 39.6 & 1005.40 & 101.5 & 1007.44 \\
\hline 40.0 & 1005.37 & 103.0 & 1007.85 \\
\hline 41.0 & 1005.41 & 104.0 & 1007.94 \\
\hline 42.6 & 1005.43 & 105.0 & 1007.69 \\
\hline 43.6 & 1005.50 & 106.0 & 1007.50 \\
\hline 44.8 & 1005.39 & 107.0 & 1007.38 \\
\hline 46.0 & 1005.42 & 109.4 & 1007.23 \\
\hline 46.9 & 1005.34 & 111.6 & 1007.32 \\
\hline 47.5 & 1004.96 & 113.0 & 1007.50 \\
\hline 49.0 & 1004.88 & 115.0 & 1007.53 \\
\hline 51.0 & 1004.58 & 117.1 & 1007.59 \\
\hline 53.0 & 1004.44 & & \\
\hline 55.0 & 1004.32 & & \\
\hline 57.0 & 1004.30 & & \\
\hline 59.0 & 1004.38 & & \\
\hline 61.0 & 1004.37 & & \\
\hline
\end{tabular}




\section{Description of Cross Section PR130}

Location: Township 8 South/Range 48 East--section 14

U.S. Geological Survey quadrangle $(1: 24,000)$ : Bloom Creek

Landowners--left bank: Gay Ranch

right bank: Jim Bowers

Access: left bank

Permission from: Gay Ranch (T7S/R49E--20)

Reference pins

\begin{tabular}{|c|c|c|}
\hline $\begin{array}{l}\text { Station } \\
\text { (meters) }\end{array}$ & $\begin{array}{c}\text { Elevation } \\
\text { (meters } \\
\text { above } \\
\text { sea level) }\end{array}$ & Comments \\
\hline & & $\begin{array}{l}\text { Pins }-1.1 \text { and } 0.0 \text { were closest to } \\
\text { leveling instrument. }\end{array}$ \\
\hline-1.1 & $1,000.706$ & $\begin{array}{l}\text { Bent over, } 0.02 \text { meter above } 1987 \\
\text { ground level. }\end{array}$ \\
\hline 0.0 & $1,000.669$ & $\begin{array}{l}\text { Bent over, } 0.02 \text { meter above } 1987 \\
\text { ground level. }\end{array}$ \\
\hline 101.9 & $1,000.641$ & 0.06 meter above 1987 ground level. \\
\hline 108.4 & $1,000.924$ & 0.01 meter above 1987 ground level. \\
\hline 110.6 & $1,000.932$ & 0.20 meter above 1987 ground level. \\
\hline
\end{tabular}




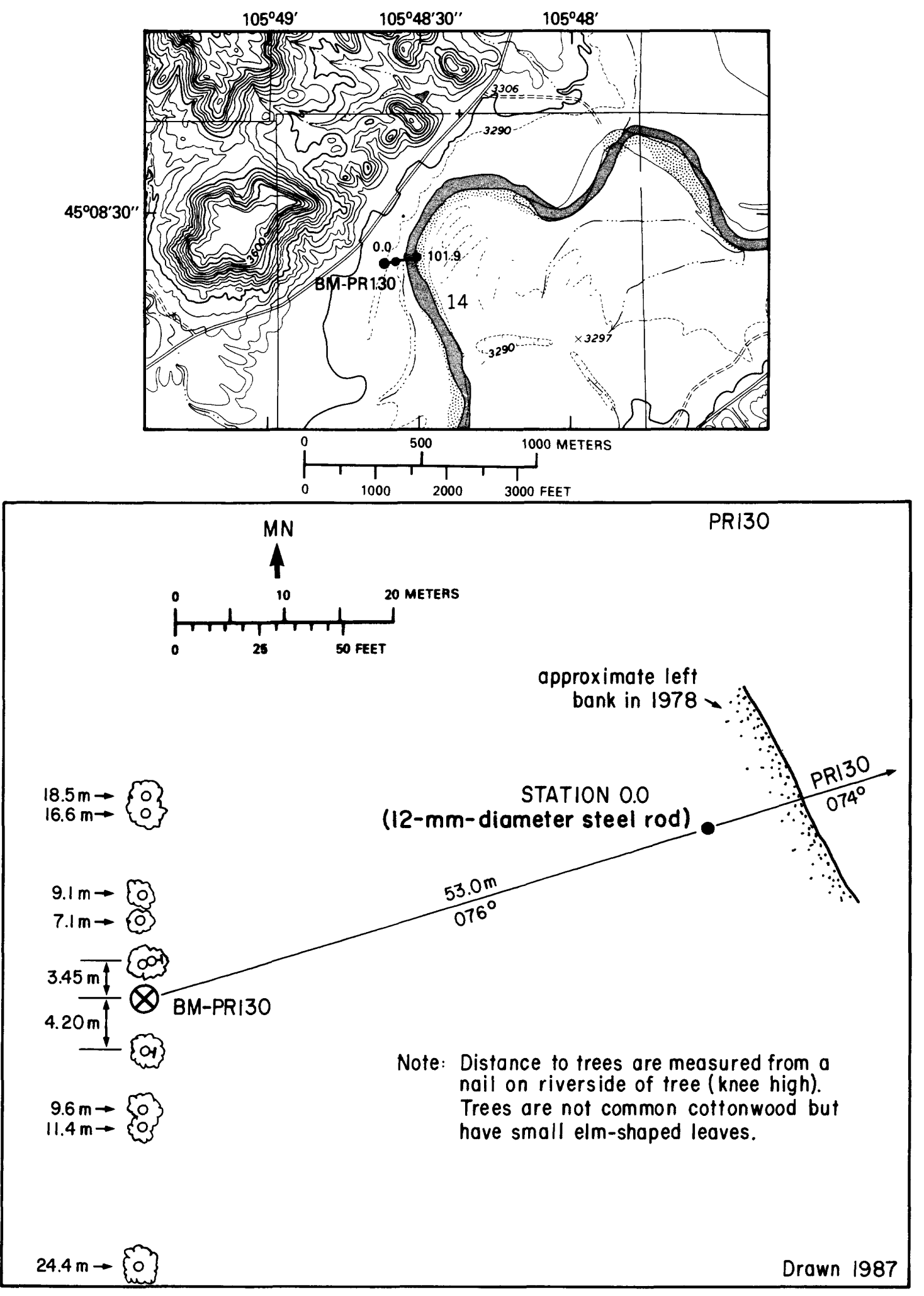

Figure 31.--Location of cross section PR130, bench mark BM-PR130, and left and right bank reference pins (tables 2 and 3 ) in the Bloom Creek quadrangle. Lower: Location of bench mark on the left bank. MN is magnetic north. 

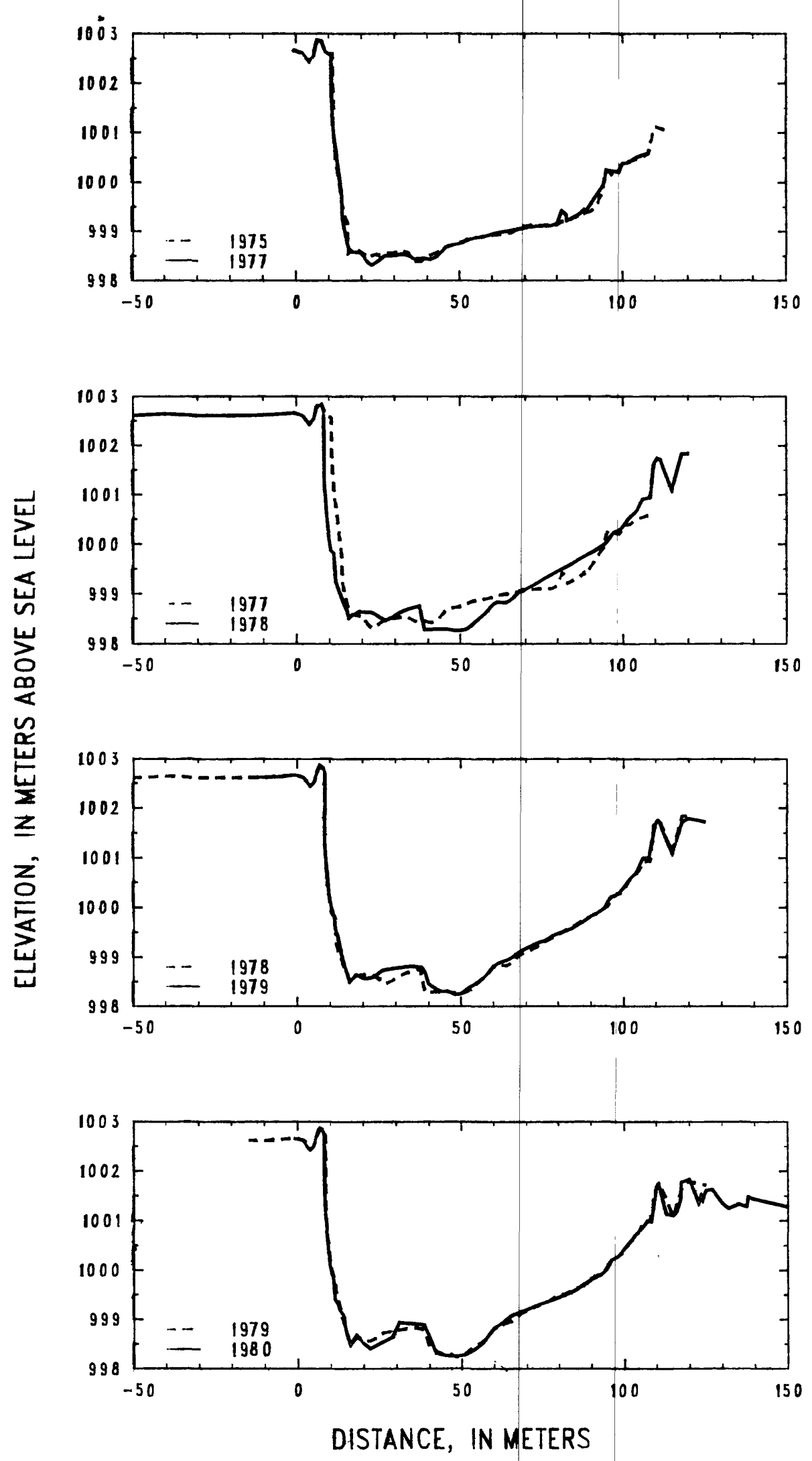

Figure 32.--Profiles of cross section PR130 from 1975 to 1980. 

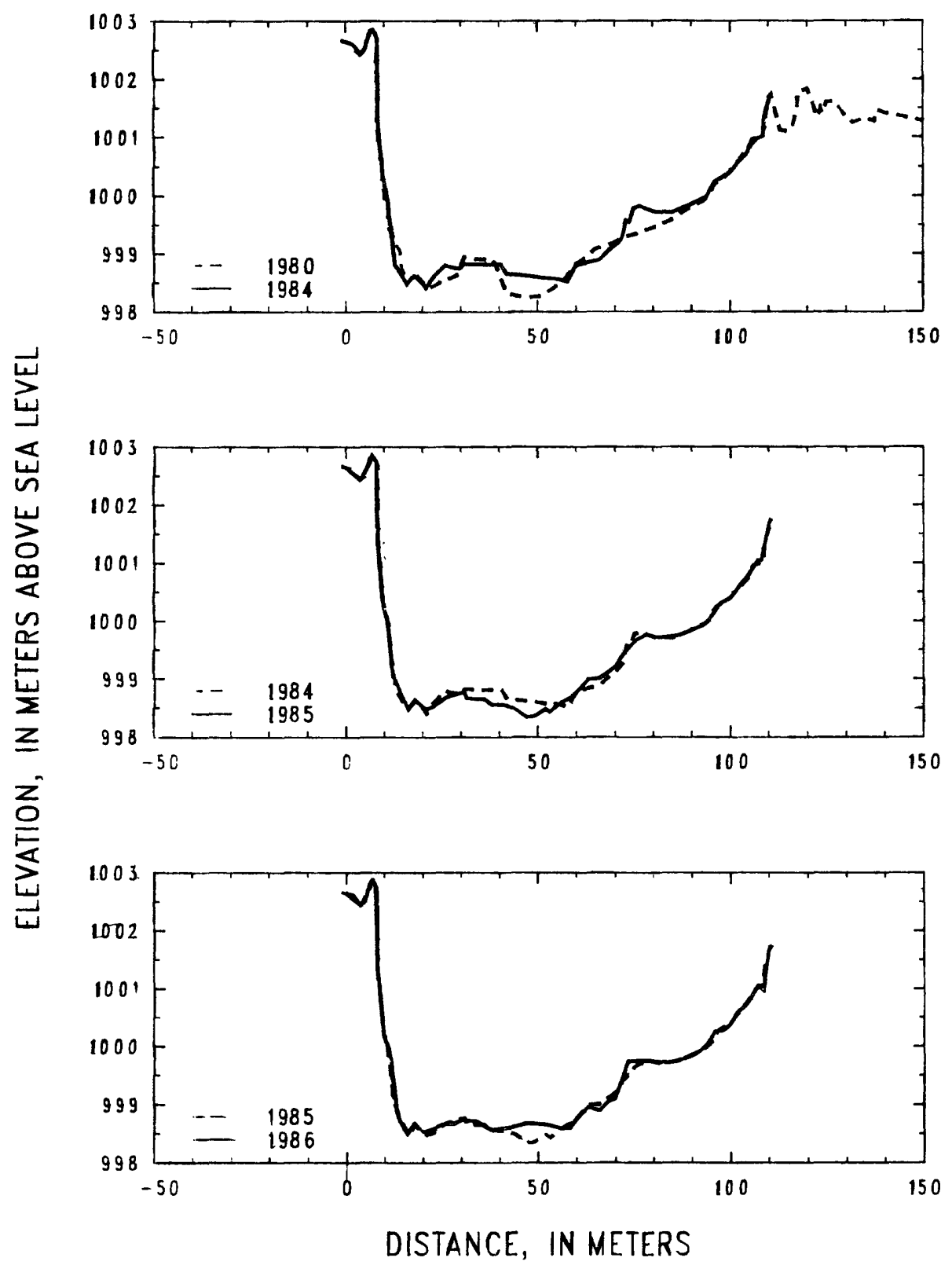

Figure 33. --Profiles of cross section PR130 from 1980 to 1986. 


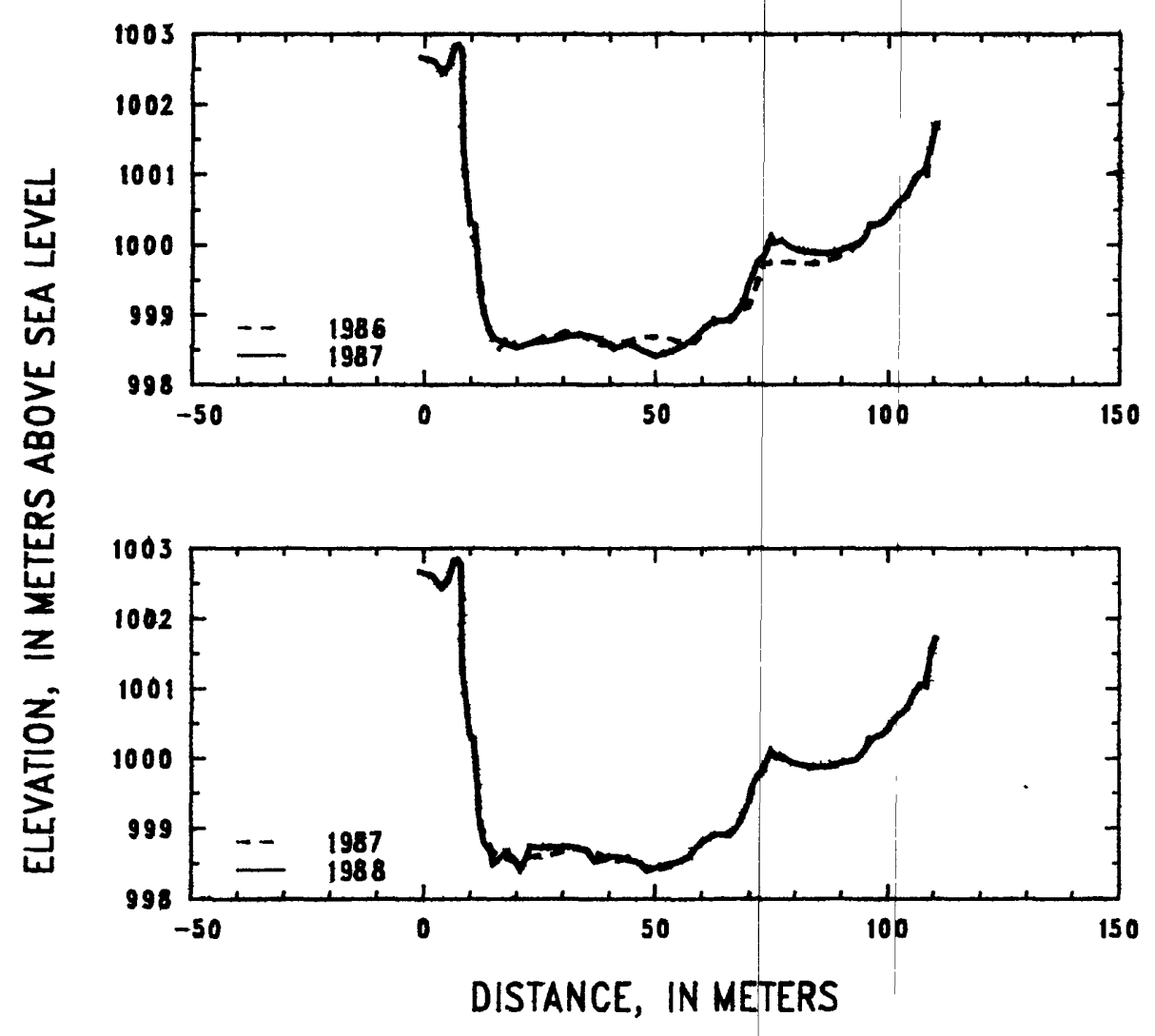

Figure 34. --Profiles of cross section PR130 from 1986 to 1988. 
Table 13.--Listing of horizontal stations and elevations for cross section PR130 [Sta., station, distance in meters from a reference point on the left bank; Elev., elevation, in meters above sea levell.

\begin{tabular}{|c|c|c|c|c|c|c|c|c|c|}
\hline \multicolumn{2}{|c|}{1975} & \multicolumn{2}{|c|}{1975} & \multicolumn{2}{|c|}{1977} & \multicolumn{2}{|c|}{1977} & \multicolumn{2}{|c|}{1978} \\
\hline Sta. & Elev. & Sta. & Elev. & Sta. & Elev. & Sta. & Elev. & Sta. & Elev. \\
\hline-1.1 & 1002.66 & 80.0 & 999.12 & -1.1 & 1002.68 & 92.0 & 999.74 & -50.0 & 1002.61 \\
\hline 0.0 & 1002.65 & 82.0 & 999.23 & 0.0 & 1002.67 & 94.1 & 999.93 & -40.0 & 1002.65 \\
\hline 2.0 & 1002.60 & 84.0 & 999.24 & 2.0 & 1002.62 & 95.1 & 1000.25 & -30.0 & 1002.60 \\
\hline 4.0 & 1002.43 & 86.0 & 999.30 & 4.0 & 1002.43 & 99.0 & 1000.20 & -20.0 & 1002.61 \\
\hline 7.0 & 1002.90 & 88.0 & 999.37 & 5.4 & 1002.54 & 100.0 & 1000.39 & -10.0 & 1002.62 \\
\hline 8.0 & 1002.81 & 90.0 & 999.39 & 6.6 & 1002.88 & 101.8 & 1000.40 & -1.1 & 1002.66 \\
\hline 9.0 & 1002.63 & 92.0 & 999.51 & 7.8 & 1002.86 & 104.0 & 1000.50 & 0.0 & 1002.66 \\
\hline 10.0 & 1002.60 & 94.0 & 999.85 & 9.0 & 1002.64 & 106.0 & 1000.55 & 2.0 & 1002.60 \\
\hline 11.3 & 1002.61 & 95.0 & 1000.24 & 10.0 & 1002.60 & 108.0 & 1000.59 & 4.0 & 1002.43 \\
\hline 11.5 & 1001.12 & 97.0 & 1000.13 & 10.8 & 1002.57 & 108.4 & 1000.66 & 5.4 & 1002.54 \\
\hline 12.2 & 1000.38 & 98.0 & 1000.13 & 10.9 & 1001.63 & & & 6.6 & 1002.81 \\
\hline 12.7 & 1000.24 & 100.0 & 1000.38 & 11.6 & 1001.01 & & & 8.0 & 1002.81 \\
\hline 13.1 & 1000.05 & 105.0 & 1000.49 & 14.0 & 999.69 & & & 8.4 & 1002.69 \\
\hline 14.0 & 999.57 & 108.0 & 1000.58 & 14.1 & 999.25 & & & 8.5 & 1001.24 \\
\hline 15.0 & 999.29 & 110.0 & 1001.12 & 15.9 & 998.66 & & & 9.4 & 1000.40 \\
\hline 15.6 & 999.18 & 115.0 & 1001.01 & 17.0 & 998.58 & & & 10.5 & 999.86 \\
\hline 15.9 & 998.71 & & & 20.0 & 998.56 & & & 11.5 & 999.80 \\
\hline 16.0 & 998.55 & & & 22.0 & 998.36 & & & 12.0 & 999.24 \\
\hline 18.0 & 998.66 & & & 23.0 & 998.31 & & & 14.0 & 998.85 \\
\hline 20.0 & 998.57 & & & 25.0 & 998.39 & & & 15.4 & 998.66 \\
\hline 22.0 & 998.46 & & & 27.0 & 998.50 & & & 15.9 & 998.51 \\
\hline 24.0 & 998.53 & & & 30.0 & 998.51 & & & 17.0 & 998.57 \\
\hline 26.0 & 998.52 & & & 32.0 & 998.54 & & & 19.0 & 998.65 \\
\hline 28.0 & 998.56 & & & 34.0 & 998.52 & & & 21.0 & 998.64 \\
\hline 30.0 & 998.56 & & & 36.0 & 998.44 & & & 23.0 & 998.63 \\
\hline 32.0 & 998.60 & & & 39.0 & 998.45 & & & 25.0 & 998.54 \\
\hline 34.0 & 998.57 & & & 41.0 & 998.42 & & & 27.0 & 998.45 \\
\hline 36.0 & 998.37 & & & 43.0 & 998.48 & & & 29.0 & 998.53 \\
\hline 38.0 & 998.39 & & & 45.4 & 998.66 & & & 31.0 & 998.61 \\
\hline 40.0 & 998.49 & & & 48.0 & 998.73 & & & 33.0 & 998.67 \\
\hline 42.0 & 998.55 & & & 50.0 & 998.75 & & & 35.0 & 998.71 \\
\hline 44.0 & 998.60 & & & 54.0 & 998.86 & & & 37.4 & 998.77 \\
\hline 46.0 & 998.70 & & & 58.0 & 998.91 & & & 39.0 & 998.28 \\
\hline 47.0 & 998.70 & & & 62.0 & 998.98 & & & 40.0 & 998.28 \\
\hline 50.0 & 998.77 & & & 66.0 & 999.02 & & & 42.0 & 998.29 \\
\hline 54.0 & 998.86 & & & 70.0 & 999.08 & & & 44.0 & 998.29 \\
\hline 58.0 & 998.90 & & & 74.0 & 999.11 & & & 46.0 & 998.29 \\
\hline 62.0 & 998.94 & & & 78.0 & 999.11 & & & 48.0 & 998.26 \\
\hline 66.0 & 998.97 & & & 80.0 & 999.17 & & & 50.0 & 998.26 \\
\hline 68.0 & 999.09 & & & 81.5 & 999.42 & & & 52.0 & 998.29 \\
\hline 70.0 & 999.10 & & & 82.6 & 999.35 & & & 54.0 & 998.40 \\
\hline 72.0 & 999.13 & & & 83.0 & 999.21 & & & 56.0 & 998.52 \\
\hline 74.0 & 999.11 & & & 87.0 & 999.36 & & & 58.0 & 998.63 \\
\hline 76.0 & 999.13 & & & 88.1 & 999.44 & & & 60.0 & 998.79 \\
\hline 78.0 & 999.13 & & & 88.4 & 999.37 & & & 61.5 & 998.84 \\
\hline
\end{tabular}


Table 13.--Listing of horizontal stations and elevations for cross section PR130 --Continued.

\begin{tabular}{|c|c|c|c|c|c|c|c|c|c|}
\hline \multicolumn{2}{|c|}{1978} & \multicolumn{2}{|c|}{1979} & \multicolumn{2}{|c|}{1979} & \multicolumn{2}{|c|}{1980} & \multicolumn{2}{|c|}{1980} \\
\hline Sta. & Elev. & Sta. & Elev. & Sta. & Elev. & Sta. & Elev. & Sta. & Elev. \\
\hline 64.0 & 998.82 & -15.0 & 1002.63 & 62.0 & 998.89 & -1.1 & 1002.66 & 96.0 & 1000.20 \\
\hline 66.0 & 998.90 & -10.0 & 1002.62 & 64.0 & 998.93 & 0.0 & 1002.65 & 98.0 & 1000.27 \\
\hline 68.0 & 999.04 & -5.0 & 1002.64 & 66.0 & 999.00 & 2.0 & 1002.60 & 100.0 & 1000.41 \\
\hline 70.0 & 999.09 & -1.1 & 1002.67 & 68.0 & 999.11 & 3.0 & 1002.48 & 101.8 & 1000.56 \\
\hline 72.0 & 999.16 & 0.0 & 1002.66 & 70.0 & 999.18 & 4.0 & 1002.42 & 103.0 & 1000.67 \\
\hline 76.0 & 999.31 & 2.0 & 1002.61 & 72.0 & 999.24 & 5.0 & 1002.49 & 105.0 & 1000.80 \\
\hline 80.0 & 999.45 & 4.0 & 1002.43 & 74.0 & 999.30 & 6.6 & 1002.83 & 107.0 & 1000.99 \\
\hline 84.0 & 999.59 & 5.0 & 1002.50 & 76.0 & 999.33 & 7.6 & 1002.85 & 108.5 & 1000.97 \\
\hline 88.0 & 999.76 & 6.0 & 1002.69 & 78.0 & 999.43 & 8.3 & 1002.72 & 110.0 & 1001.70 \\
\hline 92.0 & 999.89 & 7.0 & 1002.87 & 80.0 & 999.48 & 8.4 & 1001.24 & 110.6 & 1001.75 \\
\hline 95.0 & 1000.03 & 8.0 & 1002.81 & 82.0 & 999.52 & 10.0 & 1000.14 & 113.0 & 1001.12 \\
\hline 97.0 & 1000.23 & 8.5 & 1002.69 & 84.0 & 999.57 & 10.5 & 999.92 & 115.0 & 1001.09 \\
\hline 99.0 & 1000.29 & 8.5 & 1001.20 & 86.0 & 999.65 & 11.1 & 999.85 & 116.0 & 1001.15 \\
\hline 100.0 & 1000.35 & 10.0 & 1000.16 & 88.0 & 999.73 & 11.5 & 999.41 & 117.4 & 1001.45 \\
\hline 101.8 & 1000.54 & 11.0 & 999.88 & 90.0 & 999.83 & 12.6 & 999.29 & 117.5 & 1001.77 \\
\hline 104.0 & 1000.66 & 11.5 & 999.78 & 92.0 & 999.89 & 12.7 & 999.20 & 120.0 & 1001.83 \\
\hline 106.0 & 1000.91 & 11.6 & 999.57 & 94.0 & 999.98 & 14.2 & 999.06 & 122.8 & 1001.34 \\
\hline 108.0 & 1000.94 & 12.4 & 999.42 & 96.0 & 1000.20 & 14.7 & 998.79 & 123.5 & 1001.50 \\
\hline 108.4 & 1000.93 & 12.6 & 999.30 & 98.0 & 1000.27 & 16.1 & 998.47 & 124.0 & 1001.40 \\
\hline 109.6 & 1001.65 & 14.0 & 998.92 & 100.0 & 1000.39 & 18.0 & 998.68 & 125.0 & 1001.60 \\
\hline 110.6 & 1001.74 & 15.0 & 998.70 & 101.8 & 1000.57 & 20.0 & 998.51 & 127.0 & 1001.62 \\
\hline 111.5 & 1001.71 & 16.0 & 998.49 & 104.0 & 1000.70 & 22.0 & 998.41 & 130.0 & 1001.35 \\
\hline 115.0 & 1001.08 & 18.0 & 998.64 & 106.0 & 1000.99 & 25.0 & 998.51 & 132.0 & 1001.24 \\
\hline 118.0 & 1001.83 & 20.0 & 998.56 & 108.0 & 1001.00 & 29.0 & 998.64 & 135.0 & 1001.33 \\
\hline \multirow[t]{8}{*}{120.0} & 1001.84 & 22.0 & 998.56 & 110.0 & 1001.70 & 31.0 & 998.94 & 137.5 & 1001.27 \\
\hline & & 24.0 & 998.63 & 110.6 & 1001.76 & 34.0 & 998.91 & 138.0 & 1001.47 \\
\hline & & 26.0 & 998.73 & 111.0 & 1001.74 & 38.5 & 998.89 & 140.0 & 1001.42 \\
\hline & & 28.0 & 998.76 & 115.0 & 1001.08 & 40.0 & 998.67 & 150.0 & 1001.27 \\
\hline & & 30.0 & 998.78 & 118.0 & 1001.72 & 42.0 & 998.31 & & \\
\hline & & 32.0 & 998.80 & 120.0 & 1001.79 & 45.0 & 998.26 & & \\
\hline & & 34.0 & 998.82 & 125.0 & 1001.71 & 48.0 & 998.24 & & \\
\hline & & 36.0 & 998.82 & & & 51.0 & 998.27 & & \\
\hline \multirow[t]{13}{*}{ - } & & 38.0 & 998.79 & & & 54.0 & 998.39 & & \\
\hline & & 39.0 & 998.68 & & & 57.0 & 998.54 & & \\
\hline & & 40.0 & 998.46 & & & 60.0 & 998.82 & & \\
\hline & & 42.0 & 998.36 & & & 63.0 & 998.94 & & \\
\hline & & 44.0 & 998.29 & & & 65.0 & 999.07 & & \\
\hline & & 46.0 & 998.31 & & & 70.0 & 999.19 & & \\
\hline & & 48.0 & 998.23 & & & 73.0 & 999.28 & & \\
\hline & & 50.0 & 998.25 & & & 77.0 & 999.37 & & \\
\hline & & 52.0 & 998.35 & & & 81.0 & 999.47 & & \\
\hline & & 54.0 & 998.44 & & & 85.0 & 999.58 & & \\
\hline & & 56.0 & 998.52 & & & 90.0 & 999.81 & & \\
\hline & & 58.0 & 998.63 & & & 93.0 & 999.90 & & \\
\hline & & 60.0 & 998.81 & & & 95.0 & 1000.05 & & \\
\hline
\end{tabular}


Table 13.--Listing of horizontal stations and elevations for cross section PR130 --Continued.

\begin{tabular}{|c|c|c|c|c|c|c|c|c|c|}
\hline \multicolumn{2}{|c|}{1982} & \multicolumn{2}{|c|}{1982} & \multicolumn{2}{|c|}{1984} & \multicolumn{2}{|c|}{1984} & \multicolumn{2}{|c|}{1985} \\
\hline Sta. & Elev. & Sta. & Elev. & Sta. & Elev. & Sta. & Elev. & Sta. & Elev. \\
\hline-1.1 & 1002.67 & 100.0 & 1000.40 & -1.1 & 1002.67 & 94.0 & 999.98 & 0.0 & 1002.65 \\
\hline 0.0 & 1002.65 & 101.9 & 1000.57 & 0.0 & 1002.65 & 96.0 & 1000.24 & 4.0 & 1002.43 \\
\hline 2.0 & 1002.60 & 106.0 & 1000.98 & 2.0 & 1002.60 & 100.0 & 1000.40 & 6.0 & 1002.68 \\
\hline 4.0 & 1002.42 & 108.5 & 1000.97 & 4.0 & 1002.44 & 101.9 & 1000.56 & 7.0 & 1002.86 \\
\hline 6.0 & 1002.67 & 110.0 & 1001.69 & 5.0 & 1002.50 & 104.0 & 1000.69 & 8.2 & 1002.74 \\
\hline 7.0 & 1002.85 & 110.6 & 1001.74 & 6.6 & 1002.84 & 106.0 & 1000.96 & 8.5 & 1001.31 \\
\hline 8.0 & 1002.80 & & & 7.3 & 1002.86 & 108.5 & 1001.01 & 10.0 & 1000.22 \\
\hline 8.4 & 1002.68 & & & 8.4 & 1002.70 & 109.0 & 1001.38 & 11.0 & 999.99 \\
\hline 8.5 & 1001.20 & & & 8.6 & 1001.30 & 110.6 & 1001.74 & 12.5 & 999.05 \\
\hline 10.0 & 1000.27 & & & 10.0 & 1000.27 & & & 14.7 & 998.69 \\
\hline 10.3 & 1000.06 & & & 11.0 & 1000.01 & & & 16.3 & 998.47 \\
\hline 10.5 & 1000.01 & & & 13.0 & 998.81 & & & 18.0 & 998.64 \\
\hline 11.2 & 1000.02 & & & 14.0 & 998.71 & & & 20.0 & 998.51 \\
\hline 11.3 & 999.98 & & & 16.0 & 998.48 & & & 21.0 & 998.47 \\
\hline 11.8 & 999.68 & & & 18.0 & 998.62 & & & 23.0 & 998.53 \\
\hline 12.1 & 999.68 & & & 20.0 & 998.50 & & & 26.0 & 998.68 \\
\hline 12.7 & 999.31 & & & 21.0 & 998.40 & & & 30.0 & 998.76 \\
\hline 13.0 & 999.37 & & & 23.0 & 998.61 & & & 31.0 & 998.77 \\
\hline 13.5 & 999.08 & & & 26.0 & 998.80 & & & 31.5 & 998.66 \\
\hline 15.0 & 998.65 & & & 28.0 & 998.75 & & & 34.0 & 998.64 \\
\hline 16.0 & 998.61 & & & 30.0 & 998.74 & & & 36.0 & 998.65 \\
\hline 17.0 & 998.51 & & & 31.0 & 998.83 & & & 38.0 & 998.55 \\
\hline 20.0 & 998.57 & & & 33.0 & 998.82 & & & 41.0 & 998.55 \\
\hline 22.0 & 998.73 & & & 36.0 & 998.81 & & & 44.0 & 998.48 \\
\hline 25.0 & 998.61 & & & 40.5 & 998.82 & & & 47.0 & 998.33 \\
\hline 25.0 & 998.63 & & & 42.0 & 998.65 & & & 49.0 & 998.35 \\
\hline 30.0 & 998.70 & & & 43.0 & 998.64 & & & 52.0 & 998.48 \\
\hline 35.0 & 998.77 & & & 46.0 & 998.62 & & & 53.0 & 998.42 \\
\hline 39.0 & 998.65 & & & 49.0 & 998.60 & & & 56.0 & 998.59 \\
\hline 42.0 & 998.61 & & & 52.0 & 998.57 & & & 59.0 & 998.69 \\
\hline 45.0 & 998.64 & & & 55.0 & 998.56 & & & 62.0 & 998.91 \\
\hline 48.0 & 998.33 & & & 58.0 & 998.51 & & & 63.0 & 998.99 \\
\hline 50.0 & 998.31 & & & 60.0 & 998.79 & & & 66.0 & 999.01 \\
\hline 54.0 & 998.40 & & & 63.0 & 998.84 & & & 70.0 & 999.20 \\
\hline 57.0 & 998.53 & & & 66.0 & 998.89 & & & 72.0 & 999.41 \\
\hline 60.0 & 998.81 & & & 69.0 & 999.08 & & & 75.0 & 999.64 \\
\hline 65.0 & 998.88 & & & 71.0 & 999.18 & & & 78.0 & 999.76 \\
\hline 67.4 & 999.08 & & & 72.0 & 999.28 & & & 81.0 & 999.71 \\
\hline 69.7 & 999.30 & & & 73.0 & 999.57 & & & 84.0 & 999.72 \\
\hline 73.0 & 999.52 & & & 74.0 & 999.54 & & & 87.0 & 999.76 \\
\hline 77.0 & 999.62 & & & 75.0 & 999.78 & & & 90.0 & 999.85 \\
\hline 80.0 & 999.58 & & & 76.5 & 999.82 & & & 93.0 & 999.93 \\
\hline 85.0 & 999.66 & & & 80.0 & 999.72 & & & 95.0 & 1000.08 \\
\hline 90.0 & 999.84 & & & 85.0 & 999.70 & & & 97.0 & 1000.29 \\
\hline 95.0 & 1000.06 & & & 90.0 & 999.85 & & & 100.0 & 1000.40 \\
\hline
\end{tabular}


Table 13.--Listing of horizontal stations and elevations for cross section PR130 --Continued.

\begin{tabular}{|c|c|c|c|c|c|c|c|c|c|}
\hline \multicolumn{2}{|c|}{1985} & \multicolumn{2}{|c|}{1986} & \multicolumn{2}{|c|}{1986} & \multicolumn{2}{|c|}{1987} & \multicolumn{2}{|c|}{1987} \\
\hline Sta. & Elev. & Sta. & Elev. & Sta. & Eler. & Sta. & Elev. & Sta. & Elev. \\
\hline 101.9 & 1000.56 & -1.1 & 1002.67 & 94.0 & 1000.05 & -1.1 & 1002.67 & 87.0 & 999.87 \\
\hline 105.0 & 1000.82 & 0.0 & 1002.65 & 96.0 & 1000.26 & 0.0 & 1002.65 & 89.0 & 999.90 \\
\hline 107.0 & 1001.05 & 2.0 & 1002.61 & 98.0 & 1000.28 & 2.0 & 1002.61 & 91.0 & 999.95 \\
\hline 108.0 & 1001.04 & 4.0 & 1002.44 & 100.0 & 1000.39 & 4.0 & 1002.44 & 93.0 & 999.99 \\
\hline 110.0 & 1001.67 & 5.0 & 1002.50 & 101.9 & 1000.60 & 5.4 & 1002.57 & 95.0 & 1000.11 \\
\hline \multirow[t]{40}{*}{110.6} & 1001.75 & 6.6 & 1002.84 & 103.0 & 1000.65 & 6.5 & 1002.83 & 96.0 & 1000.29 \\
\hline & & 7.2 & 1002.88 & 105.0 & 1000.81 & 7.3 & 1002.85 & 98.0 & 1000.29 \\
\hline & & 8.1 & 1002.74 & 107.0 & 1001.04 & 8.2 & 1002.76 & 100.0 & 1000.41 \\
\hline & & 8.4 & 1001.33 & 108.5 & 1000.96 & 8.3 & 1002.40 & 101.9 & 1000.58 \\
\hline & & 10.1 & 1000.14 & 110.0 & 1001.68 & 8.4 & 1001.37 & 104.0 & 1000.70 \\
\hline & & 11.3 & 999.98 & 110.6 & 1001.74 & 10.0 & 1000.34 & 106.0 & 1000.96 \\
\hline & & 13.6 & 998.80 & & & 11.0 & 1000.28 & 108.0 & 1001.05 \\
\hline & & 16.0 & 998.49 & & & 12.2 & 999.29 & 109.0 & 1001.36 \\
\hline & & 18.0 & 998.68 & & & 12.8 & 999.00 & 110.6 & 1001.73 \\
\hline & & 20.0 & 998.53 & & & 15.0 & 998.66 & & \\
\hline & & 22.0 & 998.57 & & & 17.0 & 998.59 & & \\
\hline & & 25.0 & 998.66 & & & 20.0 & 998.52 & & \\
\hline & & 28.0 & 998.67 & & & 23.0 & 998.60 & & \\
\hline & & 30.0 & 998.74 & & & 26.0 & 998.61 & & \\
\hline & & 31.0 & 998.74 & & & 29.0 & 998.65 & & \\
\hline & & 34.0 & 998.71 & & & 32.0 & 998.70 & & \\
\hline & & 36.0 & 998.63 & & & 35.0 & 998.70 & & \\
\hline & & 38.0 & 998.56 & & & 38.0 & 998.63 & & \\
\hline & & 41.0 & 998.59 & & & 41.0 & 998.50 & & \\
\hline & & 44.0 & 998.62 & & & 44.0 & 998.59 & & \\
\hline & & 47.0 & 998.68 & & & 47.0 & 998.47 & & \\
\hline & & 50.0 & 998.67 & & & 50.0 & 998.39 & & \\
\hline & & 53.0 & 998.64 & & & 53.0 & 998.45 & & \\
\hline & & 56.0 & 998.58 & & & 56.0 & 998.54 & & \\
\hline & & 58.5 & 998.59 & & & 59.0 & 998.70 & & \\
\hline & & 60.5 & 998.79 & & & 61.0 & 998.84 & & \\
\hline & & 63.0 & 998.96 & & & 63.0 & 998.90 & & \\
\hline & & 66.0 & 998.89 & & & 65.0 & 998.91 & & \\
\hline & & 68.0 & 999.05 & & & 67.2 & 999.00 & & \\
\hline & & 69.9 & 999.10 & & & 68.8 & 999.16 & & \\
\hline & & 72.0 & 999.48 & & & 70.4 & 999.51 & & \\
\hline & & 73.3 & 999.74 & & & 72.0 & 999.76 & & \\
\hline & & 75.0 & 999.74 & & & 73.3 & 999.83 & & \\
\hline & & 77.0 & 999.75 & & & 74.8 & 1000.11 & & \\
\hline & & 79.0 & 999.75 & & & 75.3 & 1000.02 & & \\
\hline & & 81.0 & 999.73 & & & 77.0 & 1000.06 & & \\
\hline & & 84.0 & 999.72 & & & 79.0 & 999.95 & & \\
\hline & & 87.0 & 999.77 & & & 81.0 & 999.90 & & \\
\hline & & 90.0 & 999.85 & & & 83.0 & 999.88 & & \\
\hline & & 92.0 & 999.91 & & & 85.0 & 999.87 & & \\
\hline
\end{tabular}


Table 13.--Listing of horizontal stations and elevations for cross section PR130 --Continued.

\begin{tabular}{|c|c|c|c|}
\hline \multicolumn{2}{|c|}{1988} & \multicolumn{2}{|c|}{1988} \\
\hline Sta. & Elev. & Sta. & Elev. \\
\hline-1.0 & 1002.67 & 69.0 & 999.21 \\
\hline 0.0 & 1002.65 & 70.5 & 999.42 \\
\hline 2.0 & 1002.60 & 71.0 & 999.64 \\
\hline 4.0 & 1002.43 & 73.0 & 999.82 \\
\hline 5.0 & 1002.51 & 74.0 & 999.97 \\
\hline 6.0 & 1002.67 & 74.8 & 1000.11 \\
\hline 6.5 & 1002.83 & 76.0 & 1000.01 \\
\hline 7.5 & 1002.85 & 78.0 & 999.99 \\
\hline 8.1 & 1002.77 & 80.0 & 999.92 \\
\hline 8.3 & 1002.42 & 83.0 & 999.88 \\
\hline 8.4 & 1001.26 & 86.0 & 999.87 \\
\hline $9: 9$ & 1000.43 & 89.0 & 999.92 \\
\hline 10.5 & 1000.26 & 91.0 & 999.95 \\
\hline 11.0 & 1000.24 & 93.0 & 999.98 \\
\hline 11.8 & 999.48 & 95.0 & 1000.13 \\
\hline 12.2 & 999.11 & 97.0 & 1000.30 \\
\hline 13.1 & 998.79 & 99.0 & 1000.35 \\
\hline 14.5 & 998.66 & 101.9 & 1000.59 \\
\hline 15.0 & 998.48 & 104.0 & 1000.72 \\
\hline 18.0 & 998.68 & 106.0 & 1000.96 \\
\hline 20.0 & 998.52 & 107.0 & 1001.06 \\
\hline 20.9 & 998.40 & 108.5 & 1001.03 \\
\hline 22.7 & 998.64 & 109.4 & 1001.54 \\
\hline 23.0 & 998.75 & 110.6 & 1001.73 \\
\hline 25.0 & 998.72 & & \\
\hline 27.0 & 998.74 & & \\
\hline 29.0 & 998.72 & & \\
\hline 31.0 & 998.74 & & \\
\hline 33.0 & 998.72 & & \\
\hline 35.2 & 998.68 & & \\
\hline 37.0 & 998.52 & & \\
\hline 39.0 & 998.56 & & \\
\hline 41.0 & 998.61 & & \\
\hline 44.0 & 998.55 & & \\
\hline 47.0 & 998.50 & & \\
\hline 48.0 & 998.39 & & \\
\hline 50.0 & 998.43 & & \\
\hline 53.0 & 998.50 & & \\
\hline 55.0 & 998.52 & & \\
\hline 57.0 & 998.57 & & \\
\hline 58.3 & 998.68 & & \\
\hline 60.0 & 998.80 & & \\
\hline 63.0 & 998.91 & & \\
\hline 66.0 & 998.90 & & \\
\hline 67.7 & 999.02 & & \\
\hline
\end{tabular}


Location: Township 8 South/Range 49 East--section 6

U.S. Geological Survey quadrangle $(1: 24,000)$ : Bloom Creek

Landowners--left bank: Gay Ranch right bank: Jim Bowers

Access: left bank

Permission from: Gay Ranch (T7S/R49E--20)

Reference pins

\begin{tabular}{|c|c|c|}
\hline $\begin{array}{l}\text { Station } \\
\text { (meters) }\end{array}$ & $\begin{array}{c}\text { Elevation } \\
\text { (meters } \\
\text { above } \\
\text { sea level) }\end{array}$ & Comments \\
\hline & & $\begin{array}{l}\text { Pins }-1.5,0.0,8.4 \text { were closest to } \\
\text { leveling instrument. }\end{array}$ \\
\hline-1.5 & 994.728 & $\begin{array}{l}\text { New pin, 1978, } 0.21 \text { meter above } 1987 \\
\text { ground level. }\end{array}$ \\
\hline-1.5 & 994.531 & $\begin{array}{l}\text { old pin, } 0.01 \text { meter above } 1987 \text { ground } \\
\text { level. }\end{array}$ \\
\hline 0.0 & 994.600 & 0.02 meter above ground level. \\
\hline 8.4 & 994.327 & $\begin{array}{l}\text { Slow decrease in elevation, } 0.40 \text { meter } \\
\text { above } 1987 \text { ground level. }\end{array}$ \\
\hline 112.0 & 994.459 & 0.06 meter above 1987 ground level. \\
\hline 115.0 & 994.799 & 0.13 meter above 1987 ground level. \\
\hline 117.8 & 994.859 & 0.18 meter above 1980 ground level. \\
\hline
\end{tabular}




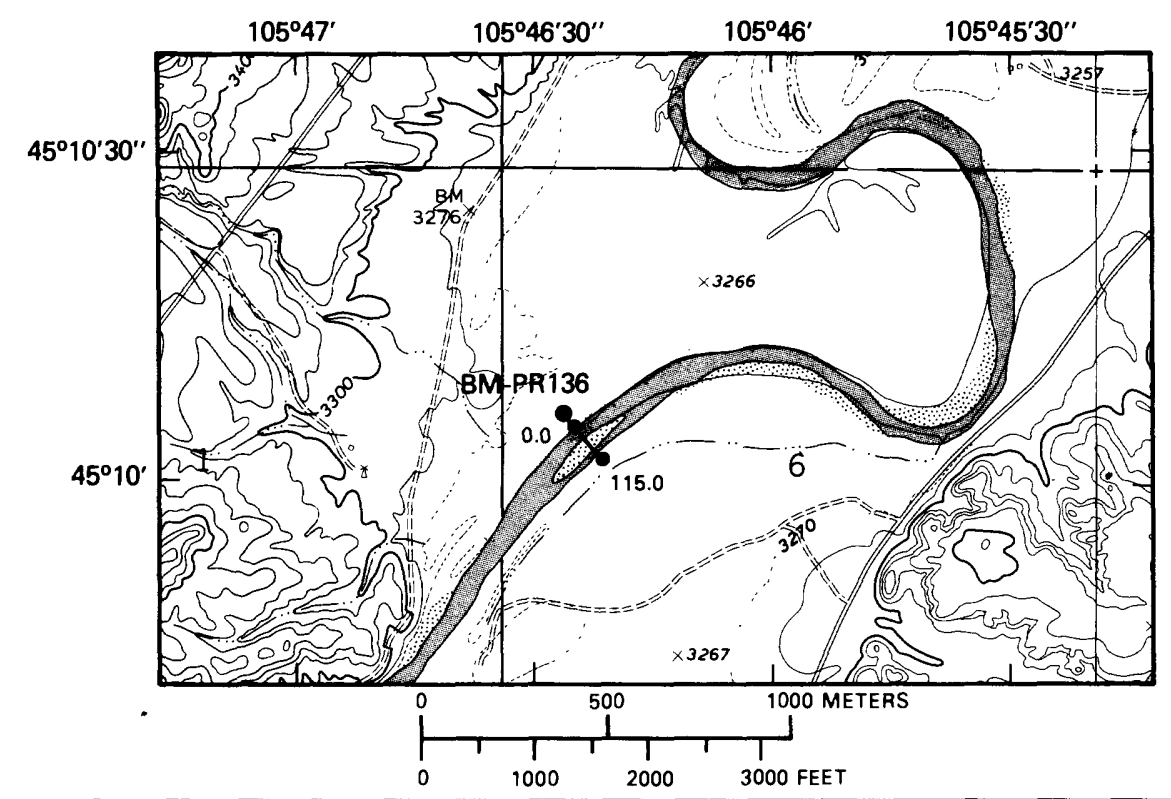

PRI36

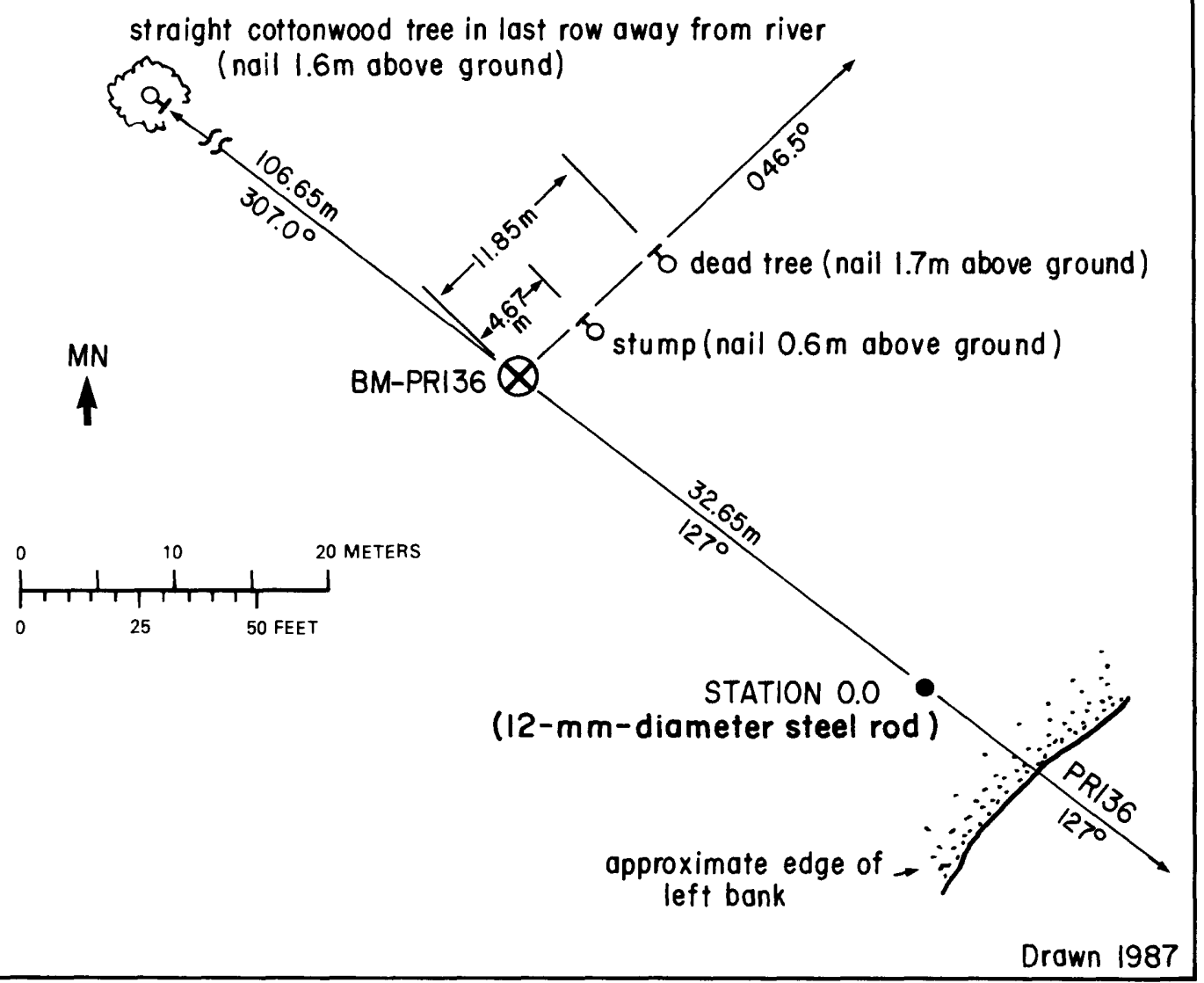

Figure 35.--Upper: Location of cross section PR136, bench mark BM-PR136, and left and right bank reference pins (tables 2 and 3 ) in the Bloom Creek quadrangle. Lower: Location of bench mark on the left bank. MN is magnetic north. 

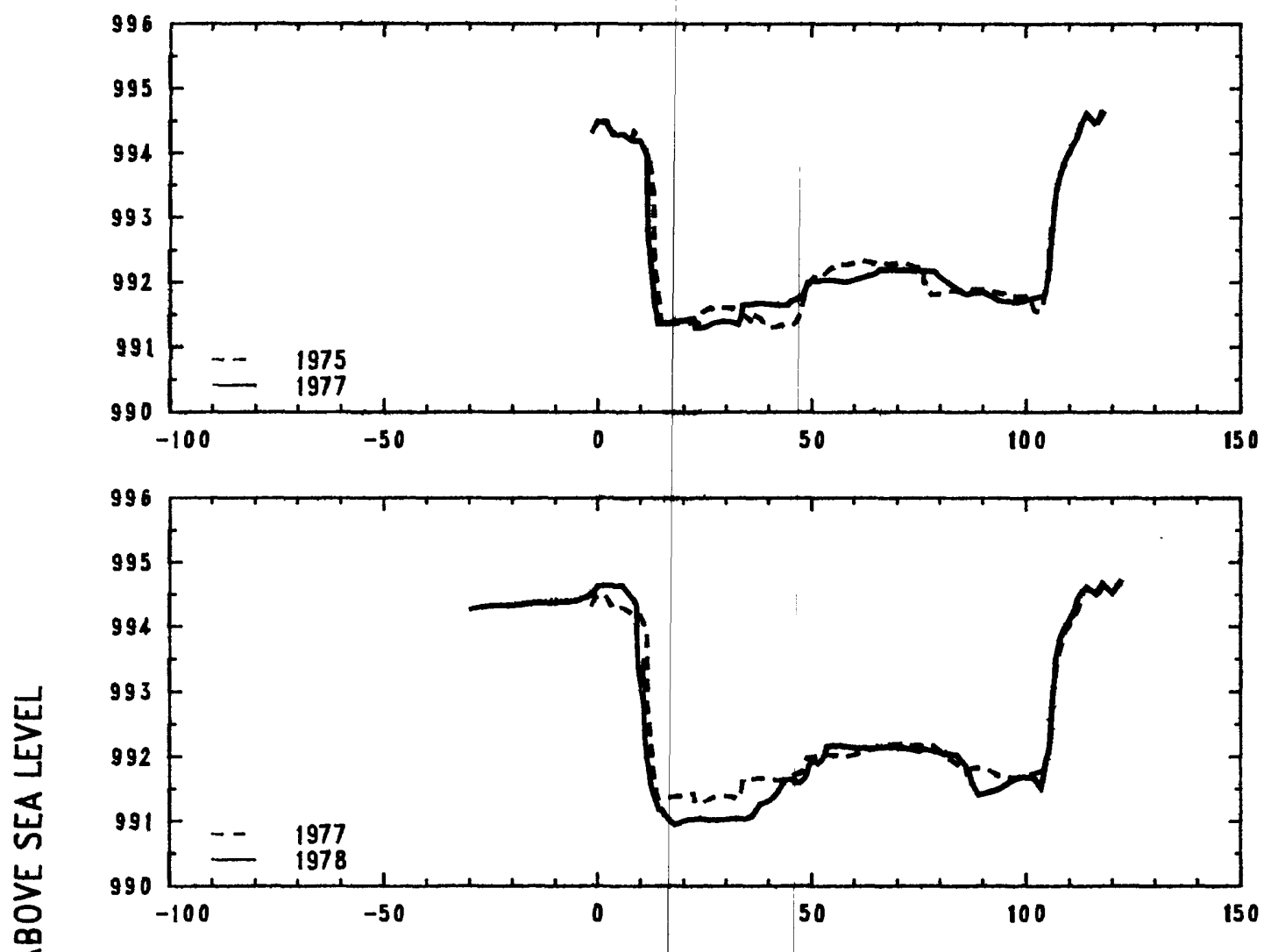

立
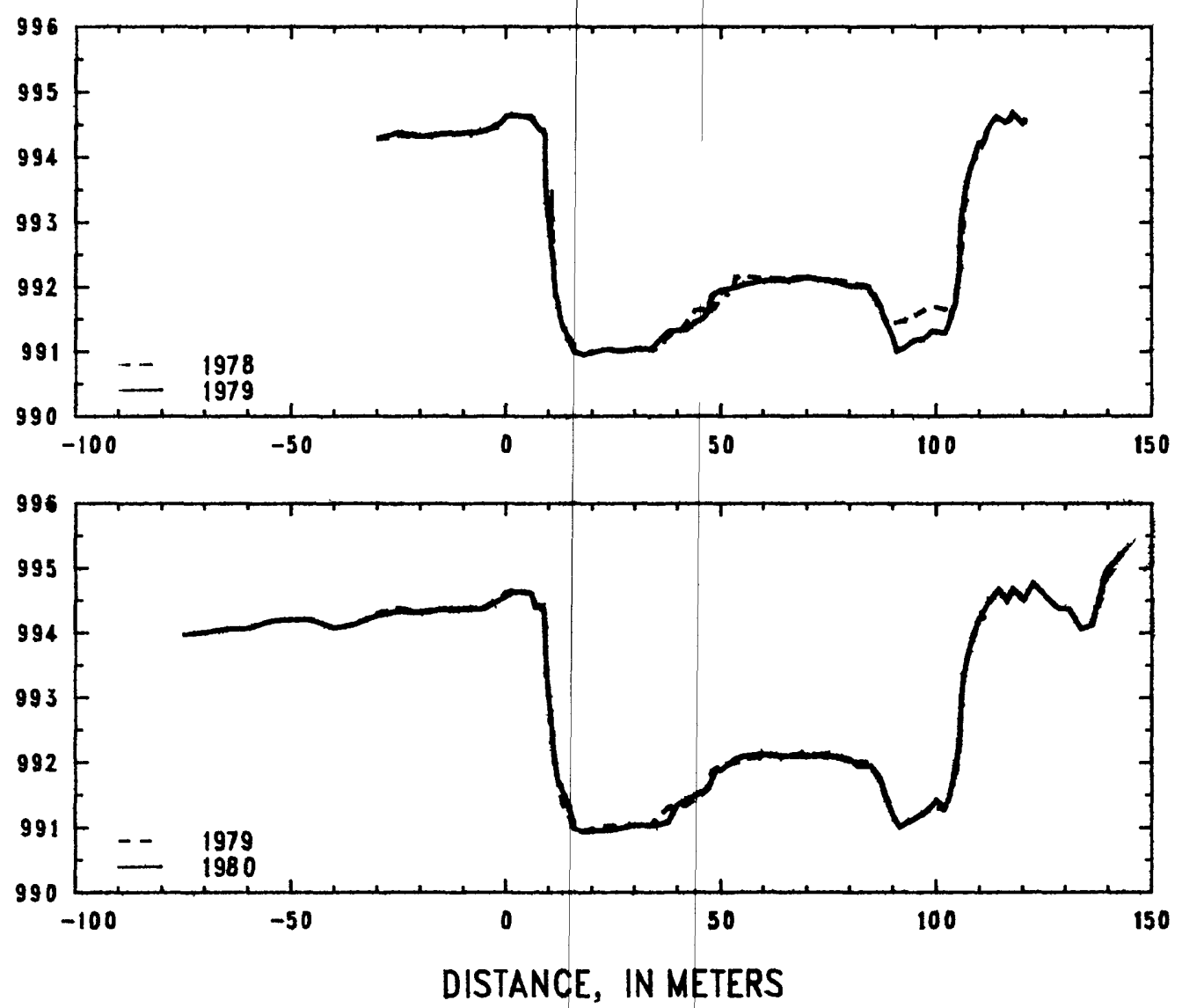

Figure 36.--Profiles of cross section PR136 from 1975 to 1980. 

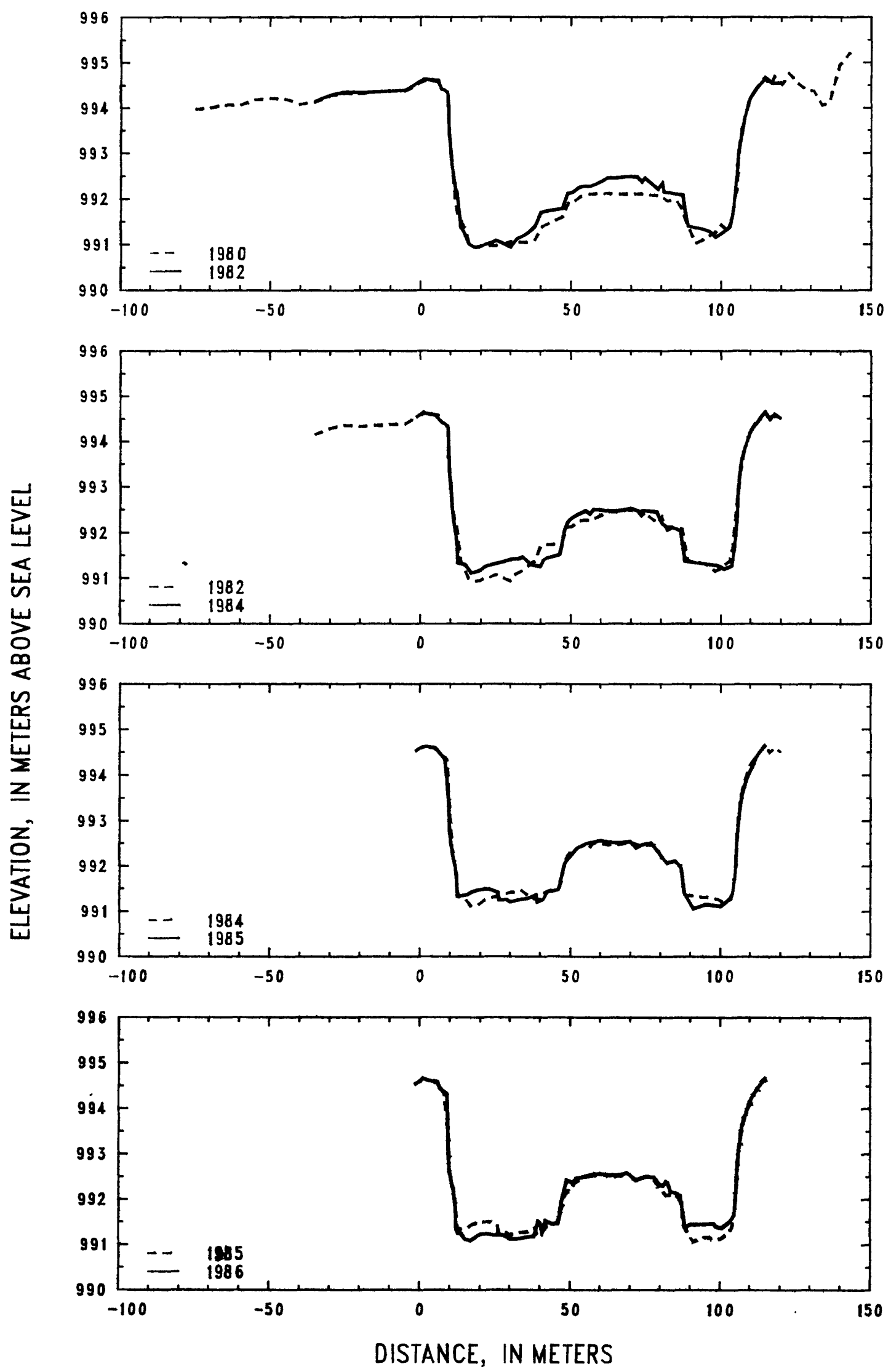

Figure 37. --Profiles of cross section PR136 from 1980 to 1986. 


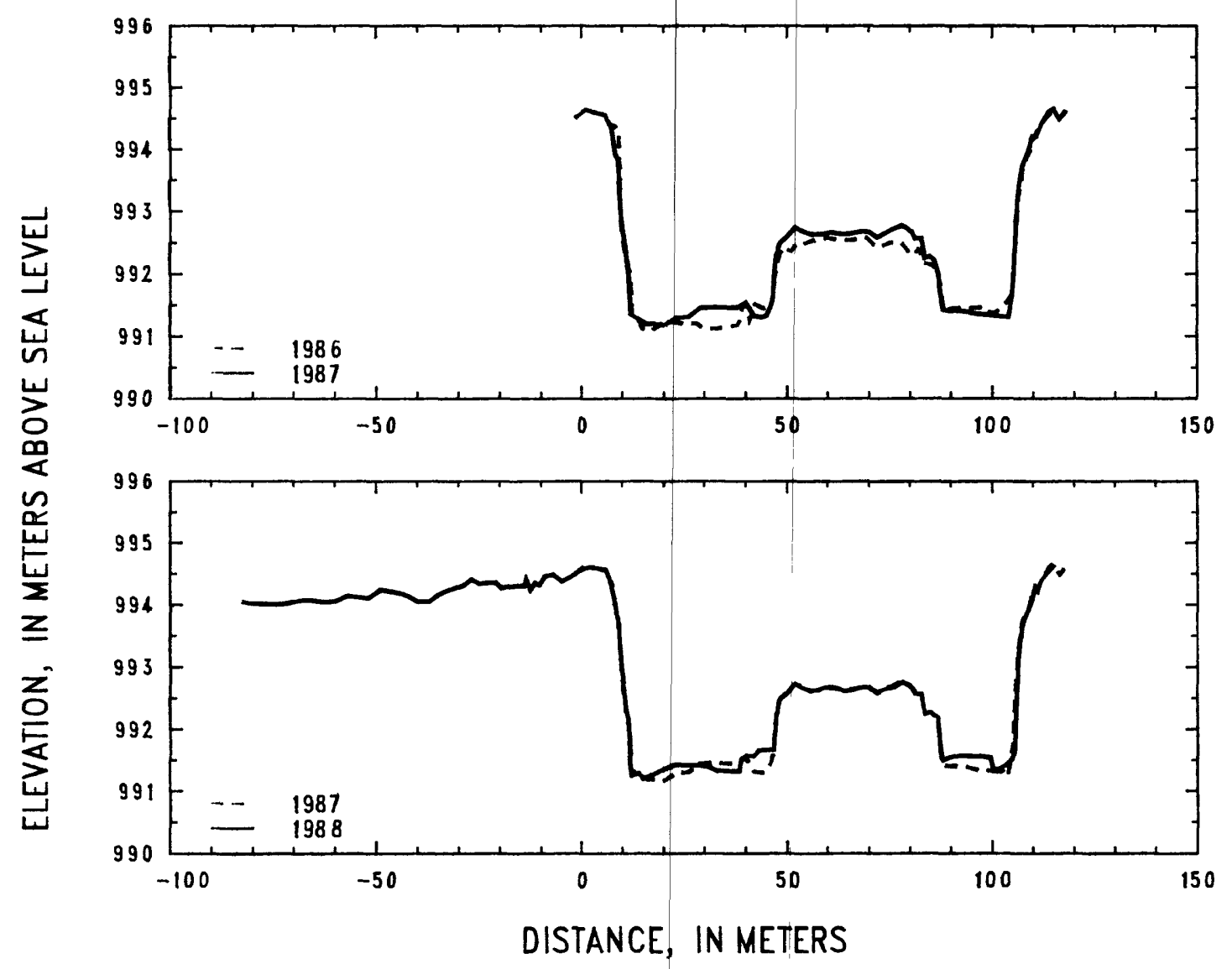

Figure 38.--Profiles of cross section PR136 from 1986 to 1988. 
Table 14.--Listing of horizontal stations and elevations for cross section PR136 [Sta., station, distance in meters from a reference point on the left bank; Elev., elevation, in meters above sea levell.

\begin{tabular}{|c|c|c|c|c|c|c|c|c|c|}
\hline \multicolumn{2}{|c|}{1975} & \multicolumn{2}{|c|}{1975} & \multicolumn{2}{|c|}{1977} & \multicolumn{2}{|c|}{1977} & \multicolumn{2}{|c|}{1978} \\
\hline Sta. & Elev. & Sta. & Elev. & Sta. & Elev. & Sta. & Elev. & Sta. & Elev. \\
\hline 0.0 & 994.49 & 68.0 & 992.28 & -1.5 & 994.32 & 98.0 & 991.67 & -30.0 & 994.27 \\
\hline 2.0 & 994.50 & 70.0 & 992.28 & 0.0 & 994.49 & 102.0 & 991.75 & -25.0 & 994.34 \\
\hline 4.0 & 994.29 & 72.0 & 992.29 & 2.0 & 994.47 & 104.4 & 991.78 & -20.0 & 994.32 \\
\hline 6.0 & 994.29 & 74.0 & 992.23 & 3.6 & 994.28 & 105.2 & 992.07 & -15.0 & 994.37 \\
\hline 8.0 & 994.20 & 76.0 & 992.22 & 6.0 & 994.29 & 107.0 & 993.38 & -10.0 & 994.37 \\
\hline 8.5 & 994.33 & 76.2 & 991.97 & 8.0 & 994.19 & 108.0 & 993.65 & -5.0 & 994.40 \\
\hline 10.0 & 994.19 & 78.0 & 991.80 & 10.0 & 994.18 & 109.0 & 993.89 & -1.5 & 994.50 \\
\hline 12.0 & 993.93 & 80.0 & 991.81 & 11.6 & 993.94 & 111.0 & 994.15 & 0.0 & 994.63 \\
\hline 12.3 & 993.85 & 82.0 & 991.86 & 11.7 & 992.82 & 111.9 & 994.25 & 2.0 & 994.64 \\
\hline 13.0 & 993.37 & 84.0 & 991.86 & 13.2 & 991.69 & 113.0 & 994.47 & 4.0 & 994.63 \\
\hline 13.4 & 992.20 & 86.0 & 991.90 & 13.8 & 991.48 & 114.0 & 994.61 & 6.0 & 994.63 \\
\hline 14.0 & 991.94 & 88.0 & 991.88 & 14.0 & 991.35 & 116.0 & 994.46 & 8.0 & 994.44 \\
\hline 14.7 & 991.53 & 90.0 & 991.88 & 15.0 & 991.36 & 117.0 & 994.52 & 8.5 & 994.41 \\
\hline 15.0 & 991.27 & 94.0 & 991.82 & 17.0 & 991.37 & 117.8 & 994.65 & 9.1 & 994.33 \\
\hline 16.0 & 991.34 & 98.0 & 991.77 & 19.0 & 991.39 & & & 9.5 & 993.31 \\
\hline 18.0 & 991.37 & 100.5 & 991.78 & 21.0 & 991.42 & & & 10.5 & 992.95 \\
\hline 20.0 & 991.36 & 102.0 & 991.55 & 22.4 & 991.43 & & & 10.7 & 993.15 \\
\hline 22.0 & 991.37 & 103.0 & 991.53 & 22.7 & 991.28 & & & 11.2 & 992.16 \\
\hline 24.0 & 991.37 & 104.0 & 991.66 & 25.0 & 991.30 & & & 12.0 & 991.81 \\
\hline 24.5 & 991.53 & 105.0 & 992.08 & 27.0 & 991.37 & & & 12.4 & 991.58 \\
\hline 26.0 & 991.59 & 106.0 & 992.89 & 29.0 & 991.39 & & & 13.1 & 991.42 \\
\hline 28.0 & 991.59 & 107.0 & 993.36 & 31.0 & 991.38 & & & 14.3 & 991.18 \\
\hline 30.0 & 991.60 & 108.0 & 993.68 & 33.0 & 991.34 & & & 15.0 & 991.17 \\
\hline 32.0 & 991.59 & 110.0 & 993.99 & 33.8 & 991.49 & & & 16.0 & 991.06 \\
\hline 33.5 & 991.51 & 114.0 & 994.63 & 34.0 & 991.63 & & & 18.0 & 990.94 \\
\hline 34.0 & 991.49 & 116.5 & 994.42 & 38.0 & 991.67 & & & 20.0 & 991.01 \\
\hline 36.0 & 991.39 & 118.3 & 994.66 & 42.0 & 991.63 & & & 22.0 & 991.03 \\
\hline 36.2 & 991.50 & & & 44.5 & 991.63 & & & 24.0 & 991.04 \\
\hline 38.0 & 991.44 & & & 45.0 & 991.69 & & & 26.0 & 991.01 \\
\hline 40.0 & 991.29 & & & 48.0 & 991.78 & & & 28.0 & 991.02 \\
\hline 42.0 & 991.31 & & & 49.1 & 991.99 & & & 30.0 & 991.03 \\
\hline 44.0 & 991.36 & & & 54.0 & 992.03 & & & 32.0 & 991.04 \\
\hline 46.0 & 991.35 & & & 58.0 & 991.99 & & & 34.0 & 991.03 \\
\hline 47.0 & 991.46 & & & 62.0 & 992.07 & & & 36.0 & 991.07 \\
\hline 47.6 & 991.50 & & & 65.0 & 992.13 & & & 38.0 & 991.26 \\
\hline 48.5 & 991.87 & & & 66.0 & 992.19 & & & 40.0 & 991.30 \\
\hline 50.5 & 992.07 & & & 70.0 & 992.18 & & & 41.8 & 991.42 \\
\hline 52.0 & 992.05 & & & 74.0 & 992.17 & & & 44.0 & 991.64 \\
\hline 54.0 & 992.19 & & & 78.0 & 992.16 & & & 46.0 & 991.65 \\
\hline 56.0 & 992.27 & & & 78.5 & 992.16 & & & 46.1 & 991.59 \\
\hline 58.0 & 992.26 & & & 80.0 & 992.07 & & & 47.5 & 991.60 \\
\hline 60.0 & 992.30 & & & 82.2 & 991.96 & & & 48.7 & 991.68 \\
\hline 62.0 & 992.33 & & & 86.0 & 991.80 & & & 49.6 & 991.88 \\
\hline 64.0 & 992.30 & & & 90.0 & 991.84 & & & 50.7 & 991.95 \\
\hline 66.0 & 992.29 & & & 94.0 & 991.69 & & & 51.6 & 991.88 \\
\hline
\end{tabular}


Table 14.--Listing of horizontal stations and elevations for cross section PR136 --Continued.

\begin{tabular}{|c|c|c|c|c|c|c|c|c|c|}
\hline \multicolumn{2}{|c|}{1978} & \multicolumn{2}{|c|}{1979} & \multicolumn{2}{|c|}{1979} & \multicolumn{2}{|c|}{1980} & \multicolumn{2}{|c|}{1980} \\
\hline Sta. & Elev. & Sta. & Elev. & Sta. & Elev. & Sta. & Elev. & Sta. & Elev. \\
\hline 52.8 & 991.96 & -30.0 & 994.29 & 64.0 & 992.10 & -75.0 & 993.97 & 80.0 & 992.05 \\
\hline 53.6 & 992.15 & -25.0 & 994.39 & 68.0 & 992.12 & -70.0 & 994.00 & 82.0 & 991.94 \\
\hline 56.0 & 992.17 & -20.0 & 994.31 & 70.0 & 992.15 & -65.0 & 994.06 & 85.0 & 991.95 \\
\hline 60.0 & 992.13 & -15.0 & 994.36 & 72.0 & 992.12 & -60.0 & 994.06 & 87.0 & 991.76 \\
\hline 64.0 & 992.12 & -10.0 & 994.37 & 75.0 & 992.10 & -55.0 & 994.18 & 88.0 & 991.54 \\
\hline 68.0 & 992.15 & -5.0 & 994.40 & 80.0 & 992.01 & -50.0 & 994.21 & 90.0 & 991.20 \\
\hline 72.0 & 992.13 & -1.5 & 994.52 & 84.0 & 992.00 & -45.0 & 994.20 & 91.5 & 991.00 \\
\hline 75.0 & 992.08 & 0.0 & 994.62 & 84.5 & 992.00 & -40.0 & 994.07 & 94.0 & 991.10 \\
\hline 76.0 & 992.12 & 2.0 & 994.64 & 85.0 & 991.91 & -35.0 & 994.13 & 97.0 & 991.21 \\
\hline 80.0 & 992.05 & 4.0 & 994.63 & 87.0 & 991.73 & -30.0 & 994.27 & 99.0 & 991.32 \\
\hline 84.0 & 992.02 & 6.0 & 994.60 & 88.0 & 991.49 & -25.0 & 994.33 & 100.0 & 991.42 \\
\hline 86.0 & 991.87 & 8.0 & 994.41 & 89.0 & 991.37 & -20.0 & 994.31 & 102.0 & 991.28 \\
\hline 86.4 & 991.73 & 8.8 & 994.42 & 90.0 & 991.21 & -15.0 & 994.36 & 103.2 & 991.48 \\
\hline 86.9 & 991.74 & 9.2 & 994.24 & 91.0 & 991.00 & -10.0 & 994.37 & 104.0 & 991.76 \\
\hline 87.4 & 991.60 & 9.3 & 993.51 & 93.0 & 991.06 & -5.0 & 994.37 & 105.3 & 992.20 \\
\hline 89.0 & 991.40 & 10.0 & 993.06 & 95.0 & 991.18 & -1.5 & 994.51 & 106.0 & 993.06 \\
\hline 91.0 & 991.45 & 11.0 & 992.38 & 97.0 & 991.19 & 2.0 & 994.63 & 107.0 & 993.53 \\
\hline 94.0 & 991.50 & 11.7 & 991.84 & 99.0 & 991.31 & 4.0 & 994.63 & 108.0 & 993.80 \\
\hline 96.0 & 991.60 & 11.9 & 991.82 & 100.0 & 991.30 & 6.0 & 994.60 & 110.0 & 994.22 \\
\hline 98.0 & 991.67 & 13.0 & 991.39 & 102.0 & 991.28 & 7.0 & 994.39 & 111.9 & 994.43 \\
\hline 100.0 & 991.69 & 15.0 & 991.16 & 103.0 & 991.42 & 8.0 & 994.40 & 114.6 & 994.67 \\
\hline 102.0 & 991.65 & 16.0 & 990.99 & 103.6 & 991.63 & 9.0 & 994.32 & 116.5 & 994.48 \\
\hline 103.5 & 991.50 & 18.0 & 990.94 & 104.4 & 991.71 & 9.5 & 993.46 & 117.8 & 994.68 \\
\hline 104.2 & 991.70 & 20.0 & 990.98 & 105.4 & 992.21 & 11.0 & 992.34 & 120.3 & 994.50 \\
\hline 104.6 & 991.89 & 22.0 & 991.02 & 105.6 & 992.71 & 12.0 & 991.73 & 122.5 & 994.76 \\
\hline 105.4 & 992.13 & 24.0 & 991.03 & 106.0 & 993.09 & 13.5 & 991.51 & 126.0 & 994.51 \\
\hline 107.0 & 993.52 & 26.0 & 991.01 & 107.0 & 993.55 & 15.2 & 991.25 & 128.5 & 994.37 \\
\hline 108.0 & 993.82 & 28.0 & 991.01 & 108.0 & 993.81 & 16.0 & 990.98 & 131.0 & 994.36 \\
\hline 109.0 & 993.98 & 30.0 & 991.04 & 109.0 & 994.00 & 18.0 & 990.93 & 133.8 & 994.05 \\
\hline 111.0 & 994.22 & 32.0 & 991.03 & 110.0 & 994.21 & 20.0 & 990.95 & 136.3 & 994.12 \\
\hline 111.9 & 994.43 & 34.0 & 991.03 & 111.0 & 994.23 & 25.0 & 990.96 & 139.5 & 994.94 \\
\hline 113.0 & 994.55 & 36.0 & 991.19 & 111.9 & 994.43 & 30.0 & 991.04 & 143.0 & 995.21 \\
\hline 114.0 & 994.61 & 38.0 & 991.31 & 113.0 & 994.54 & 35.0 & 991.03 & & \\
\hline 116.0 & 994.52 & 40.0 & 991.33 & 114.0 & 994.62 & 38.0 & 991.09 & & \\
\hline 117.0 & 994.57 & 42.0 & 991.34 & 116.0 & 994.53 & 40.0 & 991.35 & & \\
\hline 117.8 & 994.67 & 44.0 & 991.45 & 117.0 & 994.57 & 43.0 & 991.46 & & \\
\hline 120.0 & 994.51 & 46.0 & 991.52 & 117.8 & 994.68 & 47.0 & 991.59 & & \\
\hline \multirow[t]{8}{*}{122.0} & 994.70 & 47.0 & 991.61 & 120.0 & 994.51 & 49.0 & 991.89 & & \\
\hline & & 48.0 & 991.86 & & & 50.0 & 991.87 & & \\
\hline & & 50.0 & 991.96 & & & 53.0 & 992.03 & & \\
\hline & & 52.0 & 991.97 & & & 55.0 & 992.09 & & \\
\hline & & 54.0 & 992.01 & & & 60.0 & 992.12 & & \\
\hline & & 56.0 & 992.05 & & & 65.0 & 992.09 & & \\
\hline & & 58.0 & 992.07 & & & 70.0 & 992.09 & & \\
\hline & & 60.0 & 992.10 & & & 75.0 & 992.10 & & \\
\hline
\end{tabular}


Table 14.--Listing of horizontal stations and elevations for cross section PR136 --Continued.

\begin{tabular}{|c|c|c|c|c|c|c|c|c|c|}
\hline \multicolumn{2}{|c|}{1982} & \multicolumn{2}{|c|}{1982} & \multicolumn{2}{|c|}{1984} & \multicolumn{2}{|c|}{1984} & \multicolumn{2}{|c|}{1985} \\
\hline Sta. & Elev. & Sta. & Elev. & Sta. & Elev. & Sta. & Elev. & Sta. & Elev. \\
\hline-35.0 & 994.14 & 65.0 & 992.45 & -1.5 & 994.53 & 86.7 & 992.03 & -1.5 & 994.52 \\
\hline-30.0 & 994.28 & 69.0 & 992.49 & 0.0 & 994.57 & 88.0 & 991.36 & 0.0 & 994.58 \\
\hline-25.0 & 994.35 & 72.0 & 992.47 & 1.5 & 994.62 & 90.0 & 991.34 & 2.0 & 994.62 \\
\hline-20.0 & 994.32 & 73.5 & 992.36 & 3.0 & 994.60 & 93.0 & 991.32 & 5.0 & 994.58 \\
\hline-15.0 & 994.35 & 74.5 & 992.45 & 5.0 & 994.57 & 96.0 & 991.29 & 8.4 & 994.34 \\
\hline-10.0 & 994.37 & 77.0 & 992.30 & 7.0 & 994.42 & 99.0 & 991.26 & 9.4 & 993.73 \\
\hline-5.0 & 994.38 & 79.0 & 992.20 & 8.4 & 994.37 & 101.0 & 991.19 & 10.0 & 992.66 \\
\hline-1.5 & 994.54 & 80.4 & 992.34 & 9.2 & 994.29 & 103.7 & 991.27 & 11.0 & 992.22 \\
\hline 0.0 & 994.59 & 81.0 & 992.14 & 9.7 & 993.40 & 105.0 & 991.81 & 12.0 & 991.89 \\
\hline 1.0 & 994.64 & 84.0 & 992.10 & 10.8 & 992.42 & 105.6 & 992.30 & 12.5 & 991.42 \\
\hline 3.0 & 994.62 & 87.3 & 992.07 & 12.3 & 991.80 & 105.8 & 992.88 & 13.0 & 991.33 \\
\hline 6.0 & 994.57 & 88.0 & 991.70 & 12.5 & 991.32 & 107.0 & 993.56 & 16.0 & 991.37 \\
\hline 7.0 & 994.41 & 89.0 & 991.38 & 15.0 & 991.28 & 108.0 & 993.81 & 17.0 & 991.42 \\
\hline 8.4 & 994.37 & 93.0 & 991.33 & 17.0 & 991.10 & 110.0 & 994.22 & 20.0 & 991.49 \\
\hline 9.0 & 994.34 & 96.0 & 991.27 & 20.0 & 991.17 & 111.9 & 994.39 & 23.0 & 991.49 \\
\hline 9.3 & 994.17 & 98.0 & 991.14 & 21.5 & 991.24 & 115.0 & 994.65 & 26.0 & 991.41 \\
\hline 9.4 & 993.60 & 100.0 & 991.23 & 24.0 & 991.30 & 116.4 & 994.48 & 26.4 & 991.24 \\
\hline 10.6 & 992.60 & 103.0 & 991.38 & 27.0 & 991.34 & 118.0 & 994.60 & 29.0 & 991.26 \\
\hline 11.7 & 992.18 & 104.0 & 991.69 & 30.0 & 991.41 & 120.0 & 994.50 & 30.0 & 991.20 \\
\hline 12.2 & 992.13 & 105.0 & 992.29 & 33.0 & 991.42 & & & 33.0 & 991.26 \\
\hline 13.0 & 991.69 & 106.0 & 993.04 & 34.0 & 991.46 & & & 36.0 & 991.28 \\
\hline 13.4 & 991.36 & 108.0 & 993.82 & 37.0 & 991.28 & $\cdot$ & & 38.6 & 991.37 \\
\hline 14.0 & 991.30 & 110.0 & 994.23 & 40.0 & 991.24 & & & 39.0 & 991.20 \\
\hline 16.0 & 990.99 & 111.9 & 994.42 & 41.0 & 991.39 & & & 41.0 & 991.26 \\
\hline 18.0 & 990.92 & 115.0 & 994.66 & 42.0 & 991.43 & & & 42.4 & 991.43 \\
\hline 20.0 & 990.94 & 117.0 & 994.54 & 46.2 & 991.51 & & & 45.0 & 991.46 \\
\hline 21.0 & 990.99 & 120.0 & 994.55 & 47.0 & 991.71 & & & 46.0 & 991.48 \\
\hline 23.0 & 991.01 & & & 48.0 & 992.07 & & & 48.0 & 992.06 \\
\hline 25.0 & 991.07 & & & 49.0 & 992.22 & & & 49.0 & 992.15 \\
\hline 27.0 & 991.02 & & & 50.0 & 992.29 & & & 50.0 & 992.20 \\
\hline 29.0 & 990.95 & & & 52.0 & 992.38 & & & 52.0 & 992.39 \\
\hline 30.0 & 990.93 & & & 55.0 & 992.47 & & & 55.0 & 992.49 \\
\hline 32.0 & 991.11 & & & 56.4 & 992.40 & & & 60.0 & 992.57 \\
\hline 35.0 & 991.21 & & & 57.5 & 992.50 & & & 65.0 & 992.50 \\
\hline 38.0 & 991.38 & & & 60.0 & 992.49 & & & 70.0 & 992.54 \\
\hline 40.0 & 991.69 & & & 63.0 & 992.46 & & & 72.0 & 992.40 \\
\hline 43.0 & 991.73 & & & 67.0 & 992.47 & & & 74.0 & 992.48 \\
\hline 47.0 & 991.78 & & & 70.0 & 992.52 & & & 77.0 & 992.51 \\
\hline 48.8 & 992.11 & & & 73.6 & 992.43 & & & 80.0 & 992.26 \\
\hline 50.4 & 992.13 & & & 74.2 & 992.47 & & & 82.0 & 992.08 \\
\hline 52.5 & 992.24 & & & 78.7 & 992.44 & & & 85.0 & 992.11 \\
\hline 54.7 & 992.27 & & & 80.5 & 992.18 & & & 87.0 & 991.95 \\
\hline 56.0 & 992.26 & & & 81.7 & 992.13 & & & 88.0 & 991.40 \\
\hline 59.0 & 992.34 & & & 82.3 & 992.05 & & & 91.0 & 991.06 \\
\hline 62.0 & 992.45 & & & 84.0 & 992.11 & & & 95.0 & 991.16 \\
\hline
\end{tabular}


Table 14.--Listing of horizontal stations and elevations for cross section PR136 --Continued.

\begin{tabular}{|c|c|c|c|c|c|c|c|c|c|}
\hline \multicolumn{2}{|c|}{1985} & \multicolumn{2}{|c|}{1986} & \multicolumn{2}{|c|}{1986} & \multicolumn{2}{|c|}{1987} & \multicolumn{2}{|c|}{1987} \\
\hline Sta. & Elev. & Sta. & Elev. & Sta. & Elev. & Sta. & Elev. & Sta. & Elev. \\
\hline 100.0 & 991.11 & -1.5 & 994.52 & 75.0 & 992.50 & -1.5 & 994.51 & 78.0 & 992.77 \\
\hline 103.0 & 991.27 & 0.0 & 994.58 & 78.0 & 992.48 & 0.0 & 994.58 & 79.0 & 992.74 \\
\hline 104.0 & 991.38 & 1.0 & 994.65 & 80.0 & 992.31 & 1.0 & 994.65 & 80.0 & 992.68 \\
\hline 105.0 & 991.80 & 3.0 & 994.60 & 81.0 & 992.29 & 3.0 & 994.61 & 80.6 & 992.66 \\
\hline 106.0 & 992.92 & 6.0 & 994.57 & 82.0 & 992.39 & 6.0 & 994.55 & 81.3 & 992.56 \\
\hline 107.0 & 993.49 & 7.0 & 994.42 & 82.6 & 992.36 & 7.0 & 994.40 & 82.8 & 992.56 \\
\hline 109.0 & 993.95 & 8.4 & 994.36 & 83.5 & 992.16 & 7.5 & 994.35 & 83.6 & 992.24 \\
\hline 111.0 & 994.21 & 9.2 & 994.31 & 85.0 & 992.14 & 8.4 & 993.91 & 85.0 & 992.27 \\
\hline 112.0 & 994.40 & 9.8 & 992.84 & 86.8 & 992.07 & 9.0 & 993.84 & 86.0 & 992.21 \\
\hline \multirow[t]{36}{*}{115.0} & 994.66 & 10.4 & 992.45 & 88.3 & 991.38 & 9.8 & 992.92 & 87.0 & 991.97 \\
\hline & & 10.7 & 992.34 & 90.0 & 991.44 & 10.7 & 992.47 & 87.2 & 991.76 \\
\hline & & 11.3 & 992.24 & 92.0 & 991.44 & 11.5 & 992.07 & 88.0 & 991.41 \\
\hline & & 12.2 & 991.80 & 95.0 & 991.45 & 11.8 & 991.84 & 90.0 & 991.40 \\
\hline & & 12.3 & 991.34 & 97.8 & 991.46 & 12.0 & 991.34 & 93.0 & 991.40 \\
\hline & & 13.0 & 991.23 & 98.6 & 991.39 & 14.0 & 991.27 & 96.0 & 991.35 \\
\hline & & 14.0 & 991.24 & 100.5 & 991.36 & 16.0 & 991.18 & 99.0 & 991.33 \\
\hline & & 15.0 & 991.12 & 102.8 & 991.48 & 18.0 & 991.20 & 102.0 & 991.30 \\
\hline & & 17.0 & 991.07 & 104.8 & 991.66 & 20.0 & 991.17 & 104.0 & 991.29 \\
\hline & & 20.0 & 991.22 & 105.3 & 992.06 & 23.0 & 991.30 & 105.0 & 991.76 \\
\hline & & 22.0 & 991.24 & 106.2 & 993.04 & 26.0 & 991.31 & 106.0 & 992.94 \\
\hline & & 25.0 & 991.19 & 107.6 & 993.72 & 29.0 & 991.46 & 106.3 & 993.21 \\
\hline & & 28.0 & 991.20 & 109.5 & 994.02 & 32.0 & 991.47 & 107.4 & 993.72 \\
\hline & & 30.0 & 991.11 & 110.0 & 994.15 & 35.0 & 991.45 & 109.0 & 993.97 \\
\hline & & 33.0 & 991.12 & 112.0 & 994.40 & 38.0 & 991.46 & 110.0 & 994.21 \\
\hline & & 36.0 & 991.16 & 115.0 & 994.65 & 40.0 & 991.54 & 111.0 & 994.20 \\
\hline & & 38.2 & 991.18 & & & 42.0 & 991.31 & 112.0 & 994.40 \\
\hline & & 39.5 & 991.51 & & & 44.0 & 991.29 & 114.0 & 994.61 \\
\hline & & 40.2 & 991.47 & & & 45.0 & 991.32 & 115.0 & 994.66 \\
\hline & & 40.9 & 991.26 & & & 46.4 & 991.51 & 116.5 & 994.48 \\
\hline & & 42.0 & 991.52 & & & 46.8 & 991.75 & 118.0 & 994.62 \\
\hline & & 44.0 & 991.44 & & & 47.0 & 992.02 & & \\
\hline & & 46.0 & 991.46 & & & 47.6 & 992.33 & & \\
\hline & & 46.6 & 991.64 & & & 48.2 & 992.48 & & \\
\hline & & 47.0 & 991.94 & & & 50.0 & 992.59 & & \\
\hline & & 48.8 & 992.41 & & & 52.0 & 992.74 & & \\
\hline & & 50.0 & 992.38 & & & 54.0 & 992.67 & & \\
\hline & & 51.0 & 992.35 & & & 56.0 & 992.62 & & \\
\hline & & 52.0 & 992.45 & & & 59.0 & 992.63 & & \\
\hline & & 54.0 & 992.48 & & & 61.0 & 992.66 & & \\
\hline & & 57.0 & 992.54 & & & 63.0 & 992.64 & & \\
\hline & & 60.0 & 992.57 & & & 65.0 & 992.63 & & \\
\hline & & 63.0 & 992.53 & & & 68.0 & 992.67 & & \\
\hline & & 66.0 & 992.53 & & & 70.0 & 992.67 & & \\
\hline & & 69.0 & 992.58 & & & 72.0 & 992.57 & & \\
\hline & & 72.0 & 992.41 & & & 75.0 & 992.69 & & \\
\hline
\end{tabular}


Table 14.--Listing of horizontal stations and elevations for cross section PR136 --Continued.

\begin{tabular}{|c|c|c|c|c|c|c|}
\hline \multicolumn{2}{|c|}{1988} & \multicolumn{2}{|c|}{1988} & \multicolumn{2}{|c|}{1988} & \\
\hline Sta. & Elev. & Sta. & Elev. & Sta. & Elev. & \\
\hline-82.5 & 994.04 & 9.0 & 993.79 & 80.0 & 992.70 & \\
\hline-81.0 & 994.02 & 9.3 & 993.63 & 81.0 & 992.63 & \\
\hline-78.0 & 994.01 & 9.6 & 993.19 & 81.3 & 992.56 & \\
\hline-75.0 & 994.00 & 11.0 & 992.28 & 82.9 & 992.55 & \\
\hline-72.0 & 994.01 & 11.4 & 992.19 & 83.6 & 992.25 & \\
\hline-69.0 & 994.07 & 11.8 & 991.91 & 85.0 & 992.27 & \\
\hline-66.0 & 994.08 & 11.9 & 991.44 & 86.8 & 992.18 & \\
\hline-63.0 & 994.04 & 12.3 & 991.25 & 87.5 & 991.57 & \\
\hline-60.0 & 994.05 & 14.0 & 991.29 & 88.0 & 991.49 & \\
\hline-57.0 & 994.15 & 15.0 & 991.19 & 90.0 & 991.55 & \\
\hline-54.0 & 994.11 & 17.0 & 991.26 & 93.0 & 991.57 & \\
\hline-52.0 & 994.10 & 20.0 & 991.36 & 95.0 & 991.56 & \\
\hline-49.0 & 994.24 & 23.0 & 991.43 & 97.0 & 991.56 & \\
\hline-46.0 & 994.20 & 26.0 & 991.42 & 99.5 & 991.53 & \\
\hline-43.0 & 994.16 & 29.0 & 991.42 & 99.6 & 991.51 & \\
\hline-40.0 & 994.05 & 31.0 & 991.39 & 100.3 & 991.32 & \\
\hline-37.0 & 994.05 & 33.0 & 991.32 & 102.0 & 991.37 & \\
\hline-35.0 & 994.16 & 35.0 & 991.34 & 104.0 & 991.45 & \\
\hline-33.0 & 994.23 & 37.0 & 991.32 & 104.8 & 991.52 & \\
\hline-31.0 & 994.27 & 38.5 & 991.32 & 105.4 & 991.65 & \\
\hline-29.0 & 994.31 & 39.0 & 991.52 & 105.9 & 992.50 & \\
\hline-27.0 & 994.42 & 39.1 & 991.53 & 106.5 & 993.23 & \\
\hline-25.0 & 994.33 & 40.0 & 991.57 & 107.6 & 993.71 & \\
\hline-23.0 & 994.36 & 42.0 & 991.56 & 109.5 & 994.02 & \\
\hline-21.0 & 994.36 & 43.0 & 991.65 & 110.5 & 994.29 & \\
\hline-19.6 & 994.27 & 44.0 & 991.66 & 111.2 & 994.22 & \\
\hline-18.0 & 994.29 & 46.0 & 991.67 & 112.0 & 994.39 & \\
\hline-16.0 & 994.30 & 46.8 & 991.67 & 115.0 & 994.64 & \\
\hline-14.0 & 994.31 & 47.4 & 992.18 & & & \\
\hline-13.6 & 994.42 & 48.2 & 992.47 & & & \\
\hline-12.6 & 994.23 & 50.0 & 992.57 & • & & \\
\hline-11.4 & 994.35 & 52.0 & 992.74 & & & \\
\hline-10.4 & 994.31 & 54.0 & 992.66 & & & \\
\hline-9.0 & 994.46 & 56.0 & 992.60 & & & \\
\hline-7.0 & 994.49 & 58.0 & 992.65 & & & \\
\hline-5.0 & 994.37 & 60.0 & 992.68 & & & \\
\hline-3.0 & 994.45 & 62.0 & 992.67 & & & \\
\hline-1.5 & 994.50 & 64.0 & 992.60 & & & \\
\hline 0.0 & 994.58 & 66.0 & 992.63 & & & . \\
\hline 2.0 & 994.61 & 68.0 & 992.67 & & & \\
\hline 4.0 & 994.58 & 70.0 & 992.68 & & & \\
\hline 6.0 & 994.56 & 72.0 & 992.59 & & & \\
\hline 7.0 & 994.40 & 74.0 & 992.65 & & & \\
\hline 8.0 & 994.12 & 76.0 & 992.67 & & & \\
\hline 8.4 & 993.92 & 78.0 & 992.76 & & & \\
\hline
\end{tabular}


Description of Cross Section PR141

Location: Township 7 South/Range 49 East--section 29

U.S. Geological Survey quadrangle $(1: 24,000)$ : Bloom Creek

Landowners--left bank: U.S. Government

right bank: U.S. Government

Access: left bank

Permission from: Gay Ranch (T7S/R49E--20)

Reference pins

\begin{tabular}{lll}
\hline $\begin{array}{l}\text { Station } \\
\text { (meters) }\end{array}$ & $\begin{array}{c}\text { Elevation } \\
\text { (meters } \\
\text { above } \\
\text { sea level) }\end{array}$ & Comments \\
& & $\begin{array}{r}\text { Pins } 100.0 \text { and } 100.9 \text { were closest to } \\
\text { leveling instrument. }\end{array}$ \\
-26.5 & 988.467 & 0.12 meter above 1980 ground level. \\
-0.8 & 988.305 & 0.04 meter above 1984 ground leve1. \\
100.0 & 989.055 & 0.09 meter above 1987 ground level. \\
100.9 & 989.189 & 0.21 meter above 1984 ground level. \\
\hline
\end{tabular}



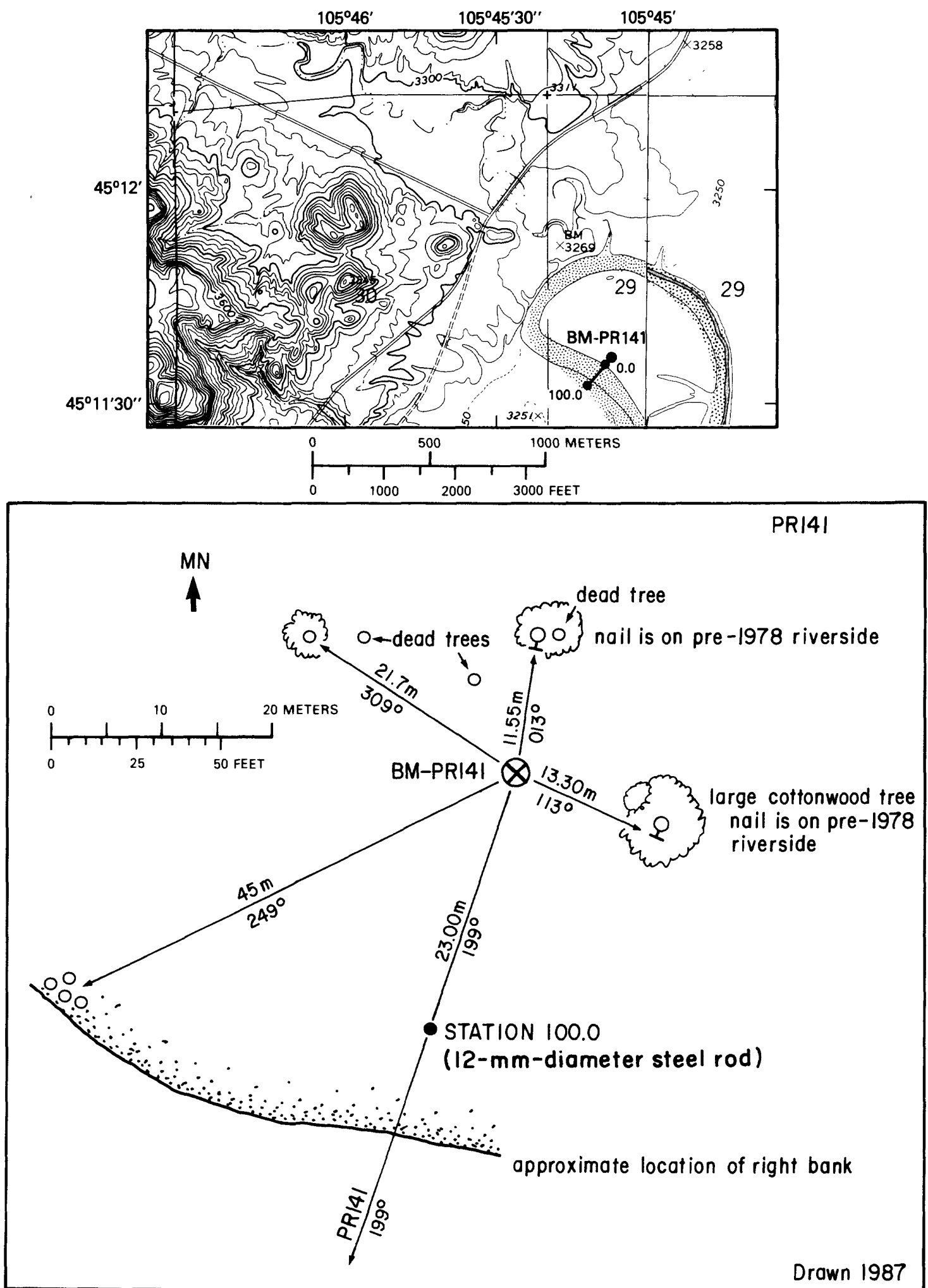

Figure 39.--Upper: Location of cross section PR141, bench mark BM-PR141, and left and right bank reference pins (tables 2 and 3 ) in the Bloom Creek quadrangle. Lower: Location of bench mark on the right bank of an abandoned channel. 


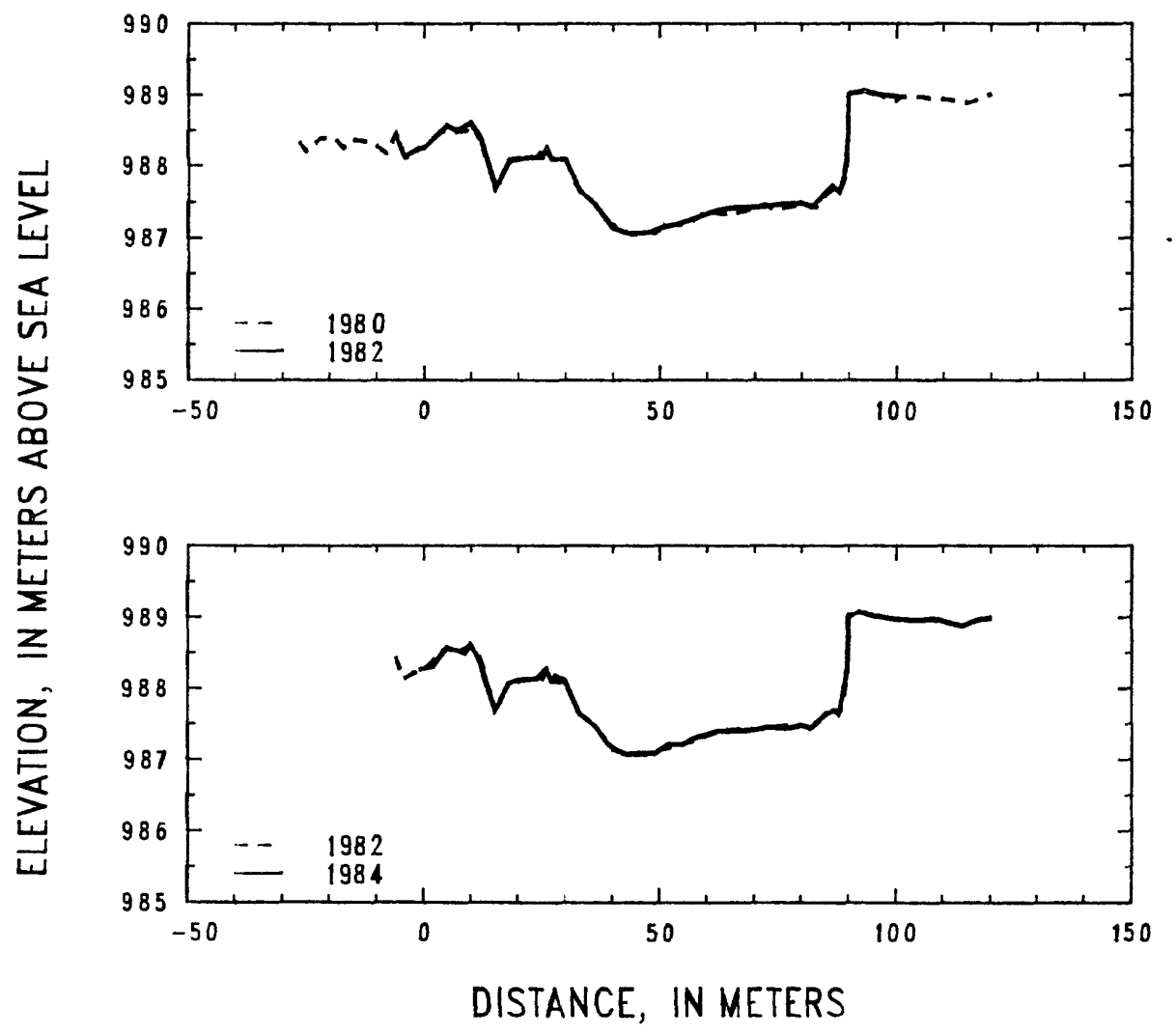

Figure 41.--Profiles of cross section PR141 from 1980 to 1984. 
Table 15.--Listing of horizontal stations and elevations for cross section PR141 [Sta., station, distance in meters from a reference point on the left bank; Elev., elevation, in meters above sea level].

\begin{tabular}{|c|c|c|c|c|c|c|c|c|c|}
\hline \multicolumn{2}{|c|}{1975} & \multicolumn{2}{|c|}{1975} & \multicolumn{2}{|c|}{1977} & \multicolumn{2}{|c|}{1977} & \multicolumn{2}{|c|}{1978} \\
\hline Sta. & Elev. & Sta. & Elev. & Sta. & Elev. & Sta. & Elev. & Sta. & Elev. \\
\hline-30.0 & 988.25 & 64.0 & 986.35 & -26.4 & 988.28 & 48.0 & 986.47 & -40.0 & 988.66 \\
\hline-20.0 & 988.37 & 66.0 & 986.36 & -25.0 & 988.21 & 50.0 & 986.53 & -36.0 & 988.69 \\
\hline-10.0 & 988.17 & 68.0 & 986.36 & -24.0 & 988.14 & 52.0 & 986.58 & -35.0 & 988.32 \\
\hline-6.0 & 988.30 & 70.0 & 986.41 & -21.0 & 988.36 & 55.0 & 986.72 & -33.0 & 988.14 \\
\hline-4.0 & 987.96 & 72.0 & 986.45 & -18.0 & 988.24 & 58.0 & 986.80 & -32.0 & 988.40 \\
\hline 0.0 & 988.08 & 74.0 & 986.48 & -15.0 & 988.30 & 61.0 & 986.82 & -30.0 & 988.32 \\
\hline 2.0 & 988.07 & 76.0 & 986.49 & -11.0 & 988.25 & 62.5 & 986.82 & -26.5 & 988.34 \\
\hline 4.0 & 988.11 & 78.0 & 986.47 & -7.0 & 988.17 & 64.4 & 986.70 & -24.0 & 988.21 \\
\hline 6.0 & 988.18 & 80.0 & 986.46 & -6.0 & 988.34 & 65.5 & 986.49 & -22.0 & 988.40 \\
\hline 8.0 & 988.22 & 82.0 & 986.42 & -4.0 & 988.01 & 67.0 & 986.42 & -21.0 & 988.43 \\
\hline 10.0 & 988.21 & 84.0 & 986.36 & -0.8 & 988.10 & 70.0 & 986.37 & -18.0 & 988.32 \\
\hline 12.0 & 988.13 & 85.0 & 986.52 & 0.0 & 988.10 & 73.0 & 986.39 & -15.0 & 988.39 \\
\hline 13.0 & 988.08 & 86.0 & 986.97 & 4.0 & 988.12 & 75.0 & 986.40 & -13.0 & 988.36 \\
\hline 14.0 & 987.74 & 87.0 & 987.81 & 8.0 & 988.23 & 77.0 & 986.40 & -11.0 & 988.34 \\
\hline 15.8 & 987.78 & 87.1 & 989.02 & 10.0 & 988.26 & 80.0 & 986.40 & -8.0 & 988.19 \\
\hline 17.0 & 987.02 & 89.0 & 989.05 & 11.0 & 988.14 & 82.0 & 986.39 & -7.0 & 988.36 \\
\hline 17.7 & 987.17 & 91.0 & 989.06 & 13.0 & 988.10 & 84.0 & 986.33 & -6.0 & 988.45 \\
\hline 19.0 & 986.83 & 93.0 & 989.07 & 14.0 & 987.77 & 85.1 & 986.23 & -4.0 & 988.14 \\
\hline 20.0 & 986.90 & 95.0 & 989.02 & 14.7 & 987.67 & 85.9 & 986.49 & -2.0 & 988.21 \\
\hline 22.0 & 987.00 & 97.0 & 988.97 & 15.0 & 987.52 & 86.5 & 986.65 & -0.9 & 988.27 \\
\hline 24.0 & 987.16 & 100.0 & 988.97 & 16.0 & 987.27 & 87.7 & 987.47 & 0.0 & 988.27 \\
\hline 25.0 & 986.92 & & & 18.0 & 987.28 & 87.8 & 989.00 & 2.0 & 988.31 \\
\hline 27.0 & 986.68 & & & 18.7 & 987.35 & 89.0 & 989.05 & 4.0 & 988.52 \\
\hline 29.0 & 986.54 & & & 19.0 & 987.30 & 91.0 & 989.01 & 8.0 & 988.50 \\
\hline 31.0 & 986.49 & & & 20.0 & 987.20 & 93.0 & 989.08 & 10.0 & 988.59 \\
\hline 32.0 & 986.44 & & & 21.7 & 986.97 & 95.0 & 989.04 & 11.0 & 988.53 \\
\hline 34.0 & 986.45 & & & 23.6 & 987.04 & 97.0 & 988.98 & 13.0 & 988.13 \\
\hline 36.0 & 986.48 & & & 24.0 & 987.15 & 100.0 & 988.98 & 15.5 & 987.70 \\
\hline 38.0 & 986.41 & & & 24.5 & 986.92 & & & 17.9 & 988.10 \\
\hline 40.0 & 986.47 & & & 25.7 & 986.71 & & & 20.0 & 988.11 \\
\hline 40.5 & 986.49 & & & 27.0 & 986.68 & & & 24.0 & 988.15 \\
\hline 42.0 & 986.55 & & & 29.0 & 986.55 & & & 25.0 & 988.15 \\
\hline 43.5 & 986.59 & & & 30.7 & 986.47 & & & 26.0 & 988.28 \\
\hline 45.0 & 986.49 & & & 32.0 & 986.43 & & & 27.0 & 988.10 \\
\hline 46.0 & 986.62 & & & 34.0 & 986.41 & & & 29.0 & 988.16 \\
\hline 47.1 & 986.52 & & & 36.0 & 986.41 & & & 30.0 & 988.12 \\
\hline 49.0 & 986.56 & & & 38.0 & 986.41 & & & 32.0 & 987.78 \\
\hline 49.8 & 986.52 & & & 40.0 & 986.37 & & & 34.0 & 987.57 \\
\hline 50.0 & 986.47 & & & 42.0 & 986.39 & & & 36.0 & 987.50 \\
\hline 52.0 & 986.38 & & & 42.2 & 986.48 & & & 38.0 & 987.31 \\
\hline 54.0 & 986.37 & & & 42.8 & 986.52 & & & 40.0 & 987.16 \\
\hline 56.0 & 986.37 & & & 43.5 & 986.47 & & & 42.0 & 987.12 \\
\hline 58.0 & 986.36 & & & 45.0 & 986.45 & & & 44.0 & 987.07 \\
\hline 60.0 & 986.35 & & & 46.0 & 986.41 & & & 46.0 & 987.09 \\
\hline 62.0 & 986.33 & & & 47.0 & 986.42 & & & 48.0 & 987.08 \\
\hline
\end{tabular}


Table 15.--Listing of horizontal stations and elevations for cross section PR141 --Continued.

\begin{tabular}{|c|c|c|c|c|c|c|c|c|c|}
\hline \multicolumn{2}{|c|}{1978} & \multicolumn{2}{|c|}{1979} & \multicolumn{2}{|c|}{1979} & \multicolumn{2}{|c|}{1980} & \multicolumn{2}{|c|}{1980} \\
\hline Sta. & Elev. & Sta. & Elev. & Sta. & Elev. & Sta. & Elev. & Sta. & Elev. \\
\hline 50.0 & 987.09 & -36.0 & 988.73 & 56.0 & 987.21 & -26.5 & 988.34 & 83.0 & 987.43 \\
\hline 52.0 & 987.18 & -35.0 & 988.34 & 58.0 & 987.31 & -25.0 & 988.22 & 85.0 & 987.60 \\
\hline 54.0 & 987.16 & -30.0 & 988.29 & 60.0 & 987.38 & -22.0 & 988.40 & 87.0 & 987.71 \\
\hline 56.0 & 987.23 & -26.5 & 988.34 & 62.0 & 987.29 & -19.0 & 988.41 & 88.0 & 987.64 \\
\hline 58.0 & 987.31 & -24.0 & 988.18 & 64.0 & 987.39 & -17.0 & 988.27 & 88.9 & 987.78 \\
\hline 60.0 & 987.44 & -22.0 & 988.39 & 66.0 & 987.38 & -15.0 & 988.39 & 89.8 & 988.25 \\
\hline 62.0 & 987.23 & -20.0 & 988.41 & 68.0 & 987.36 & -11.0 & 988.34 & 89.8 & 989.00 \\
\hline 64.0 & 987.40 & -17.0 & 988.27 & 70.0 & 987.42 & -8.0 & 988.19 & 90.0 & 989.02 \\
\hline 66.0 & 987.39 & -14.0 & 988.38 & 72.0 & 987.46 & -6.0 & 988.46 & 93.0 & 989.06 \\
\hline 68.0 & 987.36 & -11.0 & 988.34 & 74.0 & 987.39 & -4.0 & 988.13 & 96.0 & 989.01 \\
\hline 70.0 & 987.42 & -8.0 & 988.19 & 76.0 & 987.41 & -2.0 & 988.18 & 100.0 & 988.92 \\
\hline 72.0 & 987.48 & -6.0 & 988.46 & 78.0 & 987.44 & -0.9 & 988.27 & 100.9 & 988.98 \\
\hline 74.0 & 987.39 & -5.0 & 988.30 & 80.0 & 987.49 & 0.0 & 988.27 & 105.0 & 988.98 \\
\hline 76.0 & 987.42 & -2.0 & 988.19 & 82.0 & 987.41 & 4.0 & 988.51 & 110.0 & 988.94 \\
\hline 78.0 & 987.44 & -0.9 & 988.26 & 84.0 & 987.53 & 7.0 & 988.49 & 115.0 & 988.89 \\
\hline 80.0 & 987.49 & 0.0 & 988.25 & 86.0 & 987.69 & 9.0 & 988.50 & 120.0 & 989.02 \\
\hline 82.0 & 987.40 & 2.0 & 988.31 & 87.0 & 987.70 & 10.0 & 988.61 & & \\
\hline 84.0 & 987.53 & 4.0 & 988.50 & 88.5 & 987.67 & 12.0 & 988.37 & & \\
\hline 86.0 & 987.71 & 6.0 & 988.57 & 89.2 & 987.89 & 14.0 & 987.93 & & \\
\hline 88.0 & 987.64 & 8.0 & 988.50 & 89.7 & 989.15 & 15.4 & 987.69 & & \\
\hline 89.0 & 987.79 & 10.0 & 988.60 & 89.8 & 988.99 & 18.0 & 988.11 & & \\
\hline 89.6 & 988.06 & 11.0 & 988.53 & 90.0 & .989 .02 & 21.0 & 988.12 & & \\
\hline 89.6 & 988.97 & 12.0 & 988.38 & 92.0 & 989.09 & 24.0 & 988.14 & & \\
\hline 90.0 & 989.02 & 14.0 & 987.94 & 94.0 & 989.06 & 26.0 & 988.28 & & \\
\hline 91.0 & 989.02 & 15.6 & 987.69 & 96.0 & 989.01 & 27.0 & 988.11 & & \\
\hline 93.0 & 989.06 & 18.0 & 988.10 & 98.0 & 988.97 & 30.0 & 988.10 & & \\
\hline 95.0 & 989.02 & 20.0 & 988.11 & 100.0 & 988.97 & 32.0 & 987.78 & & \\
\hline 97.0 & 988.96 & 22.0 & 988.12 & 100.9 & 988.97 & 34.0 & 987.57 & & \\
\hline 100.0 & 988.98 & 25.0 & 988.13 & 105.0 & 988.98 & 36.0 & 987.50 & & \\
\hline 100.9 & 988.96 & 26.0 & 988.27 & 110.0 & 988.94 & 39.0 & 987.22 & & \\
\hline 105.0 & 988.99 & 27.0 & 988.10 & 115.0 & 988.86 & 42.0 & 987.11 & & \\
\hline 110.0 & 988.94 & 28.0 & 988.16 & 120.0 & 988.99 & 44.0 & 987.05 & & \\
\hline 115.0 & 988.86 & 30.0 & 988.10 & & & 47.0 & 987.10 & & \\
\hline 120.0 & 989.01 & 32.0 & 987.79 & & & 49.0 & 987.07 & & \\
\hline & & 34.0 & 987.57 & & & 51.0 & 987.17 & & \\
\hline & & 36.0 & 987.50 & & & 55.0 & 987.20 & & \\
\hline & & 38.0 & 987.32 & & & 58.0 & 987.28 & & \\
\hline & & 40.0 & 987.16 & & & 60.0 & 987.39 & & \\
\hline & & 42.0 & 987.12 & & & 63.0 & 987.34 & & \\
\hline & & 44.0 & 987.07 & & & 67.0 & 987.37 & & \\
\hline & & 46.0 & 987.08 & & & 70.0 & 987.42 & & \\
\hline & & 48.0 & 987.08 & & & 72.0 & 987.47 & & \\
\hline & & 50.0 & 987.12 & & & 74.0 & 987.40 & & \\
\hline & & 52.0 & 987.18 & & & 77.0 & 987.43 & & \\
\hline & & 54.0 & 987.18 & & & 80.0 & 987.49 & & \\
\hline
\end{tabular}


Table 15.--Listing of horizontal stations and elevations for cross section PR141 --Continued.

\begin{tabular}{|c|c|c|c|c|c|}
\hline \multicolumn{2}{|c|}{1982} & \multicolumn{2}{|c|}{1984} & \multicolumn{2}{|c|}{1984} \\
\hline Sta. & Elev. & Sta. & Elev. & Sta. & Elev. \\
\hline-6.0 & 988.44 & -0.9 & 988.27 & 108.0 & 988.79 \\
\hline-4.0 & 988.14 & 0.0 & 988.28 & 111.0 & 988.93 \\
\hline-0.9 & 988.27 & 2.0 & 988.32 & 114.0 & 988.88 \\
\hline 0.0 & 988.27 & 5.0 & 988.58 & 117.0 & 988.97 \\
\hline 5.0 & 988.59 & 9.0 & 988.50 & 120.0 & 989.00 \\
\hline 7.0 & 988.51 & 10.0 & 988.62 & & \\
\hline 10.0 & 988.62 & 12.0 & 988.39 & & \\
\hline 12.0 & 988.42 & 13.0 & 988.14 & & \\
\hline 15.2 & 987.69 & 15.2 & 987.71 & & \\
\hline 18.0 & 988.08 & 16.0 & 987.77 & & \\
\hline 22.0 & 988.13 & 18.0 & 988.08 & & \\
\hline 25.0 & 988.13 & 20.0 & 988.12 & & \\
\hline 26.0 & 988.26 & 24.0 & 988.14 & & \\
\hline 27.0 & 988.10 & 26.0 & 988.28 & & \\
\hline 30.0 & 988.10 & 27.0 & 988.11 & & \\
\hline 33.0 & 987.66 & 28.0 & 988.19 & & \\
\hline 36.0 & 987.50 & 30.0 & 988.11 & & \\
\hline 40.0 & 987.15 & 33.0 & 987.66 & & \\
\hline 43.0 & 987.07 & 36.0 & 987.50 & & \\
\hline 48.0 & 987.08 & 39.0 & 987.23 & & \\
\hline 50.0 & 987.14 & 40.0 & 987.16 & & \\
\hline 54.0 & 987.20 & 43.0 & 987.07 & & \\
\hline 58.0 & 987.29 & 46.0 & 987.10 & & \\
\hline 61.0 & 987.37 & 49.0 & 987.09 & & \\
\hline 65.0 & 987.43 & 52.0 & 987.22 & & \\
\hline 70.0 & 987.43 & 55.0 & 987.21 & & \\
\hline 75.0 & 987.47 & 57.0 & 987.30 & & \\
\hline 80.0 & 987.49 & 60.0 & 987.35 & & \\
\hline 82.0 & 987.43 & 62.0 & 987.40 & & \\
\hline 86.5 & 987.73 & 65.0 & 987.40 & & \\
\hline 88.0 & 987.64 & 70.0 & 987.42 & & \\
\hline 89.0 & 987.84 & 73.0 & 987.46 & & \\
\hline 89.7 & 988.33 & 77.0 & 987.44 & & \\
\hline 89.8 & 989.01 & 80.0 & 987.49 & & \\
\hline 90.0 & 989.03 & 82.0 & 987.44 & & \\
\hline 93.0 & 989.07 & 85.0 & 987.63 & & \\
\hline 95.0 & 989.02 & 87.0 & 987.70 & & \\
\hline 100.0 & 988.98 & 88.2 & 987.67 & & \\
\hline \multirow[t]{7}{*}{101.0} & 988.97 & 89.7 & 988.24 & & \\
\hline & & 90.0 & 989.02 & & \\
\hline & & 92.0 & 989.08 & & \\
\hline & & 95.0 & 989.02 & & \\
\hline & & 100.0 & 988.97 & & \\
\hline & & 100.9 & 988.98 & & \\
\hline & & 104.0 & 988.95 & & \\
\hline
\end{tabular}


Description of Cross Section PR141A

Location: Township 7 South/Range 49 East--section 29

U.S. Geological Survey quadrangle $(1: 24,000)$ : Huckins School

Landowners--left bank: U.S. Government right bank: U.S. Government

Access: $\quad$ left bank

Permission from: Gay Ranch (T7S/R49E--20)

Reference pins

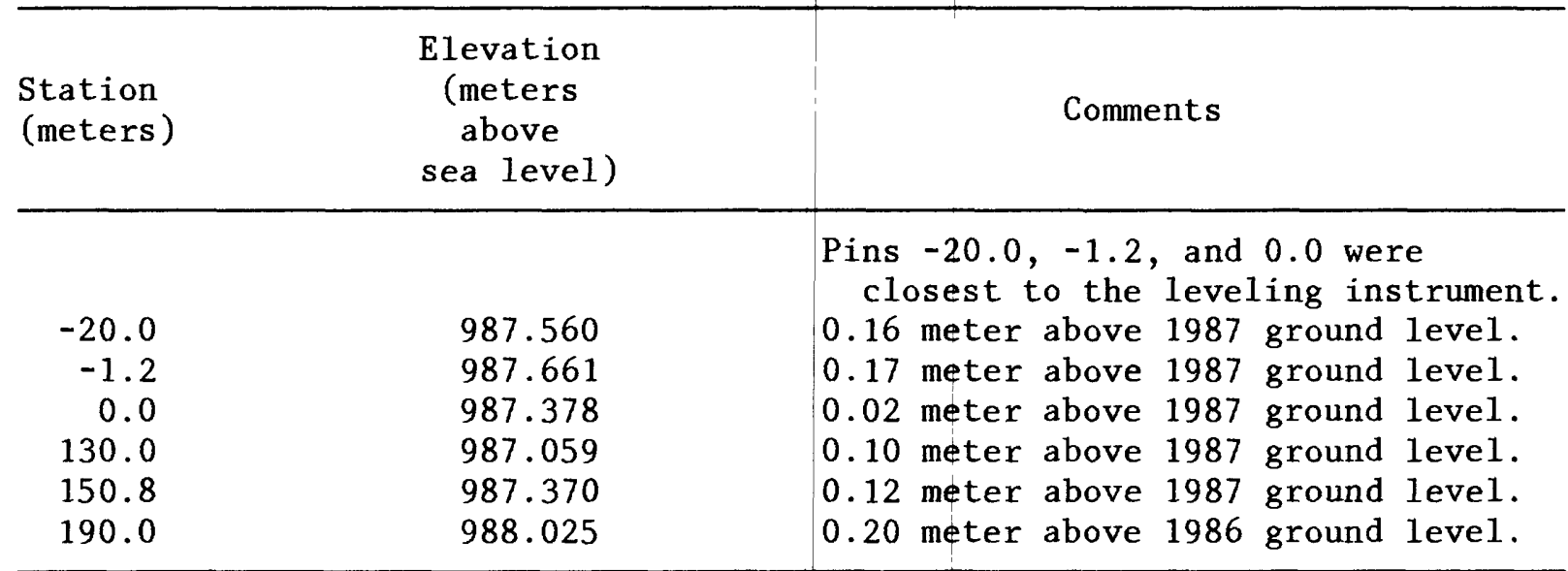




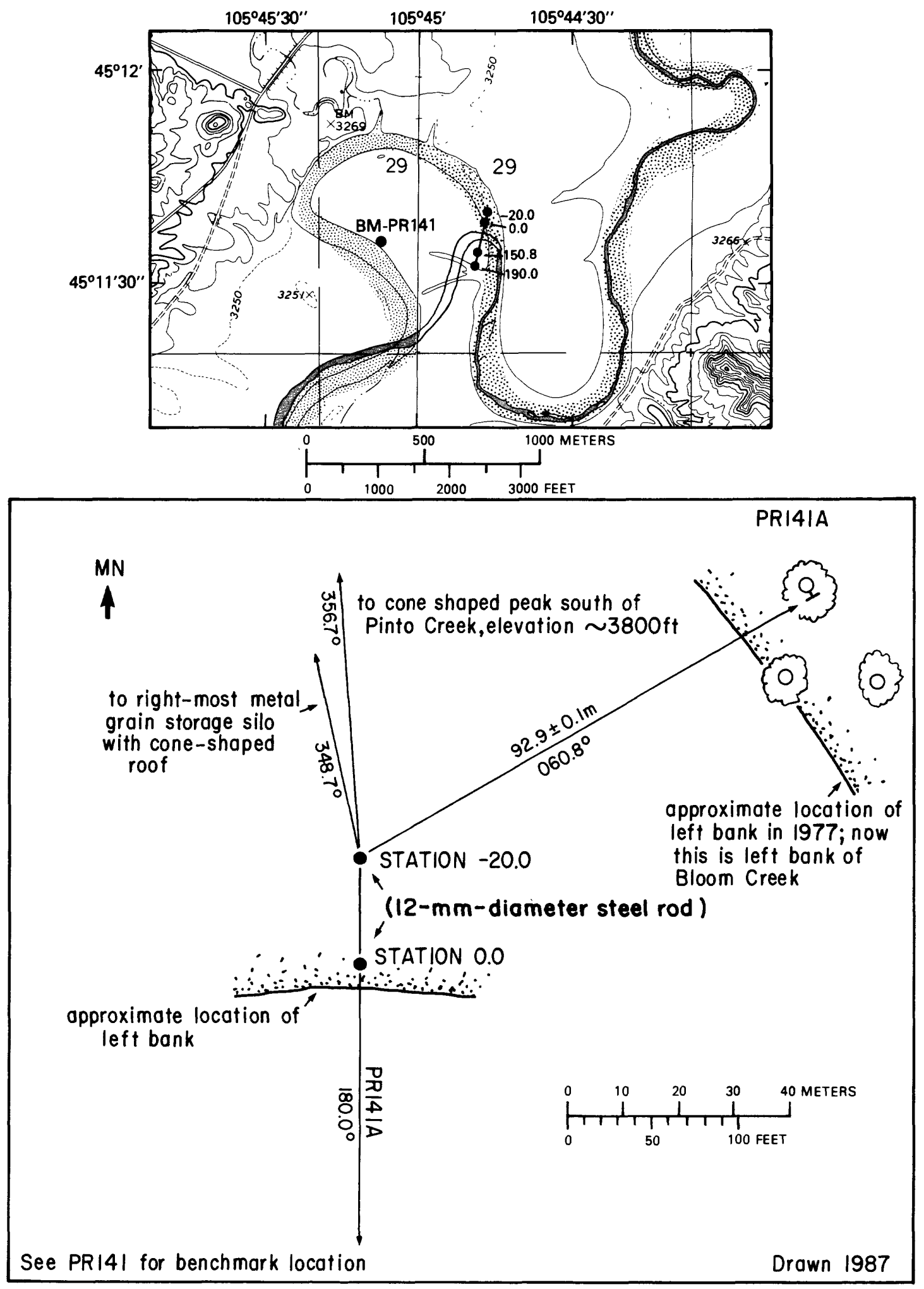

Figure 42.--Upper: Location of cross section PR141A, bench mark BM-PR141, and left and right bank reference pins (tables 2 and 3 ) in the Huckins School quadrangle. New channel is shown in its approximate location upstream and downstream from the section. Lower: Location of secondary bench marks (12-mm-diameter steel rod) at station 0.0 and $-20.0 \mathrm{~m}$ on the left bank. 

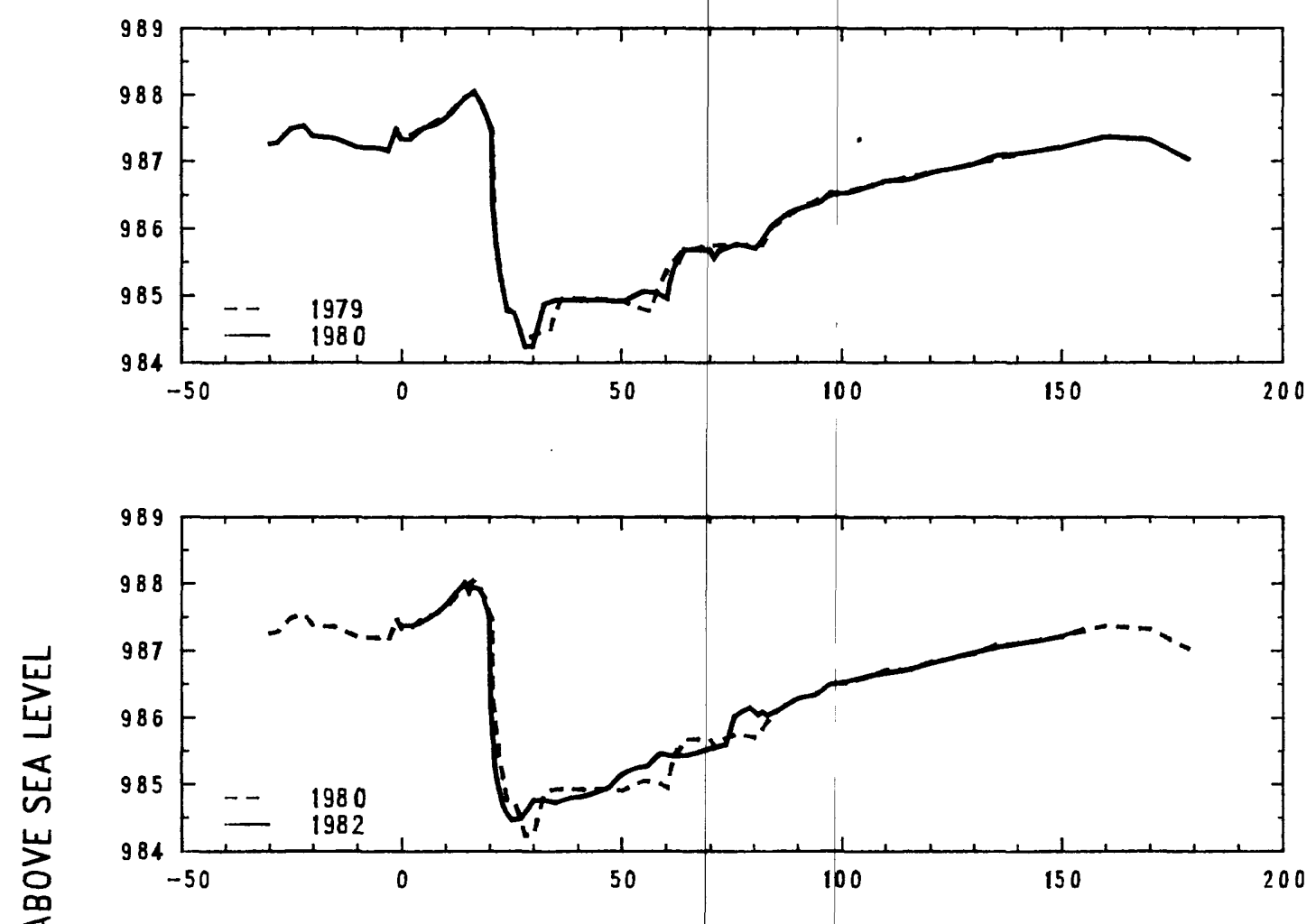

立
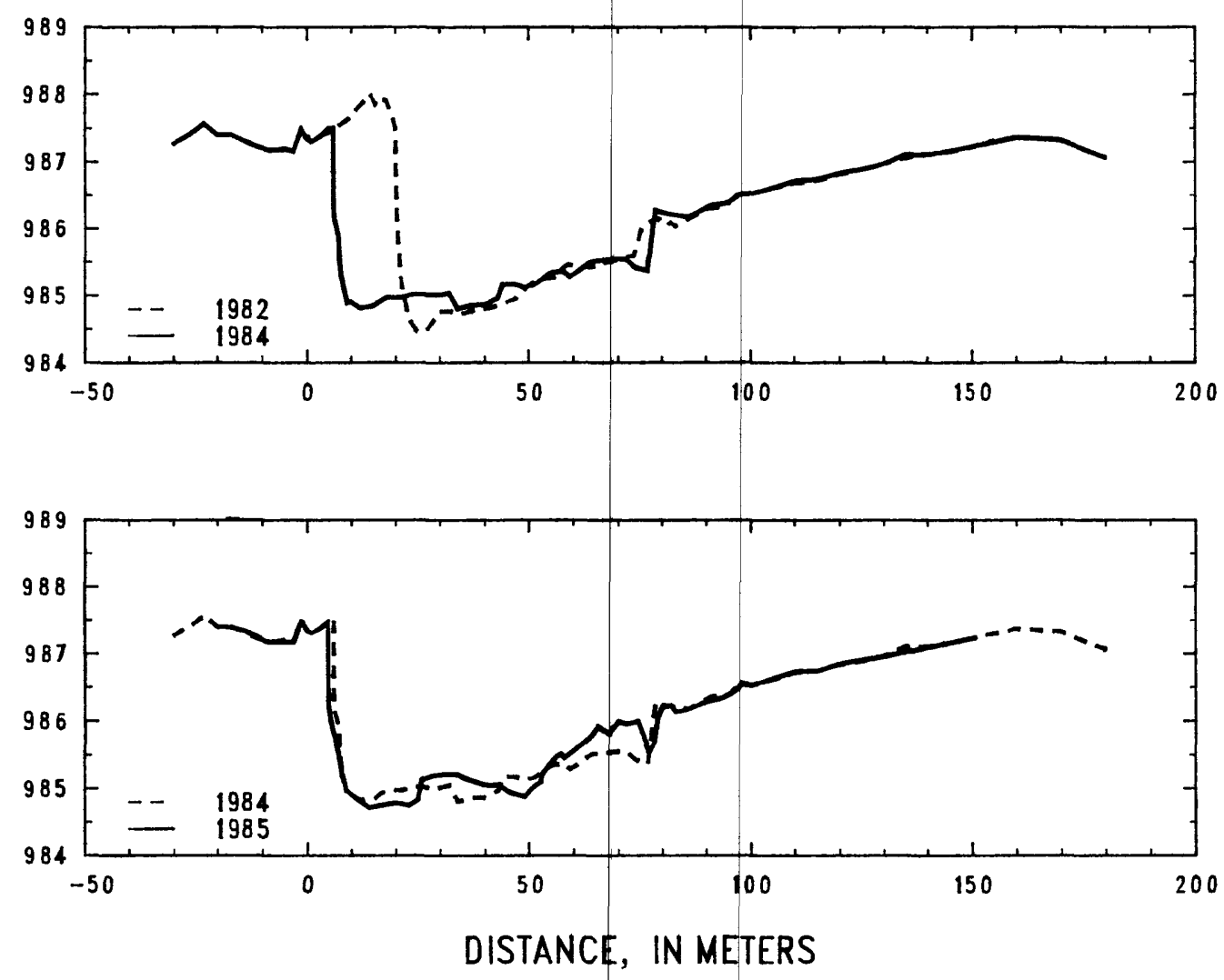

Figure 43. - Profiles of cross section PR141A from 1979 to 1985. 

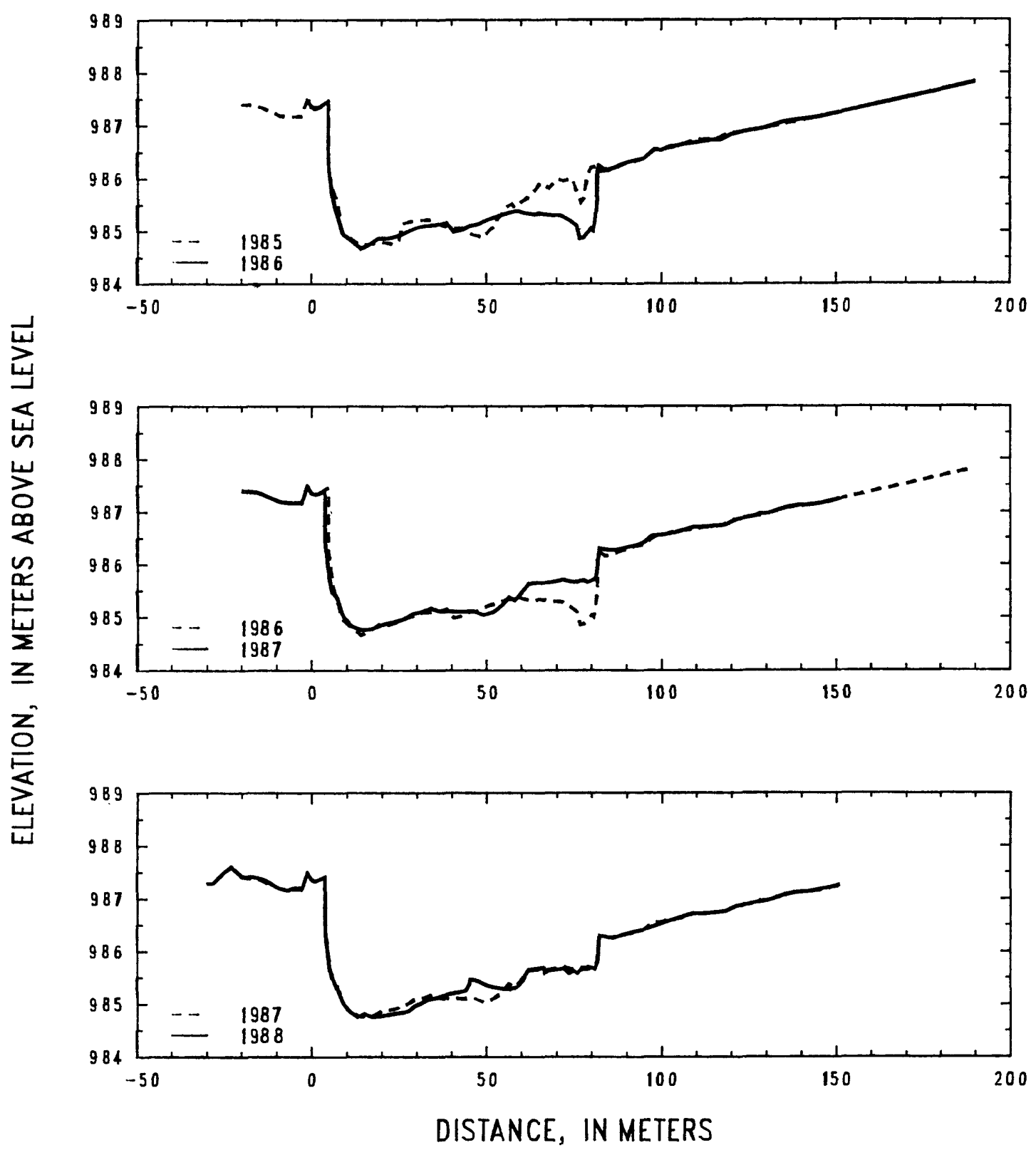

Figure 44.--Profiles of cross section PR141A from 1985 to 1988. 
Table 16.--Listing of horizontal stations and elevations for cross section PR141A

[Sta., station, distance in meters from a reference point on

the left bank; Elev., elevation, in meters above sea levell.

\begin{tabular}{|c|c|c|c|c|c|c|c|c|c|}
\hline \multicolumn{2}{|c|}{1979} & \multicolumn{2}{|c|}{1980} & \multicolumn{2}{|c|}{1980} & \multicolumn{2}{|c|}{1982} & \multicolumn{2}{|c|}{1982} \\
\hline Sta. & Elev. & Sta. & Elev. & Sta. & Elev. & Sta. & Elev. & Sta. & Elev. \\
\hline-1.2 & 987.50 & -30.0 & 987.27 & 88.0 & 986.24 & -1.2 & 987.47 & 105.0 & 986.61 \\
\hline 0.0 & 987.33 & -28.0 & 987.29 & 92.0 & 986.33 & 0.0 & 987.38 & 110.0 & 986.68 \\
\hline 5.0 & 987.51 & -25.0 & 987.50 & 95.0 & 986.39 & 3.0 & 987.37 & 115.0 & 986.72 \\
\hline 10.0 & 987.67 & -22.0 & 987.55 & 97.5 & 986.53 & 7.0 & 987.51 & 120.0 & 986.81 \\
\hline 15.0 & 988.00 & -20.0 & 987.39 & 100.0 & 986.51 & 10.0 & 987.66 & 125.0 & 986.89 \\
\hline 17.0 & 988.02 & -15.0 & 987.36 & 105.0 & 986.60 & 13.0 & 987.90 & 130.0 & 986.97 \\
\hline 20.7 & 987.49 & -10.0 & 987.21 & 110.0 & 986.72 & 14.3 & 988.01 & 135.0 & 987.05 \\
\hline 21.3 & 985.82 & -5.0 & 987.20 & 115.0 & 986.73 & 15.5 & 987.86 & 140.0 & 987.11 \\
\hline 21.9 & 985.50 & -3.0 & 987.16 & 120.0 & 986.84 & 16.0 & 987.95 & 145.0 & 987.15 \\
\hline 22.9 & 985.16 & -1.2 & 987.49 & 125.0 & 986.90 & 18.0 & 987.91 & 150.0 & 987.22 \\
\hline 24.0 & 984.82 & 0.0 & 987.34 & 130.0 & 986.96 & 20.0 & 987.52 & 150.8 & 987.24 \\
\hline 26.0 & 984.71 & 2.0 & 987.33 & 135.0 & 987.09 & 20.4 & 986.00 & 155.0 & 987.32 \\
\hline 27.0 & 984.47 & 5.0 & 987.51 & 140.0 & 987.12 & 21.5 & 985.12 & & \\
\hline 29.0 & 984.37 & 8.0 & 987.56 & 145.0 & 987.17 & 23.0 & 984.66 & & \\
\hline 31.0 & 984.44 & 11.0 & 987.70 & 150.0 & 987.22 & 24.0 & 984.54 & & \\
\hline 34.0 & 984.49 & 14.0 & 987.94 & 150.8 & 987.24 & 25.0 & 984.46 & & \\
\hline 35.0 & 984.75 & 16.6 & 988.06 & 160.0 & 987.38 & 27.0 & 984.48 & & \\
\hline 36.5 & 984.96 & 19.0 & 987.77 & 170.0 & 987.33 & 29.0 & 984.66 & & \\
\hline 40.0 & 984.96 & 20.7 & 987.46 & 179.0 & 987.03 & 30.0 & 984.76 & & \\
\hline 45.0 & 984.95 & 20.7 & 986.30 & & & 32.0 & 984.77 & & \\
\hline 50.0 & 984.92 & 22.0 & 985.49 & & & 35.0 & 984.72 & & \\
\hline 55.0 & 984.79 & 24.0 & 984.77 & & & 38.0 & 984.80 & & \\
\hline 56.6 & 984.76 & 25.7 & 984.74 & & & 41.0 & 984.81 & & \\
\hline 58.7 & 985.16 & 27.0 & 984.49 & & & 44.0 & 984.88 & & \\
\hline 61.0 & 985.46 & 28.0 & 984.24 & & & 47.0 & 984.95 & & \\
\hline 64.0 & 985.67 & 30.0 & 984.25 & & & 50.0 & 985.15 & & \\
\hline 70.0 & 985.75 & 32.5 & 984.88 & & & 53.0 & 985.24 & & \\
\hline 82.0 & 985.75 & 35.0 & 984.94 & & & 56.0 & 985.27 & & \\
\hline 84.0 & 986.01 & 40.0 & 984.93 & & & 58.4 & 985.44 & & \\
\hline 91.0 & 986.33 & 45.0 & 984.94 & & & 59.0 & 985.46 & & \\
\hline 97.0 & 986.48 & 50.0 & 984.91 & & & 62.0 & 985.41 & & \\
\hline 110.0 & 986.70 & 55.0 & 985.06 & & & 65.0 & 985.44 & & \\
\hline 120.0 & 986.84 & 58.0 & 985.04 & & & 70.0 & 985.54 & & \\
\hline 130.0 & 986.96 & 60.5 & 984.95 & & & 73.5 & 985.60 & & \\
\hline 140.0 & 987.11 & 61.0 & 985.19 & & & 75.5 & 986.02 & & \\
\hline 150.0 & 987.23 & 62.5 & 985.52 & & & 77.0 & 986.09 & & \\
\hline \multirow[t]{9}{*}{150.8} & 987.24 & 63.0 & 985.50 & & & 79.0 & 986.15 & & \\
\hline & & 64.2 & 985.69 & & & 81.0 & 986.04 & & \\
\hline & & 70.0 & 985.68 & & & 82.0 & 986.09 & & \\
\hline & & 71.0 & 985.56 & & & 83.0 & 986.03 & & \\
\hline & & 72.0 & 985.67 & & & 86.0 & 986.14 & & \\
\hline & & 76.0 & 985.77 & & & 90.0 & 986.29 & & \\
\hline & & 80.4 & 985.70 & & & 94.0 & 986.34 & & \\
\hline & & 82.1 & 985.85 & & & 97.0 & 986.49 & & \\
\hline & & 84.0 & 986.04 & & & 100.0 & 986.52 & & \\
\hline
\end{tabular}


Table 16.--Listing of horizontal stations and elevations for cross section PR141A--Continued.

\begin{tabular}{|c|c|c|c|c|c|c|c|c|c|}
\hline \multicolumn{2}{|c|}{1984} & \multicolumn{2}{|c|}{1984} & \multicolumn{2}{|c|}{1985} & \multicolumn{2}{|c|}{1985} & \multicolumn{2}{|c|}{1986} \\
\hline Sta. & Elev. & Sta. & Elev. & Sta. & Elev. & Sta. & Elev. & Sta. & $\overline{\text { Elev. }}$ \\
\hline-30.0 & 987.27 & 70.0 & 985.55 & -20.0 & 987.41 & 70.0 & 985.99 & -1.2 & 987.48 \\
\hline-26.0 & 987.42 & 72.0 & 985.54 & -17.0 & 987.40 & 72.0 & 985.95 & 0.0 & 987.35 \\
\hline-23.0 & 987.57 & 74.0 & 985.42 & -14.0 & 987.34 & 74.5 & 986.00 & 2.0 & 987.34 \\
\hline-20.0 & 987.40 & 76.8 & 985.37 & -11.0 & 987.25 & 77.0 & 985.54 & 4.0 & 987.43 \\
\hline-17.0 & 987.41 & 78.0 & 986.10 & -9.0 & 987.18 & 78.0 & 985.64 & 4.8 & 987.45 \\
\hline-14.0 & 987.31 & 78.5 & 986.28 & -6.0 & 987.17 & 79.0 & 986.06 & 4.8 & 986.27 \\
\hline-11.0 & 987.22 & 80.0 & 986.24 & -3.0 & 987.18 & 80.0 & 986.21 & 6.0 & 985.65 \\
\hline-8.0 & 987.17 & 84.0 & 986.19 & -2.0 & 987.36 & 82.0 & 986.23 & 6.6 & 985.44 \\
\hline-5.0 & 987.20 & 86.0 & 986.17 & -1.2 & 987.48 & 83.0 & 986.13 & 8.2 & 985.11 \\
\hline-3.0 & 987.16 & 91.0 & 986.36 & 0.0 & 987.35 & 86.0 & 986.17 & 8.7 & 984.98 \\
\hline-1.2 & 987.49 & 95.0 & 986.39 & 1.0 & 987.31 & 90.0 & 986.29 & 9.0 & 984.94 \\
\hline 0.0 & 987.34 & 97.0 & 986.51 & 3.0 & 987.38 & 93.0 & 986.33 & 11.0 & 984.83 \\
\hline 1.0 & 987.30 & 100.0 & 986.53 & 4.8 & 987.47 & 96.0 & 986.42 & 14.0 & 984.66 \\
\hline 3.0 & 987.38 & 105.0 & 986.61 & 4.8 & 986.26 & 98.0 & 986.56 & 16.0 & 984.72 \\
\hline 5.0 & 987.50 & 110.0 & 986.72 & 5.3 & 986.04 & 100.0 & 986.53 & 19.0 & 984.86 \\
\hline 6.1 & 987.51 & 115.0 & 986.74 & 6.0 & 985.83 & 105.0 & 986.63 & 22.0 & 984.86 \\
\hline 6.1 & 986.28 & 120.0 & 986.84 & 7.3 & 985.55 & 110.0 & 986.74 & 25.0 & 984.92 \\
\hline 6.4 & 986.13 & 125.0 & 986.89 & 8.2 & 985.15 & 115.0 & 986.74 & 28.0 & 985.01 \\
\hline 7.3 & 985.91 & 130.0 & 986.97 & 9.0 & 984.97 & 120.0 & 986.86 & 31.0 & 985.09 \\
\hline 7.5 & 985.54 & 135.0 & 987.11 & 11.0 & 984.86 & 125.0 & 986.91 & 34.0 & 985.10 \\
\hline 8.0 & 985.26 & 140.0 & 987.11 & 14.0 & 984.72 & 130.0 & 986.96 & 36.0 & 985.12 \\
\hline 9.2 & 984.89 & 145.0 & 987.16 & 17.0 & 984.76 & 150.8 & 987.25 & 38.4 & 985.16 \\
\hline 10.0 & 984.91 & 150.8 & 987.25 & 20.0 & 984.79 & & & 40.5 & 984.98 \\
\hline 12.0 & 984.82 & 160.0 & 987.37 & 23.0 & 984.75 & & & 43.0 & 985.02 \\
\hline 15.0 & 984.85 & 170.0 & 987.32 & 25.0 & 984.84 & & & 45.0 & 985.10 \\
\hline 18.0 & 984.98 & 180.0 & 987.06 & 25.9 & 985.14 & & & 47.0 & 985.11 \\
\hline 21.0 & 984.97 & & & 28.0 & 985.19 & & & 50.0 & 985.21 \\
\hline 24.0 & 985.03 & & & 31.0 & 985.22 & & & 53.0 & 985.27 \\
\hline 27.0 & 985.02 & & & 34.0 & 985.21 & & & 56.0 & 985.34 \\
\hline 30.0 & 985.01 & & & 35.5 & 985.15 & & & 59.0 & 985.38 \\
\hline 32.0 & 985.05 & & & 40.0 & 985.05 & & & 61.0 & 985.33 \\
\hline 34.0 & 984.80 & & & 43.0 & 985.05 & & & 64.0 & 985.31 \\
\hline 37.0 & 984.87 & & & 46.0 & 984.93 & & & 65.0 & 985.34 \\
\hline 40.0 & 984.87 & & & 49.0 & 984.88 & & & 68.0 & 985.29 \\
\hline 43.0 & 984.97 & & & 51.0 & 985.03 & & & 71.0 & 985.29 \\
\hline 44.0 & 985.17 & & & 52.6 & 985.10 & & & 74.0 & 985.19 \\
\hline 47.0 & 985.17 & & & 53.0 & 985.22 & & & 75.7 & 985.10 \\
\hline 49.0 & 985.12 & & & 56.0 & 985.47 & & & 76.7 & 984.85 \\
\hline 51.0 & 985.15 & & & 57.3 & 985.52 & & & 77.8 & 984.87 \\
\hline 55.0 & 985.34 & & & 58.0 & 985.45 & & & 80.0 & 985.05 \\
\hline 58.0 & 985.37 & & & 60.0 & 985.56 & & & 80.6 & 985.00 \\
\hline 59.3 & 985.28 & & & 62.0 & 985.66 & & & 81.7 & 985.53 \\
\hline 62.0 & 985.41 & & & 64.0 & 985.78 & & & 81.8 & 986.08 \\
\hline 64.0 & 985.51 & & & 65.4 & 985.92 & & & 82.3 & 986.24 \\
\hline 66.0 & 985.53 & & & 68.0 & 985.81 & & & 84.0 & 986.15 \\
\hline
\end{tabular}


Table 16.--Listing of horizontal stations and elevations for cross section PR141A--Continued.

\begin{tabular}{|c|c|c|c|c|c|c|c|c|c|}
\hline \multicolumn{2}{|c|}{1986} & \multicolumn{2}{|c|}{1987} & \multicolumn{2}{|c|}{1987} & \multicolumn{2}{|c|}{1988} & \multicolumn{2}{|c|}{1988} \\
\hline Sta. & Elev. & Sta. & Elev. & Sta. & Elev. & Sta. & $\overline{\text { Elev. }}$ & Sta. & Elev. \\
\hline 86.0 & 986.18 & -20.0 & 987.40 & 78.0 & 985.70 & -30.0 & 987.28 & 50.0 & 985.35 \\
\hline 89.0 & 986.28 & -15.0 & 987.36 & 79.0 & 985.66 & -28.0 & 987.29 & 53.0 & 985.30 \\
\hline 92.0 & 986.34 & -12.0 & 987.26 & 81.0 & 985.73 & -26.0 & 987.43 & 55.0 & 985.28 \\
\hline 95.0 & 986.38 & -9.0 & 987.18 & 81.5 & 985.80 & -25.0 & 987.50 & 56.7 & 985.28 \\
\hline 98.0 & 986.54 & -6.0 & 987.16 & 82.3 & 986.30 & -23.0 & 987.60 & 57.0 & 985.30 \\
\hline 100.0 & 986.53 & -3.0 & 987.17 & 84.0 & 986.27 & -21.0 & 987.47 & 58.0 & 985.30 \\
\hline 102.0 & 986.60 & -1.2 & 987.48 & 87.0 & 986.27 & -20.0 & 987.40 & 59.0 & 985.34 \\
\hline 105.0 & 986.62 & 0.0 & 987.36 & 90.0 & 986.33 & -17.0 & 987.40 & 60.5 & 985.45 \\
\hline 108.0 & 986.66 & 1.0 & 987.32 & 93.0 & 986.37 & -15.0 & 987.38 & 60.9 & 985.53 \\
\hline 111.0 & 986.69 & 2.0 & 987.34 & 95.0 & 986.42 & -13.0 & 987.33 & 62.0 & 985.65 \\
\hline 114.0 & 986.73 & 3.9 & 987.42 & 97.0 & 986.54 & -11.0 & 987.24 & 64.0 & 985.67 \\
\hline 117.0 & 986.73 & 3.9 & 986.45 & 100.0 & 986.56 & -9.0 & 987.17 & 66.3 & 985.69 \\
\hline 120.0 & 986.83 & 5.0 & 985.86 & 103.0 & 986.60 & -7.0 & 987.14 & 66.6 & 985.58 \\
\hline 125.0 & 986.90 & 5.4 & 985.64 & 106.0 & 986.65 & -5.0 & 987.20 & 67.6 & 985.63 \\
\hline 130.0 & 986.95 & 6.0 & 985.46 & 109.0 & 986.72 & -2.7 & 987.20 & 70.0 & 985.65 \\
\hline 135.0 & 987.07 & 7.0 & 985.39 & 112.0 & 986.71 & -1.2 & 987.48 & 72.0 & 985.68 \\
\hline 140.0 & 987.11 & 9.0 & 985.01 & 115.0 & 986.74 & 0.0 & 987.35 & 73.5 & 985.58 \\
\hline 145.0 & 987.16 & 11.0 & 984.84 & 118.0 & 986.75 & 1.0 & 987.32 & 75.0 & 985.63 \\
\hline 150.0 & 987.23 & 14.0 & 984.76 & 121.0 & 986.85 & 3.0 & 987.39 & 76.0 & 985.58 \\
\hline 150.8 & 987.24 & 17.0 & 984.77 & 123.0 & 986.87 & 3.8 & 987.41 & 77.0 & 985.66 \\
\hline \multirow[t]{25}{*}{190.0} & 987.82 & 20.0 & 984.87 & 125.0 & 986.90 & 3.9 & 986.38 & 78.5 & 985.65 \\
\hline & & 23.0 & 984.90 & 126.5 & 986.92 & 5.3 & 985.62 & 79.0 & 985.72 \\
\hline & & 26.0 & 984.96 & 128.0 & 986.97 & 5.7 & 985.57 & 81.0 & 985.66 \\
\hline & & 29.0 & 985.08 & 130.0 & 986.96 & 5.9 & 985.50 & 81.9 & 985.84 \\
\hline & & 31.0 & 985.10 & 133.0 & 987.03 & 7.0 & 985.31 & 82.1 & 986.21 \\
\hline & & 34.0 & 985.17 & 136.0 & 987.09 & 9.0 & 985.01 & 82.3 & 986.30 \\
\hline & & 37.0 & 985.10 & 139.0 & 987.12 & 11.0 & 984.84 & 84.0 & 986.27 \\
\hline & & 40.0 & 985.12 & 142.0 & 987.12 & 13.0 & 984.76 & 86.0 & 986.25 \\
\hline & & 43.0 & 985.10 & 145.0 & 987.16 & 15.0 & 984.81 & 89.0 & 986.32 \\
\hline & & 46.0 & 985.12 & 148.0 & 987.20 & 17.0 & 984.75 & 92.0 & 986.37 \\
\hline & & 49.0 & 985.03 & 150.8 & 987.25 & 20.0 & 984.78 & 95.0 & 986.42 \\
\hline & & 52.0 & 985.10 & & & 23.0 & 984.81 & 97.4 & 986.48 \\
\hline & & 55.0 & 985.27 & & & 26.0 & 984.84 & 100.0 & 986.54 \\
\hline & & 56.4 & 985.38 & & & 27.7 & 984.87 & 103.0 & 986.61 \\
\hline & & 58.0 & 985.32 & & & 28.8 & 984.93 & 106.0 & 986.66 \\
\hline & & 59.2 & 985.40 & & & 30.0 & 984.98 & 109.0 & 986.72 \\
\hline & & 61.0 & 985.54 & & & 31.0 & 985.01 & 112.0 & 986.71 \\
\hline & & 62.0 & 985.63 & & & 33.0 & 985.09 & 115.0 & 986.74 \\
\hline & & 64.0 & 985.66 & & & 36.0 & 985.13 & 118.0 & 986.75 \\
\hline & & 67.0 & 985.65 & & & 39.0 & 985.19 & 121.0 & 986.86 \\
\hline & & 70.0 & 985.68 & & & 42.0 & 985.22 & 124.0 & 986.89 \\
\hline & & 72.0 & 985.72 & & & 44.0 & 985.25 & 126.0 & 986.92 \\
\hline & & 74.0 & 985.67 & & & 45.0 & 985.35 & 128.0 & 986.94 \\
\hline & & 76.0 & 985.67 & & & 45.3 & 985.46 & 130.0 & 986.96 \\
\hline & & 77.0 & 985.70 & & & 47.0 & 985.45 & 133.0 & 987.03 \\
\hline
\end{tabular}


Table 16.--Listing. of horizontal stations and elevations for cross section PR141A--Continued. 1988

Sta. Elev.

$136.0 \quad 987.09$

$139.0 \quad 987.12$

$142.0 \quad 987.13$

$145.0 \quad 987.17$

$148.0 \quad 987.20$

$150.8 \quad 987.26$ 


\section{Description of Cross Section PR147}

Location: Township 7 South/Range 49 East--section 21

U.S. Geological Survey quadrangle $(1: 24,000)$ : Huckins School

Landowners--1eft bank: Gay Ranch

right bank Huckins Ranch

Access: left or right bank

Permission from: left bank, Gay Ranch (T7S/R49E--20)

right bank, Huckins Ranch (T7S/R49E--28)

Reference pins

\begin{tabular}{|c|c|c|}
\hline $\begin{array}{l}\text { Station } \\
\text { (meters) }\end{array}$ & $\begin{array}{l}\text { Elevation } \\
\text { (meters } \\
\text { above } \\
\text { sea leve1) }\end{array}$ & Comments \\
\hline & & $\begin{array}{l}\text { Pin } 100.7 \text { was closest to the leveling } \\
\text { instrument from } 1975 \text { to } 1985 \text {. Since } \\
1985 \text { pins }-68.9,-0.9 \text {, and } 0.0 \text { were } \\
\text { the closest. }\end{array}$ \\
\hline-68.9 & 984.012 & $\begin{array}{l}\text { 0.19 meter above } 1987 \text { ground level, } \\
\text { on fenceline. }\end{array}$ \\
\hline-0.9 & 983.979 & Pin is bent. \\
\hline 0.0 & 983.906 & 0.06 meter above 1987 ground level. \\
\hline 100.7 & 983.266 & 0.10 meter above 1987 ground level. \\
\hline
\end{tabular}




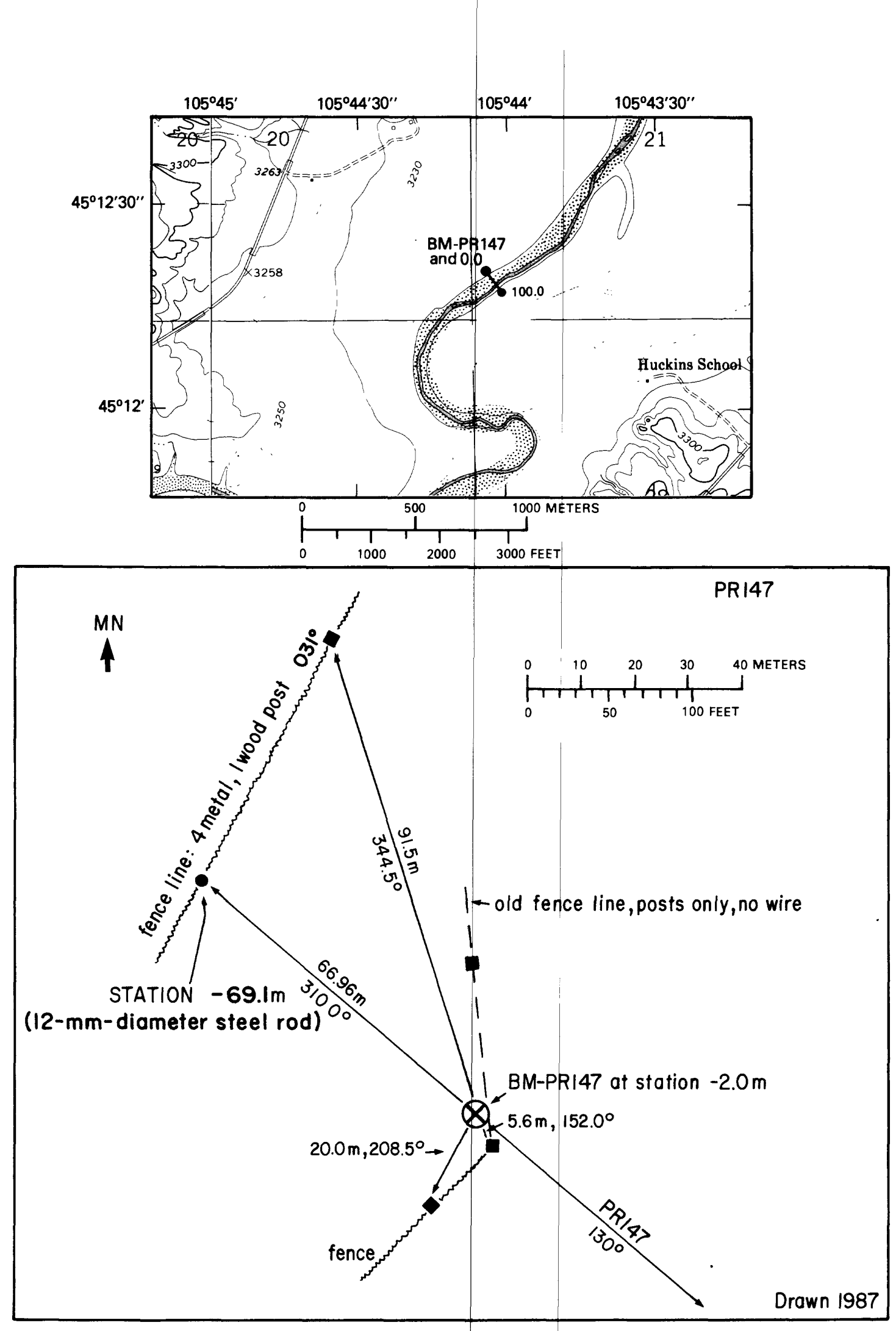

Figure 45.--Upper: Location of cross section PR147, bench mark BM-PR147, and left and right bank reference pins (tables 2 and 3 ) in the Huckins School quadrangle. Lower: Location of bench mark on the left bank. $M N$ is magnetic north. 

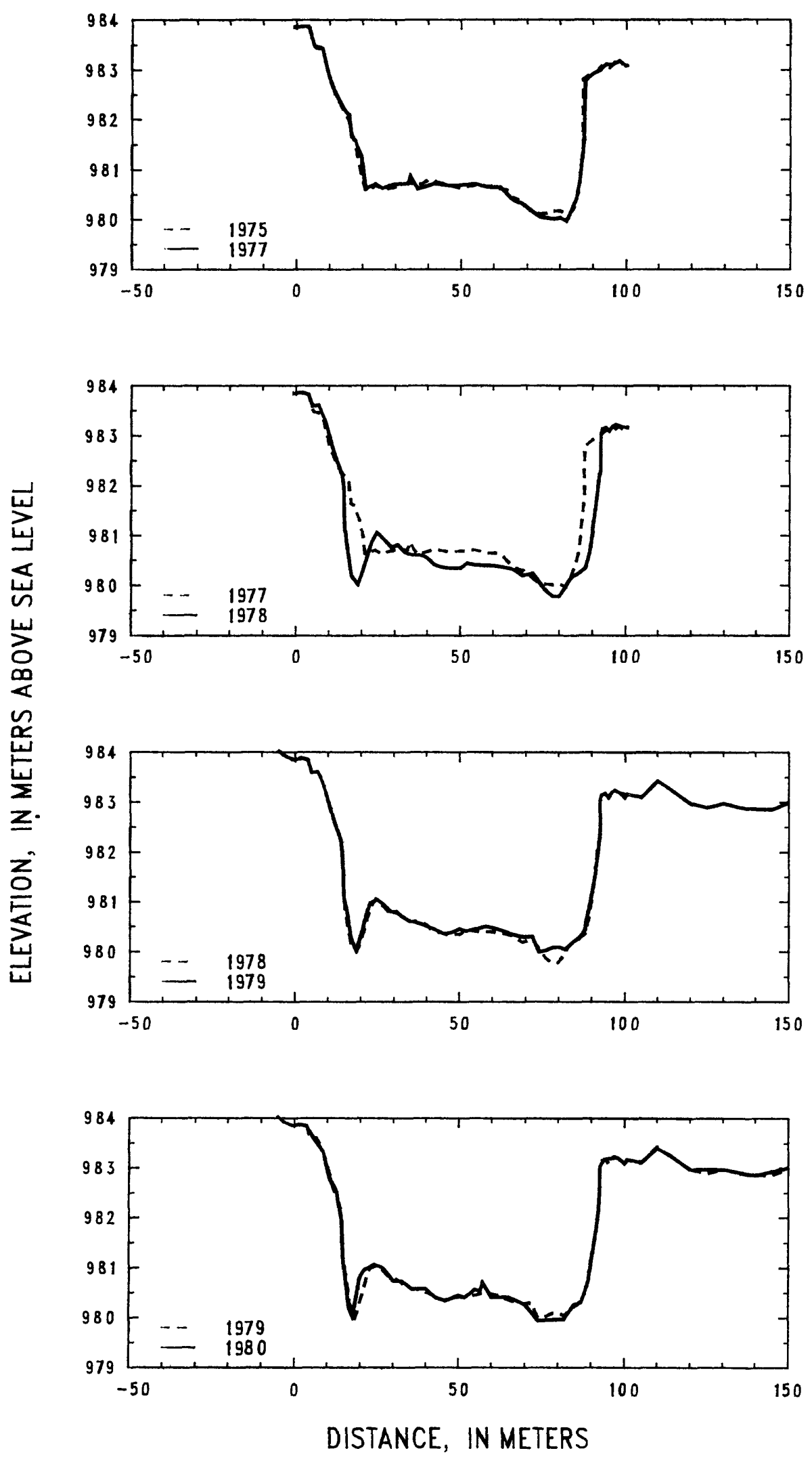

Figure 46.--Profiles of cross section PR147 from 1975 to 1980. 


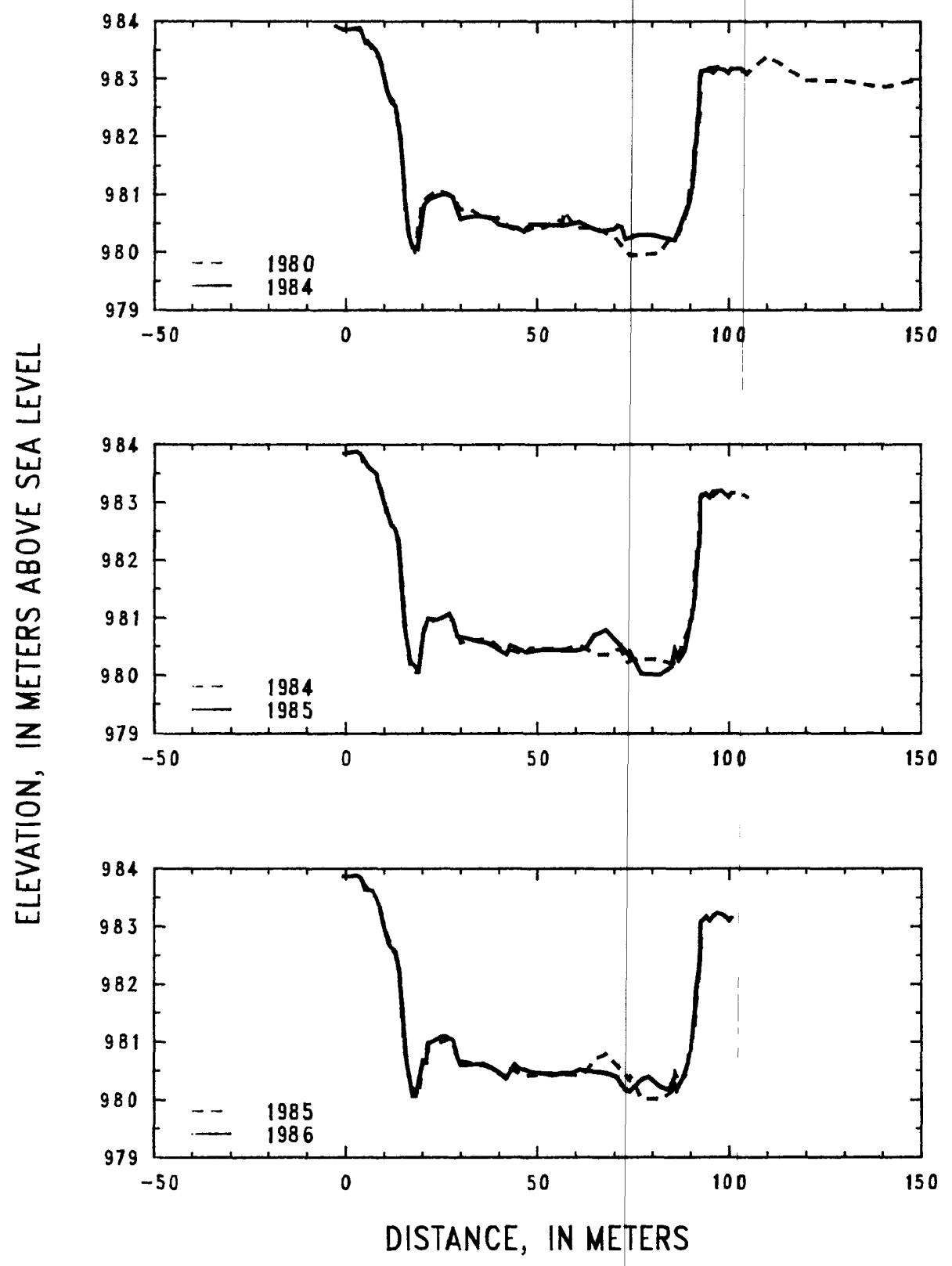

Figure 47. - Profiles of cross section PR 147 from 1980101986. 


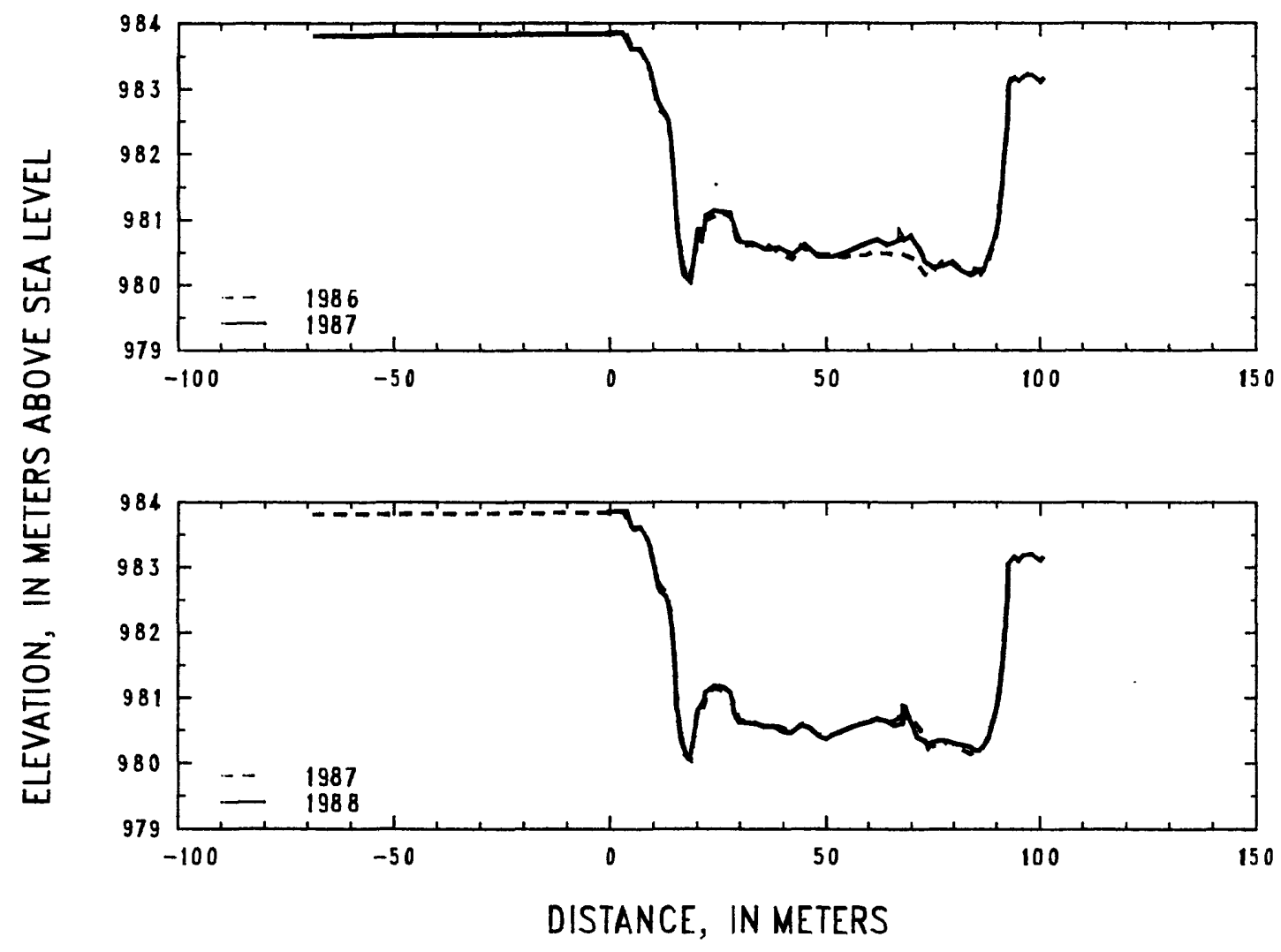

Figure 48. --Profiles of cross section PR147 from 1986 to 1988 
Table 17.--Listing. of horizontal stations and elevations for cross section PR147

[Sta., station, distance in meters from a reference point on

the left bank; Elev., elevation, in meters above sea level].

\begin{tabular}{|c|c|c|c|c|c|c|c|c|c|}
\hline \multicolumn{2}{|c|}{1975} & \multicolumn{2}{|c|}{1975} & \multicolumn{2}{|c|}{1977} & \multicolumn{2}{|c|}{1977} & \multicolumn{2}{|c|}{1978} \\
\hline Sta. & Elev. & Sta. & Elev. & Sta. & Elev. & Sta. & Elev. & Sta. & Elev. \\
\hline-0.9 & 983.86 & 78.0 & 980.17 & -0.9 & 983.85 & 76.0 & 980.02 & -0.9 & 983.86 \\
\hline 0.0 & 983.87 & 80.0 & 980.17 & 0.0 & 983.85 & 78.0 & 980.00 & 0.0 & 983.86 \\
\hline 2.0 & 983.87 & 82.0 & 980.09 & 2.0 & 983.87 & 80.0 & 980.03 & 2.0 & 983.87 \\
\hline 4.0 & 983.86 & 83.0 & 980.12 & 3.7 & 983.87 & 82.0 & 979.96 & 4.0 & 983.82 \\
\hline 5.0 & 983.56 & 84.0 & 980.20 & 5.5 & 983.47 & 83.0 & 980.09 & 5.2 & 983.59 \\
\hline 6.0 & 983.43 & 85.0 & 980.38 & 7.0 & 983.46 & 84.2 & 980.30 & 7.0 & 983.61 \\
\hline 8.0 & 983.43 & 85.8 & 980.61 & 8.0 & 983.42 & 84.8 & 980.36 & 9.0 & 983.31 \\
\hline 10.0 & 982.90 & 87.0 & 981.42 & 10.0 & 982.90 & 86.0 & 980.70 & 12.0 & 982.58 \\
\hline 12.0 & 982.46 & 87.1 & 982.82 & 11.0 & 982.66 & 87.5 & 981.65 & 14.0 & 982.26 \\
\hline 14.0 & 982.24 & 89.0 & 982.90 & 13.0 & 982.38 & 87.7 & 982.78 & 14.6 & 981.96 \\
\hline 16.0 & 981.97 & 91.0 & 982.96 & 15.0 & 982.16 & 90.0 & 982.92 & 14.9 & 981.36 \\
\hline 18.0 & 981.42 & 93.0 & 983.10 & 16.0 & 982.09 & 92.0 & 982.98 & 15.0 & 981.06 \\
\hline 20.0 & 980.81 & 95.0 & 983.03 & 16.8 & 981.64 & 94.0 & 983.11 & 16.0 & 980.59 \\
\hline 21.0 & 980.57 & 97.0 & 983.19 & 17.9 & 981.58 & 96.0 & 983.10 & 17.0 & 980.18 \\
\hline 22.0 & 980.58 & 100.0 & 983.08 & 18.0 & 981.52 & 98.0 & 983.18 & 19.0 & 980.01 \\
\hline 24.0 & 980.68 & & & 19.7 & 981.26 & 100.0 & 983.07 & 21.0 & 980.42 \\
\hline 26.0 & 980.61 & & & 19.8 & 981.14 & 100.6 & 983.09 & 23.0 & 980.88 \\
\hline 28.0 & 980.62 & & & 20.1 & 981.09 & & & 24.6 & 981.06 \\
\hline 30.0 & 980.66 & & & 21.0 & 980.61 & & & 27.2 & 980.89 \\
\hline 32.0 & 980.71 & & & 22.0 & 980.65 & & & 29.0 & 980.76 \\
\hline 34.0 & 980.73 & & & 24.0 & 980.71 & & & 31.0 & 980.81 \\
\hline 36.0 & 980.72 & & & 26.0 & 980.62 & & & 33.0 & 980.67 \\
\hline 38.0 & 980.71 & & & 28.0 & 980.67 & & & 35.0 & 980.60 \\
\hline 40.0 & 980.79 & & & 30.0 & 980.71 & & & 38.0 & 980.60 \\
\hline 42.0 & 980.78 & & & 32.0 & 980.71 & & & 40.0 & 980.54 \\
\hline 44.0 & 980.72 & & & 34.0 & 980.71 & & & 42.0 & 980.42 \\
\hline 46.0 & 980.71 & & & 34.7 & 980.87 & & & 44.0 & 980.37 \\
\hline 48.0 & 980.64 & & & 35.7 & 980.74 & & & 46.0 & 980.34 \\
\hline 50.0 & 980.63 & & & 37.0 & 980.61 & & & 48.0 & 980.34 \\
\hline 52.0 & 980.66 & & & 40.0 & 980.67 & & & 50.0 & 980.34 \\
\hline 54.0 & 980.69 & & & 42.0 & 980.72 & & & 52.0 & 980.44 \\
\hline 56.0 & 980.69 & & & 44.0 & 980.70 & & & 54.0 & 980.41 \\
\hline 58.0 & 980.66 & & & 46.0 & 980.67 & & & 57.0 & 980.39 \\
\hline 60.0 & 980.67 & & & 48.0 & 980.67 & & & 60.0 & 980.39 \\
\hline 62.0 & 980.61 & & & 50.0 & 980.69 & & & 63.0 & 980.36 \\
\hline 64.0 & 980.58 & & & 52.0 & 980.69 & & & 66.0 & 980.32 \\
\hline 64.7 & 980.55 & & & 54.0 & 980.71 & & & 69.0 & 980.19 \\
\hline 64.9 & 980.42 & & & 58.0 & 980.64 & & & 72.0 & 980.24 \\
\hline 66.0 & 980.44 & & & 62.0 & 980.64 & & & 74.0 & 980.06 \\
\hline 67.3 & 980.38 & & & 64.0 & 980.50 & & & 76.0 & 979.87 \\
\hline 68.0 & 980.31 & & & 66.0 & 980.37 & & & 78.0 & 979.77 \\
\hline 70.0 & 980.23 & & & 69.0 & 980.29 & & & 80.0 & 979.77 \\
\hline 72.0 & 980.12 & & & 70.0 & 980.24 & & & 82.0 & 979.99 \\
\hline 74.0 & 980.11 & & & 72.0 & 980.13 & & & 84.0 & 980.18 \\
\hline 76.0 & 980.12 & & & 74.0 & 980.04 & & & 86.0 & 980.25 \\
\hline
\end{tabular}


Table 17.--Listing. of horizontal stations and elevations for cross section PR147 --Continued.

\begin{tabular}{|c|c|c|c|c|c|c|c|c|c|}
\hline \multicolumn{2}{|c|}{1978} & \multicolumn{2}{|c|}{1979} & \multicolumn{2}{|c|}{1979} & \multicolumn{2}{|c|}{1980} & \multicolumn{2}{|c|}{1980} \\
\hline Sta. & Elev. & Sta. & Elev. & Sta. & Elev. & Sta. & Elev. & Sta. & Elev. \\
\hline 88.0 & 980.35 & -5.0 & 984.03 & 76.0 & 980.02 & -3.0 & 983.93 & 100.7 & 983.18 \\
\hline 89.2 & 980.65 & -3.0 & 983.92 & 78.0 & 980.09 & -1.0 & 983.86 & 105.0 & 983.11 \\
\hline 91.3 & 981.70 & -0.9 & 983.86 & 80.0 & 980.10 & 0.0 & 983.85 & 110.0 & 983.40 \\
\hline 92.0 & 982.20 & 0.0 & 983.85 & 82.0 & 980.04 & 2.0 & 983.87 & 115.0 & 983.20 \\
\hline 92.5 & 982.26 & 2.0 & 983.88 & 84.0 & 980.18 & 4.0 & 983.84 & 120.0 & 982.97 \\
\hline 92.6 & 983.04 & 4.0 & 983.84 & 86.0 & 980.27 & 6.0 & 983.61 & 130.0 & 982.97 \\
\hline 93.0 & 983.13 & 5.2 & 983.59 & 88.0 & 980.43 & 9.0 & 983.33 & 140.0 & 982.85 \\
\hline 94.0 & 983.16 & 7.0 & 983.61 & 90.0 & 981.11 & 11.0 & 982.77 & 150.0 & 983.01 \\
\hline 95.0 & 983.08 & 9.0 & 983.32 & 91.5 & 981.75 & 13.0 & 982.55 & & \\
\hline 96.0 & 983.19 & 12.0 & 982.60 & 92.5 & 982.31 & 14.5 & 981.98 & & \\
\hline 97.0 & 983.23 & 14.0 & 982.26 & 92.6 & 983.05 & 15.2 & 981.12 & & \\
\hline 98.0 & 983.20 & 14.6 & 981.92 & 93.0 & 983.14 & 16.8 & 980.19 & & \\
\hline 99.0 & 983.17 & 14.9 & 981.25 & 94.0 & 983.18 & 18.0 & 980.02 & & \\
\hline \multirow[t]{32}{*}{100.7} & 983.15 & 15.0 & 981.06 & 95.0 & 983.09 & 20.0 & 980.82 & & \\
\hline & & 16.0 & 980.80 & 96.0 & 983.20 & 21.3 & 980.96 & & \\
\hline & & 17.0 & 980.30 & 97.0 & 983.24 & 24.5 & 981.07 & & \\
\hline & & 18.8 & 980.01 & 99.0 & 983.16 & 27.0 & 981.00 & & \\
\hline & & 21.0 & 980.54 & 100.0 & 983.08 & 29.0 & 980.85 & & \\
\hline & & 22.0 & 980.80 & 100.7 & 983.17 & 30.0 & 980.74 & & \\
\hline & & 23.0 & 980.98 & 105.0 & 983.09 & 33.0 & 980.73 & & \\
\hline & & 25.0 & 981.05 & 110.0 & 983.43 & 36.0 & 980.58 & & \\
\hline & & 27.0 & 980.95 & 115.0 & 983.20 & 40.0 & 980.59 & & \\
\hline & & 29.0 & 980.81 & 120.0 & 982.97 & 43.0 & 980.41 & & \\
\hline & & 31.0 & 980.78 & 125.0 & 982.88 & 46.0 & 980.34 & & \\
\hline & & 33.0 & 980.69 & 130.0 & 982.98 & 49.0 & 980.43 & & \\
\hline & & 35.0 & 980.61 & 135.0 & 982.88 & 52.0 & 980.42 & & \\
\hline & & 37.0 & 980.58 & 140.0 & 982.86 & 55.0 & 980.57 & & \\
\hline & & 39.0 & 980.54 & 145.0 & 982.85 & 56.6 & 980.53 & & \\
\hline & & 41.0 & 980.48 & 150.0 & 983.00 & 57.3 & 980.71 & & \\
\hline & & 43.0 & 980.43 & & & 60.0 & 980.41 & & \\
\hline & & 45.0 & 980.36 & & & 65.0 & 980.42 & & \\
\hline & & 47.0 & 980.37 & & & 70.0 & 980.27 & & \\
\hline & & 50.0 & 980.46 & & & 74.0 & 979.94 & & \\
\hline & & 52.0 & 980.41 & & & 78.0 & 979.96 & & \\
\hline & & 54.0 & 980.44 & & & 82.0 & 979.98 & & \\
\hline & & 56.0 & 980.47 & & & 85.0 & 980.27 & & \\
\hline & & 58.0 & 980.51 & & & 87.0 & 980.31 & & \\
\hline & & 60.0 & 980.48 & & & 89.0 & 980.67 & & \\
\hline & & 62.0 & 980.44 & & & 91.5 & 981.75 & & \\
\hline & & 54.0 & 980.39 & & & 92.5 & 982.33 & & \\
\hline & & 06.0 & 980.34 & & & 92.7 & 983.04 & & \\
\hline & & 68.0 & 980.31 & & & 94.0 & 983.18 & & \\
\hline & & 70.0 & 980.29 & & & 96.0 & 983.20 & & \\
\hline & & 72.0 & 980.30 & & & 98.0 & 983.21 & & \\
\hline & & 74.0 & 980.00 & & & 100.0 & 983.09 & & \\
\hline
\end{tabular}


Table 17.--Listing of horizontal stations and elevations for cross section PR147 --Continued.

\begin{tabular}{|c|c|c|c|c|c|c|c|c|c|}
\hline \multicolumn{2}{|c|}{1984} & \multicolumn{2}{|c|}{1984} & \multicolumn{2}{|c|}{1985} & \multicolumn{2}{|c|}{1985} & \multicolumn{2}{|c|}{1986} \\
\hline Sta. & Elev. & Sta. & Elev. & Sta. & Elev. & Sta. & Elev. & Sta. & Elev. \\
\hline-0.9 & 983.86 & 91.0 & 981.34 & 0.0 & 983.87 & 88.5 & 980.43 & -0.9 & 983.87 \\
\hline 0.0 & 983.86 & 91.2 & 981.79 & 3.0 & 983.89 & 91.0 & 981.26 & 0.0 & 983.86 \\
\hline 3.6 & 983.89 & 91.6 & 981.87 & 4.0 & 983.83 & 92.0 & 982.12 & 3.0 & 983.88 \\
\hline 5.0 & 983.62 & 92.2 & 982.39 & 6.0 & 983.60 & 92.6 & 982.49 & 4.0 & 983.83 \\
\hline 6.0 & 983.61 & 92.6 & 983.07 & 8.0 & 983.50 & 92.7 & 983.09 & 5.0 & 983.63 \\
\hline 8.0 & 983.49 & 93.0 & 983.14 & 10.0 & 983.04 & 94.0 & 983.17 & 7.0 & 983.61 \\
\hline 9.0 & 983.32 & 95.0 & 983.16 & 12.0 & 982.62 & 95.0 & 983.09 & 9.0 & 983.34 \\
\hline 11.0 & 982.75 & 96.0 & 983.10 & 13.0 & 982.54 & 96.0 & 983.20 & 10.0 & 983.03 \\
\hline 12.0 & 982.59 & 97.0 & 983.19 & 14.0 & 982.28 & 98.0 & 983.22 & 11.4 & 982.67 \\
\hline 13.0 & 982.54 & 99.0 & 983.15 & 15.3 & 980.92 & 100.0 & 983.10 & 13.0 & 982.57 \\
\hline 14.0 & 982.18 & 100.0 & 983.10 & 17.0 & 980.24 & 100.7 & 983.17 & 14.0 & 982.24 \\
\hline 14.5 & 981.88 & 100.7 & 983.17 & 19.0 & 980.08 & & & 14.7 & 981.75 \\
\hline 15.0 & 981.39 & 103.0 & 983.18 & 20.0 & 980.59 & & & 15.7 & 980.75 \\
\hline 15.5 & 980.94 & 105.0 & 983.08 & 20.3 & 980.76 & & & 17.5 & 980.06 \\
\hline 16.6 & 980.28 & & & 21.0 & 980.87 & & & 18.3 & 980.06 \\
\hline 17.5 & 980.07 & & & 21.4 & 980.99 & & & 20.0 & 980.64 \\
\hline 18.7 & 980.04 & & & 24.0 & 980.97 & & & 21.1 & 980.63 \\
\hline 20.5 & 980.82 & & & 27.0 & 981.07 & & & 21.5 & 980.98 \\
\hline 21.6 & 980.91 & & & 28.0 & 980.96 & & & 23.0 & 981.02 \\
\hline 24.0 & 980.98 & & & 29.2 & 980.68 & & & 25.0 & 981.10 \\
\hline 26.0 & 981.01 & & & 31.0 & 980.66 & & & 26.0 & 981.10 \\
\hline 28.0 & 980.95 & & & 34.0 & 980.60 & & & 27.9 & 981.04 \\
\hline 30.0 & 980.57 & & & 37.0 & 980.57 & & & 29.1 & 980.74 \\
\hline 32.0 & 980.61 & & & 39.0 & 980.49 & & & 30.0 & 980.59 \\
\hline 35.0 & 980.62 & & & 42.0 & 980.36 & & & 32.0 & 980.60 \\
\hline 38.0 & 980.59 & & & 43.0 & 980.53 & & & 34.0 & 980.61 \\
\hline 40.0 & 980.47 & & & 45.0 & 980.46 & & & 36.0 & 980.62 \\
\hline 45.0 & 980.40 & & & 47.0 & 980.41 & & & 38.0 & 980.54 \\
\hline 46.5 & 980.34 & & & 50.0 & 980.44 & & & 40.0 & 980.45 \\
\hline 48.0 & 980.48 & & & 53.0 & 980.46 & & & 42.0 & 980.38 \\
\hline 50.0 & 980.48 & & & 56.0 & 980.44 & & & 44.0 & 980.61 \\
\hline 53.0 & 980.46 & & & 59.0 & 980.43 & & & 45.0 & 980.54 \\
\hline 57.0 & 980.45 & & & 61.0 & 980.44 & & & 47.0 & 980.51 \\
\hline 61.0 & 980.52 & & & 63.0 & 980.50 & & & 48.0 & 980.47 \\
\hline 64.0 & 980.41 & & & 65.0 & 980.71 & & & 50.0 & 980.46 \\
\hline 66.0 & 980.36 & & & 68.0 & 980.80 & & & 52.0 & 980.45 \\
\hline 70.0 & 980.39 & & & 71.0 & 980.57 & & & 54.0 & 980.42 \\
\hline 71.0 & 980.46 & & & 74.0 & 980.35 & & & 56.0 & 980.46 \\
\hline 72.0 & 980.43 & & & 74.2 & 980.40 & & & 59.0 & 980.45 \\
\hline 73.0 & 980.22 & & & 77.0 & 980.03 & & & 61.0 & 980.52 \\
\hline 76.0 & 980.29 & & & 80.0 & 980.02 & & & 62.0 & 980.50 \\
\hline 80.0 & 980.29 & & & 82.0 & 980.01 & & & 65.0 & 980.48 \\
\hline 83.0 & 980.23 & & & 85.0 & 980.16 & & & 68.0 & 980.46 \\
\hline 86.0 & 980.20 & & & 86.0 & 980.46 & & & 70.5 & 980.40 \\
\hline 90.0 & 980.89 & & & 87.0 & 980.27 & & & 73.0 & 980.16 \\
\hline
\end{tabular}


Table 17.--Listing of horizontal stations and elevations for cross section PR147 --Continued.

\begin{tabular}{|c|c|c|c|c|c|c|c|c|c|}
\hline \multicolumn{2}{|c|}{1986} & \multicolumn{2}{|c|}{1987} & \multicolumn{2}{|c|}{1987} & \multicolumn{2}{|c|}{1988} & \multicolumn{2}{|c|}{1988} \\
\hline Sta. & Elev. & Sta. & Elev. & Sta. & Elev. & Sta. & Elev. & Sta. & Elev. \\
\hline 74.0 & 980.14 & -68.9 & 983.82 & 79.0 & 980.34 & -0.9 & 983.87 & 58.7 & 980.61 \\
\hline 77.0 & 980.35 & 0.0 & 983.85 & 81.0 & 980.24 & 0.0 & 983.85 & 60.0 & 980.63 \\
\hline 79.0 & 980.40 & 2.0 & 983.87 & 84.0 & 980.14 & 2.0 & 983.87 & 62.0 & 980.68 \\
\hline 82.0 & 980.21 & 2.9 & 983.87 & 87.0 & 980.25 & 3.8 & 983.86 & 64.0 & 980.65 \\
\hline 84.4 & 980.17 & 5.0 & 983.61 & 89.5 & 980.72 & 5.0 & 983.62 & 66.0 & 980.57 \\
\hline 85.4 & 980.34 & 7.0 & 983.61 & 90.2 & 980.95 & 5.7 & 983.57 & 67.9 & 980.61 \\
\hline 86.3 & 980.17 & 9.0 & 983.37 & 91.2 & 981.51 & 7.0 & 983.61 & 68.0 & 980.87 \\
\hline 88.3 & 980.42 & 11.0 & 982.79 & 91.4 & 981.83 & 8.8 & 983.43 & 68.6 & 980.85 \\
\hline 89.8 & 980.77 & 13.3 & 982.54 & 92.5 & 982.56 & 10.0 & 983.11 & 68.7 & 980.81 \\
\hline 91.3 & 981.60 & 14.0 & 982.29 & 92.6 & 983.07 & 11.4 & 982.67 & 70.2 & 980.58 \\
\hline 91.4 & 981.81 & 14.5 & 981.88 & 93.0 & 983.15 & 12.0 & 982.60 & 71.3 & 980.40 \\
\hline 92.5 & 982.49 & 14.9 & 981.57 & 94.0 & 983.17 & 13.0 & 982.55 & 73.2 & 980.34 \\
\hline 92.6 & 983.07 & 15.5 & 980.85 & 95.0 & 983.11 & 14.0 & 982.31 & 74.0 & 980.23 \\
\hline 94.0 & 983.17 & 17.0 & 980.15 & 96.0 & 983.20 & 15.0 & 981.57 & 75.0 & 980.33 \\
\hline 95.0 & 983.09 & 18.6 & 980.04 & 98.0 & 983.22 & 15.5 & 980.84 & 77.0 & 980.36 \\
\hline 96.0 & 983.20 & 20.3 & 980.85 & 100.0 & 983.10 & 15.8 & 980.72 & 78.5 & 980.34 \\
\hline 97.0 & 983.24 & 21.6 & 980.84 & 100.7 & 983.17 & 16.3 & 980.38 & 80.0 & 980.30 \\
\hline 99.0 & 983.17 & 22.1 & 981.07 & & & 18.0 & 980.07 & 82.0 & 980.27 \\
\hline 100.0 & 983.09 & 24.0 & 981.14 & & & 18.6 & $980.07^{\circ}$ & 84.0 & 980.25 \\
\hline \multirow[t]{26}{*}{100.7} & 983.16 & 26.0 & 981.12 & & & 19.6 & 980.49 & 85.0 & 980.20 \\
\hline & & 27.7 & 981.10 & & & 20.0 & 980.71 & 86.0 & 980.19 \\
\hline & & 28.8 & 980.86 & & & 20.2 & 980.79 & 87.7 & 980.34 \\
\hline & & 30.0 & 980.65 & & & 21.0 & 980.88 & 88.2 & 980.40 \\
\hline & & 33.0 & 980.63 & & & 21.6 & 980.92 & 90.2 & 980.89 \\
\hline & & 36.0 & 980.54 & & & 22.0 & 981.09 & 91.5 & 981.64 \\
\hline & & 39.0 & 980.57 & & & 24.0 & 981.18 & 91.6 & 981.82 \\
\hline & & 42.0 & 980.46 & & & 26.0 & 981.17 & 92.6 & 982.61 \\
\hline & & 45.0 & 980.62 & & & 27.0 & 981.12 & 92.6 & 983.06 \\
\hline & & 48.0 & 980.44 & & & 28.0 & 981.07 & 94.0 & 983.17 \\
\hline & & 51.0 & 980.43 & & & 28.8 & 980.76 & 95.0 & 983.10 \\
\hline & & 54.0 & 980.49 & & & 29.3 & 980.70 & 96.0 & 983.20 \\
\hline & & 57.0 & 980.57 & & & 30.0 & 980.62 & 98.0 & 983.21 \\
\hline & & 60.0 & 980.66 & & & 32.0 & 980.62 & 100.0 & 983.10 \\
\hline & & 62.0 & 980.70 & & & 34.0 & 980.60 & 100.7 & 983.17 \\
\hline & & 64.0 & 980.61 & & & 36.0 & 980.55 & & \\
\hline & & 66.0 & 980.65 & & & 38.0 & 980.56 & & \\
\hline & & 67.0 & 980.70 & & & 40.0 & 980.48 & & \\
\hline & & 67.1 & 980.81 & & & 42.0 & 980.46 & & \\
\hline & & 68.0 & 980.68 & & & 44.0 & 980.58 & & \\
\hline & & 69.7 & 980.76 & & & 46.0 & 980.55 & & \\
\hline & & 70.0 & 980.71 & & & 48.0 & 980.42 & & \\
\hline & & 72.0 & 980.52 & & & 50.0 & 980.37 & & \\
\hline & & 73.0 & 980.35 & & & 52.0 & 980.45 & & \\
\hline & & 75.0 & 980.26 & & & 54.0 & 980.51 & & \\
\hline & & 77.0 & 980.28 & & & 56.0 & 980.55 & & \\
\hline
\end{tabular}




\section{Description of Cross Section PR151}

Location: Township 7 South/Range 49 East--section 15

U.S. Geological Survey quadrangle $(1: 24,000)$ : Huckins School

Landowners--left bank: Gay Ranch

right bank: Huckins Ranch

Access: right bank

Permission from: right bank--Huckins Ranch (T7S/R49E--28)

Reference pins

\begin{tabular}{|c|c|c|}
\hline $\begin{array}{l}\text { Station } \\
\text { (meters) }\end{array}$ & $\begin{array}{l}\text { Elevation } \\
\text { (meters } \\
\text { above } \\
\text { sea level) }\end{array}$ & Comments \\
\hline & & $\begin{array}{l}\text { Pins } 100.0,100.8,101.1 \text {, and } 101.2 \\
\text { were closest to the leveling } \\
\text { instruments. }\end{array}$ \\
\hline-0.9 & 977.634 & 0.15 meter above 1987 ground level. \\
\hline 0.0 & 977.544 & 0.10 meter above 1987 ground level. \\
\hline 100.0 & 977.115 & 0.07 meter above 1987 ground level. \\
\hline 101.1 & 976.980 & $\begin{array}{l}\text { Old pin, } 0.02 \text { meter above } 1987 \text { ground } \\
\text { level. }\end{array}$ \\
\hline 101.2 & 977.056 & $\begin{array}{l}\text { New pin, } 0.10 \text { meter above } 1987 \text { ground } \\
\text { level. }\end{array}$ \\
\hline
\end{tabular}




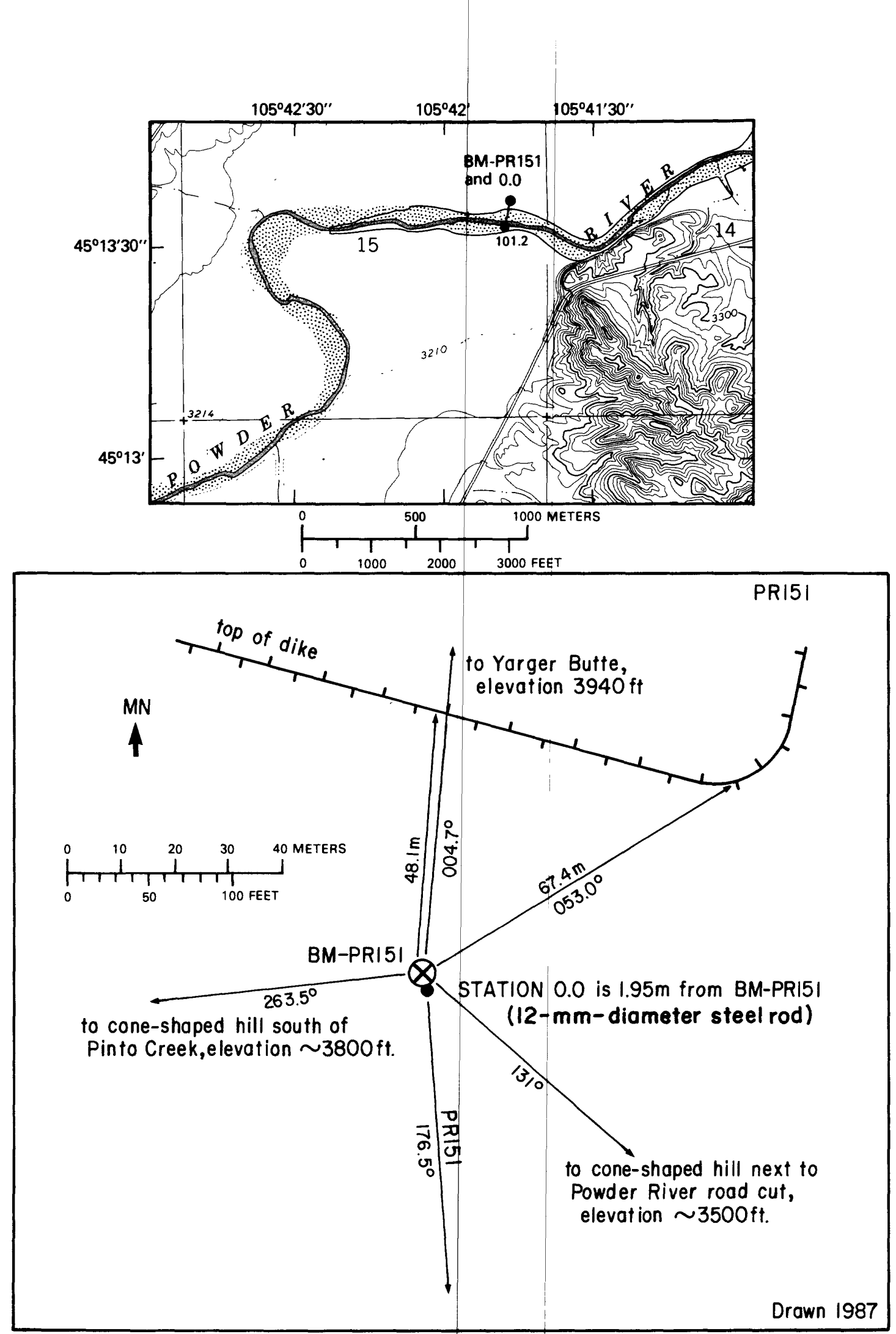

Figure 49.--Upper: Location of cross section PR151, bench mark BM-PR151, and left and right bank reference pins (tables 2 and 3 ) in the Huckins School quadrangle. Lower: Location of bench mark on the left bank. MN is magnetic north. 

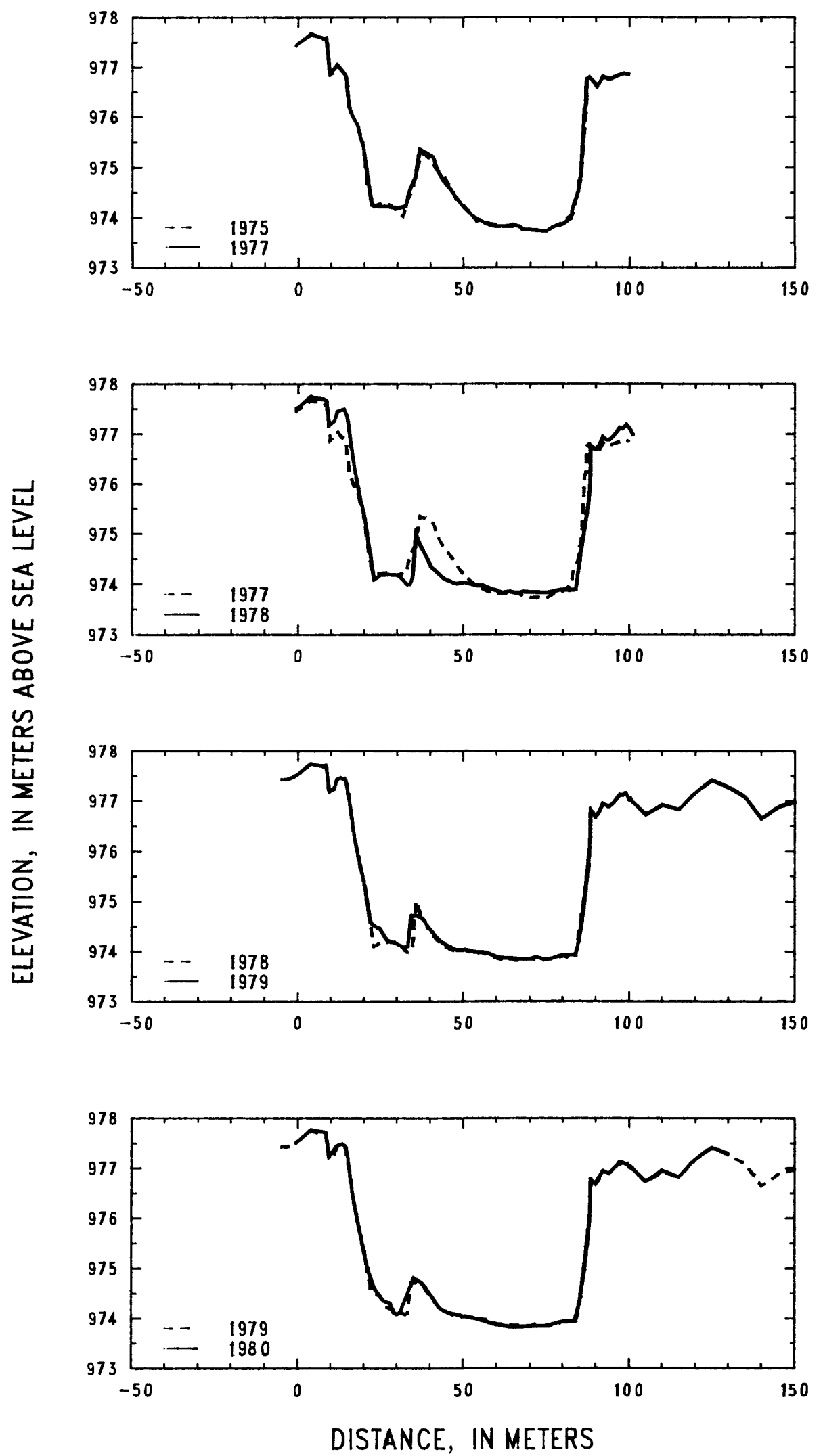

Figure 50.--Profiles of cross section PR151 from 1975 to 1980. 


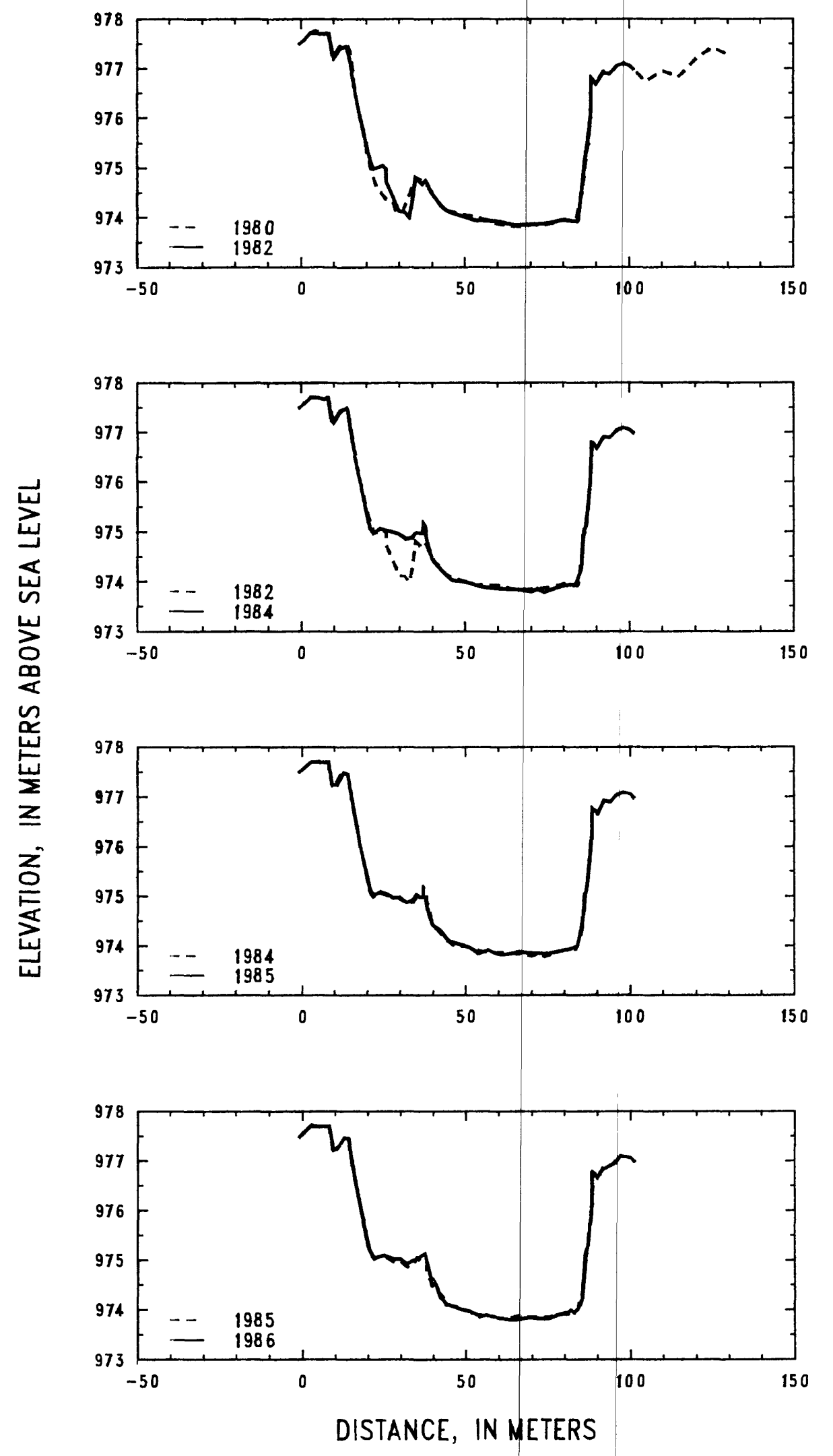

Figure 51.--Profiles of cross section PR151from 1980 to 1986. 


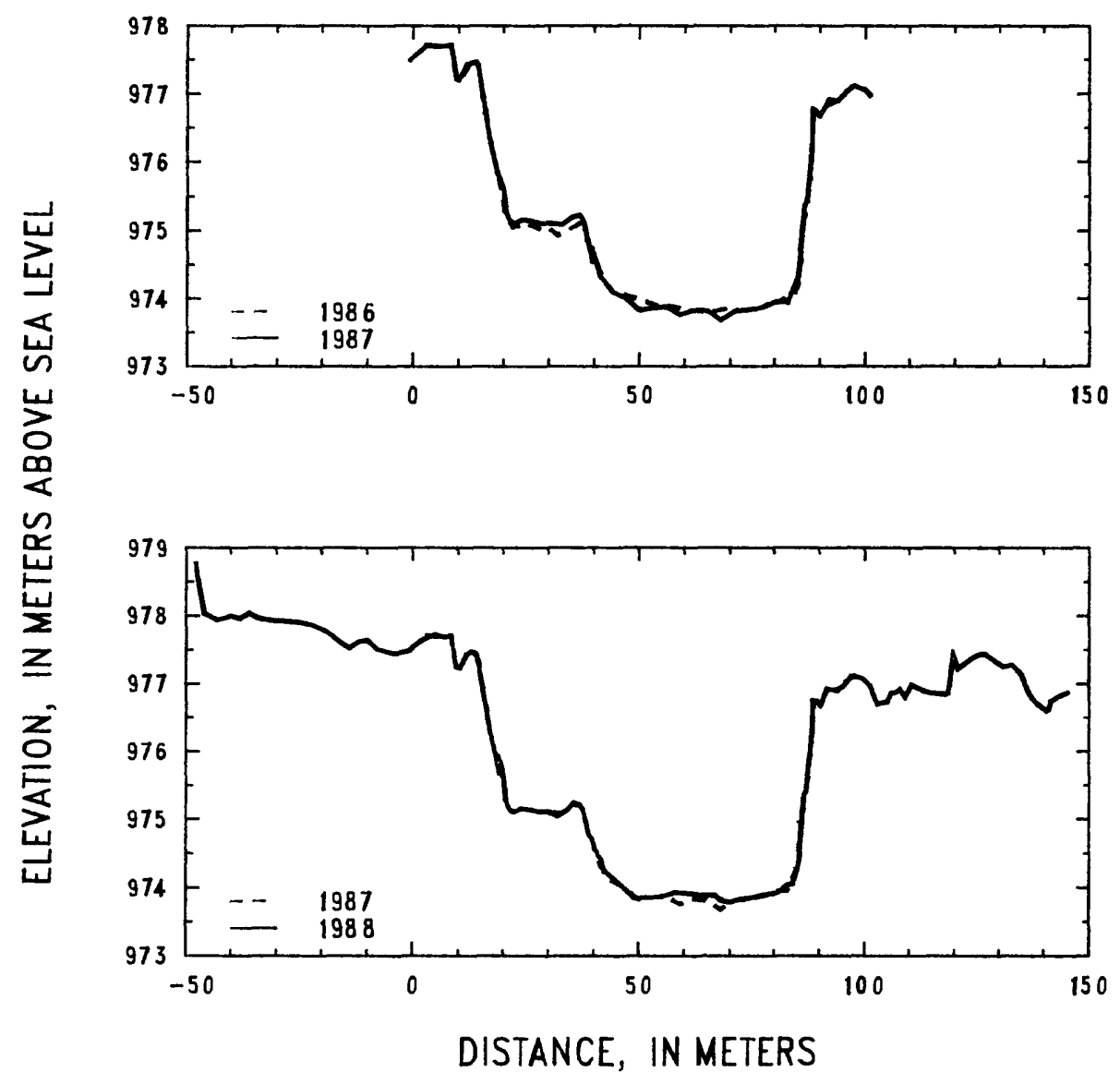

Figure 52.--Profiles of cross section PR151 from 1986 to 1988. 
Table 18.--Listing of horizontal stations and elevations for cross section PR151 [Sta., station, distance in meters from a reference point on the left bank; Elev., elevation, in meters above sea level].

\begin{tabular}{|c|c|c|c|c|c|c|c|c|c|}
\hline \multicolumn{2}{|c|}{1975} & \multicolumn{2}{|c|}{1975} & \multicolumn{2}{|c|}{1977} & \multicolumn{2}{|c|}{1977} & \multicolumn{2}{|c|}{1978} \\
\hline Sta. & Elev. & Sta. & Elev. & Sta. & Elev. & Sta. & Elev. & Sta. & Elev. \\
\hline-0.8 & 977.43 & 66.0 & 973.81 & -0.8 & 977.44 & 85.6 & 974.81 & -0.9 & 977.51 \\
\hline 0.0 & 977.48 & 68.0 & 973.74 & 0.0 & 977.48 & 86.6 & 976.16 & 0.0 & 977.54 \\
\hline 2.0 & 977.56 & 70.0 & 973.75 & 4.0 & 977.67 & 87.0 & 976.20 & 2.0 & 977.64 \\
\hline 4.0 & 977.69 & 72.0 & 973.74 & 8.0 & 977.58 & 87.2 & 976.75 & 4.0 & 977.76 \\
\hline 6.0 & 977.62 & 74.0 & 973.71 & 8.7 & 977.60 & 88.0 & 976.80 & 6.0 & 977.71 \\
\hline 8.0 & 977.57 & 76.0 & 973.75 & 9.8 & 976.87 & 90.0 & 976.65 & 8.0 & 977.69 \\
\hline 8.8 & 977.56 & 78.0 & 973.84 & 12.0 & 977.05 & 91.0 & 976.67 & 8.8 & 977.66 \\
\hline 9.9 & 976.85 & 80.0 & 973.88 & 14.5 & 976.83 & 92.0 & 976.81 & 9.4 & 977.23 \\
\hline 11.0 & 976.91 & 81.0 & 973.95 & 15.7 & 976.17 & 94.0 & 976.75 & 10.0 & 977.20 \\
\hline 12.0 & 977.04 & 83.0 & 974.07 & 17.0 & 975.96 & 96.0 & 976.82 & 11.0 & 977.25 \\
\hline 14.0 & 976.90 & 84.0 & 974.36 & 18.0 & 975.85 & 98.0 & 976.87 & 12.0 & 977.45 \\
\hline 14.7 & 976.78 & 85.0 & 974.54 & 19.0 & 975.58 & 100.0 & 976.85 & 13.0 & 977.48 \\
\hline 15.8 & 976.16 & 85.7 & 974.87 & 19.7 & 975.44 & & & 14.0 & 977.50 \\
\hline 17.0 & 975.96 & 87.1 & 976.23 & 22.4 & 974.25 & & & 15.0 & 977.32 \\
\hline 18.0 & 975.87 & 87.2 & 976.70 & 24.0 & 974.22 & & & 17.0 & 976.38 \\
\hline 19.0 & 975.59 & 87.3 & 976.76 & 27.0 & 974.23 & & & 19.0 & 975.66 \\
\hline 19.4 & 975.59 & 88.0 & 976.80 & 30.0 & 974.18 & & & 20.0 & 975.41 \\
\hline 20.0 & 975.33 & 90.3 & 976.60 & 32.8 & 974.24 & & & 22.0 & 974.56 \\
\hline 21.1 & 974.55 & 92.0 & 976.82 & 34.0 & 974.56 & & & 23.0 & 974.10 \\
\hline 21.5 & 974.53 & 94.0 & 976.76 & 35.6 & 974.78 & & & 25.0 & 974.19 \\
\hline 22.5 & 974.20 & 96.0 & 976.82 & 36.9 & 975.36 & & & 27.0 & 974.20 \\
\hline 24.0 & 974.26 & 98.0 & 976.87 & 39.0 & 975.28 & & & 30.0 & 974.19 \\
\hline 26.0 & 974.29 & 100.0 & 976.86 & 41.0 & 975.20 & & & 31.2 & 974.14 \\
\hline 28.0 & 974.25 & 100.8 & 976.84 & 42.0 & 974.98 & & & 33.0 & 974.00 \\
\hline 30.0 & 974.14 & & & 44.0 & 974.74 & & & 34.0 & 974.00 \\
\hline 31.9 & 974.02 & & & 47.0 & 974.49 & & & 34.9 & 974.13 \\
\hline 33.0 & 974.27 & & & 50.0 & 974.20 & & & 35.8 & 975.02 \\
\hline 35.0 & 974.63 & & & 53.0 & 974.01 & & & 37.0 & 974.78 \\
\hline 37.2 & 975.25 & & & 56.0 & $973.90^{\circ}$ & & & 39.0 & 974.54 \\
\hline 38.0 & 975.28 & & & 57.0 & 973.87 & & & 40.3 & 974.35 \\
\hline 40.0 & 975.17 & & & 59.0 & 973.82 & & & 42.0 & 974.24 \\
\hline 42.0 & 974.94 & & & 61.0 & 973.82 & & & 44.0 & 974.12 \\
\hline 44.0 & 974.85 & & & 63.0 & 973.82 & & & 46.0 & 974.06 \\
\hline 46.0 & 974.61 & & & 65.0 & 973.85 & & & 48.0 & 974.01 \\
\hline 48.0 & 974.35 & & & 67.0 & 973.81 & & & 50.0 & 974.04 \\
\hline 50.0 & 974.23 & & & 68.0 & 973.75 & & & 52.0 & 974.00 \\
\hline 52.0 & 974.09 & & & 71.0 & 973.73 & & & 54.0 & 973.97 \\
\hline 53.7 & 973.95 & & & 73.0 & 973.73 & & & 56.0 & 973.97 \\
\hline 54.0 & 973.92 & & & 75.0 & 973.71 & & & 58.0 & 973.92 \\
\hline 55.0 & 973.95 & & & 77.0 & 973.81 & & & 50.0 & 973.87 \\
\hline 56.0 & 973.93 & & & 79.9 & 973.86 & & & 62.0 & 973.83 \\
\hline 58.0 & 973.87 & & & 82.5 & 973.98 & & & 64.0 & 973.86 \\
\hline 60.0 & 973.84 & & & 83.2 & 974.26 & & & 66.0 & 973.82 \\
\hline 62.0 & 973.81 & & & 84.8 & 974.51 & & & 68.0 & 973.86 \\
\hline 64.0 & 973.81 & & & 85.1 & 974.75 & & & 70.0 & 973.85 \\
\hline
\end{tabular}


Table 18.--Listing of horizontal stations and elevations for cross section PR151 --Continued.

\begin{tabular}{|c|c|c|c|c|c|c|c|c|c|}
\hline \multicolumn{2}{|c|}{1978} & \multicolumn{2}{|c|}{1979} & \multicolumn{2}{|c|}{1979} & \multicolumn{2}{|c|}{1980} & \multicolumn{2}{|c|}{1980} \\
\hline Sta. & Elev. & Sta. & Elev. & Sta. & Elev. & Sta. & Elev. & Sta. & Elev. \\
\hline 72.0 & 973.85 & -5.0 & 977.43 & 74.0 & 973.84 & -0.8 & 977.50 & 110.0 & 976.95 \\
\hline 74.0 & 973.83 & -3.0 & 977.43 & 76.0 & 973.84 & 0.0 & 977.53 & 115.0 & 976.82 \\
\hline 76.0 & 973.85 & -0.9 & 977.50 & 78.0 & 973.89 & 2.0 & 977.64 & 120.0 & 977.19 \\
\hline 78.0 & 973.88 & 0.0 & 977.54 & 80.0 & 973.93 & 4.0 & 977.77 & 125.0 & 977.41 \\
\hline 80.0 & 973.91 & 2.0 & 977.64 & 82.0 & 973.92 & 8.7 & 977.71 & 130.0 & 977.29 \\
\hline 82.0 & 973.88 & 4.0 & 977.75 & 84.0 & 973.94 & 9.6 & 977.22 & & \\
\hline 84.0 & 973.90 & 6.0 & 977.71 & 84.8 & 974.15 & 12.0 & 977.45 & & \\
\hline 84.9 & 974.35 & 8.0 & 977.69 & 85.0 & 974.20 & 13.7 & 977.49 & & \\
\hline 88.0 & 975.72 & 8.6 & 977.71 & 86.5 & 974.92 & 14.7 & 977.42 & & \\
\hline 88.3 & 976.11 & 9.6 & 977.19 & 88.3 & 976.16 & 15.0 & 977.32 & & \\
\hline 88.4 & 976.74 & 11.0 & 977.24 & 88.5 & 976.82 & 17.0 & 976.36 & & \\
\hline 90.0 & 976.69 & 12.0 & 977.44 & 90.0 & 976.67 & 19.0 & 975.71 & & \\
\hline 91.0 & 976.78 & 13.0 & 977.47 & 91.0 & 976.79 & 21.0 & 975.06 & & \\
\hline 92.0 & 976.95 & 14.5 & 977.41 & 92.0 & 976.95 & 23.0 & 974.62 & & \\
\hline 93.0 & 976.89 & 16.0 & 976.84 & 93.0 & 976.91 & 26.0 & 974.35 & & \\
\hline 94.0 & 976.88 & 17.0 & 976.33 & 94.0 & 976.89 & 28.0 & 974.30 & & \\
\hline 95.0 & 976.95 & 19.0 & 975.68 & 95.0 & 976.95 & 29.0 & 974.12 & & \\
\hline 96.0 & 977.03 & 20.0 & 975.43 & 96.0 & 977.03 & 30.0 & 974.08 & & \\
\hline 97.0 & 977.13 & 22.0 & 974.58 & 97.0 & 977.13 & 31.0 & 974.11 & & \\
\hline 98.0 & 977.11 & 24.0 & 974.47 & 98.0 & 977.12 & 33.0 & 974.45 & & \\
\hline 99.0 & 977.19 & 25.0 & 974.45 & 99.0 & 977.16 & 35.2 & 974.81 & & \\
\hline 100.0 & 977.12 & 27.0 & 974.22 & 100.0 & 977.01 & 38.0 & 974.68 & & \\
\hline \multirow[t]{23}{*}{101.1} & 976.97 & 30.0 & 974.15 & 101.1 & 976.97 & 40.0 & 974.49 & & \\
\hline & & 32.5 & 974.08 & 101.2 & 976.96 & 42.5 & 974.22 & & \\
\hline & & 33.4 & 974.11 & 105.0 & 976.72 & 45.0 & 974.12 & & \\
\hline & & 34.5 & 974.72 & 110.0 & 976.92 & 50.0 & 974.04 & & \\
\hline & & 36.0 & 974.72 & 115.0 & 976.82 & 55.0 & 973.97 & & \\
\hline & & 38.0 & 974.65 & 120.0 & 977.17 & 60.0 & 973.86 & & \\
\hline & & 40.0 & 974.45 & 125.0 & 977.41 & 65.0 & 973.81 & & \\
\hline & & 42.0 & 974.27 & 130.0 & 977.27 & 70.0 & 973.84 & & \\
\hline & & 44.3 & 974.15 & 135.0 & 977.08 & 75.0 & 973.85 & & \\
\hline & & 46.0 & 974.10 & 140.0 & 976.64 & 80.0 & 973.94 & & \\
\hline & & 48.0 & 974.03 & 145.0 & 976.88 & 83.6 & 973.94 & & \\
\hline & & 50.0 & 974.04 & 150.0 & 976.98 & 85.0 & 974.26 & & \\
\hline & & 52.0 & 974.01 & & & 86.5 & 974.94 & & \\
\hline & & 54.0 & 973.98 & & & 88.2 & 976.00 & & \\
\hline & & 56.0 & 973.98 & & & 88.5 & 976.77 & & \\
\hline & & 58.0 & 973.95 & & & 90.0 & 976.69 & & \\
\hline & & 60.0 & 973.88 & & & 92.0 & 976.94 & & \\
\hline & & 62.0 & 973.87 & & & 94.0 & 976.88 & & \\
\hline & & 64.0 & 973.87 & & & 96.0 & 977.04 & & \\
\hline & & 66.0 & 973.85 & & & 98.0 & 977.11 & & \\
\hline & & 68.0 & 973.84 & & & 100.0 & 977.05 & & \\
\hline & & 70.0 & 973.84 & & & 101.2 & 976.97 & & \\
\hline & & 72.0 & 973.89 & & & 105.0 & 976.73 & & \\
\hline
\end{tabular}


Table 18.--Listing of horizontal stations and elevations for cross section PR151 --Continued.

\begin{tabular}{|c|c|c|c|c|c|c|c|c|c|}
\hline \multicolumn{2}{|c|}{1982} & \multicolumn{2}{|c|}{1982} & \multicolumn{2}{|c|}{1984} & \multicolumn{2}{|c|}{1984} & \multicolumn{2}{|c|}{1985} \\
\hline Sta. & Elev. & Sta. & Elev. & Sta. & Elev. & Sta. & Elev. & Sta. & Elev. \\
\hline-0.8 & 977.50 & 96.0 & 977.05 & -0.9 & 977.50 & 86.8 & 975.13 & -0.9 & 977.50 \\
\hline 0.0 & 977.53 & 98.0 & 977.10 & 0.0 & 977.53 & 88.0 & 975.93 & 0.0 & 977.53 \\
\hline 3.0 & 977.73 & 100.0 & 977.05 & 3.0 & 977.70 & 88.4 & 976.31 & 3.0 & 977.71 \\
\hline 6.0 & 977.70 & 101.2 & 976.97 & 5.0 & 977.71 & 88.5 & 976.79 & 6.0 & 977.70 \\
\hline 8.5 & 977.71 & & & 7.0 & 977.67 & 89.0 & 976.77 & 8.5 & 977.70 \\
\hline 9.0 & 977.53 & & & 8.4 & 977.69 & 90.0 & 976.66 & 9.5 & 977.24 \\
\hline 10.0 & 977.20 & & & 9.4 & 977.23 & 92.0 & 976.91 & 11.0 & 977.25 \\
\hline 12.0 & 977.42 & & & 10.0 & 977.18 & 94.0 & 976.89 & 13.0 & 977.49 \\
\hline 14.0 & 977.45 & & & 12.0 & 977.43 & 96.0 & 977.03 & 14.2 & 977.46 \\
\hline 15.0 & 977.14 & & & 14.0 & 977.49 & 98.0 & 977.09 & 16.0 & 976.73 \\
\hline 17.0 & 976.36 & & & 14.4 & 977.42 & 100.0 & 977.04 & 18.0 & 976.00 \\
\hline 20.0 & 975.43 & & & 15.4 & 976.93 & 101.2 & 976.97 & 20.0 & 975.43 \\
\hline 22.0 & 974.97 & & & 17.0 & 976.34 & & & 21.0 & 975.12 \\
\hline 25.0 & 975.05 & & & 18.0 & 976.00 & & & 22.0 & 975.03 \\
\hline 26.0 & 974.98 & & & 20.0 & 975.35 & & & 24.0 & 975.10 \\
\hline 26.0 & 974.73 & & & 21.0 & 975.06 & & & 26.0 & 975.05 \\
\hline 30.0 & 974.12 & & & 22.0 & 974.98 & & & 28.0 & 974.97 \\
\hline 31.5 & 974.11 & & & 24.0 & 975.07 & & & 30.0 & 974.99 \\
\hline 33.0 & 973.99 & & & 25.0 & 975.04 & & & 32.0 & 974.87 \\
\hline 34.0 & 974.29 & & & 27.0 & 975.01 & & & 34.0 & 974.93 \\
\hline 35.0 & 974.81 & & & 30.0 & 974.95 & & & 35.0 & 975.05 \\
\hline 37.0 & 974.67 & & & 32.0 & 974.85 & & & 36.0 & 974.99 \\
\hline 38.0 & 974.75 & & & 34.0 & 974.88 & & & 37.8 & 975.00 \\
\hline 38.4 & 974.70 & & & 35.0 & 974.98 & & & 38.1 & 974.78 \\
\hline 40.0 & 974.47 & & & 37.0 & 974.97 & & & 40.0 & 974.42 \\
\hline 43.0 & 974.20 & & & 37.2 & 975.17 & & & 42.0 & 974.33 \\
\hline 45.0 & 974.11 & & & 37.9 & 975.09 & & & 43.3 & 974.22 \\
\hline 48.0 & 974.04 & & & 38.2 & 974.89 & & & 45.0 & 974.10 \\
\hline 50.0 & 974.00 & & & 40.0 & 974.43 & & & 48.0 & 974.04 \\
\hline 53.0 & 973.93 & & & 43.0 & 974.19 & & & 51.0 & 973.98 \\
\hline 55.0 & 973.93 & & & 46.0 & 974.02 & & & 54.0 & 973.85 \\
\hline 60.0 & 973.92 & & & 50.0 & 973.98 & & & 57.0 & 973.92 \\
\hline 65.0 & 973.84 & & & 55.0 & 973.88 & & & 60.0 & 973.83 \\
\hline 70.0 & 973.85 & & & 60.0 & 973.86 & & & 63.0 & 973.84 \\
\hline 75.0 & 973.88 & & & 64.0 & 973.84 & & & 66.0 & 973.89 \\
\hline 80.0 & 973.95 & & & 66.0 & 973.84 & & & 69.0 & 973.86 \\
\hline 84.0 & 973.91 & & & 70.0 & 973.79 & & & 72.0 & 973.85 \\
\hline 84.4 & 973.94 & & & 72.0 & 973.85 & & & 75.0 & 973.85 \\
\hline 85.5 & 974.54 & & & 74.0 & 973.77 & & & 78.0 & 973.89 \\
\hline 87.0 & 975.33 & & & 76.0 & 973.84 & & & 81.0 & 973.93 \\
\hline 88.3 & 976.09 & & & 79.0 & 973.90 & & & 84.0 & 974.00 \\
\hline 88.5 & 976.81 & & & 82.0 & 973.94 & & & 84.8 & 974.21 \\
\hline 90.0 & 976.68 & & & 83.6 & 973.93 & & & 85.6 & 974.44 \\
\hline 92.0 & 976.91 & & & 85.4 & 974.27 & & & 86.4 & 975.07 \\
\hline 94.0 & 976.89 & & & 86.1 & 975.02 & & & 87.0 & 975.20 \\
\hline
\end{tabular}


Table 18.--Listing of horizontal stations and elevations for cross section PR151 --Continued.

\begin{tabular}{|c|c|c|c|c|c|c|c|c|c|}
\hline \multicolumn{2}{|c|}{1985} & \multicolumn{2}{|c|}{1986} & \multicolumn{2}{|c|}{1986} & \multicolumn{2}{|c|}{1987} & \multicolumn{2}{|c|}{1987} \\
\hline Sta. & Elev. & Sta. & Elev. & Sta. & Elev. & Sta. & Elev. & Sta. & Elev. \\
\hline 88.3 & 976.14 & -0.9 & 977.48 & 88.0 & 975.88 & -0.8 & 977.49 & 86.7 & 975.36 \\
\hline 88.5 & 976.78 & 0.0 & 977.53 & 88.3 & 976.33 & 0.0 & 977.53 & 87.0 & 975.41 \\
\hline 90.3 & 976.66 & 3.0 & 977.72 & 88.4 & 976.77 & 3.0 & 977.70 & 88.0 & 975.92 \\
\hline 92.0 & 976.93 & 6.0 & 977.69 & 89.0 & 976.76 & 6.0 & 977.69 & 88.4 & 976.18 \\
\hline 94.0 & 976.90 & 8.5 & 977.70 & 90.0 & 976.66 & 8.5 & 977.71 & 88.5 & 976.77 \\
\hline 96.0 & 977.05 & 9.7 & 977.21 & 91.0 & 976.80 & 9.7 & 977.21 & 90.0 & 976.68 \\
\hline 98.0 & 977.10 & 11.0 & 977.26 & 93.0 & 976.88 & 10.2 & 977.19 & 91.0 & 976.79 \\
\hline 100.0 & 977.06 & 13.0 & 977.47 & 95.0 & 976.94 & 12.0 & 977.43 & 92.0 & 976.91 \\
\hline \multirow[t]{37}{*}{101.2} & 976.97 & 14.3 & 977.45 & 97.0 & 977.10 & 14.0 & 977.45 & 94.0 & 976.89 \\
\hline & & 17.0 & 976.37 & 100.0 & 977.06 & 14.6 & 977.35 & 96.0 & 977.04 \\
\hline & & 19.0 & 975.72 & 101.2 & 976.97 & 17.0 & 976.32 & 97.6 & 977.11 \\
\hline & & 20.6 & 975.20 & & & 19.0 & 975.77 & 100.0 & 977.04 \\
\hline & & 22.0 & 975.04 & & & 20.0 & 975.61 & 101.2 & 976.96 \\
\hline & & 25.0 & 975.10 & & & 20.7 & 975.23 & & \\
\hline & & 28.0 & 975.02 & & & 21.3 & 975.15 & & \\
\hline & & 30.0 & 975.03 & & & 22.4 & 975.09 & & \\
\hline & & 32.0 & 974.92 & & & 24.0 & 975.16 & & \\
\hline & & 34.0 & 975.00 & & & 26.0 & 975.14 & & \\
\hline & & 36.0 & 975.07 & & & 28.0 & 975.10 & & \\
\hline & & 37.5 & 975.13 & & & 30.0 & 975.10 & & \\
\hline & & 39.7 & 974.52 & . & & 33.0 & 975.08 & & \\
\hline & & 40.0 & 974.60 & & & 35.0 & 975.19 & & \\
\hline & & 40.7 & 974.51 & & & 36.8 & 975.22 & & \\
\hline & & 42.0 & 974.25 & & & 37.7 & 975.13 & & \\
\hline & & 43.3 & 974.18 & & & 39.2 & 974.71 & & \\
\hline & & 44.2 & 974.09 & & & 39.7 & 974.73 & & \\
\hline & & 45.2 & 974.08 & & & 41.4 & 974.31 & & \\
\hline & & 48.0 & 974.01 & & & 42.0 & 974.28 & & \\
\hline & & 51.0 & 973.96 & & & 44.0 & 974.09 & & \\
\hline & & 54.0 & 973.88 & & & 47.0 & 973.99 & & \\
\hline & & 57.0 & 973.88 & & & 50.0 & 973.82 & & \\
\hline & & 60.0 & 973.84 & & & 53.0 & 973.86 & & \\
\hline & & 63.0 & 973.80 & & & 56.0 & 973.88 & & \\
\hline & & 66.0 & 973.80 & & & 59.0 & 973.75 & & \\
\hline & & 69.0 & 973.85 & & & 62.0 & 973.83 & & \\
\hline & & 72.0 & 973.82 & & & 65.0 & 973.81 & & \\
\hline & & 75.0 & 973.82 & & & 68.0 & 973.67 & & \\
\hline & & 78.0 & 973.90 & & & 71.0 & 973.81 & & \\
\hline & & 81.0 & 973.95 & & & 74.0 & 973.84 & & \\
\hline & & 82.0 & 974.00 & & & 77.0 & 973.86 & & \\
\hline & & 83.0 & 973.93 & & & 80.0 & 973.95 & & \\
\hline & & 84.7 & 974.08 & & & 83.0 & 973.95 & & \\
\hline & & 85.3 & 974.19 & & & 84.0 & 974.11 & & \\
\hline & & 86.5 & 975.17 & & & 85.0 & 974.27 & & \\
\hline & & 87.0 & 975.27 & & & 85.3 & 974.30 & & \\
\hline
\end{tabular}


Table 18.--Listing of horizontal stations and elevations for cross section PR151 --Continued.

\begin{tabular}{|c|c|c|c|c|c|}
\hline \multicolumn{2}{|c|}{1988} & \multicolumn{2}{|c|}{1988} & \multicolumn{2}{|c|}{1988} \\
\hline Sta. & Elev. & Sta. & Elev. & Sta. & Elev. \\
\hline-48.0 & 978.78 & 35.5 & 975.24 & 106.0 & 976.86 \\
\hline-46.0 & 978.02 & 37.0 & 975.20 & 107.0 & 976.87 \\
\hline-43.0 & 977.93 & 37.7 & 975.12 & 108.0 & 976.92 \\
\hline-40.0 & 977.99 & 39.0 & 974.76 & 109.2 & 976.79 \\
\hline-38.0 & 977.95 & 39.6 & 974.73 & 110.4 & 976.98 \\
\hline-36.0 & 978.03 & 41.0 & 974.46 & 112.0 & 976.93 \\
\hline-34.0 & 977.96 & 41.7 & 974.40 & 114.0 & 976.86 \\
\hline-31.0 & 977.92 & 42.2 & 974.22 & 116.0 & 976.84 \\
\hline-28.0 & 977.91 & 43.0 & 974.21 & 118.0 & 976.83 \\
\hline-25.0 & 977.89 & 45.6 & 974.05 & 118.7 & 976.86 \\
\hline-22.0 & 977.86 & 47.0 & 973.97 & 119.7 & 977.41 \\
\hline-19.0 & 977.76 & 49.0 & 973.84 & 120.7 & 977.21 \\
\hline-16.0 & 977.60 & 51.0 & 973.86 & 122.0 & 977.28 \\
\hline-14.0 & 977.52 & 53.0 & 973.84 & 124.0 & 977.39 \\
\hline-12.0 & 977.62 & 56.0 & 973.88 & 126.0 & 977.43 \\
\hline-10.0 & 977.64 & 58.0 & 973.93 & 127.0 & 977.43 \\
\hline-8.0 & 977.50 & 61.0 & 973.92 & 129.0 & 977.32 \\
\hline-6.0 & 977.47 & 64.0 & 973.88 & 131.0 & 977.24 \\
\hline-4.0 & 977.43 & 67.0 & 973.88 & 133.0 & 977.28 \\
\hline-0.8 & 977.48 & 68.0 & 973.81 & 135.0 & 977.14 \\
\hline 0.0 & 977.53 & 70.0 & 973.77 & 137.0 & 976.82 \\
\hline 2.0 & 977.64 & 72.0 & 973.83 & 138.6 & 976.69 \\
\hline 5.0 & 977.73 & 75.0 & 973.85 & 140.6 & 976.60 \\
\hline 7.0 & 977.67 & 78.0 & 973.90 & 141.1 & 976.61 \\
\hline 8.6 & 977.70 & 81.0 & 973.93 & 141.6 & 976.74 \\
\hline 9.6 & 977.23 & 83.0 & 974.04 & 144.0 & 976.83 \\
\hline 10.5 & 977.22 & 84.2 & 974.06 & 145.5 & 976.87 \\
\hline 12.0 & 977.43 & 85.1 & 974.22 & & \\
\hline 13.0 & 977.48 & 85.7 & 974.42 & & \\
\hline 14.0 & 977.43 & 86.7 & 975.36 & & \\
\hline 14.7 & 977.28 & 87.1 & 975.41 & & \\
\hline 17.0 & 976.34 & 88.4 & 976.18 & & \\
\hline 18.0 & 976.04 & 88.6 & 976.75 & & \\
\hline 19.0 & 975.75 & 89.5 & 976.75 & & \\
\hline 19.2 & 975.88 & 90.3 & 976.66 & & \\
\hline 19.9 & 975.77 & 91.7 & 976.92 & & \\
\hline 20.6 & 975.28 & 93.0 & 976.89 & & \\
\hline 21.2 & 975.17 & 95.0 & 976.94 & & \\
\hline 22.0 & 975.10 & 97.0 & 977.10 & & \\
\hline 24.0 & 975.15 & 99.0 & 977.08 & & \\
\hline 26.0 & 975.14 & 100.0 & 977.05 & & \\
\hline 28.0 & 975.10 & 101.2 & 976.96 & & \\
\hline 30.0 & 975.11 & 102.8 & 976.69 & & \\
\hline 32.0 & 975.04 & 104.0 & 976.72 & & \\
\hline 34.0 & 975.12 & 105.0 & 976.72 & & \\
\hline
\end{tabular}




\section{Description of Cross Section PR156A}

Location: Township 7 South/Range 49 East--section 12

U.S. Geological Survey quadrangle $(1: 24,000)$ : Huckins School

Landowners--left bank: Gay Ranch

right bank: Swope Ranch

Access: right bank

Permission from: Swope Ranch (T7S/R49E--12)

Reference pins

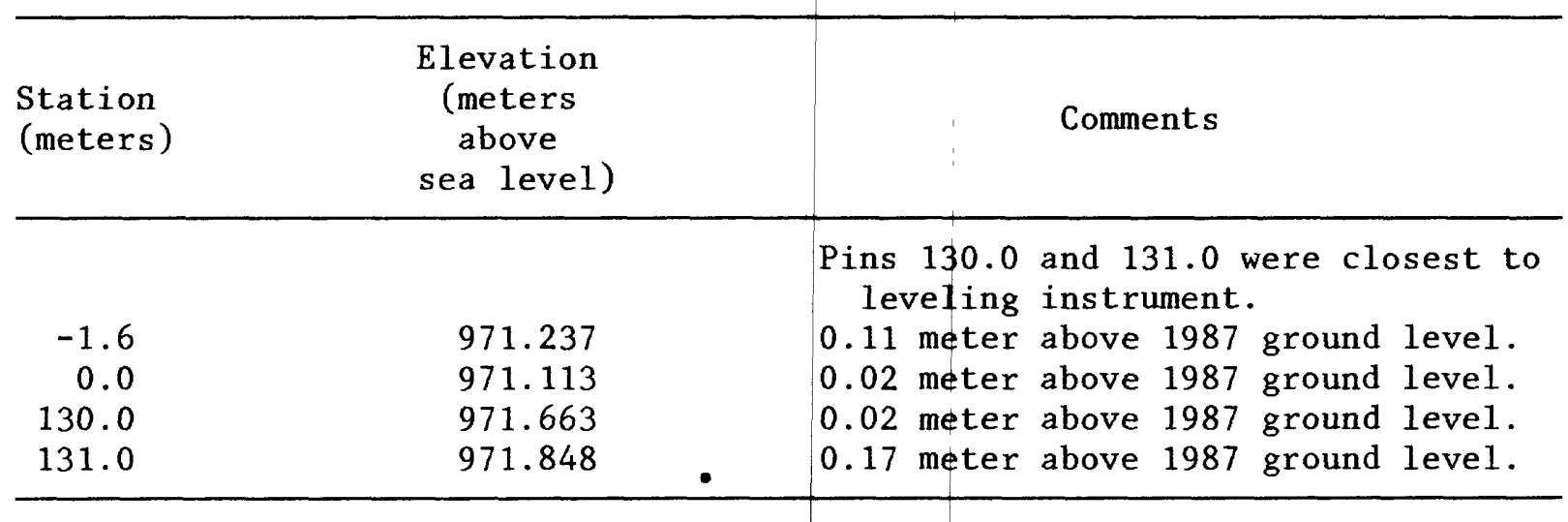




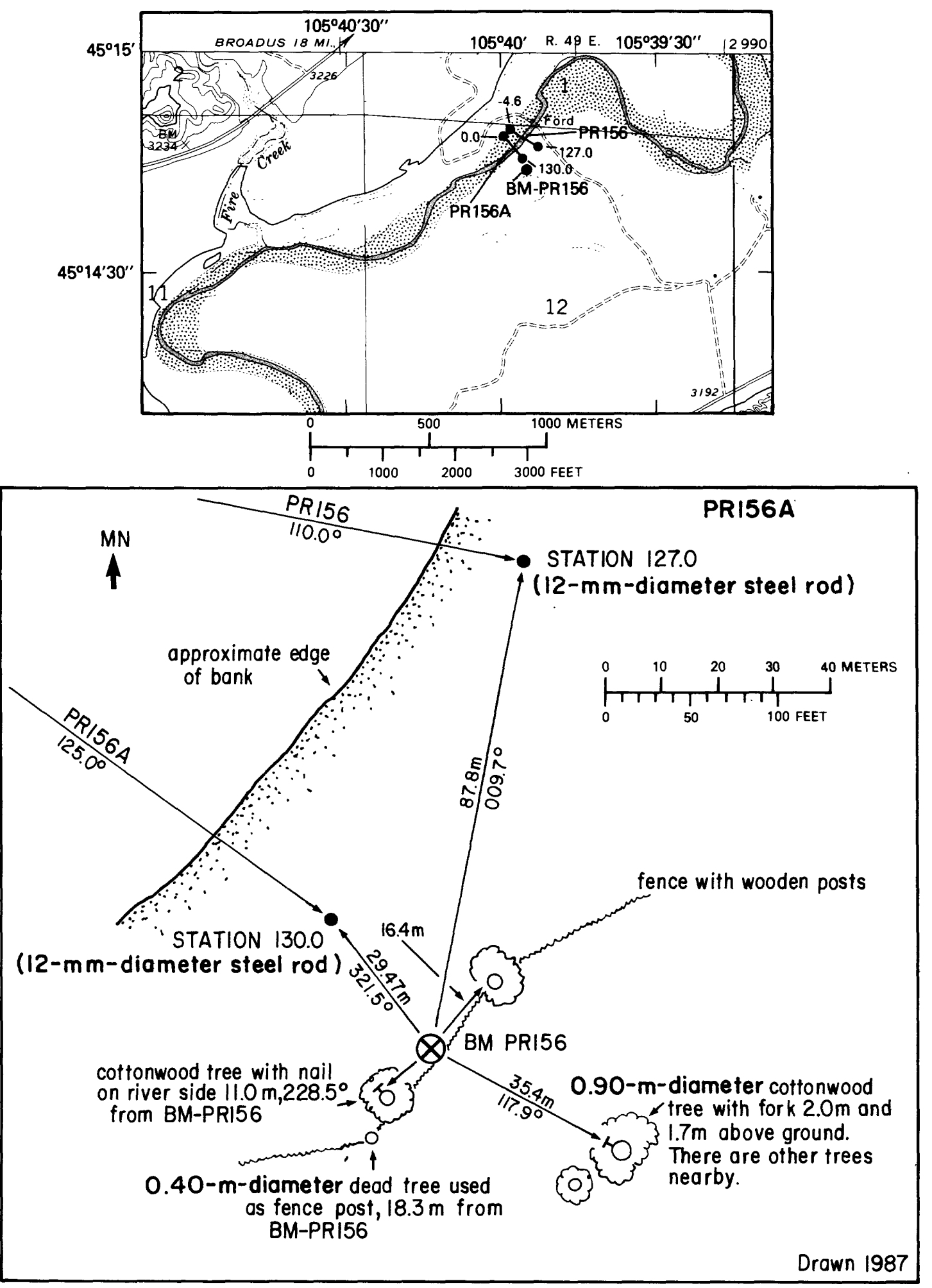

Figure 53.--Upper: Location of cross section PR156A, bench mark BM-PR151, and left and right bank reference pins (tables 2 and 3 ) in the Huckins School quadrangle. Lower: Location of bench mark on the left bank. MN is magnetic north. 

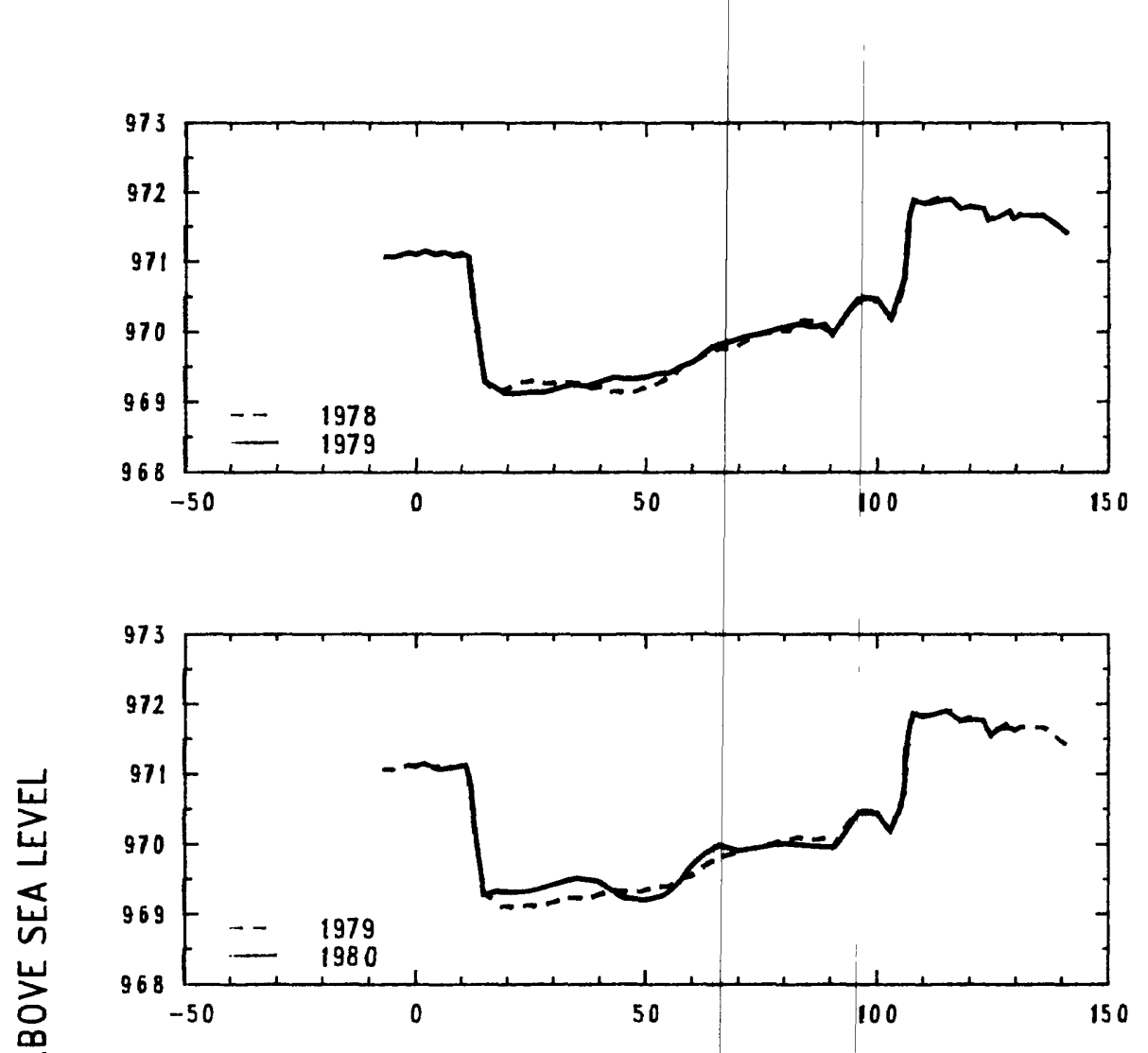

告
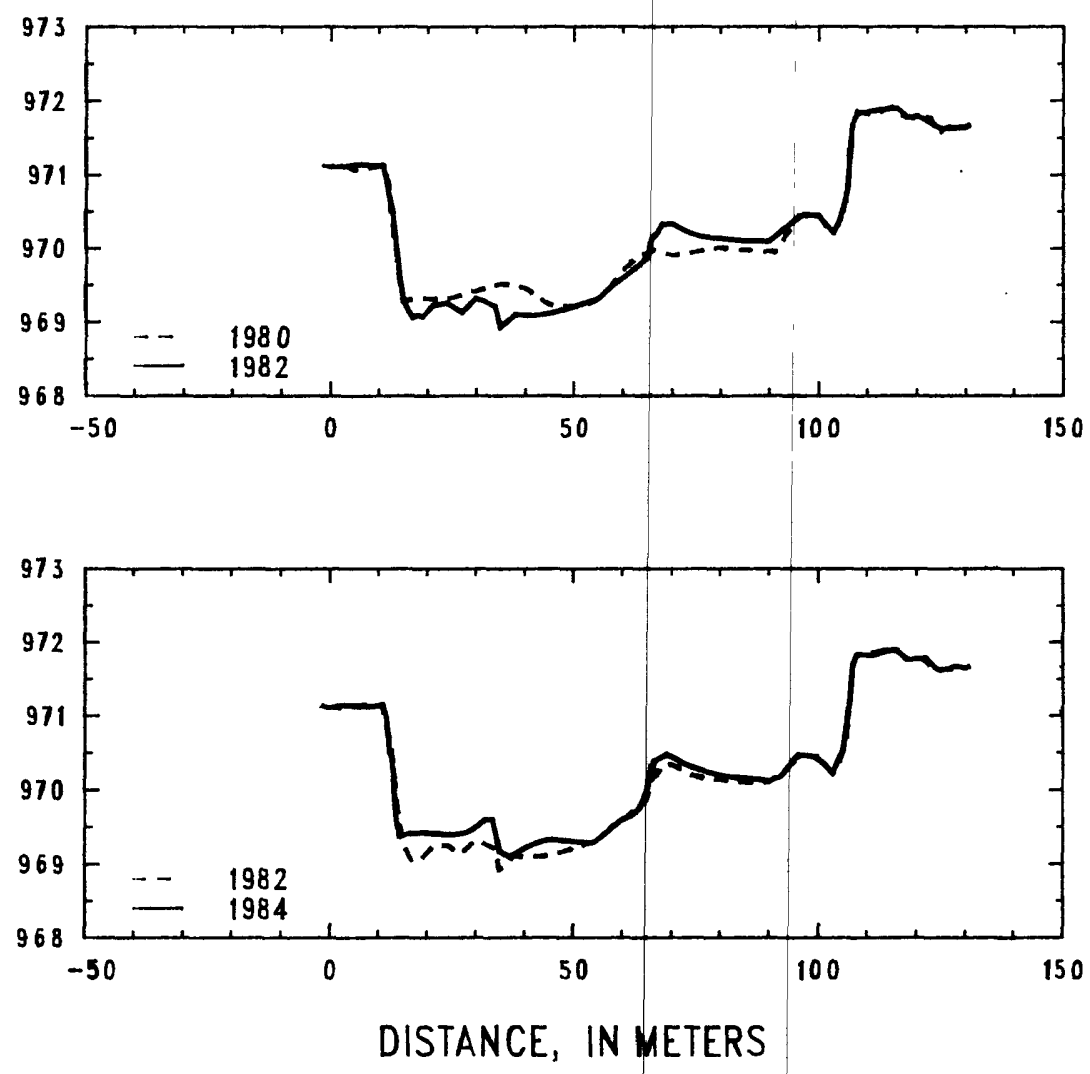

Figure 54. - Profiles of cross section PR156A from 1978 to 1984. 


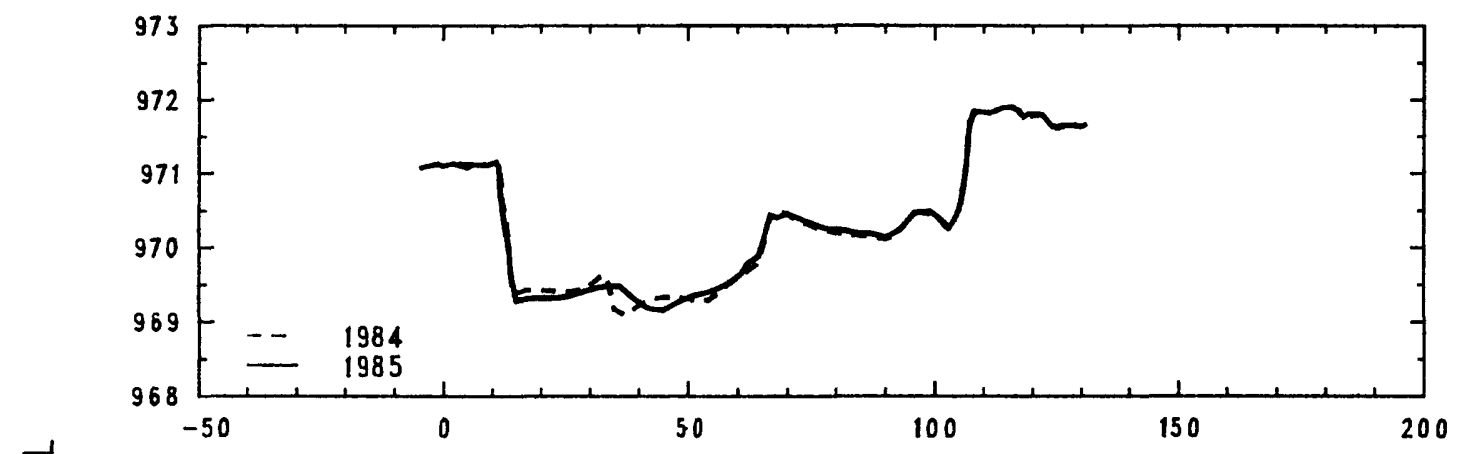

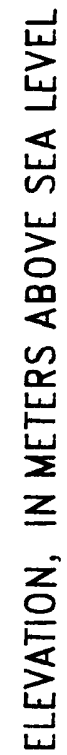
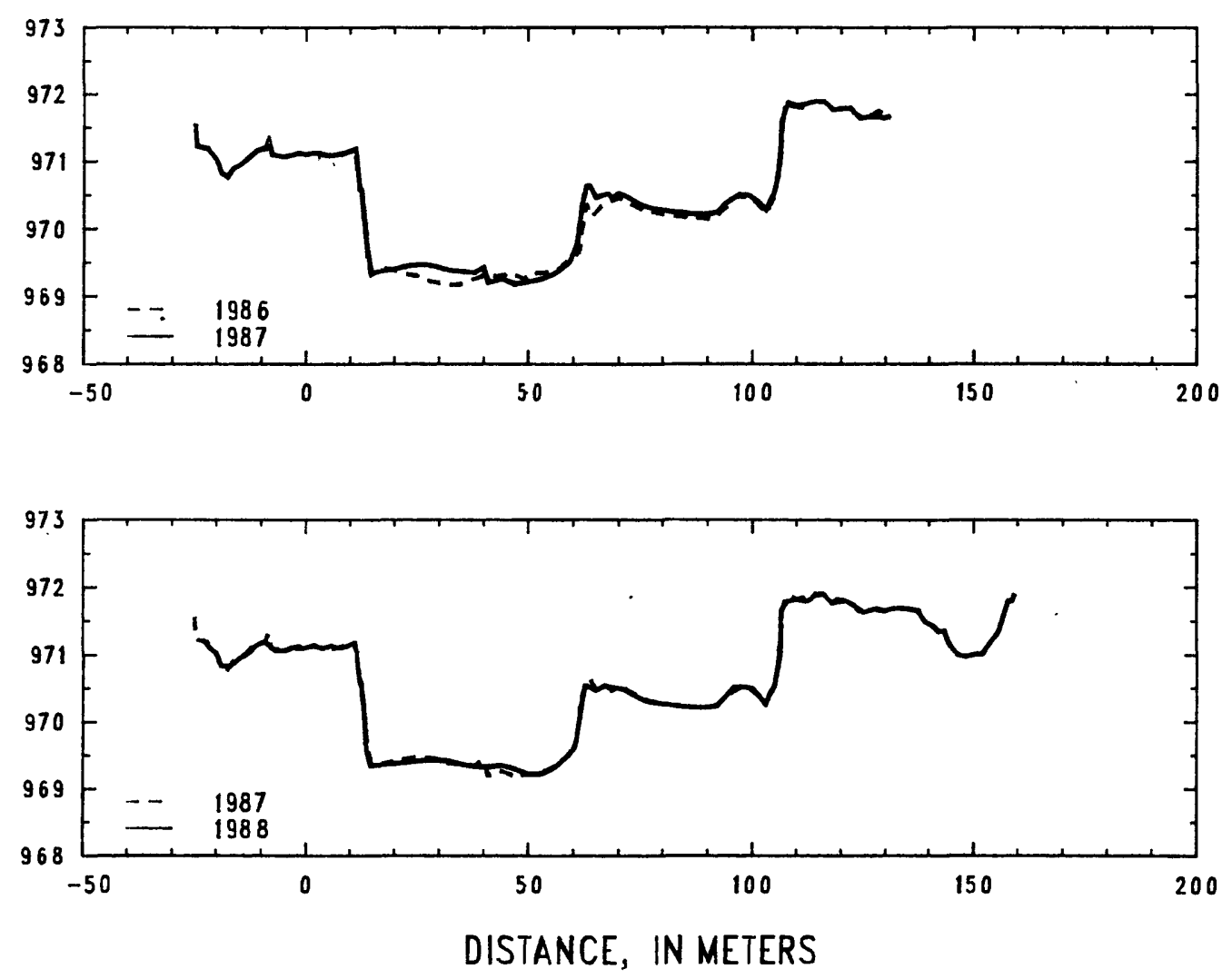

Figure 55.--Profiles of cross section PR156A from 1984 to 1988. 
Table 19.--Listing of horizontal stations and elevations for cross section PR156A [Sta., station, distance in meters from a reference point on the left bank; Elev., elevation, in meters above sea level].

\begin{tabular}{|c|c|c|c|c|c|c|c|c|c|}
\hline \multicolumn{2}{|c|}{1978} & \multicolumn{2}{|c|}{1978} & \multicolumn{2}{|c|}{1979} & \multicolumn{2}{|c|}{1979} & \multicolumn{2}{|c|}{1980} \\
\hline Sta. & Elev. & Sta. & Elev. & Sta. & Elev. & Sta. & Elev. & Sta. & Elev. \\
\hline-1.5 & 971.12 & 90.0 & 969.99 & -7.0 & 971.06 & 106.0 & 970.74 & -1.6 & 971.12 \\
\hline 0.0 & 971.09 & 92.0 & 970.08 & -5.0 & 971.05 & 107.0 & 971.66 & 0.0 & 971.10 \\
\hline 2.0 & 971.14 & 94.0 & 970.31 & -1.6 & 971.12 & 108.0 & 971.87 & 2.0 & 971.15 \\
\hline 4.0 & 971.08 & 95.8 & 970.47 & 0.0 & 971.09 & 110.0 & 971.83 & 5.0 & 971.06 \\
\hline 6.0 & 971.13 & 98.0 & 970.45 & 2.0 & 971.14 & 112.0 & 971.85 & 8.0 & 971.09 \\
\hline 8.0 & 971.07 & 100.5 & 970.42 & 4.0 & 971.09 & 114.0 & 971.89 & 11.0 & 971.13 \\
\hline 10.0 & 971.09 & 102.0 & 970.24 & 6.0 & 971.13 & 116.0 & 971.90 & 12.0 & 970.90 \\
\hline 11.5 & 971.11 & 103.0 & 970.16 & 8.0 & 971.08 & 118.0 & 971.76 & 13.0 & 970.22 \\
\hline 12.0 & 970.87 & 105.0 & 970.48 & 10.0 & 971.11 & 120.0 & 971.80 & 15.0 & 969.27 \\
\hline 13.0 & 970.08 & 106.0 & 970.70 & 11.5 & 971.05 & 123.0 & 971.76 & 18.0 & 969.33 \\
\hline 13.6 & 969.89 & 106.5 & 971.42 & 13.0 & 970.14 & 124.0 & 971.59 & 21.0 & 969.31 \\
\hline 15.0 & 969.30 & 107.0 & 971.62 & 13.5 & 970.01 & 126.0 & 971.64 & 25.0 & 969.33 \\
\hline 17.0 & 969.12 & 108.0 & 971.89 & 14.0 & 969.70 & 128.6 & 971.73 & 30.0 & 969.43 \\
\hline 20.0 & 969.19 & 110.0 & 971.83 & 15.0 & 969.27 & 129.5 & 971.61 & 35.0 & 969.52 \\
\hline 22.0 & 969.27 & 112.0 & 971.88 & 17.0 & 969.20 & 130.0 & 971.63 & 40.0 & 969.46 \\
\hline 24.0 & 969.29 & 114.0 & 971.94 & 19.0 & 969.10 & 131.0 & 971.67 & 45.0 & 969.23 \\
\hline 26.0 & 969.30 & 116.0 & 971.90 & 22.0 & 969.10 & 136.0 & 971.66 & 50.0 & 969.20 \\
\hline 28.0 & 969.25 & 118.0 & 971.76 & 25.0 & 969.13 & 141.0 & 971.40 & 54.0 & 969.27 \\
\hline 30.0 & 969.26 & 120.0 & 971.79 & 28.0 & 969.13 & & & 57.0 & 969.43 \\
\hline 32.0 & 969.27 & 123.2 & 971.76 & 31.0 & 969.19 & & & 60.0 & 969.70 \\
\hline 34.0 & 969.27 & 124.0 & 971.59 & 34.0 & 969.24 & & & 63.0 & 969.88 \\
\hline 36.0 & 969.25 & 126.0 & 971.68 & 37.0 & 969.21 & & & 66.0 & 969.99 \\
\hline 38.0 & 969.18 & 128.6 & 971.72 & 40.0 & 969.28 & & & 70.0 & 969.90 \\
\hline 40.0 & 969.21 & 129.5 & 971.58 & 43.0 & 969.34 & & & 75.0 & 969.96 \\
\hline 42.0 & 969.13 & 130.0 & 971.64 & 46.0 & 969.31 & & & 80.0 & 970.02 \\
\hline 44.0 & 969.14 & 131.0 & 971.70 & 49.0 & 969.32 & & & 85.0 & 969.98 \\
\hline 46.0 & 969.13 & & & 52.0 & 969.39 & & & 88.0 & 969.96 \\
\hline 48.0 & 969.14 & & & 55.0 & 969.40 & & & 91.0 & 969.95 \\
\hline 50.0 & 969.21 & & & 58.0 & 969.51 & & & 93.0 & 970.14 \\
\hline 52.0 & 969.24 & & & 60.0 & 969.55 & & & 96.0 & 970.45 \\
\hline 54.0 & 969.31 & & & 65.0 & 969.80 & & & 100.0 & 970.44 \\
\hline 56.0 & 969.36 & & & 68.0 & 969.85 & & & 103.0 & 970.18 \\
\hline 58.0 & 969.51 & & & 71.0 & 969.92 & & & 105.0 & 970.49 \\
\hline 60.0 & 969.56 & & & 74.0 & 969.95 & & & 106.0 & 970.80 \\
\hline 63.0 & 969.67 & & & 77.0 & 970.00 & & & 106.3 & 971.28 \\
\hline 66.0 & 969.76 & & & 80.0 & 970.06 & & & 107.0 & 971.65 \\
\hline 69.0 & 969.76 & & & 83.0 & 970.10 & & & 108.0 & 971.86 \\
\hline 72.0 & 969.89 & & & 86.0 & 970.05 & & & 110.0 & 971.82 \\
\hline 75.0 & 969.96 & & & 88.8 & 970.09 & & & 112.0 & 971.85 \\
\hline 78.0 & 970.00 & & & 90.5 & 969.94 & & & 115.0 & 971.91 \\
\hline 81.0 & 970.00 & & & 92.0 & 970.11 & & & 118.0 & 971.76 \\
\hline 82.0 & 970.11 & & & 95.0 & 970.38 & & & 121.0 & 971.78 \\
\hline 84.0 & 970.17 & & & 97.0 & 970.48 & & & 123.0 & 971.76 \\
\hline 86.0 & 970.14 & & & 100.0 & 970.45 & & & 124.6 & 971.55 \\
\hline 88.0 & 970.06 & & & 103.0 & 970.17 & & & 126.0 & 971.64 \\
\hline
\end{tabular}


Table 19.--Listing of horizontal stations and elevations for cross section PR156A--Cont inued.

\begin{tabular}{|c|c|c|c|c|c|c|c|c|c|}
\hline \multicolumn{2}{|c|}{1980} & \multicolumn{2}{|c|}{1982} & \multicolumn{2}{|c|}{1982} & \multicolumn{2}{|c|}{1984} & \multicolumn{2}{|c|}{1984} \\
\hline Sta. & Elev. & Sta. & Elev. & Sta. & Elev. & Sta. & Elev. & Sta. & Elev. \\
\hline 128.0 & 971.67 & 0.0 & 971.10 & 110.0 & 971.85 & -1.6 & 971.13 & 96.0 & 970.47 \\
\hline 130.0 & 971.63 & 3.0 & 971.11 & 113.0 & 971.89 & 0.0 & 971.10 & 99.0 & 970.45 \\
\hline \multirow[t]{42}{*}{130.9} & 971.67 & 6.0 & 971.14 & 116.0 & 971.90 & 3.0 & 971.14 & 101.0 & 970.36 \\
\hline & & 10.0 & 971.12 & 118.0 & 971.77 & 6.0 & 971.12 & 102.0 & 970.28 \\
\hline & & 11.0 & 971.13 & 120.0 & 971.79 & 9.0 & 971.12 & 103.0 & 970.23 \\
\hline & & 13.0 & 970.49 & 125.0 & 971.62 & 11.0 & 971.15 & 104.0 & 970.38 \\
\hline & & 13.0 & 970.47 & 130.0 & 971.64 & 11.8 & 970.99 & 105.0 & 970.51 \\
\hline & & 13.9 & 969.89 & 130.9 & 971.67 & 12.5 & 970.49 & 105.7 & 970.79 \\
\hline & & 14.0 & 969.84 & & & 12.9 & 970.44 & 106.5 & 971.22 \\
\hline & & 15.0 & 969.36 & & & 13.4 & 970.07 & 107.0 & 971.67 \\
\hline & & 15.5 & 969.22 & & & 13.8 & 969.63 & 108.0 & 971.84 \\
\hline & & 17.0 & 969.05 & & & 14.5 & 969.37 & 109.0 & 971.84 \\
\hline & & 18.0 & 969.09 & & & 17.0 & 969.42 & 111.0 & 971.81 \\
\hline & & 19.0 & 969.06 & & & 20.0 & 969.42 & 114.0 & 971.89 \\
\hline & & 21.0 & 969.22 & & & 23.0 & 969.40 & 116.0 & 971.90 \\
\hline & & 24.0 & 969.25 & & & 25.0 & 969.39 & 118.0 & 971.76 \\
\hline & & 27.0 & 969.12 & & & 28.0 & 969.42 & 120.0 & 971.78 \\
\hline & & 30.0 & 969.32 & & & 30.0 & 969.49 & 122.0 & 971.79 \\
\hline & & 34.0 & 969.20 & & & 32.0 & 969.60 & 124.0 & 971.64 \\
\hline & & 35.0 & 968.92 & & & 33.5 & 969.60 & 125.0 & 971.61 \\
\hline & & 38.0 & 969.10 & & & 35.0 & 969.16 & 128.0 & 971.68 \\
\hline & & 41.0 & 969.08 & & & 37.0 & 969.09 & 130.0 & 971.64 \\
\hline & & 45.0 & 969.11 & & & 40.0 & 969.22 & 130.9 & 971.67 \\
\hline & & 50.0 & 969.21 & & & 43.0 & 969.30 & & \\
\hline & & 55.0 & 969.31 & & & 45.0 & 969.33 & & \\
\hline & & 58.0 & 969.50 & & & 48.0 & 969.32 & & \\
\hline & & 61.0 & 969.64 & & & 51.0 & 969.29 & & \\
\hline & & 65.0 & 969.87 & & & 54.0 & 969.28 & & \\
\hline & & 66.0 & 970.13 & & & 57.0 & 969.44 & & \\
\hline & & 67.0 & 970.20 & & & 60.0 & 969.60 & & \\
\hline & & 68.0 & 970.33 & . & & 61.0 & 969.62 & & \\
\hline & & 70.0 & 970.34 & & & 63.5 & 969.75 & & \\
\hline & & 74.0 & 970.19 & & & 65.0 & 969.96 & & \\
\hline & & 77.0 & 970.14 & & & 66.5 & 970.39 & & \\
\hline & & 80.0 & 970.13 & & & 69.0 & 970.48 & & \\
\hline & & 85.0 & 970.09 & & & 70.0 & 970.44 & & \\
\hline & & 90.0 & 970.09 & & & 73.0 & 970.33 & & \\
\hline & & 95.0 & 970.38 & & & 76.0 & 970.26 & & \\
\hline & & 97.0 & 970.46 & & & 80.0 & 970.19 & & \\
\hline & & 100.0 & 970.44 & & & 83.0 & 970.16 & & \\
\hline & & 103.0 & 970.21 & & & 87.0 & 970.14 & & \\
\hline & & 104.0 & 970.31 & & & 90.0 & 970.11 & & \\
\hline & & 105.8 & 970.68 & & & 92.0 & 970.17 & & \\
\hline & & $\begin{array}{l}107.0 \\
108.0\end{array}$ & $\begin{array}{l}971.66 \\
971.83\end{array}$ & & & $\begin{array}{l}93.0 \\
95.0\end{array}$ & $\begin{array}{l}970.24 \\
970.40\end{array}$ & & \\
\hline
\end{tabular}


Table 19.--Listing of horizontal stations and elevations for cross section PR156A--Continued.

\begin{tabular}{|c|c|c|c|c|c|c|c|c|c|}
\hline \multicolumn{2}{|c|}{1985} & \multicolumn{2}{|c|}{1985} & \multicolumn{2}{|c|}{1986} & \multicolumn{2}{|c|}{1986} & \multicolumn{2}{|c|}{1987} \\
\hline Sta. & Elev. & Sta. & Elev. & Sta. & Elev. & Sta. & $\overline{\text { Elev. }}$ & Sta. & $\overline{\text { Elev. }}$ \\
\hline-5.0 & 971.07 & 101.0 & 970.39 & -1.6 & 971.12 & 94.0 & 970.33 & -25.0 & 971.54 \\
\hline-1.5 & 971.13 & 103.0 & 970.25 & 0.0 & 971.10 & 96.0 & 970.47 & -24.4 & 971.22 \\
\hline 0.0 & 971.10 & 105.0 & 970.50 & 2.0 & 971.15 & 98.0 & 970.49 & -22.0 & 971.18 \\
\hline 2.0 & 971.14 & 106.0 & 970.86 & 5.0 & 971.09 & 100.0 & 970.45 & -20.0 & 971.02 \\
\hline 5.0 & 971.07 & 106.6 & 971.26 & 7.0 & 971.08 & 102.0 & 970.31 & -19.0 & 970.82 \\
\hline 6.0 & 971.12 & 107.0 & 971.64 & 9.0 & 971.12 & 103.0 & 970.23 & -17.6 & 970.76 \\
\hline 9.0 & 971.11 & 108.0 & 971.81 & 11.2 & 971.18 & 104.7 & 970.46 & -16.3 & 970.90 \\
\hline 11.0 & 971.16 & 109.0 & 971.84 & 12.4 & 970.44 & 105.7 & 970.79 & -15.0 & 970.94 \\
\hline 11.5 & 971.09 & 111.0 & 971.81 & 12.8 & 970.44 & 106.3 & 971.06 & -13.0 & 971.05 \\
\hline 11.7 & 970.74 & 113.0 & 971.88 & 13.5 & 970.05 & 106.6 & 971.55 & -11.0 & 971.16 \\
\hline 12.6 & 970.30 & 115.0 & 971.90 & 13.9 & 969.59 & 107.5 & 971.81 & -9.0 & 971.20 \\
\hline 13.5 & 969.99 & 117.0 & 971.86 & 14.7 & 969.32 & 108.2 & 971.89 & -8.4 & 971.31 \\
\hline 13.8 & 969.69 & 118.0 & 971.76 & 17.0 & 969.43 & 109.0 & 971.82 & -7.6 & 971.09 \\
\hline 15.0 & 969.28 & 119.0 & 971.81 & 19.0 & 969.41 & 111.0 & 971.80 & -5.0 & 971.06 \\
\hline 18.0 & 969.31 & 122.0 & 971.79 & 22.0 & 969.34 & 114.0 & 971.90 & -1.6 & 971.13 \\
\hline 21.0 & 969.32 & 124.0 & 971.63 & 25.0 & 969.30 & 116.0 & 971.89 & 0.0 & 971.10 \\
\hline 24.0 & 969.32 & 127.0 & 971.66 & 28.0 & 969.22 & 118.0 & 971.76 & 2.0 & 971.13 \\
\hline 27.0 & 969.37 & 130.0 & 971.63 & 31.0 & 969.19 & 120.0 & 971.79 & 5.0 & 971.08 \\
\hline 30.0 & 969.43 & 130.9 & 971.67 & 34.0 & 969.17 & 123.0 & 971.72 & 7.0 & 971.09 \\
\hline 33.0 & 969.48 & & & 37.0 & 969.25 & 125.0 & 971.61 & 10.0 & 971.16 \\
\hline 36.0 & 969.48 & & & 40.0 & 969.31 & 128.3 & 971.75 & 11.4 & 971.19 \\
\hline 39.0 & 969.30 & & & 43.0 & 969.31 & 130.0 & 971.63 & 11.7 & 970.92 \\
\hline 42.0 & 969.17 & & & 46.0 & 969.34 & 130.9 & 971.67 & 12.3 & 970.60 \\
\hline 45.0 & 969.15 & & & 49.0 & 969.26 & & & 12.6 & 970.58 \\
\hline 48.0 & 969.27 & & & 51.0 & 969.36 & & & 13.8 & 969.77 \\
\hline 51.0 & 969.35 & & & 54.0 & 969.35 & & & 14.8 & 969.35 \\
\hline 54.0 & 969.39 & & & 57.0 & 969.42 & & & 17.0 & 969.38 \\
\hline 57.0 & 969.47 & & & 58.0 & 969.49 & & & 20.0 & 969.41 \\
\hline 60.0 & 969.60 & & & 60.0 & 969.59 & & & 23.0 & 969.46 \\
\hline 61.3 & 969.68 & & & 61.3 & 969.67 & & & 26.0 & 969.47 \\
\hline 62.0 & 969.78 & & & 61.6 & 969.82 & & & 29.0 & 969.45 \\
\hline 64.3 & 969.88 & & & 62.7 & 970.35 & & & 32.0 & 969.39 \\
\hline 66.6 & 970.44 & & & 63.0 & 970.36 & & & 35.0 & 969.37 \\
\hline 68.0 & 970.40 & & & 63.5 & 970.20 & & & 38.0 & 969.35 \\
\hline 70.0 & 970.46 & & & 64.2 & 970.19 & & & 39.0 & 969.41 \\
\hline 72.0 & 970.40 & & & 66.0 & 970.33 & & & 40.0 & 969.44 \\
\hline 75.0 & 970.32 & & & 68.0 & 970.41 & & & 41.0 & 969.20 \\
\hline 78.0 & 970.24 & & & 70.0 & 970.47 & & & 44.0 & 969.27 \\
\hline 81.0 & 970.23 & & & 73.0 & 970.36 & & & 47.0 & 969.18 \\
\hline 84.0 & 970.18 & & & 76.0 & 970.27 & & & 50.0 & 969.22 \\
\hline 87.0 & 970.18 & & & 79.0 & 970.23 & & & 53.0 & 969.26 \\
\hline 90.0 & 970.13 & & & 82.0 & 970.20 & & & 56.0 & 969.34 \\
\hline 93.0 & 970.24 & & & 85.0 & 970.18 & & & 59.0 & 969.51 \\
\hline 96.0 & 970.47 & & & 88.0 & 970.16 & & & 60.8 & 969.75 \\
\hline 99.0 & 970.49 & & & 91.0 & 970.14 & & & 62.0 & 970.39 \\
\hline
\end{tabular}


Table 19.--Listing of horizontal stations and elevations for cross section PR156A--Continued.

\begin{tabular}{|c|c|c|c|c|c|c|c|}
\hline \multicolumn{2}{|c|}{1987} & \multicolumn{2}{|c|}{1988} & \multicolumn{2}{|c|}{1988} & \multicolumn{2}{|c|}{1988} \\
\hline Sta. & Elev. & Sta. & Elev. & Sta. & Elev. & Sta. & Elev. \\
\hline 63.0 & 970.65 & -23.0 & 971.21 & 65.0 & 970.47 & 144.4 & 971.17 \\
\hline 63.7 & 970.65 & -20.0 & 971.01 & 67.0 & 970.55 & 146.3 & 971.00 \\
\hline 65.0 & 970.46 & -19.0 & 970.83 & 69.0 & 970.51 & 148.0 & 970.97 \\
\hline 66.0 & 970.49 & -17.0 & 970.81 & 71.0 & 970.49 & 150.0 & 971.01 \\
\hline 67.7 & 970.53 & -15.0 & 970.94 & 74.0 & 970.38 & 152.0 & 971.02 \\
\hline 68.4 & 970.47 & -13.0 & 971.02 & 76.0 & 970.31 & 154.0 & 971.20 \\
\hline 70.0 & 970.53 & -11.0 & 971.14 & 78.0 & 970.29 & 155.5 & 971.32 \\
\hline 72.0 & 970.48 & -9.0 & 971.20 & 80.0 & 970.27 & 157.0 & 971.62 \\
\hline 74.0 & 970.38 & -7.0 & 971.06 & 82.0 & 970.25 & 157.8 & 971.80 \\
\hline 77.0 & 970.31 & -5.0 & 971.06 & 84.0 & 970.23 & 158.7 & 971.80 \\
\hline 80.0 & 970.27 & -3.0 & 971.11 & 86.0 & 970.22 & 159.4 & 971.89 \\
\hline 82.0 & 970.25 & -1.6 & 971.09 & 88.0 & 970.21 & & \\
\hline 84.0 & 970.24 & 0.0 & 971.10 & 90.0 & 970.22 & & \\
\hline 87.0 & 970.21 & 2.0 & 971.14 & 92.0 & 970.24 & & \\
\hline 90.0 & 970.21 & 4.0 & 971.09 & 94.0 & 970.39 & & \\
\hline 92.0 & 970.25 & 6.0 & 971.14 & 96.0 & 970.52 & & \\
\hline 94.0 & 970.39 & 8.0 & 971.09 & 98.0 & 970.53 & & \\
\hline 97.0 & 970.51 & 10.0 & 971.15 & 100.0 & 970.49 & & \\
\hline 99.0 & 970.50 & 11.0 & 971.18 & 102.0 & 970.33 & & \\
\hline 101.0 & 970.42 & 11.4 & 971.13 & 103.0 & 970.26 & & \\
\hline 103.0 & 970.28 & 12.2 & 970.62 & 104.0 & 970.42 & & \\
\hline 104.2 & 970.45 & 12.6 & 970.57 & 105.0 & 970.52 & & \\
\hline 104.9 & 970.52 & 13.4 & 970.11 & 106.5 & 971.10 & & \\
\hline 105.8 & 970.79 & 13.8 & 969.59 & 106.7 & 971.65 & & \\
\hline 106.3 & 971.10 & 14.7 & 969.34 & 108.0 & 971.79 & & \\
\hline 106.7 & 971.59 & 17.0 & 969.37 & 110.0 & 971.82 & & \\
\hline 107.0 & 971.70 & 20.0 & 969.38 & 112.0 & 971.79 & & \\
\hline 108.0 & 971.88 & 23.0 & 969.41 & 113.0 & 971.81 & & \\
\hline 110.0 & 971.82 & 26.0 & 969.42 & 114.0 & 971.88 & & \\
\hline 112.0 & 971.86 & 29.0 & 969.44 & 116.0 & 971.90 & & \\
\hline 114.0 & 971.90 & 32.0 & 969.42 & 118.0 & 971.76 & & \\
\hline 116.0 & 971.90 & 35.0 & 969.37 & 119.0 & 971.82 & & \\
\hline 118.0 & 971.76 & 38.0 & 969.34 & 121.0 & 971.80 & & \\
\hline 120.0 & 971.79 & 41.0 & 969.33 & 123.0 & 971.73 & & \\
\hline 122.0 & 971.80 & 44.0 & 969.36 & 125.0 & 971.63 & & \\
\hline 124.0 & 971.65 & 47.0 & 969.30 & 128.0 & 971.69 & & \\
\hline 127.0 & 971.67 & 50.0 & 969.21 & 130.0 & 971.65 & & \\
\hline 130.0 & 971.64 & 53.0 & 969.23 & 130.9 & 971.67 & & \\
\hline \multirow[t]{7}{*}{130.9} & 971.68 & 55.0 & 969.29 & 133.0 & 971.70 & & \\
\hline & & 57.0 & 969.39 & 136.0 & 971.67 & & \\
\hline & & 59.0 & 969.52 & 137.5 & 971.64 & & \\
\hline & & 60.0 & 969.59 & 139.0 & 971.49 & & \\
\hline & & 60.6 & 969.71 & 141.0 & 971.42 & & \\
\hline & & 62.4 & 970.48 & 142.1 & 971.34 & & \\
\hline & & 63.0 & 970.55 & 143.4 & 971.35 & & \\
\hline
\end{tabular}




\section{Description of Cross Section PR156}

Location: Township 7 South/Range 49 East--section 12

U.S. Geological Survey quadrangle $(1: 24,000)$ : Huckins School

Landowners--left bank: Gay Ranch right bank: Swope Ranch

Access: right bank

Permission from: Swope Ranch (T7S/R49E--12)

Reference pins

\begin{tabular}{|c|c|c|}
\hline $\begin{array}{l}\text { Station } \\
\text { (meters) }\end{array}$ & $\begin{array}{l}\text { Elevation } \\
\text { (meters } \\
\text { above } \\
\text { sea level) }\end{array}$ & Comments \\
\hline $\begin{array}{r}-4.2 \\
-1.6 \\
127.0\end{array}$ & $\begin{array}{l}971.304 \\
971.039 \\
971.779\end{array}$ & $\begin{array}{l}\text { Pin } 127.0 \text { was closest to leveling } \\
\text { instrument. } \\
0.19 \text { meter above } 1987 \text { ground level. } \\
0.07 \text { meter above } 1987 \text { ground level. } \\
\pm 0.07 \text { meter, } 0.16 \text { meter above } 1987 \\
\quad \text { ground level. }\end{array}$ \\
\hline
\end{tabular}




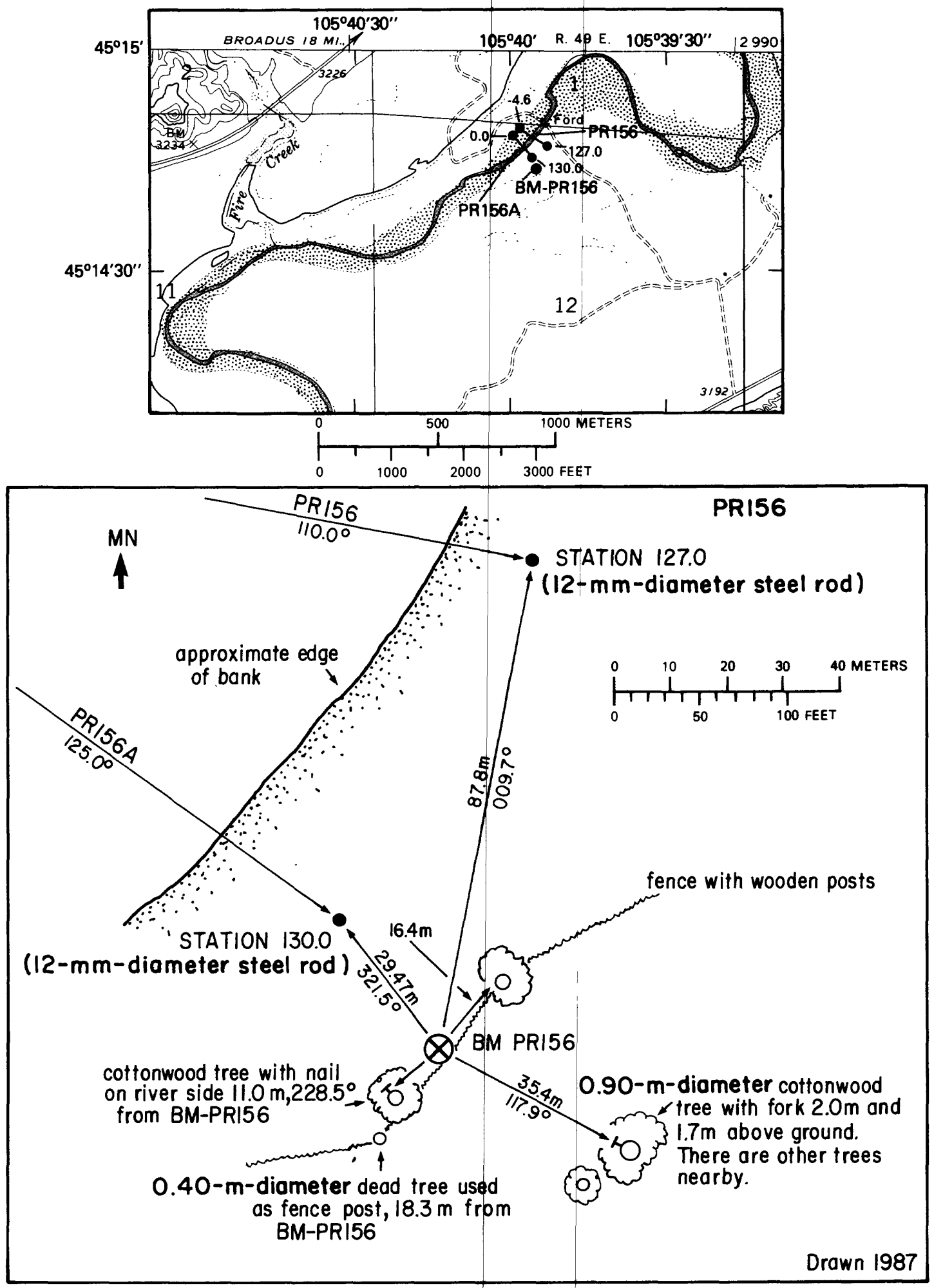

Figure 56.--Upper: Location of cross section PR156, bench mark BM-PR156, and left and right bank reference pins (tables 2 and 3 ) in the Huckins School quadrangle. Lower: Location of bench mark on the left bank. MN is magnetic north. 

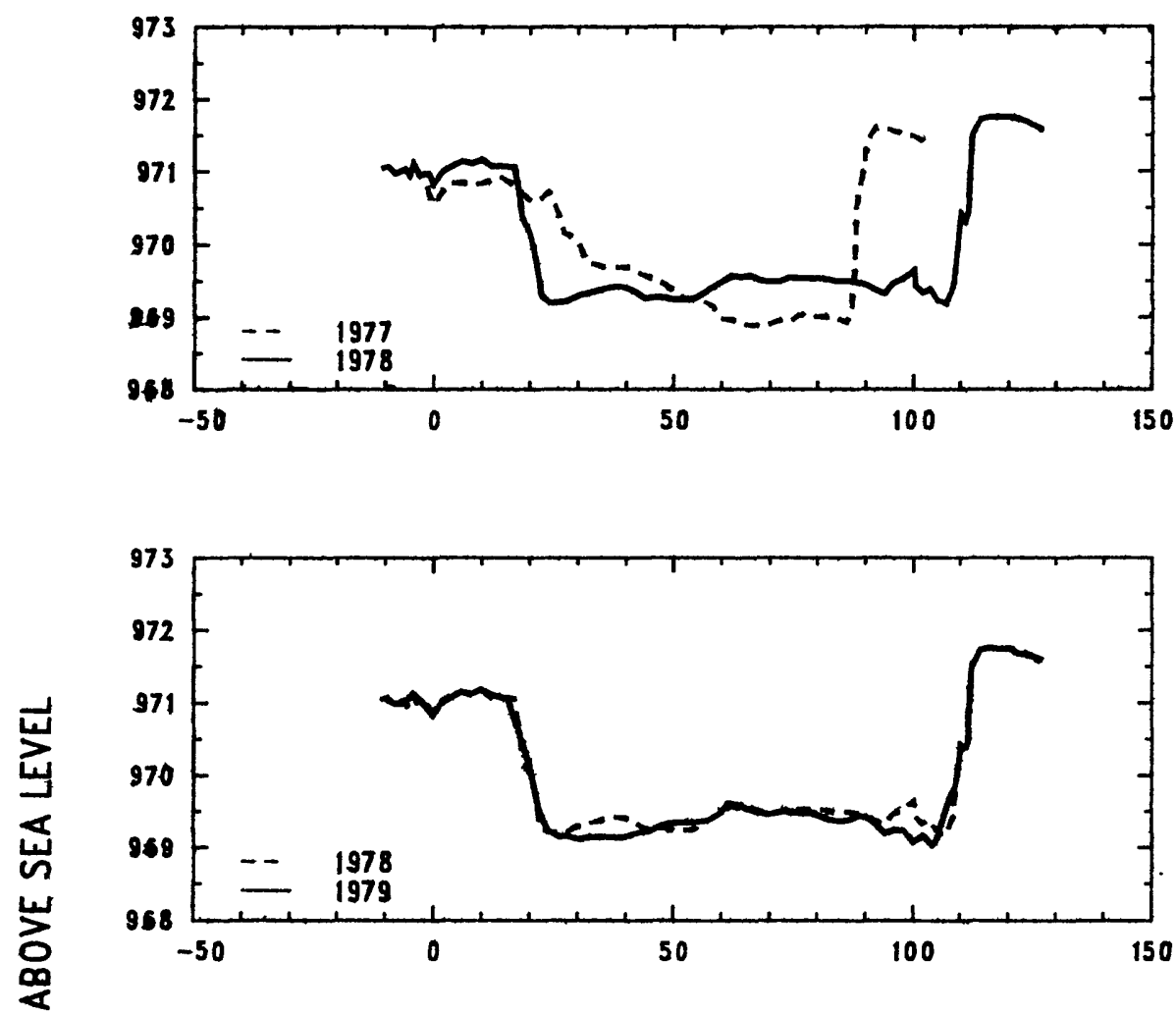

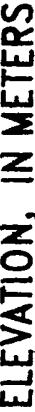
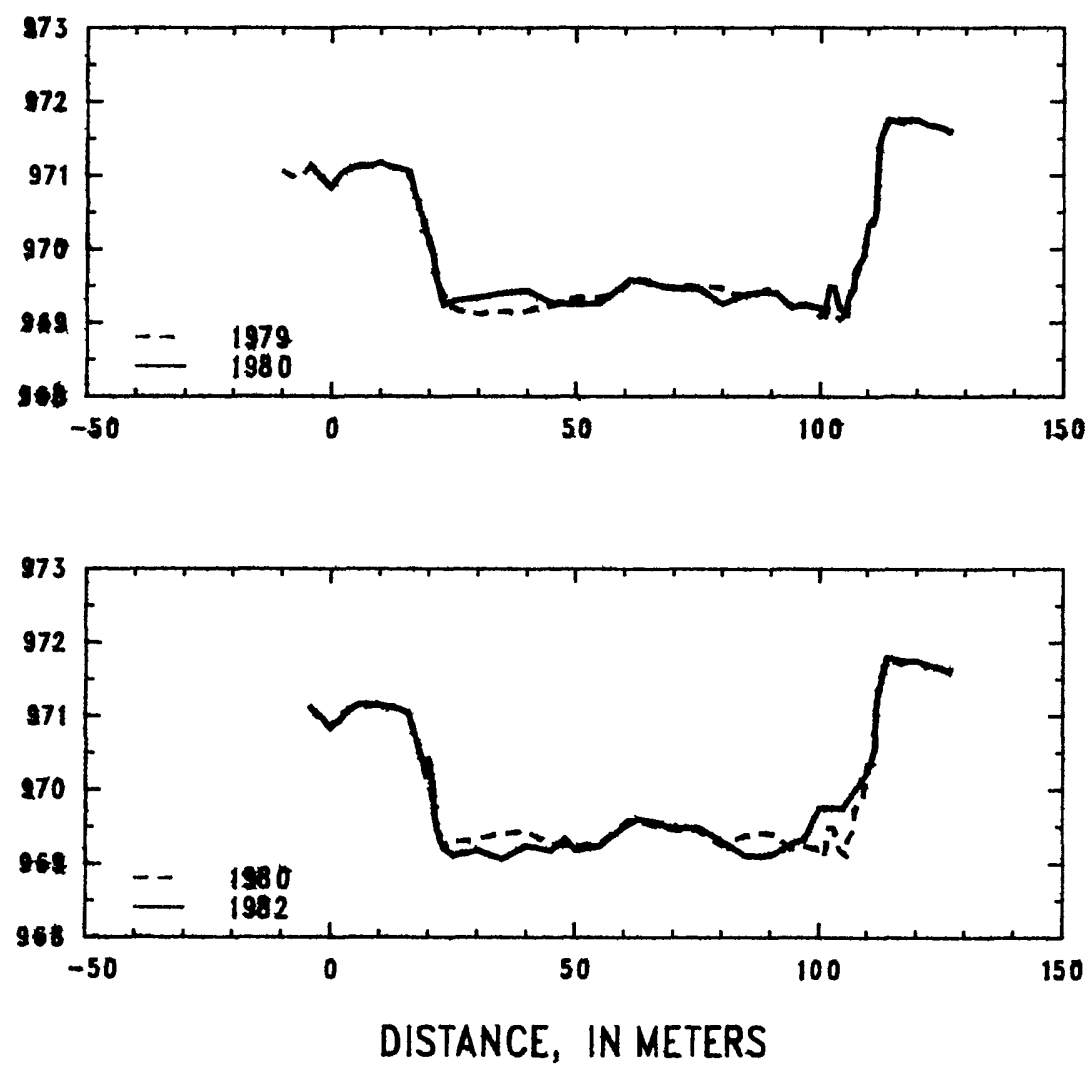

Figure 57.--Profiles of cross section PR 156 from 1977 to 1982. 


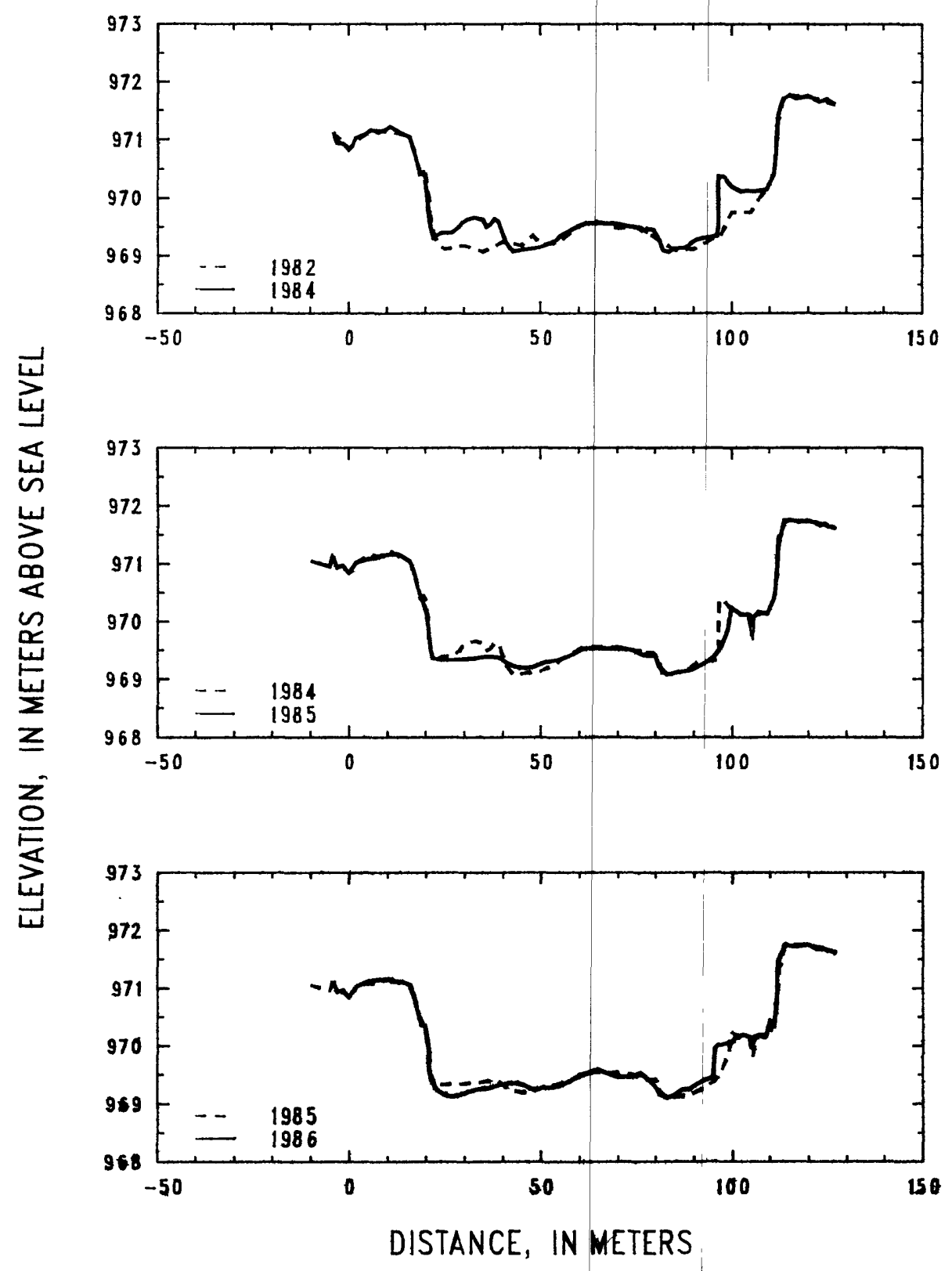

Figure 58.--Profiles of cross section PR156 from 1982 to 1986. 


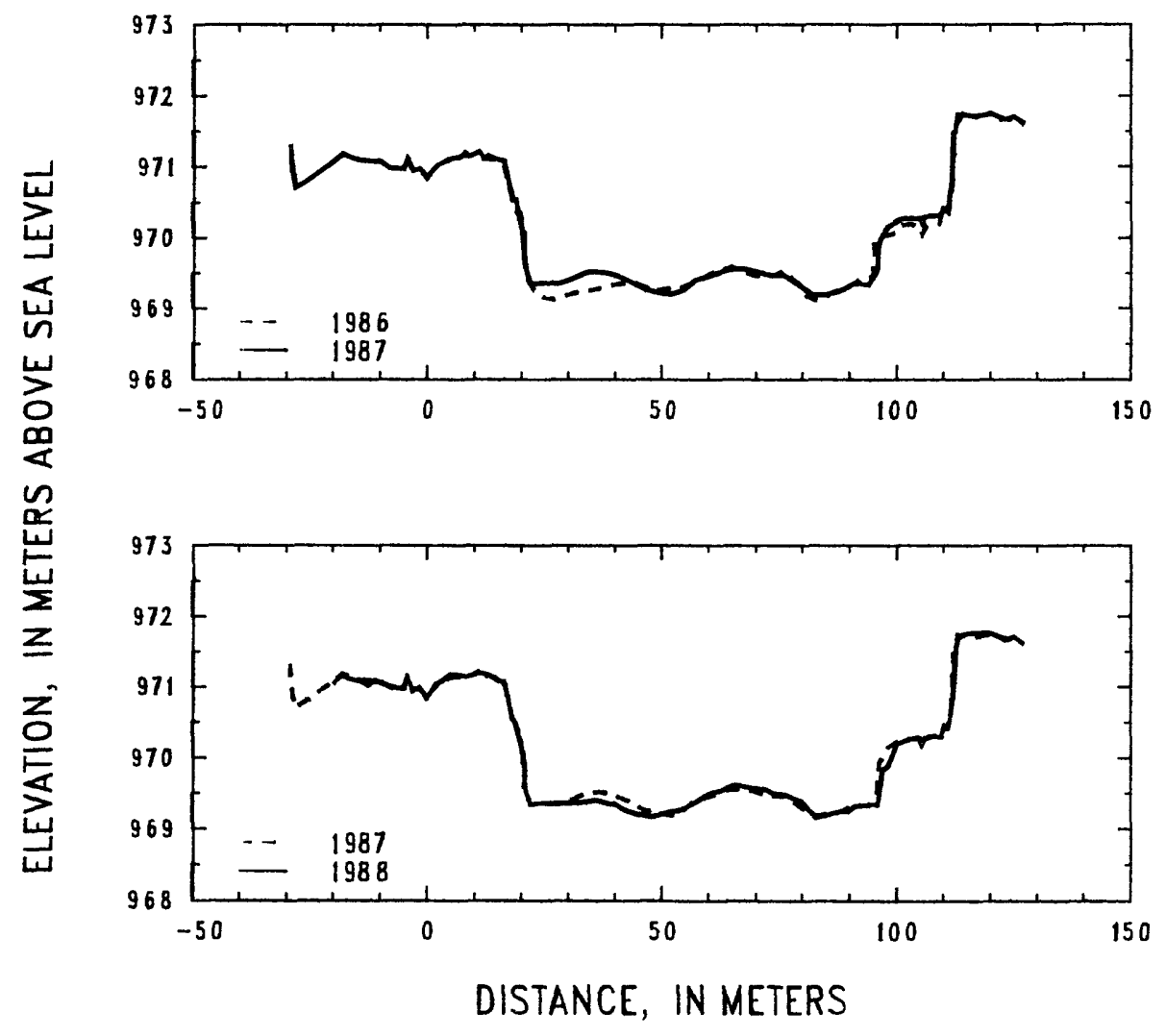

Figure 59.--Profiles of cross section PR156 trom 1986 to 1988. 
Table 20.--Listing of horizontal stations and elevations for cross section PR156

[Sta., station, distance in meters from a reference point on

the left bank; Elev., elevation, in meters above sea leve1].

\begin{tabular}{|c|c|c|c|c|c|c|c|c|c|}
\hline \multicolumn{2}{|c|}{1977} & \multicolumn{2}{|c|}{1977} & \multicolumn{2}{|c|}{1978} & \multicolumn{2}{|c|}{1978} & \multicolumn{2}{|c|}{1979} \\
\hline Sta. & Elev. & Sta. & Elev. & Sta. & Elev. & Sta. & Elev. & Sta. & Elev. \\
\hline-1.6 & 970.78 & 84.0 & 968.97 & -11.0 & 971.05 & 66.0 & 969.57 & -10.0 & 971.05 \\
\hline 0.0 & 970.54 & 86.0 & 968.92 & -9.5 & 971.07 & 68.0 & 969.50 & -8.0 & 970.98 \\
\hline 2.0 & 970.73 & 87.2 & 969.15 & -8.0 & 970.97 & 70.0 & 969.49 & -6.0 & 970.99 \\
\hline 4.0 & 970.84 & 87.8 & 969.65 & -6.0 & 971.04 & 72.0 & 969.49 & -4.2 & 971.13 \\
\hline 6.0 & 970.84 & 88.0 & 970.49 & -5.0 & 970.94 & 74.0 & 969.55 & -2.0 & 970.98 \\
\hline 8.0 & 970.82 & 89.7 & 971.06 & -4.2 & 971.12 & 76.0 & 969.54 & 0.0 & 970.82 \\
\hline 10.0 & 970.83 & 90.1 & 971.40 & -3.0 & 970.94 & 78.0 & 969.53 & 2.0 & 971.03 \\
\hline 12.0 & 970.87 & 92.0 & 971.61 & -2.0 & 970.97 & 80.0 & 969.54 & 4.0 & 971.08 \\
\hline 14.0 & 970.92 & 94.0 & 971.60 & -1.0 & 970.97 & 82.0 & 969.52 & 6.0 & 971.15 \\
\hline 16.0 & 970.83 & 96.0 & 971.54 & 0.0 & 970.82 & 84.0 & 969.49 & 8.0 & 971.12 \\
\hline 18.0 & 970.72 & 98.0 & 971.53 & 2.0 & 971.01 & 86.0 & 969.49 & 10.0 & 971.18 \\
\hline 20.0 & 970.59 & 100.0 & 971.48 & 4.0 & 971.08 & 88.0 & 969.48 & 12.0 & 971.10 \\
\hline 22.0 & 970.61 & 101.8 & 971.42 & 6.0 & 971.15 & 90.0 & 969.45 & 14.0 & 971.08 \\
\hline 24.0 & 970.71 & & & 8.0 & 971.11 & 92.0 & 969.37 & 15.5 & 971.06 \\
\hline 26.0 & 970.39 & & & 10.0 & 971.17 & 94.0 & 969.32 & 18.0 & 970.56 \\
\hline 27.1 & 970.15 & & & 12.0 & 971.07 & 95.5 & 969.46 & 20.0 & 970.14 \\
\hline 28.0 & 970.13 & & & 14.0 & 971.08 & 98.0 & 969.54 & 22.0 & 969.50 \\
\hline 30.0 & 970.03 & & & 16.8 & 971.05 & 100.2 & 969.65 & 24.0 & 969.23 \\
\hline 31.7 & 969.76 & & & 17.7 & 970.76 & 100.4 & 969.44 & 26.0 & 969.15 \\
\hline 34.0 & 969.71 & & & 18.0 & 970.53 & 100.6 & 969.42 & 28.0 & 969.16 \\
\hline 36.0 & 969.67 & & & 18.5 & 970.34 & 102.0 & 969.33 & 30.0 & 969.11 \\
\hline 38.0 & 969.68 & & & 19.8 & 970.18 & 103.5 & 969.39 & 33.0 & 969.15 \\
\hline 40.0 & 969.68 & & & 21.2 & 969.79 & 105.0 & 969.23 & 36.0 & 969.15 \\
\hline 42.0 & 969.61 & & & 22.4 & 969.30 & 107.0 & 969.18 & 39.0 & 969.14 \\
\hline 44.0 & 969.56 & & & 24.0 & 969.20 & 108.6 & 969.43 & 42.0 & 969.20 \\
\hline 46.0 & 969.52 & & & 26.0 & 969.21 & 110.0 & 970.42 & 45.0 & 969.23 \\
\hline 48.0 & 969.45 & & & 28.0 & 969.23 & 111.0 & 970.31 & 48.0 & 969.32 \\
\hline 50.0 & 969.35 & & & 30.0 & 969.30 & 111.6 & 970.45 & 51.0 & 969.35 \\
\hline 52.0 & 969.26 & & & 32.0 & 969.33 & 112.3 & 971.51 & 54.0 & 969.35 \\
\hline 54.0 & 969.24 & & & 34.0 & 969.37 & 114.0 & 971.73 & 57.0 & 969.37 \\
\hline 56.0 & 969.22 & & & 36.0 & 969.40 & 116.0 & 971.76 & 60.0 & 969.49 \\
\hline 58.0 & 969.15 & & & 38.0 & 969.43 & 120.0 & 971.76 & 61.4 & 969.62 \\
\hline 59.0 & 969.03 & & & 40.0 & 969.41 & 123.0 & 971.72 & 64.0 & 969.58 \\
\hline 60.0 & 968.97 & & & 42.0 & 969.34 & 126.0 & 971.62 & 67.0 & 969.49 \\
\hline 62.0 & 968.96 & & & 44.0 & 969.26 & 127.0 & 971.58 & 70.0 & 969.46 \\
\hline 64.0 & 968.90 & & & 46.0 & 969.27 & & & 73.0 & 969.51 \\
\hline 66.0 & 968.88 & & & 48.0 & 969.28 & & & 76.0 & 969.48 \\
\hline 68.0 & 968.87 & & & 50.0 & 969.24 & & & 79.0 & 969.48 \\
\hline 70.0 & 968.91 & & & 52.0 & 969.24 & & & 82.0 & 969.39 \\
\hline 72.0 & 968.95 & & & 54.0 & 969.25 & & & 85.0 & 969.36 \\
\hline 74.0 & 968.93 & & & 56.0 & 969.32 & & & 87.0 & 969.38 \\
\hline 76.0 & 969.03 & & & 58.0 & 969.42 & & & 89.0 & 969.45 \\
\hline 78.0 & 969.01 & & & 60.0 & 969.49 & & & 90.0 & 969.42 \\
\hline 80.0 & 969.00 & & & 62.0 & 969.57 & & & 92.0 & 969.34 \\
\hline 82.0 & 968.99 & & & 64.0 & 969.55 & & & 94.0 & 969.19 \\
\hline
\end{tabular}


Table 20.--Listing of horizontal stations and elevations for cross section PR156 --Continued.

\begin{tabular}{|c|c|c|c|c|c|c|c|c|c|}
\hline \multicolumn{2}{|c|}{1979} & \multicolumn{2}{|c|}{1980} & \multicolumn{2}{|c|}{1980} & \multicolumn{2}{|c|}{1982} & \multicolumn{2}{|c|}{1984} \\
\hline Sta. & Elev. & Sta. & Elev. & Sta. & Elev. & Sta. & Elev. & Sta. & Elev. \\
\hline 96.0 & 969.25 & -4.2 & 971.12 & 111.0 & 970.35 & -4.2 & 971.12 & -4.2 & 971.11 \\
\hline 98.0 & 969.23 & -1.6 & 970.92 & 111.6 & 970.49 & -1.6 & 970.96 & -3.0 & 970.93 \\
\hline 100.0 & 969.07 & 0.0 & 970.83 & 112.3 & 971.45 & 0.0 & 970.82 & -1.6 & 970.93 \\
\hline 102.0 & 969.17 & 2.0 & 971.03 & 114.0 & 971.76 & 3.0 & 971.02 & 0.0 & 970.83 \\
\hline 104.0 & 969.03 & 5.0 & 971.12 & 117.0 & 971.71 & 6.0 & 971.16 & 1.0 & 970.88 \\
\hline 105.0 & 969.11 & 8.0 & 971.13 & 118.0 & 971.76 & 10.0 & 971.15 & 2.0 & 971.03 \\
\hline 106.0 & 969.33 & 10.0 & 971.18 & 120.0 & 971.76 & 14.0 & 971.09 & 4.0 & 971.08 \\
\hline 108.0 & 969.73 & 12.0 & 971.11 & 122.0 & 971.68 & 16.0 & 971.04 & 6.0 & 971.16 \\
\hline 109.0 & 969.83 & 14.0 & 971.10 & 125.0 & 971.65 & 19.0 & 970.36 & 8.0 & 971.12 \\
\hline 110.0 & 970.32 & 16.0 & 971.05 & 127.0 & 971.59 & 20.0 & 970.40 & 11.0 & 971.22 \\
\hline 111.6 & 970.46 & 18.0 & 970.54 & & & 21.0 & 970.03 & 13.0 & 971.13 \\
\hline 112.4 & 971.49 & 20.0 & 970.10 & & & 21.5 & 969.55 & 15.0 & 971.07 \\
\hline 114.0 & 971.73 & 21.0 & 969.87 & & & 23.0 & 969.21 & $1 \therefore .0$ & 971.04 \\
\hline 116.0 & 971.76 & 21.1 & 969.67 & & & 25.0 & 969.11 & 17.0 & 970.83 \\
\hline 118.0 & 971.74 & 22.0 & 969.49 & & & 30.0 & 969.18 & 18.0 & 970.55 \\
\hline 120.0 & 971.75 & 23.0 & 969.24 & & & 35.0 & 969.06 & 18.5 & 970.40 \\
\hline 122.0 & 971.68 & 25.0 & 969.30 & & & 40.0 & 969.24 & 19.5 & 970.44 \\
\hline 124.0 & 971.66 & 30.0 & 969.34 & & & 45.0 & 969.17 & 19.9 & 970.38 \\
\hline 126.0 & 971.62 & 35.0 & 969.41 & & & 48.0 & 969.35 & 20.6 & 970.02 \\
\hline \multirow[t]{26}{*}{127.0} & 971.59 & 40.0 & 969.44 & & & 50.0 & 969.18 & 21.0 & 969.61 \\
\hline & & 45.0 & 969.27 & & & 55.0 & 969.24 & 22.0 & 969.30 \\
\hline & & 50.0 & 969.24 & & & 60.0 & 969.50 & 24.0 & 969.39 \\
\hline & & 55.0 & 969.27 & & & 63.0 & 969.60 & 27.0 & 969.40 \\
\hline & & 61.0 & 969.59 & & & 66.0 & 969.57 & 29.0 & 969.50 \\
\hline & & 65.0 & 969.54 & & & 70.0 & 969.47 & 29.3 & 969.55 \\
\hline & & 70.0 & 969.46 & & & 75.0 & 969.49 & 31.0 & 969.63 \\
\hline & & 75.0 & 969.46 & & & 80.0 & 969.31 & 33.0 & 969.66 \\
\hline & & 80.0 & 969.25 & & & 85.0 & 969.10 & 35.0 & 969.61 \\
\hline & & 83.0 & 969.34 & & & 90.0 & 969.11 & 36.0 & 969.49 \\
\hline & & 87.0 & 969.42 & & & 95.0 & 969.30 & 37.0 & 969.54 \\
\hline & & 89.0 & 969.42 & & & 97.0 & 969.33 & 38.0 & 969.63 \\
\hline & & 91.0 & 969.40 & & & 100.0 & 969.75 & 39.0 & 969.59 \\
\hline & & 94.0 & 969.21 & & & 105.0 & 969.75 & 41.0 & 969.21 \\
\hline & & 97.0 & 969.24 & & & 107.0 & 969.95 & 43.0 & 969.06 \\
\hline & & 100.0 & 969.19 & & & 110.0 & 970.22 & 45.0 & 969.09 \\
\hline & & 101.0 & 969.11 & & & 111.5 & 970.52 & 50.0 & 969.14 \\
\hline & & 102.0 & 969.49 & & & 112.0 & 971.22 & 54.0 & 969.26 \\
\hline & & 103.0 & 969.48 & & & 114.0 & 971.80 & 55.0 & 969.33 \\
\hline & & 104.0 & 969.20 & & & 117.0 & 971.74 & 60.0 & 969.52 \\
\hline & & 105.5 & 969.12 & & & 120.0 & 971.74 & 62.0 & 969.56 \\
\hline & & 105.9 & 969.32 & & & 123.0 & 971.68 & 65.0 & 969.56 \\
\hline & & 107.0 & 969.45 & & & 127.0 & 971.60 & 70.0 & 969.55 \\
\hline & & 107.4 & 969.72 & & & & & 73.0 & 969.50 \\
\hline & & 109.2 & 969.91 & & & & & 76.0 & 969.48 \\
\hline & & 110.0 & 970.34 & & & & & 77.5 & 969.41 \\
\hline
\end{tabular}


Table 20.--Listing of horizontal stations and elevations for cross section PR156 --Continued.

\begin{tabular}{|c|c|c|c|c|c|c|c|c|c|}
\hline \multicolumn{2}{|c|}{1984} & \multicolumn{2}{|c|}{1985} & \multicolumn{2}{|c|}{1985} & \multicolumn{2}{|c|}{1986} & \multicolumn{2}{|c|}{1986} \\
\hline Sta. & Elev. & Sta. & Elev. & Sta. & Elev. & Sta. & Elev. & Sta. & Elev. \\
\hline 77.8 & 969.47 & -10.0 & 971.05 & 99.0 & 969.86 & -4.2 & 971.12 & 91.0 & 969.36 \\
\hline 79.5 & 969.44 & -5.0 & 970.96 & 99.7 & 970.24 & -3.0 & 970.95 & 93.0 & 969.42 \\
\hline 81.0 & 969.28 & -4.2 & 971.12 & 102.0 & 970.13 & -1.6 & 970.95 & 94.9 & 969.45 \\
\hline 82.0 & 969.08 & -3.0 & 970.93 & 104.5 & 970.11 & 0.0 & 970.84 & 95.3 & 969.96 \\
\hline 83.5 & 969.05 & -1.6 & 970.97 & 105.4 & 969.80 & 2.0 & 971.03 & 96.0 & 970.01 \\
\hline 85.0 & 969.12 & 0.0 & 970.84 & 105.8 & 970.08 & 5.0 & 971.12 & 98.0 & 970.04 \\
\hline 88.0 & 969.14 & 2.0 & 971.02 & 107.0 & 970.17 & 7.0 & 971.15 & 100.0 & 970.08 \\
\hline 90.0 & 969.25 & 4.0 & 971.07 & 109.3 & 970.13 & 10.0 & 971.17 & 101.0 & 970.17 \\
\hline 92.0 & 969.30 & 7.0 & 971.10 & 111.2 & 970.45 & 12.0 & 971.11 & 103.0 & 970.19 \\
\hline 94.0 & 969.31 & 10.0 & 971.16 & 111.8 & 970.79 & 14.0 & 971.11 & 105.0 & 970.13 \\
\hline 96.1 & 969.34 & 13.0 & 971.16 & 112.4 & 971.34 & 16.0 & 971.05 & 105.4 & 970.04 \\
\hline 96.5 & 969.51 & 16.0 & 971.04 & 113.0 & 971.48 & 17.0 & 970.83 & 106.0 & 970.16 \\
\hline 96.5 & 970.37 & 17.0 & 970.87 & 113.7 & 971.75 & 18.8 & 970.36 & 107.0 & 970.19 \\
\hline 98.0 & 970.35 & 18.0 & 970.56 & 115.0 & 971.76 & 19.8 & 970.35 & 109.0 & 970.19 \\
\hline 100.0 & 970.19 & 20.0 & 970.20 & 117.0 & 971.73 & 20.6 & 970.04 & 110.0 & 970.43 \\
\hline 102.5 & 970.10 & 20.8 & 969.91 & 121.0 & 971.73 & 20.9 & 969.55 & 111.0 & 970.35 \\
\hline 104.0 & 970.12 & 21.2 & 969.51 & 124.0 & 971.67 & 21.5 & 969.38 & 111.8 & 970.78 \\
\hline 106.0 & 970.11 & 22.0 & 969.36 & 127.0 & 971.61 & 23.0 & 969.23 & 112.0 & 971.46 \\
\hline 108.0 & 970.13 & 24.0 & 969.33 & & & 25.0 & 969.14 & 114.0 & 971.75 \\
\hline 109.4 & 970.15 & 27.0 & 969.33 & & & 27.0 & 969.12 & 115.0 & 971.72 \\
\hline 111.1 & 970.39 & 30.0 & 969.35 & & & 30.0 & 969.19 & 117.0 & 971.71 \\
\hline 111.9 & 970.78 & 33.0 & 969.37 & & & 33.0 & 969.25 & 120.0 & 971.75 \\
\hline 112.4 & 971.44 & 36.0 & 969.40 & & & 36.0 & 969.27 & 122.0 & 971.67 \\
\hline 113.6 & 971.69 & 39.0 & 969.38 & & & 39.0 & 969.32 & 124.0 & 971.67 \\
\hline 115.0 & 971.76 & 42.0 & 969.24 & & & 42.0 & 969.37 & 127.0 & 971.60 \\
\hline 117.0 & 971.70 & 45.0 & 969.19 & & & 45.0 & 969.33 & & \\
\hline 120.0 & 971.75 & 48.0 & 969.21 & & & 48.0 & 969.24 & & \\
\hline 123.0 & 971.65 & 51.0 & 969.30 & & & 51.0 & 969.28 & & \\
\hline 125.0 & 971.70 & 54.0 & 969.33 & & & 54.0 & 969.29 & & \\
\hline 127.0 & 971.61 & 57.0 & 969.39 & & & 57.0 & 969.37 & & \\
\hline & & 61.0 & 969.53 & & & 60.0 & 969.48 & & \\
\hline & & 64.0 & 969.55 & & & 63.0 & 969.54 & & \\
\hline & & 67.0 & 969.53 & & & 64.8 & 969.59 & & \\
\hline & & 71.0 & 969.53 & & & 66.0 & 969.55 & & \\
\hline & & 74.0 & 969.50 & & & 69.0 & 969.47 & & \\
\hline & & 77.0 & 969.41 & & & 72.0 & 969.46 & & \\
\hline & & 80.0 & 969.41 & & & 74.0 & 969.47 & & \\
\hline & & 81.0 & 969.22 & & & 76.0 & 969.53 & & \\
\hline & & 83.0 & 969.08 & & & 79.0 & 969.36 & & \\
\hline & & 86.0 & 969.13 & & & 80.4 & 969.24 & & \\
\hline & & 89.0 & 969.16 & & & 81.0 & 969.16 & & \\
\hline & & 92.0 & 969.25 & & & 83.0 & 969.10 & & \\
\hline & & 95.0 & 969.38 & & & 85.0 & 969.16 & & \\
\hline & & 96.0 & 969.47 & & & 86.6 & 969.24 & & \\
\hline & & 97.0 & 969.55 & & & 89.0 & 969.25 & & \\
\hline
\end{tabular}


Table 20.--Listing of horizontal stations and elevations for cross section PR156 --Continued.

\begin{tabular}{|c|c|c|c|c|c|c|c|}
\hline \multicolumn{2}{|c|}{1987} & \multicolumn{2}{|c|}{1987} & \multicolumn{2}{|c|}{1988} & \multicolumn{2}{|c|}{1988} \\
\hline Sta. & Elev. & Sta. & Elev. & Sta. & Elev. & Sta. & Elev. \\
\hline-29.5 & 971.31 & 70.0 & 969.52 & -20.0 & 971.04 & 66.0 & 969.62 \\
\hline-28.0 & 970.70 & 73.0 & 969.46 & -18.0 & 971.16 & 68.0 & 969.59 \\
\hline-25.0 & 970.83 & 76.0 & 969.47 & -16.0 & 971.09 & 70.0 & 969.56 \\
\hline-20.0 & 971.07 & 79.0 & 969.34 & -14.0 & 971.08 & 72.0 & 969.56 \\
\hline-18.0 & 971.19 & 82.0 & 969.19 & -12.6 & 971.01 & 74.0 & 969.49 \\
\hline-15.0 & 971.10 & 85.0 & 969.19 & -11.0 & 971.09 & 76.0 & 969.49 \\
\hline-10.0 & 971.07 & 88.0 & 969.24 & -8.0 & 971.00 & 78.0 & 969.44 \\
\hline-8.0 & 970.98 & 91.0 & 969.35 & -5.0 & 970.97 & 80.0 & 969.38 \\
\hline-5.0 & 970.97 & 94.0 & 969.33 & -4.2 & 971.13 & 81.0 & 969.29 \\
\hline-4.2 & 971.12 & 95.9 & 969.53 & -3.0 & 970.94 & 82.0 & 969.23 \\
\hline-3.0 & 970.94 & 96.2 & 969.94 & -1.6 & 970.99 & 83.0 & 969.16 \\
\hline-1.6 & 970.98 & 98.0 & 970.16 & 0.0 & 970.83 & 84.0 & 969.18 \\
\hline 0.0 & 970.85 & 99.6 & 970.22 & 2.0 & 971.04 & 86.0 & 969.21 \\
\hline 2.0 & 971.02 & 101.0 & 970.27 & 3.0 & 971.06 & 88.0 & 969.25 \\
\hline 5.0 & 971.13 & 103.0 & 970.28 & 5.0 & 971.17 & 89.8 & 969.25 \\
\hline 7.0 & 971.14 & 104.9 & 970.27 & 7.0 & 971.15 & 91.0 & 969.32 \\
\hline 8.0 & 971.20 & 105.4 & 970.20 & 9.0 & 971.14 & 93.0 & 969.34 \\
\hline 9.0 & 971.14 & 106.0 & 970.29 & 11.0 & 971.20 & 95.0 & 969.35 \\
\hline 11.0 & 971.22 & 107.0 & 970.31 & 13.0 & 971.17 & 96.0 & 969.34 \\
\hline 12.0 & 971.11 & 109.4 & 970.31 & 15.0 & 971.08 & 97.0 & 969.84 \\
\hline 13.0 & 971.16 & 110.0 & 970.42 & 16.3 & 971.07 & 98.0 & 969.88 \\
\hline 15.0 & 971.10 & 111.0 & 970.41 & 17.0 & 970.91 & 99.0 & 970.03 \\
\hline 16.3 & 971.08 & 111.7 & 970.77 & 18.0 & 970.59 & 100.0 & 970.20 \\
\hline 17.0 & 970.89 & 112.0 & 971.48 & 19.0 & 970.46 & 101.0 & 970.22 \\
\hline 18.2 & 970.53 & 112.5 & 971.50 & 20.0 & 970.16 & 103.0 & 970.28 \\
\hline 18.9 & 970.53 & 113.0 & 971.74 & 20.7 & 969.92 & 104.6 & 970.29 \\
\hline 19.8 & 970.17 & 115.0 & 971.72 & 20.8 & 969.57 & 105.4 & 970.18 \\
\hline 20.3 & 970.03 & 117.0 & 971.71 & 22.0 & 969.33 & 106.0 & 970.27 \\
\hline 20.7 & 969.59 & 118.0 & 971.72 & 24.0 & 969.36 & 108.0 & 970.31 \\
\hline 22.0 & 969.34 & 120.0 & 971.75 & 27.0 & 969.37 & 109.5 & 970.30 \\
\hline 25.0 & 969.36 & 123.0 & 971.67 & 30.0 & 969.36 & 110.2 & 970.46 \\
\hline 28.0 & 969.34 & 125.0 & 971.71 & 33.0 & 969.38 & 111.0 & 970.42 \\
\hline 31.0 & 969.42 & 127.0 & 971.61 & 36.0 & 969.41 & 111.4 & 970.52 \\
\hline 34.0 & 969.51 & & & 39.0 & 969.35 & 112.0 & 970.87 \\
\hline 37.0 & 969.52 & & & 40.0 & 969.35 & 112.5 & 971.47 \\
\hline 40.0 & 969.47 & & & 42.0 & 969.26 & 113.0 & 971.69 \\
\hline 43.0 & 969.38 & & & 45.0 & 969.20 & 114.0 & 971.75 \\
\hline 46.0 & 969.27 & & & 48.0 & 969.16 & 116.0 & 971.77 \\
\hline 49.0 & 969.22 & & & 51.0 & 969.23 & 118.0 & 971.76 \\
\hline 52.0 & 969.19 & & & 54.0 & 969.26 & 120.0 & 971.77 \\
\hline 55.0 & 969.27 & & & 57.0 & 969.36 & 122.0 & 971.71 \\
\hline 57.0 & 969.40 & & & 60.0 & 969.50 & 124.0 & 971.68 \\
\hline 61.0 & 969.48 & & & 62.0 & 969.53 & 125.0 & 971.71 \\
\hline 64.0 & 969.55 & & & 64.0 & 969.58 & 127.0 & 971.62 \\
\hline 67.0 & 969.57 & & & 65.0 & 969.61 & & \\
\hline
\end{tabular}




\section{Description of Cross Section PR163}

Location: Township 6 South/Range 50 East--section 32

U.S. Geological Survey quadrangle $(1: 24,000)$ : Yarger Butte

Landowners--left bank: Gay Ranch

right bank: Gay Ranch

Access: left bank

Permission from: Gay Ranch (T7S/R49E--20).

Reference pins

\begin{tabular}{lcl}
\hline $\begin{array}{l}\text { Station } \\
\text { (meters) }\end{array}$ & $\begin{array}{c}\text { Elevation } \\
\text { (meters } \\
\text { above } \\
\text { sea level) }\end{array}$ & Comments \\
& & Pin -73.0 was closest to leveling \\
& & instrument. \\
73.0 & 965.431 & 0.14 meter above 1987 ground level. \\
75.0 & 964.417 & 0.15 meter above 1987 ground level. \\
110.9 & 964.549 & 0.15 meter above 1987 ground level. \\
\hline
\end{tabular}




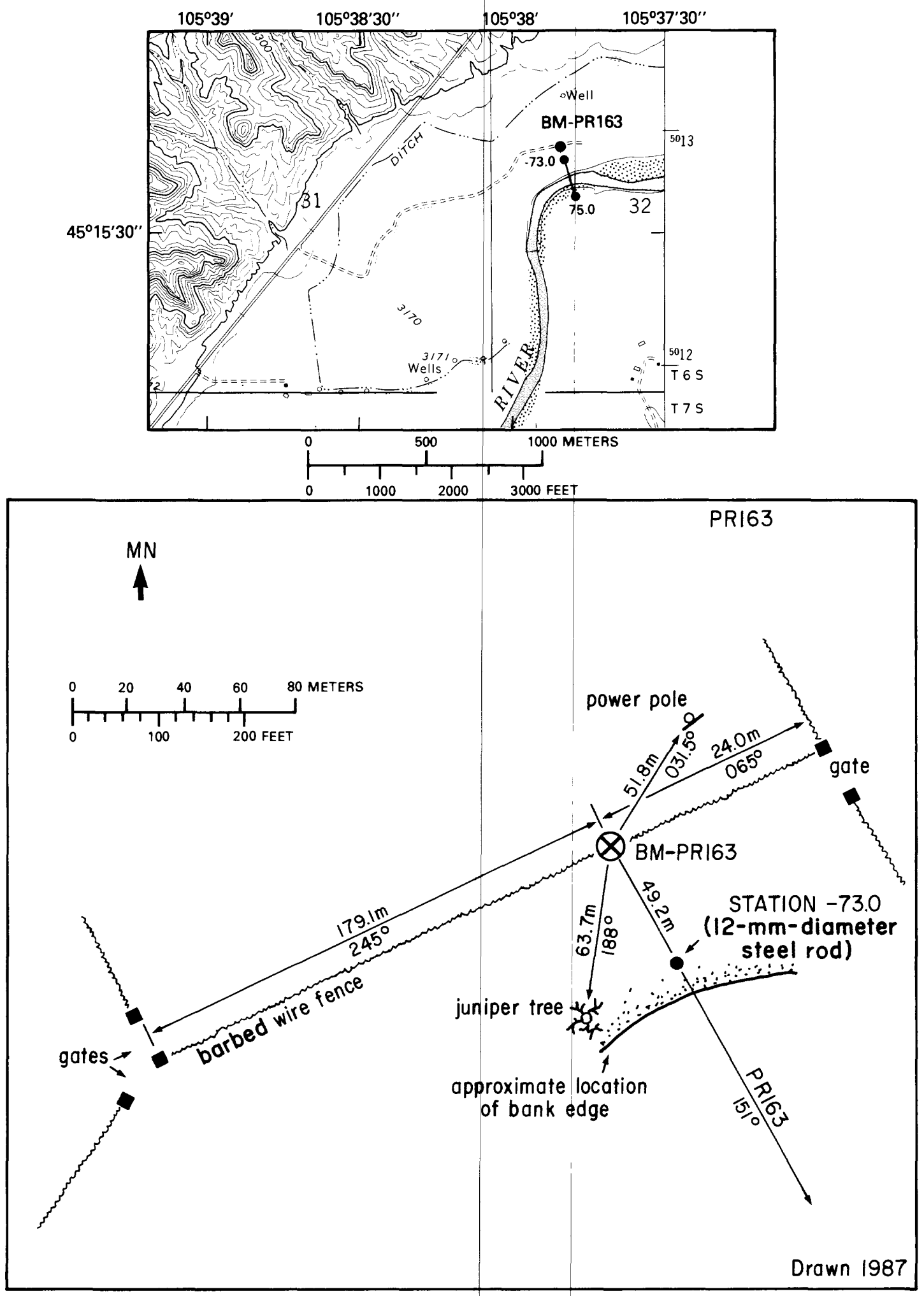

Figure 60.--Upper: Location of cross section PR163, bench mark BM-PR163, and left and right bank reference pins (tables 2 and 3 ) in the Yarger Butte quadrangle. Lower: Location of bench mark on the left bank. $M N$ is magnetic north. 

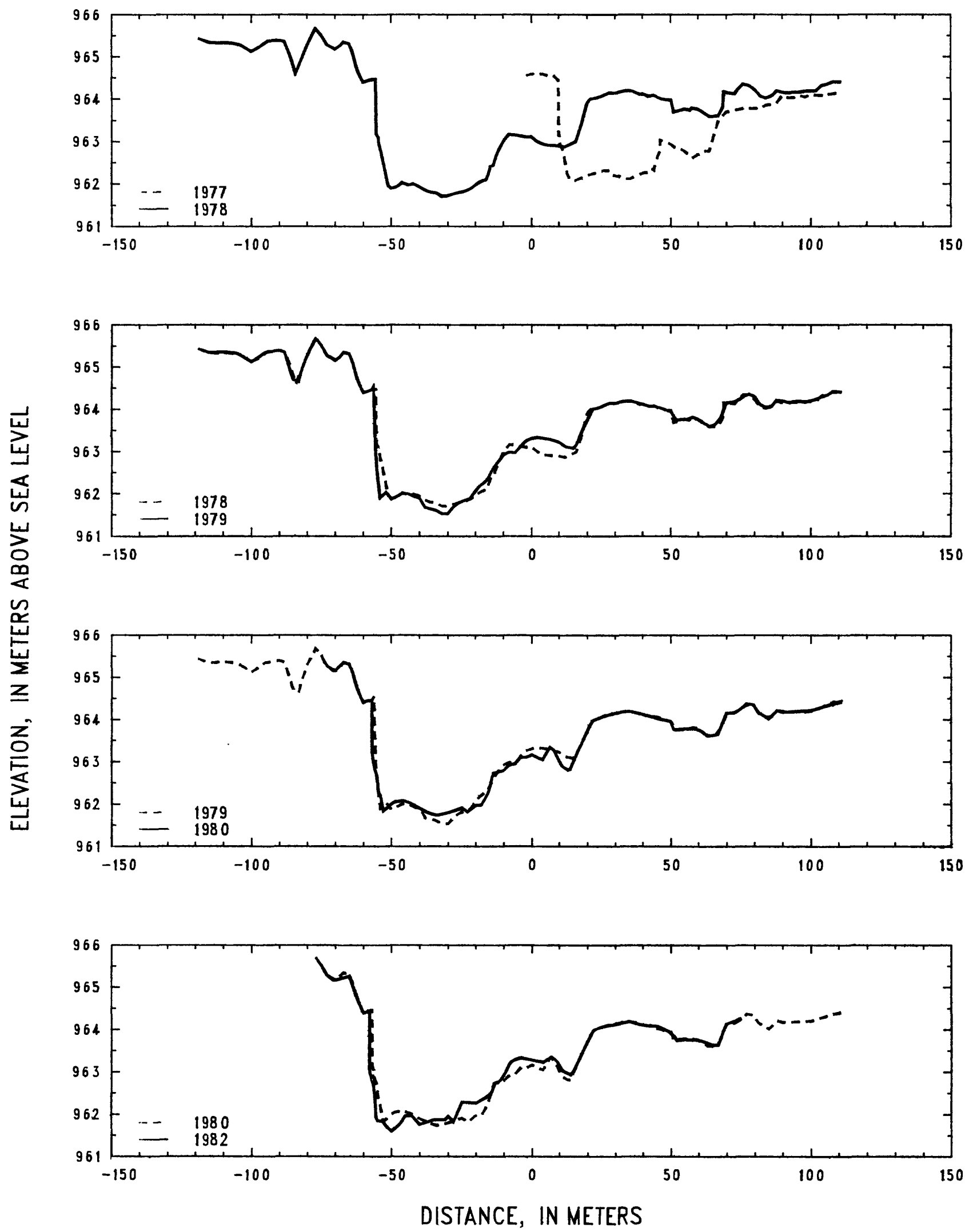

Figure 61.--Profiles of cross section PR163 from 1977 to 1982. 


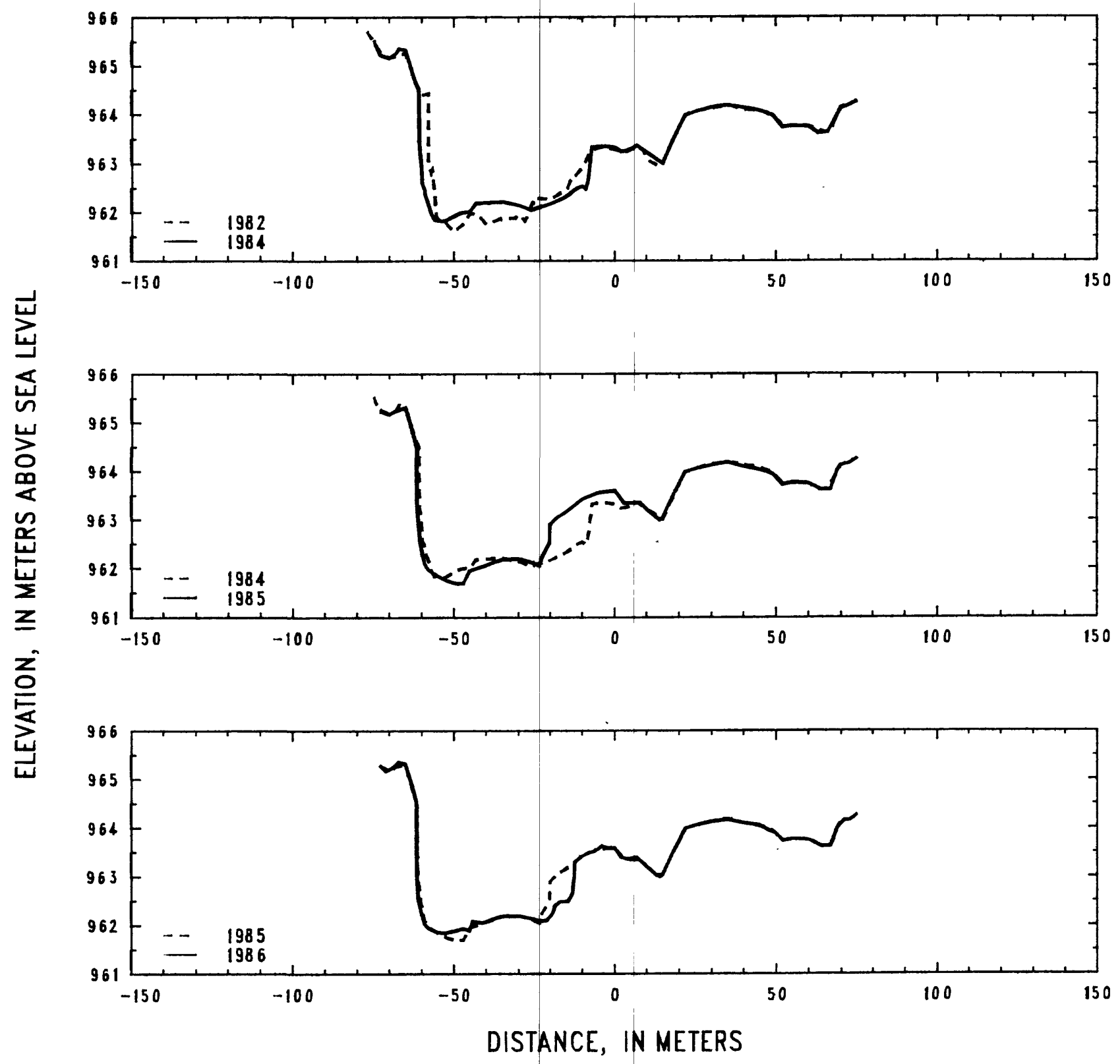

Figure 62.--Profiles of cross section PR163 from 1982 to 1986. 


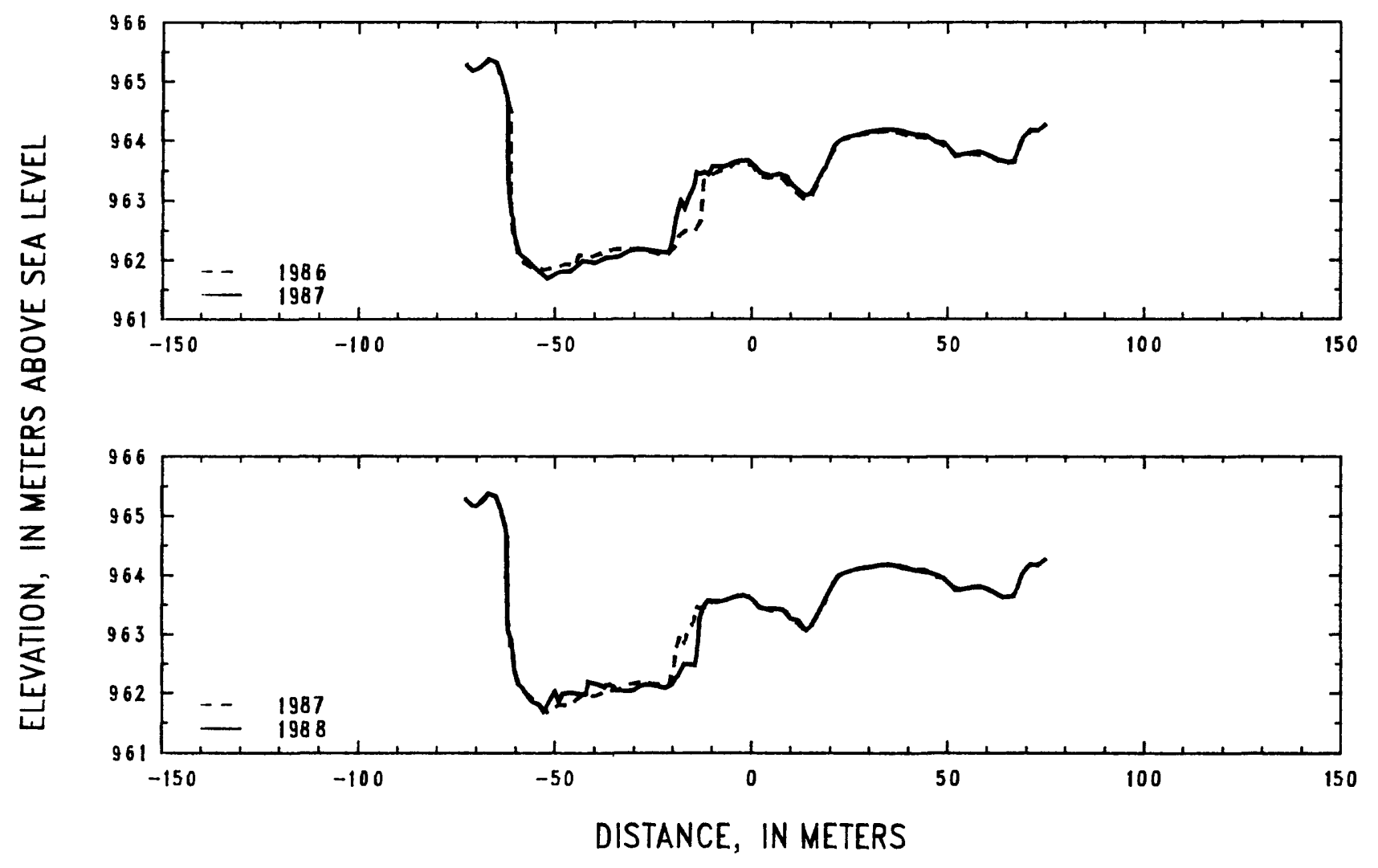

Figure 63.--Profiles of cross section PR163 from 1986 to 1988. 
Table 21.--Listing of horizontal stations and elevations for cross section PR163 [Sta., station, distance in meters from a reference point on the left bank; Elev., elevation, in meters above sea level].

\begin{tabular}{|c|c|c|c|c|c|c|c|c|c|}
\hline \multicolumn{2}{|c|}{1977} & \multicolumn{2}{|c|}{1977} & \multicolumn{2}{|c|}{1978} & \multicolumn{2}{|c|}{1978} & \multicolumn{2}{|c|}{1978} \\
\hline Sta. & Elev. & Sta. & Elev. & Sta. & Elev. & Sta. & Elev. & Sta. & Elev. \\
\hline-1.6 & 964.56 & 67.1 & 963.48 & -119.0 & 965.44 & -24.0 & 961.82 & 68.8 & 963.81 \\
\hline 0.0 & 964.59 & 70.0 & 963.70 & -115.0 & 965.34 & -22.0 & 961.88 & 69.2 & 964.18 \\
\hline 2.0 & 964.58 & 72.0 & 963.73 & -112.0 & 965.33 & -20.0 & 961.97 & 70.0 & 964.16 \\
\hline 4.0 & 964.60 & 74.0 & 963.75 & -108.0 & 965.34 & -18.0 & 962.06 & 73.0 & 964.12 \\
\hline 6.0 & 964.55 & 76.0 & 963.78 & -104.0 & 965.29 & -16.0 & 962.11 & 74.0 & 964.22 \\
\hline 8.0 & 964.56 & 78.0 & 963.79 & -100.0 & 965.12 & -15.0 & 962.30 & 76.0 & 964.35 \\
\hline 9.8 & 964.45 & 80.0 & 963.78 & -97.0 & 965.24 & -14.6 & 962.41 & 78.0 & 964.33 \\
\hline 9.8 & 963.18 & 82.0 & 963.78 & -94.0 & 965.38 & -13.5 & 962.44 & 80.0 & 964.23 \\
\hline 10.1 & 963.20 & 84.0 & 963.85 & -90.0 & 965.40 & -12.0 & 962.75 & 82.0 & 964.07 \\
\hline 10.3 & 962.95 & 86.0 & 963.87 & -88.0 & 965.36 & -10.0 & 963.00 & 84.0 & 964.02 \\
\hline 11.1 & 962.85 & 88.0 & 963.91 & -86.0 & 965.03 & -8.0 & 963.17 & 86.0 & 964.07 \\
\hline 12.6 & 962.37 & 90.0 & 964.09 & -84.0 & 964.60 & -6.0 & 963.15 & 88.0 & 964.19 \\
\hline 13.0 & 962.23 & 92.0 & 964.03 & -82.0 & 964.93 & -2.0 & 963.10 & 90.0 & 964.15 \\
\hline 14.0 & 962.10 & 94.0 & 964.04 & -80.0 & 965.28 & 0.0 & 963.11 & 92.0 & 964.14 \\
\hline 15.0 & 962.07 & 96.0 & 964.09 & -77.0 & 965.67 & 2.0 & 962.99 & 94.0 & 964.17 \\
\hline 16.0 & 962.08 & 98.0 & 964.05 & -75.0 & 965.52 & 4.0 & 962.93 & 96.0 & 964.15 \\
\hline 18.0 & 962.14 & 100.0 & 964.11 & -73.0 & 965.29 & 6.0 & 962.90 & 98.0 & 964.19 \\
\hline 20.0 & 962.20 & 102.0 & 964.09 & -70.0 & 965.17 & 8.0 & 962.90 & 100.0 & 964.19 \\
\hline 22.0 & 962.24 & 104.0 & 964.09 & -68.0 & 965.27 & 10.0 & 962.89 & 102.0 & 964.20 \\
\hline 24.0 & 962.29 & 110.8 & 964.16 & -67.0 & 965.35 & 12.0 & 962.86 & 104.0 & 964.33 \\
\hline 26.0 & 962.31 & & & -65.0 & 965.31 & 14.0 & 962.93 & 106.0 & 964.35 \\
\hline 28.0 & 962.31 & & & -64.0 & 965.16 & 16.0 & 962.99 & 108.0 & 964.41 \\
\hline 29.0 & 962.21 & & & -62.0 & 964.67 & 18.0 & 963.39 & 110.9 & 964.40 \\
\hline 30.0 & 962.19 & & & -60.0 & 964.40 & 20.0 & 963.91 & & \\
\hline 32.0 & 962.17 & & & -57.0 & 964.47 & 21.0 & 964.00 & & \\
\hline 34.0 & 962.12 & & & -55.5 & 964.47 & 24.0 & 964.04 & & \\
\hline 36.0 & 962.12 & & & -55.4 & 963.17 & 28.0 & 964.15 & & \\
\hline 38.0 & 962.20 & & & -54.6 & 963.10 & 30.0 & 964.14 & & \\
\hline 40.0 & 962.25 & & & -54.5 & 962.98 & 34.0 & 964.20 & & \\
\hline 42.0 & 962.27 & & & -53.8 & 962.80 & 36.0 & 964.20 & & \\
\hline 43.0 & 962.36 & & & -52.2 & 962.30 & 39.0 & 964.11 & & \\
\hline 44.0 & 962.31 & & & -51.0 & 961.94 & 40.0 & 964.12 & & \\
\hline 44.1 & 962.37 & & & -50.0 & 961.89 & 42.0 & 964.06 & & \\
\hline 45.4 & 962.65 & & & -48.0 & 961.93 & 44.0 & 964.09 & & \\
\hline 46.2 & 962.76 & & & -46.0 & 962.03 & 46.0 & 964.01 & & \\
\hline 46.3 & 963.03 & & & -44.0 & 961.97 & 48.0 & 963.98 & & \\
\hline 48.0 & 962.98 & & & -42.0 & 962.00 & 50.0 & 963.97 & & \\
\hline 50.0 & 962.92 & & & -40.0 & 961.93 & 51.0 & 963.69 & & \\
\hline 52.0 & 962.83 & & & -38.0 & 961.85 & 55.0 & 963.77 & & \\
\hline 54.0 & 962.80 & & & -36.0 & 961.80 & 57.0 & 963.74 & & \\
\hline 56.0 & 962.71 & & & -34.0 & 961.78 & 57.6 & 963.79 & & \\
\hline 58.0 & 962.61 & & & -32.0 & 961.70 & 60.0 & 963.76 & & \\
\hline $60.0^{\prime}$ & 962.69 & & & -30.0 & 961.71 & 64.0 & 963.58 & & \\
\hline 62.0 & 962.77 & & & -28.0 & 961.76 & 67.1 & 963.61 & & \\
\hline 64.0 & 962.78 & & & -26.0 & 961.80 & 68.0 & 963.72 & & \\
\hline
\end{tabular}


Table 21.--Listing. of horizontal stations and elevations for cross section PRI63 --Continued.

\begin{tabular}{|c|c|c|c|c|c|c|c|c|c|}
\hline \multicolumn{2}{|c|}{1979} & \multicolumn{2}{|c|}{1979} & \multicolumn{2}{|c|}{1979} & \multicolumn{2}{|c|}{1980} & \multicolumn{2}{|c|}{1980} \\
\hline Sta. & Elev. & Sta. & Elev. & Sta. & Elev. & Sta. & Elev. & Sta. & Elev. \\
\hline-119.0 & 965.44 & -16.0 & 962.32 & 82.0 & 964.11 & -75.0 & 965.52 & 14.0 & 962.82 \\
\hline-115.0 & 965.34 & -14.0 & 962.58 & 84.0 & 964.05 & -73.0 & 965.27 & 16.0 & 963.15 \\
\hline-110.0 & 965.37 & -12.0 & 962.76 & 86.0 & 964.07 & -71.0 & 965.16 & 18.0 & 963.44 \\
\hline-105.0 & 965.33 & -10.0 & 962.93 & 88.0 & 964.22 & -70.0 & 965.15 & 21.0 & 963.88 \\
\hline-100.0 & 965.13 & -8.0 & 962.99 & 90.0 & 964.20 & -69.0 & 965.22 & 22.0 & 963.98 \\
\hline-95.0 & 965.35 & -6.0 & 962.97 & 92.0 & 964.16 & -67.0 & 965.34 & 25.0 & 964.06 \\
\hline-90.0 & 965.40 & -4.0 & 963.13 & 94.0 & 964.18 & '-65.0 & 965.31 & 30.0 & 964.15 \\
\hline-88.0 & 965.35 & -2.0 & 963.26 & 96.0 & 964.20 & -64.0 & 965.14 & 35.0 & 964.20 \\
\hline-85.0 & 964.70 & 0.0 & 963.31 & 98.0 & 964.17 & -62.0 & 964.67 & 40.0 & 964.11 \\
\hline-83.0 & 964.65 & 2.0 & 963.34 & 100.0 & 964.21 & -60.0 & 964.39 & 43.0 & 964.06 \\
\hline-82.0 & 964.93 & 4.0 & 963.32 & 102.0 & 964.24 & -59.0 & 964.44 & 46.0 & 964.00 \\
\hline-80.0 & 965.29 & 6.0 & 963.29 & 104.0 & 964.31 & -57.0 & 964.46 & 49.8 & 963.95 \\
\hline-77.0 & 965.68 & 8.0 & 963.26 & 106.0 & 964.34 & -56.9 & 963.17 & 51.0 & 963.75 \\
\hline-75.0 & 965.52 & 10.0 & 963.22 & 108.0 & 964.43 & -56.0 & 962.84 & 55.0 & 963.78 \\
\hline-73.0 & 965.27 & 12.0 & 963.12 & 110.9 & 964.40 & -55.0 & 962.63 & 59.0 & 963.77 \\
\hline-70.0 & 965.15 & 15.0 & 963.08 & & & -54.8 & 962.30 & 61.0 & 963.72 \\
\hline-68.0 & 965.26 & 16.0 & 963.17 & & & -54.0 & 962.19 & 63.0 & $963.6 \mathbf{i}$ \\
\hline-67.0 & 965.36 & 19.0 & 963.60 & & & -53.0 & 961.83 & 65.0 & 963.62 \\
\hline-65.0 & 965.31 & 21.0 & 963.89 & & & -51.0 & 961.95 & 67.0 & 963.65 \\
\hline-64.0 & 965.15 & 22.0 & 964.01 & & & -49.0 & 962.05 & 69.0 & 964.00 \\
\hline-62.0 & 964.67 & 24.0 & 964.04 & & & -46.0 & 962.08 & 70.0 & 964.15 \\
\hline-60.0 & 964.39 & 28.0 & 964.15 & & & -43.0 & 962.01 & 73.0 & 964.16 \\
\hline-57.0 & 964.47 & 30.0 & 964.15 & & & -40.0 & 961.89 & 75.0 & 964.25 \\
\hline-56.3 & 964.55 & 34.0 & 964.20 & & & -37.0 & 961.79 & 77.0 & 964.37 \\
\hline-55.6 & 962.90 & 36.0 & 964.19 & & & -34.0 & 961.73 & 79.5 & 964.34 \\
\hline-55.0 & 962.42 & 40.0 & 964.12 & & & -31.0 & 961.78 & 81.0 & 964.17 \\
\hline-54.0 & 961.90 & 42.0 & 964.07 & & & -28.0 & 961.84 & 85.0 & 964.01 \\
\hline-52.0 & 962.04 & 45.0 & 964.06 & & & -25.0 & 961.91 & 88.0 & 964.21 \\
\hline-50.0 & 961.87 & 48.0 & 963.99 & & & -23.0 & 961.81 & 90.0 & 964.17 \\
\hline-48.0 & 961.94 & 50.0 & 963.94 & & & -20.0 & 961.97 & 95.0 & 964.19 \\
\hline-46.0 & 962.01 & 52.0 & 963.74 & & & -18.0 & 961.98 & 100.0 & 964.21 \\
\hline-44.0 & 962.00 & 54.0 & 963.77 & & & -15.8 & 962.24 & 105.0 & 964.32 \\
\hline-42.0 & 961.93 & 56.0 & 963.77 & & & -13.6 & 962.73 & 110.9 & 964.41 \\
\hline-40.0 & 961.89 & 58.0 & 963.82 & & & -10.0 & 962.79 & & \\
\hline-38.0 & 961.67 & 60.0 & 963.77 & & & -8.0 & 962.92 & & \\
\hline-36.0 & 961.63 & 62.0 & 963.67 & & & -6.0 & 962.95 & & \\
\hline-34.0 & 961.60 & 64.0 & 963.60 & & & -4.0 & 963.10 & & \\
\hline-32.0 & 961.52 & 66.0 & 963.64 & & & -2.0 & 963.10 & & \\
\hline-30.0 & 961.53 & 68.0 & 963.80 & & & 0.0 & 963.16 & & \\
\hline-28.0 & 961.69 & 70.0 & 964.16 & & & 2.0 & 963.08 & & \\
\hline-26.0 & 961.79 & 73.0 & 964.18 & & & 4.0 & 963.04 & & \\
\hline-24.0 & 961.85 & 75.0 & 964.25 & & & 6.6 & 963.34 & & \\
\hline-22.0 & 961.89 & 76.0 & 964.31 & & & 8.0 & 963.27 & & \\
\hline-20.0 & 962.07 & 78.0 & 964.36 & & & 11.0 & 962.88 & & \\
\hline-19.0 & 962.16 & 80.0 & 964.30 & & & 13.0 & 962.80 & & \\
\hline
\end{tabular}


Table 21.--Listing of horizontal stations and elevations for cross section PR163 --Continued.

\begin{tabular}{|c|c|c|c|c|c|c|c|c|c|}
\hline \multicolumn{2}{|c|}{1982} & \multicolumn{2}{|c|}{1982} & \multicolumn{2}{|c|}{1984} & \multicolumn{2}{|c|}{1984} & \multicolumn{2}{|c|}{1985} \\
\hline Sta. & Elev. & Sta. & Elev. & Sta. & Elev. & Sta. & Elev. & Sta. & Elev. \\
\hline-77.0 & 965.70 & 22.0 & 963.98 & -75.0 & 965.53 & 7.0 & 963.36 & -73.0 & 965.28 \\
\hline-75.0 & 965.51 & 25.0 & 964.06 & -73.0 & 965.23 & 10.0 & 963.21 & -70.0 & 965.17 \\
\hline-73.0 & 965.28 & 30.0 & 964.12 & -72.0 & .965 .20 & 12.0 & 963.13 & -68.0 & 965.25 \\
\hline-70.0 & 965.16 & 35.0 & 964.19 & -70.0 & 965.16 & 15.0 & 962.99 & -65.0 & 965.31 \\
\hline-65.0 & 965.26 & 40.0 & 964.09 & -68.0 & 965.25 & 17.0 & 963.30 & -61.4 & 964.49 \\
\hline-62.0 & 964.68 & 45.0 & 964.07 & -67.0 & 965.36 & 20.0 & 963.72 & -61.3 & 963.31 \\
\hline-60.0 & 964.39 & 49.0 & 963.95 & -65.0 & 965.32 & 22.0 & 963.98 & -60.3 & 962.49 \\
\hline-57.9 & 964.43 & 51.0 & 963.83 & -63.0 & 964.90 & 25.0 & 964.06 & -59.0 & 962.08 \\
\hline-57.9 & 963.04 & 52.0 & 963.73 & -62.0 & 964.68 & 30.0 & 964.15 & -58.0 & 961.97 \\
\hline-57.7 & 962.96 & 55.0 & 963.76 & -61.0 & 964.51 & 35.0 & 964.19 & -55.0 & 961.84 \\
\hline-57.0 & 962.79 & 60.0 & 963.75 & -60.8 & 964.48 & 45.0 & 964.07 & -52.0 & 961.74 \\
\hline-56.7 & 962.85 & 65.0 & 963.62 & -60.6 & 963.27 & 49.0 & 963.97 & -49.0 & 961.68 \\
\hline-56.2 & 962.52 & 67.0 & 963.63 & -60.0 & 962.91 & 52.0 & 963.73 & -47.0 & 961.70 \\
\hline-56.0 & 962.43 & 69.0 & 964.01 & -59.8 & 962.57 & 55.0 & 963.76 & -45.0 & 961.95 \\
\hline-55.5 & 961.91 & 70.0 & 964.14 & -59.1 & 962.46 & 60.0 & 963.75 & -41.0 & 962.04 \\
\hline-55.0 & 961.84 & 75.0 & 964.26 & -58.9 & 962.35 & 63.0 & 963.61 & -39.0 & 962.09 \\
\hline-53.0 & 961.83 & & & -58.0 & 962.20 & 66.0 & 963.63 & -35.0 & 962.19 \\
\hline-52.0 & 961.72 & & & -57.0 & 961.96 & 68.0 & 963.86 & -30.0 & 962.20 \\
\hline-50.0 & 961.59 & & & -56.0 & 961.84 & 70.0 & 964.12 & -26.0 & 962.12 \\
\hline-47.0 & 961.76 & & & -55.0 & 961.82 & 75.0 & 964.26 & -23.5 & 962.05 \\
\hline-45.0 & 961.96 & & & -53.0 & 961.81 & & & -22.5 & 962.26 \\
\hline-43.0 & 961.97 & & & -50.0 & 961.92 & & & -20.2 & 962.53 \\
\hline-40.0 & 961.75 & & & -48.0 & 961.99 & & & -20.0 & 962.90 \\
\hline-35.0 & 961.87 & & & -45.0 & 962.01 & & & -18.0 & 963.04 \\
\hline-31.0 & 961.87 & & & -43.0 & 962.18 & & & -15.0 & 963.16 \\
\hline-30.0 & 961.95 & & & -42.0 & 962.18 & & & -10.0 & 963.43 \\
\hline-28.0 & 961.81 & & & -40.0 & 962.20 & & & -5.0 & 963.56 \\
\hline-25.0 & 962.29 & & & -39.0 & 962.19 & & & 0.0 & 963.60 \\
\hline-20.0 & 962.26 & & & -38.0 & 962.21 & & & 3.0 & 963.34 \\
\hline-16.0 & 962.42 & & & -35.0 & 962.22 & & & 8.0 & 963.35 \\
\hline-14.5 & 962.50 & & & -33.0 & 962.18 & & & 10.0 & 963.20 \\
\hline-13.0 & 962.74 & & & -29.0 & 962.12 & & & 14.0 & 962.99 \\
\hline-12.0 & 962.74 & & & -26.0 & 962.03 & & & 15.0 & 963.04 \\
\hline-10.0 & 962.91 & & & -24.0 & 962.09 & & & 18.0 & 963.48 \\
\hline-7.6 & 963.22 & & & -20.0 & 962.17 & & & 22.0 & 963.99 \\
\hline-6.0 & 963.29 & & & -15.0 & 962.32 & & & 25.0 & 964.05 \\
\hline-4.0 & 963.33 & & & -12.0 & 962.48 & & & 30.0 & 964.13 \\
\hline 0.0 & 963.27 & & & -10.0 & 962.54 & & & 35.0 & 964.19 \\
\hline 4.0 & 963.22 & & & -9.0 & 962.48 & & & 40.0 & 964.09 \\
\hline 7.0 & 963.35 & & & -8.2 & 962.64 & & & 45.0 & 964.03 \\
\hline 9.0 & 963.25 & & & -7.0 & 963.33 & & & 49.0 & 963.94 \\
\hline 11.0 & 963.02 & & & -3.0 & 963.35 & & & 52.0 & 963.72 \\
\hline 14.0 & 962.92 & & & 0.0 & 963.32 & & & 55.0 & 963.77 \\
\hline 15.0 & 963.01 & & & 2.0 & 963.23 & & & 60.0 & 963.75 \\
\hline 20.0 & 963.72 & & & 4.0 & 963.26 & & & 64.0 & 963.61 \\
\hline
\end{tabular}


Table 21.--Listing of horizontal stations and elevations for cross section PR163 --Continued.

\begin{tabular}{|c|c|c|c|c|c|c|c|c|c|}
\hline \multicolumn{2}{|c|}{1985} & \multicolumn{2}{|c|}{1986} & \multicolumn{2}{|c|}{1986} & \multicolumn{2}{|c|}{1987} & \multicolumn{2}{|c|}{1987} \\
\hline Sta. & Elev. & Sta. & Elev. & Sta. & Elev. & Sta. & Elev. & Sta. & Elev. \\
\hline 67.0 & 963.63 & -73.0 & 965.28 & 15.0 & 963.05 & -73.0 & 965.29 & 9.0 & 963.38 \\
\hline 69.0 & 964.03 & -71.0 & 965.16 & 16.0 & 963.19 & -71.0 & 965.17 & 10.0 & 963.26 \\
\hline 70.0 & 964.12 & -69.0 & 965.24 & 18.0 & 963.48 & -69.0 & 965.24 & 13.5 & 963.07 \\
\hline 73.0 & 964.17 & -67.0 & 965.35 & 20.0 & 963.73 & -67.0 & 965.37 & 15.0 & 963.11 \\
\hline \multirow[t]{41}{*}{75.0} & 964.26 & -65.0 & 965.31 & 22.0 & 963.99 & -65.0 & 965.30 & 16.0 & 963.23 \\
\hline & & -63.0 & 964.90 & 25.0 & 964.05 & -64.0 & 965.15 & 18.0 & 963.50 \\
\hline & & -61.8 & 964.63 & 30.0 & 964.13 & -62.1 & 964.69 & 18.6 & 963.53 \\
\hline & & -61.4 & 964.48 & 35.0 & 964.17 & -62.0 & 963.26 & 19.7 & 963.71 \\
\hline & & -61.3 & 963.01 & 40.0 & 964.08 & -61.1 & 962.75 & 21.0 & 963.91 \\
\hline & & -60.8 & 962.49 & 45.0 & 964.04 & -60.7 & 962.70 & 23.0 & 964.03 \\
\hline & & -60.0 & 962.26 & 47.0 & 963.96 & -60.5 & 962.49 & 26.0 & 964.09 \\
\hline & & -59.0 & 962.02 & 50.0 & 963.88 & -60.3 & 962.40 & 29.0 & 964.14 \\
\hline & & -58.0 & 961.95 & 52.0 & 963.73 & -59.0 & 962.09 & 32.0 & 964.17 \\
\hline & & -56.0 & 961.87 & 55.0 & 963.77 & -57.0 & 961.98 & 35.0 & 964.19 \\
\hline & & -53.0 & 961.83 & 60.0 & 963.75 & -55.0 & 961.85 & 38.0 & 964.16 \\
\hline & & -50.0 & 961.88 & 62.0 & 963.68 & -52.0 & 961.68 & 41.0 & 964.10 \\
\hline & & -47.0 & 961.93 & 64.0 & 963.61 & -49.0 & 961.80 & 44.0 & 964.05 \\
\hline & & -45.0 & 961.89 & 66.8 & 963.63 & -46.0 & 961.81 & 47.0 & 963.97 \\
\hline & & -44.0 & 962.08 & 68.0 & 963.83 & -43.0 & 961.98 & 49.0 & 963.95 \\
\hline & & -41.0 & 962.05 & 69.0 & 964.01 & -40.0 & 961.95 & 49.6 & 963.92 \\
\hline & & -38.0 & 962.13 & 71.0 & 964.16 & -37.0 & 962.04 & 52.0 & 963.74 \\
\hline & & -35.0 & 962.19 & 73.0 & 964.16 & -34.0 & 962.05 & 55.0 & 963.78 \\
\hline & & -32.0 & 962.19 & 75.0 & 964.27 & -31.0 & 962.16 & 58.0 & 963.81 \\
\hline & & -29.0 & 962.19 & & & -28.0 & 962.19 & 61.0 & 963.73 \\
\hline & & -26.0 & 962.13 & & & -25.0 & 962.15 & 63.0 & 963.66 \\
\hline & & -23.0 & 962.08 & & & -22.0 & 962.12 & 65.0 & 963.63 \\
\hline & & -21.0 & 962.10 & & & -21.0 & 962.13 & 66.8 & 963.64 \\
\hline & & -19.2 & 962.24 & & & -20.0 & 962.38 & 68.0 & 963.86 \\
\hline & & -18.5 & 962.40 & & & -19.2 & 962.73 & 69.0 & 964.03 \\
\hline & & -17.0 & 962.48 & & & -18.0 & 962.98 & 71.0 & 964.18 \\
\hline & & -14.4 & 962.49 & & & -17.0 & 962.85 & 73.0 & 964.17 \\
\hline & & -13.1 & 962.64 & & & -16.0 & 963.06 & 75.0 & 964.27 \\
\hline & & -12.4 & 963.07 & & & -14.6 & 963.24 & & \\
\hline & & -12.3 & 963.31 & & & -14.0 & 963.47 & & \\
\hline & & -10.0 & 963.43 & & & -13.0 & 963.43 & & \\
\hline & & -8.0 & 963.49 & & & -12.0 & 963.47 & & \\
\hline & & -6.0 & 963.52 & & & -11.0 & 963.42 & & \\
\hline & & -4.0 & 963.63 & & & -10.0 & 963.56 & & \\
\hline & & -2.0 & 963.58 & & & -7.0 & 963.56 & & \\
\hline & & 0.0 & 963.58 & & & -4.0 & 963.65 & & \\
\hline & & 2.0 & 963.40 & & & -1.0 & 963.66 & & \\
\hline & & 4.0 & 963.36 & & & 1.0 & 963.54 & & \\
\hline & & 7.0 & 963.39 & & & 2.0 & 963.45 & & \\
\hline & & 10.0 & 963.20 & & & 5.0 & 963.39 & & \\
\hline & & 13.0 & 963.02 & & & 7.0 & 963.44 & & \\
\hline
\end{tabular}


Table 21.--Listing of horizontal stations and elevations for cross section PR163 --Continued.

\begin{tabular}{|c|c|c|c|c|c|c|}
\hline \multicolumn{2}{|c|}{1988} & \multicolumn{2}{|c|}{1988} & \multicolumn{2}{|c|}{1988} & \\
\hline Sta. & Elev. & Sta. & Elev. & Sta. & Elev. & \\
\hline-73.0 & 965.29 & -14.4 & 962.47 & 69.0 & 964.02 & \\
\hline-71.0 & 965.17 & -14.0 & 962.61 & 71.0 & 964.18 & \\
\hline-70.0 & 965.16 & -13.3 & 962.97 & 73.0 & 964.17 & \\
\hline-68.0 & 965.27 & -13.2 & 963.26 & 75.0 & 964.27 & \\
\hline-67.0 & 965.38 & -12.0 & 963.49 & & & \\
\hline-65.0 & 965.32 & -11.0 & 963.57 & & & \\
\hline-64.0 & 965.15 & -9.0 & 963.54 & & & \\
\hline-63.0 & 964.90 & -7.0 & 963.56 & & & \\
\hline-62.3 & 964.71 & -5.0 & 963.62 & & & \\
\hline-62.0 & 963.14 & -2.0 & 963.67 & & & \\
\hline-61.7 & 963.01 & 0.0 & 963.60 & & & \\
\hline-61.0 & 962.90 & 2.0 & 963.45 & & & \\
\hline-60.5 & 962.41 & 4.0 & 963.42 & & & \\
\hline-59.4 & 962.15 & 6.0 & 963.43 & & & \\
\hline-58.5 & 962.10 & 8.0 & 963.41 & & & \\
\hline-57.7 & 962.01 & 10.0 & 963.27 & & & \\
\hline-56.0 & 961.86 & 12.0 & 963.23 & & & \\
\hline-54.0 & 961.81 & 13.0 & 963.12 & & & \\
\hline-53.0 & 961.68 & 14.0 & 963.06 & & & \\
\hline-51.0 & 961.92 & 15.0 & 963.12 & & & \\
\hline-50.0 & 962.03 & 17.0 & 963.36 & & & \\
\hline-49.0 & 961.85 & 19.0 & 963.63 & & & - \\
\hline-48.0 & 961.99 & 21.0 & 963.89 & & & \\
\hline-46.0 & 962.01 & 22.0 & 963.99 & & & \\
\hline-44.0 & 961.98 & 24.0 & 964.06 & & & \\
\hline-42.1 & 961.98 & 27.0 & 964.11 & & & \\
\hline-41.7 & 962.09 & 30.0 & 964.14 & & & \\
\hline-41.6 & 962.20 & 33.0 & 964.18 & & & \\
\hline-39.0 & 962.14 & 36.0 & 964.18 & & & \\
\hline-37.4 & 962.09 & 39.0 & 964.11 & & & \\
\hline-37.0 & 962.13 & 42.0 & 964.08 & & & \\
\hline-36.0 & 962.15 & 45.0 & 964.04 & & & \\
\hline-35.0 & 962.09 & 48.0 & 963.98 & & & \\
\hline-34.6 & 962.04 & 50.0 & 963.88 & & & \\
\hline-32.0 & 962.02 & 51.0 & 963.81 & & & \\
\hline-30.0 & 962.05 & 53.0 & 963.75 & & & \\
\hline-29.0 & 962.10 & 55.0 & 963.78 & & & \\
\hline-27.0 & 962.15 & 58.0 & 963.82 & & & \\
\hline-24.5 & 962.12 & 60.0 & 963.77 & & & \\
\hline-22.0 & 962.08 & 62.0 & 963.70 & & & \\
\hline-20.5 & 962.13 & 64.0 & 963.62 & & & \\
\hline-20.0 & 962.18 & 65.0 & 963.63 & & & \\
\hline-19.5 & 962.24 & 66.4 & 963.64 & & & \\
\hline-18.7 & 962.28 & 67.0 & 963.68 & & & \\
\hline-17.1 & 962.49 & 68.0 & 963.83 & & & \\
\hline
\end{tabular}




\section{Description of Cross Section PR167}

Location: Township 6 South/Range 50 East--section 28

U.S. Geological Survey quadrangle $(1: 24,000)$ : Lonesome Peak

Landowners--left bank: Gay Ranch

right bank Gay Ranch

Access: right bank, across Daily Ranch

Permission from: John Daily (T6S/R50E--22)

Reference pins

\begin{tabular}{lll}
\hline $\begin{array}{l}\text { Station } \\
\text { (meters) }\end{array}$ & $\begin{array}{c}\text { Elevation } \\
\text { (meters } \\
\text { above } \\
\text { sea leve1) }\end{array}$ & Comments \\
& & $\begin{array}{c}\text { Pins } 150.0 \text { and } 150.6 \text { were closest to } \\
\text { leveling instrument. }\end{array}$ \\
-1.6 & 959.633 & 0.06 meter above 1987 ground leve1. \\
150.0 & 959.489 & 0.04 meter above 1987 ground level. \\
150.6 & 959.615 & 0.18 meter above 1987 ground lesvel. \\
\hline
\end{tabular}




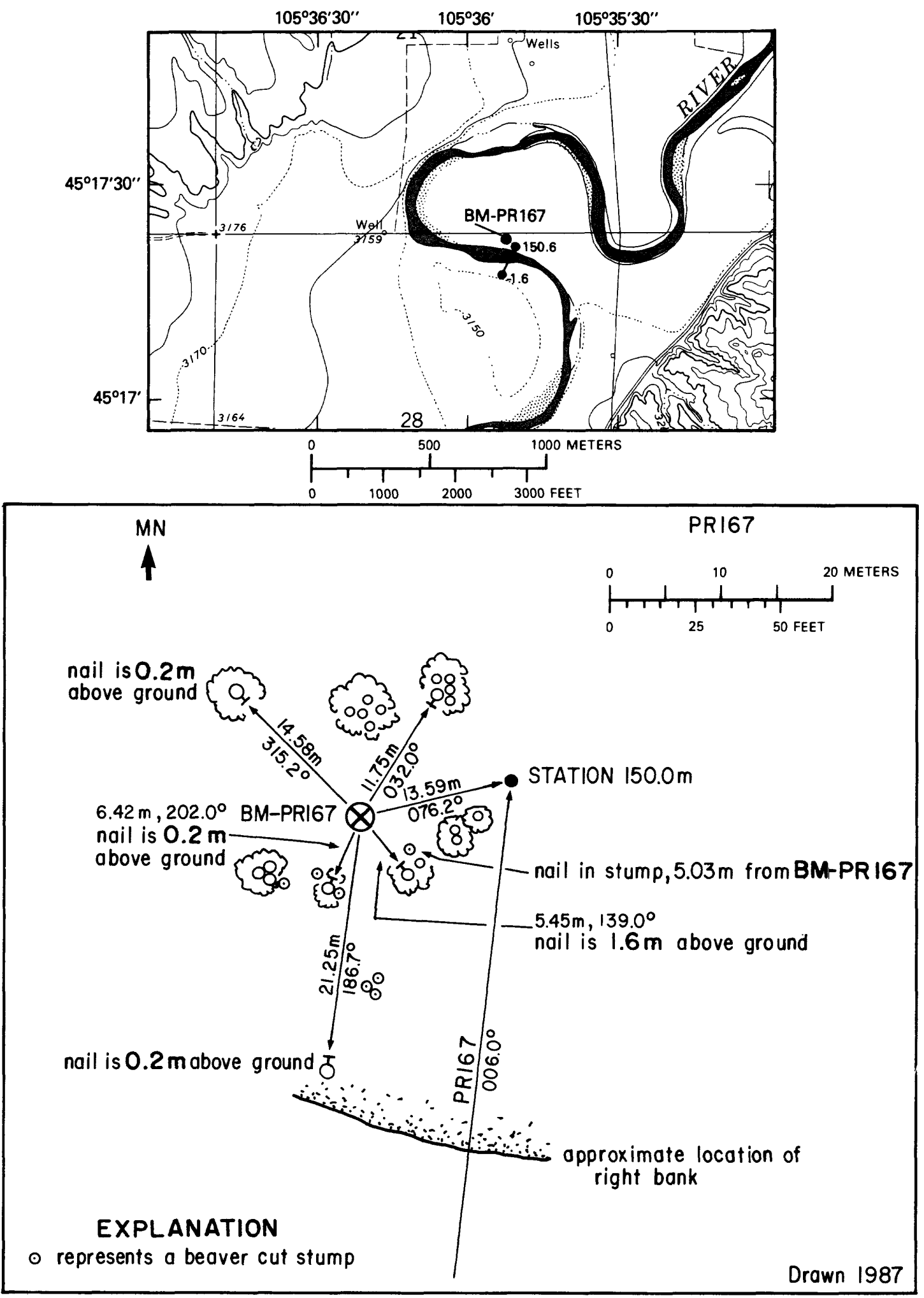

Figure 64.--Upper: Location of cross section PR167, bench mark BM-PR167, and left and right bank reference pins (tables 2 and 3 ) in the Lonesome Peak quadrangle. Lower: Location of bench mark on tile left bank. MN is magnetic north. 


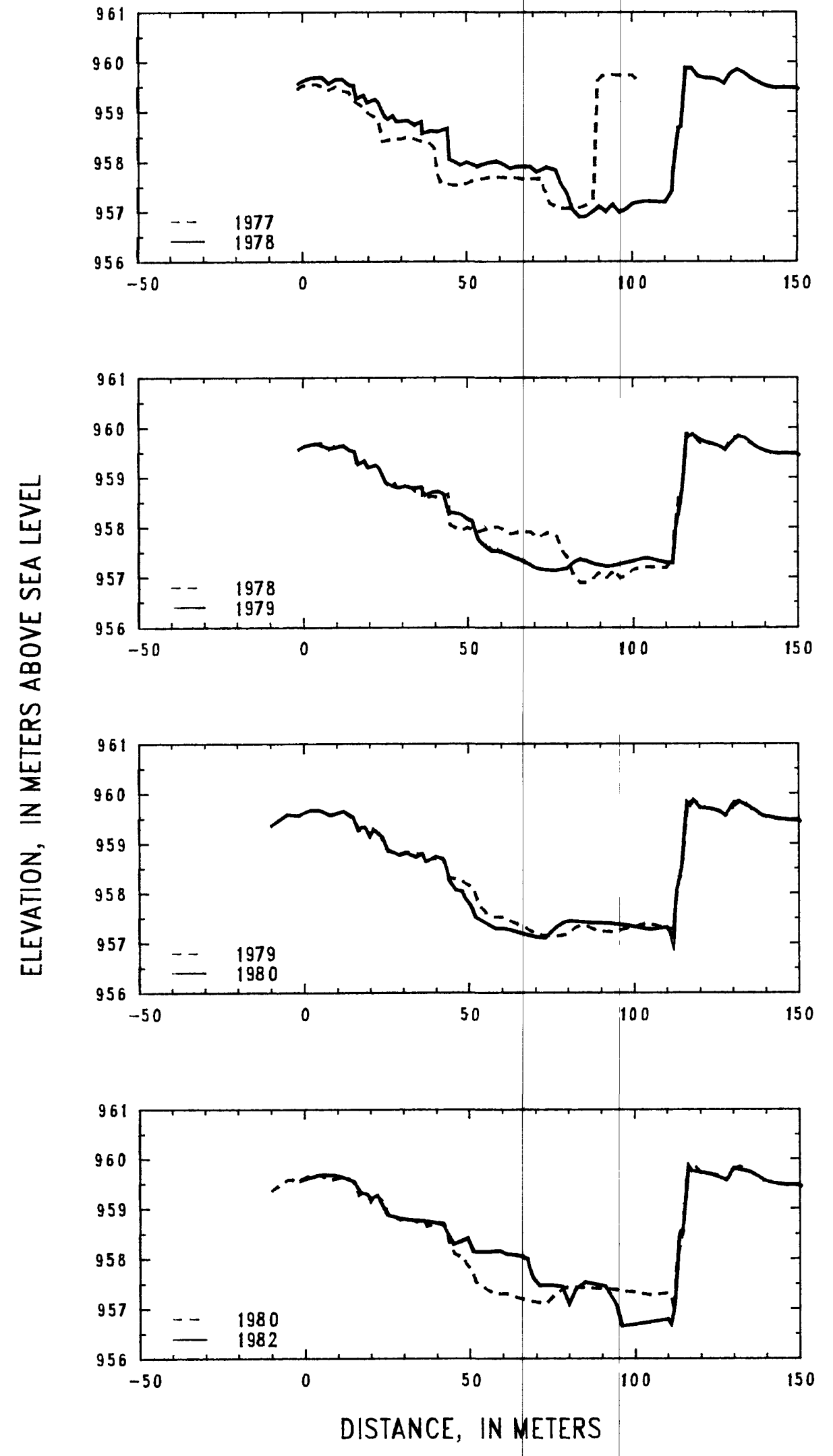

Figure 65.--Profiles of cross section PR167 from 1977 to 1982. 


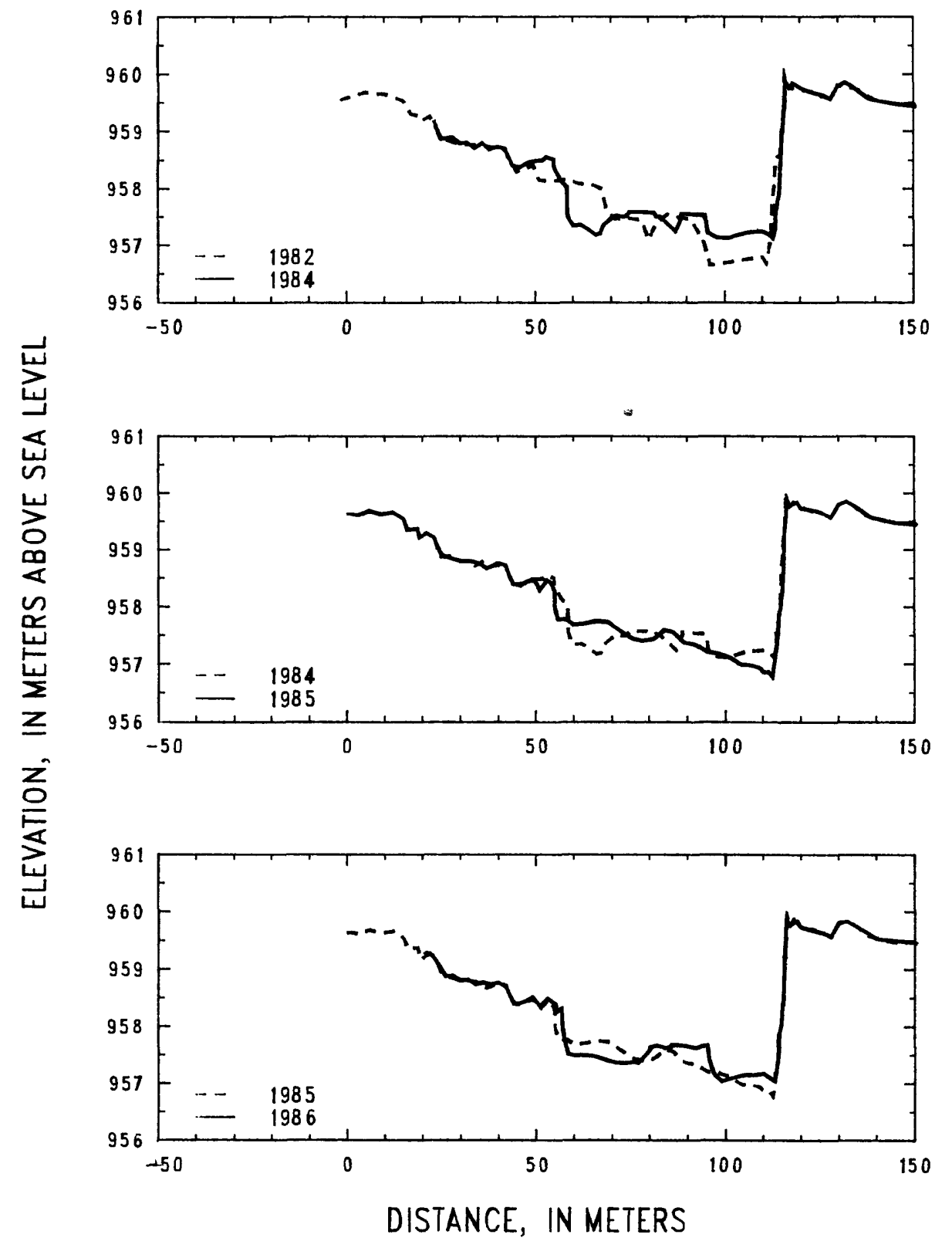

Figure 66.--Profiles of cross section PR167 from 1982 to 1986. 


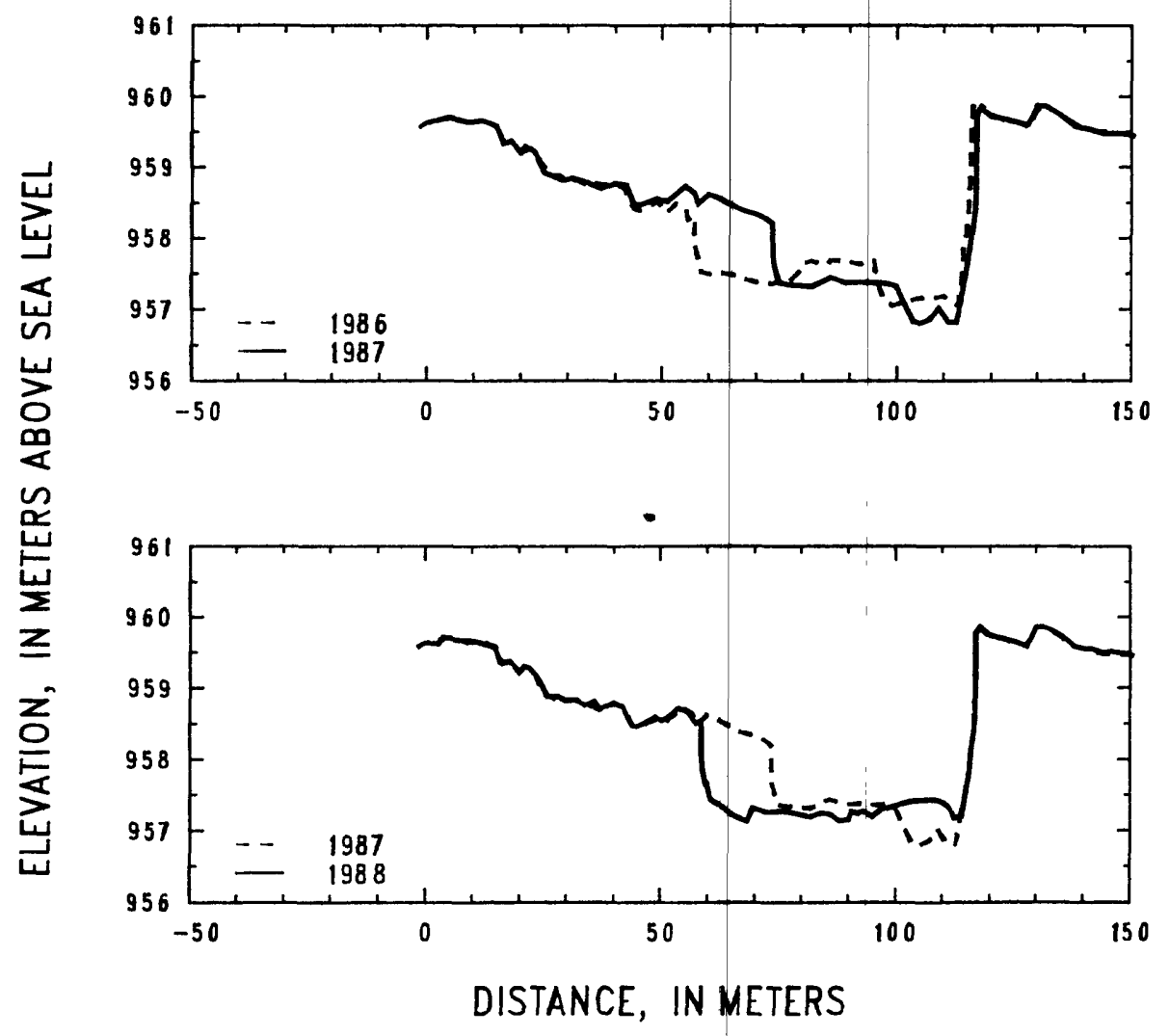

Figure 67.--Profiles of cross section PR167 from 1986 to 1988. 
Table 22.--Listing of horizontal stations and elevations for cross section PR167

[Sta., station, distance in meters from a reference point on

the left bank; Elev., elevation, in meters above sea levell.

\begin{tabular}{|c|c|c|c|c|c|c|c|c|c|}
\hline \multicolumn{2}{|c|}{1977} & \multicolumn{2}{|c|}{1977} & \multicolumn{2}{|c|}{1978} & \multicolumn{2}{|c|}{1978} & \multicolumn{2}{|c|}{1979} \\
\hline Sta. & Elev. & Sta. & Elev. & Sta. & Elev. & Sta. & Elev. & Sta. & Elev. \\
\hline-1.6 & 959.45 & 79.0 & 957.05 & -1.6 & 959.56 & 84.0 & 956.89 & -1.6 & 959.56 \\
\hline 0.0 & 959.52 & 81.0 & 957.06 & 0.0 & 959.62 & 86.0 & 956.90 & 0.0 & 959.62 \\
\hline 2.0 & 959.54 & 83.0 & 957.06 & 2.0 & 959.67 & 88.0 & 956.99 & 2.0 & 959.66 \\
\hline 4.0 & 959.55 & 85.0 & 957.09 & 4.0 & 959.69 & 90.0 & 957.10 & 4.0 & 959.68 \\
\hline 6.0 & 959.50 & 87.0 & 957.16 & 6.0 & 959.69 & 92.0 & 956.99 & 6.0 & 959.63 \\
\hline 8.0 & 959.43 & 88.0 & 957.25 & 8.0 & 959.56 & 94.0 & 957.14 & 8.0 & 959.57 \\
\hline 10.0 & 959.50 & 88.4 & 957.41 & 10.0 & 959.65 & 96.0 & 956.98 & 10.0 & 959.61 \\
\hline 12.0 & 959.41 & 89.6 & 959.62 & 12.0 & 959.66 & 98.0 & 957.04 & 12.0 & 959.64 \\
\hline 14.0 & 959.38 & 91.0 & 959.71 & 14.0 & 959.54 & 100.0 & 957.16 & 14.0 & 959.54 \\
\hline 16.0 & 959.18 & 93.0 & 959.74 & 15.4 & 959.52 & 102.0 & 957.19 & 15.4 & 959.52 \\
\hline 18.0 & 959.10 & 95.0 & 959.73 & 16.3 & 959.26 & 104.0 & 957.21 & 16.6 & 959.26 \\
\hline 20.0 & 958.96 & 97.0 & 959.72 & 18.6 & 959.33 & 106.0 & 957.21 & 18.6 & 959.34 \\
\hline 22.0 & 958.87 & 100.0 & 959.73 & 19.6 & 959.18 & 108.0 & 957.19 & 19.7 & 959.20 \\
\hline 23.3 & 958.77 & 101.6 & 959.63 & 22.0 & 959.25 & 110.0 & 957.19 & 22.0 & 959.25 \\
\hline 24.4 & 958.40 & & & 23.0 & 959.19 & 112.0 & 957.40 & 23.0 & 959.20 \\
\hline 26.0 & 958.43 & & & 25.0 & 958.89 & 112.4 & 957.83 & 25.0 & 958.89 \\
\hline 28.0 & 958.47 & & & 26.0 & 958.85 & 114.0 & 958.68 & 27.0 & 958.82 \\
\hline 30.0 & 958.45 & & & 27.5 & 958.92 & 114.8 & 958.70 & 29.0 & 958.79 \\
\hline 32.0 & 958.50 & & & 28.5 & 958.80 & 115.5 & 959.30 & 31.0 & 958.83 \\
\hline 34.0 & 958.45 & & & 30.0 & 958.81 & 116.2 & 959.88 & 33.0 & 958.78 \\
\hline 36.0 & 958.41 & & & 32.0 & 958.83 & 118.0 & 959.87 & 36.0 & 958.80 \\
\hline 38.0 & 958.40 & & & 34.0 & 958.73 & 120.0 & 959.71 & 37.0 & 958.63 \\
\hline 40.0 & 958.27 & & & 36.2 & 958.80 & 122.0 & 959.67 & 39.0 & 958.70 \\
\hline 41.0 & 957.74 & & & 36.5 & 958.56 & 124.0 & 959.67 & 41.0 & 958.72 \\
\hline 42.0 & 957.57 & & & 39.0 & 958.62 & 126.0 & 959.64 & 42.5 & 958.66 \\
\hline 44.0 & 957.55 & & & 41.0 & 958.60 & 128.0 & 959.56 & 44.0 & 958.31 \\
\hline 46.0 & 957.52 & & & 44.0 & 958.67 & 130.0 & 959.77 & 46.0 & 958.28 \\
\hline 48.0 & 957.54 & & & 44.6 & 958.04 & 132.0 & 959.85 & 48.0 & 958.25 \\
\hline 50.0 & 957.56 & & & 46.0 & 958.00 & 134.0 & 959.78 & 50.0 & 958.15 \\
\hline 52.0 & 957.62 & & & 48.0 & 957.93 & 136.0 & 959.67 & 51.3 & 958.12 \\
\hline 54.0 & 957.65 & & & 50.0 & 957.99 & 138.0 & 959.60 & 53.0 & 957.76 \\
\hline 56.0 & 957.67 & & & 53.0 & 957.90 & 140.0 & 959.53 & 55.0 & 957.62 \\
\hline 58.0 & 957.69 & & & 56.0 & 957.98 & 142.0 & 959.49 & 57.0 & 957.52 \\
\hline 60.0 & 957.68 & & & 59.0 & 958.01 & 144.0 & 959.47 & 60.0 & 957.51 \\
\hline 62.0 & 957.67 & & & 61.0 & 957.94 & 146.0 & 959.49 & 62.0 & 957.45 \\
\hline 64.0 & 957.67 & & & 63.0 & 957.86 & 148.0 & 959.48 & 64.0 & 957.39 \\
\hline 66.0 & 957.65 & & & 65.0 & 957.90 & 150.0 & 959.47 & 66.0 & 957.34 \\
\hline 68.0 & 957.64 & & & 67.0 & 957.91 & 150.6 & 959.44 & 68.0 & 957.27 \\
\hline 70.0 & 957.65 & & & 69.0 & 957.90 & & & 70.0 & 957.19 \\
\hline 72.5 & 957.65 & & & 71.0 & 957.79 & & & 72.0 & 957.15 \\
\hline 73.3 & 957.43 & & & 74.0 & 957.89 & & & 74.0 & 957.14 \\
\hline 73.8 & 957.41 & & & 76.9 & 957.82 & & & 76.0 & 957.13 \\
\hline 74.0 & 957.31 & & & 78.5 & 957.54 & & & 78.0 & 957.15 \\
\hline 75.0 & 957.16 & & & 80.0 & 957.39 & & & 80.0 & 957.18 \\
\hline 77.0 & 957.08 & & & 82.0 & 957.03 & & & 82.0 & 957.30 \\
\hline
\end{tabular}


Table 22.--Listing of horizontal stations and elevations for cross section PR167 --Continued.

\begin{tabular}{|c|c|c|c|c|c|c|c|c|c|}
\hline \multicolumn{2}{|c|}{1979} & \multicolumn{2}{|c|}{1980} & \multicolumn{2}{|c|}{1980} & \multicolumn{2}{|c|}{1982} & \multicolumn{2}{|c|}{1982} \\
\hline Sta. & Elev. & Sta. & Elev. & Sta. & Elev. & Sta. & Elev. & Sta. & Elev. \\
\hline 84.0 & 957.36 & -10.0 & 959.35 & 100.0 & 957.32 & -1.6 & 959.56 & 123.0 & 959.69 \\
\hline 86.0 & 957.32 & -5.0 & 959.58 & 105.0 & 957.26 & 5.0 & 959.69 & 128.0 & 959.57 \\
\hline 88.0 & 957.26 & -1.6 & 959.56 & 110.0 & 957.31 & 10.0 & 959.66 & 130.0 & 959.81 \\
\hline 90.0 & 957.24 & 0.0 & 959.62 & 111.0 & 957.21 & 15.0 & 959.54 & 135.0 & 959.74 \\
\hline 92.0 & 957.21 & 2.0 & 959.67 & 111.9 & 956.96 & 17.0 & 959.31 & 140.0 & 959.54 \\
\hline 94.0 & 957.23 & 5.0 & 959.66 & 112.4 & $9 \$ 7.59$ & 19.0 & 959.28 & 145.0 & 959.48 \\
\hline 96.0 & 957.26 & 8.0 & 959.56 & 112.8 & 958.03 & 20.0 & 959.19 & 150.0 & 959.48 \\
\hline 98.0 & 957.30 & 12.0 & 959.65 & 113.3 & 958.14 & 22.0 & 959.27 & 150.6 & 959.44 \\
\hline 100.0 & 957.32 & 14.8 & 959.53 & 114.5 & 958.53 & 25.0 & 958.88 & & \\
\hline 102.0 & 957.36 & 16.4 & 959.26 & 115.4 & 959.30 & 30.0 & 958.78 & & \\
\hline 104.0 & 957.39 & 17.6 & 959.33 & 116.1 & 959.82 & 35.0 & 958.76 & & \\
\hline 106.0 & 957.36 & 18.6 & 959.33 & 117.0 & 959.75 & 39.0 & 958.72 & & \\
\hline 108.0 & 957.31 & 20.0 & 959.14 & 118.0 & 959.87 & 42.0 & 958.67 & & \\
\hline 110.0 & 957.29 & 21.0 & 959.29 & 120.0 & 959.71 & 45.0 & 958.29 & & \\
\hline 112.0 & 957.28 & 23.7 & 959.13 & 123.0 & 959.69 & 49.5 & 958.41 & & \\
\hline 113.0 & 958.05 & 25.5 & 958.84 & 126.0 & 959.64 & 51.0 & 958.14 & & \\
\hline 114.0 & 958.35 & 27.0 & 958.83 & 127.8 & 959.55 & 55.0 & 958.13 & & \\
\hline 115.0 & 958.82 & 29.0 & 958.76 & 130.5 & 959.82 & 59.0 & 958.16 & & \\
\hline 116.2 & 959.80 & 31.0 & 958.81 & 132.0 & 959.84 & 61.0 & 958.09 & & \\
\hline 118.0 & 959.87 & 34.0 & 958.72 & 135.0 & 959.72 & 65.0 & 958.06 & & \\
\hline 120.0 & 959.76 & 36.0 & 958.79 & 137.0 & 959.65 & 67.5 & 957.99 & & \\
\hline 122.0 & 959.70 & 37.0 & 958.64 & 139.0 & 959.55 & 67.7 & 957.89 & & \\
\hline 124.0 & 959.68 & 40.0 & 958.73 & 142.0 & 959.51 & 69.0 & 957.62 & & \\
\hline 126.0 & 959.63 & 42.0 & 958.69 & 145.0 & 959.47 & 71.0 & 957.46 & & \\
\hline 128.0 & 959.55 & 43.0 & 958.55 & 150.0 & 959.45 & 74.0 & 957.48 & & \\
\hline 130.0 & 959.71 & 44.0 & 958.26 & 150.6 & 959.42 & 78.0 & 957.44 & & \\
\hline 132.0 & 959.83 & 46.0 & 958.06 & & & 80.0 & 957.09 & & \\
\hline 134.0 & 959.80 & 48.0 & 958.03 & & & 82.0 & 957.40 & & \\
\hline 136.0 & 959.68 & 49.0 & 957.88 & & & 85.0 & 957.54 & & \\
\hline 138.0 & 959.59 & 50.0 & 957.80 & & & 88.0 & 957.49 & & \\
\hline 140.0 & 959.53 & 51.0 & 957.69 & & & 91.0 & 957.45 & & \\
\hline 142.0 & 959.49 & 52.0 & 957.51 & & & 94.0 & 957.11 & & \\
\hline 144.0 & 959.47 & 55.0 & 957.40 & & & 95.0 & 956.92 & & \\
\hline 146.0 & 959.48 & 58.0 & 957.29 & & & 96.0 & 956.65 & & \\
\hline 148.0 & 959.47 & 61.0 & 957.29 & & & 110.0 & 956.79 & & \\
\hline 150.0 & 959.47 & 65.0 & 957.20 & & & 111.0 & 956.68 & & \\
\hline 150.6 & 959.43 & 70.0 & 957.12 & & & 112.0 & 957.04 & & \\
\hline & & 73.0 & 957.09 & & & 113.0 & 957.95 & & \\
\hline & & 76.0 & 957.30 & & & 113.4 & 958.46 & & \\
\hline & & 79.0 & 957.42 & & & 114.0 & 958.56 & & \\
\hline & & 81.0 & 957.44 & & & 114.6 & 958.59 & & \\
\hline & & 84.0 & 957.42 & & & 115.5 & 959.29 & & \\
\hline & & 87.0 & 957.40 & & & 116.2 & 959.86 & & \\
\hline & & 90.0 & 957.40 & & & 117.0 & 959.76 & & \\
\hline & & 95.0 & 957.37 & & & 120.0 & 959.73 & & \\
\hline
\end{tabular}


Table 22.--Listing of horizontal stations and elevations for cross section PR167 --Continued.

\begin{tabular}{|c|c|c|c|c|c|c|c|c|c|}
\hline \multicolumn{2}{|c|}{1984} & \multicolumn{2}{|c|}{1984} & \multicolumn{2}{|c|}{1985} & \multicolumn{2}{|c|}{1985} & \multicolumn{2}{|c|}{1986} \\
\hline Sta. & Elev. & Sta. & Elev. & Sta. & Elev. & Sta. & Elev. & Sta. & Elev. \\
\hline 23.0 & 959.18 & 101.0 & 957.11 & 0.0 & 959.63 & 104.0 & 956.98 & 20.0 & 959.19 \\
\hline 25.0 & 958.86 & 104.0 & 957.19 & 3.0 & 959.61 & 107.0 & 956.97 & 22.0 & 959.27 \\
\hline 28.0 & 958.89 & 107.0 & 957.23 & 6.0 & 959.68 & 109.0 & 956.93 & 24.0 & 959.10 \\
\hline 30.0 & 958.78 & 110.0 & 957.24 & 9.0 & 959.62 & 110.0 & 956.86 & 26.0 & 958.87 \\
\hline 32.0 & 958.80 & 112.0 & 957.17 & 12.0 & 959.66 & 111.5 & 956.86 & 28.0 & 958.88 \\
\hline 34.0 & 958.70 & 112.7 & 957.11 & 15.0 & 959.54 & 112.5 & 956.77 & 30.0 & 958.79 \\
\hline 36.0 & 958.79 & 113.2 & 957.23 & 16.0 & 959.35 & 114.0 & 957.60 & 32.0 & 958.81 \\
\hline 38.0 & 958.68 & 113.6 & 957.63 & 18.6 & 959.37 & 114.3 & 957.90 & 34.0 & 958.73 \\
\hline 40.0 & 958.73 & 114.5 & 957.90 & 19.3 & 959.21 & 114.6 & 957.96 & 36.0 & 958.77 \\
\hline 42.0 & 958.69 & 115.0 & 958.81 & 21.0 & 959.29 & 115.4 & 958.46 & 38.0 & 958.73 \\
\hline 44.0 & 958.39 & 115.9 & 959.50 & 23.0 & 959.21 & 115.6 & 959.27 & 40.0 & 958.76 \\
\hline 46.0 & 958.36 & 116.0 & 959.91 & 25.0 & 958.91 & 115.9 & 959.45 & 42.0 & 958.69 \\
\hline 48.0 & 958.45 & 116.2 & 959.83 & 27.0 & 958.85 & 116.3 & 959.89 & 44.0 & 958.41 \\
\hline 50.0 & 958.48 & 117.0 & 959.75 & 30.0 & 958.79 & 116.9 & 959.76 & 45.0 & 958.39 \\
\hline 52.0 & 958.49 & 118.0 & 959.84 & 33.0 & 958.79 & 119.0 & 959.84 & 47.0 & 958.43 \\
\hline 53.0 & 958.55 & 120.0 & 959.75 & 35.0 & 958.75 & 120.0 & 959.72 & 49.0 & 958.51 \\
\hline 54.9 & 958.50 & 123.0 & 959.68 & 37.0 & 958.66 & 123.0 & 959.69 & 51.0 & 958.35 \\
\hline 55.0 & 958.36 & 126.0 & 959.63 & 40.0 & 958.76 & 126.0 & 959.63 & 53.0 & 958.49 \\
\hline 57.0 & 958.11 & 128.0 & 959.56 & 42.0 & 958.71 & 127.9 & 959.56 & 55.2 & 958.38 \\
\hline 58.5 & 958.01 & 130.0 & 959.79 & 44.0 & 958.39 & 130.0 & 959.78 & 55.6 & 958.25 \\
\hline 58.6 & 957.54 & 132.0 & 959.85 & 46.0 & 958.39 & 132.0 & 959.85 & 56.8 & 958.31 \\
\hline 59.1 & 957.43 & 135.0 & 959.72 & 49.0 & 958.47 & 135.0 & 959.73 & 57.2 & 957.89 \\
\hline 60.0 & 957.33 & 138.0 & 959.57 & 50.0 & 958.42 & 138.0 & 959.58 & 58.4 & 957.51 \\
\hline 62.0 & 957.35 & 140.0 & 959.53 & 51.0 & 958.28 & 141.0 & 959.52 & 60.0 & 957.48 \\
\hline 64.0 & 957.26 & 145.0 & 959.47 & 53.0 & 958.46 & 144.0 & 959.47 & 63.0 & 957.50 \\
\hline 66.0 & 957.17 & 150.0 & 959.44 & 54.0 & 958.41 & 147.0 & 959.46 & 66.0 & 957.46 \\
\hline 67.0 & 957.19 & 150.6 & 959.43 & 55.0 & 958.26 & 150.0 & 959.47 & 68.0 & 957.41 \\
\hline 68.0 & 957.35 & & & 55.1 & 957.98 & 150.6 & 959.43 & 70.0 & 957.37 \\
\hline 69.5 & 957.43 & & & 56.0 & 957.77 & & & 73.0 & 957.35 \\
\hline 70.7 & 957.50 & & & 58.0 & 957.77 & & & 76.0 & 957.38 \\
\hline 71.6 & 957.52 & & & 60.0 & 957.68 & & & 77.0 & 957.36 \\
\hline 73.0 & 957.49 & & & 63.0 & 957.70 & & & 77.7 & 957.42 \\
\hline 74.0 & 957.50 & & & 66.0 & 957.75 & & & 80.0 & 957.58 \\
\hline 75.0 & 957.58 & & & 69.0 & 957.73 & & & 80.2 & 957.64 \\
\hline 78.0 & 957.58 & & & 71.7 & 957.60 & & & 82.0 & 957.67 \\
\hline 81.5 & 957.56 & & & 75.0 & 957.45 & & & 84.0 & 957.62 \\
\hline 82.0 & 957.49 & & & 78.0 & 957.40 & & & 85.6 & 957.64 \\
\hline 83.0 & 957.49 & & & 81.0 & 957.43 & & & 85.8 & 957.68 \\
\hline 87.0 & 957.23 & & & 84.0 & 957.59 & & & 88.0 & 957.67 \\
\hline 88.3 & 957.42 & & & 86.0 & 957.56 & & & 90.0 & 957.65 \\
\hline 88.8 & 957.55 & & & 89.0 & 957.37 & & & 92.0 & 957.62 \\
\hline 90.5 & 957.54 & & & 92.0 & 957.33 & & & 94.0 & 957.66 \\
\hline 94.8 & 957.51 & & & 95.0 & 957.20 & & & 95.2 & 957.67 \\
\hline 95.5 & 957.21 & & & 98.0 & 957.18 & & & 95.5 & 957.43 \\
\hline 98.0 & 957.12 & & & 101.0 & 957.11 & & & 97.0 & 957.15 \\
\hline
\end{tabular}


Table 22.--Listing of horizontal stations and elevations for cross section PR167 --Continued.

\begin{tabular}{|c|c|c|c|c|c|c|c|c|c|}
\hline \multicolumn{2}{|c|}{1986} & \multicolumn{2}{|c|}{1987} & \multicolumn{2}{|c|}{1987} & \multicolumn{2}{|c|}{1988} & \multicolumn{2}{|c|}{1988} \\
\hline Sta. & Elev. & Sta. & Elev. & Sta. & Elev. & Sta. & Elev. & Sta. & $\overline{\text { Elev. }}$ \\
\hline 99.0 & 957.04 & -1.6 & 959.57 & 100.0 & 957.30 & -1.6 & 959.58 & 68.4 & 957.12 \\
\hline 102.0 & 957.11 & 0.0 & 959.63 & 101.5 & 957.08 & 0.0 & 959.63 & 69.6 & 957.32 \\
\hline 105.0 & 957.16 & 5.0 & 959.71 & 103.5 & 956.81 & 3.0 & 959.61 & 72.0 & 957.26 \\
\hline 108.0 & 957.15 & 9.0 & 959.63 & 105.0 & 956.79 & 4.0 & 959.71 & 74.0 & 957.26 \\
\hline 110.0 & 957.18 & 12.0 & 959.66 & 107.0 & 956.85 & 7.0 & 959.67 & 76.0 & 957.28 \\
\hline 112.0 & 957.09 & 15.0 & 959.57 & 109.0 & 957.01 & 10.0 & 959.66 & 78.0 & 957.25 \\
\hline 113.0 & 957.04 & 16.5 & 959.33 & 111.0 & 956.81 & 13.0 & 959.60 & 80.0 & 957.22 \\
\hline 113.9 & 957.43 & 18.0 & 959.37 & 112.8 & 956.80 & 15.0 & 959.55 & 82.0 & 957.19 \\
\hline 114.2 & 957.87 & 20.0 & 959.20 & 115.0 & 957.61 & 16.0 & 959.36 & 84.0 & 957.25 \\
\hline 114.6 & 957.97 & 21.0 & 959.29 & 116.8 & 958.45 & 18.0 & 959.38 & 86.0 & 957.23 \\
\hline 115.2 & 958.34 & 23.0 & 959.21 & 117.0 & 959.76 & 20.0 & 959.21 & 88.0 & 957.13 \\
\hline 115.5 & 958.76 & 25.0 & 958.91 & 118.0 & 959.83 & 21.0 & 959.31 & 90.0 & 957.15 \\
\hline 115.8 & 959.45 & 27.0 & 958.86 & 120.0 & 959.71 & 22.0 & 959.28 & 90.6 & 957.27 \\
\hline 116.2 & 959.89 & 29.0 & 958.80 & 122.0 & 959.70 & 24.0 & 959.12 & 92.0 & 957.24 \\
\hline 116.5 & 959.81 & 31.0 & 958.84 & 125.0 & 959.64 & 26.0 & 958.88 & 93.0 & 957.28 \\
\hline 117.0 & 959.76 & 34.0 & 958.78 & 128.0 & 959.60 & 28.0 & 958.89 & 95.0 & 957.20 \\
\hline 118.0 & 959.87 & 37.0 & 958.69 & 130.0 & 959.87 & 30.0 & 958.81 & 97.0 & 957.31 \\
\hline 120.0 & 959.73 & 40.0 & 958.78 & 132.0 & 959.86 & 32.0 & 958.84 & 99.0 & 957.33 \\
\hline 123.0 & 959.67 & 42.5 & 958.74 & 135.0 & 959.73 & 34.0 & 958.75 & 101.1 & 957.38 \\
\hline 126.0 & 959.63 & 44.0 & 958.47 & 138.0 & 959.58 & 35.0 & 958.78 & 103.0 & 957.41 \\
\hline 128.0 & 959.57 & 45.0 & 958.46 & 141.0 & 959.54 & 36.0 & 958.81 & 105.5 & 957.43 \\
\hline 130.0 & 959.81 & 47.0 & 958.50 & 144.0 & 959.47 & 37.0 & 958.69 & 107.0 & 957.43 \\
\hline 132.0 & 959.84 & 49.0 & 958.56 & 147.0 & 959.48 & 38.0 & 958.73 & 109.0 & 957.43 \\
\hline 135.0 & 959.74 & 51.0 & 958.51 & 150.0 & 959.45 & 40.0 & 958.79 & 110.4 & 957.38 \\
\hline 140.0 & 959.53 & 53.0 & 958.62 & 150.6 & 959.43 & 42.0 & 958.73 & 111.4 & 957.32 \\
\hline 145.0 & 959.49 & 55.0 & 958.73 & & & 44.0 & 958.46 & 112.5 & 957.18 \\
\hline 150.0 & 959.46 & 57.0 & 958.63 & & & 45.0 & 958.45 & 114.0 & 957.20 \\
\hline \multirow[t]{18}{*}{150.6} & 959.45 & 57.8 & 958.48 & & & 47.0 & 958.52 & 114.5 & 957.38 \\
\hline & & 60.0 & 958.62 & & & 49.0 & 958.60 & 115.8 & 957.87 \\
\hline & & 62.0 & 958.56 & & & 50.4 & 958.53 & 116.1 & 958.16 \\
\hline & & 64.0 & 958.49 & & & 52.0 & 958.61 & 116.9 & 958.50 \\
\hline & & 67.0 & 958.39 & & & 54.0 & 958.71 & 117.0 & 959.78 \\
\hline & & 70.0 & 958.33 & & & 56.0 & 958.66 & 118.0 & 959.86 \\
\hline & & 72.0 & 958.28 & & & 57.7 & 958.50 & 119.0 & 959.79 \\
\hline & & 73.5 & 958.20 & & & 58.7 & 958.54 & 120.0 & 959.73 \\
\hline & & 73.8 & 957.62 & & & 58.8 & 958.10 & 122.0 & 959.71 \\
\hline & & 75.0 & 957.36 & & & 59.0 & 957.84 & 124.0 & 959.67 \\
\hline & & 77.0 & 957.33 & & & 59.7 & 957.64 & 126.0 & 959.63 \\
\hline & & 82.0 & 957.31 & & & 60.0 & 957.65 & 128.0 & 959.58 \\
\hline & & 83.0 & 957.34 & & & 60.3 & 957.53 & 130.0 & 959.86 \\
\hline & & 86.0 & 957.44 & & & 60.7 & 957.43 & 132.0 & 959.86 \\
\hline & & 89.0 & 957.36 & & & 61.7 & 957.39 & 134.0 & 959.78 \\
\hline & & 92.0 & 957.37 & & & 63.0 & 957.35 & 136.0 & 959.70 \\
\hline & & 95.0 & 957.36 & & & 65.0 & 957.23 & 138.0 & 959.59 \\
\hline & & 98.0 & 957.35 & & & 67.0 & 957.16 & 140.0 & 959.54 \\
\hline
\end{tabular}


Table 22.--Listing of horizontal stations and elevations for cross section PR167 --Continued. 1988

Sta. Elev.

$142.0 \quad 959.55$

$144.0 \quad 959.48$

$146.0 \quad 959.51$

$148.0 \quad 959.48$

$150.0 \quad 959.47$

$150.6 \quad 959.44$ 


\section{Description of Cross Section PR175}

Location: Township 6 South/Range 50 East--section 15

U.S. Geological Survey quadrangle $(1: 24,000)$ : Lonesome Peak

Landowners--left bank: Frances Rice

right bank: Frances Rice

Access: left bank

Permission from: Frances Rice (residence in Broadus)

Reference pins

\begin{tabular}{|c|c|c|}
\hline $\begin{array}{l}\text { Station } \\
\text { (meters) }\end{array}$ & $\begin{array}{l}\text { Elevation } \\
\text { (meters } \\
\text { above } \\
\text { sea level) }\end{array}$ & Comments \\
\hline & & $\begin{array}{l}\text { Pins at stations less than } 200.0 \\
\text { were closest to leveling instrument. }\end{array}$ \\
\hline-39.3 & 953.486 & $\begin{array}{l}\text { 0.20 meter above } 1980 \text { ground level, on } \\
\text { fenceline. }\end{array}$ \\
\hline-1.7 & 953.653 & 0.08 meter above 1982 ground level. \\
\hline-0.7 & 953.769 & 0.19 meter above 1982 ground level. \\
\hline 0.0 & 953.522 & At 1982 ground level. \\
\hline 100.0 & 952.793 & 0.03 meter above 1987 ground level. \\
\hline 124.0 & 952.845 & 0.04 meter above 1987 ground level. \\
\hline 250.0 & 952.106 & Bent over, possibly covered. \\
\hline 300.0 & 953.184 & 0.03 meter above 1987 ground level. \\
\hline 320.0 & 953.563 & $\begin{array}{l} \pm 0.03 \text { meter, long level sight; } 0.14 \\
\text { meter above } 1987 \text { ground level. }\end{array}$ \\
\hline
\end{tabular}




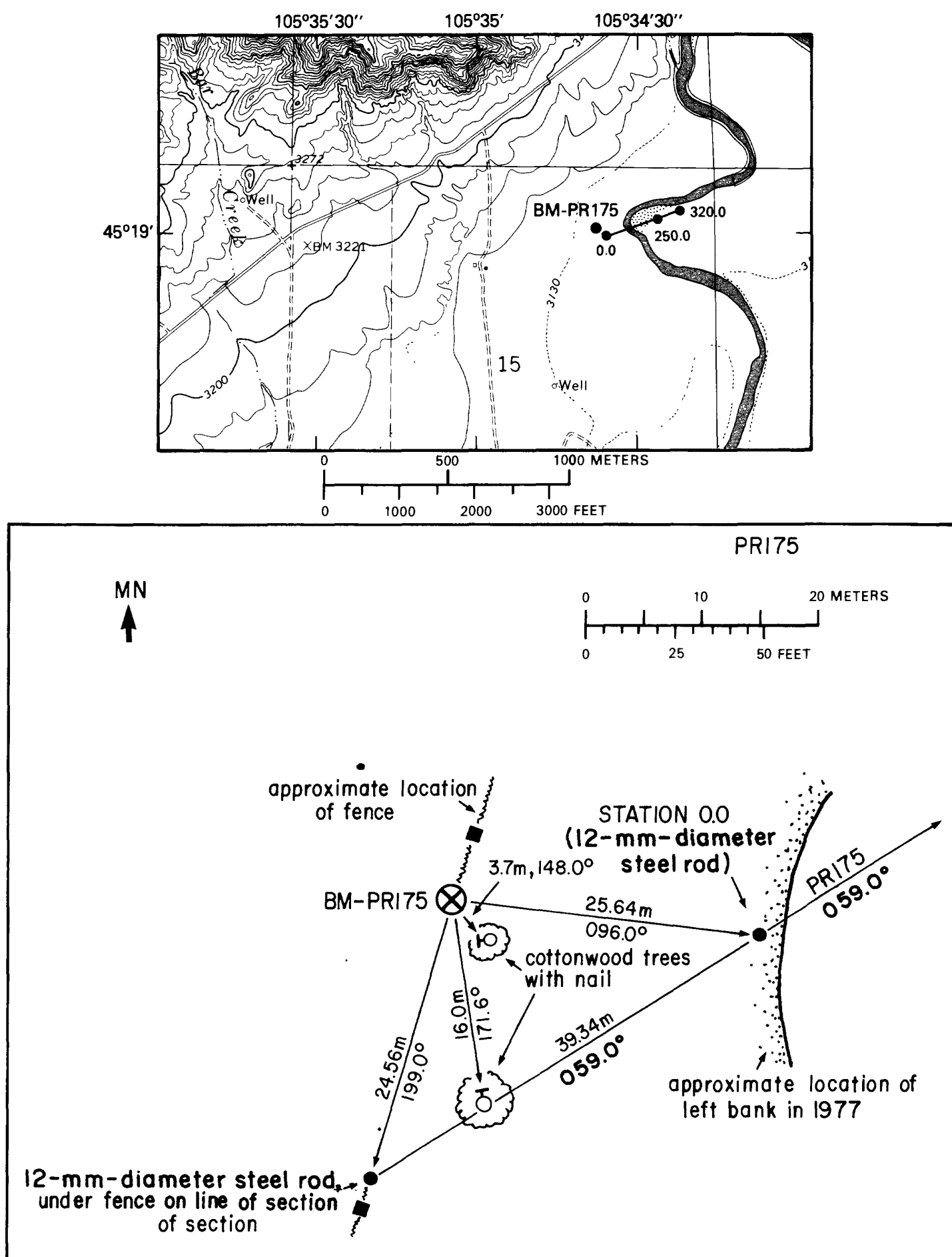

Drawn 1987

Figure 68.--Upper: Location of cross section PR175, bench mark BM-PR175, and left and right bank reference pins (tables 2 and 3 ) in the Lonesome Peak quadrangle. Lower: Location of bench mark on the left bank. MN is magnetic north. 

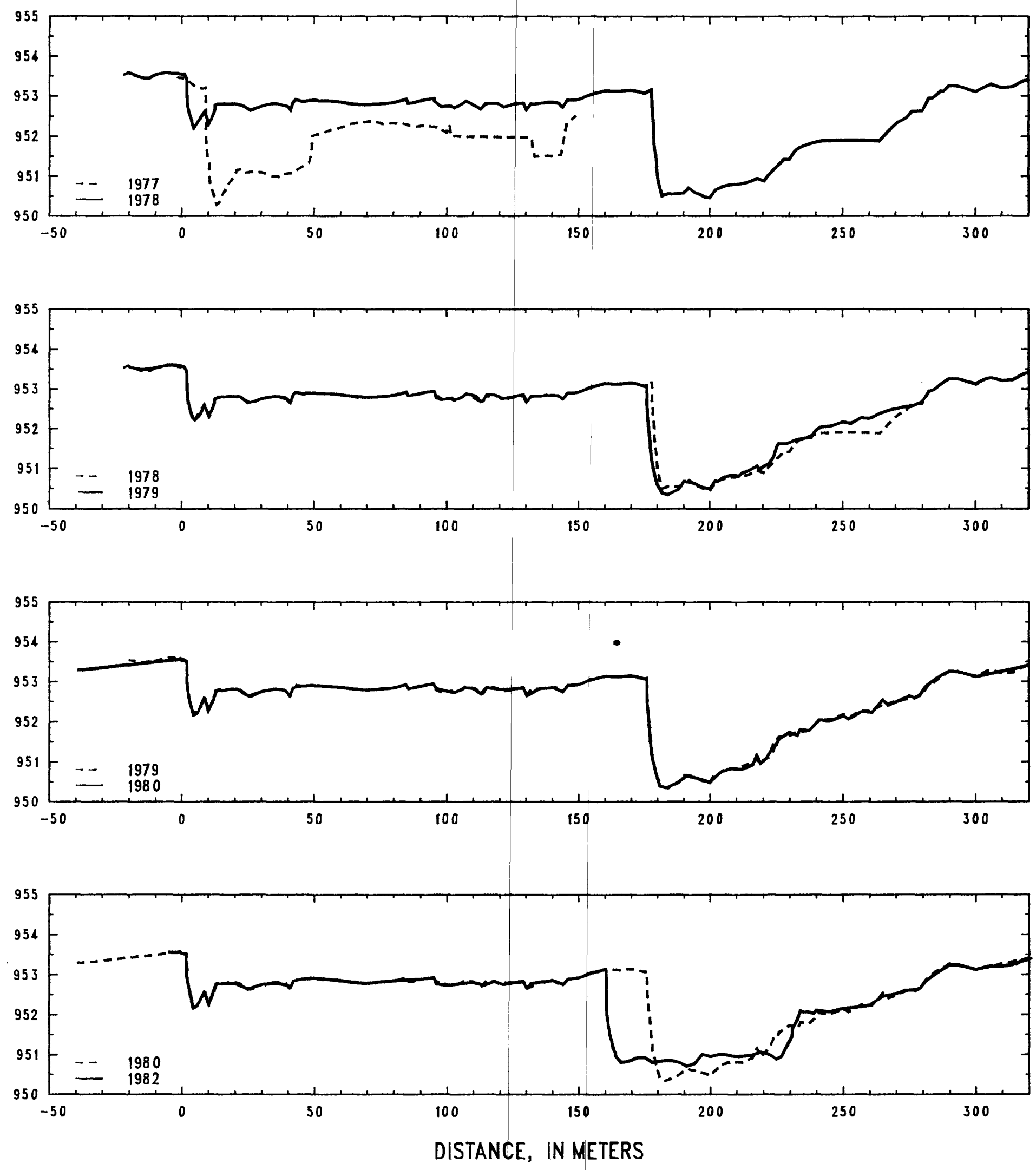

Figure 69.--Profiles of cross section PR175 from 1977 to 1982. 

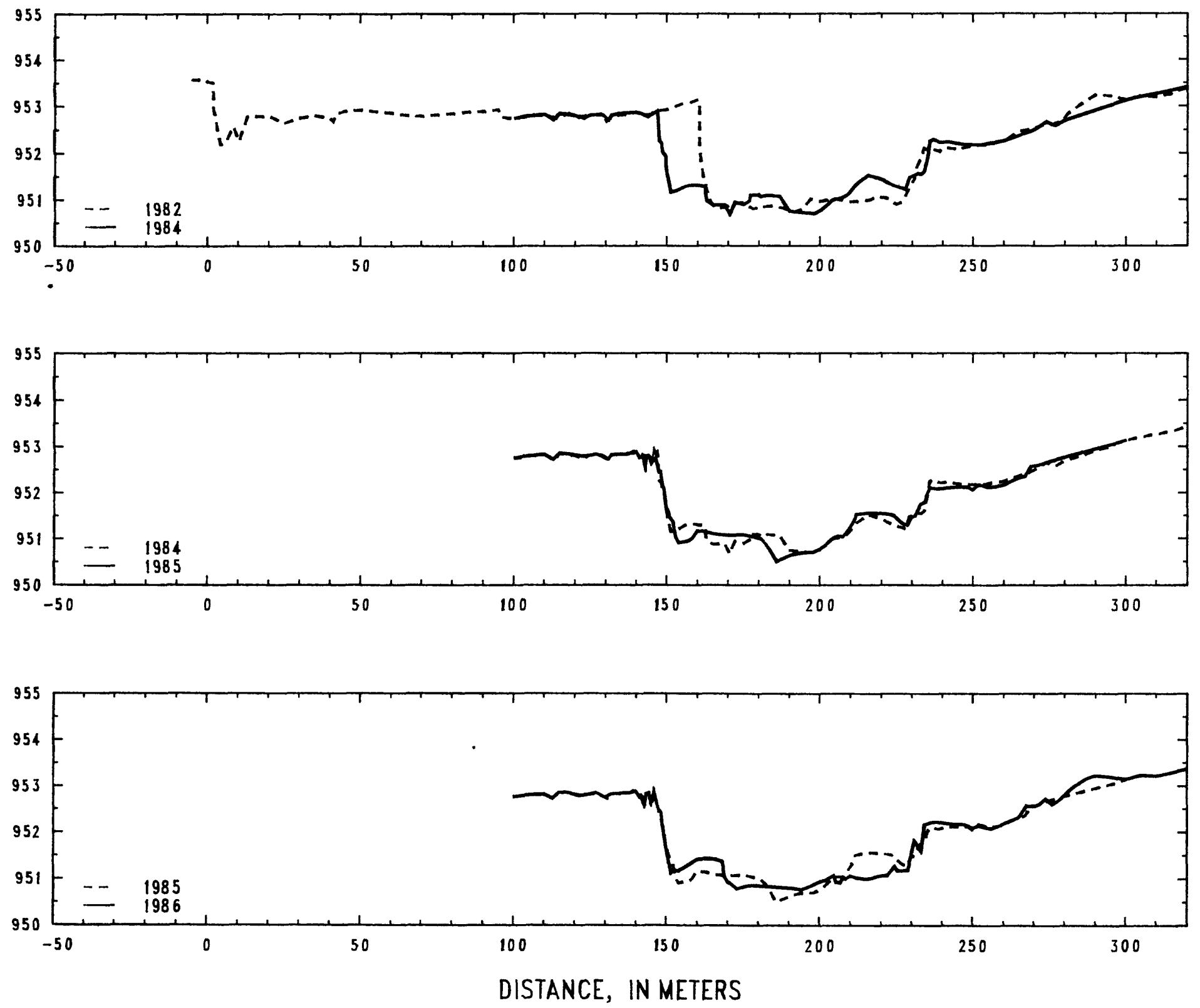

igure 70.--Profiles of cross section PR175 from 1982 to 1986. 


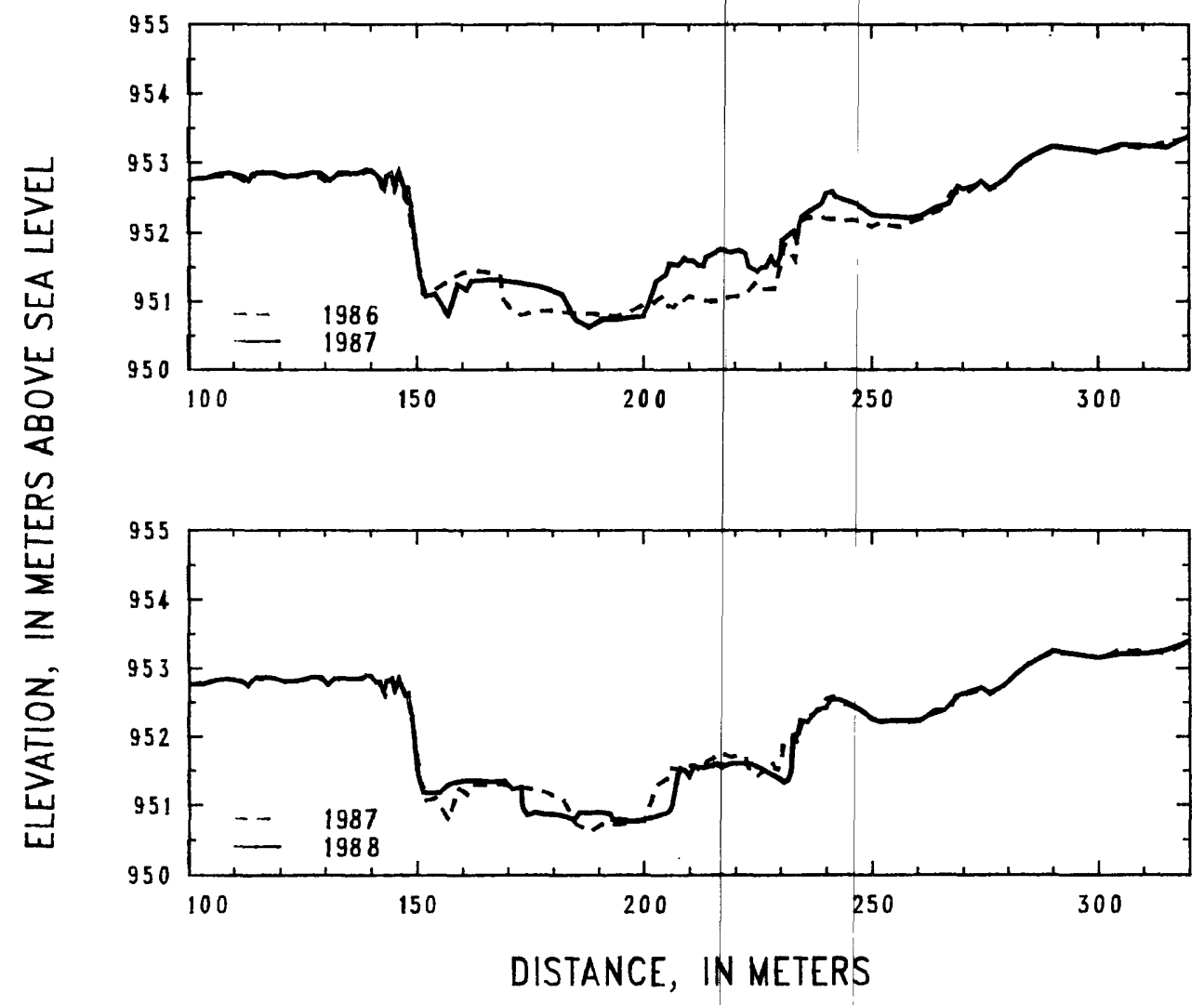

Figure 71.--Profiles of cross section PR175 from 1986 to 1988. 
Table 23.--Listing of horizontal stations and elevations for cross section PR175

[Sta., station, distance in meters from a reference point on

the left bank; Elev., elevation, in meters above sea level].

\begin{tabular}{|c|c|c|c|c|c|c|c|c|c|}
\hline \multicolumn{2}{|c|}{1977} & \multicolumn{2}{|c|}{1977} & \multicolumn{2}{|c|}{1978} & \multicolumn{2}{|c|}{1978} & \multicolumn{2}{|c|}{1978} \\
\hline Sta. & Elev. & Sta. & Elev. & Sta. & Elev. & Sta. & $\overline{\text { Elev. }}$ & Sta. & Elev. \\
\hline-1.7 & 953.46 & 76.0 & 952.30 & -22.0 & 953.53 & 95.3 & 952.95 & 198.0 & 950.47 \\
\hline 0.0 & 953.46 & 80.0 & 952.33 & -20.2 & 953.58 & 95.4 & 952.87 & 200.0 & 950.45 \\
\hline 2.0 & 953.42 & 84.0 & 952.30 & -18.0 & 953.53 & 98.0 & 952.73 & 202.0 & 950.65 \\
\hline 4.0 & 953.30 & 88.0 & 952.23 & -16.0 & 953.47 & 100.0 & 952.75 & 204.0 & 950.71 \\
\hline 6.0 & 953.20 & 92.0 & 952.26 & -14.0 & 953.45 & 101.5 & 952.75 & 206.0 & 950.76 \\
\hline 8.0 & 953.18 & 96.0 & 952.22 & -12.0 & 953.45 & 103.1 & 952.70 & 210.0 & 950.80 \\
\hline 9.0 & 953.21 & 100.0 & 952.09 & -10.2 & 953.53 & 107.5 & 952.88 & 214.0 & 950.84 \\
\hline 9.3 & 951.79 & 101.2 & 952.28 & -8.0 & 953.57 & 113.0 & 952.67 & 218.0 & 950.95 \\
\hline 9.8 & 951.41 & 101.6 & 952.00 & -6.0 & 953.59 & 114.7 & 952.81 & 220.5 & 950.88 \\
\hline 10.4 & 951.32 & 132.2 & 951.96 & -4.0 & 953.57 & 116.0 & 952.82 & 221.5 & 950.98 \\
\hline 10.6 & 950.90 & 133.3 & 951.49 & -1.7 & 953.56 & 119.0 & 952.82 & 224.0 & 951.15 \\
\hline 11.0 & 950.79 & 143.3 & 951.54 & 1.0 & 953.55 & 121.5 & 952.73 & 228.0 & 951.43 \\
\hline 12.0 & 950.53 & 145.7 & 952.34 & 2.0 & 953.45 & 126.0 & 952.82 & 230.0 & 951.42 \\
\hline 13.0 & 950.27 & 148.8 & 952.50 & 2.1 & 952.80 & 129.2 & 952.83 & 232.0 & 951.63 \\
\hline 14.0 & 950.32 & & & 2.7 & 952.57 & 130.3 & 952.65 & 234.0 & 951.73 \\
\hline 15.0 & 950.50 & & & 3.7 & 952.38 & 132.0 & 952.81 & 238.0 & 951.83 \\
\hline 16.0 & 950.60 & & & 4.6 & 952.19 & 133.3 & 952.80 & 242.0 & 951.89 \\
\hline 17.0 & 950.73 & & & 8.5 & 952.61 & 140.0 & 952.86 & 246.0 & 951.91 \\
\hline 18.0 & 950.83 & & & 10.3 & 952.29 & 142.5 & 952.82 & 250.0 & 951.91 \\
\hline 19.0 & 950.93 & & & 12.4 & 952.65 & 143.3 & 952.75 & 260.0 & 951.90 \\
\hline 20.1 & 951.01 & & & 12.5 & 952.77 & 144.0 & 952.72 & 264.0 & 951.89 \\
\hline 20.8 & 951.16 & & & 13.0 & 952.79 & 145.7 & 952.91 & 268.0 & 952.18 \\
\hline 22.0 & 951.17 & & & 16.0 & 952.80 & 148.8 & 952.90 & 271.0 & 952.36 \\
\hline 24.0 & 951.08 & & & 20.0 & 952.81 & 150.0 & 952.91 & 274.0 & 952.46 \\
\hline 26.0 & 951.11 & & & 23.0 & 952.76 & 155.0 & 953.05 & 276.0 & 952.62 \\
\hline 28.0 & 951.10 & & & 26.0 & 952.64 & 160.0 & 953.14 & 280.0 & 952.64 \\
\hline 30.0 & 951.09 & & & 30.0 & 952.75 & 165.0 & 953.12 & 282.5 & 952.95 \\
\hline 31.7 & 951.05 & & & 35.0 & 952.82 & 170.0 & 953.15 & 284.0 & 952.96 \\
\hline 33.0 & 951.02 & & & 40.0 & 952.74 & 175.0 & 953.07 & 287.0 & 953.13 \\
\hline 35.0 & 950.98 & & & 41.0 & 952.64 & 178.0 & 953.17 & 288.0 & 953.12 \\
\hline 37.0 & 950.98 & & & 42.0 & 952.85 & 178.4 & 952.45 & 290.0 & 953.26 \\
\hline 38.0 & 951.04 & & & 43.0 & 952.92 & 178.7 & 952.33 & 293.0 & 953.26 \\
\hline 40.0 & 951.07 & & & 45.3 & 952.86 & 178.9 & 951.86 & 296.0 & 953.19 \\
\hline 42.0 & 951.09 & & & 50.0 & 952.90 & 179.1 & 951.66 & 300.0 & 953.11 \\
\hline 44.0 & 951.18 & & & 52.0 & 952.88 & 179.5 & 951.54 & 304.0 & 953.25 \\
\hline 46.0 & 951.26 & & & 56.0 & 952.88 & 180.2 & 950.97 & 306.0 & 953.30 \\
\hline 48.0 & 951.33 & & & 60.0 & 952.85 & 181.0 & 950.71 & 310.0 & 953.20 \\
\hline 49.0 & 951.56 & & & 64.0 & 952.80 & 182.0 & 950.50 & 314.0 & 953.24 \\
\hline 49.4 & 952.00 & & & 68.0 & 952.78 & 184.0 & 950.56 & 318.0 & 953.39 \\
\hline 52.0 & 952.05 & & & 72.0 & 952.80 & 186.0 & 950.55 & 320.0 & 953.41 \\
\hline 56.0 & 952.15 & & & 76.0 & 952.81 & 188.0 & 950.56 & & \\
\hline 60.0 & 952.21 & & & 80.0 & 952.84 & 190.0 & 950.58 & & \\
\hline 64.0 & 952.31 & & & 84.9 & 952.93 & 192.0 & 950.71 & & \\
\hline 68.0 & 952.34 & & & 85.5 & 952.81 & 194.0 & 950.59 & & \\
\hline 72.0 & 952.39 & & & 90.0 & 952.89 & 196.0 & 950.54 & & \\
\hline
\end{tabular}


Table 23.--Listing of horizontal stations and elevations for cross section PR175 --Continued.

\begin{tabular}{|c|c|c|c|c|c|c|c|c|c|}
\hline \multicolumn{2}{|c|}{1979} & \multicolumn{2}{|c|}{1979} & \multicolumn{2}{|c|}{1979} & \multicolumn{2}{|c|}{1979} & \multicolumn{2}{|c|}{1980} \\
\hline Sta. & Elev. & Sta. & Elev. & Sta. & Elev. & Sta. & Elev. & Sta. & Elev. \\
\hline-20.0 & 953.55 & 100.0 & 952.75 & 200.0 & 950.50 & 320.0 & 953.41 & -39.3 & 953.28 \\
\hline-15.0 & 953.48 & 102.0 & 952.77 & 202.0 & 950.69 & & & -1.7 & 953.55 \\
\hline-10.0 & 953.52 & 103.0 & 952.72 & 203.0 & 950.67 & & & -0.7 & 953.58 \\
\hline-5.0 & 953.61 & 106.0 & 952.80 & 205.0 & 950.78 & & & 0.0 & 953.52 \\
\hline-0.7 & 953.59 & 107.5 & 952.89 & 208.0 & 950.83 & & & 1.0 & 953.53 \\
\hline 1.0 & 953.55 & 111.0 & 952.82 & 209.0 & 950.85 & & & 1.8 & 953.51 \\
\hline 2.0 & 953.45 & 113.0 & 952.68 & 211.0 & 950.81 & & & 2.0 & 953.42 \\
\hline 2.1 & 952.87 & 114.0 & 952.71 & 212.0 & 950.89 & & & 2.1 & 952.88 \\
\hline 3.0 & 952.52 & 115.0 & 952.86 & 215.0 & 950.96 & & & 3.0 & 952.49 \\
\hline 4.0 & 952.28 & 118.0 & 952.85 & 217.9 & 951.07 & & & 4.4 & 952.15 \\
\hline 5.0 & 952.21 & 121.0 & 952.76 & 218.7 & 950.95 & & & 6.0 & 952.21 \\
\hline 7.0 & 952.35 & 124.0 & 952.79 & 220.0 & 951.05 & & & 8.4 & 952.58 \\
\hline 8.5 & 952.60 & 127.0 & 952.84 & 222.0 & 951.11 & & & 8.7 & 952.57 \\
\hline 10.3 & 952.31 & 129.0 & 952.86 & 224.0 & 951.27 & & & 10.0 & 952.25 \\
\hline 12.0 & 952.56 & 130.4 & 952.67 & 225.4 & 951.56 & & & 12.0 & 952.56 \\
\hline 12.5 & 952.76 & 132.0 & 952.82 & 226.0 & 951.63 & & & 13.0 & 952.77 \\
\hline 14.0 & 952.79 & 135.0 & 952.82 & 229.0 & 951.63 & & & 15.0 & 952.76 \\
\hline 15.0 & 952.80 & 138.0 & 952.85 & 232.0 & 951.72 & & & 18.0 & 952.81 \\
\hline 18.0 & 952.82 & 142.0 & 952.85 & 235.0 & 951.76 & & & 21.0 & 952.80 \\
\hline 22.0 & 952.80 & 144.0 & 952.75 & 238.0 & 951.79 & & & 24.0 & 952.67 \\
\hline 25.0 & 952.65 & 145.0 & 952.82 & 240.0 & 951.98 & & & 26.0 & 952.62 \\
\hline 28.0 & 952.70 & 146.0 & 952.91 & 242.0 & 952.05 & & & 30.0 & 952.74 \\
\hline 30.0 & 952.75 & 148.0 & 952.90 & 246.0 & 952.08 & & & 35.0 & 952.81 \\
\hline 35.0 & 952.82 & 150.0 & 952.93 & 250.0 & 952.18 & & & 39.0 & 952.78 \\
\hline 39.0 & 952.78 & 151.0 & 952.95 & 253.0 & 952.13 & & & 40.8 & 952.63 \\
\hline 41.0 & 952.65 & 155.0 & 953.06 & 257.0 & 952.28 & & & 42.0 & 952.84 \\
\hline 42.0 & 952.86 & 160.0 & 953.14 & 260.0 & 952.25 & & & 45.0 & 952.88 \\
\hline 43.0 & 952.92 & 165.0 & 953.12 & 264.0 & 952.40 & & & 50.0 & 952.90 \\
\hline 45.0 & 952.89 & 170.0 & 953.16 & 268.0 & 952.48 & & & 55.0 & 952.87 \\
\hline 50.0 & 952.90 & 175.0 & 953.07 & 271.0 & 952.52 & & & 60.0 & 952.84 \\
\hline 54.0 & 952.88 & 176.1 & 953.10 & 274.0 & 952.57 & & & 65.0 & 952.80 \\
\hline 58.0 & 952.87 & 176.3 & 952.47 & 276.0 & 952.56 & & & 70.0 & 952.78 \\
\hline 62.0 & 952.83 & 177.0 & 951.89 & 280.0 & 952.69 & & & 75.0 & 952.81 \\
\hline 66.0 & 952.80 & 178.0 & 951.20 & 282.5 & 952.96 & & & 80.0 & 952.83 \\
\hline 70.0 & 952.79 & 179.0 & 950.85 & 284.0 & 952.97 & & & 84.7 & 952.92 \\
\hline 74.0 & 952.80 & 180.0 & 950.58 & 287.0 & 953.13 & & & 85.6 & 952.81 \\
\hline 78.0 & 952.82 & 182.0 & 950.39 & 290.0 & 953.26 & & & 90.0 & 952.88 \\
\hline 82.0 & 952.86 & 184.0 & 950.34 & 293.0 & 953.26 & & & 95.0 & 952.94 \\
\hline 84.7 & 952.93 & 186.0 & 950.42 & 296.0 . & 953.19 & & & 96.0 & 952.82 \\
\hline 85.5 & 952.83 & 188.0 & 950.48 & 300.0 & 953.13 & & & 100.0 & 952.74 \\
\hline 89.0 & 952.88 & 190.0 & 950.68 & 304.0 & 953.26 & & & 103.0 & 952.71 \\
\hline 92.0 & 952.92 & 192.0 & 950.66 & 306.0 & 953.29 & & & 107.0 & 952.85 \\
\hline 95.3 & 952.95 & 194.0 & 950.63 & 310.0 & 953.20 & & & 110.0 & 952.82 \\
\hline 96.0 & 952.78 & 196.0 & 950.56 & 314.0 & 953.23 & & & 113.0 & 952.68 \\
\hline 98.0 & 952.73 & 198.0 & 950.51 & 318.0 & 953.38 & & & 115.0 & 952.84 \\
\hline
\end{tabular}


Table 23.---Listing of horizontal stations and elevations for cross section PR175 --Continued.

\begin{tabular}{|c|c|c|c|c|c|c|c|c|c|}
\hline \multicolumn{2}{|c|}{1980} & \multicolumn{2}{|c|}{1980} & \multicolumn{2}{|c|}{1982} & \multicolumn{2}{|c|}{1982} & \multicolumn{2}{|c|}{1982} \\
\hline Sta. & $\overline{\text { Elev. }}$ & Sta. & Elev. & Sta. & Elev. & Sta. & Elev. & Sta. & Elev. \\
\hline 120.0 & 952.79 & 252.0 & 952.06 & -5.0 & 953.57 & 146.0 & 952.89 & 239.0 & 952.02 \\
\hline 125.0 & 952.80 & 257.0 & 952.24 & -1.7 & 953.57 & 150.0 & 952.92 & 240.0 & 952.11 \\
\hline 129.0 & 952.84 & 261.0 & 952.23 & -0.7 & 953.58 & 151.0 & 952.94 & 245.0 & 952.07 \\
\hline 130.4 & 952.64 & 265.0 & 952.54 & 0.0 & 953.52 & 155.0 & 953.05 & 250.0 & 952.16 \\
\hline 135.0 & 952.81 & 267.0 & 952.40 & 1.9 & 953.50 & 158.0 & 953.09 & 255.0 & 952.18 \\
\hline 140.0 & 952.85 & 270.0 & 952.49 & 2.0 & 952.95 & 160.0 & 953.14 & 260.0 & 952.24 \\
\hline 144.0 & 952.73 & 275.0 & 952.63 & 4.3 & 952.18 & 160.3 & 953.12 & 265.0 & 952.46 \\
\hline 146.0 & 952.90 & 277.0 & 952.58 & 6.0 & 952.22 & 160.4 & 953.11 & 270.0 & 952.52 \\
\hline 150.0 & 952.92 & 279.0 & 952.66 & 8.4 & 952.56 & 160.6 & 952.16 & 273.0 & 952.60 \\
\hline 151.0 & 952.94 & 281.0 & 952.86 & 10.3 & 952.25 & 160.6 & 952.15 & 276.0 & 952.61 \\
\hline 155.0 & 953.05 & 285.0 & 953.07 & 13.0 & 952.77 & 160.8 & 951.96 & 279.0 & 952.66 \\
\hline 160.0 & 953.13 & 290.0 & 953.26 & 16.0 & 952.78 & 161.5 & 951.61 & 281.0 & 952.83 \\
\hline 165.0 & 953.11 & 295.0 & 953.21 & 20.0 & 952.79 & 162.0 & 951.45 & 285.0 & 953.02 \\
\hline 170.0 & 953.14 & 300.0 & 953.11 & 25.0 & 952.63 & 162.4 & 951.36 & 290.0 & 953.24 \\
\hline 175.0 & 953.06 & 320.0 & 953.40 & 30.0 & 952.75 & 163.0 & 951.18 & 295.0 & 953.21 \\
\hline 176.0 & 953.08 & & & 35.0 & 952.80 & 164.0 & 951.03 & 300.0 & 953.12 \\
\hline 176.0 & 952.52 & & & 40.0 & 952.73 & 164.0 & 950.96 & 305.0 & 953.21 \\
\hline 177.0 & 951.92 & & & 41.0 & 952.67 & 166.0 & 950.80 & 310.0 & 953.19 \\
\hline 178.0 & 951.10 & & & 42.0 & 952.85 & 169.0 & 950.83 & 315.0 & 953.27 \\
\hline 181.0 & 950.38 & & & 45.0 & 952.89 & 172.0 & 950.92 & 320.0 & 953.40 \\
\hline 183.0 & 950.34 & & & 50.0 & 952.92 & 175.0 & 950.93 & & \\
\hline 185.0 & 950.39 & & & 55.0 & 952.88 & 178.0 & 950.80 & & \\
\hline 188.0 & 950.49 & & & 60.0 & 952.85 & 181.0 & 950.84 & & \\
\hline 191.0 & 950.64 & & & 65.0 & 952.80 & 185.0 & 950.86 & & \\
\hline 195.0 & 950.58 & & & 70.0 & 952.78 & 188.0 & 950.81 & & \\
\hline 200.0 & 950.47 & & & 75.0 & 952.82 & 191.0 & 950.71 & & \\
\hline 201.0 & 950.55 & & & 80.0 & 952.85 & 194.0 & 950.78 & & \\
\hline 204.0 & 950.73 & & & 85.0 & 952.87 & 197.0 & 951.01 & & \\
\hline 208.0 & 950.83 & & & 90.0 & 952.89 & 200.0 & 950.95 & & \\
\hline 212.0 & 950.80 & & & 90.0 & 952.88 & 204.0 & 951.02 & & \\
\hline 216.0 & 950.92 & & & 95.0 & 952.93 & 207.0 & 950.98 & & \\
\hline 217.6 & 951.16 & & & 96.0 & 952.76 & 210.0 & 950.95 & & \\
\hline 219.0 & 950.94 & & & 100.0 & 952.72 & 213.0 & 950.97 & & \\
\hline 221.0 & 951.06 & & & 105.0 & 952.78 & 216.0 & 950.99 & & \\
\hline 223.0 & 951.26 & & & 110.0 & 952.81 & 219.0 & 951.07 & & \\
\hline 224.0 & 951.42 & & & 114.0 & 952.73 & 222.0 & 951.01 & & \\
\hline 225.0 & 951.41 & & & 118.0 & 952.84 & 225.0 & 950.89 & & \\
\hline 226.0 & 951.56 & & & 121.0 & 952.75 & 227.0 & 950.95 & & \\
\hline 230.0 & 951.73 & & & 125.0 & 952.80 & 229.7 & 951.32 & & \\
\hline 233.0 & 951.65 & & & 129.0 & 952.83 & 230.8 & 951.45 & & \\
\hline 234.0 & 951.80 & & & 130.3 & 952.66 & 231.0 & 951.65 & & \\
\hline 237.5 & 951.77 & & & 132.0 & 952.78 & 233.0 & 951.88 & & \\
\hline 241.0 & 952.04 & & & 136.0 & 952.82 & 234.0 & 952.10 & & \\
\hline 245.0 & 952.01 & & & 140.0 & 952.86 & 235.0 & 952.06 & & \\
\hline 250.0 & 952.14 & & & 144.0 & 952.75 & 237.0 & 952.07 & & \\
\hline
\end{tabular}


Table 23.--Listing of horizontal stations and elevations for cross section PR175 --Continued.

\begin{tabular}{|c|c|c|c|c|c|c|c|c|c|}
\hline \multicolumn{2}{|c|}{1984} & \multicolumn{2}{|c|}{1984} & \multicolumn{2}{|c|}{1985} & \multicolumn{2}{|c|}{1985} & \multicolumn{2}{|c|}{1986} \\
\hline Sta. & Elev. & Sta. & Elev. & Sta. & Elev. & Sta. & Elev. & Sta. & Elev. \\
\hline 100.0 & 952.73 & 200.0 & 950.75 & 100.0 & 952.74 & 186.0 & 950.49 & 100.0 & 952.75 \\
\hline 105.0 & 952.79 & 202.0 & 950.86 & 105.0 & 952.79 & 190.0 & 950.63 & 105.0 & 952.80 \\
\hline 110.0 & 952.82 & 204.0 & 950.99 & 110.0 & 952.82 & 194.0 & 950.69 & 110.0 & 952.81 \\
\hline 113.0 & 952.70 & 207.0 & 951.03 & 113.0 & 952.71 & 198.0 & 950.70 & 113.0 & 952.71 \\
\hline 115.0 & 952.84 & 209.0 & 951.10 & 115.0 & 952.85 & 201.0 & 950.80 & 115.0 & 952.86 \\
\hline 120.0 & 952.79 & 212.0 & 951.35 & 120.0 & 952.81 & 204.0 & 950.99 & 118.0 & 952.86 \\
\hline 123.0 & 952.73 & 215.6 & 951.52 & 123.0 & 952.77 & 206.0 & 951.05 & 121.0 & 952.78 \\
\hline 124.0 & 952.77 & 218.0 & 951.47 & 124.0 & 952.79 & 207.5 & 951.03 & 124.0 & 952.79 \\
\hline 125.0 & 952.81 & 220.7 & 951.41 & 127.0 & 952.83 & 210.0 & 951.26 & 127.0 & 952.85 \\
\hline 129.0 & 952.81 & 221.3 & 951.37 & 130.0 & 952.73 & 211.2 & 951.39 & 130.0 & 952.74 \\
\hline 131.0 & 952.69 & 224.0 & 951.29 & 131.0 & 952.71 & 211.7 & 951.52 & 132.0 & 952.82 \\
\hline 132.0 & 952.81 & 228.0 & 951.21 & 132.0 & 952.82 & 214.0 & 951.54 & 135.0 & 952.84 \\
\hline 135.0 & 952.84 & 229.2 & 951.48 & 135.0 & 952.83 & 217.0 & 951.56 & 138.0 & 952.85 \\
\hline 140.0 & 952.87 & 232.0 & 951.56 & 137.0 & 952.82 & 220.0 & 951.54 & 140.0 & 952.89 \\
\hline 143.0 & 952.78 & 233.0 & 951.54 & 139.0 & 952.89 & 224.0 & 951.50 & 141.2 & 952.75 \\
\hline 145.0 & 952.81 & 234.0 & 951.60 & 140.0 & 952.88 & 227.0 & 951.31 & 141.6 & 952.79 \\
\hline 146.9 & 952.89 & 235.0 & 951.88 & 141.0 & 952.75 & 228.7 & 951.28 & 142.7 & 952.57 \\
\hline 147.6 & 952.24 & 235.9 & 952.25 & 142.0 & 952.77 & 230.0 & 951.47 & 143.4 & 952.82 \\
\hline 148.3 & 952.20 & 237.0 & 952.29 & 143.0 & 952.53 & 231.0 & 951.49 & 144.3 & 952.85 \\
\hline 148.7 & 951.99 & 239.0 & 952.21 & 143.3 & 952.77 & 233.0 & 951.75 & 145.1 & 952.62 \\
\hline 149.6 & 951.92 & 242.0 & 952.23 & 144.5 & 952.77 & 234.5 & 951.79 & 146.1 & 952.87 \\
\hline 149.8 & 951.67 & 245.0 & 952.18 & 145.0 & 952.61 & 235.0 & 951.98 & 147.4 & 952.47 \\
\hline 151.3 & 951.15 & 250.0 & 952.17 & 145.6 & 952.68 & 236.0 & 952.10 & 148.2 & 952.42 \\
\hline 153.0 & 951.18 & 252.0 & 952.16 & 146.0 & 952.86 & 239.0 & 952.07 & 149.1 & 951.98 \\
\hline 156.0 & 951.29 & 255.0 & 952.20 & 146.3 & 952.74 & 242.0 & 952.10 & 150.2 & 951.58 \\
\hline 159.0 & 951.32 & 260.0 & 952.25 & 147.5 & 952.43 & 245.0 & 952.12 & 151.5 & 951.13 \\
\hline 162.4 & 951.27 & 263.0 & 952.32 & 148.0 & 952.44 & 248.0 & 952.12 & 152.0 & 951.17 \\
\hline 162.7 & 951.06 & 266.0 & 952.39 & 148.6 & 952.16 & 250.0 & 952.05 & 154.0 & 951.17 \\
\hline 163.0 & 950.96 & 270.0 & 952.49 & 149.2 & 952.06 & 252.0 & 952.17 & 157.0 & 951.29 \\
\hline 165.0 & 950.88 & 274.0 & 952.66 & 150.0 & 951.64 & 255.0 & 952.10 & 160.0 & 951.41 \\
\hline 168.0 & 950.89 & 277.0 & 952.57 & 150.4 & 951.60 & 260.0 & 952.15 & 163.0 & 951.44 \\
\hline 169.0 & 950.88 & 280.0 & 952.69 & 151.0 & 951.44 & 265.0 & 952.32 & 166.0 & 951.42 \\
\hline 170.5 & 950.67 & 300.0 & 953.13 & 152.0 & 951.36 & 267.0 & 952.35 & 168.3 & 951.37 \\
\hline 172.3 & 950.94 & 320.0 & 953.41 & 153.0 & 951.01 & 269.0 & 952.57 & 168.7 & 951.11 \\
\hline 175.0 & 950.89 & & & 154.0 & 950.90 & 271.0 & 952.58 & 169.0 & 951.03 \\
\hline 177.0 & 950.97 & & & 157.0 & 950.94 & 275.0 & 952.68 & 170.0 & 950.92 \\
\hline 177.4 & 951.10 & & & 158.5 & 951.00 & 300.0 & 953.13 & 171.0 & 950.89 \\
\hline 180.0 & 951.10 & & & 160.0 & 951.17 & & & 173.0 & 950.78 \\
\hline 180.5 & 951.05 & & & 162.0 & 951.15 & & & 176.0 & 950.85 \\
\hline 183.0 & 951.09 & & & 165.0 & 951.09 & & & 179.0 & 950.86 \\
\hline 187.0 & 951.06 & & & 170.0 & 951.06 & & & 182.0 & 950.83 \\
\hline 188.0 & 950.95 & & & 175.0 & 951.09 & & & 185.0 & 950.82 \\
\hline 190.0 & 950.74 & & & 180.0 & 951.00 & & & 188.0 & 950.81 \\
\hline 193.0 & 950.72 & & & 180.8 & 950.99 & & & 191.0 & 950.80 \\
\hline 198.0 & 950.69 & & & 183.0 & 950.83 & & & 194.0 & 950.76 \\
\hline
\end{tabular}


Table 23.--Listing of horizontal stations and elevations for cross section PR175 --Continued.

\begin{tabular}{|c|c|c|c|c|c|c|c|c|c|}
\hline \multicolumn{2}{|c|}{1986} & \multicolumn{2}{|c|}{1986} & \multicolumn{2}{|c|}{1987} & \multicolumn{2}{|c|}{1987} & \multicolumn{2}{|c|}{1987} \\
\hline Sta. & Elev. & Sta. & Elev. & Sta. & Elev. & Sta. & Elev. & Sta. & Elev. \\
\hline 197.0 & 950.85 & 295.0 & 953.19 & 100.0 & 952.76 & 182.0 & 951.09 & 255.0 & 952.23 \\
\hline 200.0 & 950.95 & 300.0 & 953.14 & 104.0 & 952.78 & 185.0 & 950.72 & 258.0 & 952.20 \\
\hline 202.0 & 950.97 & 305.0 & 953.24 & 106.0 & 952.82 & 188.0 & 950.61 & 261.0 & 952.24 \\
\hline 203.2 & 951.03 & 310.0 & 953.21 & 109.0 & 952.85 & 191.0 & 950.74 & 264.0 & 952.35 \\
\hline 204.6 & 951.08 & 315.0 & 953.29 & 112.0 & 952.80 & 194.0 & 950.72 & 267.0 & 952.41 \\
\hline 205.4 & 950.92 & 320.0 & 953.37 & 113.2 & 952.73 & 197.0 & 950.76 & 269.0 & 952.65 \\
\hline 206.8 & 950.91 & & & 114.1 & 952.84 & 200.0 & 950.77 & 270.0 & 952.62 \\
\hline 207.7 & 951.04 & & & 116.0 & 952.85 & 202.8 & 951.29 & 273.0 & 952.67 \\
\hline 208.5 & 951.00 & & & 119.0 & 952.86 & 205.0 & 951.39 & 274.0 & 952.74 \\
\hline 210.0 & 951.07 & & & 121.0 & 952.80 & 205.7 & 951.54 & 276.0 & 952.62 \\
\hline 212.0 & 951.02 & & & 124.0 & 952.81 & 207.0 & 951.53 & 279.0 & 952.73 \\
\hline 215.0 & 950.99 & & & 127.0 & 952.86 & 207.8 & 951.52 & 282.0 & 952.93 \\
\hline 216.5 & 951.00 & & & 129.0 & 952.85 & 209.0 & 951.62 & 285.0 & 953.09 \\
\hline 219.0 & 951.07 & & & 131.0 & 952.74 & 210.0 & 951.58 & 290.0 & 953.24 \\
\hline 222.0 & 951.08 & & & 133.0 & 952.85 & 211.0 & 951.59 & 295.0 & 953.20 \\
\hline 224.6 & 951.27 & & & 135.0 & 952.85 & 212.0 & 951.52 & 300.0 & 953.15 \\
\hline 225.4 & 951.17 & & & 137.0 & 952.84 & 213.2 & 951.51 & 305.0 & 953.27 \\
\hline 227.0 & 951.17 & & & 139.0 & 952.90 & 213.7 & 951.64 & 310.0 & 953.25 \\
\hline 229.0 & 951.19 & & & 140.1 & 952.89 & 215.0 & 951.68 & 315.0 & 953.22 \\
\hline 231.0 & 951.82 & & & 142.0 & 952.76 & 217.0 & 951.76 & 320.0 & 953.39 \\
\hline 233.3 & 951.59 & & & 142.9 & 952.62 & 219.0 & 951.70 & & \\
\hline 234.3 & 952.16 & & & 143.5 & 952.81 & 221.0 & 951.74 & & \\
\hline 236.0 & 952.20 & & & 144.4 & 952.86 & 222.4 & 951.68 & & \\
\hline 238.0 & 952.23 & & & 145.1 & 952.67 & 223.0 & 951.50 & & \\
\hline 240.0 & 952.19 & & & 146.0 & 952.85 & 225.0 & 951.43 & & \\
\hline 242.0 & 952.18 & & & 147.3 & 952.62 & 226.0 & 951.49 & & \\
\hline 244.0 & 952.16 & & & 148.0 & 952.64 & 227.0 & 951.50 & & \\
\hline 246.0 & 952.17 & & & 148.3 & 952.48 & 228.0 & 951.63 & & \\
\hline 248.0 & 952.15 & & & 148.6 & 952.40 & 228.8 & 951.53 & & \\
\hline 250.0 & 952.08 & & & 149.5 & 951.93 & 229.4 & 951.52 & & \\
\hline 252.0 & 952.14 & & & 150.7 & 951.35 & 230.2 & 951.66 & & \\
\hline 253.0 & 952.11 & & & 151.0 & 951.31 & 230.6 & 951.89 & & \\
\hline 256.0 & 952.07 & & & 152.0 & 951.06 & 233.0 & 952.02 & & \\
\hline 259.0 & 952.15 & & & 154.0 & 951.10 & 233.6 & 951.88 & & \\
\hline 262.0 & 952.24 & & & 157.0 & 950.78 & 234.7 & 952.21 & & \\
\hline 265.0 & 952.32 & & & 159.0 & 951.24 & 237.0 & 952.33 & & \\
\hline 267.6 & 952.57 & & & 161.0 & 951.16 & 239.0 & 952.41 & & \\
\hline 270.0 & 952.55 & & & 162.0 & 951.29 & 240.0 & 952.56 & & \\
\hline 272.0 & 952.61 & & & 164.0 & 951.30 & 241.4 & 952.59 & & \\
\hline 274.0 & 952.72 & & & 167.0 & 951.32 & 241.9 & 952.51 & & \\
\hline 276.0 & 952.60 & & & 170.0 & 951.29 & 244.0 & 952.47 & & \\
\hline 279.0 & 952.72 & & & 172.0 & 951.27 & 246.0 & 952.42 & & \\
\hline 282.0 & 952.93 & & & 174.0 & 951.24 & 248.0 & 952.35 & & \\
\hline 287.0 & 953.16 & & & 176.0 & 951.23 & 250.0 & 952.25 & & \\
\hline 290.0 & 953.23 & & & 179.0 & 951.16 & 252.0 & 952.23 & & \\
\hline
\end{tabular}


Table 23.--Listing of horizontal stations and elevations for cross section PR175 --Continued.

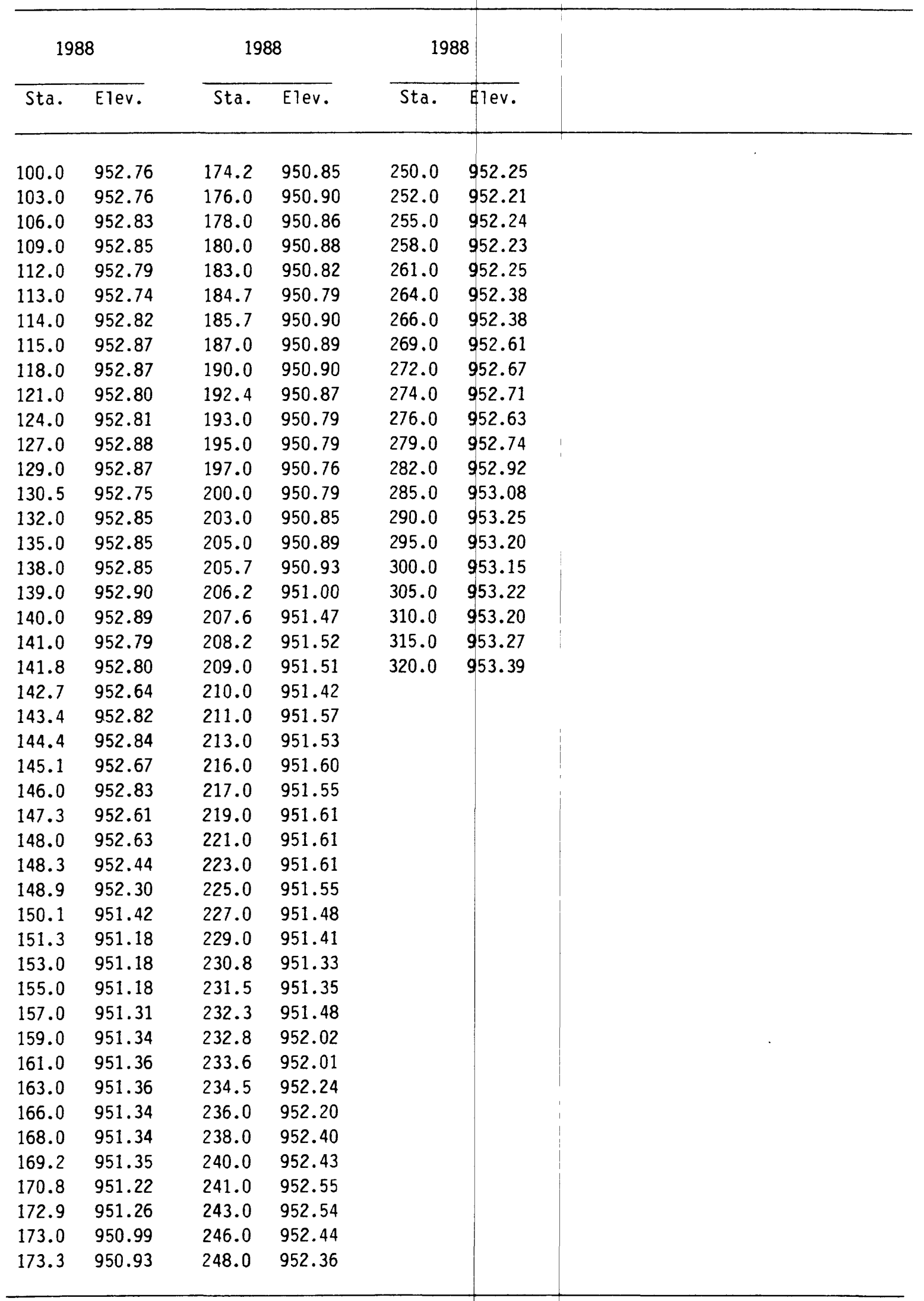




\section{Description of Cross Section PR180}

Location: Township 6 South/Range 50 East--section 12

U.S. Geological Survey quadrangle $(1: 24,000)$ : Lonesome Peak

Landowners--left bank: EB Ranch

right bank: U.S. Government

Access: left bank

Permission from: J.L. Wilson or Howard Best (T6S/R50E--1)

Reference pins

\begin{tabular}{|c|c|c|}
\hline $\begin{array}{l}\text { Station } \\
\text { (meters) }\end{array}$ & $\begin{array}{c}\text { Elevation } \\
\text { (meters } \\
\text { above } \\
\text { sea level) }\end{array}$ & Comments \\
\hline & & $\begin{array}{l}\text { Pins }-40.0 \text { and }-30.0 \text { were closest } \\
\text { to leveling instrument. }\end{array}$ \\
\hline-40.0 & 949.987 & $\begin{array}{l}\text { Bent, } 0.15 \text { meter above } 1987 \text { ground } \\
\text { level. }\end{array}$ \\
\hline-30.0 & 949.789 & $\begin{array}{l}\text { Bent, } 0.02 \text { meter above } 1987 \text { ground } \\
\text { level. }\end{array}$ \\
\hline 102.0 & 948.906 & At 1978 ground level. \\
\hline 103.4 & 948.966 & 0.06 meter above 1987 ground level \\
\hline 111.3 & 949.773 & 0.20 meter above 1987 ground level \\
\hline
\end{tabular}



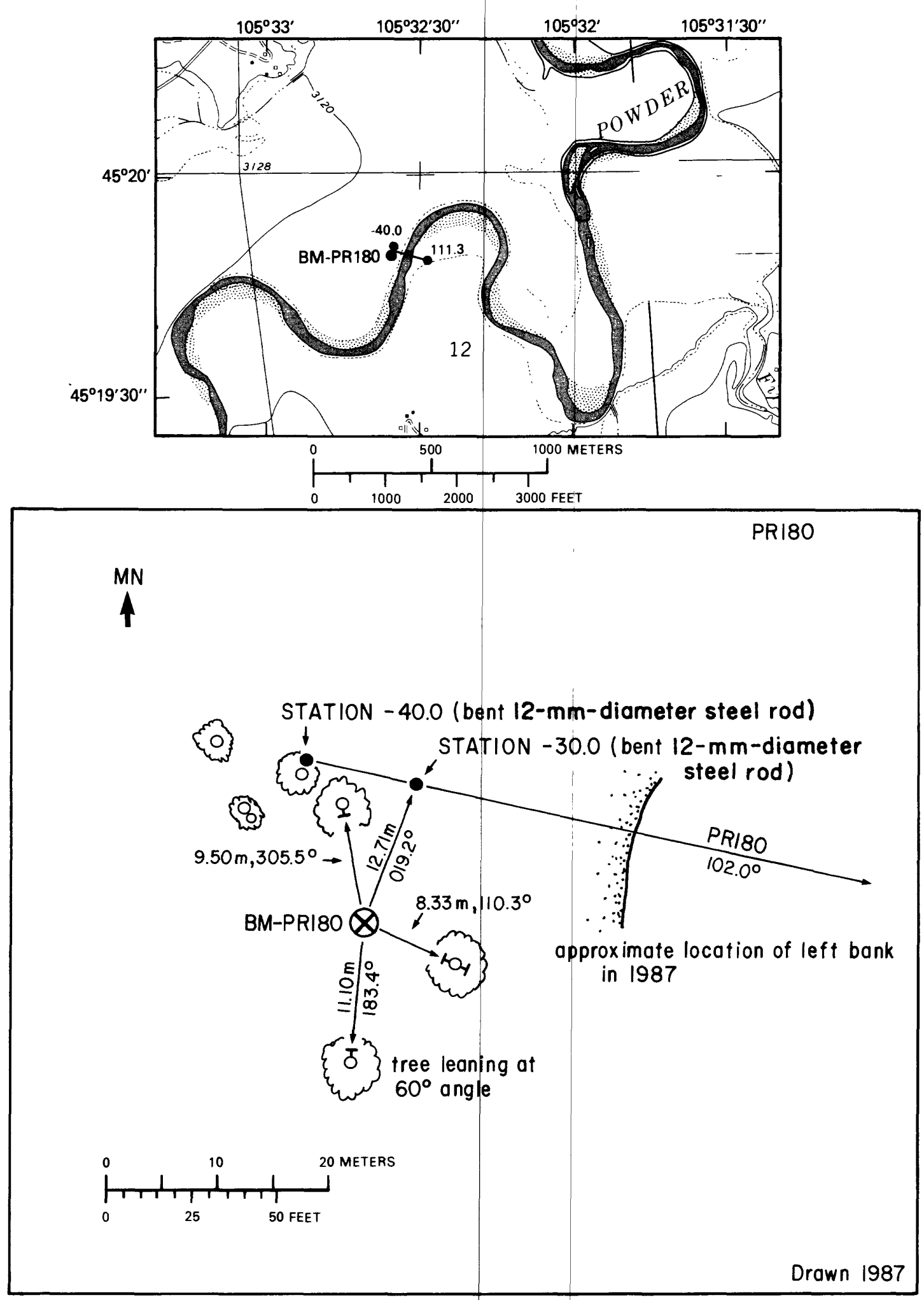

Figure 72.--Upper: Location of cross section PR180, bench mark BM-PR180, and left and right bank reference pins (tables 2 and 3) in the Lonesome Peak quadrangle. Lower: Location of bench mark on the left bank. MN is magnetic north. 

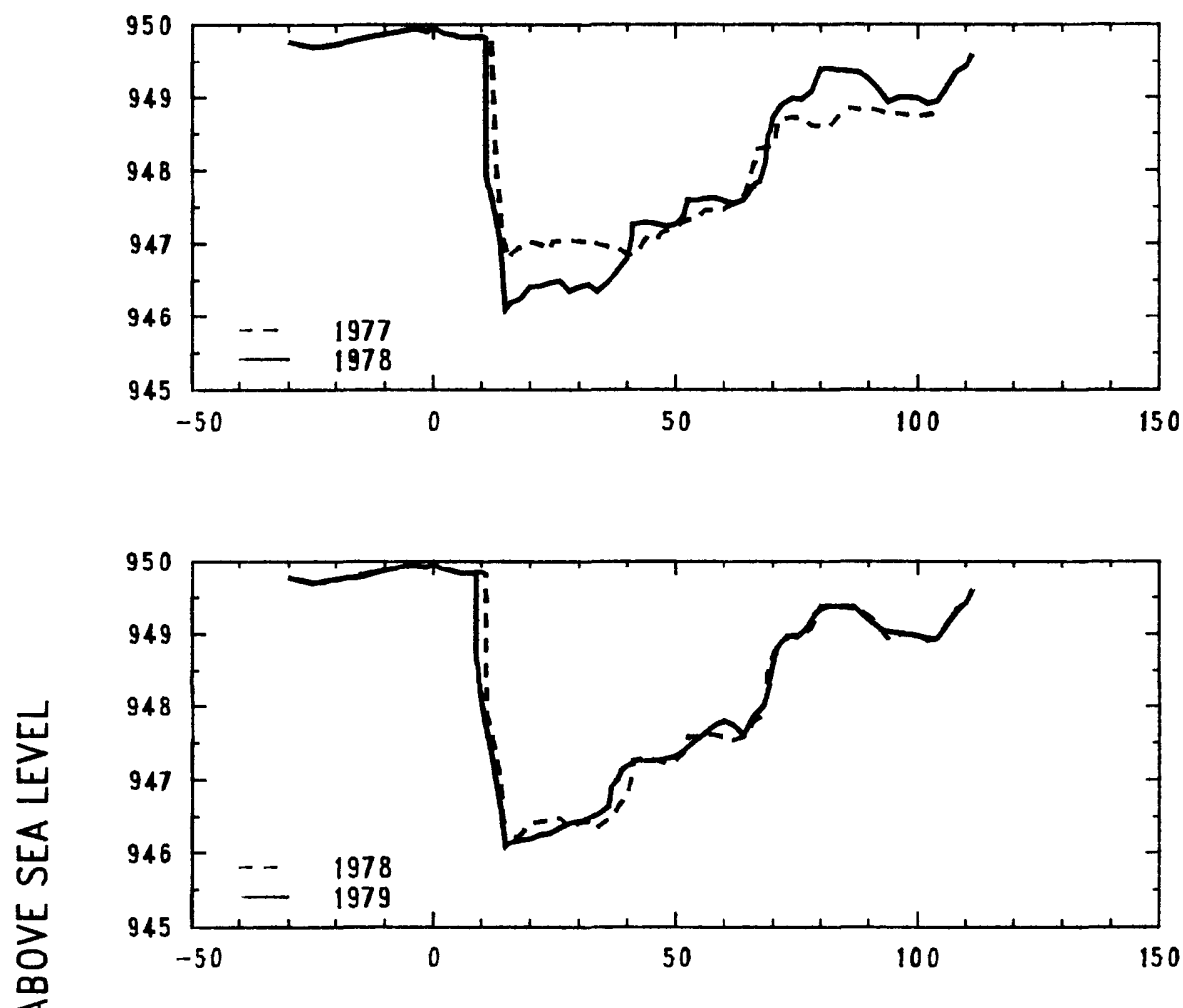

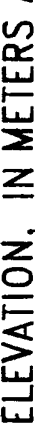
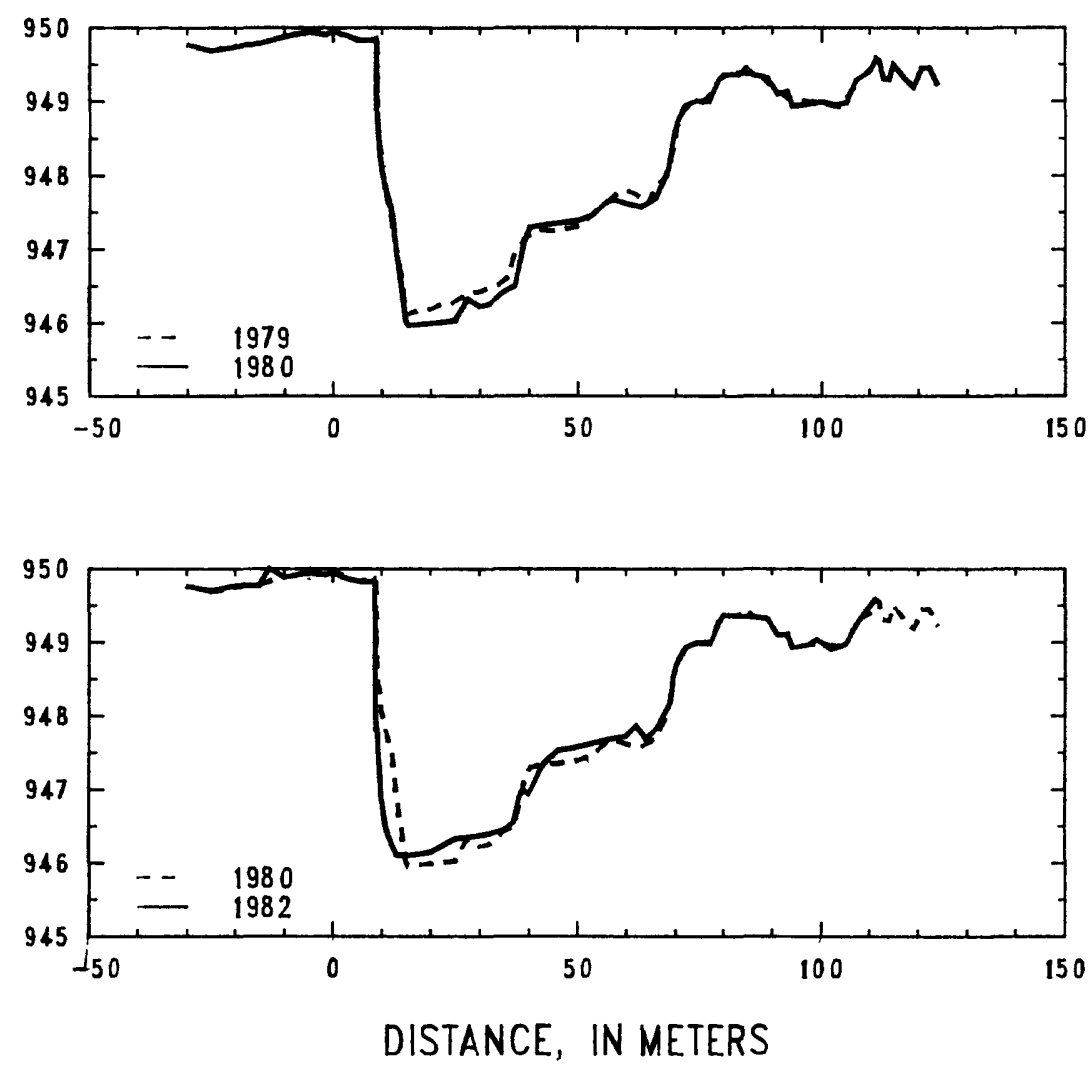

Figure 73.--Profiles of cross section PR180 from 1977 to 1982. 


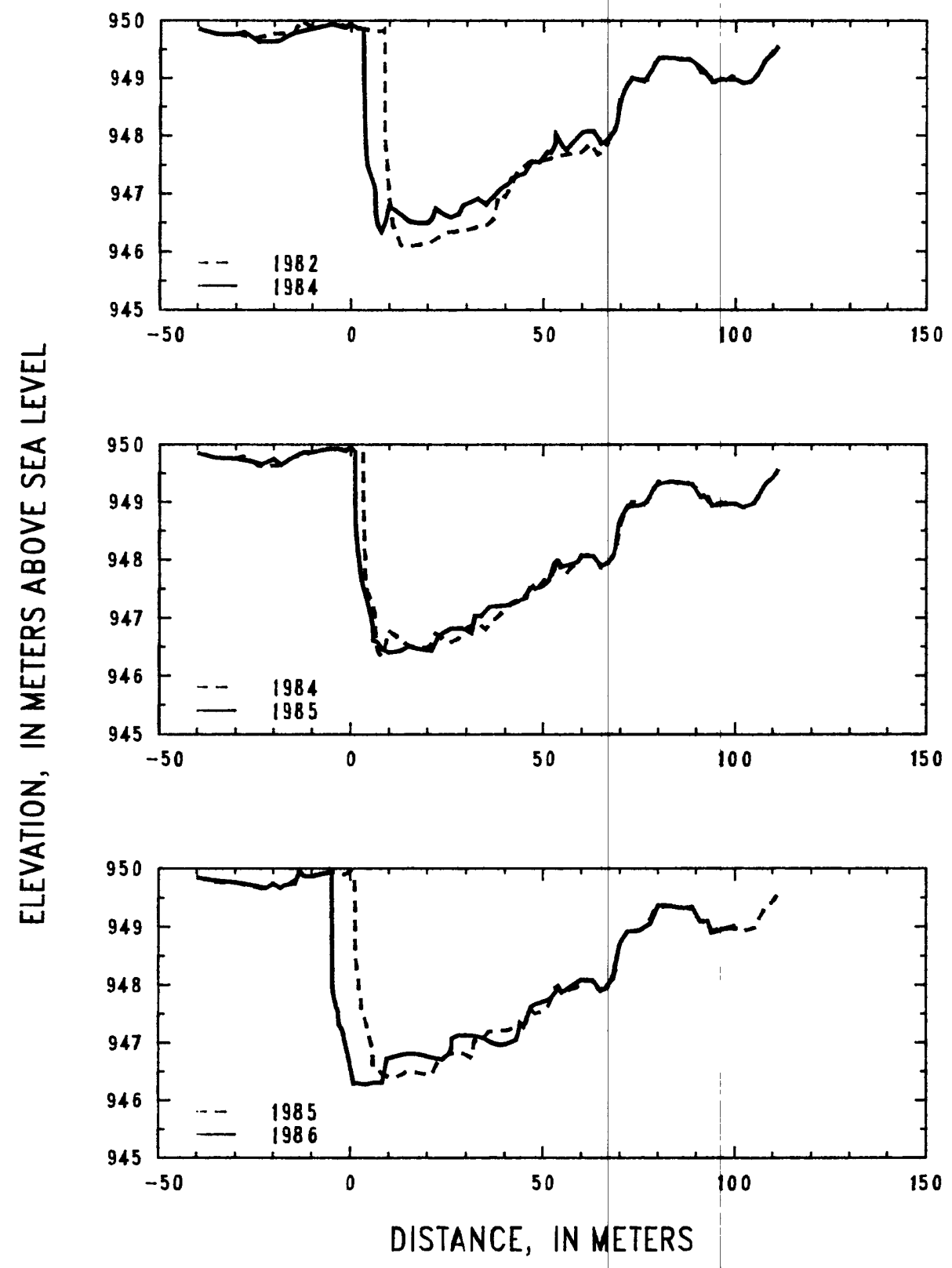

Figure 74.--Profiles of cross section PR180 from 1982 to 1986. 


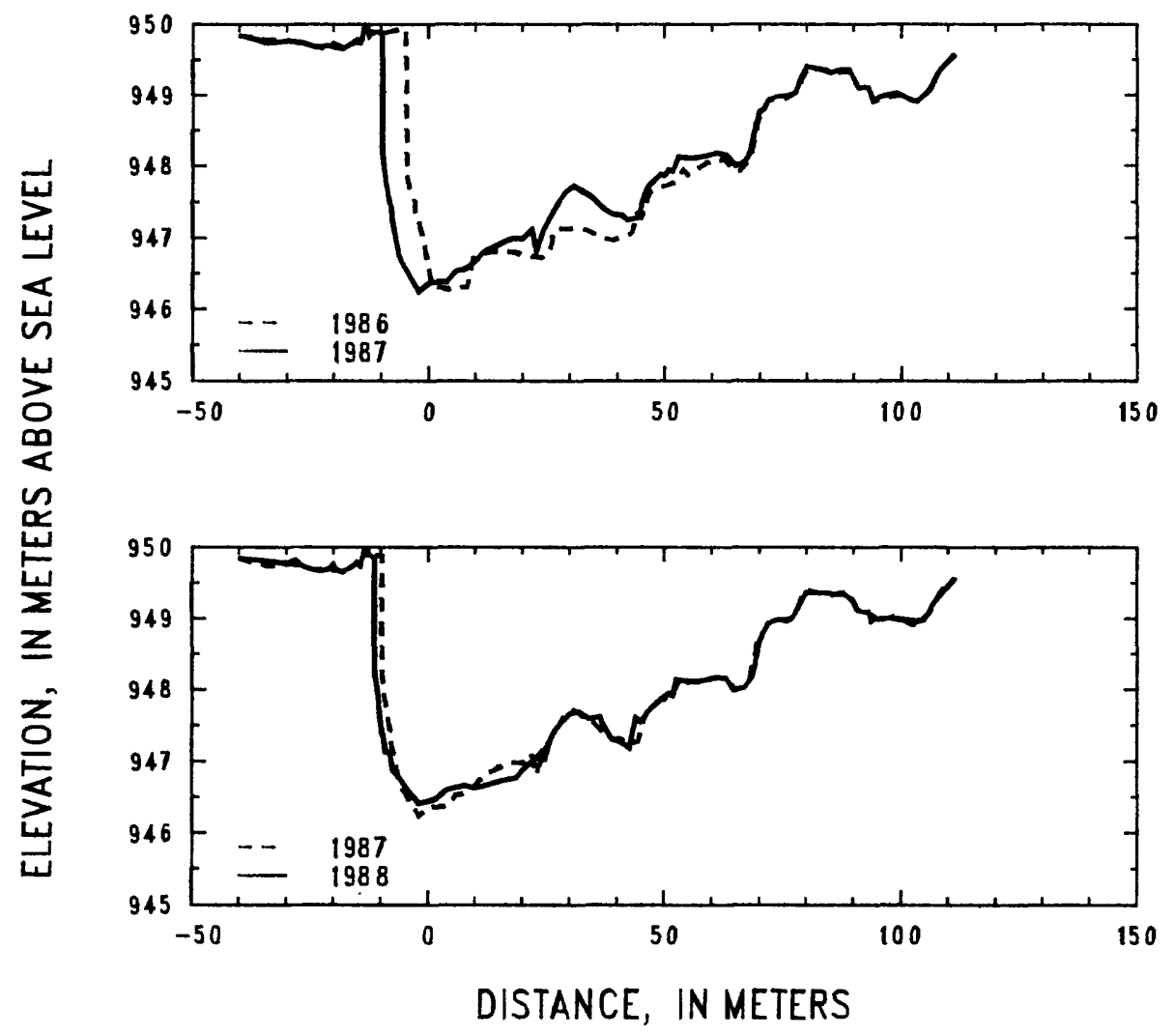

Figure 75. --Profiles of cross section PR180 from 1986 to 1988. 
Table 24.--Listing of horizontal stations and elevations for cross section PR180 [Sta., station, distance in meters from a reference point on the left bank; Elev., elevation, in meters above sea level].

\begin{tabular}{|c|c|c|c|c|c|c|c|c|c|}
\hline \multicolumn{2}{|c|}{1977} & \multicolumn{2}{|c|}{1977} & \multicolumn{2}{|c|}{1978} & \multicolumn{2}{|c|}{1978} & \multicolumn{2}{|c|}{1979} \\
\hline Sta. & Elev. & Sta. & Elev. & Sta. & Elev. & Sta. & Elev. & Sta. & Elev. \\
\hline-1.5 & 949.90 & 70.6 & 948.39 & -30.0 & 949.77 & 62.0 & 947.53 & -30.0 & 949.78 \\
\hline 0.0 & 949.98 & 71.2 & 948.69 & -25.0 & 949.69 & 64.0 & 947.59 & -25.0 & 949.69 \\
\hline 2.0 & 949.88 & 74.0 & 948.72 & -20.0 & 949.74 & 66.4 & 947.82 & -20.0 & 949.76 \\
\hline 4.0 & 949.86 & 76.0 & 948.71 & -15.0 & 949.82 & 67.4 & 947.85 & -15.0 & 949.79 \\
\hline 6.0 & 949.81 & 78.0 & 948.60 & -10.0 & 949.88 & 68.7 & 948.12 & -10.0 & 949.89 \\
\hline 8.0 & 949.82 & 80.0 & 948.59 & -7.0 & 949.91 & 69.0 & 948.47 & -5.0 & 949.95 \\
\hline 10.0 & 949.83 & 82.0 & 948.60 & -5.0 & 949.94 & 69.6 & 948.55 & -1.5 & 949.91 \\
\hline 12.1 & 949.78 & 84.0 & 948.76 & -4.0 & 949.96 & 70.0 & 948.72 & 0.0 & 949.96 \\
\hline 13.1 & 948.05 & 86.0 & 948.85 & -1.5 & 949.91 & 72.0 & 948.91 & 2.0 & 949.89 \\
\hline 13.4 & 947.78 & 88.0 & 948.82 & 0.0 & 949.97 & 74.0 & 948.99 & 4.0 & 949.86 \\
\hline 14.3 & 947.13 & 90.0 & 948.83 & 2.0 & 949.88 & 76.0 & 948.97 & 6.0 & 949.83 \\
\hline 15.0 & 946.93 & 92.0 & 948.81 & 4.0 & 949.86 & 78.0 & 949.08 & 8.0 & 949.83 \\
\hline 15.5 & 946.78 & 94.0 & 948.75 & 6.0 & 949.82 & 80.0 & 949.39 & 9.0 & 949.84 \\
\hline 16.0 & 946.84 & 96.0 & 948.77 & 8.0 & 949.84 & 82.0 & 949.39 & 9.0 & 948.68 \\
\hline 18.0 & 946.96 & 98.0 & 948.75 & 10.0 & 949.85 & 84.0 & 949.36 & 9.5 & 948.53 \\
\hline 20.0 & 947.01 & 100.0 & 948.73 & 11.0 & 949.82 & 86.0 & 949.35 & 10.0 & 948.12 \\
\hline 22.0 & 946.98 & 103.4 & 948.77 & 11.1 & 947.93 & 88.0 & 949.34 & 11.0 & 947.74 \\
\hline 24.0 & 946.90 & & & 11.6 & 947.76 & 90.0 & 949.25 & 12.0 & 947.40 \\
\hline 24.6 & 947.02 & & & 13.9 & 947.03 & 92.0 & 949.10 & 13.0 & 946.99 \\
\hline 26.0 & 947.03 & & & 15.0 & 946.09 & 94.0 & 948.93 & 14.0 & 946.64 \\
\hline 28.0 & 947.03 & & & 16.0 & 946.19 & 96.0 & 949.00 & 15.0 & 946.10 \\
\hline 30.0 & 947.02 & & & 18.0 & 946.25 & 98.0 & 949.00 & 16.0 & 946.14 \\
\hline 32.0 & 947.01 & & & 20.0 & 946.41 & 100.0 & 948.99 & 18.0 & 946.18 \\
\hline 34.0 & 946.98 & & & 22.0 & 946.42 & 102.0 & 948.91 & 20.0 & 946.19 \\
\hline 36.0 & 946.94 & & & 24.0 & 946.47 & 104.0 & 948.94 & 22.0 & 946.25 \\
\hline 38.0 & 946.93 & & & 26.0 & 946.49 & 106.0 & 949.13 & 24.0 & 946.26 \\
\hline 40.0 & 946.84 & & & 28.0 & 946.34 & 108.0 & 949.34 & 26.0 & 946.34 \\
\hline 42.0 & 946.85 & & & 30.0 & 946.41 & 110.0 & 949.43 & 28.0 & 946.41 \\
\hline 44.0 & 947.07 & & & 32.0 & 946.44 & 111.2 & 949.59 & 30.0 & 946.43 \\
\hline 46.0 & 947.05 & & & 34.0 & 946.34 & & & 32.0 & 946.49 \\
\hline 47.0 & 947.14 & & & 36.0 & 946.46 & & & 34.0 & 946.53 \\
\hline 48.0 & 947.16 & & & 38.0 & 946.61 & & & 36.2 & 946.64 \\
\hline 50.0 & 947.22 & & & 40.0 & 946.79 & & & 36.9 & 946.91 \\
\hline 52.0 & 947.30 & & & 40.9 & 947.03 & & & 38.0 & 946.99 \\
\hline 54.0 & 947.33 & & & 41.2 & 947.26 & & & 39.0 & 947.14 \\
\hline 56.0 & 947.44 & & & 44.0 & 947.29 & & & 40.0 & 947.19 \\
\hline 58.0 & 947.45 & & & 46.0 & 947.26 & & & 42.0 & 947.26 \\
\hline 60.0 & 947.46 & & & 48.0 & 947.22 & & & 45.0 & 947.25 \\
\hline 62.0 & 947.54 & & & 50.0 & 947.26 & & & 48.0 & 947.29 \\
\hline 64.0 & 947.63 & & & 51.5 & 947.36 & & & 50.0 & 947.32 \\
\hline 65.3 & 947.83 & & & 52.5 & 947.59 & & & 52.0 & 947.42 \\
\hline 66.0 & 948.04 & & & 54.0 & 947.58 & & & 54.0 & 947.54 \\
\hline 66.5 & 948.11 & & & 56.0 & 947.61 & & & 56.0 & 947.63 \\
\hline 66.8 & 948.29 & & & 58.0 & 947.62 & & & 58.0 & 947.75 \\
\hline 69.0 & 948.32 & & & 60.0 & 947.57 & & & 60.0 & 947.80 \\
\hline
\end{tabular}


Table 24.--Listing of horizontal stations and elevations for cross section PR180 --Continued.

\begin{tabular}{|c|c|c|c|c|c|c|c|c|c|}
\hline \multicolumn{2}{|c|}{1979} & \multicolumn{2}{|c|}{1980} & \multicolumn{2}{|c|}{1980} & \multicolumn{2}{|c|}{1982} & \multicolumn{2}{|c|}{1982} \\
\hline Sta. & Elev. & Sta. & Elev. & Sta. & Elev. & Sta. & Elev. & Sta. & Elev. \\
\hline 62.0 & 947.74 & -30.0 & 949.77 & 84.6 & 949.45 & -30.0 & 949.76 & 80.0 & 949.37 \\
\hline 64.0 & 947.60 & -25.0 & 949.68 & 86.0 & 949.35 & -25.0 & 949.69 & 83.0 & 949.35 \\
\hline 66.0 & 947.85 & -20.0 & 949.75 & 89.0 & 949.31 & -20.0 & 949.77 & 86.0 & 949.33 \\
\hline 68.0 & 948.00 & -15.0 & 949.79 & 91.0 & 949.09 & -15.0 & 949.77 & 89.0 & 949.31 \\
\hline 69.0 & 948.21 & -10.0 & 949.88 & 93.0 & 949.13 & -13.0 & 950.00 & 91.0 & 949.09 \\
\hline 70.0 & 948.58 & -5.0 & 949.94 & 94.0 & 948.93 & -10.0 & 949.87 & 93.0 & 949.10 \\
\hline 71.0 & 948.82 & -1.5 & 949.90 & 97.0 & 948.96 & -5.0 & 949.94 & 94.0 & 948.92 \\
\hline 73.0 & 948.98 & 0.0 & 949.96 & 100.0 & 948.99 & -1.5 & 949.90 & 97.0 & 948.95 \\
\hline 75.0 & 948.96 & 5.0 & 949.82 & 103.0 & 948.94 & 0.0 & 949.95 & 99.0 & 949.03 \\
\hline 77.0 & 949.08 & 8.0 & 949.83 & 105.3 & 948.98 & 2.0 & 949.87 & 102.0 & 948.90 \\
\hline 79.0 & 949.30 & 8.9 & 949.85 & 107.2 & 949.28 & 5.0 & 949.82 & 105.0 & 948.97 \\
\hline 81.0 & 949.38 & 9.0 & 948.68 & 110.0 & 949.40 & 6.0 & 949.81 & 108.0 & 949.32 \\
\hline 83.0 & 949.39 & 10.0 & 948.09 & 111.3 & 949.58 & 8.5 & 949.82 & 111.3 & 949.58 \\
\hline 85.0 & 949.37 & 11.0 & 947.77 & 112.0 & 949.54 & 8.6 & 949.83 & & \\
\hline 87.0 & 949.37 & 11.7 & 947.59 & 112.8 & 949.30 & 8.7 & 948.10 & & \\
\hline 90.0 & 949.19 & 12.4 & 947.36 & 114.0 & 949.29 & 9.0 & 947.78 & & \\
\hline 93.0 & 949.04 & 12.7 & 947.08 & 114.8 & 949.50 & 9.2 & 947.75 & & \\
\hline 100.0 & 948.97 & 14.0 & 946.52 & 117.0 & 949.31 & 10.0 & 946.96 & . & \\
\hline 102.0 & 948.94 & 15.0 & 946.00 & 119.0 & 949.18 & 10.0 & 946.93 & & \\
\hline 104.0 & 948.93 & 15.5 & 945.96 & 120.7 & 949.45 & 11.0 & 946.44 & & \\
\hline 106.0 & 949.12 & 25.0 & 946.03 & 122.2 & 949.45 & 13.0 & 946.10 & & \\
\hline 108.0 & 949.31 & 27.5 & 946.33 & 123.8 & 949.22 & 15.0 & 946.09 & & \\
\hline 110.0 & 949.43 & 30.0 & 946.22 & & & 20.0 & 946.14 & & \\
\hline \multirow[t]{22}{*}{111.3} & 949.60 & 32.0 & 946.25 & & & 25.0 & 946.33 & & \\
\hline & & 35.0 & 946.43 & & & 30.0 & 946.37 & & \\
\hline & &,$\quad 37.3$ & 946.51 & & & 35.0 & 946.44 & & \\
\hline & & 39.0 & 947.08 & & & 37.0 & 946.55 & & \\
\hline & & 40.2 & 947.30 & & & 38.2 & 946.92 & & \\
\hline & & 45.0 & 947.35 & & & 39.0 & 946.99 & & \\
\hline & & 50.0 & 947.39 & & & 40.0 & 946.93 & & \\
\hline & & 53.0 & 947.46 & & & 43.0 & 947.35 & & \\
\hline & & 56.0 & 947.65 & & & 46.0 & 947.53 & & \\
\hline & & 58.0 & 947.66 & & & 50.0 & 947.57 & & \\
\hline & & 60.0 & 947.61 & & & 55.0 & 947.66 & & \\
\hline & & 63.0 & 947.57 & & & 60.0 & 947.72 & & \\
\hline & & 66.0 & 947.69 & & & 62.0 & 947.86 & & \\
\hline & & 68.5 & 948.08 & & & 64.0 & 947.67 & & \\
\hline & & 69.6 & 948.54 & & & 66.0 & 947.81 & & \\
\hline & & 70.4 & 948.74 & & & 69.0 & 948.19 & & \\
\hline & & 72.0 & 948.94 & & & 69.5 & 948.53 & & \\
\hline & & 74.0 & 949.00 & & & 70.0 & 948.67 & & \\
\hline & & 77.0 & 949.00 & & & 72.0 & 948.92 & & \\
\hline & & 79.0 & 949.30 & & & 74.0 & 948.99 & & \\
\hline & & 80.0 & 949.36 & & & 77.0 & 948.97 & & \\
\hline & & 83.0 & 949.36 & & & 79.0 & 949.27 & & \\
\hline
\end{tabular}


Table 24.--Listing of horizontal stations and elevations for cross section PR180 --Continued.

\begin{tabular}{|c|c|c|c|c|c|c|c|c|c|}
\hline \multicolumn{2}{|c|}{1984} & \multicolumn{2}{|c|}{1984} & \multicolumn{2}{|c|}{1985} & \multicolumn{2}{|c|}{1985} & \multicolumn{2}{|c|}{1986} \\
\hline Sta. & Elev. & Sta. & Elev. & Sta. & Elev. & Sta. & Elev. & Sta. & Elev. \\
\hline-40.0 & 949.86 & 52.0 & 947.71 & -40.0 & 949.85 & 54.0 & 947.98 & -40.0 & 949.85 \\
\hline-35.0 & 949.76 & 53.0 & 947.84 & -35.0 & 949.76 & 55.0 & 947.87 & -35.0 & 949.79 \\
\hline-30.0 & 949.76 & 53.2 & 948.03 & -30.0 & 949.75 & 57.0 & 947.91 & -30.0 & 949.77 \\
\hline-28.0 & 949.80 & 55.0 & 947.80 & -26.0 & 949.70 & 59.0 & 947.96 & -25.0 & 949.70 \\
\hline-24.0 & 949.63 & 56.0 & 947.75 & -23.0 & 949.65 & 60.0 & 948.07 & -22.0 & 949.66 \\
\hline-19.0 & 949.64 & 58.0 & 947.91 & -20.0 & 949.75 & 63.0 & 948.06 & -20.0 & 949.74 \\
\hline-15.0 & 949.78 & 60.0 & 948.06 & -18.0 & 949.64 & 65.0 & 947.88 & -18.0 & 949.66 \\
\hline-10.0 & 949.87 & 61.0 & 948.08 & -15.0 & 949.78 & 67.0 & 947.96 & -17.0 & 949.72 \\
\hline-5.0 & 949.94 & 63.0 & 948.08 & -12.0 & 949.87 & 68.6 & 948.13 & -14.3 & 949.74 \\
\hline-1.5 & 949.88 & 64.0 & 947.97 & -10.0 & 949.86 & 69.7 & 948.61 & -13.0 & 949.97 \\
\hline 0.0 & 949.94 & 65.0 & 947.86 & -7.0 & 949.91 & 70.3 & 948.73 & -12.3 & 949.88 \\
\hline 2.0 & 949.86 & 66.0 & 947.89 & -4.0 & 949.94 & 72.0 & 948.93 & -11.0 & 949.86 \\
\hline 3.2 & 949.86 & 68.0 & 948.04 & -1.5 & 949.89 & 75.0 & 948.94 & -8.0 & 949.90 \\
\hline 3.4 & 948.69 & 69.0 & 948.19 & 0.0 & 949.95 & 77.0 & 949.00 & -6.0 & 949.93 \\
\hline 3.7 & 948.03 & 70.0 & 948.60 & 1.2 & 949.87 & 79.0 & 949.28 & -4.8 & 949.93 \\
\hline 4.0 & 947.67 & 71.0 & 948.79 & 1.3 & 948.63 & 81.0 & 949.34 & -4.5 & 947.89 \\
\hline 4.3 & 947.47 & 73.0 & 949.01 & 2.0 & 948.14 & 84.0 & 949.37 & -3.7 & 947.63 \\
\hline 5.3 & 947.30 & 76.0 & 948.94 & 3.0 & 947.60 & 86.0 & 949.33 & -3.1 & 947.56 \\
\hline 6.4 & 947.09 & 78.0 & 949.13 & 4.0 & 947.38 & 89.0 & 949.30 & -2.9 & 947.31 \\
\hline 6.5 & 946.62 & 80.0 & 949.36 & 5.9 & 946.89 & 91.0 & 949.08 & -2.1 & 947.24 \\
\hline 7.0 & 946.44 & 81.0 & 949.36 & 6.0 & 946.60 & 93.0 & 949.08 & -0.6 & 946.80 \\
\hline 8.0 & 946.33 & 84.0 & 949.36 & 7.0 & 946.58 & 94.0 & 948.93 & 1.0 & 946.29 \\
\hline 9.0 & 946.49 & 86.0 & 949.32 & 8.0 & 946.47 & 97.0 & 948.96 & 2.0 & 946.30 \\
\hline 10.0 & 946.78 & 88.0 & 949.34 & 10.0 & 946.40 & 100.0 & 948.98 & 4.0 & 946.27 \\
\hline 13.0 & 946.63 & 90.0 & 949.23 & 13.0 & 946.43 & 102.0 & 948.91 & 6.0 & 946.31 \\
\hline 15.0 & 946.52 & 92.0 & 949.11 & 15.0 & 946.51 & 105.0 & 948.98 & 8.4 & 946.31 \\
\hline 17.0 & 946.49 & 94.0 & 948.93 & 18.0 & 946.46 & 107.0 & 949.21 & 9.7 & 946.73 \\
\hline 20.0 & 946.49 & 96.0 & 948.99 & 21.0 & 946.43 & 108.0 & 949.33 & 12.0 & 946.78 \\
\hline 21.0 & 946.55 & 98.0 & 948.98 & 23.0 & 946.70 & 110.0 & 949.43 & 15.0 & 946.82 \\
\hline 22.0 & 946.74 & 100.0 & 948.98 & 26.0 & 946.81 & 111.3 & 949.56 & 18.0 & 946.80 \\
\hline 24.0 & 946.63 & 102.0 & 948.91 & 29.0 & 946.82 & & & 21.0 & 946.74 \\
\hline 26.0 & 946.58 & 104.0 & 948.93 & 31.4 & 946.73 & & & 24.0 & 946.71 \\
\hline 28.0 & 946.64 & 106.0 & 949.09 & 32.3 & 947.03 & & & 25.6 & 946.81 \\
\hline 29.2 & 946.80 & 108.0 & 949.33 & 34.0 & 947.04 & & & 26.3 & 946.92 \\
\hline 33.0 & 946.91 & 110.0 & 949.43 & 36.0 & 947.19 & & & 26.4 & 947.08 \\
\hline 35.0 & 946.80 & 111.3 & 949.55 & 38.0 & 947.20 & & & 28.0 & 947.13 \\
\hline 35.8 & 946.87 & & & 41.0 & 947.22 & & & 31.0 & 947.13 \\
\hline 37.0 & 946.94 & & & 44.0 & 947.30 & & & 34.0 & 947.09 \\
\hline 39.0 & 947.09 & & & 45.7 & 947.35 & & & 37.0 & 946.99 \\
\hline 41.1 & 947.19 & & & 46.1 & 947.46 & & & 39.0 & 946.96 \\
\hline 41.6 & 947.26 & & & 47.4 & 947.55 & & & 41.0 & 947.00 \\
\hline 45.0 & 947.35 & & & 48.0 & 947.49 & & & 43.0 & 947.06 \\
\hline 47.0 & 947.56 & & & 50.0 & 947.55 & & & 43.7 & 947.20 \\
\hline 49.0 & 947.54 & & & 51.5 & 947.64 & & & 44.0 & 947.35 \\
\hline 51.0 & 947.72 & & & 53.0 & 947.94 & & & 45.0 & 947.27 \\
\hline
\end{tabular}


Table 24.--Listing of horizontal stations and elevations for cross section PR180 --Continued.

\begin{tabular}{|c|c|c|c|c|c|c|c|c|c|}
\hline \multicolumn{2}{|c|}{1986} & \multicolumn{2}{|c|}{1987} & \multicolumn{2}{|c|}{1987} & \multicolumn{2}{|c|}{1988} & \multicolumn{2}{|c|}{1988} \\
\hline Sta. & Elev. & Sta. & Elev. & Sta. & Elev. & Sta. & Elev. & Sta. & Elev. \\
\hline 47.0 & 947.63 & -40.0 & 949.84 & 41.0 & 947.30 & -40.0 & 949.85 & 30.0 & 947.66 \\
\hline 48.0 & 947.67 & -37.0 & 949.79 & 42.0 & 947.24 & -30.0 & 949.77 & 32.0 & 947.70 \\
\hline 50.0 & 947.72 & -34.0 & 949.73 & 44.4 & 947.27 & -28.0 & 949.82 & 34.0 & 947.59 \\
\hline 52.0 & 947.76 & -32.0 & 949.74 & 45.6 & 947.56 & -27.0 & 949.74 & 36.5 & 947.62 \\
\hline 54.0 & 947.94 & -30.0 & 949.77 & 46.6 & 947.71 & -25.0 & 949.69 & 37.0 & 947.51 \\
\hline 55.0 & 947.87 & -27.0 & 949.74 & 48.0 & 947.80 & -23.0 & 949.66 & 39.0 & 947.30 \\
\hline 57.0 & 947.96 & -24.0 & 949.68 & 49.2 & 947.88 & -21.0 & 949.69 & 41.0 & 947.26 \\
\hline 60.0 & 948.09 & -21.0 & 949.70 & 50.0 & 947.86 & -20.0 & 949.76 & 42.7 & 947.18 \\
\hline 63.0 & 948.08 & -18.0 & 949.65 & 51.0 & 947.94 & -19.5 & 949.67 & 42.9 & 947.24 \\
\hline 64.5 & 947.93 & -15.0 & 949.78 & 51.9 & 947.92 & -18.0 & 949.65 & 44.0 & 947.61 \\
\hline 66.0 & 947.92 & -14.2 & 949.73 & 53.0 & 948.13 & -16.0 & 949.72 & 45.0 & 947.54 \\
\hline 68.1 & 948.10 & -13.3 & 949.99 & 55.0 & 948.10 & -15.0 & 949.79 & 47.0 & 947.73 \\
\hline 70.0 & 948.72 & -12.8 & 949.96 & 57.0 & 948.12 & -14.3 & 949.73 & 49.0 & 947.85 \\
\hline 72.0 & 948.93 & -12.4 & 949.85 & 59.0 & 948.14 & -13.3 & 949.99 & 51.0 & 947.95 \\
\hline 75.0 & 948.93 & -12.0 & 949.90 & 61.0 & 948.18 & -12.8 & 949.99 & 51.8 & 947.91 \\
\hline 78.0 & 949.06 & -9.7 & 949.87 & 63.0 & 948.15 & -12.4 & 949.88 & 52.6 & 948.14 \\
\hline 80.0 & 949.38 & -9.7 & 948.20 & 65.0 & 947.99 & -11.3 & 949.85 & 55.0 & 948.11 \\
\hline 83.0 & 949.37 & -8.8 & 947.71 & 67.0 & 948.05 & -11.3 & 948.22 & 58.0 & 948.12 \\
\hline 86.0 & 949.32 & -7.9 & 947.41 & 68.0 & 948.16 & -10.6 & 947.99 & 61.0 & 948.17 \\
\hline 89.0 & 949.34 & -7.6 & 947.20 & 69.0 & 948.48 & -10.1 & 947.60 & 63.0 & 948.17 \\
\hline 91.0 & 949.10 & -7.3 & 947.13 & 70.0 & 948.76 & -9.8 & 947.39 & 64.7 & 947.99 \\
\hline 93.0 & 949.11 & -6.0 & 946.69 & 71.0 & 948.80 & -9.5 & 947.35 & 67.0 & 948.04 \\
\hline 94.0 & 948.90 & -4.0 & 946.46 & 72.0 & 948.94 & -9.0 & 947.13 & 68.6 & 948.18 \\
\hline 97.0 & 948.97 & -2.0 & 946.24 & 74.0 & 948.98 & -8.0 & 947.12 & 70.0 & 948.67 \\
\hline \multirow[t]{22}{*}{100.0} & 949.01 & 0.0 & 946.36 & 76.0 & 948.98 & -7.3 & 946.86 & 71.0 & 948.80 \\
\hline & & 2.0 & 946.38 & 77.6 & 949.03 & -6.0 & 946.77 & 72.0 & 948.94 \\
\hline & & 4.0 & 946.37 & 78.8 & 949.25 & -4.0 & 946.56 & 74.0 & 948.99 \\
\hline & & 6.0 & 946.53 & 80.0 & 949.40 & -2.0 & 946.41 & 76.0 & 948.96 \\
\hline & & 8.0 & 946.56 & 82.0 & 949.37 & 0.0 & 946.44 & 77.0 & 948.99 \\
\hline & & 10.0 & 946.66 & 85.0 & 949.31 & 2.0 & 946.50 & 79.0 & 949.26 \\
\hline & & 12.0 & 946.81 & 87.0 & 949.36 & 4.0 & 946.60 & 80.0 & 949.36 \\
\hline & & 14.0 & 946.87 & 89.0 & 949.36 & 6.0 & 946.63 & 82.0 & 949.36 \\
\hline & & 16.0 & 946.94 & 91.0 & 949.09 & 8.0 & 946.66 & 84.0 & 949.37 \\
\hline & & 18.0 & 946.99 & 93.0 & 949.10 & 10.0 & 946.62 & 86.0 & 949.34 \\
\hline & & 20.0 & 946.98 & 94.0 & 948.92 & 12.0 & 946.66 & 88.0 & 949.34 \\
\hline & & 22.0 & 947.11 & 96.0 & 948.99 & 14.0 & 946.70 & 90.0 & 949.25 \\
\hline & & 23.0 & 946.81 & 99.0 & 949.03 & 16.0 & 946.74 & 91.0 & 949.10 \\
\hline & & 24.5 & 947.12 & 102.0 & 948.92 & 18.7 & 946.77 & 93.0 & 949.09 \\
\hline & & 27.0 & 947.42 & 103.4 & 948.90 & 20.5 & 946.91 & 95.0 & 948.99 \\
\hline & & 29.0 & 947.62 & 106.0 & 949.07 & 23.0 & 947.04 & 98.0 & 949.01 \\
\hline & & 31.0 & 947.72 & 108.0 & 949.34 & 24.1 & 947.13 & 100.1 & 948.98 \\
\hline & & 33.0 & 947.62 & 111.3 & 949.57 & 25.0 & 947.04 & 103.5 & 948.96 \\
\hline & & 35.0 & 947.54 & & & 25.7 & 947.23 & 105.0 & 948.98 \\
\hline & & 37.0 & 947.40 & & & 26.5 & 947.38 & 107.0 & 949.22 \\
\hline & & 39.0 & 947.32 & & & 28.0 & 947.53 & 109.0 & 949.38 \\
\hline & & & & & & & & 111.3 & 949.56 \\
\hline
\end{tabular}


Description of Cross Section PR183

Location: Township 6 South/Range 50 East--section 1

U.S. Geological Survey quadrangle $(1: 24,000)$ : Lonesome Peak

Landowners--left bank: EB Ranch

right bank: EB Ranch

Access: $\quad$ left bank

Permission from: J.L. Wilson or Howard Best (T6S/R50E--1)

Reference pins

\begin{tabular}{|c|c|c|}
\hline $\begin{array}{l}\text { Station } \\
\text { (meters) }\end{array}$ & $\begin{array}{c}\text { Elevation } \\
\text { (meters } \\
\text { above } \\
\text { sea level) }\end{array}$ & Comments \\
\hline & & $\begin{array}{l}\text { Pins } 100.0 \text { and } 101.1 \text { were closest } \\
\text { to leveling instrument. }\end{array}$ \\
\hline-7.0 & 945.955 & 0.17 above 1987 ground level. \\
\hline 0.0 & 945.669 & $\begin{array}{l}\text { Elevation increased } 0.07 \text { meter between } \\
1980 \text { and } 1985,0.06 \text { meter above } 1987 \\
\text { ground level. }\end{array}$ \\
\hline 100.0 & 945.648 & At 1987 ground level. \\
\hline 101.1 & 945.857 & 0.10 meter above 1987 ground level. \\
\hline
\end{tabular}




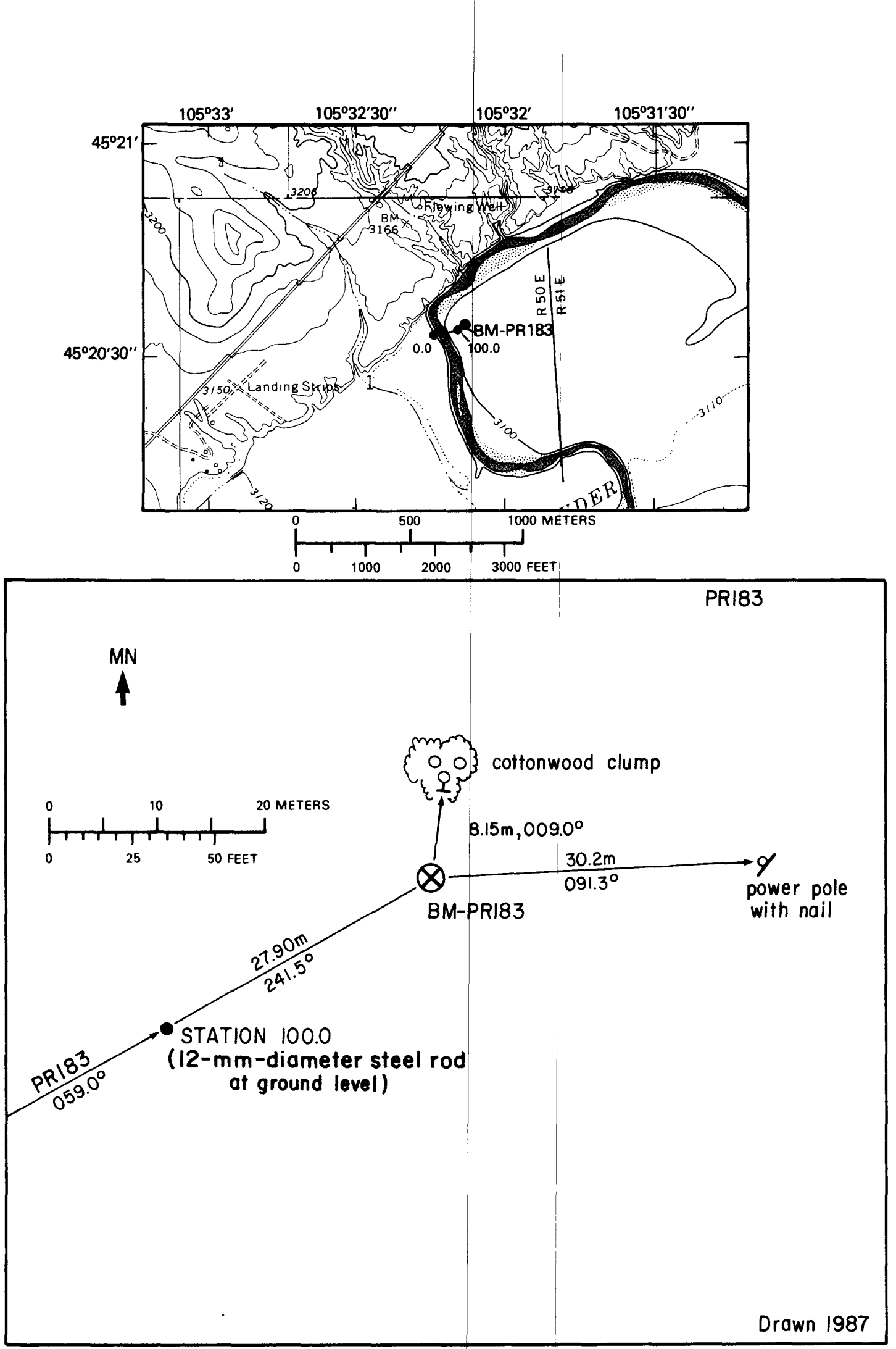

Figure 76.--Upper: Location of cross section PR183, bench mark BM-PR183, and left and right bank reference pins (tables 2 and 3 ) in the Lonesome Peak quadrangle. Lower: Location of bench mark on the left bank. MN is magnetic north. 

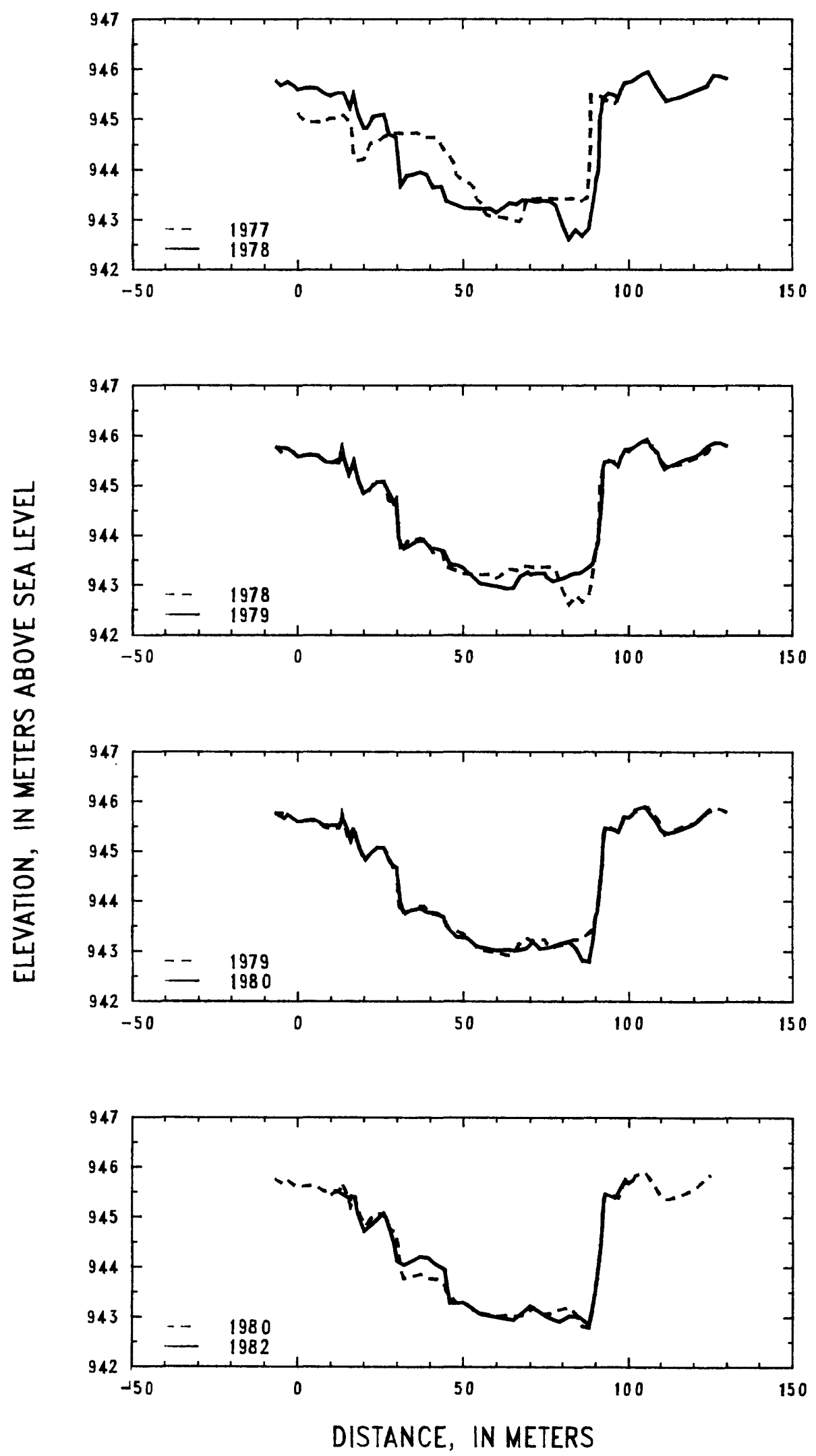

Figure 77.--Profiles of cross section PR183 from 1977 to 1982. 


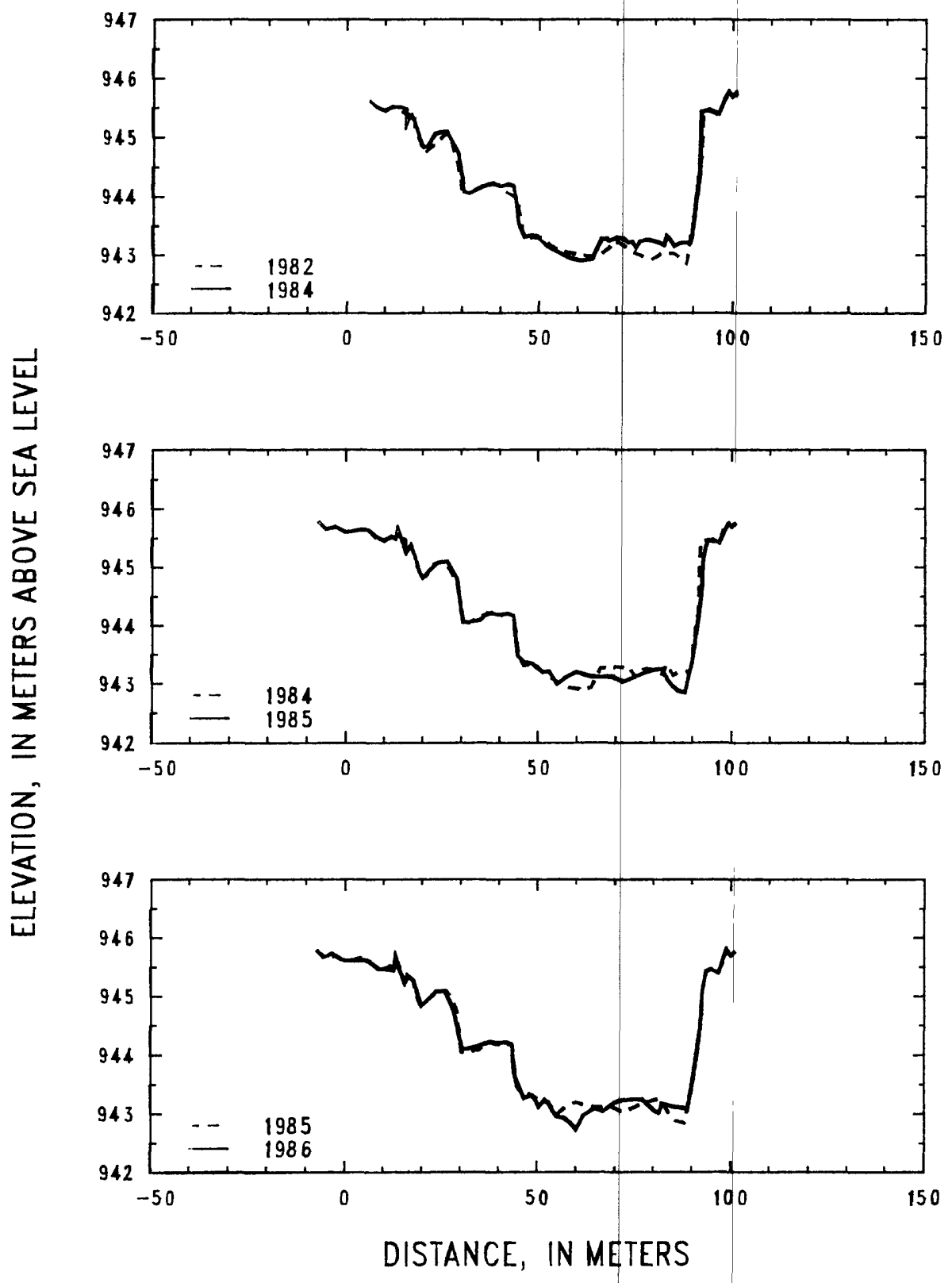

Figure 78.--Profiles of cross section PR183 from 1982 to 1986. 


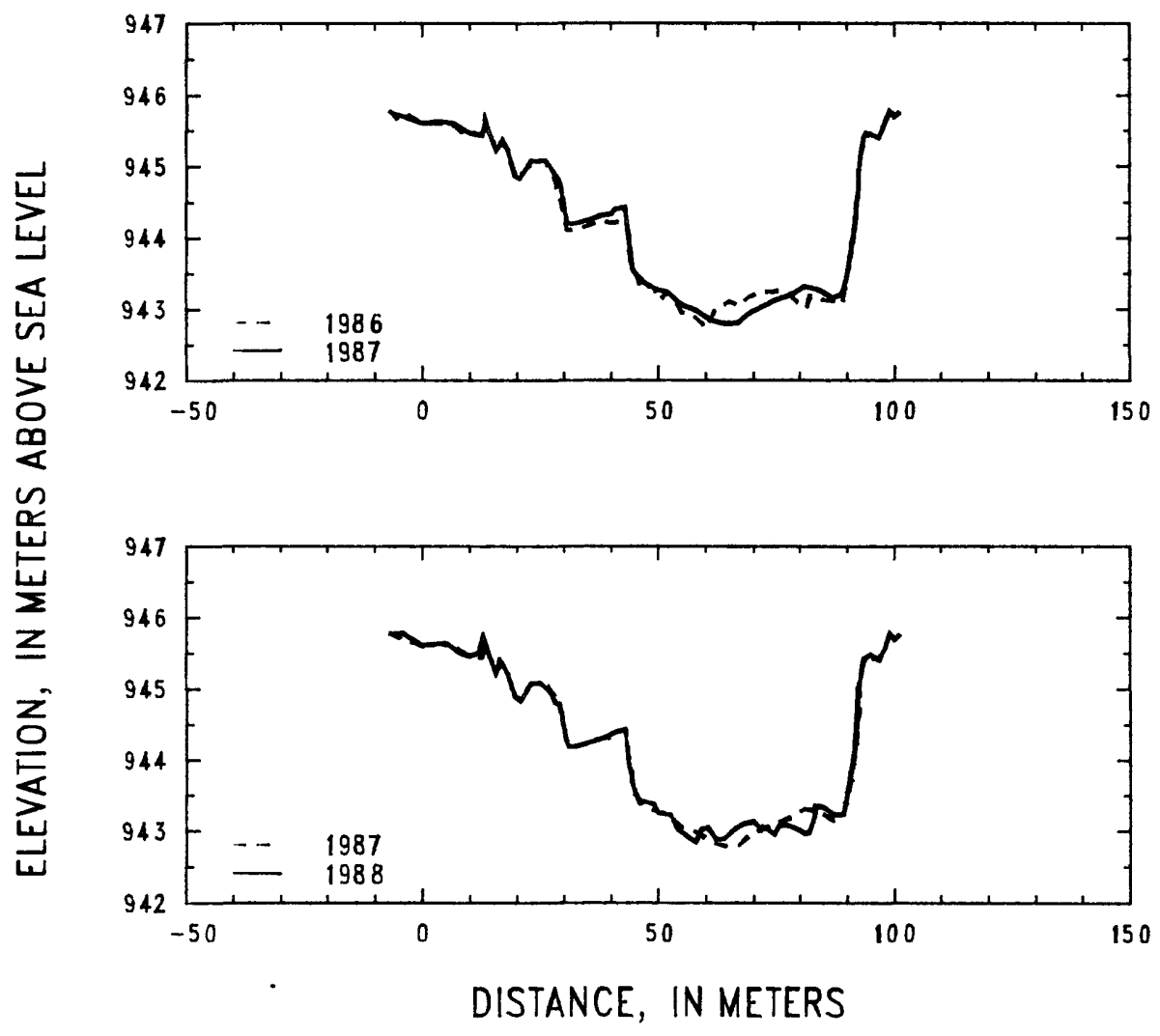

Figure 79.--Profiles of cross section PR183 from 1986 to 1988.

207

(followed by p. 209) 
Table 25.--Listing of horizontal stations and elevations for cross section PR183

[Sta., station, distance in meters from a reference point on

the left bank; Elev., elevation, in meters above sea leve1].

\begin{tabular}{|c|c|c|c|c|c|c|c|c|c|}
\hline \multicolumn{2}{|c|}{1977} & \multicolumn{2}{|c|}{1977} & \multicolumn{2}{|c|}{1978} & \multicolumn{2}{|c|}{1978} & \multicolumn{2}{|c|}{1979} \\
\hline Sta. & Elev. & Sta. & Elev. & Sta. & Elev. & Sta. & Elev. & Sta. & Elev. \\
\hline 0.0 & 945.11 & 75.0 & 943.42 & -7.0 & 945.77 & 76.0 & 943.37 & -7.0 & 945.77 \\
\hline 2.0 & 944.96 & 77.0 & 943.42 & -5.0 & 945.67 & 78.0 & 943.28 & -3.0 & 945.74 \\
\hline 4.0 & 944.94 & 79.0 & 943.40 & -3.0 & 945.75 & 80.0 & 942.90 & 0.0 & 945.59 \\
\hline 6.0 & 944.94 & 81.0 & 943.41 & -1.0 & 945.66 & 82.0 & 942.60 & 2.0 & 945.60 \\
\hline 8.0 & 944.94 & 83.0 & 943.42 & 0.0 & 945.59 & 84.0 & 942.80 & 4.0 & 945.62 \\
\hline 10.0 & 945.02 & 85.0 & 943.34 & 2.0 & 945.62 & 86.0 & 942.67 & 6.0 & 945.60 \\
\hline 12.0 & 945.00 & 87.5 & 943.43 & 4.0 & 945.63 & 88.0 & 942.81 & 8.0 & 945.48 \\
\hline 14.0 & 945.08 & 88.3 & 944.23 & 6.0 & 945.62 & 89.8 & 943.57 & 10.0 & 945.46 \\
\hline 16.3 & 944.88 & 88.6 & 945.47 & 8.0 & 945.51 & 90.3 & 943.83 & 12.5 & 945.46 \\
\hline 16.8 & 944.36 & 90.0 & 945.46 & 10.0 & 945.46 & 90.8 & 943.93 & 13.5 & 945.76 \\
\hline 18.0 & 944.17 & 92.0 & 945.43 & 12.0 & 945.52 & 91.0 & 944.15 & 14.0 & 945.54 \\
\hline 20.0 & 944.20 & 94.0 & 945.32 & 14.0 & 945.52 & 91.3 & 945.01 & 15.5 & 945.21 \\
\hline 22.0 & 944.52 & 96.0 & 945.30 & 16.0 & 945.22 & 91.7 & 945.11 & 17.0 & 945.50 \\
\hline 24.0 & 944.54 & 98.1 & 945.63 & 17.0 & 945.50 & 92.3 & 945.41 & 18.5 & 945.07 \\
\hline 26.0 & 944.65 & 99.2 & 945.72 & 18.0 & 945.20 & 94.0 & 945.51 & 20.0 & 944.84 \\
\hline 28.0 & 944.70 & 100.0 & 945.64 & 19.0 & 944.98 & 96.0 & 945.46 & 22.0 & 944.95 \\
\hline 30.0 & 944.73 & 101.1 & 945.60 & 20.0 & 944.82 & 96.7 & 945.38 & 24.0 & 945.08 \\
\hline 32.0 & 944.71 & & & 21.0 & 944.83 & 97.6 & 945.57 & 26.0 & 945.08 \\
\hline 34.0 & 944.72 & & & 23.0 & 945.06 & 98.7 & 945.72 & 28.0 & 944.80 \\
\hline 36.0 & 944.73 & & & 26.1 & 945.10 & 101.1 & 945.75 & 29.0 & 944.64 \\
\hline 38.0 & 944.64 & & & 27.5 & 944.71 & 104.0 & 945.89 & 29.8 & 944.73 \\
\hline 40.0 & 944.63 & & & 29.7 & 944.64 & 106.0 & 945.94 & 30.6 & 943.90 \\
\hline 42.0 & 944.64 & & & 31.2 & 943.67 & 108.0 & 945.70 & 32.0 & 943.72 \\
\hline 44.0 & 944.40 & & & 33.0 & 943.88 & 111.6 & 945.36 & 34.0 & 943.81 \\
\hline 46.0 & 944.23 & & & 35.0 & 943.91 & 114.0 & 945.41 & 36.0 & 943.89 \\
\hline 47.3 & 944.10 & & & 37.0 & 943.95 & 115.7 & 945.43 & 38.0 & 943.90 \\
\hline 47.8 & 943.88 & & & 39.0 & 943.89 & 124.0 & 945.66 & 40.0 & 943.74 \\
\hline 49.0 & 943.82 & & & 41.0 & 943.64 & 126.0 & 945.87 & 42.0 & 943.73 \\
\hline 51.0 & 943.76 & & & 43.5 & 943.66 & 128.0 & 945.86 & 44.0 & 943.68 \\
\hline 53.0 & 943.63 & & & 43.9 & 943.58 & 130.0 & 945.80 & 46.0 & 943.41 \\
\hline 54.0 & 943.41 & & & 45.0 & 943.36 & & & 48.0 & 943.40 \\
\hline 55.0 & 943.34 & & & 47.0 & 943.31 & & & 50.0 & 943.34 \\
\hline 56.0 & 943.19 & & & 50.0 & 943.23 & & & 51.0 & 943.27 \\
\hline 57.0 & 943.09 & & & 52.0 & 943.22 & & & 53.0 & 943.15 \\
\hline 58.0 & 943.06 & & & 54.0 & 943.22 & & & 55.0 & 943.03 \\
\hline 59.0 & 943.05 & & & 56.0 & 943.21 & & & 57.0 & 943.00 \\
\hline 60.0 & 943.05 & & & 58.0 & 943.23 & & & 59.0 & 942.98 \\
\hline 62.0 & 943.03 & & & 60.0 & 943.14 & & & 61.0 & 942.96 \\
\hline 64.0 & 943.00 & & & 62.0 & 943.24 & & & 63.0 & 942.92 \\
\hline 66.0 & 942.96 & & & 64.0 & 943.33 & & & 65.0 & 942.94 \\
\hline 67.0 & 942.95 & & & 66.0 & 943.30 & & & 67.0 & 943.18 \\
\hline 68.0 & 943.09 & & & 68.0 & 943.39 & & & 69.4 & 943.26 \\
\hline 69.1 & 943.40 & & & 70.0 & 943.38 & & & 70.2 & 943.21 \\
\hline 71.0 & 943.41 & & & 72.0 & 943.35 & & & 72.0 & 943.24 \\
\hline 73.0 & 943.42 & & & 74.0 & 943.37 & & & 74.5 & 943.24 \\
\hline
\end{tabular}


Table 25.--Listing of horizontal stations and elevations for cross section PR183 --Continued.

\begin{tabular}{|c|c|c|c|c|c|c|c|c|c|}
\hline \multicolumn{2}{|c|}{1979} & \multicolumn{2}{|c|}{1980} & \multicolumn{2}{|c|}{1980} & \multicolumn{2}{|c|}{1982} & \multicolumn{2}{|c|}{1984} \\
\hline Sta. & Elev. & Sta. & Elev. & Sta. & Elev. & Sta. & Elev. & Sta. & Elev. \\
\hline 75.0 & 943.16 & -7.0 & 945.76 & 90.5 & 943.79 & 10.0 & 945.44 & 6.0 & 945.61 \\
\hline 77.0 & 943.07 & -4.0 & 945.65 & 91.9 & 944.68 & 12.0 & 945.52 & 8.0 & 945.50 \\
\hline 80.0 & 943.14 & -3.0 & 945.73 & 92.2 & 945.15 & 16.0 & 945.36 & 10.0 & 945.45 \\
\hline 83.0 & 943.23 & 0.0 & 945.59 & 92.8 & 945.47 & 16.3 & 945.42 & 12.0 & 945.51 \\
\hline 85.0 & 943.24 & 5.0 & 945.63 & 95.0 & 945.44 & 17.4 & 945.40 & 13.0 & 945.51 \\
\hline 87.0 & 943.31 & 8.0 & 945.51 & 96.8 & 945.38 & 18.0 & 945.09 & 14.0 & 945.51 \\
\hline 89.0 & 943.42 & 11.0 & 945.52 & 98.6 & 945.70 & 20.2 & 944.72 & 15.5 & 945.47 \\
\hline 90.0 & 943.70 & 12.8 & 945.51 & 100.0 & 945.67 & 26.0 & 945.08 & 15.5 & 945.22 \\
\hline 90.8 & 943.86 & 13.5 & 945.68 & 101.1 & 945.72 & 27.0 & 944.95 & 16.0 & 945.31 \\
\hline 91.0 & 944.10 & 14.9 & 945.45 & 102.0 & 945.84 & 28.6 & 944.59 & 17.0 & 945.37 \\
\hline 91.9 & 944.71 & 16.0 & 945.20 & 105.0 & 945.90 & 30.0 & 944.12 & 18.0 & 945.20 \\
\hline 92.2 & 945.32 & 17.0 & 945.41 & 108.0 & 945.63 & 32.0 & 944.04 & 19.0 & 944.96 \\
\hline 92.6 & 945.47 & 19.0 & 945.00 & 110.0 & 945.42 & 37.0 & 944.21 & 20.0 & 944.82 \\
\hline 94.0 & 945.50 & 20.5 & 944.82 & 112.0 & 945.35 & 39.5 & 944.18 & 21.0 & 944.84 \\
\hline 96.0 & 945.43 & 22.0 & 944.95 & 115.0 & 945.41 & 41.0 & 944.08 & 23.0 & 945.06 \\
\hline 96.7 & 945.38 & 24.0 & 945.07 & 120.0 & 945.55 & 44.4 & 943.95 & 24.0 & 945.08 \\
\hline 97.6 & 945.56 & 26.0 & 945.06 & 125.0 & 945.85 & 45.3 & 943.53 & 26.0 & 945.10 \\
\hline 98.7 & 945.72 & 28.0 & 944.76 & & & 46.0 & 943.28 & 27.0 & 944.95 \\
\hline 100.0 & 945.68 & 29.9 & 944.66 & & & 50.0 & 943.29 & 29.0 & 944.72 \\
\hline 101.1 & 945.75 & 31.0 & 943.97 & & & 55.0 & 943.06 & 30.0 & 944.31 \\
\hline 104.0 & 945.88 & 32.0 & 943.77 & & & 60.0 & 943.00 & 30.6 & 944.06 \\
\hline 106.0 & 945.89 & 35.0 & 943.82 & & & 65.0 & 942.94 & 32.0 & 944.04 \\
\hline 107.0 & 945.76 & 37.0 & 943.85 & & & 70.0 & 943.23 & 35.0 & 944.15 \\
\hline 109.0 & 945.66 & 39.0 & 943.77 & & & 75.0 & 943.01 & 38.0 & 944.22 \\
\hline 110.0 & 945.45 & 42.0 & 943.75 & & & 79.0 & 942.91 & 40.0 & 944.16 \\
\hline 111.0 & 945.33 & 44.0 & 943.67 & & & 82.0 & 943.03 & 42.0 & 944.20 \\
\hline 112.0 & 945.38 & 45.0 & 943.48 & & & 85.0 & 943.02 & 43.4 & 944.16 \\
\hline 118.0 & 945.54 & 48.0 & 943.28 & & & 88.0 & 942.84 & 44.0 & 943.98 \\
\hline 120.0 & 945.57 & 51.0 & 943.26 & & & 90.0 & 943.54 & 44.7 & 943.51 \\
\hline 122.0 & 945.65 & 54.0 & 943.09 & & & 92.0 & 944.74 & 46.0 & 943.30 \\
\hline 123.0 & 945.72 & 57.0 & 943.05 & & & 92.4 & 945.36 & 48.0 & 943.33 \\
\hline 124.0 & 945.78 & 60.0 & 943.01 & & & 93.0 & 945.47 & 49.5 & 943.31 \\
\hline 126.0 & 945.86 & 63.0 & 943.03 & & & 96.0 & 945.39 & 50.0 & 943.26 \\
\hline 128.0 & 945.86 & 66.0 & 943.01 & & & 99.0 & 945.76 & 52.0 & 943.14 \\
\hline \multirow[t]{11}{*}{130.0} & 945.79 & 69.0 & 943.06 & & & 100.0 & 945.68 & 55.0 & 943.03 \\
\hline & & 71.0 & 943.19 & & & 101.1 & 945.74 & 58.0 & 942.93 \\
\hline & & 73.0 & 943.05 & & & & & 61.0 & 942.89 \\
\hline & & 76.0 & 943.07 & & & & & 63.8 & 942.93 \\
\hline & & 79.0 & 943.15 & & & & & 66.2 & 943.27 \\
\hline & & 82.0 & 943.19 & & & & & 67.6 & 943.27 \\
\hline & & 84.0 & 943.07 & & & & & 68.0 & 943.23 \\
\hline & & 86.0 & 942.81 & & & & & 70.0 & 943.29 \\
\hline & & 88.0 & 942.78 & & & & & 72.0 & 943.27 \\
\hline & & 89.2 & 943.20 & & & & & 73.0 & 943.20 \\
\hline & & 90.0 & 943.70 & & & & & 74.0 & 943.22 \\
\hline
\end{tabular}


Table 25.--Listing of horizontal stations and elevations for cross section PR183 --Continued.

\begin{tabular}{|c|c|c|c|c|c|c|c|c|c|}
\hline \multicolumn{2}{|c|}{1984} & \multicolumn{2}{|c|}{1985} & \multicolumn{2}{|c|}{1985} & \multicolumn{2}{|c|}{1986} & \multicolumn{2}{|c|}{1986} \\
\hline Sta. & Elev. & Sta. & Elev. & Sta. & Elev. & Sta. & Elev. & Sta. & Elev. \\
\hline 75.0 & 943.11 & -7.0 & 945.78 & 84.0 & 943.01 & -7.0 & 945.78 & 80.0 & 943.06 \\
\hline 76.0 & 943.23 & -5.0 & 945.65 & 86.0 & 942.88 & -5.0 & 945.66 & 81.5 & 943.01 \\
\hline 78.0 & 943.26 & -2.5 & 945.69 & 88.0 & 942.84 & -3.0 & 945.73 & 82.3 & 943.18 \\
\hline 81.0 & 943.20 & 0.0 & 945.60 & 89.8 & 943.33 & 0.0 & 945.60 & 85.0 & 943.12 \\
\hline 82.3 & 943.15 & 2.0 & 945.62 & 91.6 & 944.22 & 3.0 & 945.61 & 88.0 & 943.10 \\
\hline 83.0 & 943.32 & 4.0 & 945.65 & 92.4 & 944.64 & 6.0 & 945.61 & 88.9 & 943.06 \\
\hline 83.6 & 943.28 & 6.0 & 945.63 & 92.5 & 945.03 & 9.0 & 945.46 & 90.3 & 943.54 \\
\hline 85.0 & 943.14 & 8.0 & 945.52 & 93.6 & 945.43 & 11.0 & 945.47 & 91.6 & 944.16 \\
\hline 86.5 & 943.20 & 10.0 & 945.45 & 95.0 & 945.48 & 12.8 & 945.44 & 92.3 & 944.60 \\
\hline 89.3 & 943.21 & 12.0 & 945.53 & 96.7 & 945.40 & 13.3 & 945.68 & 92.6 & 945.04 \\
\hline 89.6 & 943.29 & 13.0 & 945.48 & 98.2 & 945.63 & 14.1 & 945.48 & 93.6 & 945.41 \\
\hline 91.5 & 944.30 & 13.5 & 945.65 & 99.0 & 945.73 & 15.7 & 945.22 & 95.0 & 945.46 \\
\hline 91.7 & 944.63 & 14.5 & 945.46 & 100.0 & 945.68 & 16.4 & 945.36 & 96.7 & 945.39 \\
\hline 92.0 & 945.43 & 15.8 & 945.24 & 100.9 & 945.75 & 17.8 & 945.27 & 99.0 & 945.80 \\
\hline 94.0 & 945.47 & 17.0 & 945.39 & & & 20.0 & 944.83 & 100.0 & 945.68 \\
\hline 96.5 & 945.39 & 18.0 & 945.20 & & & 22.0 & 944.97 & 101.1 & 945.75 \\
\hline 98.0 & 945.66 & 19.0 & 944.94 & & & 23.7 & 945.08 & & \\
\hline 99.0 & 945.77 & 20.0 & 944.81 & & & 26.0 & 945.09 & & \\
\hline 100.0 & 945.68 & 22.0 & 944.96 & & & 28.0 & 944.81 & & \\
\hline \multirow[t]{26}{*}{101.1} & 945.77 & 24.0 & 945.08 & & & 30.7 & 944.10 & & \\
\hline & & 26.5 & 945.10 & & & 32.0 & 944.10 & & \\
\hline & & 29.0 & 944.78 & & & 35.0 & 944.15 & & \\
\hline & & 30.5 & 944.06 & & & 38.0 & 944.23 & & \\
\hline & & 32.0 & 944.06 & & & 40.0 & 944.20 & & \\
\hline & & 35.0 & 944.09 & & & 42.0 & 944.22 & & \\
\hline & & 37.0 & 944.22 & & & 43.5 & 944.18 & & \\
\hline & & 40.0 & 944.18 & & & 44.1 & 943.66 & & \\
\hline & & 42.0 & 944.20 & & & 46.6 & 943.26 & & \\
\hline & & 43.6 & 944.16 & & & 48.5 & 943.32 & & \\
\hline & & 44.6 & 943.48 & & & 49.5 & 943.24 & & \\
\hline & & 46.0 & 943.37 & & & 50.4 & 943.11 & & \\
\hline & & 48.4 & 943.34 & & & 52.0 & 943.24 & & \\
\hline & & 51.0 & 943.20 & & & 53.0 & 943.17 & & \\
\hline & & 53.0 & 943.20 & & & 55.0 & 942.95 & & \\
\hline & & 55.0 & 942.98 & & & 57.0 & 942.93 & & \\
\hline & & 57.0 & 943.10 & & & 59.0 & 942.81 & & \\
\hline & & 60.0 & 943.20 & & & 60.0 & 942.72 & & \\
\hline & & 63.0 & 943.13 & & & 62.0 & 942.97 & & \\
\hline & & 66.0 & 943.11 & & & 65.0 & 943.10 & & \\
\hline & & 69.0 & 943.13 & & & 67.0 & 943.04 & & \\
\hline & & 72.0 & 943.03 & & & 69.0 & 943.16 & & \\
\hline & & 75.0 & 943.12 & & & 71.0 & 943.22 & & \\
\hline & & 78.0 & 943.20 & & & 73.0 & 943.24 & & \\
\hline & & 81.0 & 943.25 & & & 75.0 & 943.25 & & \\
\hline & & 82.0 & 943.25 & & & 77.0 & 943.25 & & \\
\hline
\end{tabular}


Table 25.--Listing of horizontal stations and elevations for cross section PR183 --Continued.

\begin{tabular}{|c|c|c|c|c|c|c|c|}
\hline \multicolumn{2}{|c|}{1987} & \multicolumn{2}{|c|}{1987} & \multicolumn{2}{|c|}{1988} & \multicolumn{2}{|c|}{1988} \\
\hline Sta. & Elev. & Sta. & Elev. & Sta. & Elev. & Sta. & Elev. \\
\hline-7.0 & 945.77 & 91.8 & 944.17 & -7.0 & 945.78 & 66.0 & 943.02 \\
\hline 0.0 & 945.61 & 92.0 & 944.49 & -4.0 & 945.78 & 68.0 & 943.11 \\
\hline 4.0 & 945.64 & 92.4 & 944.65 & -3.0 & 945.73 & 70.0 & 943.14 \\
\hline 7.0 & 945.59 & 92.6 & 945.03 & 0.0 & 945.61 & 71.7 & 943.01 \\
\hline 10.0 & 945.46 & 93.5 & 945.40 & 2.0 & 945.62 & 72.0 & 943.06 \\
\hline 12.8 & 945.43 & 94.0 & 945.47 & 5.0 & 945.64 & 74.5 & 942.94 \\
\hline 13.4 & 945.63 & 96.7 & 945.40 & 8.0 & 945.50 & 75.5 & 943.07 \\
\hline 14.0 & 945.50 & 99.0 & 945.77 & 10.0 & 945.46 & 77.0 & 943.09 \\
\hline 15.7 & 945.22 & 100.0 & 945.69 & 12.0 & 945.51 & 79.0 & 943.04 \\
\hline 17.0 & 945.37 & 101.1 & 945.76 & 13.0 & 945.73 & 81.0 & 942.96 \\
\hline 18.0 & 945.25 & & & 14.0 & 945.51 & 82.0 & 942.98 \\
\hline 19.6 & 944.87 & & & 15.6 & 945.22 & 83.2 & 943.21 \\
\hline 20.7 & 944.82 & & & 16.5 & 945.40 & 83.3 & 943.35 \\
\hline 23.0 & 945.08 & & & 18.0 & 945.23 & 85.0 & 943.34 \\
\hline 24.0 & 945.08 & & & 19.5 & 944.90 & 87.0 & 943.24 \\
\hline 26.2 & 945.09 & & & 21.0 & 944.85 & 88.0 & 943.22 \\
\hline 29.0 & 944.78 & & & 23.0 & 945.08 & 89.2 & 943.23 \\
\hline 29.5 & 944.67 & & & 25.0 & 945.09 & 91.7 & 944.15 \\
\hline 30.8 & 944.19 & & & 27.0 & 944.95 & 92.3 & 945.01 \\
\hline 32.0 & 944.18 & & & 28.0 & 944.80 & 92.6 & 945.06 \\
\hline 34.0 & 944.22 & & & 29.0 & 944.78 & 93.4 & 945.42 \\
\hline 36.0 & 944.25 & & & 30.5 & 944.25 & 95.0 & 945.48 \\
\hline 38.0 & 944.31 & & & 31.0 & 944.18 & 96.6 & 945.41 \\
\hline 40.0 & 944.32 & & & 33.0 & 944.19 & 97.8 & 945.55 \\
\hline 41.0 & 944.39 & & & 35.0 & 944.24 & 99.0 & 945.78 \\
\hline 43.0 & 944.42 & & & 37.0 & 944.28 & 100.0 & 945.68 \\
\hline 44.6 & 943.54 & & & 39.0 & 944.32 & 101.1 & 945.75 \\
\hline 45.4 & 943.48 & & & 41.0 & 944.40 & & \\
\hline 47.0 & 943.36 & & & 42.9 & 944.43 & & \\
\hline 49.0 & 943.28 & & & 43.9 & 943.88 & & \\
\hline 52.0 & 943.22 & & & 44.2 & 943.85 & & \\
\hline 55.0 & 943.05 & & & 44.6 & 943.61 & & \\
\hline 58.0 & 942.98 & & & 46.0 & 943.38 & & \\
\hline 61.0 & 942.84 & & & 47.0 & 943.42 & & \\
\hline 64.0 & 942.78 & & & 49.0 & 943.37 & & \\
\hline 67.0 & 942.80 & & & 50.0 & 943.24 & & \\
\hline 69.0 & 942.94 & & & 52.5 & 943.22 & & \\
\hline 72.0 & 943.03 & & & 54.0 & 943.02 & & \\
\hline 75.0 & 943.12 & & & 55.0 & 942.97 & & \\
\hline 78.0 & 943.19 & & & 57.0 & 942.87 & & \\
\hline 81.0 & 943.31 & & & 58.0 & 942.84 & & \\
\hline 84.0 & 943.27 & & & 59.0 & 943.01 & & \\
\hline 87.0 & 943.15 & & & 60.4 & 943.05 & & \\
\hline 89.0 & 943.20 & & & 62.0 & 942.87 & & \\
\hline 90.1 & 943.47 & & & 64.0 & 942.89 & & \\
\hline
\end{tabular}




\section{Description of Cross Section PR191}

Location: Township 5 South/Range 50 East--section 25

U.S. Geological Survey quadrangle $(1: 24,000)$ : Eldon Mountain

Landowners--left bank: Doug and Lucille Randall

right bank: Doug and Lucille Randall

Access: $\quad$ left bank

Permission from: Doug and Lucille Randall (T5S/R51E--8)

or Craig Randall (T5S/R51E--17)

Reference pins

\begin{tabular}{rcc}
\hline $\begin{array}{c}\text { Station } \\
\text { (meters) }\end{array}$ & $\begin{array}{c}\text { Elevation } \\
\text { (meters } \\
\text { above } \\
\text { sea level) }\end{array}$ & Comments \\
& & $\begin{array}{c}\text { Pins }-0.4 \text { and } 0.0 \text { were closest to } \\
\text { leveling instrument. }\end{array}$ \\
-0.4 & 937.994 & 0.16 meter above 1986 ground level. \\
0.0 & 937.698 & At 1987 ground level. \\
150.3 & 936.632 & 0.27 meter above 1977 ground level, \\
possibly buried in 1978. & 0.19 meter above 1987 ground level. \\
151.6 & 937.081 & 0.17 meter above 1979 ground level. \\
186.0 & 936.924 &
\end{tabular}




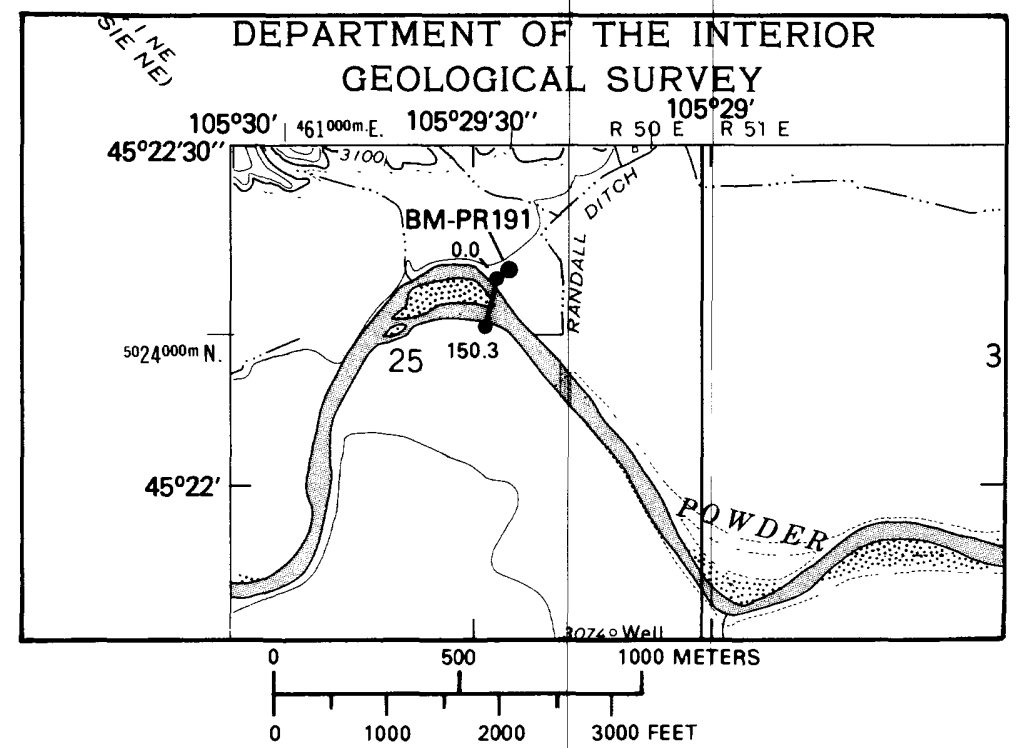

PRI9I

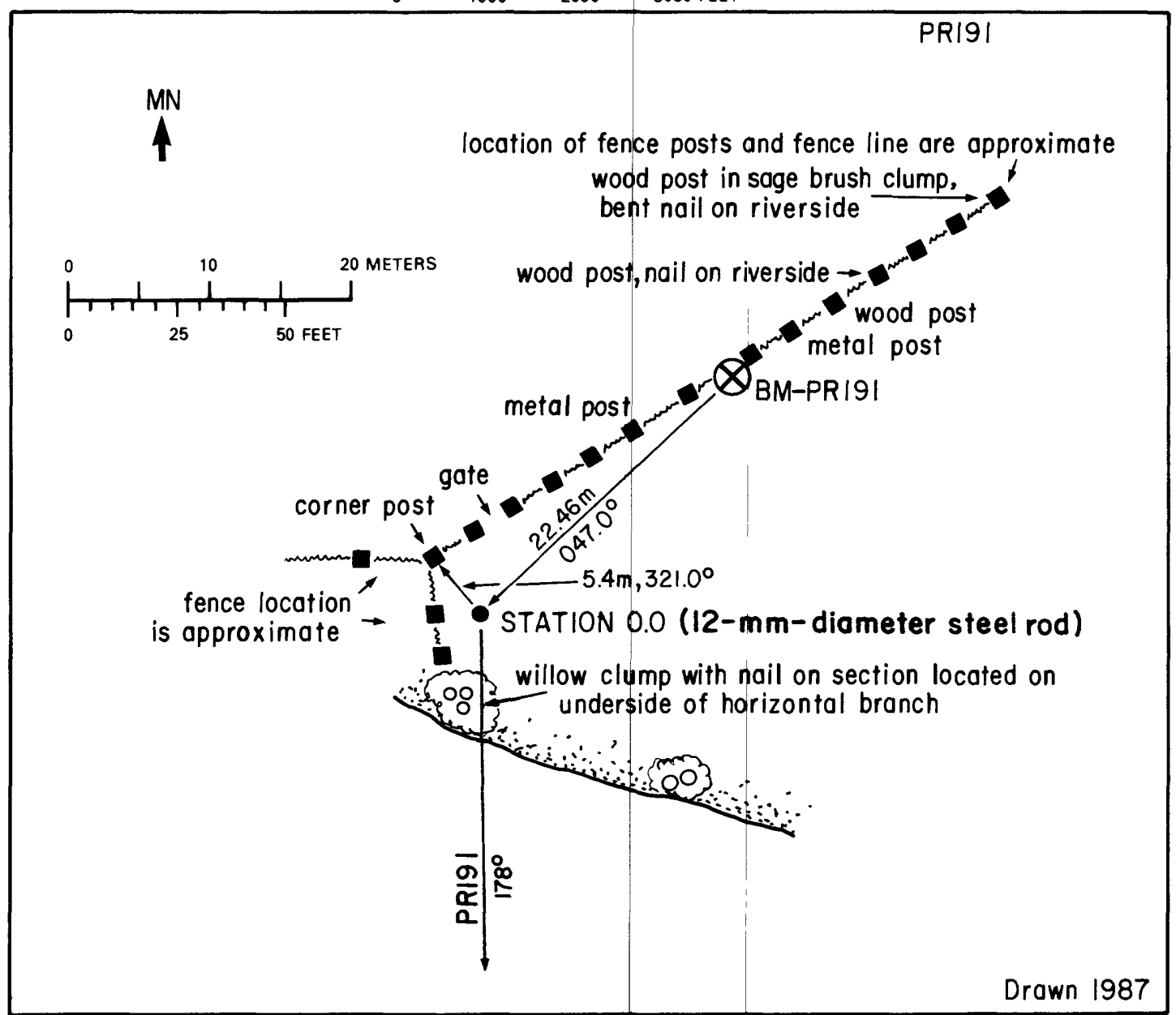

location of fence posts and fence line are approximate wood post in sage brush clump, bent nail on riverside

Figure 80.--Upper: Location of cross section PR191, bench mark BM-PR191, and left and right bank reference pins (tables 2 and 3 ) in the Eldon Mountain quadrangle. Lower: Location of bench mark on the left bank. $M N$ is magnetic north. 

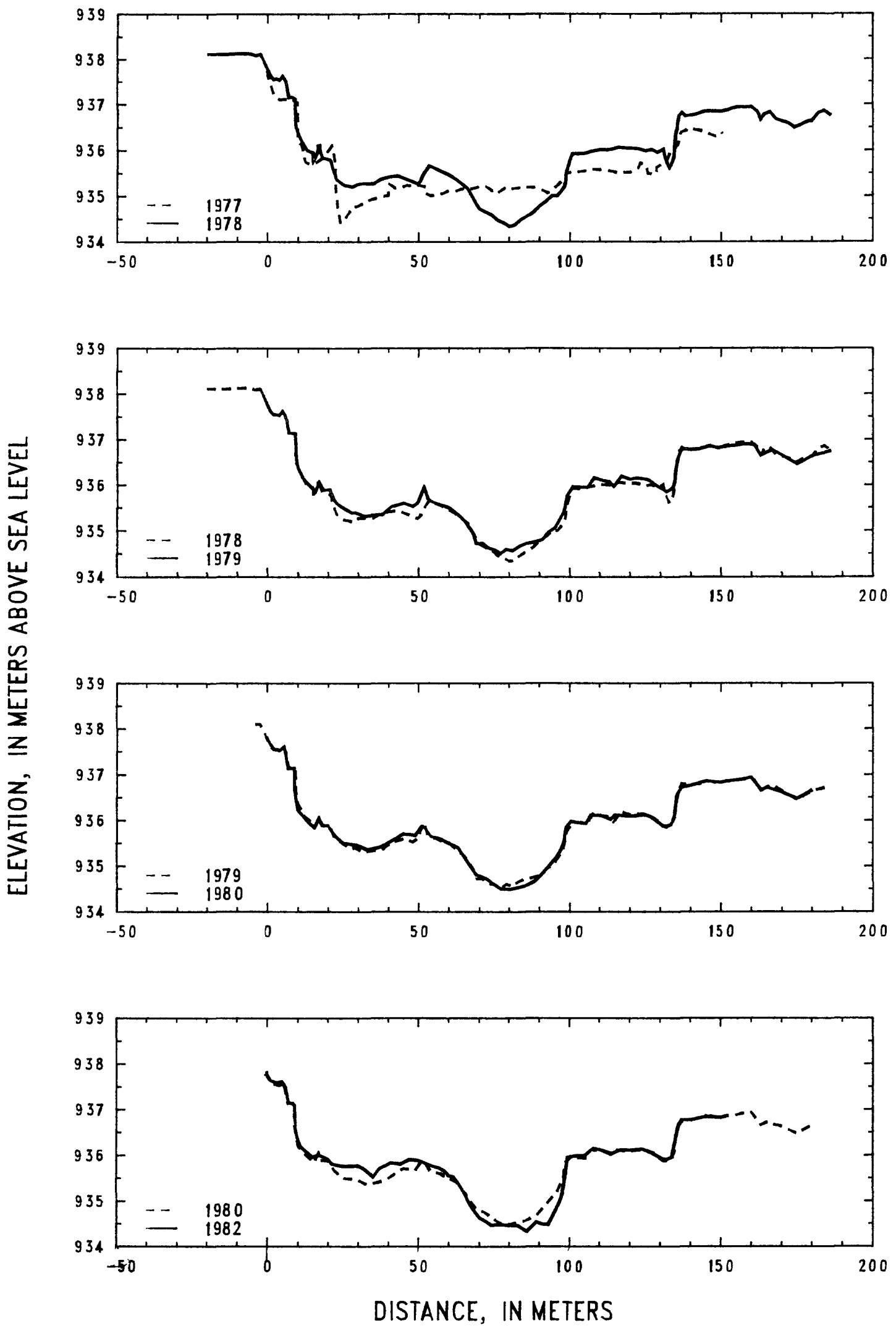

Figure 81. --Profiles of cross section PR191 from 1977 to 1982. 


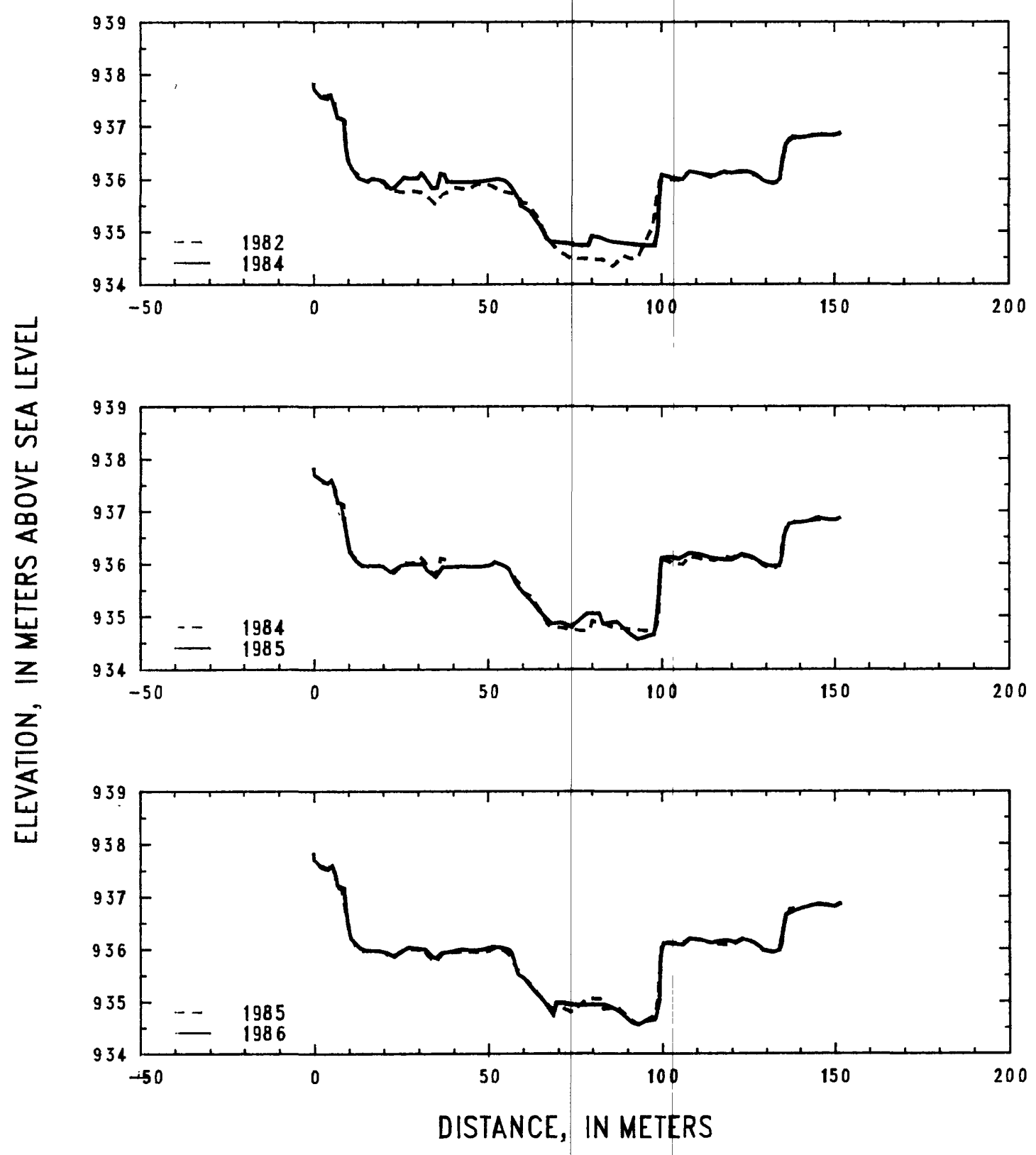

Figure 82.--Profiles of cross section PR191 from 1982 to 1986. 


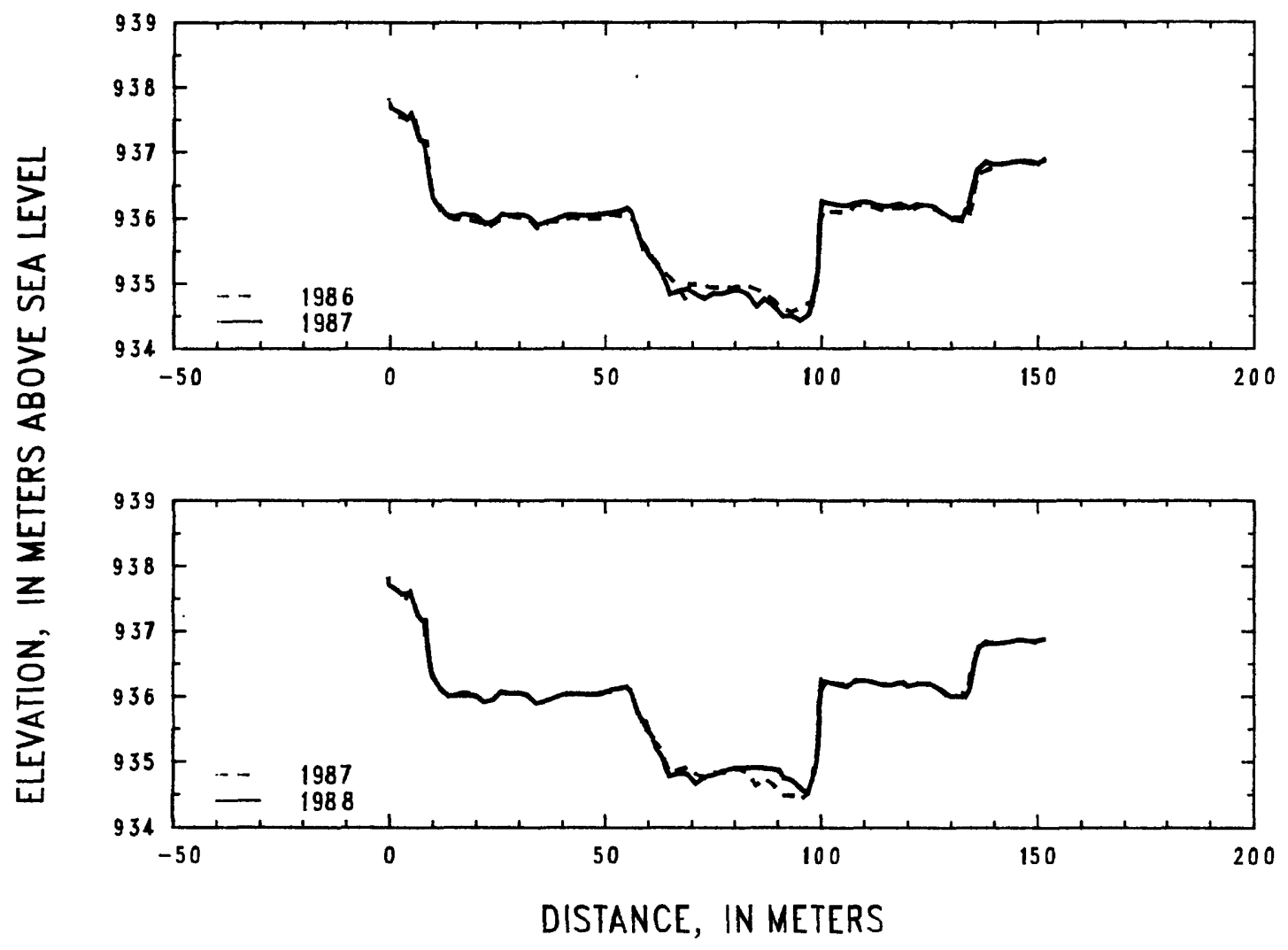

Figure 83. - Profiles of cross section PR191 from 1986 to 1988. 
Table 26.- Listing of horizontal stations and elevations for cross section PR191 [Sta., station, distance in meters from a reference point on the left bank; Elev., elevation, in meters above sea level].

\begin{tabular}{|c|c|c|c|c|c|c|c|c|c|}
\hline \multicolumn{2}{|c|}{1977} & \multicolumn{2}{|c|}{1977} & \multicolumn{2}{|c|}{1978} & \multicolumn{2}{|c|}{1978} & \multicolumn{2}{|c|}{1978} \\
\hline Sta. & Elev. & Sta. & Elev. & Sta. & Elev. & Sta. & Elev. & Sta. & Elev. \\
\hline-0.4 & 937.73 & 66.0 & 935.15 & -20.0 & 938.12 & 60.0 & 935.46 & 138.0 & 936.74 \\
\hline 2.0 & 937.23 & 68.0 & 935.17 & -15.0 & 938.10 & 62.0 & 935.37 & 140.0 & 936.76 \\
\hline 4.0 & 937.10 & 70.0 & 935.21 & -10.0 & 938.13 & 64.0 & 935.27 & 142.0 & 936.78 \\
\hline 6.0 & 937.12 & 72.0 & 935.22 & -6.0 & 938.13 & 66.0 & 935.17 & 144.0 & 936.84 \\
\hline 8.0 & 937.10 & 74.0 & 935.12 & -4.0 & 938.08 & 68.0 & 934.93 & 146.0 & 936.85 \\
\hline 9.8 & 937.04 & 75.5 & 935.10 & -2.4 & 938.11 & 70.0 & 934.71 & 148.0 & 936.84 \\
\hline 10.0 & 936.18 & 76.0 & 935.07 & -0.4 & 937.84 & 72.0 & 934.64 & 150.0 & 936.83 \\
\hline 11.1 & 936.12 & 77.0 & 935.11 & 1.0 & 937.64 & 74.0 & 934.59 & 151.6 & 936.87 \\
\hline 12.4 & 935.74 & 79.0 & 935.20 & 2.0 & 937.55 & 76.0 & 934.46 & 152.0 & 936.87 \\
\hline 14.0 & 935.69 & 80.0 & 935.16 & 3.0 & 937.57 & 78.0 & 934.41 & 154.0 & 936.92 \\
\hline 16.0 & 935.74 & 84.0 & 935.19 & 4.0 & 937.53 & 80.0 & 934.33 & 156.0 & 936.94 \\
\hline 18.0 & 935.83 & 88.0 & 935.19 & 5.0 & 937.63 & 82.0 & 934.37 & 158.0 & 936.93 \\
\hline 20.0 & 936.00 & 91.5 & 935.11 & 6.0 & 937.51 & 84.0 & 934.49 & 160.0 & 936.94 \\
\hline 21.0 & 936.09 & 92.6 & 935.06 & 7.0 & 937.15 & 86.0 & 934.56 & 162.0 & 936.82 \\
\hline 21.6 & 935.94 & 93.6 & 935.10 & 8.1 & 937.16 & 88.0 & 934.64 & 163.0 & 936.65 \\
\hline 22.2 & 935.77 & 95.6 & 935.22 & 9.1 & 937.12 & 90.0 & 934.78 & 164.0 & 936.77 \\
\hline 22.5 & 935.10 & 98.2 & 935.38 & 9.2 & 936.52 & 92.0 & 934.87 & 166.0 & 936.83 \\
\hline 22.6 & 935.00 & 98.8 & 935.50 & 11.0 & 936.20 & 94.0 & 935.00 & 168.0 & 936.67 \\
\hline 23.0 & 934.78 & 104.0 & 935.54 & 13.0 & 935.99 & 96.0 & 935.00 & 170.0 & 936.62 \\
\hline 24.0 & $=934.41$ & 108.0 & 935.58 & 14.9 & 935.94 & 98.0 & 935.18 & 172.0 & 936.59 \\
\hline 25.0 & 934.45 & 112.0 & 935.56 & 15.0 & 935.76 & 98.6 & 935.32 & 174.0 & 936.48 \\
\hline 26.0 & 934.56 & 116.0 & 935.50 & 16.5 & 935.98 & 98.7 & 935.50 & 176.0 & 936.54 \\
\hline 28.0 & 934.76 & 120.0 & 935.52 & 16.6 & 936.10 & 99.5 & 935.67 & 178.0 & 936.61 \\
\hline 30.0 & 934.78 & 122.6 & 935.61 & 17.1 & 936.11 & 100.0 & 935.79 & 180.0 & 936.64 \\
\hline 32.0 & 934.86 & 123.4 & 935.72 & 17.2 & 936.00 & 100.8 & 935.92 & 182.0 & 936.81 \\
\hline 34.0 & 934.93 & 125.0 & 935.68 & 18.0 & 935.84 & 103.0 & 935.92 & 184.0 & 936.86 \\
\hline 36.0 & 934.95 & 125.9 & 935.49 & 20.5 & 935.79 & 105.0 & 935.93 & 186.0 & 936.75 \\
\hline 38.0 & 934.99 & 128.5 & 935.48 & 21.0 & 935.73 & 107.0 & 935.97 & & \\
\hline 39.8 & 935.01 & 128.8 & 935.62 & 21.1 & 935.64 & 110.0 & 936.01 & & \\
\hline 40.0 & 935.07 & 132.0 & 935.76 & 22.7 & 935.36 & 113.0 & 936.00 & & \\
\hline 40.2 & 935.14 & 133.0 & 935.93 & 25.0 & 935.24 & 116.0 & 936.06 & & \\
\hline 40.3 & 935.26 & 135.6 & 936.11 & 28.0 & 935.20 & 119.0 & 936.04 & & \\
\hline 42.0 & 935.17 & 136.7 & 936.39 & 30.0 & 935.27 & 122.0 & 936.04 & & \\
\hline 44.0 & 935.19 & 140.0 & 936.47 & 32.0 & 935.28 & 125.0 & 935.98 & & \\
\hline 46.0 & 935.24 & 144.0 & 936.40 & 35.0 & 935.27 & 127.0 & 936.01 & & \\
\hline 48.0 & 935.21 & 148.0 & 936.29 & 37.0 & 935.36 & 129.0 & 935.94 & & \\
\hline 50.0 & 935.24 & 150.0 & 936.38 & 40.0 & 935.42 & 131.0 & 936.02 & & \\
\hline 52.3 & 935.19 & 150.3 & 936.36 & 43.0 & 935.44 & 131.4 & 935.82 & & \\
\hline 52.4 & 935.05 & & & 46.0 & 935.36 & 133.0 & 935.60 & & \\
\hline 54.0 & 935.00 & & & 49.6 & 935.26 & 134.4 & 935.80 & & \\
\hline 56.0 & 935.03 & & & 50.5 & 935.32 & 135.0 & 936.38 & & \\
\hline 58.2 & 935.06 & & & 51.4 & 935.46 & 135.3 & 936.43 & & \\
\hline 60.0 & 935.12 & & & 53.4 & 935.66 & 135.5 & 936.55 & & \\
\hline 62.0 & 935.11 & & & 56.0 & 935.58 & 136.0 & 936.75 & & \\
\hline 64.0 & 935.16 & & & 58.0 & 935.52 & 137.0 & 936.82 & & \\
\hline
\end{tabular}


Table 26.--Listing of horizontal stations and elevations for cross section PR191 --Continued.

\begin{tabular}{|c|c|c|c|c|c|c|c|c|c|}
\hline \multicolumn{2}{|c|}{1979} & \multicolumn{2}{|c|}{1979} & \multicolumn{2}{|c|}{1979} & \multicolumn{2}{|c|}{1980} & \multicolumn{2}{|c|}{1980} \\
\hline Sta. & Elev. & Sta. & Elev. & Sta. & Elev. & Sta. & Elev. & Sta. & Elev. \\
\hline-4.0 & 938.10 & 77.0 & 934.50 & 180.0 & 936.64 & -0.4 & 937.82 & 110.0 & 936.11 \\
\hline-2.4 & 938.10 & 79.0 & 934.60 & 186.0 & 936.75 & 1.0 & 937.65 & 113.6 & 936.02 \\
\hline-0.4 & 937.82 & 81.0 & 934.56 & & & 2.0 & 937.55 & 115.0 & 936.12 \\
\hline 1.0 & 937.62 & 83.0 & 934.65 & & & 4.0 & 937.52 & 120.0 & 936.08 \\
\hline 2.0 & 937.54 & 85.0 & 934.71 & & & 5.5 & 937.60 & 125.0 & 936.13 \\
\hline 3.0 & 937.55 & 88.0 & 934.75 & & & 6.9 & 937.13 & 128.0 & 936.04 \\
\hline 4.0 & 937.53 & 91.0 & 934.81 & & & 8.9 & 937.14 & 131.0 & 935.87 \\
\hline 5.0 & 937.62 & 92.6 & 934.95 & & & 9.1 & 936.62 & 133.7 & 935.90 \\
\hline 6.0 & 937.50 & 95.0 & 935.06 & & & 10.2 & 936.21 & 134.6 & 936.05 \\
\hline 7.0 & 937.14 & 97.8 & 935.37 & & & 13.0 & 935.99 & 135.5 & 936.56 \\
\hline 9.2 & 937.13 & 98.6 & 935.59 & & & 15.5 & 935.84 & 137.0 & 936.73 \\
\hline 9.4 & 936.62 & 99.0 & 935.76 & & & 17.0 & 936.05 & 140.0 & 936.76 \\
\hline 10.0 & 936.38 & 101.0 & 935.96 & & & 18.0 & 935.89 & 145.0 & 936.86 \\
\hline 12.0 & 936.10 & 103.0 & 935.95 & & & 20.0 & 935.89 & 150.0 & 936.82 \\
\hline 14.0 & 935.98 & 106.0 & 935.95 & & & 22.0 & 935.68 & 151.6 & 936.86 \\
\hline 15.7 & 935.85 & 108.0 & 936.16 & & & 25.0 & 935.50 & 155.0 & 936.88 \\
\hline 17.0 & 936.06 & 111.0 & 936.09 & & & 30.0 & 935.45 & 160.0 & 936.94 \\
\hline 18.4 & 935.88 & 113.0 & 936.07 & & & 33.0 & 935.36 & 163.3 & 936.65 \\
\hline 20.4 & 935.90 & 114.5 & 935.96 & & & 37.0 & 935.41 & 165.0 & 936.72 \\
\hline 22.0 & 935.65 & 116.0 & 936.10 & & & 40.0 & 935.51 & 168.0 & 936.66 \\
\hline 23.0 & 935.58 & 117.0 & 936.19 & & & 45.0 & 935.71 & 170.0 & 936.62 \\
\hline 25.0 & 935.49 & 120.0 & 936.12 & & & 49.0 & 935.67 & 175.0 & 936.47 \\
\hline 28.0 & 935.38 & 122.0 & 936.15 & & & 51.0 & 935.87 & 180.0 & 936.66 \\
\hline 29.0 & 935.40 & 124.0 & 936.14 & & & 52.0 & 935.84 & & \\
\hline 32.0 & 935.31 & 126.0 & 936.11 & & & 54.0 & 935.65 & & \\
\hline 35.0 & 935.34 & 128.0 & 936.01 & & & 57.0 & 935.60 & & \\
\hline 38.0 & 935.36 & 130.0 & 935.93 & & & 60.0 & 935.49 & & \\
\hline 41.0 & 935.53 & 132.0 & 935.84 & & & 63.0 & 935.40 & & \\
\hline 42.0 & 935.54 & 134.0 & 935.93 & & & 66.0 & 935.10 & & \\
\hline 45.0 & 935.60 & 134.7 & 936.05 & & & 69.0 & 934.81 & & \\
\hline 48.0 & 935.52 & 135.1 & 936.37 & & & 72.0 & 934.74 & & \\
\hline 49.7 & 935.61 & 136.0 & 936.65 & & & 75.0 & 934.59 & & \\
\hline 51.7 & 935.95 & 137.0 & 936.80 & & & 77.0 & 934.50 & & \\
\hline 53.0 & 935.70 & 140.0 & 936.77 & & & 80.0 & 934.48 & & \\
\hline 54.0 & 935.64 & 143.0 & 936.80 & & & 83.0 & 934.53 & & \\
\hline 56.0 & 935.59 & 146.0 & 936.86 & & & 86.0 & 934.58 & & \\
\hline 59.0 & 935.52 & 149.0 & 936.81 & & & 89.0 & 934.68 & & \\
\hline 60.0 & 935.50 & 151.6 & 936.85 & & & 92.0 & 934.91 & & \\
\hline 63.0 & 935.34 & 155.0 & 936.87 & & & 96.0 & 935.22 & & \\
\hline 65.0 & 935.22 & 158.0 & 936.91 & & & 98.0 & 935.49 & & \\
\hline 67.9 & 934.95 & 161.0 & 936.88 & & & 98.7 & 935.84 & & \\
\hline 69.0 & 934.72 & 163.0 & 936.64 & & & 100.5 & 935.98 & & \\
\hline 71.0 & 934.73 & 165.0 & 936.72 & & & 103.0 & 935.94 & & \\
\hline 73.0 & 934.62 & 167.0 & 936.76 & & . & 105.5 & 935.93 & & \\
\hline 75.0 & 934.59 & 175.0 & 936.45 & & & 107.0 & 936.11 & & \\
\hline
\end{tabular}


Table 26.--Listing of horizontal stations and elevations for cross section PR191 --Continued.

\begin{tabular}{|c|c|c|c|c|c|c|c|c|c|}
\hline \multicolumn{2}{|c|}{1982} & \multicolumn{2}{|c|}{1982} & \multicolumn{2}{|c|}{1984} & \multicolumn{2}{|c|}{1984} & \multicolumn{2}{|c|}{1985} \\
\hline Sta. & Elev. & Sta. & Elev. & Sta. & Elev. & Sta. & Elev. & Sta. & Elev. \\
\hline-0.4 & 937.83 & 105.0 & 935.99 & -0.4 & 937.82 & 79.0 & 934.73 & -0.4 & 937.83 \\
\hline 0.0 & 937.72 & 108.0 & 936.15 & 0.0 & 937.70 & 80.0 & 934.91 & 0.0 & 937.69 \\
\hline 1.0 & 937.64 & 111.0 & 936.10 & 2.0 & 937.56 & 82.0 & 934.90 & 3.0 & 937.56 \\
\hline 3.0 & 937.58 & 114.0 & 936.03 & 4.0 & 937.53 & 85.0 & 934.81 & 4.0 & 937.53 \\
\hline 5.0 & 937.61 & 117.0 & 936.13 & 5.0 & 937.60 & 90.0 & 934.76 & 5.0 & 937.60 \\
\hline 6.0 & 937.50 & 120.0 & 936.11 & 7.0 & 937.17 & 95.0 & 934.73 & 6.0 & 937.45 \\
\hline 7.0 & 937.17 & 124.0 & 936.14 & 8.0 & 937.16 & 98.0 & 934.73 & 7.0 & 937.16 \\
\hline 9.0 & 937.10 & 127.0 & 936.06 & 8.7 & 937.13 & 99.0 & 935.04 & 8.0 & 937.14 \\
\hline 9.5 & 936.55 & 130.0 & 935.95 & 9.3 & 936.59 & 99.6 & 935.98 & 9.0 & 936.72 \\
\hline 10.0 & 936.38 & 132.0 & 935.90 & 10.0 & 936.34 & 100.1 & 936.09 & 10.4 & 936.21 \\
\hline 11.0 & 936.19 & 134.0 & 935.96 & 11.0 & 936.20 & 103.0 & 936.02 & 12.0 & 936.07 \\
\hline 15.0 & 935.96 & 136.0 & 936.67 & 13.0 & 936.02 & 106.0 & 935.99 & 14.0 & 935.96 \\
\hline 17.0 & 936.01 & 137.0 & 936.77 & 15.6 & 935.95 & 108.0 & 936.15 & 16.0 & 935.97 \\
\hline 20.0 & 935.93 & 140.0 & 936.77 & 17.0 & 936.02 & 112.0 & 936.09 & 20.0 & 935.95 \\
\hline 21.3 & 935.81 & 145.0 & 936.85 & 20.0 & 935.96 & 115.0 & 936.06 & 22.0 & 935.84 \\
\hline 25.0 & 935.75 & 150.0 & 936.82 & 22.0 & 935.82 & 118.0 & 936.15 & 25.0 & 935.97 \\
\hline 30.0 & 935.77 & 151.6 & 936.85 & 23.0 & 935.83 & 120.0 & 936.11 & 28.0 & 936.00 \\
\hline 32.5 & 935.68 & & & 26.0 & 936.04 & 122.0 & 936.15 & 30.8 & 935.99 \\
\hline 35.0 & 935.52 & & & 27.0 & 936.02 & 125.0 & 936.14 & 31.3 & 936.03 \\
\hline 37.0 & 935.71 & & & 30.0 & 936.02 & 127.4 & 936.07 & 32.4 & 935.88 \\
\hline 41.0 & 935.84 & & & 31.0 & 936.12 & 129.0 & 935.97 & 35.0 & 935.75 \\
\hline 44.0 & 935.80 & & & 31.4 & 936.09 & 131.0 & 935.92 & 37.0 & 935.94 \\
\hline 47.0 & 935.91 & & & 34.0 & 935.83 & 133.0 & 935.92 & 39.0 & 935.94 \\
\hline 50.0 & 935.89 & & & 35.4 & 935.84 & 134.2 & 936.00 & 42.0 & 935.96 \\
\hline 55.0 & 935.75 & & & 36.4 & 936.11 & 135.0 & 936.36 & 45.0 & 935.94 \\
\hline 57.0 & 935.72 & & & 37.4 & 936.09 & 136.0 & 936.66 & 48.0 & 935.95 \\
\hline 59.0 & 935.64 & & & 38.3 & 935.95 & 138.0 & 936.80 & 50.0 & 935.96 \\
\hline 60.0 & 935.55 & & & 42.0 & 935.94 & 140.0 & 936.78 & 52.0 & 936.03 \\
\hline 61.5 & 935.53 & & & 45.0 & 935.95 & 145.0 & 936.84 & 54.0 & 935.99 \\
\hline 64.3 & 935.28 & & & 47.0 & 935.95 & 150.0 & 936.83 & 56.0 & 935.89 \\
\hline 67.0 & 934.89 & & & 50.0 & 935.98 & 151.6 & 936.87 & 58.0 & 935.63 \\
\hline 70.0 & 934.63 & & & 53.0 & 936.01 & & & 60.0 & 935.46 \\
\hline 72.0 & 934.56 & & & 55.0 & 935.97 & & & 63.0 & 935.25 \\
\hline 74.0 & 934.47 & & & 57.0 & 935.83 & & & 65.0 & 935.07 \\
\hline 77.0 & 934.48 & & & 58.0 & 935.71 & & & 68.0 & 934.86 \\
\hline 80.0 & 934.46 & & & 59.4 & 935.59 & & & 71.0 & 934.88 \\
\hline 83.0 & 934.46 & & & 59.5 & 935.49 & & & 74.0 & 934.79 \\
\hline 86.0 & 934.33 & & & 62.0 & 935.39 & & & 75.0 & 934.86 \\
\hline 89.0 & 934.54 & & & 64.0 & 935.21 & & & 77.0 & 934.97 \\
\hline 91.0 & 934.48 & & & 65.7 & 935.04 & & & 78.5 & 935.06 \\
\hline 93.0 & 934.48 & & & 67.0 & 934.86 & & & 80.0 & 935.06 \\
\hline 97.0 & 935.02 & & & 68.0 & 934.81 & & & 82.0 & 935.06 \\
\hline 98.0 & 935.29 & & & 70.0 & 934.79 & & & 83.0 & 934.85 \\
\hline 99.4 & 935.96 & & & 73.0 & 934.77 & & & 85.0 & 934.88 \\
\hline 102.0 & 935.99 & & & 76.0 & 934.73 & & & 87.0 & 934.90 \\
\hline
\end{tabular}


Table 26.--Listing of horizontal stations and elevations for cross section PR191 --Continued.

\begin{tabular}{|c|c|c|c|c|c|c|c|c|c|}
\hline \multicolumn{2}{|c|}{1985} & \multicolumn{2}{|c|}{1986} & \multicolumn{2}{|c|}{1986} & \multicolumn{2}{|c|}{1987} & \multicolumn{2}{|c|}{1987} \\
\hline Sta. & Elev. & Sta. & Elev. & Sta. & Elev. & Sta. & Elev. & Sta. & Elev. \\
\hline 90.0 & 934.69 & -0.4 & 937.83 & 89.0 & 934.73 & 0.0 & 937.70 & 81.0 & 934.89 \\
\hline 93.0 & 934.56 & 0.0 & 937.70 & 91.0 & 934.60 & 1.0 & 937.66 & 83.0 & 934.83 \\
\hline 96.0 & 934.63 & 1.0 & 937.63 & 93.0 & 934.56 & 3.0 & 937.59 & 85.0 & 934.63 \\
\hline 97.7 & 934.66 & 2.0 & 937.56 & 95.0 & 934.63 & 4.0 & 937.50 & 87.0 & 934.75 \\
\hline 98.7 & 935.07 & 4.0 & 937.52 & 97.0 & 934.70 & 5.0 & 937.60 & 89.0 & 934.63 \\
\hline 99.6 & 935.93 & 5.3 & 937.59 & 98.0 & 934.67 & 7.0 & 937.18 & 91.0 & 934.48 \\
\hline 100.0 & 936.11 & 6.3 & 937.43 & 99.0 & 935.01 & 8.0 & 937.15 & 93.0 & 934.49 \\
\hline 102.0 & 936.13 & 6.8 & 937.21 & 99.1 & 935.09 & 10.0 & 936.32 & 95.0 & 934.41 \\
\hline 105.0 & 936.09 & 8.7 & 937.16 & 99.5 & 935.88 & 11.0 & 936.22 & 97.0 & 934.51 \\
\hline 108.0 & 936.20 & 9.3 & 936.66 & 100.3 & 936.11 & 13.0 & 936.05 & 98.0 & 934.74 \\
\hline 111.0 & 936.17 & 10.5 & 936.22 & 103.0 & 936.09 & 15.0 & 936.01 & 99.0 & 935.13 \\
\hline 114.0 & 936.10 & 13.0 & 936.03 & 106.0 & 936.08 & 17.0 & 936.06 & 99.3 & 935.24 \\
\hline 117.0 & 936.08 & 15.0 & 935.97 & 108.0 & 936.20 & 20.0 & 936.03 & 99.7 & 936.01 \\
\hline 120.0 & 936.07 & 16.0 & 935.98 & 111.0 & 936.17 & 22.0 & 935.91 & 100.1 & 936.25 \\
\hline 123.0 & 936.18 & 19.0 & 935.96 & 114.0 & 936.11 & 24.0 & 935.95 & 101.0 & 936.23 \\
\hline 126.0 & 936.12 & 21.0 & 935.93 & 117.0 & 936.15 & 26.0 & 936.07 & 103.0 & 936.20 \\
\hline 129.0 & 935.99 & 23.0 & 935.85 & 119.0 & 936.16 & 28.0 & 936.04 & 105.0 & 936.18 \\
\hline 132.0 & 935.94 & 25.0 & 935.96 & 121.0 & 936.12 & 30.0 & 936.06 & 107.0 & 936.20 \\
\hline 134.0 & 935.96 & 27.0 & 936.04 & 123.0 & 936.20 & 32.0 & 936.02 & 109.0 & 936.24 \\
\hline 134.7 & 936.11 & 30.0 & 936.01 & 126.0 & 936.13 & 34.0 & 935.90 & 111.0 & 936.23 \\
\hline 135.5 & 936.55 & 32.0 & 935.99 & 128.0 & 936.05 & 36.0 & 935.93 & 113.0 & 936.19 \\
\hline 137.0 & 936.76 & 34.0 & 935.85 & 129.0 & 935.98 & 38.0 & 935.98 & 115.0 & 936.17 \\
\hline 139.0 & 936.78 & 35.0 & 935.83 & 132.0 & 935.93 & 40.0 & 936.03 & 118.0 & 936.21 \\
\hline 142.0 & 936.80 & 36.6 & 935.93 & 134.0 & 935.98 & 42.0 & 936.06 & 120.0 & 936.15 \\
\hline 145.0 & 936.87 & 39.0 & 935.95 & 136.0 & 936.66 & 44.0 & 936.04 & 122.0 & 936.21 \\
\hline 148.0 & 936.83 & 42.0 & 936.00 & 140.0 & 936.78 & 46.0 & 936.03 & 124.0 & 936.19 \\
\hline 150.0 & 936.82 & 45.0 & 935.98 & 145.0 & 936.86 & 48.0 & 936.04 & 126.0 & 936.17 \\
\hline \multirow[t]{18}{*}{151.6} & 936.87 & 48.0 & 935.99 & 150.0 & 936.81 & 50.0 & 936.07 & 128.0 & 936.07 \\
\hline & & 51.0 & 936.05 & 151.6 & 936.87 & 52.0 & 936.09 & 130.0 & 935.99 \\
\hline & & 54.0 & 936.03 & & & 54.0 & 936.13 & 132.0 & 935.99 \\
\hline & & 56.0 & 935.98 & & & 55.0 & 936.15 & 132.6 & 935.98 \\
\hline & & 56.8 & 935.91 & & & 55.9 & 936.10 & 133.3 & 936.12 \\
\hline & & 58.7 & 935.51 & & & 58.0 & 935.66 & 133.8 & 936.10 \\
\hline & & 60.0 & 935.46 & & & 59.4 & 935.54 & 135.0 & 936.44 \\
\hline & & 63.0 & 935.22 & & & 60.0 & 935.41 & 136.0 & 936.72 \\
\hline & & 65.8 & 935.01 & & & 62.4 & 935.23 & 138.0 & 936.85 \\
\hline & & 68.5 & 934.74 & & & 64.0 & 934.99 & 140.0 & 936.80 \\
\hline & & 69.5 & 934.98 & & & 65.0 & 934.82 & 142.0 & 936.81 \\
\hline & & 71.0 & 934.98 & & & 67.0 & 934.88 & 144.0 & 936.84 \\
\hline & & 74.0 & 934.94 & & & 69.0 & 934.91 & 146.0 & 936.86 \\
\hline & & 77.0 & 934.92 & & & 71.0 & 934.81 & 148.0 & 936.86 \\
\hline & & 80.0 & 934.94 & & & 73.0 & 934.75 & 150.0 & 936.81 \\
\hline & & 83.0 & 934.95 & & & 75.0 & 934.84 & 151.6 & 936.89 \\
\hline & & 85.0 & 934.90 & & & 77.0 & 934.83 & & \\
\hline & & 87.0 & 934.84 & & & 79.0 & 934.88 & & \\
\hline
\end{tabular}


Table 26.--Listing of horizontal stations and elevations for cross section PR191 --Continued.

\begin{tabular}{|c|c|c|c|c|}
\hline \multicolumn{2}{|c|}{1988} & \multicolumn{2}{|c|}{1988} & \multirow[b]{2}{*}{$\cdot$} \\
\hline Sta. & Elev. & Sta. & Elev. & \\
\hline-0.4 & 937.83 & 75.0 & 934.80 & \\
\hline 0.0 & 937.70 & 77.0 & 934.86 & \\
\hline 1.0 & 937.67 & 80.0 & 934.91 & \\
\hline 3.0 & 937.57 & 83.0 & 934.91 & \\
\hline 5.0 & 937.59 & 86.0 & 934.91 & \\
\hline 7.0 & 937.21 & 89.0 & 934.88 & \\
\hline 8.0 & 937.16 & 90.3 & 934.87 & \\
\hline 8.6 & 937.17 & 91.5 & 934.75 & \\
\hline 9.0 & 936.73 & 93.0 & 934.73 & \\
\hline 10.0 & 936.34 & 96.0 & 934.55 & \\
\hline 12.0 & 936.10 & 97.0 & 934.53 & \\
\hline 14.0 & 936.00 & 98.7 & 934.87 & \\
\hline 16.0 & 936.02 & 99.3 & 935.20 & \\
\hline 18.0 & 936.02 & 99.6 & 935.87 & \\
\hline 20.0 & 936.01 & 100.0 & 936.10 & \\
\hline 22.0 & 935.91 & 101.0 & 936.23 & \\
\hline 24.0 & 935.95 & 103.0 & 936.19 & \\
\hline 26.0 & 936.08 & 106.0 & 936.15 & \\
\hline 28.0 & 936.04 & 108.0 & 936.24 & \\
\hline 30.0 & 936.06 & 111.0 & 936.23 & \\
\hline 32.0 & 936.02 & 114.0 & 936.16 & \\
\hline 34.0 & 935.90 & 116.0 & 936.17 & \\
\hline 36.0 & 935.92 & 118.0 & 936.20 & \\
\hline 38.0 & 935.97 & 119.0 & 936.22 & \\
\hline 40.0 & 936.03 & 120.0 & 936.15 & \\
\hline 43.0 & 936.05 & 122.0 & 936.19 & \\
\hline 46.0 & 936.03 & 125.0 & 936.20 & \\
\hline 49.0 & 936.04 & 128.0 & 936.09 & \\
\hline 51.0 & 936.10 & 130.0 & 936.00 & \\
\hline 53.0 & 936.12 & 132.0 & 935.99 & \\
\hline 55.0 & 936.14 & 133.4 & 935.99 & \\
\hline 56.0 & 936.07 & 134.4 & 936.10 & \\
\hline 58.0 & 935.70 & 135.6 & 936.58 & \\
\hline 59.0 & 935.60 & 136.5 & 936.76 & \\
\hline 59.4 & 935.61 & 138.0 & 936.81 & \\
\hline 60.0 & 935.47 & 140.0 & 936.81 & \\
\hline 61.1 & 935.36 & 143.0 & 936.83 & \\
\hline 61.7 & 935.21 & 146.0 & 936.87 & \\
\hline 63.1 & 935.09 & 149.0 & 936.84 & \\
\hline 64.2 & 934.87 & 151.6 & 936.88 & \\
\hline 65.0 & 934.78 & & & \\
\hline 67.0 & 934.83 & & & \\
\hline 69.0 & 934.81 & & & \\
\hline 71.0 & 934.66 & & & \\
\hline 73.0 & 934.77 & & & \\
\hline
\end{tabular}


Location: Township 5 South/Range 51 East--section 30

U.S. Geological Survey quadrangle $(1: 24,000)$ : Eldon Mountain

Landowners--left bank: Doug and Lucille Randal1

right bank: 'Doug and Lucille Randall

Access: right bank, across Stuver Ranch

Permission from: Shirley or John Stuver (T5S/R51E--31)

Reference pins

\begin{tabular}{rl|l}
\hline $\begin{array}{l}\text { Station } \\
\text { (meters) }\end{array}$ & $\begin{array}{c}\text { Elevation } \\
\text { (meters } \\
\text { above } \\
\text { sea leve1) }\end{array}$ & \multicolumn{1}{c}{ Comments } \\
\hline-1.2 & 934.316 & $\begin{array}{c}\text { Pins } 96.0 \text { and 113.3 were closest to } \\
\text { leveling instruments. } \\
\text { Bent, } 0.13 \text { meter above } 1987 \text { ground } \\
\text { level. } \\
0.01 \text { meter above } 1987 \text { ground level. } \\
\text { Bent flush with 1988 ground leve1. } \\
0.10 \text { meter above 1988 ground level }\end{array}$ \\
96.0 & 934.186 & \\
113.3 & 934.229 &
\end{tabular}



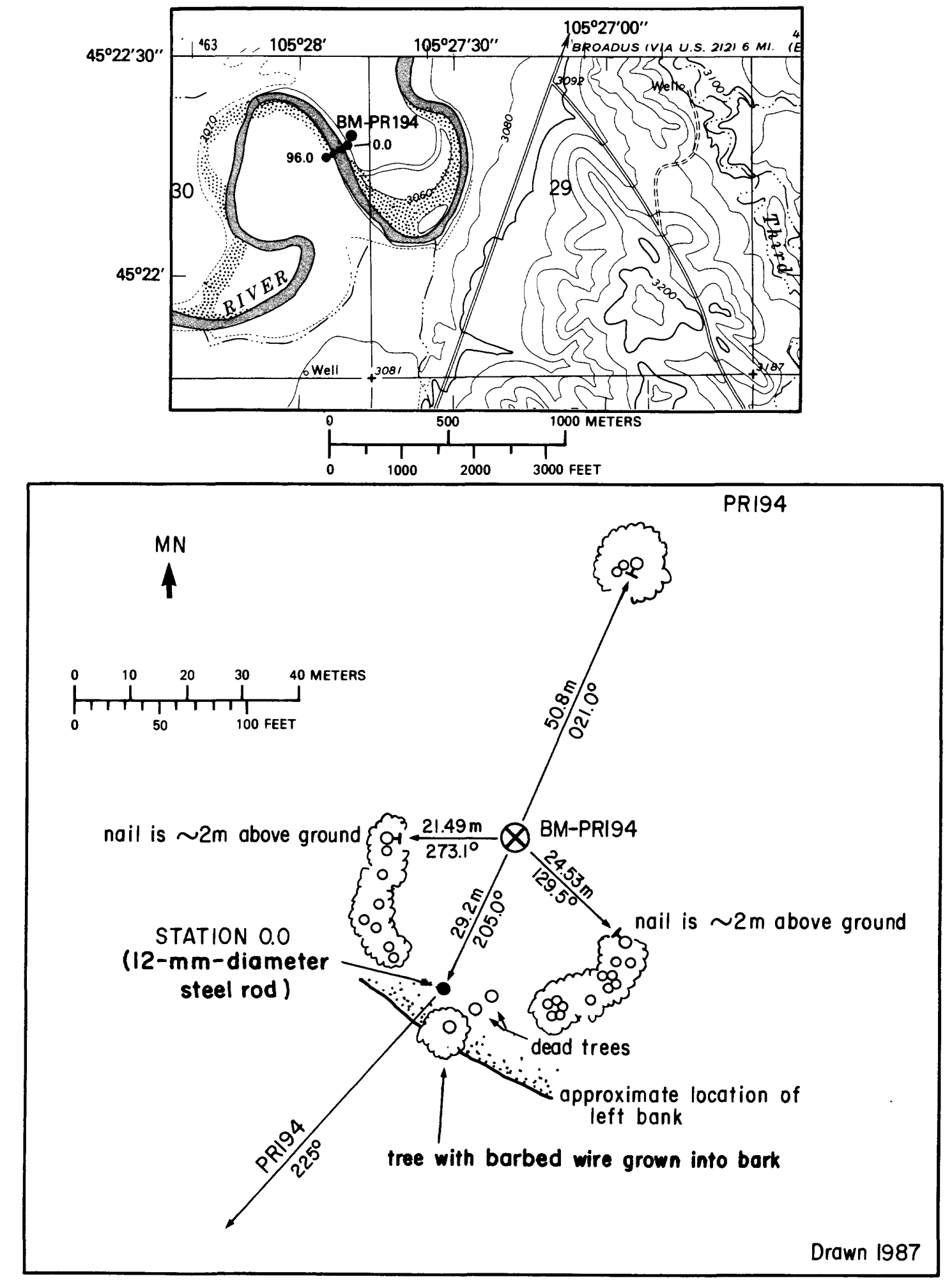

PRI94

Figure 84.--Upper: Location of cross section PR194, bench mark BM-PR194, and left and right bank reference pins (tables 2 and 3 ) in the Eldon Mountain quadrangle. Lower: Location of bench mark on the left bank. MN is magnetic north. 


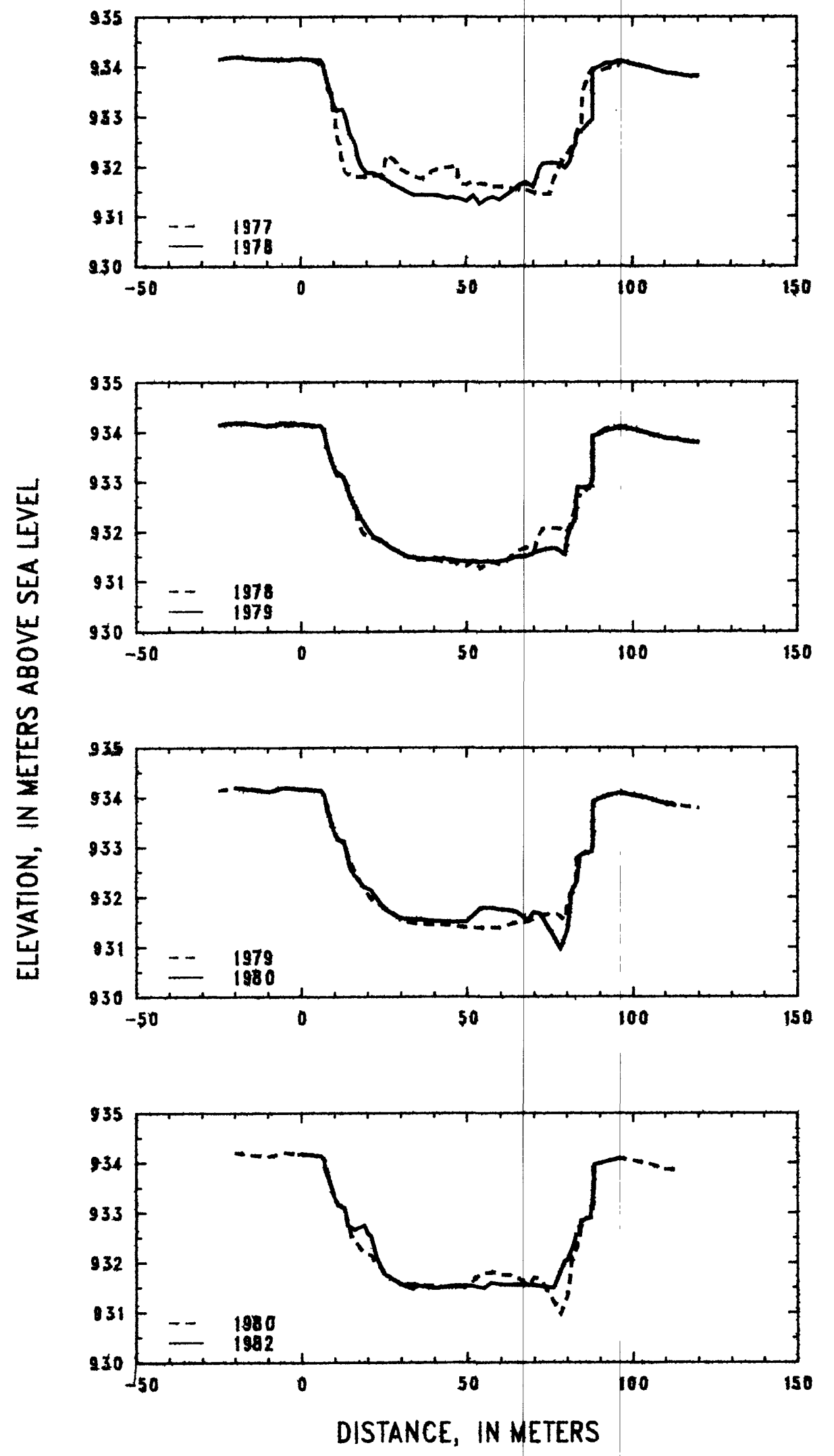

Figure 85.--Profiles of cross section PR194 from 1977 to 1982. 


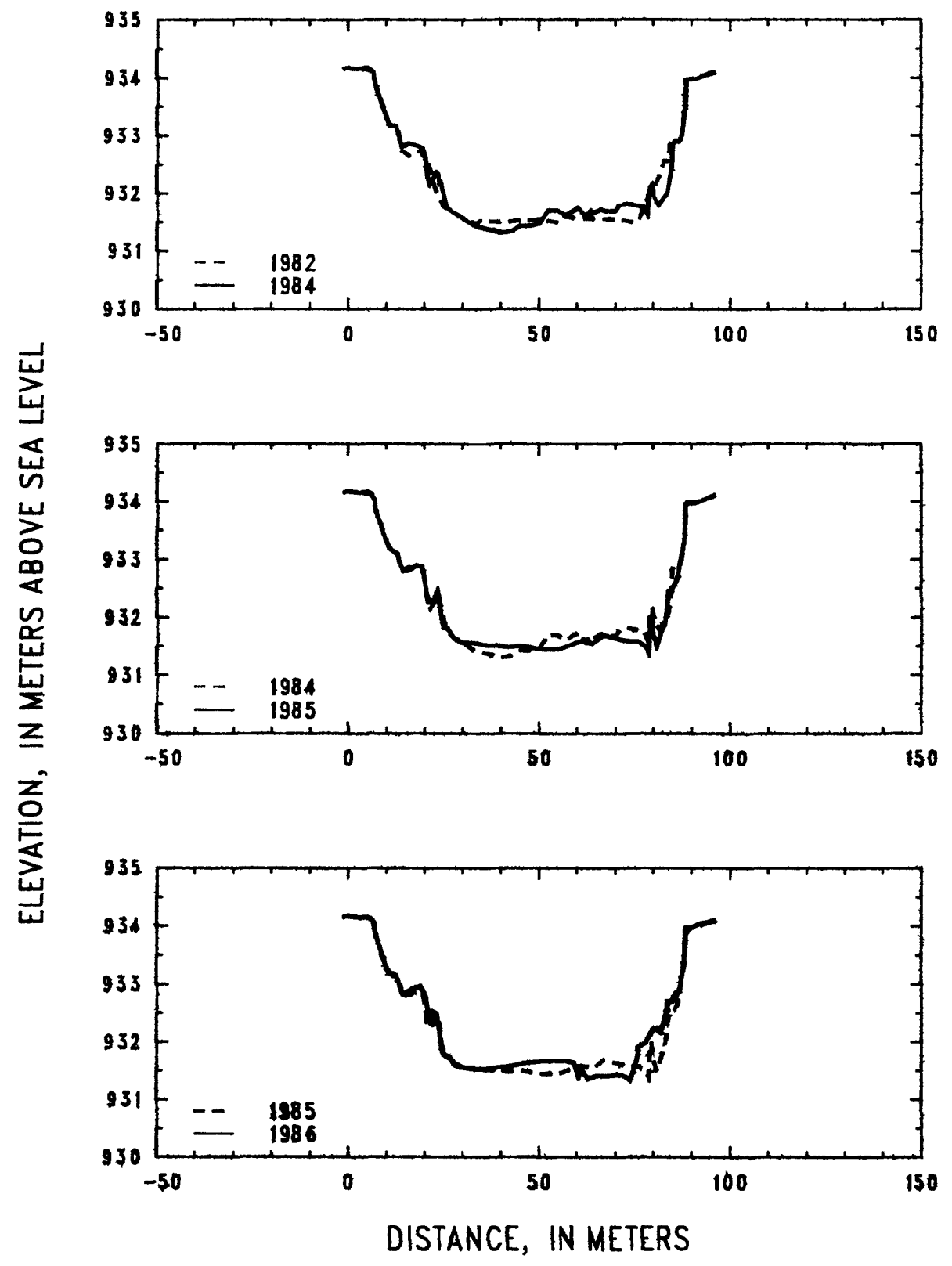

Figure 86.--Profiles of cross section PR194 from 1982 to 1986. 


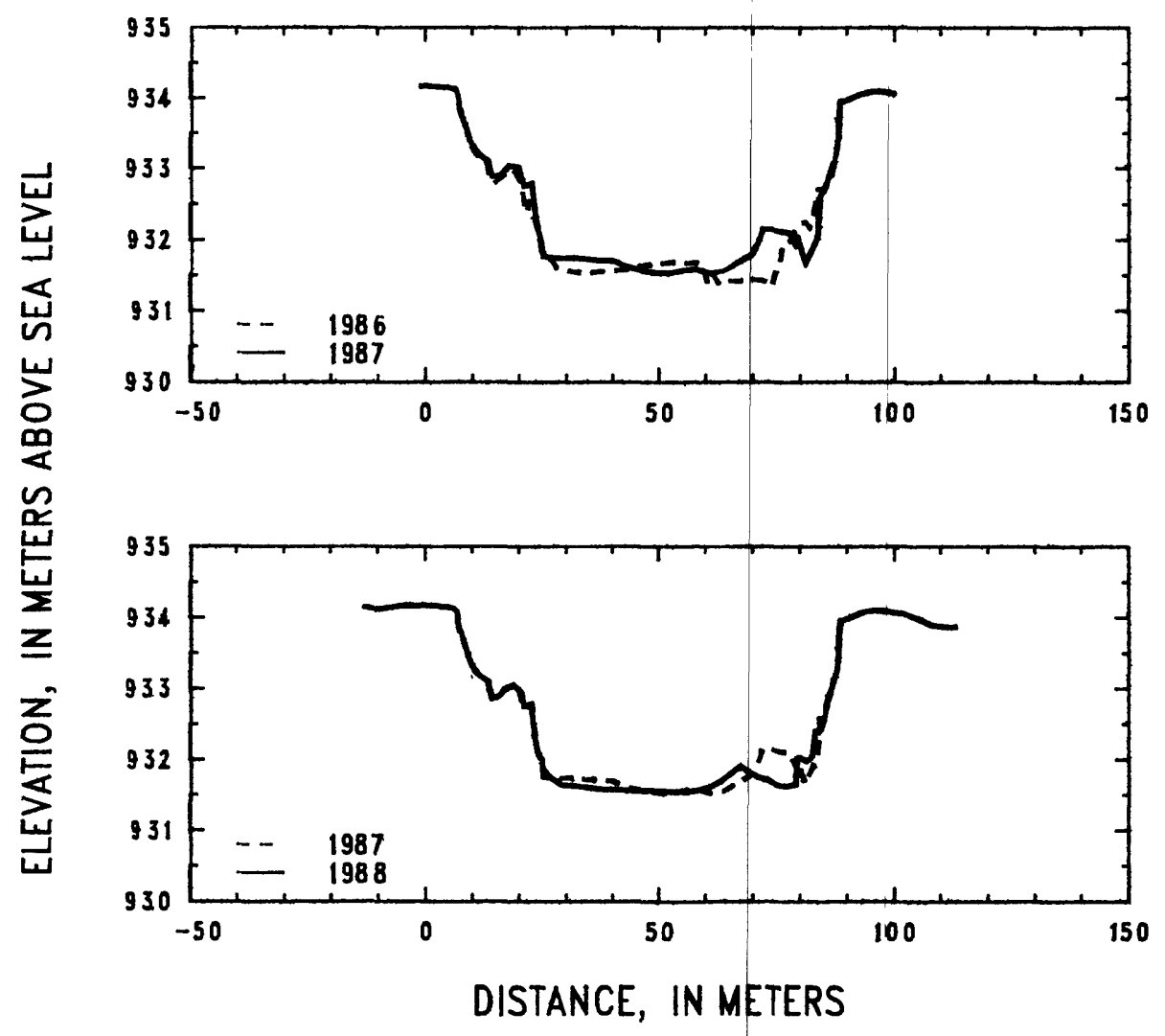

Figure 87.--Profiles of cross section PR194 from 1986 to 1988. 
Table 27.--Listing of horizontal stations and elevations for cross section PR194 [Sta., station, distance in meters from a reference point on the left bank; Elev., elevation, in meters above sea level].

\begin{tabular}{|c|c|c|c|c|c|c|c|c|c|}
\hline \multicolumn{2}{|c|}{1977} & \multicolumn{2}{|c|}{1977} & \multicolumn{2}{|c|}{1978} & \multicolumn{2}{|c|}{1978} & \multicolumn{2}{|c|}{1979} \\
\hline Sta. & Elev. & Sta. & Elev. & Sta. & Elev. & Sta. & Elev. & Sta. & Elev. \\
\hline-1.2 & 934.15 & 58.0 & 931.59 & -25.0 & 934.15 & 64.0 & 931.53 & -25.0 & 934.14 \\
\hline 0.0 & 934.16 & 60.0 & 931.60 & -20.0 & 934.20 & 66.0 & 931.64 & -20.0 & 934.19 \\
\hline 2.0 & 934.15 & 62.0 & 931.59 & -16.0 & 934.17 & 68.0 & 931.68 & -15.0 & 934.17 \\
\hline 4.0 & 934.11 & 64.0 & 931.55 & -12.0 & 934.14 & 70.0 & 931.59 & -10.0 & 934.11 \\
\hline 6.0 & 934.07 & 66.0 & 931.56 & -8.0 & 934.14 & 72.0 & 931.98 & -5.0 & 934.19 \\
\hline 7.4 & 933.98 & 68.0 & 931.53 & -4.0 & 934.13 & 73.0 & 932.06 & -1.2 & 934.15 \\
\hline 8.4 & 933.54 & 70.0 & 931.48 & -1.2 & 934.15 & 75.0 & 932.08 & 0.0 & 934.16 \\
\hline 9.4 & 933.48 & 72.0 & 931.43 & 0.0 & 934.17 & 78.0 & 932.06 & 2.0 & 934.15 \\
\hline 10.2 & 933.01 & 74.8 & 931.45 & 2.0 & 934.15 & 79.5 & 931.97 & 4.0 & 934.12 \\
\hline 10.6 & 932.94 & 76.0 & 931.72 & 4.0 & 934.14 & 81.0 & 932.09 & 6.0 & 934.13 \\
\hline 10.8 & 932.74 & 78.0 & 932.03 & 6.0 & 934.13 & 82.0 & 932.34 & 7.0 & 934.02 \\
\hline 11.3 & 932.52 & 80.0 & 932.23 & 7.0 & 934.04 & 82.9 & 932.45 & 8.0 & 933.73 \\
\hline 12.0 & 932.45 & 82.0 & 932.42 & 7.2 & 933.94 & 83.0 & 932.66 & 10.0 & 933.27 \\
\hline 12.4 & 932.17 & 84.0 & 932.74 & 9.0 & 933.53 & 83.5 & 932.74 & 12.0 & 933.12 \\
\hline 13.0 & 932.10 & 84.6 & 932.85 & 10.0 & 933.26 & 84.5 & 932.70 & 13.0 & 933.10 \\
\hline 13.3 & 931.91 & 85.0 & 933.49 & 11.0 & 933.13 & 87.0 & 932.89 & 14.0 & 932.86 \\
\hline 14.0 & 931.86 & 87.0 & 933.86 & 12.7 & 933.15 & 88.0 & 932.94 & 16.0 & 932.52 \\
\hline 16.0 & 931.80 & 88.0 & 933.96 & 13.8 & 933.00 & 88.0 & 933.95 & 18.0 & 932.29 \\
\hline 18.0 & 931.80 & 90.0 & 933.89 & 14.8 & 932.68 & 90.0 & 934.00 & 20.0 & 932.06 \\
\hline 20.0 & 931.79 & 92.0 & 933.96 & 16.5 & 932.47 & 92.0 & 934.07 & 22.0 & 931.89 \\
\hline 22.0 & 931.82 & 94.0 & 934.00 & 17.0 & 932.35 & 94.0 & 934.07 & 24.0 & 931.83 \\
\hline 24.7 & 931.85 & 95.0 & 934.02 & 17.3 & 932.19 & 96.0 & 934.12 & 26.0 & 931.72 \\
\hline 26.0 & 932.25 & 96.0 & 934.02 & 18.6 & 931.99 & 98.0 & 934.09 & 26.8 & 931.70 \\
\hline 28.5 & 932.12 & & & 20.0 & 931.88 & 100.0 & 934.05 & 29.0 & 931.60 \\
\hline 29.3 & 931.98 & & & 22.0 & 931.88 & 105.0 & 933.98 & 32.0 & 931.49 \\
\hline 32.0 & 931.88 & & & 24.0 & 931.80 & 110.0 & 933.87 & 35.0 & 931.46 \\
\hline 34.0 & 931.81 & & & 26.0 & 931.74 & 113.0 & 933.86 & 38.0 & 931.44 \\
\hline 36.0 & 931.76 & & & 28.0 & 931.64 & 116.0 & 933.81 & 41.0 & 931.46 \\
\hline 38.0 & 931.81 & & & 30.0 & 931.57 & 120.0 & 933.80 & 44.0 & 931.44 \\
\hline 39.0 & 931.84 & & & 32.0 & 931.50 & & & 47.0 & 931.41 \\
\hline 39.1 & 931.90 & & & 34.0 & 931.43 & & & 50.0 & 931.39 \\
\hline 41.0 & 931.95 & & & 36.0 & 931.43 & & & 53.0 & 931.38 \\
\hline 43.0 & 931.98 & & & 38.0 & 931.43 & & & 56.0 & 931.40 \\
\hline 45.0 & 931.99 & & & 40.0 & 931.43 & & & 59.0 & 931.38 \\
\hline 47.2 & 932.03 & & & 42.0 & 931.41 & & & 62.0 & 931.43 \\
\hline 47.4 & 931.76 & & & 44.0 & 931.36 & & & 65.0 & 931.50 \\
\hline 48.3 & 931.66 & & & 46.0 & 931.39 & & & 68.0 & 931.50 \\
\hline 49.0 & 931.64 & & & 48.0 & 931.35 & & & 71.0 & 931.59 \\
\hline 50.0 & 931.63 & & & 50.0 & 931.30 & & & 74.0 & 931.65 \\
\hline 50.5 & 931.66 & & & 52.0 & 931.42 & & & 77.0 & 931.66 \\
\hline 52.0 & 931.69 & & & 54.0 & 931.25 & & & 79.0 & 931.55 \\
\hline 53.0 & 931.64 & & & 56.0 & 931.35 & & & 79.5 & 931.53 \\
\hline 54.0 & 931.66 & & & 58.0 & 931.39 & & & 30.0 & 931.81 \\
\hline 55.2 & 931.65 & & & 60.0 & 931.33 & & & 81.0 & 932.05 \\
\hline 56.0 & 931.62 & & & 62.0 & 931.44 & & & 82.8 & 932.27 \\
\hline
\end{tabular}


Table 27.--Listing of horizontal stations and elevations for cross section PR194 --Continued.

\begin{tabular}{|c|c|c|c|c|c|c|c|c|c|}
\hline \multicolumn{2}{|c|}{1979} & \multicolumn{2}{|c|}{1980} & \multicolumn{2}{|c|}{1980} & \multicolumn{2}{|c|}{1982} & \multicolumn{2}{|c|}{1984} \\
\hline Sta. & Elev. & Sta. & Elev. & Sta. & Elev. & Sta. & Elev. & Sta. & Elev. \\
\hline 83.0 & 932.74 & -20.0 & 934.20 & 100.0 & 934.03 & -1.2 & 934.17 & -1.2 & 934.16 \\
\hline 83.5 & 932.89 & -15.0 & 934.15 & 105.0 & 933.97 & 0.0 & 934.18 & 0.0 & 934.19 \\
\hline 85.0 & 932.87 & -10.0 & 934.11 & 110.0 & 933.86 & 3.0 & 934.15 & 2.0 & 934.16 \\
\hline 87.0 & 932.90 & -5.0 & 934.20 & 113.0 & 933.84 & 6.0 & 934.13 & 5.0 & 934.18 \\
\hline 88.0 & 933.03 & -1.2 & 934.16 & & & 7.0 & 934.05 & 6.8 & 934.10 \\
\hline 88.0 & 933.91 & 0.0 & 934.17 & & & 7.2 & 933.90 & 7.2 & 933.90 \\
\hline 90.0 & 933.97 & 2.0 & 934.16 & & & 9.0 & 933.54 & 9.0 & 933.52 \\
\hline 92.0 & 934.03 & 4.0 & 934.14 & & & 10.0 & 933.30 & 11.0 & 933.18 \\
\hline 94.0 & 934.05 & 6.0 & 934.13 & & & 11.0 & 933.17 & 12.7 & 933.16 \\
\hline 96.0 & 934.09 & 7.0 & 934.05 & & & 13.0 & 933.10 & 14.0 & 932.81 \\
\hline 98.0 & 934.08 & 10.0 & 933.31 & & & 13.5 & 932.99 & 16.0 & 932.87 \\
\hline 100.0 & 934.05 & 11.0 & 933.15 & & & 14.0 & 932.74 & 18.0 & 932.83 \\
\hline 105.0 & 933.96 & 13.0 & 933.11 & & & 16.0 & 932.65 & 19.6 & 932.78 \\
\hline 110.0 & 933.87 & 15.0 & 932.52 & & & 19.0 & 932.75 & 20.3 & 932.62 \\
\hline 113.0 & 933.86 & 19.0 & 932.19 & & & 20.0 & 932.58 & 20.7 & 932.32 \\
\hline 116.0 & 933.81 & 21.0 & 932.14 & & & 21.0 & 932.53 & 21.4 & 932.15 \\
\hline \multirow[t]{29}{*}{120.0} & 933.78 & 25.0 & 931.77 & & & 23.0 & 932.03 & 23.1 & 932.37 \\
\hline & & 30.0 & 931.57 & & & 25.0 & 931.77 & 23.6 & 932.34 \\
\hline & & 35.0 & 931.55 & & & 30.0 & 931.56 & 24.0 & 932.17 \\
\hline & & 40.0 & 931.52 & & & 33.5 & 931.47 & 24.3 & 932.19 \\
\hline & & 45.0 & 931.50 & & & 35.0 & 931.54 & 25.8 & 931.77 \\
\hline & & 50.0 & 931.51 & & & 40.0 & 931.48 & 27.0 & 931.68 \\
\hline & & 54.0 & 931.78 & & & 45.0 & 931.53 & 30.0 & 931.56 \\
\hline & & 58.0 & 931.79 & & & 50.0 & 931.54 & 33.0 & 931.43 \\
\hline & & 59.0 & 931.75 & & & 55.0 & 931.48 & 36.0 & 931.38 \\
\hline & & 63.0 & 931.74 & & & 57.0 & 931.59 & 40.0 & 931.30 \\
\hline & & 65.0 & 931.70 & & & 60.0 & 931.56 & 43.0 & 931.34 \\
\hline & & 68.0 & 931.54 & & & 65.0 & 931.55 & 45.0 & 931.43 \\
\hline & & 69.0 & 931.60 & & & 70.0 & 931.54 & 48.0 & 931.44 \\
\hline & & 70.0 & 931.70 & & & 75.0 & 931.49 & 50.0 & 931.47 \\
\hline & & 72.0 & 931.66 & & & 76.0 & 931.50 & 52.4 & 931.69 \\
\hline & & 76.0 & 931.20 & & & 79.2 & 932.03 & 54.7 & 931.70 \\
\hline & & 78.0 & 930.96 & & & 80.0 & 932.05 & 57.0 & 931.60 \\
\hline & & 79.0 & 931.15 & & & 82.2 & 932.39 & 60.5 & 931.75 \\
\hline & & 80.0 & 931.31 & & & 82.7 & 932.56 & 62.3 & 931.57 \\
\hline & & 81.0 & 931.75 & & & 83.9 & 932.55 & 63.6 & 931.69 \\
\hline & & 81.5 & 932.11 & & & 84.3 & 932.84 & 63.8 & 931.64 \\
\hline & & 83.0 & 932.29 & & & 85.0 & 932.85 & 66.4 & 931.72 \\
\hline & & 84.0 & 932.83 & & & 87.0 & 932.91 & 67.2 & 931.68 \\
\hline & & 87.6 & 932.91 & & & 88.0 & 933.37 & 70.0 & 931.68 \\
\hline & & 87.9 & 933.07 & & & 88.4 & 933.97 & 70.7 & 931.74 \\
\hline & & 88.0 & 933.91 & & & 90.0 & 933.99 & 71.0 & 931.79 \\
\hline & & 90.0 & 933.98 & & & 93.0 & 934.04 & 73.0 & 931.82 \\
\hline & & 93.0 & 934.05 & & & 96.0 & 934.09 & 76.0 & 931.78 \\
\hline & & 96.0 & 934.09 & & & & & 77.5 & 931.76 \\
\hline
\end{tabular}


Table 27.--Listing of horizontal stations and elevations for cross section PR194 --Continued.

\begin{tabular}{|c|c|c|c|c|c|c|c|c|c|}
\hline \multicolumn{2}{|c|}{1984} & \multicolumn{2}{|c|}{1985} & \multicolumn{2}{|c|}{1985} & \multicolumn{2}{|c|}{1986} & \multicolumn{2}{|c|}{1986} \\
\hline Sta. & Elev. & Sta. & Elev. & Sta. & Elev. & Sta. & Elev. & Sta. & Elev. \\
\hline 78.5 & 931.61 & -1.2 & 934.17 & 81.0 & 931.49 & -1.2 & 934.17 & 78.0 & 931.98 \\
\hline 79.2 & 932.11 & 0.0 & 934.19 & 82.3 & 931.79 & 0.0 & 934.17 & 79.0 & 932.13 \\
\hline 79.8 & 932.16 & 2.0 & 934.16 & 83.0 & 931.87 & 3.0 & 934.15 & 80.0 & 932.22 \\
\hline 80.4 & 931.91 & 4.0 & 934.15 & 84.0 & 932.45 & 5.0 & 934.16 & 81.0 & 932.25 \\
\hline 81.5 & 931.79 & 6.0 & 934.13 & 84.6 & 932.43 & 6.0 & 934.13 & 82.0 & 932.16 \\
\hline 83.7 & 932.00 & 7.0 & 934.03 & 86.4 & 932.67 & 7.0 & 934.05 & 83.5 & 932.54 \\
\hline 84.9 & 932.42 & 7.3 & 933.84 & 86.9 & 932.96 & 7.2 & 933.92 & 83.8 & 932.71 \\
\hline 85.0 & 932.83 & 9.0 & 933.52 & 87.5 & 933.10 & 9.0 & 933.52 & 85.0 & 932.70 \\
\hline 85.7 & 932.91 & 10.0 & 933.31 & 88.2 & 933.36 & 10.5 & 933.23 & 86.0 & 932.86 \\
\hline 86.7 & 932.90 & 11.0 & 933.18 & 88.5 & 933.97 & 12.0 & 933.15 & 86.8 & 932.88 \\
\hline 87.3 & 932.97 & 13.0 & 933.10 & 89.0 & 933.97 & 12.8 & 933.15 & 88.0 & 933.31 \\
\hline 88.2 & 933.41 & 14.4 & 932.79 & 91.0 & 933.98 & 14.2 & 932.82 & 88.5 & 933.97 \\
\hline 88.6 & 933.96 & 16.0 & 932.81 & 93.0 & 934.03 & 15.0 & 932.80 & 90.0 & 933.98 \\
\hline 89.0 & 933.99 & 18.0 & 932.90 & 96.0 & 934.11 & 17.0 & 932.93 & 92.0 & 934.05 \\
\hline 91.0 & 933.98 & 19.5 & 932.87 & & & 19.0 & 932.98 & 94.0 & 934.07 \\
\hline 93.0 & 934.04 & 20.0 & 932.70 & & & 20.3 & 932.82 & 96.0 & 934.10 \\
\hline 95.0 & 934.09 & 21.0 & 932.26 & & & 21.5 & 932.38 & & \\
\hline \multirow[t]{28}{*}{96.0} & 934.10 & 22.4 & 932.30 & & & 22.2 & 932.53 & & \\
\hline & & 23.5 & 932.47 & & & 23.2 & 932.49 & & \\
\hline & & 24.0 & 932.30 & & & 24.8 & 931.82 & & \\
\hline & & 24.3 & 932.04 & & & 25.7 & 931.75 & & \\
\hline & & 25.0 & 931.82 & & & 26.7 & 931.72 & & \\
\hline & & 26.0 & 931.81 & & & 28.0 & 931.60 & & \\
\hline & & 28.0 & 931.62 & & & 31.0 & 931.55 & & \\
\hline & & 30.0 & 931.55 & & & 34.0 & 931.52 & & \\
\hline & & 33.0 & 931.55 & & & 37.0 & 931.55 & & \\
\hline & & 36.0 & 931.51 & & & 40.0 & 931.56 & & \\
\hline & & 39.0 & 931.51 & & & 43.0 & 931.58 & & \\
\hline & & 42.0 & 931.48 & & & 46.0 & 931.64 & & \\
\hline & & 45.0 & 931.51 & & & 49.0 & 931.66 & & \\
\hline & & 48.0 & 931.47 & & & 52.0 & 931.67 & & \\
\hline & & 51.0 & 931.44 & & & 55.0 & 931.67 & & \\
\hline & & 55.0 & 931.44 & & & 58.0 & 931.67 & & \\
\hline & & 58.0 & 931.52 & & & 59.4 & 931.64 & & \\
\hline & & 61.0 & 931.59 & & & 60.5 & 931.39 & & \\
\hline & & 64.0 & 931.54 & & & 61.5 & 931.53 & & \\
\hline & & 67.0 & 931.69 & & & 63.0 & 931.36 & & \\
\hline & & 70.0 & 931.64 & & & 65.0 & 931.42 & & \\
\hline & & 73.0 & 931.58 & & & 68.0 & 931.41 & & \\
\hline & & 76.0 & 931.58 & & & 70.0 & 931.44 & & \\
\hline & & 78.0 & 931.50 & & & 72.0 & 931.42 & & \\
\hline & & 78.8 & 931.38 & & & 74.0 & 931.33 & & \\
\hline & & 79.1 & 931.78 & & & 75.0 & 931.57 & & \\
\hline & & 79.6 & 932.00 & & & 75.9 & 931.73 & & \\
\hline & & 80.0 & 931.72 & & & 76.0 & 931.91 & & \\
\hline
\end{tabular}


Table 27.---Listing of horizontal stations and elevations for cross section PR194 --Continued.

\begin{tabular}{|c|c|c|c|c|c|c|c|}
\hline \multicolumn{2}{|c|}{1987} & \multicolumn{2}{|c|}{1987} & \multicolumn{2}{|c|}{1988} & \multicolumn{2}{|c|}{1988} \\
\hline Sta. & Elev. & Sta. & Elev. & Sta. & Elev. & Sta. & Elev. \\
\hline-1.2 & 934.18 & 85.0 & 932.67 & -13.0 & 934.16 & 67.4 & 931.90 \\
\hline 0.0 & 934.18 & 87.0 & 933.07 & -10.0 & 934.12 & 69.0 & 931.83 \\
\hline 3.0 & 934.15 & 87.4 & 933.09 & -8.0 & 934.15 & 71.0 & 931.75 \\
\hline 5.0 & 934.15 & 88.1 & 933.34 & -6.0 & 934.17 & 73.0 & 931.72 \\
\hline 6.5 & 934.13 & 88.5 & 933.95 & -4.0 & 934.18 & 75.0 & 931.64 \\
\hline 7.0 & 934.05 & 90.0 & 933.98 & -1.2 & 934.17 & 77.0 & 931.61 \\
\hline 7.4 & 933.82 & 92.0 & 934.04 & 0.0 & 934.19 & 79.2 & 931.65 \\
\hline 10.0 & 933.34 & 94.0 & 934.08 & 2.0 & 934.17 & 79.4 & 932.02 \\
\hline 12.0 & 933.16 & 96.0 & 934.11 & 4.0 & 934.15 & 80.0 & 932.04 \\
\hline 13.0 & 933.13 & 100.0 & 934.07 & 5.0 & 934.16 & 81.5 & 931.97 \\
\hline 13.6 & 933.11 & & & 6.7 & 934.10 & 82.6 & 932.04 \\
\hline 14.3 & 932.88 & & & 7.0 & 934.06 & 83.3 & 932.40 \\
\hline 16.0 & 932.90 & & & 7.2 & 933.89 & 84.2 & 932.39 \\
\hline 18.0 & 933.04 & & & 8.0 & 933.75 & 84.9 & 932.49 \\
\hline 20.0 & 933.01 & & & 10.0 & 933.32 & 86.0 & 932.80 \\
\hline 21.0 & 932.75 & & & 11.0 & 933.23 & 87.1 & 933.07 \\
\hline 22.8 & 932.79 & & & 12.0 & 933.18 & 88.0 & 933.26 \\
\hline 23.8 & 932.19 & & & 13.6 & 933.10 & 88.6 & 933.96 \\
\hline 24.4 & 932.05 & & & 14.4 & 932.86 & 90.0 & 933.99 \\
\hline 25.1 & 931.76 & & & 16.0 & 932.90 & 92.0 & 934.04 \\
\hline 28.0 & 931.73 & & & 17.0 & 933.00 & 94.0 & 934.09 \\
\hline 31.0 & 931.75 & & & 19.0 & 933.05 & 96.0 & 934.10 \\
\hline 34.0 & 931.73 & & & 20.6 & 932.94 & 98.0 & 934.09 \\
\hline 37.0 & 931.70 & & & 21.2 & 932.75 & 100.0 & 934.06 \\
\hline 40.0 & 931.70 & & & 22.7 & 932.75 & 102.0 & 934.05 \\
\hline 43.0 & 931.61 & & & 23.8 & 932.21 & 104.0 & 934.00 \\
\hline 46.0 & 931.56 & & & 24.2 & 932.07 & 106.0 & 933.95 \\
\hline 49.0 & 931.53 & & & 25.0 & 932.01 & 108.0 & 933.89 \\
\hline 52.0 & 931.52 & & & 25.1 & 931.87 & 110.0 & 933.87 \\
\hline 55.0 & 931.56 & & & 26.5 & 931.76 & 112.0 & 933.86 \\
\hline 58.0 & 931.58 & & & 29.0 & 931.64 & 113.3 & 933.87 \\
\hline 61.0 & 931.52 & & & 32.0 & 931.62 & & \\
\hline 64.0 & 931.56 & & & 35.0 & 931.59 & & \\
\hline 67.0 & 931.68 & & & 38.0 & 931.57 & & \\
\hline 70.0 & 931.80 & & & 41.0 & 931.57 & & \\
\hline 71.5 & 932.02 & & & 44.0 & 931.55 & & \\
\hline 72.0 & 932.15 & & & 47.0 & 931.56 & & \\
\hline 74.0 & 932.14 & & & 50.0 & 931.55 & & \\
\hline 76.0 & 932.11 & & & 53.0 & 931.54 & & \\
\hline 77.8 & 932.09 & & & 56.0 & 931.54 & & \\
\hline 79.0 & 931.92 & & & 59.0 & 931.58 & & \\
\hline 79.6 & 932.01 & & & 61.0 & 931.62 & & \\
\hline 81.1 & 931.66 & & & 63.0 & 931.69 & & \\
\hline 83.8 & 932.05 & & & 64.2 & 931.75 & & \\
\hline 84.3 & 932.60 & & & 66.0 & 931.84 & & \\
\hline
\end{tabular}


Description of Cross Section PR200A

Location: Township 5 South/Range 51 East--section 8

U.S. Geological Survey quadrangle $(1: 24,000)$ : Broadus

Landowners--left bank: Doug and Lucille Randall

right bank: Doug and Lucille Randa11

Access: left bank

Permission from: Doug and Lucille Randall (T5S/R51E--8)

or Craig Randall (T5S/R51E--17)

Reference pins

\begin{tabular}{|c|c|c|}
\hline $\begin{array}{l}\text { Station } \\
\text { (meters) }\end{array}$ & $\begin{array}{c}\text { Elevation } \\
\text { (meters } \\
\text { above } \\
\text { sea level) }\end{array}$ & Comments \\
\hline & & $\begin{array}{l}\text { Pin }-6.4 \text { was closest to leveling } \\
\text { instrument. }\end{array}$ \\
\hline-6.4 & 927.802 & $\begin{array}{l}0.05 \text { meter above } 1987 \text { ground level, } \\
\text { on fenceline. }\end{array}$ \\
\hline 150.0 & 926.445 & 0.07 meter above 1987 ground level. \\
\hline 150.9 & 926.520 & 0.17 meter above 1987 ground level. \\
\hline 250.0 & 926.913 & 0.10 meter above 1987 ground level. \\
\hline 275.0 & 926.849 & 0.10 meter above 1987 ground level. \\
\hline
\end{tabular}



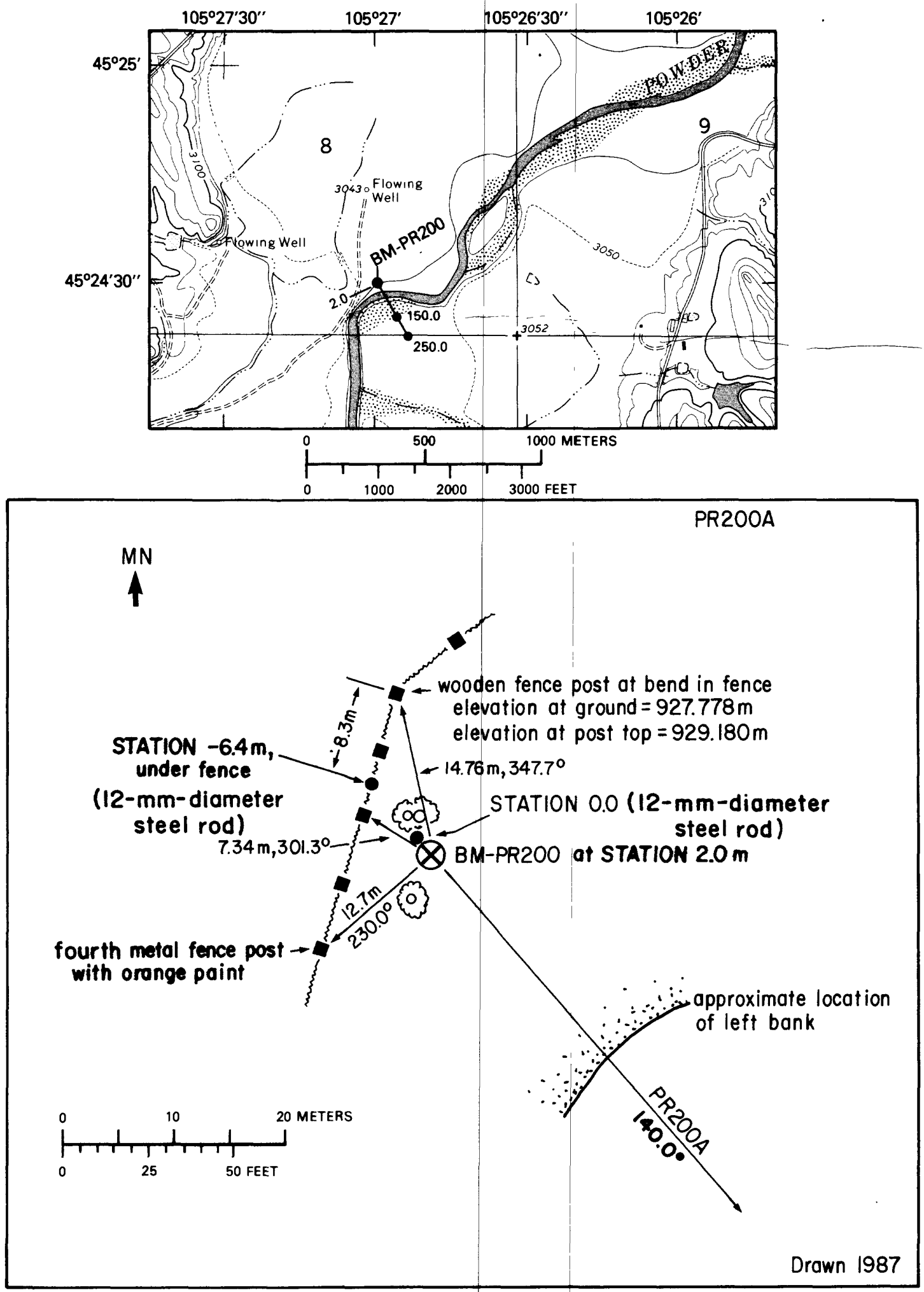

Figure 88.--Upper: Location of cross section PR200A, bench mark BM-PR200, and left and right bank reference pins (tables 2 and 3 ) in the Broadus quadrangle. Lower: Location of bench mark on the left bank. MN is magnetic north. 

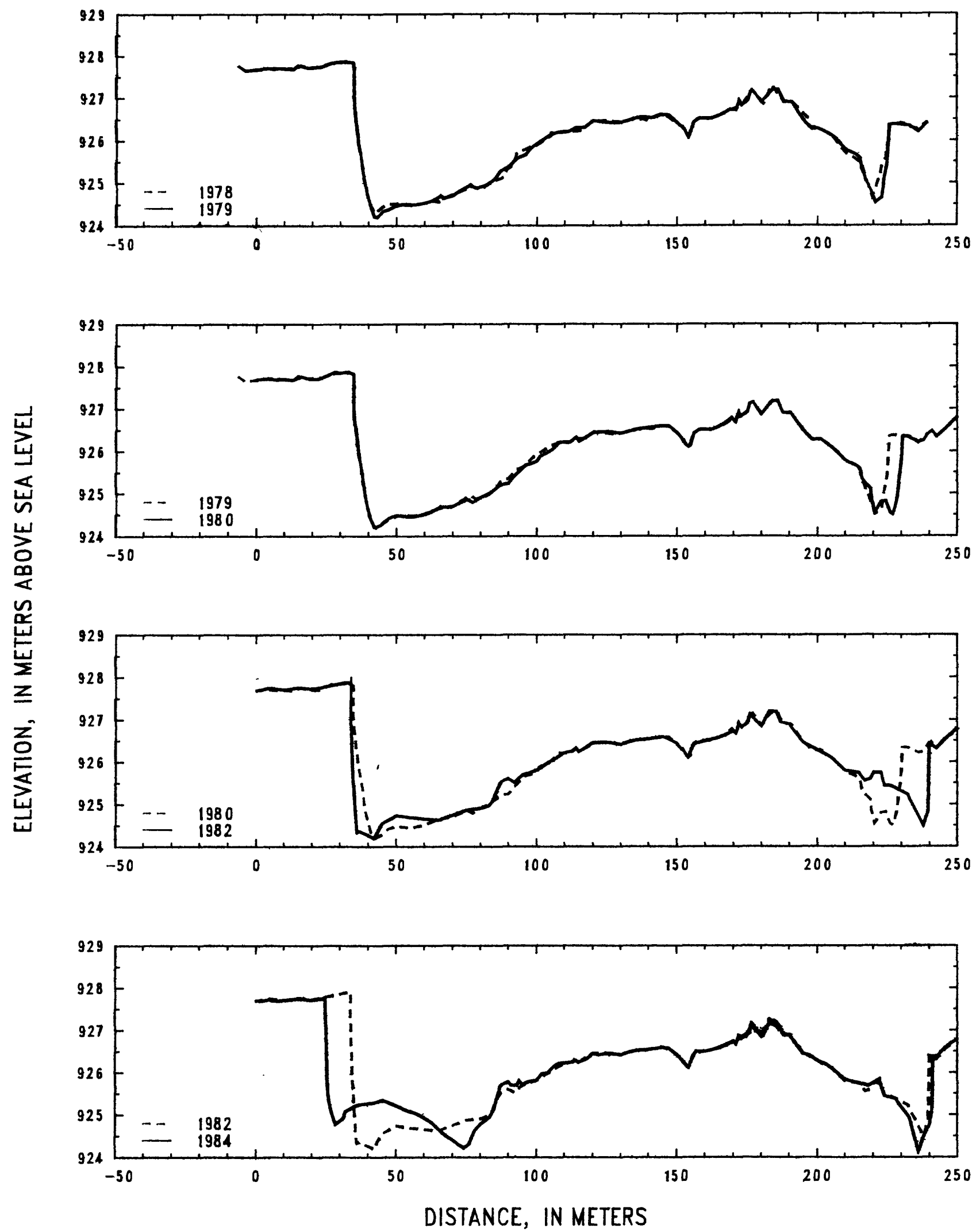

Figure 89.--Profiles of cross section PR200A from 1978 to 1984. 

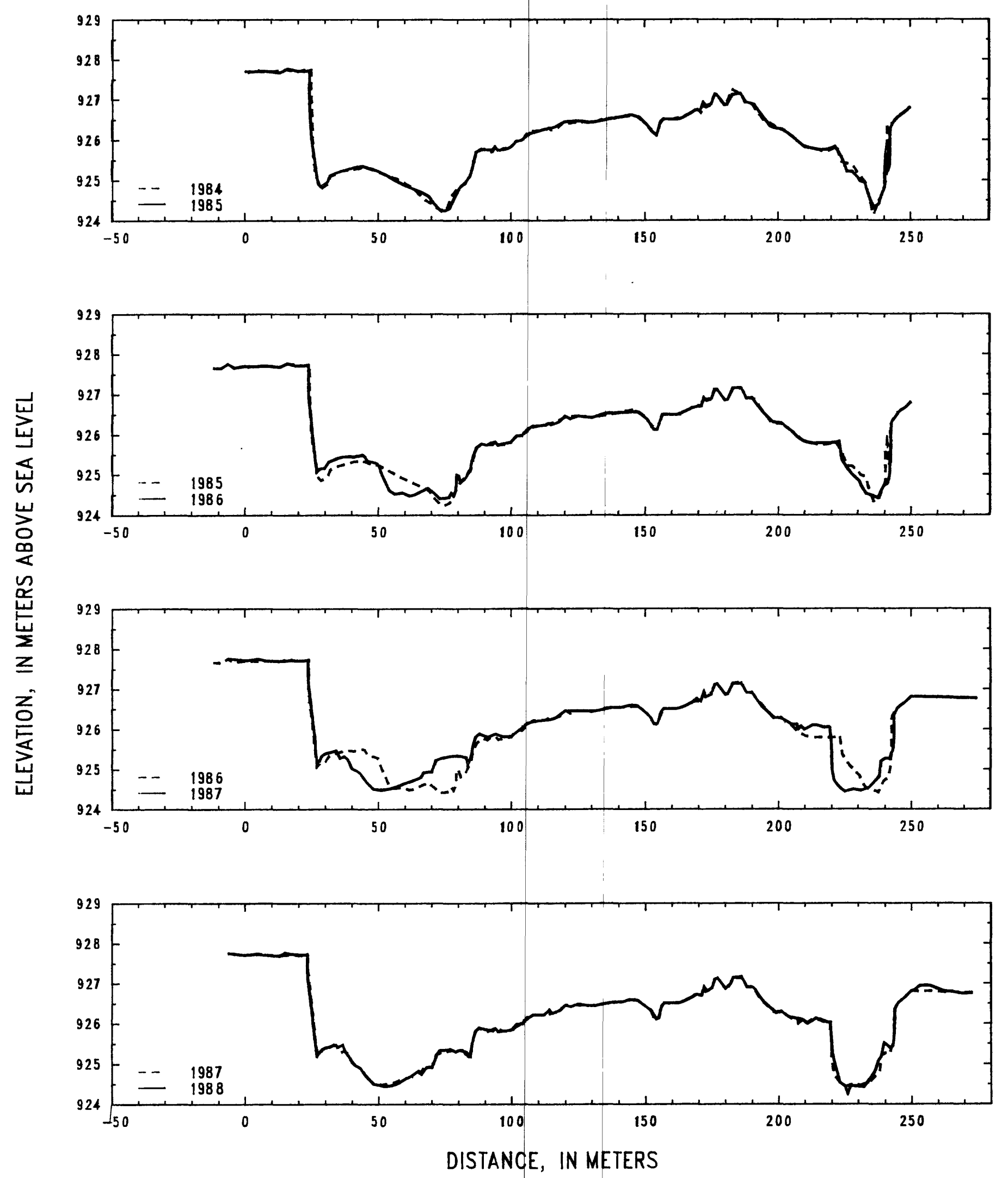

Figure 90.--Profiles of cross section PR200A from 1984 to 1988. 
Table 28.--Listing of horizontal stations and elevations for cross section PR200A [Sta., station, distance in meters from a reference point on the left bank; Elev., elevation, in meters above sea leve11.

\begin{tabular}{|c|c|c|c|c|c|c|c|c|c|}
\hline \multicolumn{2}{|c|}{1978} & \multicolumn{2}{|c|}{1978} & \multicolumn{2}{|c|}{1978} & \multicolumn{2}{|c|}{1979} & \multicolumn{2}{|c|}{1979} \\
\hline Sta. & Elev. & Sta. & Elev. & Sta. & Elev. & Sta. & Elev. & Sta. & Elev. \\
\hline 0.0 & 927.68 & 89.5 & 925.31 & 239.4 & 926.41 & -6.5 & 927.77 & 101.0 & 925.97 \\
\hline 4.0 & 927.72 & 91.5 & 925.41 & & & -4.0 & 927.65 & 105.0 & 926.14 \\
\hline 6.0 & 927.68 & 93.0 & 925.71 & & & 0.0 & 927.69 & 108.0 & 926.21 \\
\hline 8.0 & 927.71 & 100.0 & 925.89 & . & & 4.0 & 927.72 & 111.0 & 926.20 \\
\hline 10.0 & 927.70 & 104.9 & 926.10 & & & 6.0 & 927.71 & 114.0 & 926.30 \\
\hline 13.0 & 927.70 & 105.0 & 926.18 & & & 7.0 & 927.72 & 117.0 & 926.30 \\
\hline 14.0 & 927.71 & 110.0 & 926.19 & & & 10.0 & 927.70 & 120.0 & 926.46 \\
\hline 16.0 & 927.78 & 115.0 & 926.22 & & & 13.0 & 927.68 & 123.0 & 926.44 \\
\hline 18.0 & 927.73 & 120.0 & 926.45 & & & 15.0 & 927.78 & 126.0 & 926.41 \\
\hline 20.0 & 927.74 & 125.0 & 926.46 & & & 18.0 & 927.72 & 129.0 & 926.41 \\
\hline 22.0 & 927.73 & 130.0 & 926.41 & & & 21.0 & 927.73 & 132.0 & 926.45 \\
\hline 24.0 & 927.77 & 135.0 & 926.50 & & & 24.0 & 927.77 & 135.0 & 926.52 \\
\hline 26.0 & 927.82 & 140.0 & 926.47 & & & 27.0 & 927.84 & 138.0 & 926.55 \\
\hline 28.0 & 927.84 & 145.0 & 926.61 & & & 30.0 & 927.86 & 141.0 & 926.52 \\
\hline 30.0 & 927.86 & 150.0 & 926.39 & & & 33.0 & 927.86 & 144.0 & 926.61 \\
\hline 32.0 & 927.87 & 150.9 & 926.34 & & & 34.8 & 927.84 & 147.0 & 926.59 \\
\hline 34.0 & 927.86 & 152.7 & 926.24 & & & 34.9 & 927.80 & 150.0 & 926.42 \\
\hline 34.9 & 927.82 & 154.2 & 926.08 & & & 35.0 & 926.89 & 150.9 & 926.36 \\
\hline 35.0 & 926.82 & 156.0 & 926.43 & & & 36.5 & 925.90 & 152.0 & 926.30 \\
\hline 36.0 & 926.29 & 158.0 & 926.52 & & & 37.2 & 925.73 & 154.0 & 926.06 \\
\hline 36.2 & 926.14 & 163.0 & 926.50 & & & 38.2 & 925.23 & 156.0 & 926.42 \\
\hline 36.4 & 925.96 & 168.0 & 926.69 & & & 40.0 & 924.61 & 158.0 & 926.51 \\
\hline 36.7 & 925.76 & 173.0 & 926.80 & & & 42.0 & 924.21 & 162.0 & 926.50 \\
\hline 37.2 & 925.70 & 176.5 & 927.18 & & & 43.0 & 924.19 & 166.0 & 926.60 \\
\hline 37.7 & 925.46 & 180.7 & 926.85 & & & 45.0 & 924.34 & 169.0 & 926.72 \\
\hline 38.1 & 925.39 & 183.7 & 927.29 & & & 47.0 & 924.38 & 171.0 & 926.70 \\
\hline 38.5 & 925.15 & 188.0 & 926.89 & & & 50.0 & 924.47 & 172.0 & 926.93 \\
\hline 38.9 & 924.96 & 190.5 & 926.92 & & & 53.0 & 924.51 & 173.0 & 926.81 \\
\hline 40.0 & 924.59 & 196.5 & 926.51 & & & 56.0 & 924.48 & 175.0 & 926.92 \\
\hline 42.0 & 924.29 & 197.0 & 926.30 & & & 59.0 & 924.52 & 176.0 & 927.12 \\
\hline 45.0 & 924.44 & 200.0 & 926.28 & & & 62.0 & 924.57 & 177.0 & 927.15 \\
\hline 47.0 & 924.50 & 205.0 & 926.10 & & & 65.0 & 924.67 & 180.0 & 926.88 \\
\hline 50.0 & 924.51 & 210.0 & 925.70 & & & 65.7 & 924.71 & 184.0 & 927.19 \\
\hline 53.0 & 924.47 & 215.9 & 925.45 & & & 66.7 & 924.64 & 186.0 & 927.19 \\
\hline 56.0 & 924.48 & 216.7 & 925.21 & & & 70.0 & 924.70 & 188.0 & 926.89 \\
\hline 59.0 & 924.51 & 219.6 & 924.65 & & & 73.0 & 924.85 & 191.0 & 926.88 \\
\hline 62.0 & 924.57 & 222.5 & 925.21 & & & 76.4 & 924.96 & 194.0 & 926.56 \\
\hline 65.0 & 924.55 & 223.2 & 925.42 & & & 78.0 & 924.88 & 196.0 & 926.38 \\
\hline 68.0 & 924.67 & 224.5 & 925.57 & & & 82.0 & 924.95 & 198.0 & 926.25 \\
\hline 71.0 & 924.74 & 225.1 & 925.91 & & & 85.0 & 925.09 & 200.0 & 926.27 \\
\hline 74.0 & 924.84 & 225.5 & 925.92 & & & 87.0 & 925.28 & 203.0 & 926.17 \\
\hline 76.0 & 924.93 & 225.9 & 926.35 & & & 90.0 & 925.37 & 206.0 & 926.06 \\
\hline 80.0 & 924.84 & 227.0 & 926.36 & & & 93.0 & 925.58 & 210.0 & 925.77 \\
\hline 83.0 & 924.99 & 230.0 & 926.40 & & & 96.0 & 925.66 & 214.0 & 925.64 \\
\hline 88.0 & 925.12 & 235.0 & 926.26 & & & 98.0 & 925.83 & 215.3 & 925.56 \\
\hline
\end{tabular}


Table 28.--Listing of horizontal stations and elevations for cross section PR200A--Continued.

\begin{tabular}{|c|c|c|c|c|c|c|c|c|c|}
\hline \multicolumn{2}{|c|}{1979} & \multicolumn{2}{|c|}{1980} & \multicolumn{2}{|c|}{1980} & \multicolumn{2}{|c|}{1980} & \multicolumn{2}{|c|}{1982} \\
\hline Sta. & Elev. & Sta. & Elev. & Sta. & Elev. & Sta. & Elev. & Sta. & Elev. \\
\hline 216.0 & 925.28 & 0.0 & 927.70 & 125.0 & 926.47 & 230.2 & 925.82 & 0.0 & 927.70 \\
\hline 217.0 & 925.14 & 4.0 & 927.72 & 130.0 & 926.42 & 230.4 & 926.34 & 5.0 & 927.76 \\
\hline 217.2 & 925.04 & 7.0 & 927.69 & 135.0 & 926.51 & 232.0 & 926.34 & 10.0 & 927.71 \\
\hline 218.0 & 924.97 & 10.0 & 927.70 & 140.0 & 926.56 & 236.0 & 926.21 & 15.0 & 927.77 \\
\hline 220.0 & 924.65 & 13.0 & 927.68 & 144.0 & 926.59 & 238.0 & 926.24 & 20.0 & 927.73 \\
\hline 220.7 & 924.52 & 16.0 & 927.76 & 147.0 & 926.59 & 239.4 & 926.39 & 25.0 & 927.80 \\
\hline 222.0 & 924.60 & 19.0 & 927.70 & 150.0 & 926.42 & 241.0 & 926.47 & 30.0 & 927.87 \\
\hline 223.0 & 924.64 & 22.0 & 927.70 & 150.9 & 926.36 & 242.6 & 926.33 & 32.0 & 927.89 \\
\hline 224.0 & 925.12 & 25.0 & 927.78 & 153.0 & 926.15 & 246.0 & 926.54 & 33.0 & 927.89 \\
\hline 225.0 & 925.40 & 28.0 & 927.88 & 154.0 & 926.10 & 250.0 & 926.79 & 33.8 & 927.84 \\
\hline 225.7 & 926.35 & 30.0 & 927.85 & 154.8 & 926.14 & & & 33.9 & 927.87 \\
\hline 227.0 & 926.37 & 33.0 & 927.88 & 156.0 & 926.43 & & & 34.0 & 927.01 \\
\hline 230.0 & 926.36 & 34.8 & 927.81 & 158.0 & 926.51 & & & 34.9 & 925.54 \\
\hline 233.0 & 926.33 & 34.9 & 926.91 & 162.0 & 926.50 & & & 35.7 & 924.67 \\
\hline 236.0 & 926.19 & 36.7 & 925.88 & 166.0 & 926.61 & & & 36.2 & 924.34 \\
\hline \multirow[t]{30}{*}{239.4} & 926.40 & 37.2 & 925.72 & 169.5 & 926.78 & & & 37.0 & 924.37 \\
\hline & & 38.0 & 925.40 & 170.8 & 926.68 & & & 42.0 & 924.20 \\
\hline & & 39.1 & 924.83 & 172.0 & 926.92 & & & 43.0 & 924.31 \\
\hline & & 41.0 & 924.35 & 172.5 & 926.80 & & & 45.0 & 924.56 \\
\hline & & 42.4 & 924.20 & 175.0 & 926.92 & & & 50.0 & 924.74 \\
\hline & & 45.0 & 924.29 & 175.7 & 927.11 & & & 55.0 & 924.69 \\
\hline & & 47.0 & 924.42 & 177.0 & 927.15 & & & 60.0 & 924.66 \\
\hline & & 50.0 & 924.49 & 180.0 & 926.86 & & & 65.0 & 924.63 \\
\hline & & 55.0 & 924.44 & 182.0 & 927.04 & & & 70.0 & 924.75 \\
\hline & & 60.0 & 924.51 & 184.0 & 927.18 & & & 75.0 & 924.88 \\
\hline & & 65.0 & 924.66 & 186.0 & 927.18 & & & 80.0 & 924.91 \\
\hline & & 70.0 & 924.70 & 187.7 & 926.90 & & & 83.0 & 924.98 \\
\hline & & 75.0 & 924.87 & 190.5 & 926.90 & & & 84.2 & 925.09 \\
\hline & & 77.0 & 924.79 & 195.0 & 926.47 & & & 87.0 & 925.51 \\
\hline & & 80.0 & 924.92 & 198.0 & 926.26 & & & 88.0 & 925.56 \\
\hline & & 83.0 & 925.00 & 201.0 & 926.28 & & & 90.0 & 925.61 \\
\hline & & 87.0 & 925.21 & 205.0 & 926.09 & & & 92.0 & 925.52 \\
\hline & & 88.0 & 925.22 & 210.0 & 925.77 & & & 95.0 & 925.70 \\
\hline & & 90.0 & 925.24 & 214.0 & 925.65 & & & 100.0 & 925.81 \\
\hline & & 92.0 & 925.42 & 215.3 & 925.58 & & & 105.0 & 926.03 \\
\hline & & 96.0 & 925.67 & 216.0 & 925.34 & & & 110.0 & 926.22 \\
\hline & & 98.0 & 925.71 & 217.0 & 925.23 & & & 113.0 & 926.26 \\
\hline & & 100.0 & 925.77 & 219.0 & 925.10 & & & 114.0 & 926.34 \\
\hline & & 101.0 & 925.88 & 219.4 & 924.87 & & & 115.0 & 926.24 \\
\hline & & 105.0 & 926.01 & 220.5 & 924.54 & & & 120.0 & 926.46 \\
\hline & & 108.0 & 926.21 & 222.7 & 924.80 & & & 125.0 & 926.47 \\
\hline & & 112.0 & 926.21 & 224.5 & 924.82 & & & 130.0 & 926.41 \\
\hline & & 114.0 & 926.33 & 226.0 & 924.54 & & & 135.0 & 926.52 \\
\hline & & 115.0 & 926.23 & 227.0 & 924.50 & & & 140.0 & 926.55 \\
\hline & & 120.0 & $926 .: 4$ & 228.4 & 924.82 & & & 145.0 & 926.61 \\
\hline
\end{tabular}


Table 28.--Listing of horizontal stations and elevations for cross section PR200A--Continued.

\begin{tabular}{|c|c|c|c|c|c|c|c|c|c|}
\hline \multicolumn{2}{|c|}{1982} & \multicolumn{2}{|c|}{1984} & \multicolumn{2}{|c|}{1984} & \multicolumn{2}{|c|}{1984} & \multicolumn{2}{|c|}{1985} \\
\hline Sta. & Elev. & Sta. & Elev. & Sta. & Elev. & Sta. & Elev. & Sta. & Elev. \\
\hline 150.0 & 926.43 & 0.0 & 927.70 & 108.0 & 926.22 & 220.0 & 925.74 & 1.0 & 927.68 \\
\hline 150.9 & 926.35 & 3.0 & 927.69 & 110.0 & 926.22 & 222.4 & 925.84 & 4.0 & 927.70 \\
\hline 152.0 & 926.31 & 5.0 & 927.73 & 113.0 & 926.24 & 222.6 & 925.69 & 7.0 & 927.71 \\
\hline 154.0 & 926.10 & 6.0 & 927.69 & 114.0 & 926.29 & 224.0 & 925.43 & 10.0 & 927.70 \\
\hline 156.0 & 926.43 & 10.0 & 927.70 & 115.0 & 926.23 & 225.0 & 925.41 & 13.0 & 927.67 \\
\hline 160.0 & 926.50 & 15.0 & 927.75 & 118.0 & 926.32 & 228.0 & 925.36 & 16.0 & 927.77 \\
\hline 165.0 & 926.58 & 20.0 & 927.71 & 120.0 & 926.45 & 230.0 & 925.17 & 20.0 & 927.71 \\
\hline 170.0 & 926.74 & 24.8 & 927.77 & 122.0 & 926.40 & 233.0 & 924.91 & 22.0 & 927.71 \\
\hline 171.0 & 926.67 & 25.8 & 925.45 & 125.0 & 926.45 & 234.0 & 924.68 & 24.2 & 927.73 \\
\hline 172.0 & 926.91 & 27.0 & 925.07 & 130.0 & 926.41 & 236.0 & 924.13 & 24.3 & 926.95 \\
\hline 173.0 & 926.82 & 28.5 & 924.77 & 135.0 & 926.51 & 238.0 & 924.48 & 25.0 & 926.05 \\
\hline 175.0 & 926.93 & 31.4 & 924.92 & 140.0 & 926.54 & 239.5 & 924.69 & 27.6 & 924.90 \\
\hline 176.0 & 927.13 & 32.0 & 925.08 & 142.0 & 926.56 & 240.0 & 924.74 & 28.5 & 924.84 \\
\hline 180.0 & 926.84 & 36.0 & 925.22 & 145.0 & 926.59 & 241.0 & 925.09 & 30.0 & 924.90 \\
\hline 183.0 & 927.21 & 39.0 & 925.26 & 147.0 & 926.57 & 241.3 & 926.38 & 31.0 & 924.95 \\
\hline 185.5 & 927.19 & 42.0 & 925.28 & 150.0 & 926.41 & 243.0 & 926.38 & 32.0 & 925.12 \\
\hline 187.0 & 926.94 & 45.0 & 925.34 & 150.9 & 926.36 & 245.0 & 926.54 & 35.0 & 925.20 \\
\hline 190.0 & 926.89 & 50.0 & 925.21 & 153.0 & 926.19 & 247.0 & 926.65 & 38.0 & 925.27 \\
\hline 195.0 & 926.45 & 54.0 & 925.08 & 154.3 & 926.10 & 250.0 & 926.79 & 41.0 & 925.31 \\
\hline 200.0 & 926.27 & 57.0 & 925.03 & 156.0 & 926.41 & & & 44.0 & 925.35 \\
\hline 205.0 & 926.10 & 60.0 & 924.92 & 157.0 & 926.50 & & & 47.0 & 925.27 \\
\hline 210.0 & 925.79 & 63.0 & 924.80 & 160.0 & 926.49 & & & 50.0 & 925.23 \\
\hline 215.0 & 925.74 & 65.5 & 924.64 & 165.0 & 926.58 & & & 53.0 & 925.14 \\
\hline 217.0 & 925.55 & 68.0 & 924.48 & 170.0 & 926.74 & & & 56.0 & 925.01 \\
\hline 219.0 & 925.60 & 70.0 & 924.41 & 171.0 & 926.66 & & & 59.0 & 924.90 \\
\hline 220.2 & 925.75 & 72.0 & 924.30 & 172.0 & 926.83 & & & 62.0 & 924.81 \\
\hline 223.0 & 925.74 & 74.0 & 924.21 & 173.0 & 926.79 & & & 64.3 & 924.74 \\
\hline 224.0 & 925.44 & 76.0 & 924.31 & 175.0 & 926.87 & & & 67.0 & 924.66 \\
\hline 226.0 & 925.44 & 78.0 & 924.64 & 176.5 & 927.16 & & & 69.0 & 924.59 \\
\hline 232.4 & 925.22 & 80.0 & 924.78 & 179.0 & 926.92 & & & 71.0 & 924.40 \\
\hline 238.0 & 924.49 & 83.0 & 924.92 & 180.4 & 926.86 & & & 73.0 & 924.27 \\
\hline 239.0 & 924.71 & 85.0 & 925.14 & 183.0 & 927.25 & & & 75.0 & 924.23 \\
\hline 239.5 & 924.82 & 86.0 & 925.42 & 185.0 & 927.16 & & & 77.0 & 924.30 \\
\hline 240.0 & 926.43 & 87.0 & 925.68 & 186.0 & 927.16 & & & 79.0 & 924.60 \\
\hline 242.5 & 926.31 & 88.0 & 925.72 & 188.0 & 926.88 & & & 81.0 & 924.83 \\
\hline 245.0 & 926.51 & 90.0 & 925.77 & 190.0 & 926.89 & & & 83.0 & 924.95 \\
\hline \multirow[t]{9}{*}{250.0} & 926.80 & 91.0 & 925.69 & 195.0 & 926.43 & & & 84.7 & 925.10 \\
\hline & & 93.0 & 925.69 & 197.0 & 926.40 & & & 87.0 & 925.69 \\
\hline & & 94.0 & 925.80 & 200.0 & 926.26 & & & 89.0 & 925.77 \\
\hline & & 95.0 & 925.70 & 203.0 & 926.19 & & & 91.0 & 925.74 \\
\hline & & 97.0 & 925.78 & 206.0 & 926.05 & & & 93.0 & 925.75 \\
\hline & & 100.0 & 925.81 & 209.0 & 925.86 & & & 94.0 & 925.83 \\
\hline & & 102.0 & 925.96 & 212.0 & 925.78 & & & 95.0 & 925.73 \\
\hline & & 104.0 & 925.98 & 215.0 & 925.74 & & & 98.0 & 925.77 \\
\hline & & 106.0 & 926.13 & 218.0 & 925.68 & & & 100.0 & 925.82 \\
\hline
\end{tabular}


Table 28.--Listing of horizontal stations and elevations for cross section PR200A--Continued.

\begin{tabular}{|c|c|c|c|c|c|c|c|c|c|}
\hline \multicolumn{2}{|c|}{1985} & \multicolumn{2}{|c|}{1985} & \multicolumn{2}{|c|}{1986} & \multicolumn{2}{|c|}{1986} & \multicolumn{2}{|c|}{1986} \\
\hline Sta. & Elev. & Sta. & Elev. & Sta. & Elev. & Sta. & Elev. & Sta. & Elev. \\
\hline 102.0 & 925.96 & 221.5 & 925.82 & -12.0 & 927.65 & 78.8 & 924.54 & 172.0 & 926.92 \\
\hline 104.0 & 925.98 & 224.0 & 925.55 & -9.0 & 927.65 & 79.2 & 924.69 & 172.5 & 926.86 \\
\hline 107.0 & 926.15 & 226.0 & 925.20 & -6.4 & 927.76 & 79.5 & 924.99 & 175.0 & 926.90 \\
\hline 110.0 & 926.21 & 228.0 & 925.20 & -4.0 & 927.66 & 80.2 & 924.98 & 176.0 & 927.12 \\
\hline 113.0 & 926.27 & 230.0 & 925.06 & 0.0 & 927.71 & 81.0 & 924.81 & 177.0 & 927.13 \\
\hline 116.0 & 926.30 & 231.0 & 924.96 & 3.0 & 927.69 & 83.0 & 924.93 & 178.0 & 927.03 \\
\hline 120.0 & 926.44 & 232.8 & 924.92 & 7.0 & 927.71 & 84.0 & 925.08 & 180.0 & 926.85 \\
\hline 125.0 & 926.47 & 234.0 & 924.65 & 10.0 & 927.70 & 84.7 & 925.14 & 181.0 & 926.86 \\
\hline 130.0 & 926.42 & 236.0 & 924.31 & 13.0 & 927.68 & 85.2 & 925.34 & 183.0 & 927.16 \\
\hline 135.0 & 926.49 & 238.0 & 924.40 & 16.0 & 927.77 & 86.0 & 925.49 & 184.0 & 927.16 \\
\hline 140.0 & 926.55 & 239.2 & 924.67 & 19.0 & 927.71 & 87.0 & 925.66 & 186.0 & 927.16 \\
\hline 145.0 & 926.61 & 240.3 & 924.80 & 22.0 & 927.71 & 89.0 & 925.78 & 188.0 & 926.89 \\
\hline 148.0 & 926.54 & 240.4 & 925.48 & 23.8 & 927.73 & 91.0 & 925.73 & 190.0 & 926.92 \\
\hline 150.0 & 926.42 & 241.0 & 925.80 & 23.9 & 926.95 & 92.0 & 925.79 & 192.0 & 926.72 \\
\hline 150.9 & 926.36 & 241.4 & 925.52 & 27.0 & 925.07 & 94.0 & 925.82 & 195.0 & 926.49 \\
\hline 153.0 & 926.17 & 241.4 & 925.22 & 28.0 & 925.15 & 95.0 & 925.74 & 198.0 & 926.30 \\
\hline 154.5 & 926.10 & 242.2 & 925.38 & 29.8 & 925.17 & 97.0 & 925.78 & 201.0 & 926.28 \\
\hline 156.0 & 926.44 & 242.3 & 926.24 & 31.4 & 925.32 & 100.0 & 925.81 & 203.0 & 926.19 \\
\hline 157.0 & 926.52 & 243.0 & 926.35 & 34.0 & 925.39 & 102.0 & 925.97 & 205.0 & 926.09 \\
\hline 160.0 & 926.49 & 245.0 & 926.54 & 36.0 & 925.44 & 104.0 & 925.98 & 208.0 & 925.90 \\
\hline 163.0 & 926.51 & 248.0 & 926.67 & 39.0 & 925.46 & 106.0 & 926.11 & 210.0 & 925.82 \\
\hline 166.0 & 926.60 & 250.0 & 926.80 & 40.6 & 925.44 & 108.0 & 926.22 & 213.0 & 925.78 \\
\hline 169.0 & 926.73 & & & 42.0 & 925.47 & 110.0 & 926.22 & 216.0 & 925.78 \\
\hline 170.7 & 926.71 & & & 43.9 & 925.50 & 113.0 & 926.24 & 219.0 & 925.78 \\
\hline 172.0 & 926.92 & & & 44.7 & 925.48 & 114.0 & 926.29 & 222.0 & 925.80 \\
\hline 173.0 & 926.83 & & & 46.0 & 925.34 & 115.0 & 926.24 & 223.0 & 925.83 \\
\hline 175.0 & 926.89 & & & 48.0 & 925.31 & 118.0 & 926.33 & 224.0 & 925.36 \\
\hline 176.0 & 927.08 & & & 49.5 & 925.28 & 120.0 & 926.46 & 226.0 & 925.15 \\
\hline 177.0 & 927.13 & & & 50.5 & 925.19 & 122.0 & 926.39 & 228.0 & 924.98 \\
\hline 180.0 & 926.85 & & & 50.9 & 925.08 & 125.0 & 926.47 & 230.5 & 924.83 \\
\hline 181.0 & 926.86 & & & 52.0 & 924.90 & 130.0 & 926.42 & 231.2 & 924.70 \\
\hline 183.0 & 927.13 & & & 53.2 & 924.74 & 135.0 & 926.52 & 233.0 & 924.51 \\
\hline 186.0 & 927.15 & & & 54.0 & 924.61 & 140.0 & 926.54 & 235.0 & 924.49 \\
\hline 188.0 & 926.92 & & & 56.0 & 924.52 & 144.0 & 926.56 & 237.4 & 924.41 \\
\hline 191.0 & 926.86 & & & 57.0 & 924.53 & 147.0 & 926.57 & 239.5 & 924.70 \\
\hline 194.0 & 926.58 & & & 59.0 & 924.56 & 150.0 & 926.41 & 241.0 & 924.77 \\
\hline 197.0 & 926.32 & & & 61.5 & 924.47 & 150.9 & 926.37 & 242.0 & 924.98 \\
\hline 199.0 & 926.27 & & & 63.0 & 924.49 & 153.3 & 926.12 & 242.1 & 925.24 \\
\hline 202.0 & 926.24 & & & 66.0 & 924.59 & 154.6 & 926.11 & 242.5 & 925.38 \\
\hline 205.0 & 926.09 & & & 68.5 & 924.66 & 156.5 & 926.50 & 242.7 & 926.30 \\
\hline 207.0 & 925.98 & & & 71.0 & 924.53 & 159.0 & 926.50 & 244.0 & 926.43 \\
\hline 210.0 & 925.81 & & & 73.0 & 924.41 & 162.0 & 926.49 & 246.0 & 926.60 \\
\hline 213.0 & 925.77 & & & 76.5 & 924.44 & 165.0 & 926.58 & 248.0 & 926.67 \\
\hline 216.0 & 925.73 & & & 77.5 & 924.55 & 168.0 & 926.68 & 250.0 & 926.80 \\
\hline 219.0 & 925.76 & & & 78.3 & 924.47 & 171.0 & 926.71 & & \\
\hline
\end{tabular}


Table 28.--Listing of horizontal stations and elevations for cross section PR200A--Continued.

\begin{tabular}{|c|c|c|c|c|c|c|c|c|c|}
\hline \multicolumn{2}{|c|}{1987} & \multicolumn{2}{|c|}{1987} & \multicolumn{2}{|c|}{1987} & \multicolumn{2}{|c|}{1988} & \multicolumn{2}{|c|}{1988} \\
\hline Sta. & Elev. & Sta. & Elev. & Sta. & Elev. & Sta. & Elev. & Sta. & Elev. \\
\hline-6.4 & 927.76 & 86.6 & 925.82 & 188.0 & 926.90 & -6.4 & 927.76 & 83.0 & 925.23 \\
\hline 0.0 & 927.71 & 88.0 & 925.89 & 190.0 & 926.90 & 0.0 & 927.70 & 84.6 & 925.18 \\
\hline 5.0 & 927.75 & 91.0 & 925.80 & 192.0 & 926.71 & 5.0 & 927.74 & 85.1 & 925.46 \\
\hline 8.0 & 927.69 & 94.0 & 925.88 & 194.0 & 926.53 & 7.0 & 927.71 & 85.9 & 925.69 \\
\hline 11.0 & 927.69 & 97.0 & 925.81 & 196.0 & 926.41 & 10.0 & 927.71 & 86.6 & 925.81 \\
\hline 14.0 & 927.70 & 100.0 & 925.83 & 198.0 & 926.28 & 13.0 & 927.69 & 88.0 & 925.90 \\
\hline 17.0 & 927.72 & 103.0 & 925.99 & 200.0 & 926.28 & 15.0 & 927.77 & 90.0 & 925.85 \\
\hline 20.0 & 927.70 & 106.0 & 926.15 & 202.5 & 926.22 & 17.0 & 927.74 & 93.0 & 925.81 \\
\hline 22.0 & 927.71 & 109.0 & 926.21 & 205.0 & 926.11 & 20.0 & 927.72 & 94.0 & 925.88 \\
\hline 23.6 & 927.72 & 112.0 & 926.22 & 207.0 & 926.12 & 22.0 & 927.71 & 95.0 & 925.79 \\
\hline 23.6 & 927.31 & 115.0 & 926.25 & 207.6 & 926.00 & 23.3 & 927.73 & 98.0 & 925.82 \\
\hline 25.7 & 926.04 & 117.0 & 926.31 & 208.6 & 926.07 & 23.3 & 927.23 & 100.0 & 925.84 \\
\hline 26.2 & 925.79 & 120.0 & 926.46 & 209.8 & 925.99 & 26.5 & 925.45 & 102.0 & 925.98 \\
\hline 26.5 & 925.37 & 123.0 & 926.46 & 211.0 & 926.03 & 27.3 & 925.23 & 104.0 & 925.99 \\
\hline 27.0 & 925.19 & 126.0 & 926.45 & 213.0 & 926.11 & 29.0 & 925.37 & 106.0 & 926.13 \\
\hline 27.8 & 925.21 & 129.0 & 926.44 & 215.0 & 926.08 & 30.0 & 925.40 & 108.0 & 926.22 \\
\hline 28.7 & 925.34 & 132.0 & 926.45 & 217.0 & 926.04 & 32.0 & 925.43 & 110.0 & 926.22 \\
\hline 30.0 & 925.40 & 135.0 & 926.50 & 219.0 & 926.05 & 33.3 & 925.48 & 112.0 & 926.22 \\
\hline 32.0 & 925.43 & 138.0 & 926.55 & 219.4 & 926.03 & 35.0 & 925.42 & 114.0 & 926.34 \\
\hline 34.0 & 925.47 & 141.0 & 926.54 & 220.0 & 925.56 & 36.5 & 925.47 & 115.0 & 926.25 \\
\hline 35.5 & 925.27 & 144.0 & 926.59 & 220.1 & 925.07 & 37.5 & 925.29 & 118.0 & 926.33 \\
\hline 37.0 & 925.32 & 147.0 & 926.58 & 221.0 & 924.71 & 39.0 & 925.15 & 120.0 & 926.46 \\
\hline 38.1 & 925.26 & 150.0 & 926.42 & 223.0 & 924.53 & 41.0 & 924.96 & 122.0 & 926.42 \\
\hline 40.0 & 925.02 & 150.9 & 926.36 & 225.0 & 924.43 & 43.0 & 924.90 & 125.0 & 926.49 \\
\hline 42.9 & 924.91 & 152.0 & 926.30 & 228.0 & 924.50 & 45.0 & 924.72 & 128.0 & 926.44 \\
\hline 45.0 & 924.72 & 153.2 & 926.13 & 231.0 & 924.46 & 46.7 & 924.61 & 131.0 & 926.43 \\
\hline 48.0 & 924.51 & 154.7 & 926.13 & 234.0 & 924.54 & 48.0 & 924.50 & 134.0 & 926.49 \\
\hline 51.0 & 924.48 & 156.0 & 926.44 & 236.0 & 924.68 & 50.0 & 924.47 & 137.0 & 926.52 \\
\hline 54.0 & 924.51 & 157.0 & 926.51 & 238.0 & 924.83 & 52.0 & 924.44 & 140.0 & 926.55 \\
\hline 57.0 & 924.57 & 159.0 & 926.50 & 238.5 & 925.19 & 54.0 & 924.45 & 141.0 & 926.54 \\
\hline 60.0 & 924.65 & 162.0 & 926.50 & 240.0 & 925.27 & 57.0 & 924.51 & 144.0 & 926.60 \\
\hline 63.0 & 924.72 & 165.0 & 926.58 & 241.0 & 925.27 & 59.7 & 924.61 & 147.0 & 926.58 \\
\hline 66.0 & 924.81 & 167.0 & 926.65 & 241.7 & 925.25 & 62.0 & 924.69 & 150.0 & 926.42 \\
\hline 67.0 & 924.92 & 169.3 & 926.76 & 242.4 & 925.40 & 64.0 & 924.78 & 150.9 & 926.37 \\
\hline 69.0 & 924.93 & 170.8 & 926.69 & 243.0 & 925.47 & 65.0 & 924.83 & 152.0 & 926.32 \\
\hline 70.0 & 924.93 & 172.0 & 926.93 & 243.6 & 925.74 & 66.0 & 924.74 & 154.0 & 926.11 \\
\hline 71.5 & 925.23 & 172.6 & 926.79 & 243.6 & 926.35 & 68.0 & 924.92 & 155.0 & 926.13 \\
\hline 74.0 & 925.29 & 174.7 & 926.86 & 245.0 & 926.54 & 70.0 & 924.91 & 156.0 & 926.43 \\
\hline 77.0 & 925.33 & 176.0 & 927.11 & 247.0 & 926.63 & 72.0 & 925.26 & 157.0 & 926.52 \\
\hline 79.0 & 925.34 & 177.0 & 927.13 & 250.0 & 926.81 & 73.0 & 925.33 & 160.0 & 926.50 \\
\hline 81.0 & 925.33 & 180.0 & 926.87 & 275.0 & 926.75 & 75.0 & 925.35 & 163.0 & 926.52 \\
\hline 83.1 & 925.29 & 181.0 & 926.88 & & & 77.0 & 925.36 & 166.0 & 926.61 \\
\hline 83.6 & 925.14 & 183.0 & 927.14 & & & 79.0 & 925.29 & 169.0 & 926.72 \\
\hline 84.8 & 925.32 & 185.0 & 927.13 & & & 80.0 & 925.34 & 171.0 & 926.70 \\
\hline 85.6 & 925.61 & 186.0 & 927.15 & & & 81.5 & 925.35 & 172.0 & 926.92 \\
\hline
\end{tabular}


Table 28.--Listing of horizontal stations and elevations for cross section PR200A--Continued.

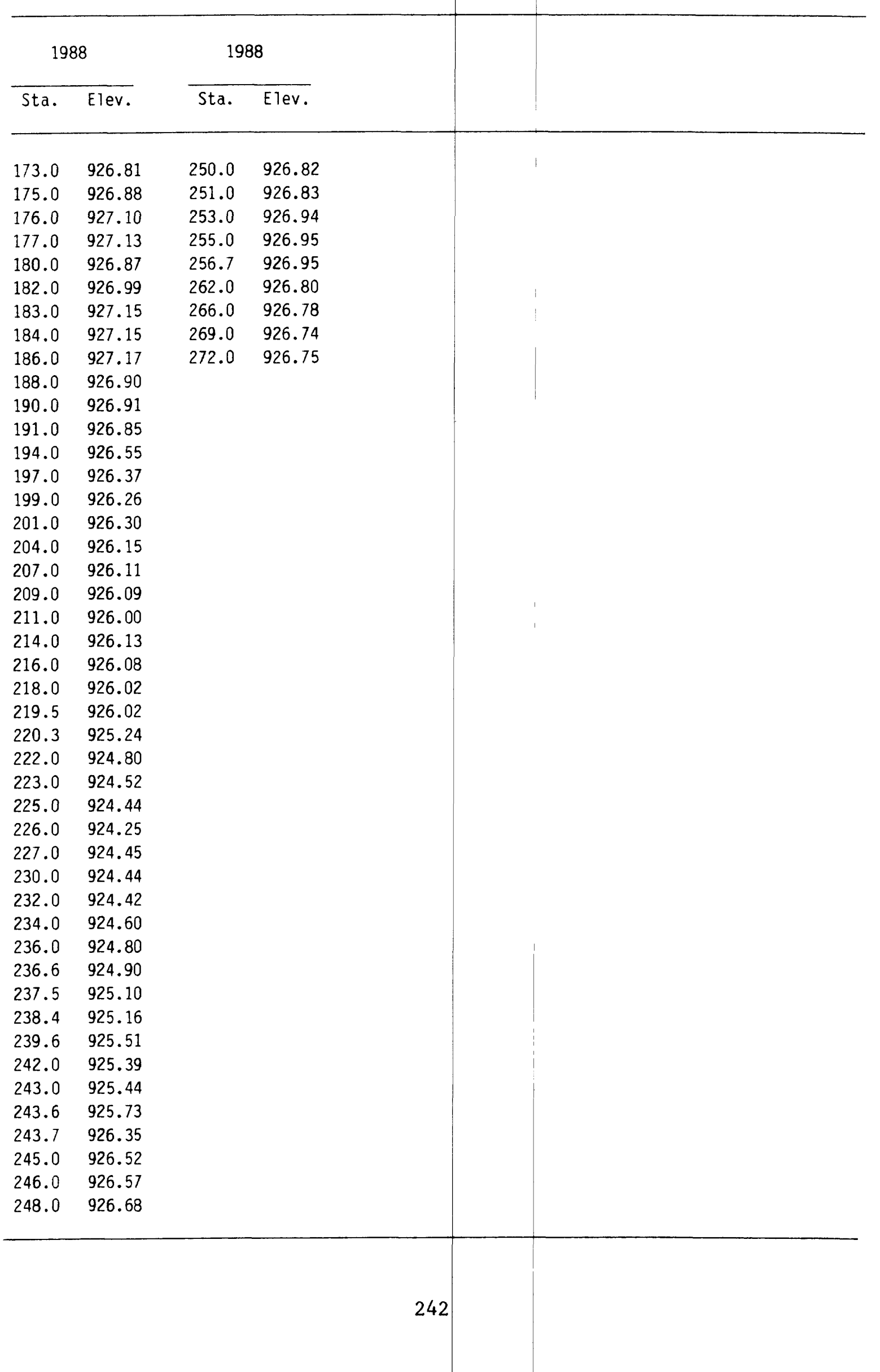




\section{Description of Cross Section PR206}

Location: Township 5 South/Range 51 East--section 2

U.S. Geological Survey quadrangle $(1: 24,000)$ : Broadus

Landowners--left bank: Ron and Twila Talcott

right bank: Charles Russell

Access: right bank

Permission from: Charles Russell (T5S/R51E--10)

Reference pins

\begin{tabular}{lcc}
\hline $\begin{array}{l}\text { Station } \\
\text { (meters) }\end{array}$ & $\begin{array}{c}\text { Elevation } \\
\text { (meters } \\
\text { above } \\
\text { sea leve1) }\end{array}$ & Comments \\
\hline-30.0 & 921.075 & $\begin{array}{c}\text { Pins } 101.9 \text { and } 139.6 \text { were closest to } \\
\text { leveling instrument. } \\
\pm 0.003 \text { meter, } 0.10 \text { meter above } 1987 \\
\text { ground level. } \\
\text { Upstream bolt in upstream circular, } \\
\text { concrete footing. } \\
\text { fence line, } 0.11 \text { meter above } 1986 \\
\text { ground level. }\end{array}$ \\
\hline
\end{tabular}




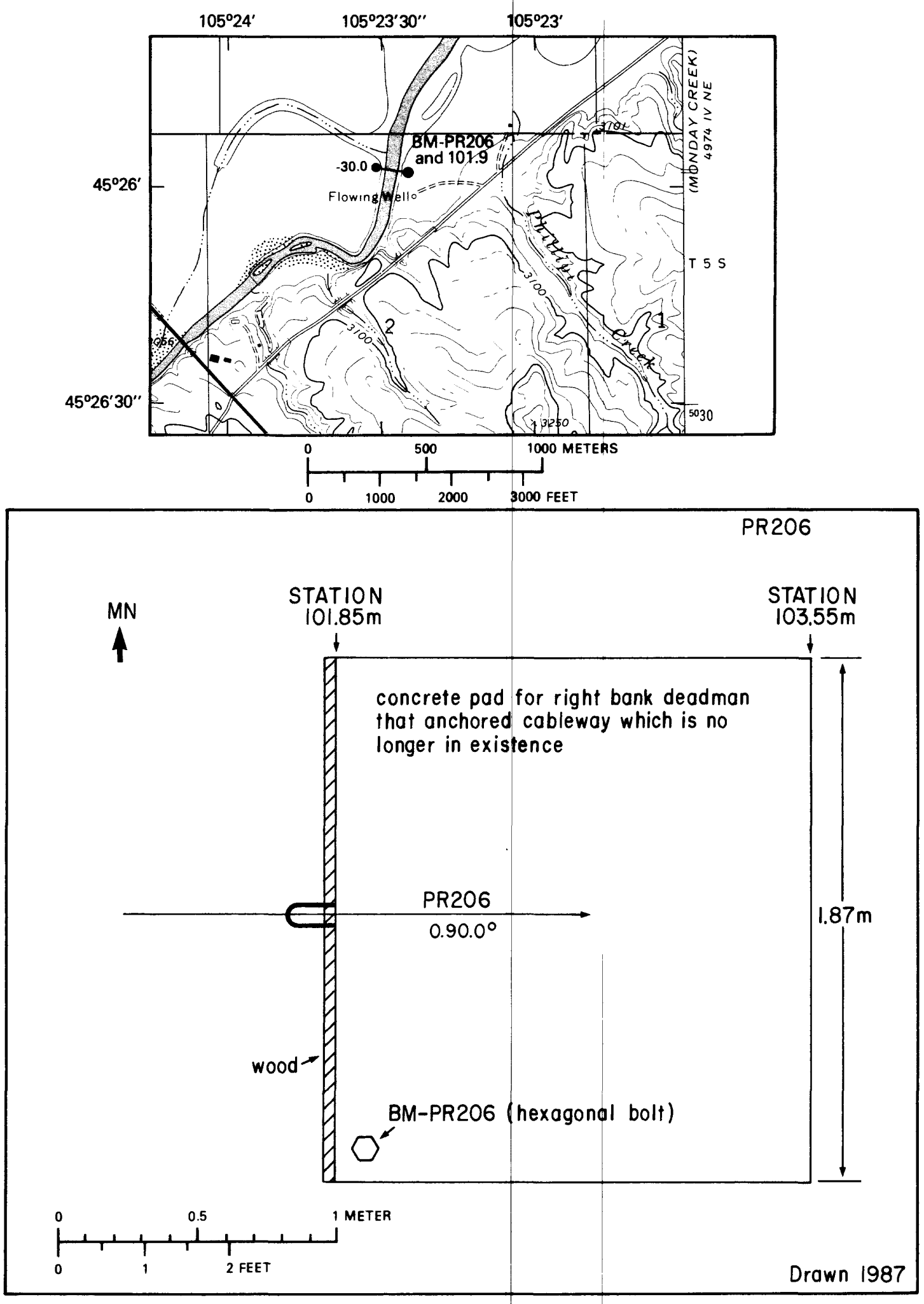

Figure 91.--Upper: Location of cross section PR206, bench mark BM-PR206, and left and right bank reference pins (tables 2 and 3 ) in the Broadus quaddrangle. Lower: Location of bench mark on the left bank. MN is magnetic north. 

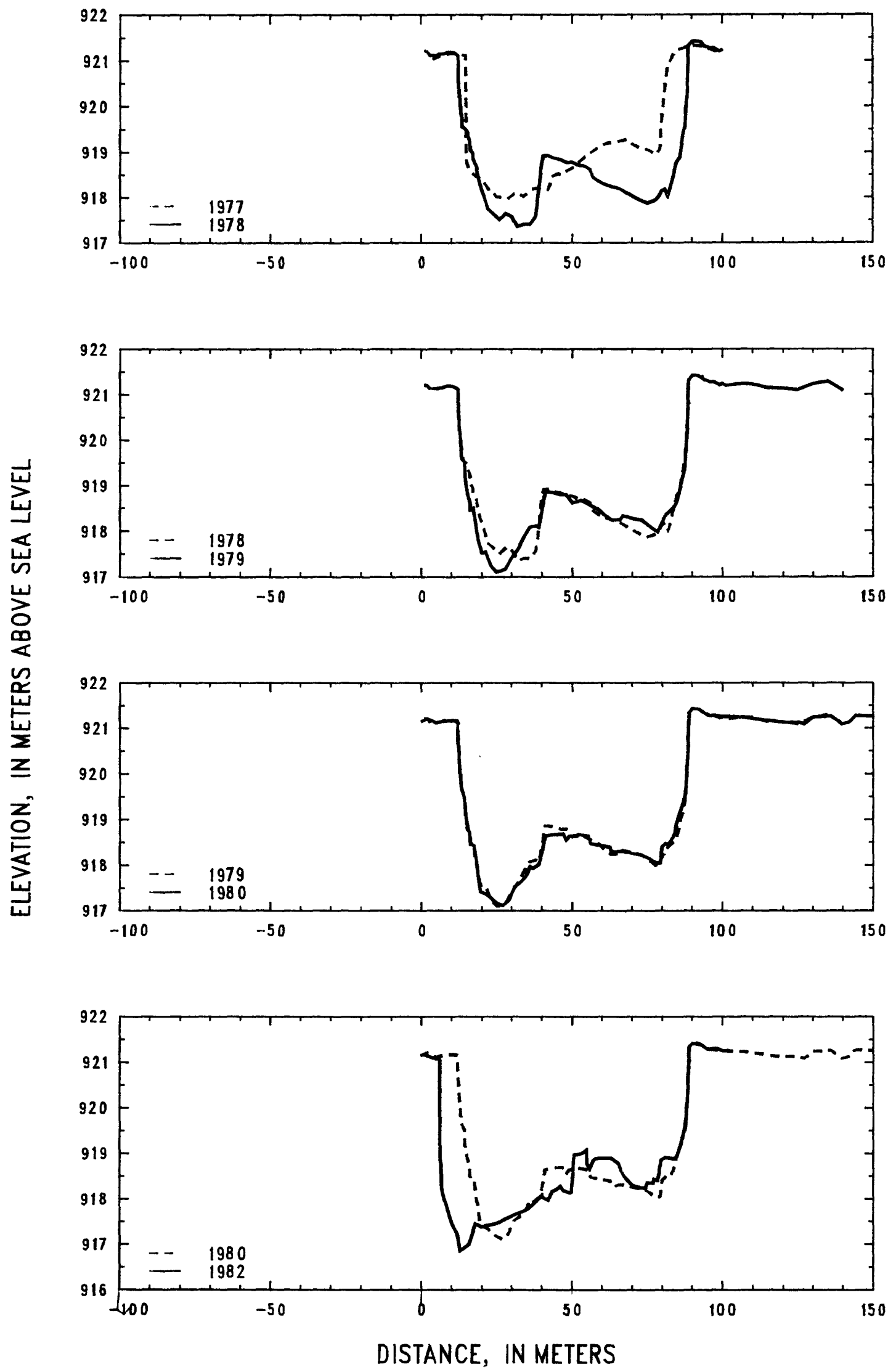

Figure 92.--Profiles of cross section PR206 from 1977 to 1982. 

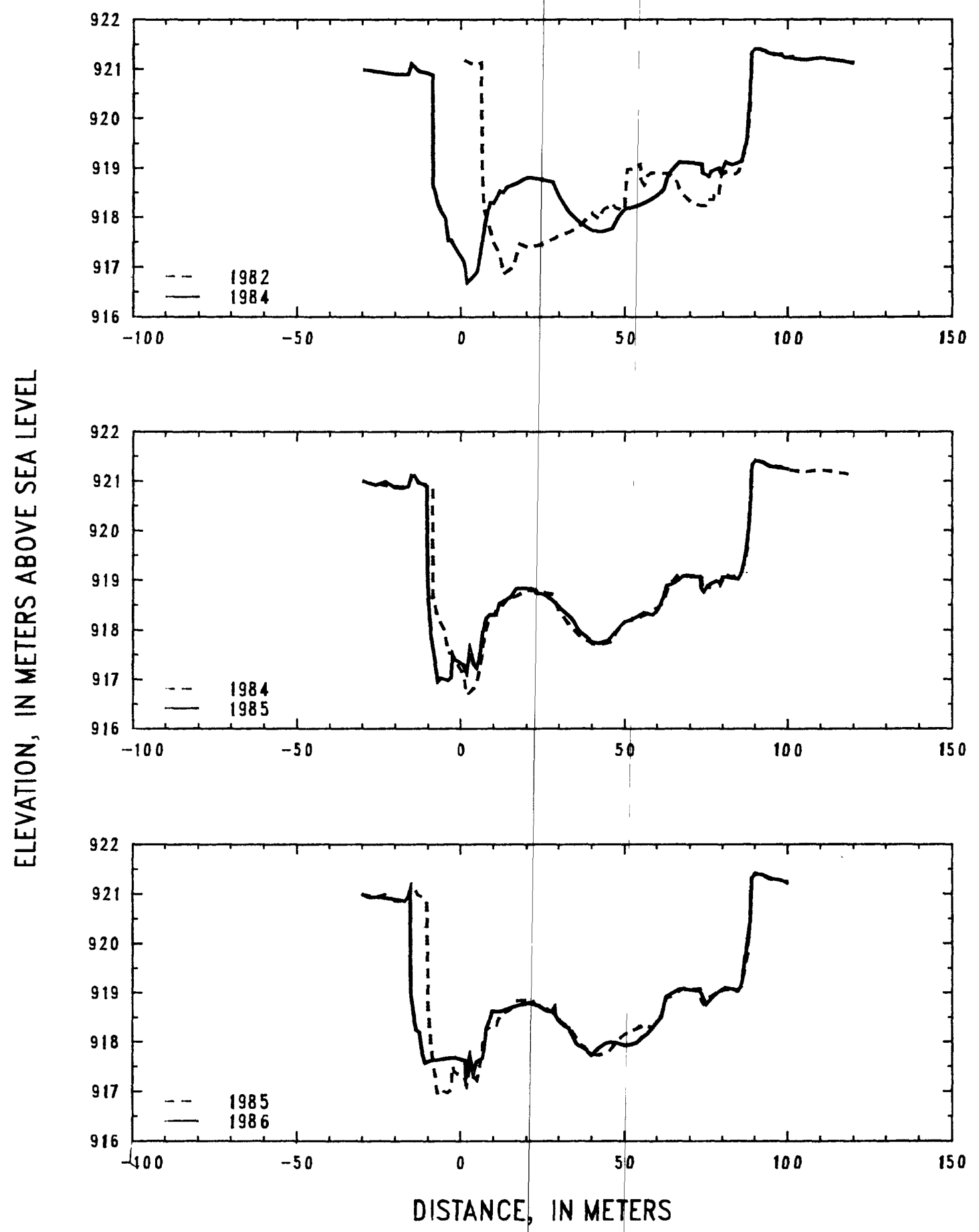

Figure 93.--Profiles of cross section PR206 from 1982 to 1986. 


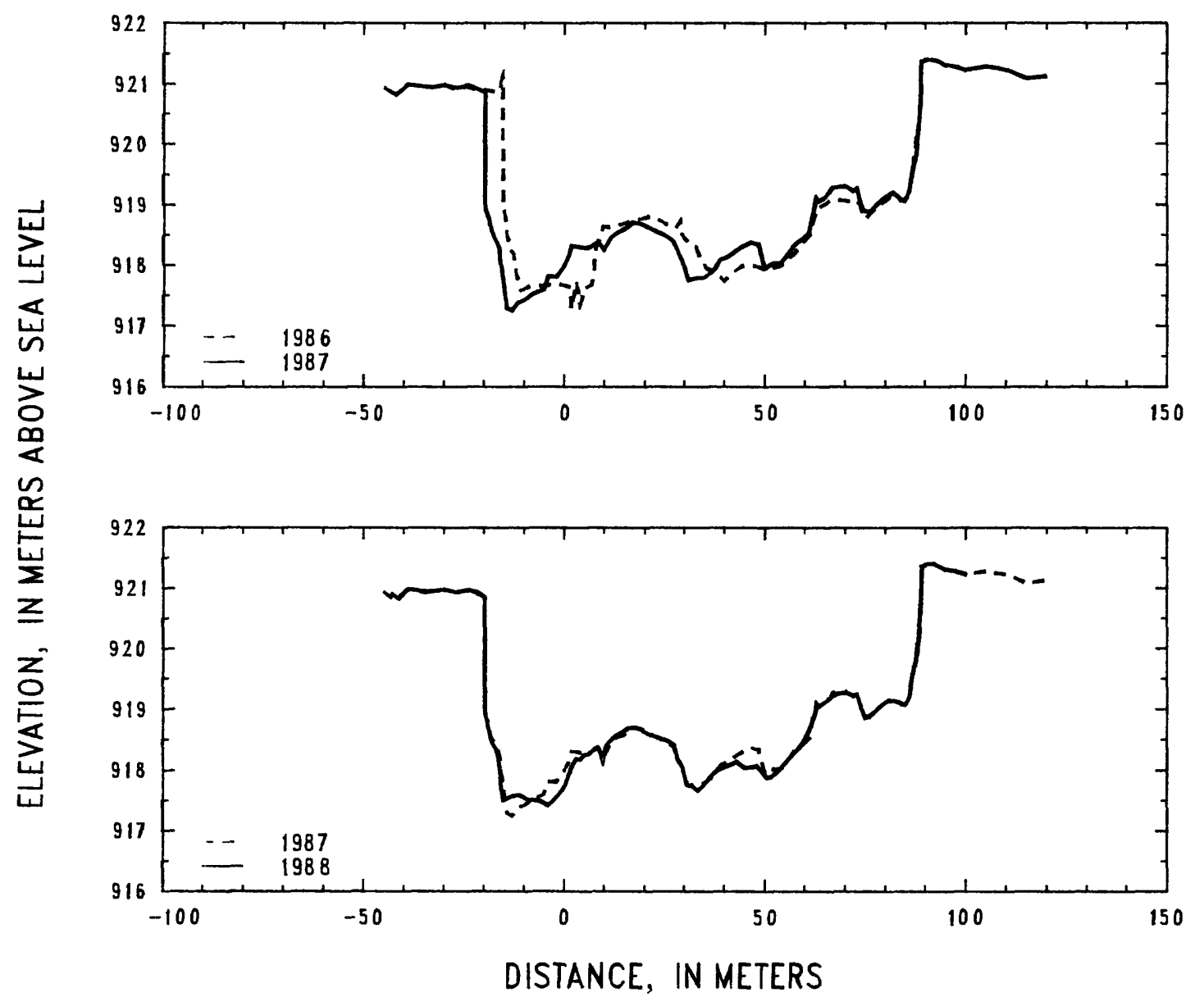

Figure 94.--Profiles of cross section PR206 from 1986 to 1988.

(followed by p. 249) 
Table 29.--Listing of horizontal stations and elevations for cross section PR206 [Sta., station, distance in meters from a reference point on the left bank; Elev., elevation, in meters above sea levell.

\begin{tabular}{|c|c|c|c|c|c|c|c|c|c|}
\hline \multicolumn{2}{|c|}{1977} & \multicolumn{2}{|c|}{1977} & \multicolumn{2}{|c|}{1978} & \multicolumn{2}{|c|}{1978} & \multicolumn{2}{|c|}{1979} \\
\hline Sta. & Elev. & Sta. & Elev. & Sta. & Elev. & Sta. & Elev. & Sta. & Elev. \\
\hline 4.0 & 921.04 & 80.0 & 919.77 & 1.0 & 921.23 & 42.0 & 918.92 & 1.0 & 921.21 \\
\hline 6.0 & 921.10 & 81.9 & 920.91 & 2.0 & 921.21 & 44.0 & 918.86 & 2.0 & 921.21 \\
\hline 8.0 & 921.14 & 84.0 & 921.16 & 3.0 & 921.12 & 46.0 & 918.84 & 3.0 & 921.13 \\
\hline 10.1 & 921.17 & 86.0 & 921.26 & 4.0 & 921.12 & 48.0 & 918.77 & 5.0 & 921.13 \\
\hline 12.0 & 921.16 & 88.0 & 921.30 & 5.0 & 921.11 & 50.0 & 918.77 & 7.0 & 921.15 \\
\hline 14.0 & 921.12 & 90.0 & 921.34 & 6.0 & 921.15 & 52.0 & 918.70 & 9.0 & 921.20 \\
\hline 14.8 & 921.12 & 94.0 & 921.32 & 7.0 & 921.15 & 54.0 & 918.69 & 11.0 & 921.17 \\
\hline 15.0 & 918.97 & 98.0 & 921.19 & 8.0 & 921.17 & 56.1 & 918.61 & 12.3 & 921.11 \\
\hline 15.2 & 918.75 & 100.0 & 921.14 & 9.0 & 921.20 & 57.0 & 918.45 & 12.4 & 920.58 \\
\hline 16.0 & 918.61 & & & 10.0 & 921.19 & 60.0 & 918.30 & 12.6 & 920.50 \\
\hline 18.0 & 918.47 & & & 11.0 & 921.18 & 63.0 & 918.24 & 12.7 & 920.29 \\
\hline 20.0 & 918.41 & & & 12.3 & 921.10 & 66.0 & 918.19 & 12.9 & 920.19 \\
\hline 22.5 & 918.16 & & & 12.4 & 920.53 & 69.0 & 918.07 & 13.0 & 919.99 \\
\hline 23.0 & 918.12 & & & 12.6 & 920.50 & 72.0 & 917.95 & 13.4 & 919.61 \\
\hline 24.0 & 918.10 & & & 12.7 & 920.22 & 75.0 & 917.87 & 14.0 & 919.59 \\
\hline 25.0 & 918.01 & & & 12.9 & 920.22 & 78.0 & 917.95 & 14.5 & 919.39 \\
\hline 27.0 & 918.00 & & & 13.0 & 919.99 & 79.0 & 918.02 & 14.7 & 919.11 \\
\hline 29.0 & 917.98 & & & 13.2 & 919.95 & 80.0 & 918.15 & 15.2 & 918.89 \\
\hline 31.0 & 918.10 & & & 13.3 & 919.82 & 81.0 & 918.18 & 15.4 & 918.79 \\
\hline 32.0 & 918.09 & & & 13.5 & 919.80 & 82.0 & 918.02 & 16.3 & 918.62 \\
\hline 34.0 & 918.01 & & & 13.6 & 919.55 & 83.7 & 918.45 & 16.4 & 918.46 \\
\hline 36.0 & 918.16 & & & 14.5 & 919.51 & 85.0 & 918.79 & 17.3 & 918.50 \\
\hline 38.0 & 918.21 & & & 15.5 & 919.44 & 86.0 & 918.88 & 18.0 & 918.12 \\
\hline 40.0 & 918.16 & & & 15.6 & 919.34 & 87.2 & 919.46 & 19.0 & 917.78 \\
\hline 42.0 & 918.16 & & & 16.2 & 919.23 & 87.7 & 919.54 & 20.0 & 917.53 \\
\hline 43.4 & 918.47 & & & 16.3 & 919.03 & 88.0 & 919.98 & 21.3 & 917.54 \\
\hline 45.0 & 918.52 & & & 17.1 & 918.97 & 88.1 & 920.02 & 23.0 & 917.23 \\
\hline 47.0 & 918.54 & & & 17.2 & 918.83 & 88.2 & 920.13 & 25.0 & 917.10 \\
\hline 48.0 & 918.58 & & & 18.4 & 918.68 & 88.4 & 920.19 & 26.0 & 917.11 \\
\hline 50.0 & 918.65 & & & 18.5 & 918.58 & 88.9 & 921.34 & 28.0 & 917.18 \\
\hline 52.0 & 918.76 & & & 19.0 & 918.40 & 90.0 & $921 .+2$ & 30.0 & 917.42 \\
\hline 54.0 & 918.88 & & & 20.0 & 918.17 & 91.0 & 921.43 & 32.0 & 917.59 \\
\hline 56.0 & 918.97 & & & 21.0 & 918.01 & 93.0 & 921.41 & 34.0 & 917.86 \\
\hline 58.0 & 919.07 & & & 22.0 & 917.74 & 94.0 & 921.32 & 36.0 & 918.09 \\
\hline 60.0 & 919.15 & & & 24.0 & 917.62 & 96.0 & 921.28 & 38.0 & 918.12 \\
\hline 62.0 & 919.20 & & & 26.0 & 917.51 & 97.0 & 921.29 & 39.0 & 918.08 \\
\hline 64.0 & 919.21 & & & 28.0 & 917.65 & 99.0 & 921.20 & 40.0 & 918.40 \\
\hline 66.0 & 919.24 & & & 30.0 & 917.58 & 100.0 & 921.24 & 41.0 & 918.86 \\
\hline 68.0 & 919.28 & & & 32.0 & 917.35 & & & 43.0 & 918.85 \\
\hline 70.0 & 919.17 & & & 34.0 & 917.40 & & & 46.0 & 918.79 \\
\hline 72.0 & 919.10 & & & 36.0 & 917.40 & & & 48.0 & 918.80 \\
\hline 74.0 & 919.07 & & & 38.0 & 917.58 & & & 50.0 & 918.61 \\
\hline 76.0 & 919.02 & & & 39.5 & 918.40 & & & 53.0 & 918.67 \\
\hline 78.0 & 918.93 & & & 40.0 & 918.75 & & & 56.0 & 918.56 \\
\hline 79.4 & 919.07 & & & 40.5 & 918.91 & & & 59.0 & 918.46 \\
\hline
\end{tabular}


Table 29.--Listing of horizontal stations and elevations for cross section PR206 --Continued.

\begin{tabular}{|c|c|c|c|c|c|c|c|c|c|}
\hline \multicolumn{2}{|c|}{1979} & \multicolumn{2}{|c|}{1980} & \multicolumn{2}{|c|}{1980} & \multicolumn{2}{|c|}{1982} & \multicolumn{2}{|c|}{1982} \\
\hline Sta. & Elev. & Sta. & Elev. & Sta. & Elev. & Sta. & Elev. & Sta. & Elev. \\
\hline 61.0 & 918.33 & 0.0 & 921.15 & 78.0 & 918.04 & 1.0 & 921.17 & 84.0 & 918.88 \\
\hline 63.0 & 918.23 & 2.0 & 921.21 & 79.4 & 918.04 & 3.0 & 921.12 & 85.0 & 918.94 \\
\hline 66.0 & 918.25 & 5.0 & 921.11 & 80.0 & 918.41 & 5.0 & 921.08 & 87.0 & 919.37 \\
\hline 67.0 & 918.34 & 8.0 & 921.17 & 81.0 & 918.47 & 6.3 & 921.13 & 88.0 & 919.78 \\
\hline 70.0 & 918.26 & 11.0 & 921.17 & 82.0 & 918.45 & 6.5 & 918.88 & 88.5 & 920.34 \\
\hline 73.0 & 918.23 & 12.0 & 921.15 & 83.0 & 918.55 & 6.8 & 918.74 & 89.0 & 921.34 \\
\hline 76.0 & 918.09 & 12.1 & 921.12 & 84.0 & 918.66 & 7.0 & 918.21 & 90.0 & 921.42 \\
\hline 78.5 & 917.97 & 12.3 & 920.62 & 85.0 & 918.95 & 10.0 & 917.50 & 93.0 & 921.38 \\
\hline 81.0 & 918.33 & 12.5 & 920.51 & 87.8 & 919.53 & 12.0 & 917.23 & 95.0 & 921.29 \\
\hline 84.0 & 918.53 & 12.6 & 920.29 & 88.5 & 920.35 & 13.0 & 916.86 & 98.0 & 921.31 \\
\hline 85.0 & 918.64 & 13.4 & 919.65 & 89.0 & 921.33 & 16.0 & 917.01 & 100.0 & 921.25 \\
\hline 87.4 & 919.33 & 14.3 & 919.52 & 90.0 & 921.43 & 18.0 & 917.46 & 101.8 & 921.26 \\
\hline 87.7 & 919.47 & 14.8 & 919.04 & 92.0 & 921.41 & 20.0 & 917.39 & & \\
\hline 88.5 & 920.35 & 15.6 & 918.83 & 93.0 & 921.38 & 25.0 & 917.46 & & \\
\hline 88.8 & 921.30 & 16.1 & 918.64 & 95.0 & 921.28 & 30.0 & 917.63 & & \\
\hline 90.0 & 921.43 & 16.3 & 918.45 & 97.0 & 921.27 & 35.0 & 917.76 & & \\
\hline 92.0 & 921.41 & 17.5 & 918.43 & 100.0 & 921.24 & 40.0 & 918.08 & & \\
\hline 94.0 & 921.32 & 19.6 & 917.42 & 105.0 & 921.24 & 40.0 & 918.05 & & \\
\hline 96.0 & 921.27 & 22.0 & 917.34 & 110.0 & 921.20 & 42.0 & 917.97 & & \\
\hline 97.0 & 921.27 & 24.5 & 917.18 & 120.0 & 921.12 & 43.5 & 918.17 & & \\
\hline 99.0 & 921.21 & 27.0 & 917.11 & 125.0 & 921.12 & 45.0 & 918.22 & & \\
\hline 100.0 & 921.24 & 29.0 & 917.23 & 127.0 & 921.08 & 46.0 & 918.29 & & \\
\hline 101.0 & 921.19 & 31.0 & 917.53 & 130.0 & 921.24 & 47.0 & 918.19 & & \\
\hline 105.0 & 921.24 & 33.0 & 917.62 & 136.0 & 921.26 & 49.5 & 918.13 & & \\
\hline 110.0 & 921.23 & 35.0 & 917.79 & 139.6 & 921.08 & 49.8 & 918.15 & & \\
\hline 115.0 & 921.14 & 36.0 & 917.97 & 142.0 & 921.13 & 50.3 & 918.56 & & \\
\hline 120.0 & 921.13 & 37.0 & 917.93 & 144.0 & 921.27 & 50.6 & 918.97 & & \\
\hline 125.0 & 921.09 & 39.0 & 918.02 & 150.0 & 921.25 & 53.0 & 919.00 & & \\
\hline 130.0 & 921.24 & 40.0 & 918.23 & & & 54.8 & 919.08 & & \\
\hline 135.0 & 921.29 & 41.0 & 918.64 & & & 55.0 & 918.77 & & \\
\hline 140.0 & 921.08 & 43.0 & 918.67 & & & 56.0 & 918.66 & & \\
\hline & & 47.0 & 918.69 & & & 57.0 & 918.85 & & \\
\hline & & 48.0 & 918.55 & & & 58.0 & 918.90 & & \\
\hline & & 49.0 & 918.64 & & & 63.0 & 918.90 & & \\
\hline & & 51.0 & 918.64 & & & 65.5 & 918.78 & & \\
\hline & & 52.0 & 918.68 & & & 67.0 & 918.53 & & \\
\hline & & 55.3 & 918.64 & & & 70.0 & 918.30 & & \\
\hline & & 56.2 & 918.47 & & & 73.0 & 918.24 & & \\
\hline & & 60.0 & 918.42 & & & 74.5 & 918.24 & & \\
\hline & & 62.7 & 918.39 & & & 75.0 & 918.36 & & \\
\hline & & 63.2 & 918.29 & & & 77.0 & 918.36 & & \\
\hline & & 66.8 & 918.31 & & & 77.2 & 918.44 & & \\
\hline & & 70.0 & 918.25 & & & 78.5 & 918.40 & & \\
\hline & & 73.0 & 918.22 & & & 79.7 & 918.86 & & \\
\hline & & 75.0 & 918.17 & & & 81.0 & 918.92 & & \\
\hline
\end{tabular}


Table 29.--Listing. of horizontal stations and elevations for cross section PR206 --Continued.

\begin{tabular}{|c|c|c|c|c|c|c|c|c|c|}
\hline \multicolumn{2}{|c|}{1986} & \multicolumn{2}{|c|}{1987} & \multicolumn{2}{|c|}{1987} & \multicolumn{2}{|c|}{1988} & \multicolumn{2}{|c|}{1988} \\
\hline Sta. & Elev. & Sta. & Elev. & Sta. & Elev. & Sta. & Elev. & Sta. & Elev. \\
\hline 40.0 & 917.72 & -45.0 & 920.93 & 37.0 & 917.89 & -43.0 & 920.91 & 30.8 & 917.75 \\
\hline 42.0 & 917.87 & -42.0 & 920.81 & 39.1 & 918.08 & -41.0 & 920.82 & 32.0 & 917.73 \\
\hline 44.0 & 917.97 & -39.0 & 920.99 & 41.0 & 918.13 & -39.0 & 920.98 & 33.5 & 917.66 \\
\hline 46.0 & 917.99 & -36.0 & 920.96 & 43.0 & 918.25 & -37.0 & 920.98 & 35.0 & 917.75 \\
\hline 48.0 & 917.97 & -33.0 & 920.94 & 45.0 & 918.32 & -35.0 & 920.93 & 37.0 & 917.93 \\
\hline 50.0 & 917.92 & -30.0 & 920.98 & 46.5 & 918.37 & -32.0 & 920.96 & 39.0 & 918.03 \\
\hline 52.0 & 917.94 & -27.0 & 920.93 & 48.5 & 918.33 & -30.0 & 920.98 & 41.0 & 918.07 \\
\hline 54.0 & 917.99 & -24.0 & 920.98 & 50.0 & 917.94 & -27.0 & 920.93 & 43.0 & 918.14 \\
\hline 55.3 & 918.08 & -22.0 & 920.91 & 52.0 & 918.02 & -24.0 & 920.97 & 45.0 & 918.03 \\
\hline 57.0 & 918.15 & -19.7 & 920.85 & 54.0 & 918.02 & -21.0 & 920.91 & 47.0 & 918.05 \\
\hline 59.0 & 918.31 & -19.7 & 918.92 & 56.0 & 918.19 & -19.7 & 920.83 & 48.0 & 918.07 \\
\hline 61.3 & 918.51 & -19.0 & 918.80 & 57.5 & 918.33 & -19.7 & 918.97 & 49.0 & 917.99 \\
\hline 62.8 & 918.92 & -18.0 & 918.53 & 59.0 & 918.40 & -18.0 & 918.46 & 50.5 & 917.87 \\
\hline 65.0 & 919.00 & -16.1 & 918.27 & 61.0 & 918.52 & -17.0 & 918.35 & 51.6 & 917.89 \\
\hline 67.0 & 919.08 & -15.9 & 918.12 & 62.9 & 919.11 & -16.0 & 918.15 & 53.0 & 917.96 \\
\hline 69.0 & 919.07 & -14.2 & 917.28 & 63.5 & 919.05 & -15.6 & 917.72 & 55.0 & 918.11 \\
\hline 72.0 & 919.04 & -13.0 & 917.24 & 65.0 & 919.11 & -15.0 & 917.50 & 57.0 & 918.27 \\
\hline 73.1 & 919.04 & -11.5 & 917.38 & 67.0 & 919.27 & -13.0 & 917.57 & 59.0 & 918.39 \\
\hline 75.0 & 918.75 & -10.0 & 917.42 & 70.0 & 919.30 & -11.0 & 917.59 & 61.0 & 918.63 \\
\hline 76.0 & 918.84 & -8.0 & 917.51 & 72.0 & 919.21 & -9.0 & 917.51 & 62.5 & 918.91 \\
\hline 78.0 & 918.97 & -5.0 & 917.60 & 73.0 & 919.26 & -6.0 & 917.49 & 63.0 & 919.07 \\
\hline 81.0 & 919.10 & -4.0 & 917.81 & 74.4 & 918.89 & -4.0 & 917.41 & 63.3 & 919.04 \\
\hline 83.0 & 919.07 & -2.0 & 917.81 & 76.0 & 918.87 & -2.0 & 917.54 & 65.0 & 919.13 \\
\hline 85.0 & 919.04 & 0.0 & 918.00 & 78.0 & 919.00 & 0.0 & 917.72 & 68.0 & 919.26 \\
\hline 86.0 & 919.17 & 1.0 & 918.12 & 80.0 & 919.12 & 2.0 & 918.07 & 70.0 & 919.27 \\
\hline 88.5 & 920.42 & 1.8 & 918.31 & 82.0 & 919.19 & 3.0 & 918.18 & 72.0 & 919.23 \\
\hline 89.1 & 921.33 & 4.0 & 918.28 & 84.0 & 919.07 & 4.0 & 918.17 & 73.0 & 919.24 \\
\hline 90.0 & 921.40 & 6.0 & 918.27 & 85.0 & 919.07 & 5.0 & 918.25 & 74.0 & 919.03 \\
\hline 92.0 & 921.40 & 8.5 & 918.37 & 86.0 & 919.18 & 6.0 & 918.26 & 75.0 & 918.86 \\
\hline 95.0 & 921.30 & 10.0 & 918.24 & 87.0 & 919.62 & 8.0 & 918.36 & 77.0 & 918.93 \\
\hline 98.3 & 921.27 & 11.6 & 918.45 & 87.6 & 919.77 & 8.8 & 918.34 & 79.0 & 919.07 \\
\hline \multirow[t]{14}{*}{100.0} & 921.21 & 13.0 & 918.53 & 88.8 & 920.60 & 9.7 & 918.14 & 81.0 & 919.16 \\
\hline & & 15.0 & 918.59 & 89.1 & 921.37 & 10.3 & 918.36 & 83.0 & 919.13 \\
\hline & & 17.0 & 918.70 & 91.0 & 921.40 & 11.0 & 918.44 & 85.0 & 919.07 \\
\hline & & 19.0 & 918.68 & 93.0 & 921.37 & 13.0 & 918.57 & 86.0 & 919.19 \\
\hline & & 21.0 & 918.60 & 95.0 & 921.30 & 14.0 & 918.60 & 86.9 & 919.58 \\
\hline & & 23.0 & 918.54 & 97.0 & 921.29 & 16.0 & 918.69 & 88.0 & 919.92 \\
\hline & & 25.0 & 918.48 & 100.0 & 921.22 & 18.0 & 918.71 & 88.8 & 920.60 \\
\hline & & 27.0 & 918.39 & 105.0 & 921.29 & 20.0 & 918.62 & 89.2 & 921.37 \\
\hline & & 28.0 & 918.26 & 110.0 & 921.23 & 22.0 & 918.56 & 90.0 & 921.40 \\
\hline & & 29.0 & 918.11 & 115.0 & 921.08 & 24.0 & 918.51 & 92.0 & 921.41 \\
\hline & & 29.4 & 918.06 & 120.0 & 921.12 & 26.0 & 918.47 & 94.0 & 921.32 \\
\hline & & 31.0 & 917.74 & & & 27.4 & 918.41 & 96.0 & 921.30 \\
\hline & & 33.0 & 917.78 & & & 28.2 & 918.17 & 98.0 & 921.25 \\
\hline & & 35.0 & 917.79 & & & 29.5 & 918.04 & 100.0 & 921.22 \\
\hline
\end{tabular}


Table 29.--Listing of horizontal stations and elevations for cross section PR206 --Continued.

\begin{tabular}{|c|c|c|c|c|c|c|c|c|c|}
\hline \multicolumn{2}{|c|}{1984} & \multicolumn{2}{|c|}{1984} & \multicolumn{2}{|c|}{1985} & \multicolumn{2}{|c|}{1985} & \multicolumn{2}{|c|}{1986} \\
\hline Sta. & $\overline{\text { Elev. }}$ & Sta. & Elev. & Sta. & Elev. & Sta. & Elev. & Sta. & Elev. \\
\hline-30.0 & 920.98 & 55.0 & 918.26 & -30.0 & 921.00 & 48.0 & 918.03 & -30.0 & 920.99 \\
\hline-25.0 & 920.94 & 58.0 & 918.36 & -26.0 & 920.92 & 50.0 & 918.16 & -28.0 & 920.92 \\
\hline-20.0 & 920.88 & 60.0 & 918.44 & -23.0 & 920.99 & 53.0 & 918.24 & -25.0 & 920.94 \\
\hline-18.0 & 920.88 & 62.0 & 918.57 & -20.0 & 920.86 & 56.0 & 918.34 & -22.0 & 920.90 \\
\hline-16.0 & 920.90 & 63.5 & 918.89 & -18.0 & 920.86 & 58.7 & 918.29 & -19.0 & 920.88 \\
\hline-15.0 & 921.11 & 66.6 & 919.12 & -16.0 & 920.89 & 60.0 & 918.39 & -17.0 & 920.85 \\
\hline-13.0 & 920.96 & 69.5 & 919.10 & -15.0 & 921.10 & 61.5 & 918.58 & -16.0 & 920.92 \\
\hline-10.0 & 920.91 & 73.5 & 919.07 & -14.0 & 921.12 & 63.0 & 918.90 & -15.3 & 921.10 \\
\hline-8.7 & 920.87 & 73.7 & 918.89 & -13.0 & 920.98 & 65.0 & 918.94 & -15.1 & 919.02 \\
\hline-8.5 & 918.61 & 75.7 & 918.82 & -10.3 & 920.89 & 68.0 & 919.09 & -14.0 & 918.42 \\
\hline-8.0 & 918.55 & 76.3 & 918.93 & -9.9 & 918.75 & 71.0 & 919.07 & -13.5 & 918.24 \\
\hline-7.0 & 918.24 & 78.7 & 918.99 & -9.0 & 917.88 & 73.2 & 919.08 & -12.5 & 918.19 \\
\hline-6.0 & 918.11 & 79.3 & 918.89 & -8.0 & 917.43 & 73.4 & 918.84 & -12.0 & 917.90 \\
\hline-4.8 & 917.98 & 81.0 & 919.12 & -7.0 & 916.95 & 74.2 & 918.76 & -11.7 & 917.80 \\
\hline-4.0 & 917.53 & 83.0 & 919.06 & -6.0 & 917.03 & 75.3 & 918.89 & -10.8 & 917.57 \\
\hline-3.0 & 917.53 & 86.0 & 919.14 & -4.0 & 916.97 & 78.0 & 918.99 & -9.0 & 917.63 \\
\hline-1.0 & 917.30 & 87.5 & 919.57 & -3.0 & 917.04 & 79.2 & 918.94 & -7.0 & 917.64 \\
\hline 0.0 & 917.19 & 88.6 & 920.60 & -2.5 & 917.50 & 80.0 & 919.07 & -4.0 & 917.67 \\
\hline 1.0 & 917.08 & 89.0 & 921.33 & -2.0 & 917.40 & 82.0 & 919.06 & -1.0 & 917.68 \\
\hline 2.0 & 916.68 & 90.0 & 921.41 & 0.0 & 917.32 & 85.0 & 919.02 & 0.0 & 917.65 \\
\hline 4.0 & 916.81 & 92.0 & 921.40 & 1.0 & 917.28 & 86.0 & 919.15 & 1.5 & 917.62 \\
\hline 5.0 & 916.91 & 94.0 & 921.32 & 2.0 & 917.12 & 87.6 & 919.71 & 1.8 & 917.27 \\
\hline 6.0 & 917.24 & 97.0 & 921.29 & 3.0 & 917.62 & 88.6 & 920.48 & 3.0 & 917.72 \\
\hline 7.0 & 917.66 & 98.0 & 921.28 & 4.0 & 917.30 & 89.0 & 921.32 & 4.0 & 917.34 \\
\hline 7.8 & 917.99 & 99.0 & 921.23 & 5.0 & 917.22 & 90.0 & 921.42 & 5.0 & 917.60 \\
\hline 9.0 & 918.29 & 100.0 & 921.23 & 6.0 & 917.50 & 92.0 & 921.40 & 6.9 & 917.67 \\
\hline 10.0 & 918.28 & 105.0 & 921.18 & 7.0 & 917.98 & 94.0 & 921.31 & 7.4 & 917.89 \\
\hline 12.0 & 918.53 & 110.0 & 921.23 & 8.0 & 918.24 & 97.0 & 921.27 & 8.0 & 918.27 \\
\hline 13.0 & 918.50 & 117.0 & 921.15 & 9.0 & 918.31 & 100.0 & 921.24 & 8.6 & 918.37 \\
\hline 14.0 & 918.61 & 120.0 & 921.11 & 11.0 & 918.31 & & & 9.7 & 918.63 \\
\hline 17.0 & 918.68 & & & 12.0 & 918.54 & & & 12.0 & 918.61 \\
\hline 20.0 & 918.80 & & & 15.0 & 918.65 & & & 15.0 & 918.69 \\
\hline 22.0 & 918.80 & & & 17.0 & 918.83 & & & 18.0 & 918.75 \\
\hline 25.0 & 918.76 & & & 20.0 & 918.84 & & & 21.0 & 918.80 \\
\hline 28.1 & 918.70 & & & 22.0 & 918.83 & & & 24.0 & 918.72 \\
\hline 30.0 & 918.40 & & & 23.0 & 918.74 & & & 26.0 & 918.63 \\
\hline 33.0 & 918.10 & & & 25.0 & 918.72 & & & 27.7 & 918.60 \\
\hline 36.0 & 917.90 & & & 28.0 & 918.60 & & & 28.9 & 918.72 \\
\hline 39.0 & 917.75 & & & 31.0 & 918.40 & & & 29.0 & 918.53 \\
\hline 40.0 & 917.71 & & & 34.0 & 918.22 & & & 30.0 & 918.43 \\
\hline 43.0 & 917.70 & & & 36.0 & 917.98 & & & 32.0 & 918.31 \\
\hline 46.0 & 917.76 & & & 38.0 & 917.87 & & & 33.1 & 918.28 \\
\hline 48.0 & 918.01 & & & 40.0 & 917.75 & & & 33.5 & 918.19 \\
\hline 50.0 & 918.16 & & & 42.0 & 917.72 & & & 35.5 & 917.94 \\
\hline 52.0 & 918.20 & & & 45.0 & 917.79 & & & 38.0 & 917.85 \\
\hline
\end{tabular}

